LIBRARY OF THE

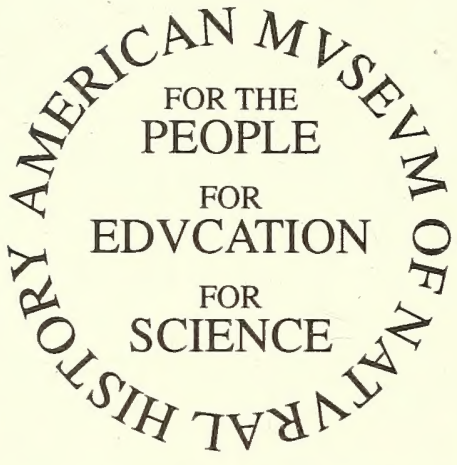




\title{
Anatomy of the Woodchuck (Marmota monax)
}

\author{
by
}

A. J. Bezuidenhout and H. E. Evans

SPECIAL PUBLICATION NO. 13

American Society of Mammalogists

Published 21 February 2005 
Price $\$ 45.00$ includes postage and handling.

American Society of Mammalogists

P.O. Box 7060

Lawrence, KS 66044-1897

ISBN: 1-891276-43-3

Library of Congress Control Number: 2005921107

Printed at Allen Press, Inc., Lawrence, Kansas 66044

Issued: 21 February 2005

Copyright (C) by the American Society of Mammalogists 2005 


\title{
SPECIAL PUBLICATIONS
}

\section{AMERICAN SOCIETY OF MAMMALOGISTS}

This series, published by the American Society of Mammalogists in association with Allen Press, Inc., has been established for peer-reviewed papers of monographic scope concerned with any aspect of the biology of mammals.

Copies of Special Publications by the Society may be ordered from: American Society of Mammalogists, \% Allen Marketing and Management, P.O. Box 7060, Lawrence, KS 66044-8897, or at www. mammalogy.org.

\author{
Dr. Joseph F. Merritt \\ Editor for Special Publications \\ Department of Biology \\ United States Air Force Academy \\ 2355 Faculty Drive \\ US Air Force Academy, CO 80840
}

\author{
Dr. David M. Leslie, Jr. \\ Chair, ASM Publications Committee \\ Oklahoma Cooperative Fish and Wildlife Research Unit \\ United States Geological Survey \\ 404 Life Sciences West \\ Oklahoma State University \\ Stillwater, OK 74078-3051
}





\section{ANATOMY OF THE WOODCHUCK}

(MARMOTA MONAX)

A. J. Bezuidenhout and H. E. Evans

Published by the American Society of Mammalogists

\section{Contents}

Acknowledgments . . . . . . . . . . . . . . . . . . . . . . .

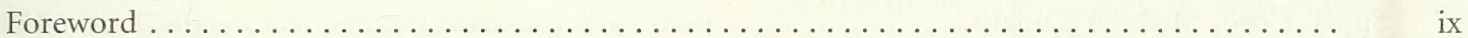

Chapter 1. Introduction

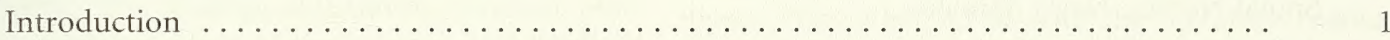

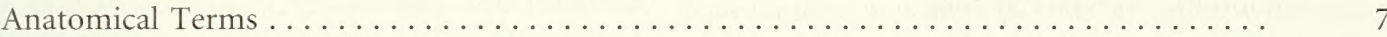

Integument $\ldots \ldots \ldots \ldots \ldots \ldots \ldots \ldots \ldots \ldots \ldots \ldots \ldots \ldots \ldots \ldots \ldots \ldots \ldots \ldots \ldots \ldots \ldots \ldots \ldots \ldots \ldots$

Chapter 2. Skeleton

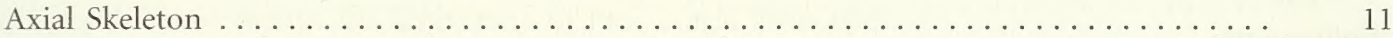

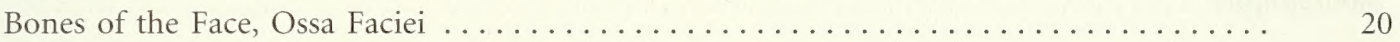

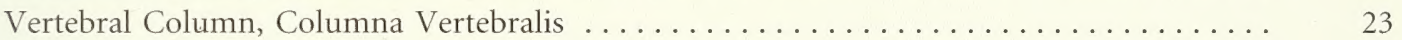

Sternum . . . . . . . . . . . . . . . . . . . . . . . . . . . . . . . 26

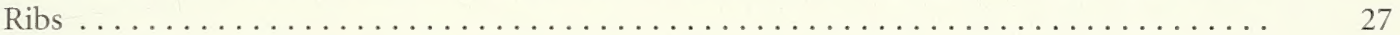

Appendicular Skeleton . . . . . . . . . . . . . . . . . . . . . . . 27

Chapter 3. Joints and Ligaments

Skull . . . . . . . . . . . . . . . . . . . . . . . . . . . . . . . . 39

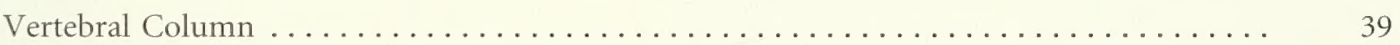

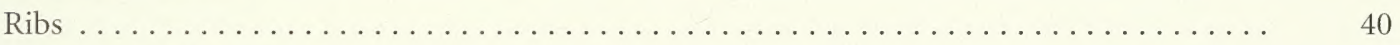

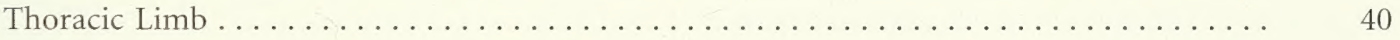

Pelvic Limb . . . . . . . . . . . . . . . . . . . . . . . . . . . . . . . . . . 42

Chapter 4. Muscular System

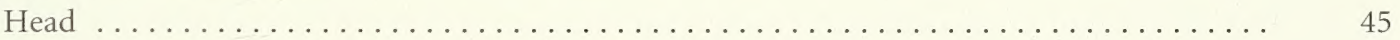

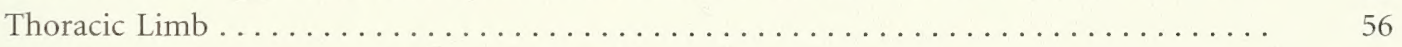

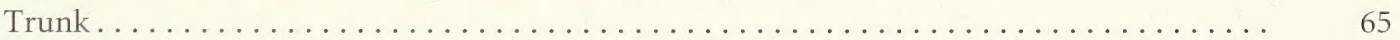

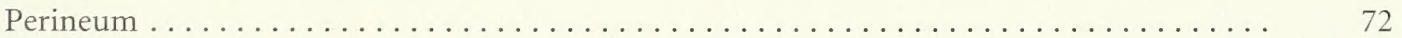

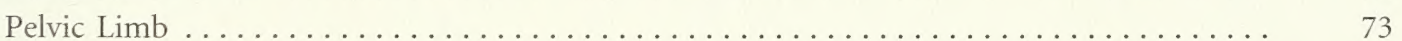

Chapter 5. Digestive System

Mouth and Associated Structures $\ldots \ldots \ldots \ldots \ldots \ldots \ldots \ldots \ldots \ldots \ldots \ldots \ldots \ldots \ldots$

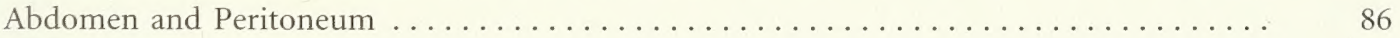

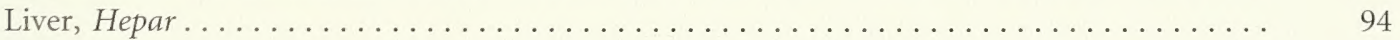

Chapter 6. Respiratory System

Nose, Nasal Cavity and Paranasal Sinuses $\ldots \ldots \ldots \ldots \ldots \ldots \ldots \ldots \ldots \ldots \ldots \ldots$

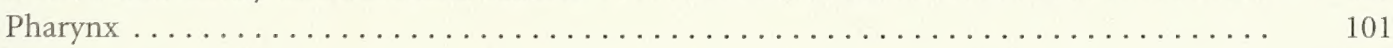

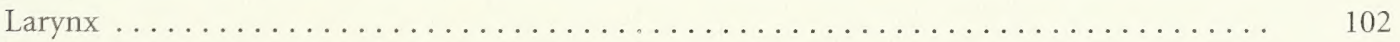

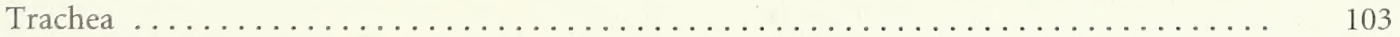

Thoracic Cavity and Pleurae ............................... 104

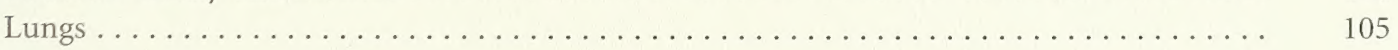

Chapter 7. Urogenital Apparatus

Urogenital Apparatus 


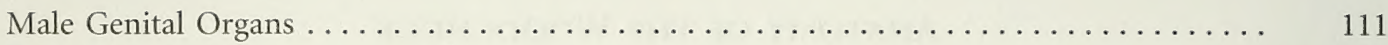

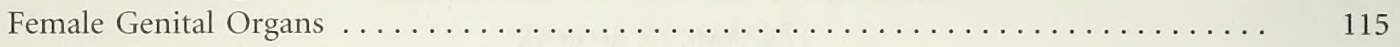

Chapter 8. Endocrine Organs

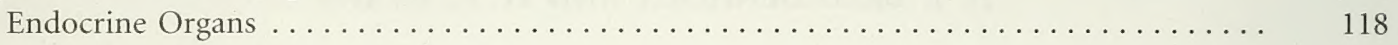

Chapter 9. Cardiovascular System

Heart and Pericardium . . . . . . . . . . . . . . . . . . . . . . . . . 119

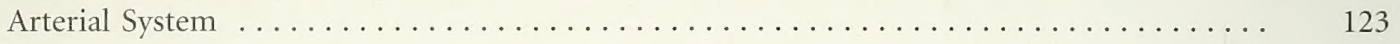

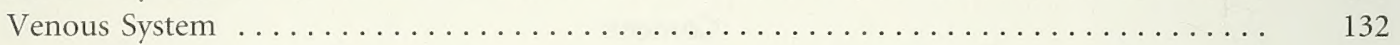

Lymphatic System . . . . . . . . . . . . . . 141

Chapter 10. Central Nervous System

Meninges . . . . . . . . . . . . . 145

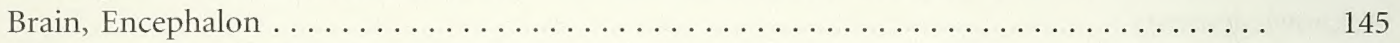

Spinal Cord, Medulla Spinalis. . . . . . . . . . . . . . . . . . . . . 153

Peripheral Nervous System, Cranial Nerves . . . . . . . . . . . . . . . . . . . 154

Spinal Nerves, Nervi Spinales . . . . . . . . . . . . . . . . . . . . . . . 161

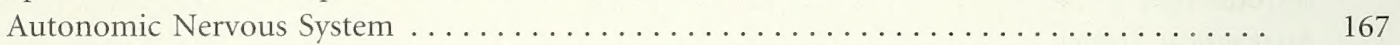

Chapter 11. Sensory Organs

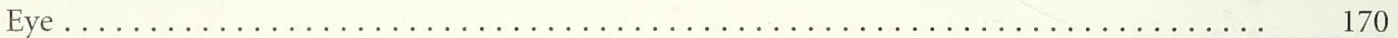

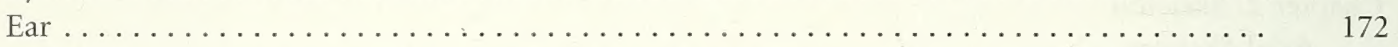

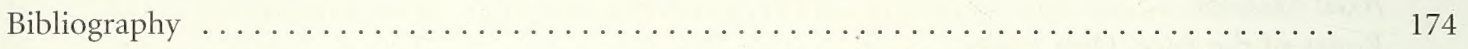




\section{Acknowledgments}

The authors thank Dr. Bud Tennant of the College of Veterinary Medicine at Cornell University for his vision to initiate this study of woodchuck anatomy and for his financial support. His laboratory supplied the skeletal material as well as the animals that were dissected and illustrated. Without his continued support and encouragement, it would not have possible to complete the work.

We thank the Department of Biomedical Sciences in the College of Veterinary Medicine at Cornell University for providing space and facilities to do the work. The College illustrator, Michael Simmons, prepared some of the artwork and finalized the numbering on all illustrations. The library and computer facilities at Cornell University aided us greatly.

Dr. Bezuidenhout thanks the University of Pre- toria, South Africa, for granting him sabbatical leave to work on the project and providing their biomedical illustrator, Christine Seegers, who drew most of the illustrations.

It was a pleasure to have the encouragement and advice of Virginia Hayssen, Joseph Merritt, and David Leslie, Jr. of the Publications Committee of the American Society of Mammalogists. We also thank the hard-working reviewers, Stuart O. Landry, Jr. and Richard W. Thorington, Jr., who caught many errors and suggested improvements. Ken Armitage of Lawrence, Kansas and Raymond Ramousse of Lyon, France helped us with the literature. Vadim Rumiantsev of Russia put us in touch with many of his colleagues within and outside the former Soviet Union. A sizeable monetary subvention for publication was provided by Marmotech, Inc., Ithaca, New York. 



\section{Foreword}

The woodchuck (Marmota monax) or, as it is known in the southern United States, the ground hog is 1 of 14 species of marmots (Rodentia: Sciuridae), all of which are found in the Northern Hemisphere. In North America, the woodchuck ranges from north Georgia to the maritime provinces of Canada, west to Oklahoma, Missouri, and Iowa, across Canada to the Yukon Territory and to southeastern Alaska. Other marmot species live in colonies and often at high elevations. The woodchuck, however, is a solitary species, the largest among marmots, and lives at low elevations.

For a century, the woodchuck has been the subject of a diverse series of studies conducted by faculty at Cornell University. These included some of the earliest investigations of the physiology of hibernation in which the possible role of brown fat was recognized. The wide-ranging field studies of Prof. William Hamilton culminated in the publication of his classic monograph on the natural history of the woodchuck. In more recent years, the woodchuck has been used in studies of the pathogenesis of viral hepatitis and primary liver cancer (hepatocellular carcinoma). The comprehensive study of woodchuck anatomy described in this volume by my colleagues Dr. Abraham J. Bezuidenhout and Prof. Howard E. Evans, 2 of the world's most distinguished and productive anatomists, was a critical part of the development of the woodchuck as a laboratory animal model for biomedical research.

In 1978, I received a telephone call from Dr. Norman Javitt, then Chief of Gastroenterology at Cornell Medical College, asking me what I knew about woodchucks. I explained that my knowledge at the time was limited to the well recognized role of the woodchuck in decimating vegetable gardens and to the alleged role of woodchuck burrows in the etiology of long bone fractures of livestock. Dr. Javitt then told me of a recent report by Dr. Jesse Summers and colleagues from the Fox Chase Cancer Center describing a virus isolated from woodchucks that appeared to be closely related to the hepatitis B virus (HBV) of humans. The woodchucks described by Dr. Summers and associates had been trapped originally in the native habitat of Pennsylvania and maintained and studied in an experimental colony at the Philadelphia Zoological Garden by Dr. Robert Snyder. Prior to the virological studies, woodchucks from the colony had experienced high rates of chronic hepatitis and hepatocellular carcinoma, complications known to be associated with $\mathrm{HBV}$ infection in humans. With Dr. Javitt's encouragement, the following spring we initiated studies of woodchucks trapped in Tompkins County, New York.

It was recognized from the beginning that to use the woodchuck as an experimental animal model for studies of the pathogenesis of viral hepatitis, a breeding colony would be required to produce pathogen-free experimental animals. A colony of WHV-free woodchucks was established at Cornell in 1979. Subsequently, support for the colony was obtained from the National Institute of Allergy and Infectious Diseases (NIAID) and the National Cancer Institute (NCI). The initial program officers from those institutes were Dr. Frank Tyeryar (NIAID) and Dr. John Cole (NCI). The successful production of laboratory-born and reared woodchucks led to collaborative experimental studies with Dr. John L. Gerin and colleagues from the Division of Molecular Virology and Immunology, Georgetown University School of Medicine, Dr. Robert H. Purcell and associates from the Hepatitis Viruses Section, Laboratory of Infectious Diseases, NIAID, and the late Dr. Hans Popper of the Mt. Sinai School of Medicine. The woodchuck now is recognized as a valuable animal model for $\mathrm{HBV}$ and liver cancer research and for preclinical evaluation of new strategies for the treatment and prevention of these important human diseases. The model is now used widely throughout the United States, Canada, Europe, and Asia.

Since discovery of WHV, members of the hepatitis B family of viruses (Hepadnaviridae) have been identified in California ground squirrels (Spermophilus beecheyi), Arctic ground squirrels (S. parryii), and Richardson's ground squirrels (S. richardsonii). In all of these species, viral infection has been associated with chronic hepatitis and hepatocellular carcinoma. Other related members of the family Sciuridae also are known to have similar liver diseases (e.g., black-tailed prairie dog, Cynomys ludovicianus) but have not yet been shown to be infected with a hepadnavirus.

Significant variations in the prevalence of $\mathrm{HBV}$ infection are recognized in different parts of the world. Very high rates of $\mathrm{HBV}$ infection and primary liver cancer exist in Sub-Saharan Africa and Asia, while the respective frequencies are low in North America and Western Europe. Interestingly, woodchuck hepatitis virus (WHV) infection is hyperendemic in the mid-Atlantic states where rates of infection as high as 59\% have been reported and 
where one-half of the infected woodchucks are chronic carriers. In Tompkins County, New York, the infection rate is about $1 \%$ and the carrier rate is $<0.1 \%$. In the other parts of the United States and Canada so far studied, WHV infection rates are either similarly low or no serologic evidence of infection has been detected. Similarly, the California ground squirrel hepatitis virus has been detected only in squirrels from a very small area on and near the campus of Stanford University.

This volume represents a quantum advance in the anatomical information currently available on the woodchuck and will be of significant value to those using this species in biomedical research. The text also should be useful to the far broader group of biologists that conduct research not only on the natural history of the woodchuck but on other species of marmots and ground squirrels. Utilizing modern methods, the phylogenetic relationships within the genus Marmota and between marmots and Spermophilus have now been established at the molecular genetic level. It seems reasonably certain that research collaboration between the 2 groups of scientists that will benefit most from this book could provide valuable and unique insight into the evolution of hepadnavirus infection in the family Sciuridae. It is hoped this volume, a direct consequence of the use of the woodchuck in biomedical research, will be used as a reference to support continued research on a global basis and to be a bridge between the biomedical research community and those studying ecology and evolution of these interesting and valuable members of our world.

The authors are to be congratulated for the sustained effort and high level of scholarship over the past years that have resulted in the completion of the work in this volume. Special thanks must be given to the American Society of Mammalogists that recognized the scientific value of the manuscript, provided editorial review, and sponsored publication of the book. Finally, Marmotech Inc. is acknowledged for providing the grant-in-aid that made publication possible.

Bud C. Tennant, DVM James Law Professor of Comparative Medicine

Gastrointestinal Unit Department of Clinical Sciences Cornell College of Veterinary Medicine Ithaca, NY 14853

\section{References}

Hamilton, W. J., Jr. 1934. The life history of the rufescent woodchuck, Marmota monax rufescens Howell. Annals of the Carnegie Museum 23:85-178.

Kruckenhauser, L., W. Pinsker, E. Haring, and W. Arnold. 1999. Marmot phylogeny revisited: molecular evidence for a diphyletic origin of sociality. Journal of Zoological Systematics and Evolutionary Research 37:49-56.

Popper, H., L. Roth, R. H. Purcell, B. C. Tennant, and J. L. Gerin. 1987. Hepatocarcinogenicity of the woodchuck hepatitis virus. Proceedings of the National Academy of Science 84:866-870.

Steppan, S. J., et al. 1999. Molecular phylogeny of the marmots (Rodentia: Sciuridae): tests of evolutionary and biogeographic hypotheses. Systematic Biology 48:715-734.

Steppan, S.J., B. L. Storz, and R. S. Hoffmann. 2004. Nuclear DNA phylogeny of the squirrels (Mammalia: Rodentia) and the evolution of arboreality from c-myc and RAG1. Molecular Phylogenetics and Evolution 30:703-719.

Summers, J., J. M. Smolec, and R. A. Snyder. 1978. A virus similar to human hepatitis B virus associated with hepatitis and hepatoma in woodchucks. Proceedings of the National Academy of Science 75:4533-4537.

Tennant, B. C., et al. 2004. Hepatocellular carcinoma in the woodchuck model of hepatitis B virus infection. Gastroenterology 127, Supplement 1:S283-S293. 


\section{Chapter 1. Introduction}

The woodchuck or marmot (Fig. 1-1) of the genus Marmota is a ground dwelling rodent in the squirrel family (Sciuridae) with a circumboreal distribution. No marmot is found in the Southern Hemisphere. All marmots are heavy bodied animals that dig their own burrows, eat green plant material, and in cold climates hibernate during the winter. They have one litter of 2 to 9 young each spring, and molt once annually. Some species of marmots are more social or communal than others. The solitary M. monax of the eastern U.S. contrasts with the more social $M$. flaviventris of the western U.S. and the almost colonial M. bobac of Eurasia. Several ecological and ethological studies have illuminated various aspects of the life history of marmots (Armitage 1975, 1991, 1999; Barash 1989; and Blumstein et al. 1997).

It is generally agreed that there are fourteen species of marmots in the world. As illustrated by Barash (1989) there are six species in North America and eight in Eurasia (Fig. 1-2). The North American species of marmots include: 1) woodchuck, Marmota monax, 2) yellow-bellied marmot, $M$. flaviventris, 3) hoary marmot, $M$. caligata, 4) Vancouver marmot (a melanistic species), M. vancouverensis, 5) Olympic marmot, $M$. olympus and 6) Arctic or Brower's marmot, M. broweri. The Old World species are: 7) black-capped marmot, $M$. camtschatica, 8) bobak or steppe marmot M. bobak, 9) tarbagan or Mongolian marmot, M. sibirica, 10) Himalayan marmot, M. himalayana, 11) Menzbier's marmot, $M$. menzbieri, 12) long-tailed or red marmot, $M$. caudata, 13) gray or Altai marmot, $M$. baibacina, and 14) Alpine marmot, M. marmota.

A detailed study of marmot phylogeny and classification by Steppan et al. (1999) employed a statistical approach using moleculār sequence data. They tested a suite of previously proposed hypotheses of phylogenetic relationships using a sequential phylogenetic estimation procedure. Having obtained complete cytochrome "b" sequences for 13 of the 14 species their results support the concept of monophyly of Marmota.

Miller and Kellogg (1955) recognized nine subspecies, including the New York woodchuck, within the monax group of North America.

Paleontological evidence suggests that marmots first arose in North America (Black 1963), and then crossed the Bering landbridge into Eurasia by the
Pleistocene. The Alaskan M. broweri has been proposed as a re-invasion of the Nearctic from the $\mathrm{Pa}$ learctic (Hoffmann et al. 1979). Steppan et al. (1999) believe a North American radiation gave rise to the monax, flaviventris, and caligata groups while a Eurasian radiation produced marmota, bobac, menzbieri, caudata, and broweri.

The proposed classification of the genus Marmota as given by Steppan et al. (1999) was based on an extensive maximum likelihood analysis of molecular sequence data. Although they recognize fourteen species of marmots their phylogenetic relationships remain unresolved.

Classification of marmots has varied over the years as they became better known. The genus Marmota was established by Blumenbach in 1779 (Handbuch der Naturgeschichte Vol. 1 p. 79). His type Marmota alpina had been called Mus marmota by Linnaeus. Other synonyms for Marmota are Glis, Artomys, Lagomys, Lipara and Marmotops.

Steppan et al. (1999) proposed a new subgenus, Petromarmota, defined as the most recent common ancestor of $M$. olympus, $M$. flaviventris, $M$. caligata, and $M$. vancouverensis. This clade is characterized by dorsal pelage with light-tipped guard hairs and a white nose and chin. A light patch is also present between or just in front of the eyes, separated from the white nose by a dark brown to black transverse stripe.

The summary topology of the classification for marmots presented by Steppan et al. (1999) is shown below:

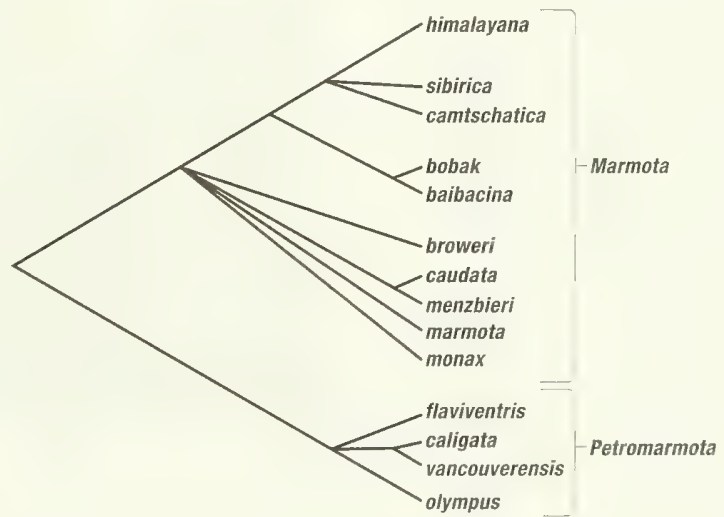

Materials. This anatomical monograph is based on dissections of wild-caught and captive 

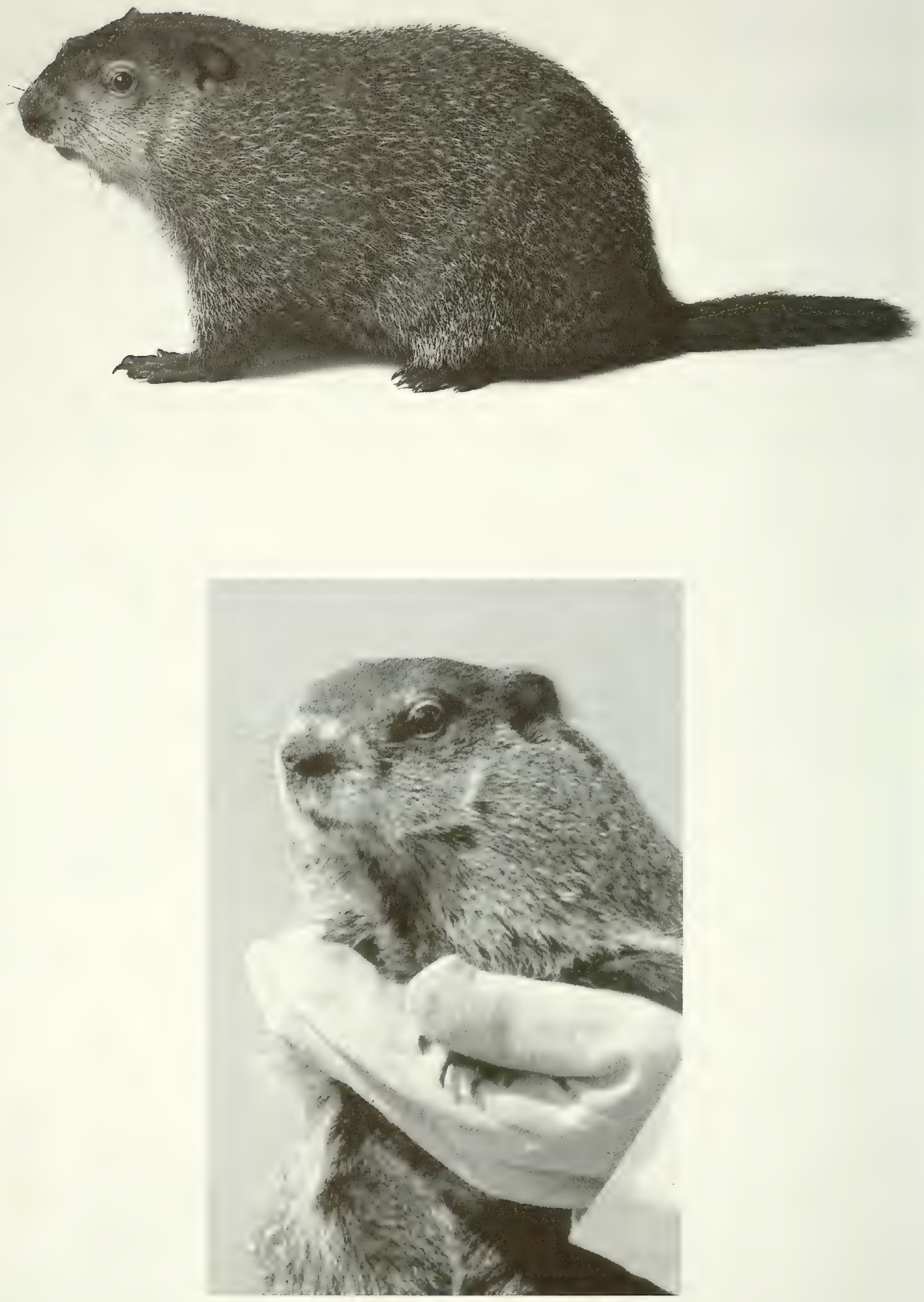

Fig. 1-1. Marmota monax, the Woodchuck of New York State. 


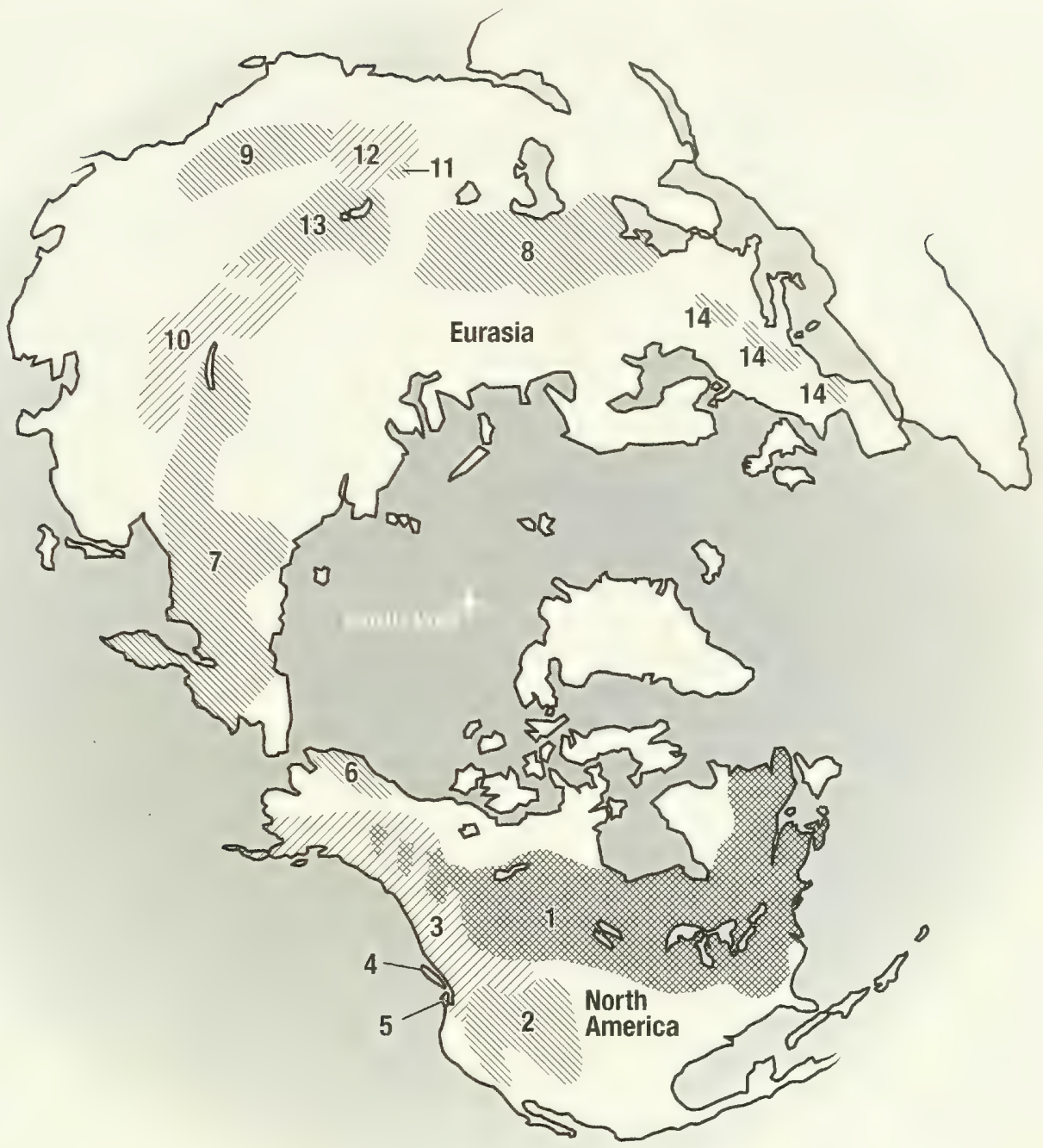

Fig. 1-2. Worldwide distribution of the genus Marmota; adapted from "Marmot's Social Behavior and Ecology" (Barash 1989).

bred woodchucks, Marmota monax, from New York State (Fig. 1-1). Christine Seegers made most of the anatomical drawings at Onderstepoort, South Africa, under the supervision of the senior author. Michael Simmons of the New York State Veterinary College, at Cornell University made additional art work and the labeling of the figures.

Description and Size. The body of the woodchuck is stocky and well furred so that the head and thorax blends with the trunk. The color of the pelage is a mixture of brown and gray (grizzle) with dark brown under-fur. Many of the guard hairs over the body are tipped with white. As the suggested New York subspecies name "rufescens" implies, the fur often has a reddish tint in front or around the shoulders. The underparts of the animal are sometimes lighter than the back. There is a rather short, hairy tail (about one-fifth to one-third of the total body length). In some areas (along the St. Lawrence Seaway) melanism is common and some animals appear completely black. White woodchucks have also been seen.

The head is short with a blunt somewhat compressed nose that is covered with hair to the edge of the nostrils. The face is well haired and has prominent facial vibrissae. The eyes are small and round. The ears are short, broad, and rounded, well covered with hair. During the breeding season a distinct ridge or swelling is present between the eye 
and the ear of male animals. There is no visible neck thus contributing to the overall thickset appearance of the animal.

The limbs are short and stout, each with five digits and slightly curved claws. The claws (Figs. 1-5a and 1-5b) of the forelimb are very effective for digging and are somewhat heavier than those of the pelvic limb. The first digit of the fore-paw is rudimentary and bears a broad, flat nail. The third digit is the longest, the second and fourth are subequal in length. The palm of the fore-paw is bare except at the carpo-metacarpal junction and bears two carpal and three metacarpal pads. The sole of the hind-paw is bare except at the heel and has two tarsal and four metatarsal pads (Figs. 1-5a and 1-5b).

When the animal runs the body is kept close to the ground and its short, furry, dark-tipped tail is dragged behind it. On occasion, when it stops running, the tail may be raised in the air or the animal may stand upright on its hind legs.

According to Whitaker and Hamilton (1998), 64 males from Indiana, had an average total length of $599 \mathrm{~mm}$; average tail length $139 \mathrm{~mm}$; and a hind foot length of $90 \mathrm{~mm}$. For 74 females average total length was $595 \mathrm{~mm}$; tail length $134 \mathrm{~mm}$; and hind foot $82 \mathrm{~mm}$.

Average measurements for 198 New York adults were: total length $555 \mathrm{~mm}$; tail length $125 \mathrm{~mm}$; and hind foot $76 \mathrm{~mm}$. The weight was 2.7 to $5.4 \mathrm{~kg}$ (6 to $10 \mathrm{lbs}$.) with an occasional individual weighing up to $6.3 \mathrm{~kg}$ (14 lbs.). A two-day hunt (Aug. 1999) near Ithaca, New York, gathered 85 woodchucks one of which weighed 13 pounds $2 \mathrm{oz}$. In the northern part of its New York range the woodchuck is smaller. Snyder, Davis, and Christian (1961) described seasonal changes in the weights of woodchucks for the immature, yearlings and adults. Their study over a three-year period included measurements of 1700 animals. They found the weight loss immediately after hibernation was much greater than the rate during hibernation. Much of the fat stored prior to hibernation was still present in late winter when the animals emerged from hibernation. Males utilize fat as a source of energy during the period of food scarcity before the females emerge. For females stored fat is an important source of energy during gestation and lactation.

Captive bred animals for this study came from Dr. Bud Tennant's marmot facility in Ithaca, New York, where they are being bred to study chronic viral hepatitis and subsequent development of primary hepatocellular carcinoma (Summers et al. 1978; Young and Sims 1979).

Distribution. Marmota monax of the Eastern United States is found from Maine to Wisconsin, south to Mississippi, across to central Alabama, northern Georgia, and the western Carolinas. This species also extends from Nova Scotia on the Atlantic coast, across southern Canada into eastern Alaska.

Habitat. The woodchuck lives in excavated burrows in open pastures, on the edges of woodlots, on brush covered dry banks along roads, or in combinations of these features. They often have more than one burrow to serve as either living quarters or retreats. Their winter and summer dens may be close to each other. Favorite spots are welldrained slopes close to a large rock or tree stump. Old sumac groves adjacent to a pasture or a vegetable garden seem to be ideal. Their burrows may be a meter or more in depth with side branches. Although they are rarely seen in water they are good swimmers.

Habits. Marmots are the largest and heaviest of sciurids. They are not adapted for climbing trees and rarely do except when chased. They are most often seen in open fields or pastures, along the shoulders of roads or crossing the road. It is obvious that many do not make it across since road kills in central New York are mostly woodchucks. They will dig under fences or even climb low ones if desirable foraging is in sight. Once they have established a path from their burrow, they will continue to use it on their daily forays. Any object placed on or near their path is usually investigated; they will even enter an unbaited live trap. They are diurnal and feed mainly in early morning and late afternoon. Feces are deposited in a dry chamber of the burrow, and only rarely are scats seen at the den or feeding site. When danger is perceived, they run for the nearest burrow that may or may not be their primary den. Upon emerging from their burrow or while foraging in a field they may stand on their hind legs to get a better view (see the figure of US postage stamp). A whistle (made by the larynx) serves as an alarm call.

The woodchuck is the most solitary of the marmot species, rarely sharing a den even with its mate. There are usually two or more entrances to their burrow. The den entrance is kept clean and free of plant growth and usually has a low pile of newly excavated earth. On a hillside the earth mound forms a small platform on the down side where the animal may stand on its hind legs to get a better view. If debris, branches, or stones are placed near the entrance to the burrow they are soon removed.

Mating occurs in the field shortly after they emerge from hibernation. Gestation is about a month and the usual litter size is four or five. The young are born naked, blind, and helpless. Toward the end of the nursing period, the female will bring 
food into the nest chamber. When the young first leave the nest they are still nursing and at times have to be carried back into the nest by the mother. After the young are weaned and feeding on their own (about 6 weeks) they are forced to disperse by aggressive behavior of both parents. In Europe where marmots ( $M$. marmota) live in colonies together, the young may remain in the den for two years, and visiting females or young can enter without aggression.

Food. Their food preferences are often in conflict with the home gardener and farmer. They like nothing better than newly sprouted beans, peas, corn or sunflowers and can eat a row very quickly. They also like tender grass or grains. During the summer they put on weight, some of which $(20 \%)$ is a fat reserve for their winter hibernation. In northern New York hibernation lasts about 7 months. In more temperate climates it is shorter, and in the southern part of their range they do not hibernate.

Territory and Population. Hamilton (1934) reported a three acre field in central New York with 30 dens and estimated that there was probably one woodchuck for every two acres or 50 per square mile. The number of woodchucks is declining primarily because of habitat destruction as a consequence of urbanization.

For information on marmot ecology, distribution and behavior there is a large body of literature. Most significant are Barash (1989), Armitage (1975 and 1991), and Blumstein and Armitage (1998). For an extensive bibliography see: "Bibliographia Marmotarum" by Ramousse (1997). This alphabetical listing of over 2000 titles on marmots contains abstracts and key words. Another source of information on marmot biology is the Proceedings of the International Conference on Marmots, four of which have been held so far.

Enemies. With the decline ${ }^{-}$of wolves, foxes, bobcats and birds of prey, the greatest impact on woodchuck populations are humans.

Malocclusion of teeth, while not a disease, may be a factor in malnutrition. Hamilton (1937) reported five animals in five hundred to have serious malocclusion of either upper or lower incisors. The upper incisor may curl and pierce the palate or reach far out of the mouth. A specimen in the Cornell Veterinary College Museum (Fig. 1-3) shows a severe example which certainly must have interfered with feeding. One of the upper incisors had pierced the palate.
Human Relations: As a Source of Food. Hamilton (1934) noted that young woodchucks properly prepared are a joy to many country folk. He cited a local Ithaca, New York, source who confirmed the canning of woodchuck meat in twoquart fruit jars for winter consumption. In some parts of the world the marmot is a regular item in the human diet. When woodchucks are eaten by humans there is a possibility of infection with tularemia, and there have been cases in the past.

As a Farm or Garden Pest. The economic impact of the woodchuck can be considerable for the home gardener. The effect on a farmers grain, corn, or bean crop is usually small but has at times resulted in bounties or organized sport hunts. During a two day summer hunt in Ithaca, New York, 85 woodchucks were brought in, 31 by a single hunter. The danger of burrows to farm machinery or to animals is minimal, because the burrow entrance is often in a thicket at the edge of a field or next to a rock-pile or stump.

External measurements of eastern woodchucks vary greatly between wild and captive animals, between young and old, between the sexes and at various times of the year. Adult males are usually larger than females. The total body length of wild adult animals is 520 to $608 \mathrm{~mm}$. Yearlings weigh 2070 to $4225 \mathrm{~g}$, and adult animals 2820 to $5390 \mathrm{~g}$. They can be bred in captivity and can attain a weight of at least $8200 \mathrm{~g}$.

Although the woodchuck is a very common animal, little has been published about its anatomy. Frink et al. (1977) studied the seasonal variation in the morphology of the thyroid gland, and that of the adrenal gland by Christian (1962). Kenagy and Trombulak (1986) reported on the size and function of the testes in relation to body weight, while Christian et al. (1972) studied the annual cycle of spermatogenesis and testes morphology. Mossman et al. (1932) compared the male reproductive tracts of Sciuridae, and Pocock (1923) used penis morphology as a basis for classification. Courser et al. (1963) studied the somatic chromosomes of the woodchuck. Rasmussen (1918) reported on the cyclic changes in the interstitial cells of the ovary and testis. Nuckle and Bergeron (1983) used eye lens weight as a criterion for age classification, while $\mathrm{Da}$ vis (1964) evaluated various characters for determining the age of woodchucks. Snyder et al. (1961) determined weight gain and loss, Davis (1967) reported on the annual rhythm of fat deposition, and Young and Sims (1979) discussed the woodchuck as a biological model for the study of obesity.

Baldwin et al. (1985) studied changes in serum testosterone concentrations of adult and yearling woodchucks. They noted that Marmota monax has 

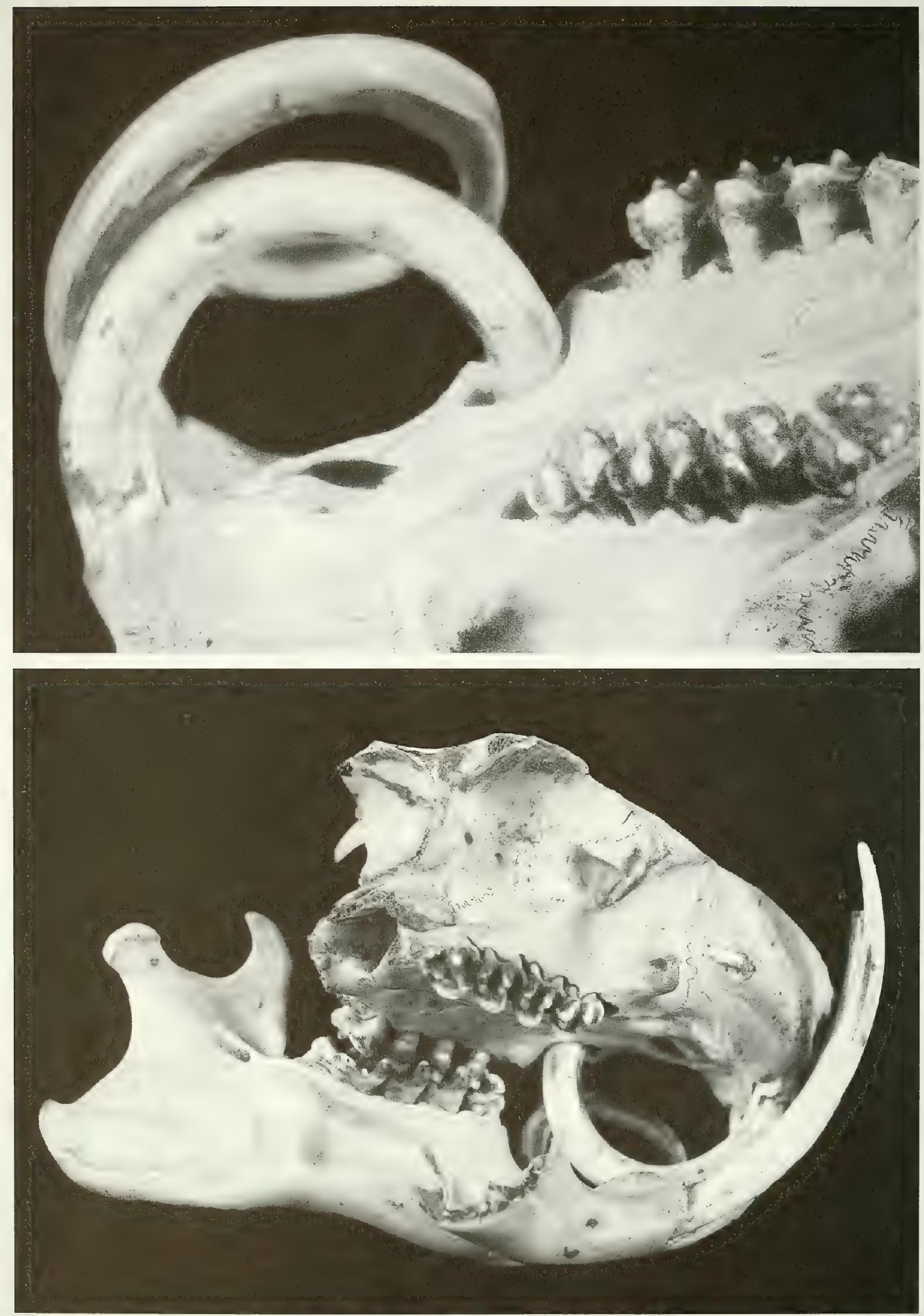

Fig. 1-3. Malocclusion in a woodchuck. Bottom) Right lateral view, right lower jaw removed. Note that the upper right incisor is piercing the left palate. The lower incisors are overgrown but maintained their normal curvature. Top) Ventral view of the palate. 
one short breeding season per year in late winter following emergence from hibernation. The testes of adults are regressed and abdominal in position prior to hibernation in early autumn and testicular recrudecesence occurs during hibernation.

In central New York State, males emerge from hibernation in mid-February to beginning of March, 1-3 weeks before females. When they emerge, they have large scrotal testes containing mature sperm. Mating occurs during the first three weeks of March as females emerge from hibernation. The testes of males are devoid of mature sperm by the end of April. Testicular volumes and serum testosterone concentrations were measured bi-weekly in 5 adults and 4 yearling captive woodchucks. Testosterone levels and testes volumes measured in newly captured woodchucks in March and April and again 2-3 months later were generally similar to those of their laboratory counterparts.

Females give birth to a single litter during the first three weeks of April following a 31-32 day gestation period. Concannon et al. (1983, 1984) studied the corpora lutea present during pregnancy and found that they are maintained or rejuvenated following parturition and lactation. They secrete amounts of progesterone equal to or greater than those of pregnancy.

The remarks of Godman (1842) on the muscles of the ear of the marmot led to further reports on the matter by Seton (1929) and Jones (1937). The only study of the osteology and myology of Marmota monax reported in the literature is the work by Gilbert (1940) on the anatomy of the forelimb of the woodchuck. There were short notes on the anatomy of the tongue by Tuckerman (1889), and another by Wood (1969) on the third upper premolar.

Anatomical Terms. In the anatomical descriptions which follow we have used Nomina Anatomica Veterinaria for domestic animals (NAV 1992) as a guide rather than the Nomina Anatomica (NA 1996) or the Terminologia Anatomica (TA 1998), both of which pertain to the human. In this monograph each term, when first introduced, is followed by its Latin equivalent in italics. If both the Latin and English terms are the same, only one term will be cited here in italics. Until the Basel Nomina Anatomica (BNA) was produced in 1895 there was no international agreement on anatomical nomenclature. Each language had its own terms for structures of the body and many structures had different names in different countries. Early writings in anatomy were in Greek, then Arabic, and later in Latin. It is common practice to translate Latin terms into the vernacular of the person using them. However, many terms are used in Latin with- out any vernacular transliteration, and thus they are the same in several languages.

The present Nomina Anatomica (NA) for humans (6th edition 1989) includes 5,640 standard terms, more than $80 \%$ of which were continued from the BNA. This NA nomenclature was opposed by veterinary anatomists because it used the old terms of direction related to the human standing position, with the forearms supinated in a posture that is impossible in most animals. "Anterior/posterior" in the human anatomical position corresponds to "ventral/dorsal" in the woodchuck.

An International Committee on Veterinary Anatomical Nomenclature (ICVAN) published Nomina Anatomica Veterinaria for domestic mammals in 1968 and revised it as a 4th edition in 1994. The guiding principles for the establishment of $\mathrm{No}^{-}$ mina Anatomica Veterinaria were similar to those of Nomina Anatomica:

1. Aside from a very limited number of exceptions, each anatomical concept should be designated by a single term.

2. Each term should be in Latin in the official list, but anatomists of each country are free to translate the official Latin terms into the language of instruction.

3. Each term should be as short and simple as possible.

4. The terms should be easy to remember and should have, above all, instructive and descriptive value.

5. Structures that are closely related topographically should have similar names.

6. Differentiating adjectives should generally be opposites, as major and minor, or superficial and deep.

7. Terms derived from proper names (eponyms) should not be used.

Unfortunately there is still no international Nomina for mammals other than the human and a few domestic animals. The authors of the present work have attempted to select terms that are consistent with the principles of past anatomical terminologies. Some rodent structures have no counterpart in human or domestic mammals, and therefore new terms or choices from the past literature have been used. At the end of this book we include a glossary of anatomical synonyms.

Directional Terms (Fig. 1-4). The terms cranialis and caudalis apply to the neck and trunk and to the limbs proximal to the carpus and tarsus. The terms dorsal and palmar are used on the manus (hand), and dorsal and plantar on the pes (foot). On the head the terms rostral, caudal, dorsal, and ventral are preferred, with the terms an- 


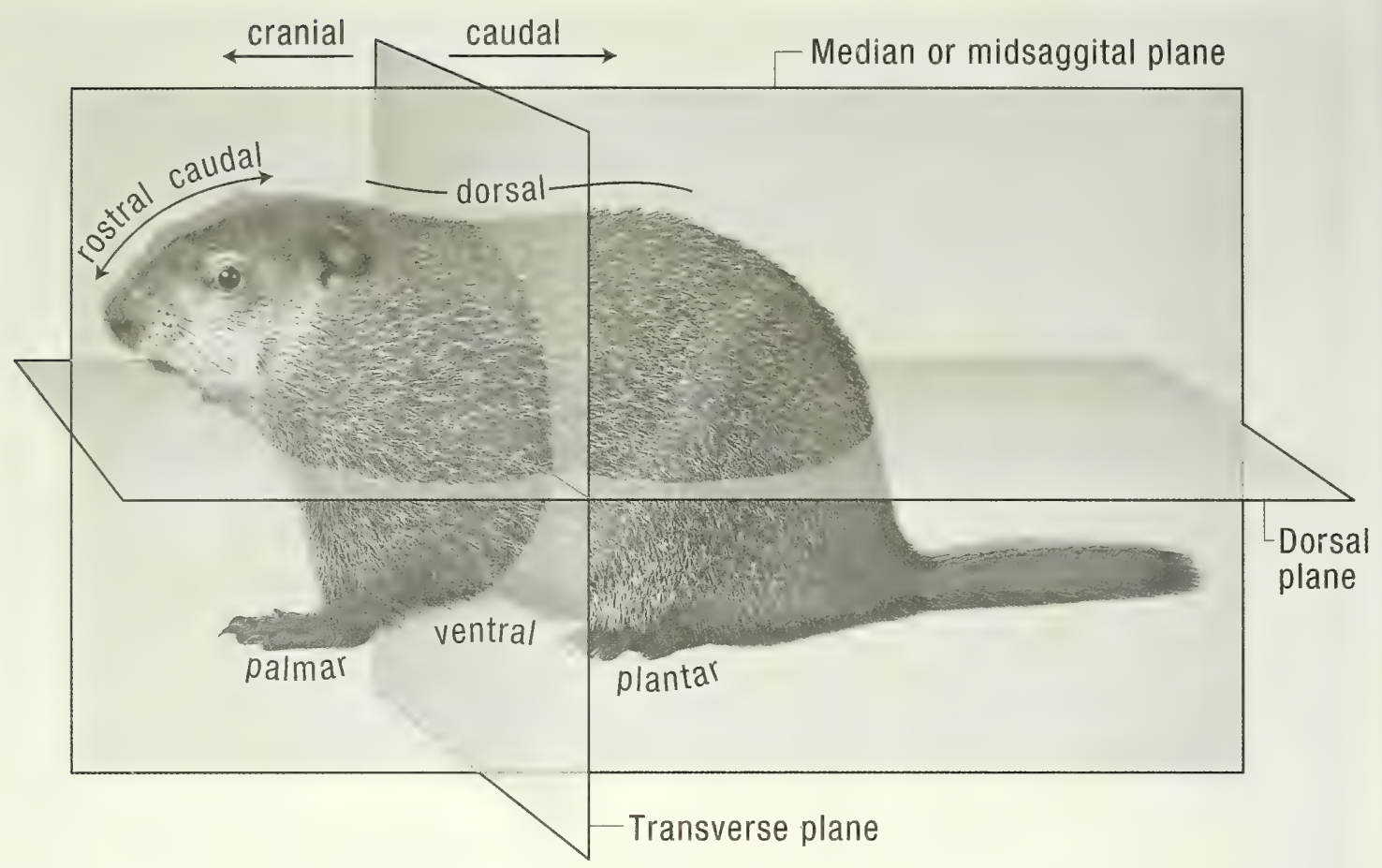

Fig. 1-4. Directional terms and planes of the body.

terior, posterior, superior, and inferior used in a few locations, such as the eyeball, eyelids, lips, and inner ear. Medial and lateral are used on the whole body, except that axial and abaxial, referring to the functional longitudinal axis of a limb may be used to designate the sides of the digits. There are a few other conventions and a brief Latin Grammar that are explained in the introduction of Nomina Anatomica Veterinaria 4th edition (ISBN 0-9600444-77).

The figure below of the planes, positions, and directions on the body of a woodchuck should aid in understanding the descriptions of the structures described in the text.

Integument. The common integument includes the skin, hair, claws, and pads of the fore and hind paws. Also included are glands derived from the skin such as the temporal gland of the head and the mammary glands of the thorax and abdomen.

Skin. The microscopic anatomy of the woodchuck's skin was described by Panic et al. (1992). The epidermis consists of four layers: stratum basale, stratum spinosum, stratum granulosum, and stratum corneum. There is no clear distinction between the superficial and deep dermis. Elastic fibers are seen throughout the dermis. Both compound and simple hair follicles are present, with com- pound being more common. Arrector pili muscles of the skin are largest over the back. Sebaceous glands are present either within the outer root sheath of hair follicles or in the dense connective tissue surrounding the hair follicles. No apocrine sweat glands are found but there are abundant eccrine sweat glands in the subcutaneous fat of the footpads.

At birth the skin is pink and unpigmented (Hamilton 1934; Baldwin 1985) without visible hair. By the second day after birth the skin has a grayish cast and pigment becomes visible. When the pup is 7 days old the skin of the back is definitively gray but the abdomen is still pinkish.

Hair Coat. Tactile hairs appear at 1-2 days and body hair 9-10 days after birth (Hamilton 1934; Baldwin 1985). In the adult most of the body surface is covered with hair. It is most dense on dorsal and lateral parts of the body, while the abdomen and the inside of the flanks are sparsely haired. All pads, nipples and the nasal surface are hairless.

The pelage of the eastern woodchuck is coarser and thinner than that of other monax subspecies. The underfur is dense, soft and somewhat woolly, and is mainly confined to the back and sides. The underfur of the upper parts varies from black to dark gray at the base, followed by a broad area of light pinkish cinnamon to strongly reddish. 

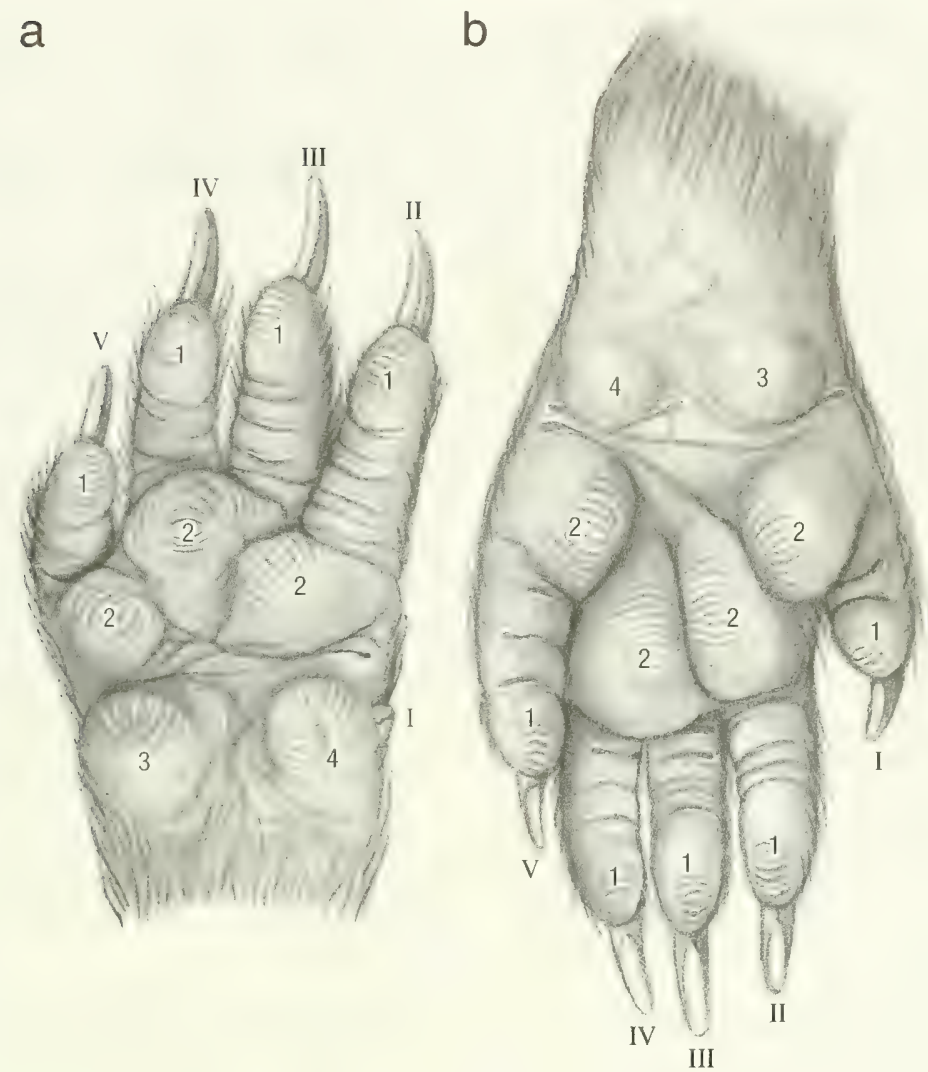

Fig. 1-5. a) Left forepaw, palmar view. 1 digital pads, 2 metacarpal pads, 3 lateral carpal pad, 4 medial carpal pad, I-V digits I to V. b) Left hindpaw, plantar view. 1 digital pads, 2 metatarsal pads, 3 medial tarsal pad, 4 lateral tarsal pad, I-V digits I to $\mathrm{V}$.

Upperfur, the longer and coarser hair that covers the whole body, is dark chestnut brown, tipped with light buff. The hair on the head and feet is shorter than that on the rest of the body. Hair on the tail is long and coarse. The under parts are covered by short, sparse fine hair. The hair of the forelegs is black overlaid by brown, while the hair of the feet is black.

Tufts of long, stiff, black whiskers or tactile hairs, pili tactless, grow out from the upper lip, lower lip, chin, caudal intermandibular region, above the medial canthus of the eye, and from the sides of the cheeks. These whiskers are usually referred to as vibrissae (hairs of the nose) rather than tactile hairs. Ahl (1987) reviewed the literature and discussed the relationship between vibrissal length and feeding habits in the Sciuridae. She concluded that the more terrestrial or fossorial species have short vibrissae but that many questions remain as to how the animal uses its vibrassae in daily life.

Foot Pads and Claws (Figs. 1-5a and 15b). A pad or torus consists of a thick epidermal covering, the underlying dermis, and the subcuta- neous cushion or pulvinus. The skin of the pads is heavily pigmented, and the areas between the pads are bare. Pads occur on the palmar/plantar surfaces of the paws and are named according to the area that they overlay. The palmar surface of the forepaw has two carpal, three metacarpal and four digital pads, while the plantar surface of the hind-paw has two tarsal, four metatarsal and five digital pads.

The claws of the woodchuck are approximately $15 \mathrm{~mm}$ long and 2-3 $\mathrm{mm}$ wide. They are curved, pigmented and laterally compressed. Each claw consists of a wall and a sole. The wall fits into the unguicular groove and is supported by the unguicular process of the distal phalanx. The sole of the claw is small. It consists of a triangular area of soft horn located between the two walls on the palmar/ plantar surface of the claw.

Glands. Eccrine sweat glands are found only in the foot pads. They are abundant and lie in the adipose tissue of the digital cushions. Apocrine sweat glands are not present in the skin of the woodchuck. Sebaceous glands are distributed over the skin associated with hair follicles. In some areas 
they enlarge to form discreet sudoriferous glandular masses or scent glands. Scent glands are present at the angle of the mouth (oral or buccal glands) and the temporal region (temporal scent glands). The temporal glands enlarge during the breeding season to form two prominent swellings between the eye and the ear. They are approximately $3 \mathrm{~cm}$ long and $2 \mathrm{~cm}$ wide and secrete a yellowish, aromatic fluid that is used for scent marking the area of the family group. Rausch and Bridgens (1989) described and illustrated the macro- and microscopic characteristics of facial glands in seven species of marmots with emphasis on $M$. broweri, $M$. caligata, and $M$. monax.

Three anal sacs open on the anocutaneous junction of the anus. One sac lies on the ventral midline and the other two on either side of it. They lie between the external and internal anal sphincter muscles. The ducts of the sacs are unpigmented and are everted when the animals are threatened. Smith and Hearn (1979) have described the anal scent gland as being composed of both apocrine and sebaceous components emptying into a common duct.

Walro et al. (1983) described the histology of scent glands associated with the oral angle. Smith and Hearn (1979) studied the ultrastructure of the anal glands.

Muscles of Skin. Two muscles are associated with the skin. The cutaneous muscles ( $m$. cutaneous trunci and $m$. platysma) lie in the hypodermis and are attached to the dermis of the skin (see Muscular System) They move the skin as a whole.

The erector pili muscles, $\mathrm{mm}$. arrectores pilorum, lie within the dermis that attaches to the connective tissue sheath surrounding the hair follicle bulb. They erect or raise the hair.

Mammary Glands. The woodchuck has eight mammae. They are arranged in two bilaterally symmetrical rows extending from the ventral thorax to the inguinal region. There are four thoracic, two abdominal and two inguinal mammary glands. The teats indicate the position of the glands.

Each mammary gland consists of a glandular complex (parenchyma) and its associated papilla mammae or teat. They are well developed in the female and rudimentary in the male. The parenchyma of each mammary gland drains into $2-5$ teat sinuses. Each teat sinus opens by means of a short teat canal onto the apex of the teat. Although the number of duct openings on teats vary between 2 5 , three duct openings are generally present.

Blood supply: The thoracic mammary glands are supplied by perforating branches of the internal thoracic artery and mammary branches of the lateral thoracic artery.

Branches from the external pudendal artery supply the abdominal and inguinal mammary glands. The venous drainage follows the pattern of the arterial supply.

Innervation: Ventral branches of the thoracic and lumbar spinal nerves innervate the mammary glands. 


\section{Chapter 2. Skeleton}

The skeleton (Fig. 2-1) consists of axial, appendicular and heterotopic bones. In the woodchuck heterotopic bones are the patella in the quadriceps muscle of the pelvic limb and the baculum or os penis. The baculum is an ossification of the distal end of the fused corpora cavernosa within the glans of the penis. It is an elongate bone, measuring approximately $5 \mathrm{~mm}$ in length and $1 \mathrm{~mm}$ in diameter. Pocock (1923) compared the os penis of various Sciuridae and found the baculum of the woodchuck (Marmota monax) to be similar to that of the European marmot (Marmota marmota).

\section{AXIAL SKELETON}

The axial skeleton (Fig. 2-1) includes the skull, mandible, hyoid apparatus, vertebrae, ribs, and sternum.

Skull (Figs. 2-2 to 2-14). Comparative osteology of the marmot skull with measurements for several species have been provided by Wehrli (1935). The craniometric points used for measurements are shown in figures 2-2 to 2-4.

In young animals the individual bones of the skull are clearly visible, but with age they fuse or overlap to such an extent that it becomes difficult to distinguish their margins.

The morphology of the skull is used extensively in the classification of sciurids, as well as in the identification of the various subspecies of Marmota monax (Howell 1915). The skulls of young, yearling, and adult woodchucks differ markedly from each other. It is thus impossible to give a general description of the skull with specific measurements applicable to all ages. The following measurements of the skull are from Howell (1915).

Adult male: Condylo-basal length, 81.3-94.4 (88.6) mm; palatal length, 50.7-54.5 (51.8) mm; postpalatal length, 32-36.4 (33.5) $\mathrm{mm}$; length of nasals, 33.9-39.8 (36.8) mm; zygomatic breadth, 53.4-64.7 (60.2) mm; breadth across mastoids, 40.3-46 (43.2) mm; least interorbital breadth, 21.725.4 (23.8) $\mathrm{mm}$; breadth of rostrum, 18-21.2 (19.4) $\mathrm{mm}$; maxillary tooth row, 18.7-21.8 (20.1) $\mathrm{mm}$.

Adult female: Condylo-basal length, 84-88.9 (85.8) mm; palatal length, 49.1-52.4 (50.6) mm; postpalatal length, 31.5-31.8 (31.6) $\mathrm{mm}$; length of nasals, 33.4-39.1 (35.6) mm; zygomatic breadth, 57.5-62 (59.9) mm; breadth across mastoids, 40.6-
43.8 (42.4) $\mathrm{mm}$; least interorbital breadth, 22.226.3 (23.6) $\mathrm{mm}$; breadth of rostrum, 18.2-20.4 (19) $\mathrm{mm}$; maxillary tooth row, 17.5-21 (19.7) $\mathrm{mm}$.

The Skull as a Whole. The skull of the woodchuck is fairly wide. The length to width ratio of animals less than one year old is approximately 1.6 to 1 . As woodchucks age, the skull becomes broader to reach a length to width ratio of 1.4 to 1. The broadening of the skull is primarily due to extensive growth of the frontal, zygomatic and temporal bones. The parietal bones of eight-week old animals are about as wide as that of adult animals. Furthermore, the ratio of cranial length to facial length of the young animal is approximately 1 to 7.1 , and that of the adult animal 2 to 4.1 (facial length is measured from the prosthion to the nasion, and cranial length from the nasion to the inion (Fig. 2-3).

The skull is flattened dorsoventrally so that the height of the cranium is almost the same as the height of the face (Fig. 2-3). The dorsal surface of the cranium (calvaria) is flattened and the left and right halves are separated from each other caudally by a low median sagittal crest. The dorsum of the nose (nasal and incisive bones) forms an even, but marked convexity (Fig. 2-3).

The zygomatic arch, arcus zygomaticus is well developed and projects laterally, giving the skull a broad appearance (Fig. 2-5). The zygomatic process of the frontal bone does not reach the zygomatic arch, leaving the bony orbit incomplete caudoventrally. The large, shallow, temporal fossa, fossa temporalis, is bounded dorsally by the temporal line, caudally by the nuchal crest, ventrally by the zygomatic arch and rostrally by the zygomatic process of the frontal bone. It provides attachment for the temporal muscle.

Numerous foramina and canals are present in or between the bones of the skull. The nomenclature used by different authors for foramina and canals is very confusing. Table $I$ is a comparison between terminology used by Wahlert (1974) and that used by Nomina Anatomica Veterinaria:

\section{BONES OF THE CRANIUM, OSSA CRANII}

The occipital bone, os occipitale, (Fig. 2-10) is a compound bone that forms a large foramen, foramen magnum, for the entrance of the spinal cord 


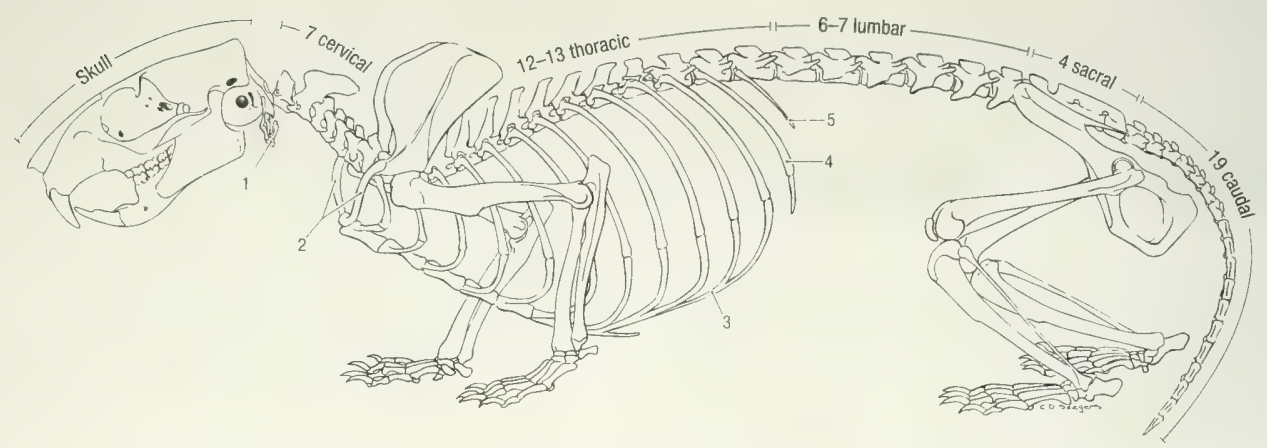

Fig. 2-1. Skeleton, left lateral view. 1 hyoid apparatus, 2 clavicles, 3 costal arch, 4 and 5 asternal ribs.

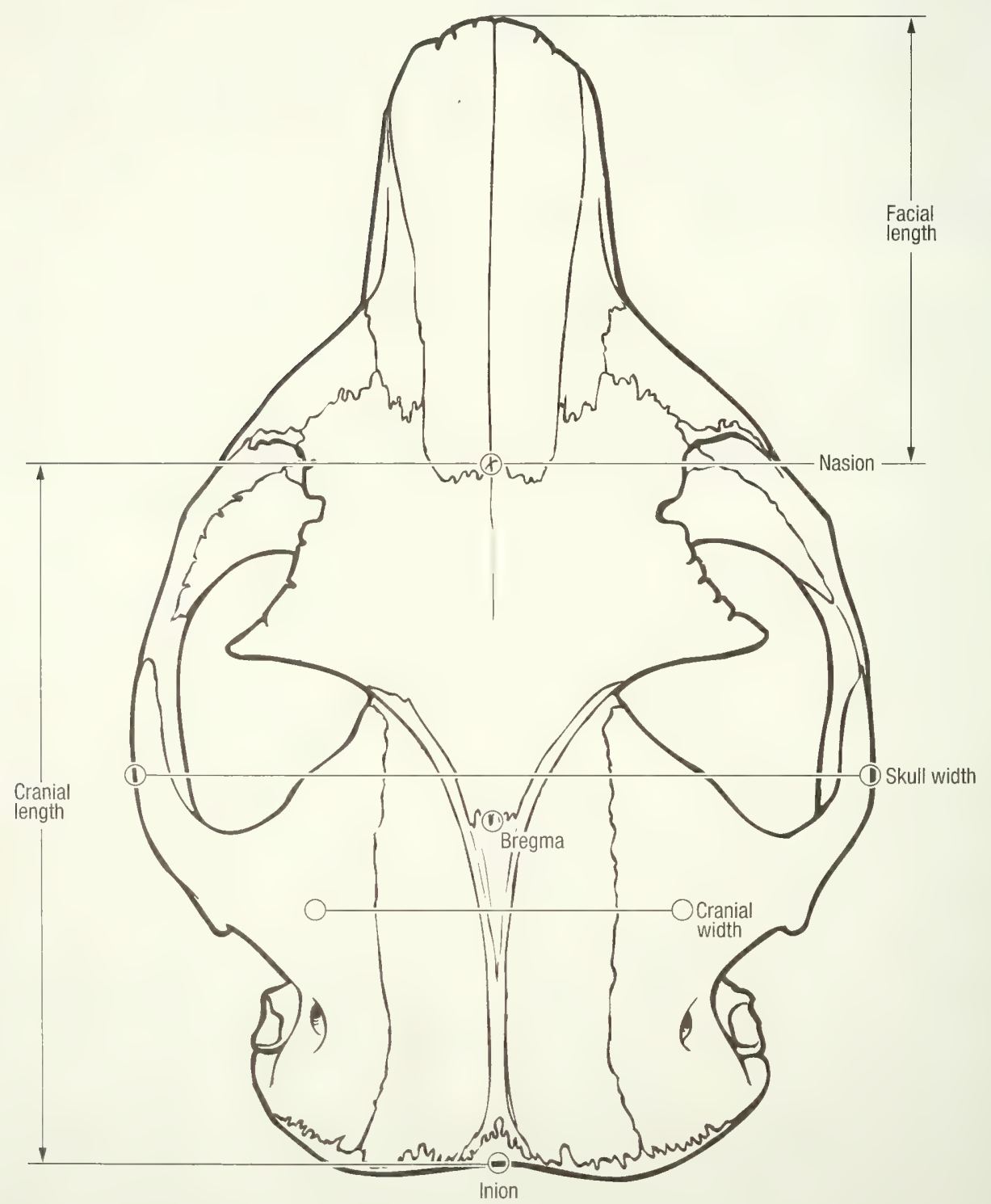

Fig. 2-2. Skull, dorsal view to show craniometric points. 


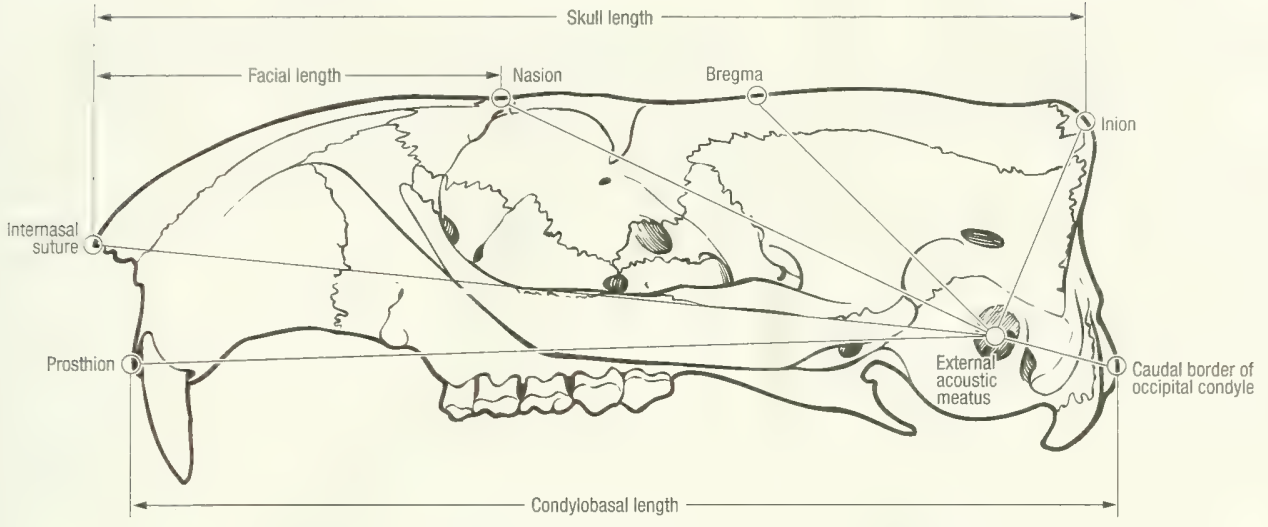

Fig. 2-3. Skull, lateral view to show craniometric points.

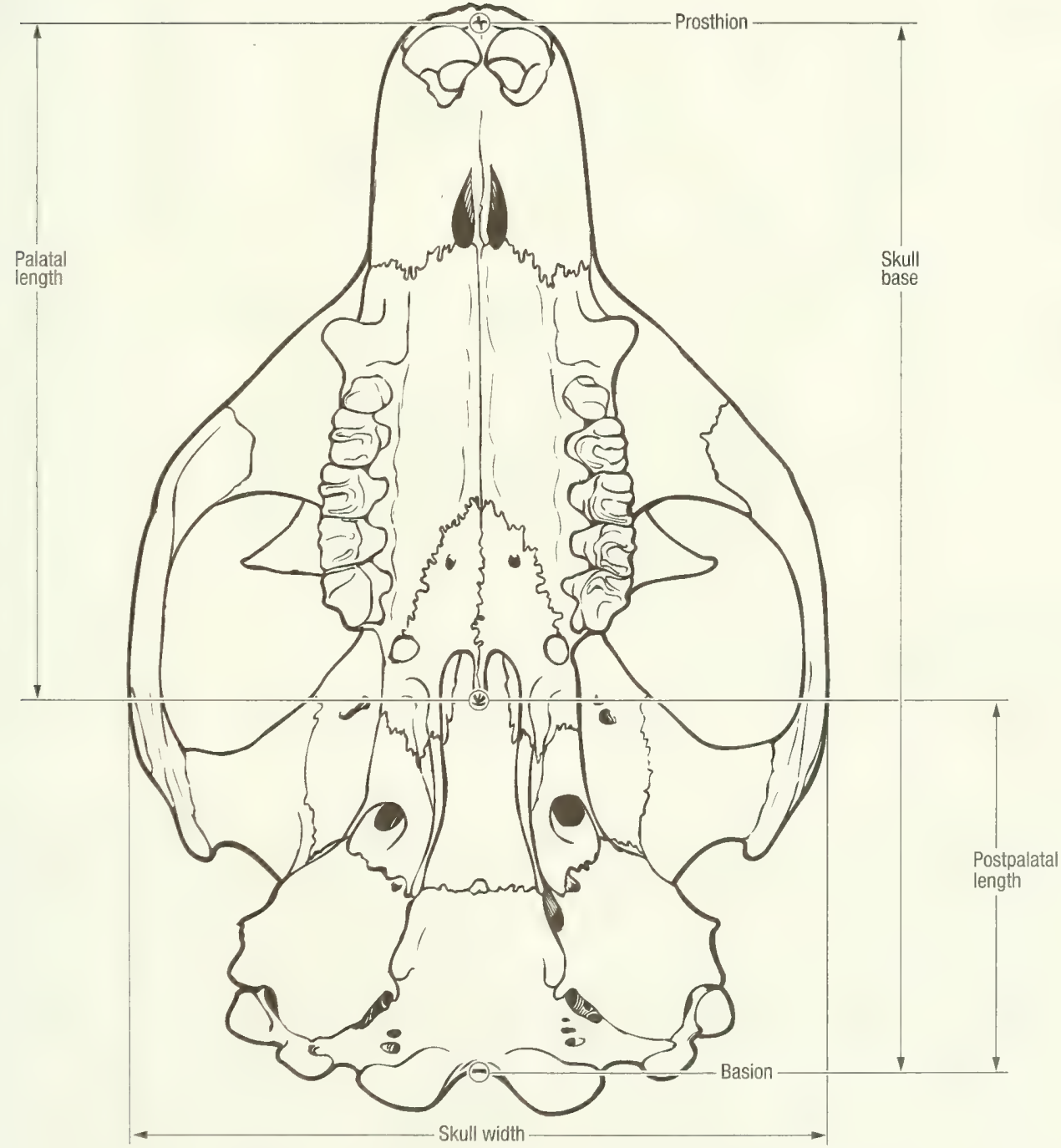

Fig. 2-4. Skull, ventral view to show craniometric points. 


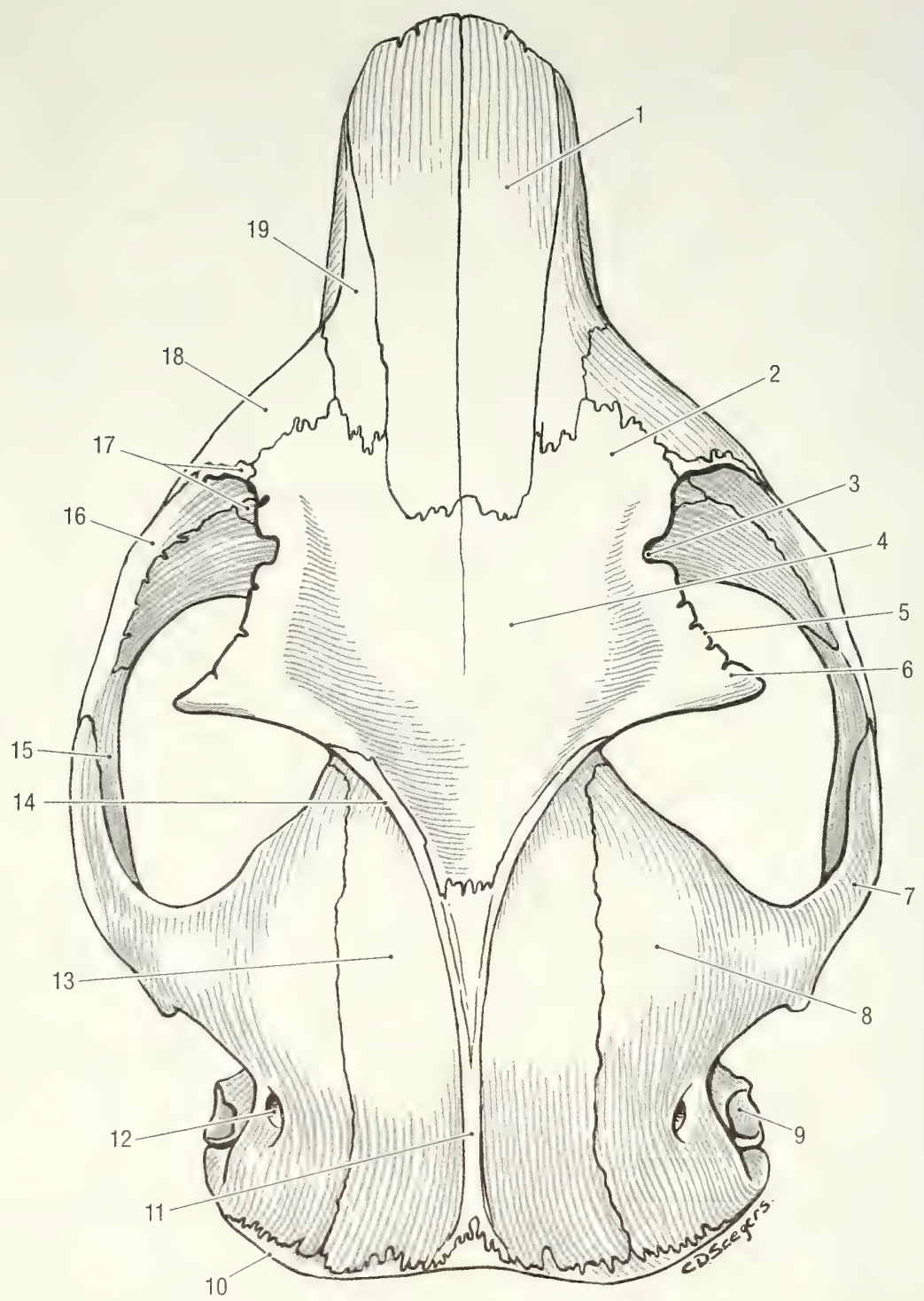

Fig. 2-5. Skull, dorsal view, mature animal. 1 nasal bone, 2 nasal part of frontal bone, 3 supraorbital incisure, 4 frontal bone, 5 supraorbital margin, 6 zygomatic process of frontal bone, 7 zygomatic process of temporal bone, 8 squamous temporal bone, 9 external acoustic pore, 10 nuchal crest, 11 external sagittal crest, 12 retroarticular foramen, 13 parietal bone, 14 temporal line, 15 temporal process of zygomatic bone, 16 zygomatic bone, 17 lachrymal bone, 18 maxilla, 19 incisive bone.

into the cranium. It develops as a dorsal supraoccipital, a ventral basioccipital, and paired lateral exoccipitals that bear the condyles for articulation with the atlas (Fig. 2-10).

The supraoccipital bone, os supraoccipitale, (Fig. 2-10) forms the vertical caudal portion of the cranium. It often has one or two vascular foramina close to the midline. The outer surface of the bone is irregular and roughened for the attachment of muscles. It fuses dorsally with the interparietal and parietal bones, and dorsolaterally with the temporal bones to form a prominent nuchal crest. Each lat- eral exoccipital bone, os exoccipitale (Figs. 2.8 and 2.10) has an elliptical occipital condyle for articulation with the atlas, and a paracondylar processes for the attachment of the digastric muscle. Ventral to each condyle is a shallow depression, the ventral condylar fossa (Fig. 2-8). The latter has a canal for the passage of the hypoglossal nerve, canalis $n$. $h y$ poglossi, and one or two canals for the passage of blood vessels. The lateral part of the occipital bone fuses with the mastoid part of the temporal bone. The basal part or basioccipital bone, os basioccipitale (Fig. 2-8), is unpaired and forms the caudal third 
Table 1. A comparison of the terminology used by Wahlert (1974) and Nomina Anatomica Veterinaria (1994).

Wahlert NAV Contents

Nasolachrymal foramen

Nutritive foramen

Orbital fissure

Optic canal

Post-alar fissure

Postglenoid foramen

Posterior maxillary notch or foramen

Posterior palatine foramen

Sphenofrontal foramen

Sphenopalatine foramen

Sphenoidal fissure

Squamoso-mastoid foramen

Stapedial foramen

Stapedial artery canal

Stylomastoid foramen

Supraorbital notch

Transverse canal

Anterior alveolar canal

Alisphenoid canal

Buccinator foramen

Carotid canal

Canal of Huguier

Dorsal palatine foramen

Eustacian canal

Ethmoid foramen

Facial canal

Oval foramen

Accessory oval foramen

Round foramen

Hypoglossal foramen

Infraorbital foramen

Incisive foramen

Jugular foramen
For. lacrimale

Fissura orbitalis

Canalis opticus

For. retroarticulare

No equivalent

For. palatinum majus

No equivalent

For. sphenopalatinum

For. alare rostrale

No equivalent

No equivalent

No equivalent

For. stylomastoideum

Incisura supraorbitale

No equivalent

Canalis alveolaris

Canalis alaris

For. alare parvum

Canalis caroticus

Fissura petrotympanica

Caudal palatine foramen

Canalis musculo-tubarius

For. ethmoidale

Canalis facialis

For. ovale

For. alare caudale

For. rotundum

Canalis n. hypoglossi

For. infraorbitale

Fissura palatina

For. Jugulare
Nasolacrimal duct

Oculo motor, trochlear and abducens nerves, and the ophthalmic and part of the maxillary divisions of the trigeminal nerve.

Optic nerve

V. emissaria for. retroarticulare

Descending palatine vein

Palatine (descending) artery, vein and nerve

Opthalmic artery

Sphenopalatine artery, vein and nerve

Nerves transmitted by the orbital fissure and round foramen

Stapedial artery

Stapedial artery

Facial nerve

Frontal branch of ophthalmic nerve and superior ophthalmic artery

Vein

Branches of the maxillary nerve, artery and vein to the upper incisor teeth

Maxillary artery, vein and nerve

Buccinator and masseteric branches of mandibular nerve

Inferior petrosal sinus

Chorda tympani

Descending palatine artery, vein and nerve

Auditory tube

Ethemoidal artery and vein, nasociliary branch of ophthalmic nerve

Facial nerve

Mandibular division of trigeminal nerve

Maxillary nerve, artery and vein

Maxillary nerve

Hypoglossal nerve

Infraorbital nerve, artery and vein

Palatine artery and vein, incisive duct

Glossopharyngeal, vagus and accessory nerves, emmissary veins, stapedial (external ophthalmic) artery

A diploic vein of the base of the cranium. It has a prominent muscular process, tuberculum musculare, for the tensor muscle of the soft palate. Externally, the basioccipital bone lies against the bulla of the temporal bone to form the tympanooccipital fissure, fissura tympanooccipitalis, and intracranially it fuses with the petrous temporal bone to form the petrooccipital fissure, fissura petrooccipitalis.

The parietal bone, os parietale, (Figs. 2-5, 2-7, 211) are paired. They form the caudodorsal roof of the cranium and fuse along the midline to form a low external sagittal crest, crista sagittalis externa.
The sagittal crest divides rostrally, and is continued onto the caudal edges of the zygomatic processes of the frontal bones. Together, the caudal edges of the zygomatic processes and the external sagittal crest form the temporal line, linea temporalis to which the temporal muscle attaches. Rostrally the parietal bones fuse with the frontal bones, laterally with the temporal bones and caudally with the supraoccipital bone.

The interparietal bone, os interparietale (Fig. 26), is a small, triangular bone wedged between the parietal and occipital bones. In an eight-week old 


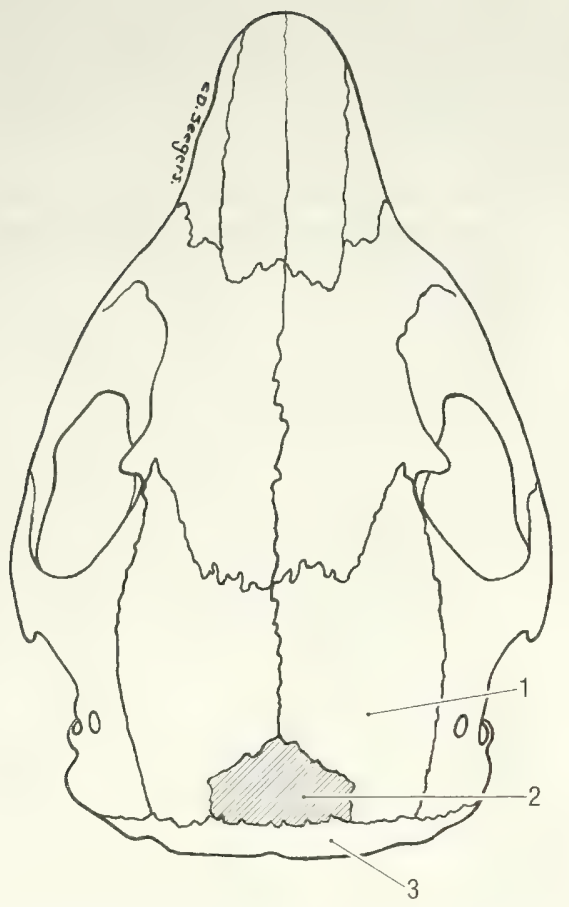

Fig. 2-6. Skull, dorsal view of an immature animal. 1 parietal bone, 2 interparietal bone, 3 occipital bone.

animal it is visible on the surface of the skull 10 $\mathrm{mm}$ wide and $8 \mathrm{~mm}$ long. It cannot be identified externally on the skull of a mature animal.

The interparietal bone is overgrown by the parietals that meet on the midline as the external sagittal crest.

Temporal bone, os temporale (Figs. 2-5 and 2-7), is paired, each consisting of squamous, petrous and tympanic parts.

The squamous part, pars squamosa, forms the caudolateral portions of the cranium. It has a large zygomatic process, processus zygomaticus, which joins the temporal process of the zygomatic bone to form the zygomatic arch. The root of the zygomatic process has a large ventral concave area, the mandibular fossa, fossa mandibularis (Fig. 2-8), which bears the articular surface for the mandible. A large retroarticular foramen, foramen retroarticulare (Figs. 2-5 and 2-7), for the passage of blood vessels lies caudal to the root of the zygomatic process, and the caudoventral border of the bone bears a shallow tympanic incisure for the tympanic bulla. The squamous part fuses dorsally with the parietal bone, rostrally with the frontal bone and rostrally and rostroventrally with the wing of the basisphenoid bone. Caudoventrally it fuses with the tympanic and petrous parts of the temporal bone and caudodorsally with the occipital bone (Fig. 2-7). The fusion of the petrous and squamous parts of the temporal bone completes the ventrolateral part of the nuchal crest. The temporal bone of the woodchuck does not have distinct retroarticular and retrotympanic processes.

The tympanic part of the temporal bone, pars tympanica, forms the caudoventral part of the cranium between the basioccipital ventrally and the supraoccipital bone dorsally (Fig. 2-8). Dorsolaterally it has a large opening, the external acoustic pore, porus acusticus externus (Fig. 2-7), that leads to a short external acoustic meatus, meatus acusticus externus. Ventral to the latter is the well-developed

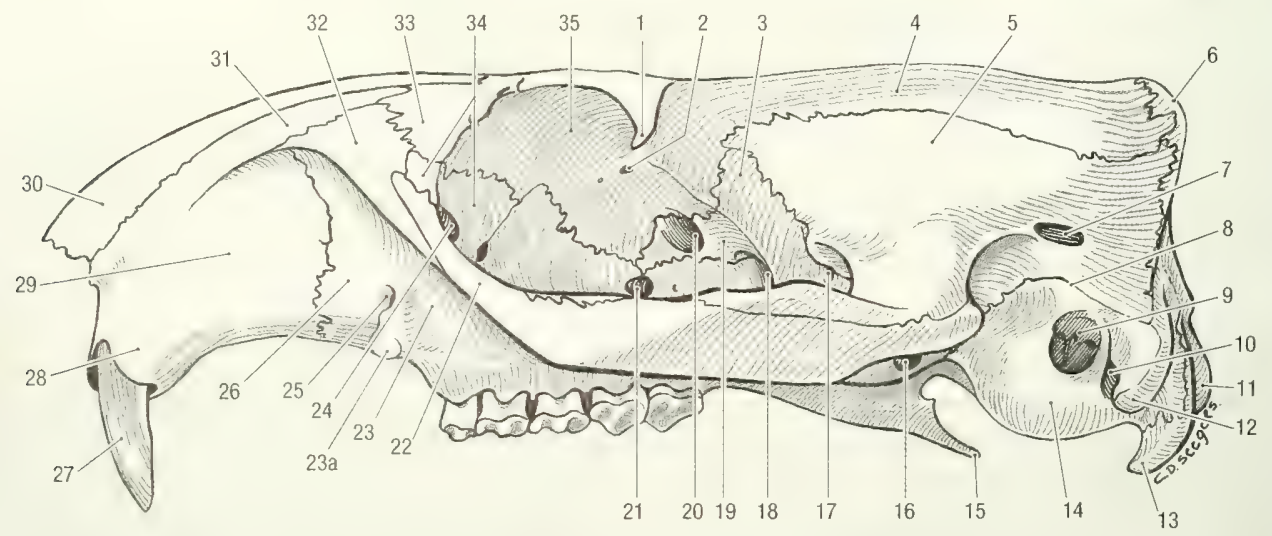

Fig. 2-7. Skull, left lateral view. 1 zygomatic process of frontal bone, 2 ethmoid foramen, 3 wing of basisphenoid bone, 4 parietal bone, 5 squamous temporal bone, 6 nuchal crest, 7 retroarticular foramen, 8 tympanic incisure, 9 external acoustic meatus, 10 stylomastoid foramen, 11 exoccipital condyle, 12 mastoid process, 13 paracondylar process, 14 tympanic bulla, 15 hamulus of pterygoid bone, 16 caudal alar foramen, 17 small alar foramen, 18 rostral alar foramen, 19 wing of presphenoid bone, 20 optic canal, 21 sphenopalatine foramen, 22 zygomatic bone, 23 alveolar process of maxilla, 23a facial tuberosity 24 lacrimal foramen, 25 infraorbital foramen, 26 maxilla, 27 upper incisor tooth, 28 alveolar process of incisive bone, 29 incisive bone, 30 nasal bone, 31 nasal process of incisive bone, 32 frontal process of maxilla, 33 frontal bone, 34 lacrimal bone, 35 orbital part of frontal bone. 


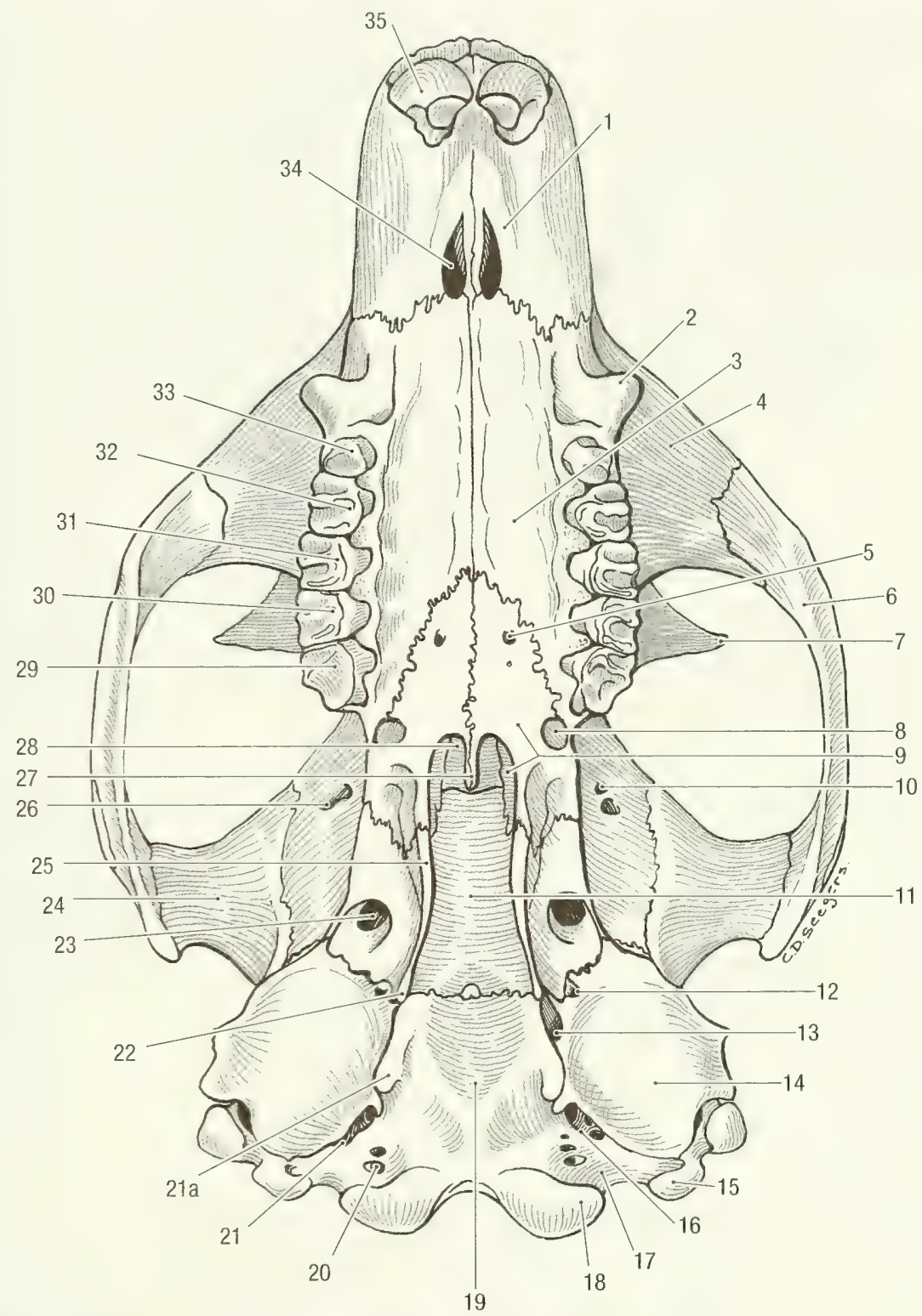

Fig. 2-8. Skull, ventral view. 1 palatine process of incisive bone, 2 facial (masseteric) tuberosity, 3 palatine process of maxilla, 4 zygomatic process of maxilla, 5 major palatine foramen, 6 zygomatic bone, 7 zygomatic process of frontal bone, 8 notch for minor palatine artery, 9 palatine bone, 10 ethmoid foramina, 11 body of basisphenoid bone, 12 musculotubal canal, 13 carotid canal, 14 tympanic bulla, 15 paracondylar process of exoccipital bone, 16 tympanooccipital fissure, 17 ventral condylar fossa, 18 exoccipital condyle, 19 basi-occipital bone, 20 hypoglossal canal, 21 jugular foramen, 21 a muscular process basioccipital bone, 22 hamulus of pterygoid bone, 23 oval foramen, 24 mandibular fossa, 25 pterygoid bone, 26 orbital fissure, 27 caudal nasal spine, 28 presphenoid bone, 29 third upper molar, 30 second upper molar, 31 first upper molar, 32 fourth upper premolar, 33 third upper premolar, 34 palatine fissure, 35 upper incisor.

tympanic bulla, bulla tympanica (Figs. 2-7 and 28). The tympanic part of the temporal bone fuses dorsally along the tympanic incisure with the squamous part (Fig. 2-7), caudally with the mastoid process of the petrous part of the temporal bone, ventromedially with the basi-occipital bone and craniomedially with the wing of the basisphenoid bone (Fig. 2-8). The fissure between the tympanic bulla and the basi-occipital has two openings for the passage of blood vessels and nerves. These are the jugular foramen, foramen jugulare, caudally and the carotid canal, canalis caroticus, rostrally (Fig. 28). Immediately lateral to the carotid canal lies the opening of the musculo-tubal canal, canalis musculotubarius, which forms the bony part of the auditory tube to the middle ear cavity. 
The petrous part of the temporal bone, pars petrosa, mainly lies intracranially and forms the ventrolateral part of the cranial cavity (Fig. 2-11). Its inner surface is divided into rostral and caudal parts by the petrosal crest, crista petrosa. The rostral part bears a foramen for the external ophthalmic artery. The caudal part bears the internal acoustic pore, porus acusticus internus and a deep cerebellar fossa, fossa cerebellaris. The pore leads to the internal acoustic meatus, meatus acusticus internus, for the passage of the facial and vestibulo-cochlear nerves, the fossa is for the cerebellar parafloccular lobe. Only a small portion of the petrous temporal bone is visible on the caudal and lateral surfaces of the skull (Figs. 2-7 and 2-10). It is bounded caudomedially by the squamous and lateral parts of the occipital bone and caudolaterally by the squamous and tympanic parts of the temporal bone. The large mastoid process, processus mastoideus (Fig. 2-7), lies between the external acoustic meatus and the paracondylar process and forms the lateral part of the externally visible petrous temporal bone. The stylomastoid foramen, foramen stylomastoideum, is located medial to the mastoid process, between the latter and the tympanic bulla. On the inner surface the petrous temporal bone fuses medially with the basi-occipital bone, rostrally and dorsally with the squamous and tympanic parts of the temporal bone, and caudally with the exoccipital bones. Externally it fuses medially with the exoccipital bone and dorsally with the supraoccipital bone (Fig. 210). Laterally it fuses with the squamous part of the temporal bone and ventrolaterally and ventrally with the tympanic part of the temporal bone.

The frontal bone, os frontale, is paired and irregular in outline (Figs. 2-5 and 2-7). Each bone consists of squamous, orbital, and nasal parts and is extensively aerated by the frontal paranasal sinus. The squamous and nasal parts form the rostrodorsal, and the orbital part forms the rostrolateral part of the cranium. The squamous part, squama frontalis is flattened and projects laterally to form a zygomatic process, processus zygomaticus. The zygomatic process of the frontal bone does not extend to the zygomatic arch leaving the bony orbit open caudally. The rostral border of the zygomatic process forms the supraorbital margin, margo supraorbitalis. The latter bears a supraorbital incisure, incisura supraorbitalis at the base of the zygomatic process for the passage of blood vessels and nerves. The caudal margin of the zygomatic process continues caudally, blending with the external sagittal crest of the parietal bone. The orbital part, pars orbitalis (Fig. 2-7), of the frontal bone projects ventromedially, forming a major portion of the medial wall of the bony orbit. Distally it bears two ethmoidal foramina, foramina ethmoidalia, for the pas- sage of blood vessels and nerves. The nasal part of the frontal bone lies dorsomedially on the cranium. It is small and fuses rostrally with the nasal bones.

Externally, the frontal bone fuses extensively with the bones of the cranium and face. Along the dorsal midline the two frontal bones fuse with each other. Rostrally they fuse with the nasal, incisive and maxillary bones (Fig. 2-5); rostroventrally with the lacrimal and maxillary bones; caudoventrally with the wing of the basisphenoid bone and caudally with the squamous temporal and parietal bones.

The basisphenoid bone, os basisphenoidale, is unpaired and consists of a body, corpus, an alisphenoid wing, ala, and a pterygoid process, processus pterygoideus. The body forms the middle part of the cranial floor (Figs. 2-8 and 2-11) upon which the hypophysis rests. Its dorsal (internal) surface is smooth and without a distinct sella turcica. It fuses with the basi-occipital bone caudally, with the tympanic bulla laterally and with the presphenoid rostrally. The wing extends dorsolaterally and rostrally from the body to form the ventrolateral part of the cranium (Fig. 2-7). It bears the round foramen rostrally and the oval foramen (Fig. 2-8) caudally along its junction with the basispheniod body. Dorsal to the alar canal the wing bears the small foramen alare parvum for branches of the mandibular nerve. The wing fuses caudally with the tympanic bulla, dorsally with the squamous temporal bone and rostrally with the frontal and palatine bones. The pterygoid process projects laterally and ventrally from the body of the basisphenoid bone (Figs. 2-7 and 2-8). A large alar canal, canalis alaris, traverses the base of the pterygoid process. The latter opens caudally at the caudal alar foramen, foramen alare caudale, and rostrally at the rostral alar foramen, foramen alare rostrale. The foramen alare parvum is connected to the caudal part of the alar canal, and the round foramen opens into the rostral part of the canal. The pterygoid process fuses rostrally with the palatine bone and medially with the pterygoid bone. The fusion with the pterygoid bone is so intimate that it is impossible to identify the suture line, even in very young animals.

The presphenoid bone, os presphenoidale, is unpaired and consists of a body and two wings (Figs. 2-7 and 2-11). The major portion of the body lies within the cranial cavity, forming the rostral part of the cranial floor. Only a small portion, the orbital wing, can be seen on the surface of the skull. The wing bears the large optic canal, canalis opticus, on each side and helps form the orbital fissure that lay caudoventral to the optic canal (Fig. 2-7). Externally, the presphenoid bone fuses dorsally and rostrally with the orbital part of the frontal bone, ventrally with the perpendicular part of the palatine 


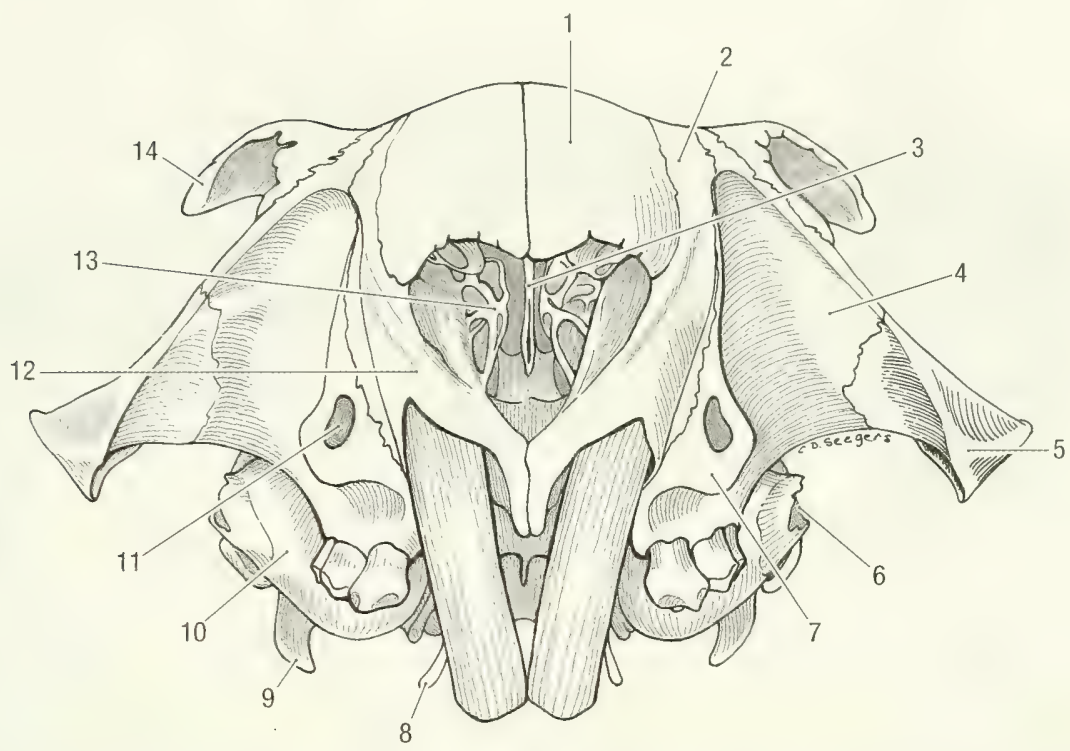

Fig. 2-9. Skull, rostral view. 1 nasal bone, 2 incisive bone, 3 vomer, 4 maxilla, 5 zygomatic bone, 6 external acoustic pore, 7 facial (masseteric) tuberosity of maxilla, 8 hamulus of pterygoid bone, 9 paracondylar process, 10 tympanic bulla, 11 infraorbital foramen, 12 incisive bone, 13 ethmoid turbinate bones, 14 zygomatic process of frontal bone.

bone and caudally with the wing of the basisphenoid bone.

The pterygoid bone, os pterygoideum, is paired and situated medial to the pterygoid process of the basisphenoid, to which it is fused. It projects caudoventrally to form the hamulus, hamulus pterygoideus (Figs. 2-7 to 2-10), for the attachment of muscles.
The ethmoid bone, os ethmoidale (Figs. 2-9 to 211 ), is a complex of bone and cartilage scrolls that is located between the facial and cranial portions of the skull. It is only visible within the nasal cavity. Although unpaired as a unit it develops as right and left dorsal, middle, and ventral nasal conchae, concha nasalis dorsalis, media, et ventralis. A transverse cribriform plate, lamina cribrosa, through which the

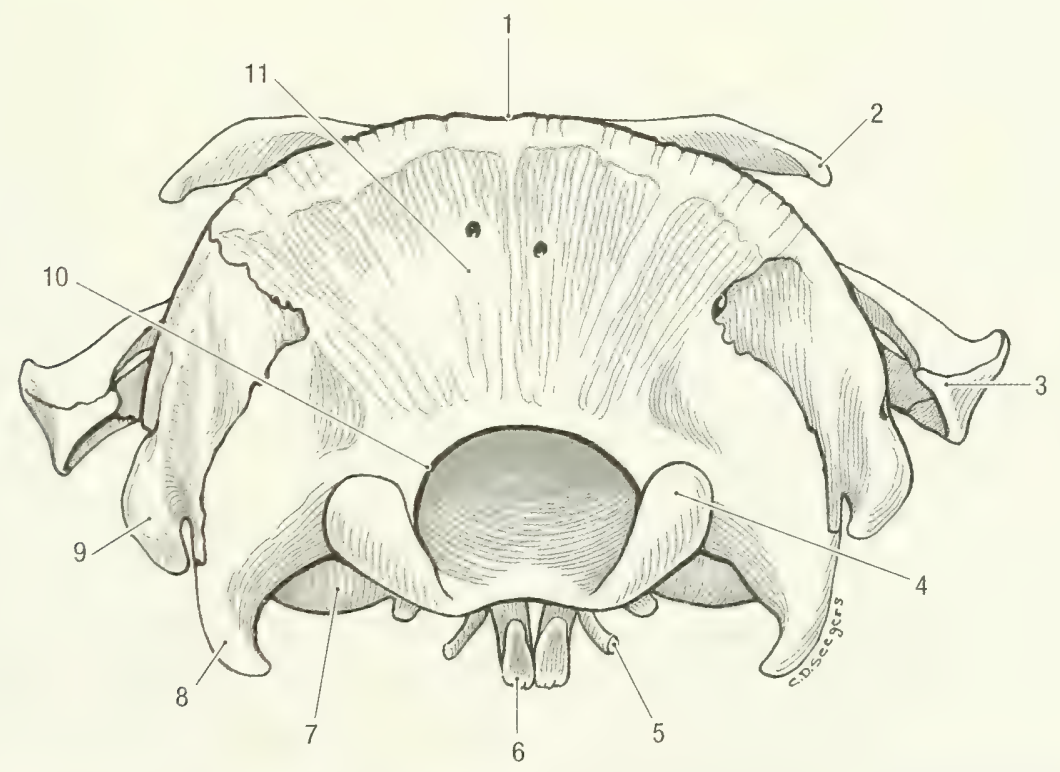

Fig. 2-10. Skull, caudal view. 1 nuchal crest, 2 zygomatic process of frontal bone, 3 zygomatic bone, 4 exoccipital condyle, 5 hamulus of pterygoid bone, 6 upper incisor, 7 tympanic bulla, 8 paracondylar process, 9 mastoid process, 10 foramen magnum, 11 supraoccipital bone. 


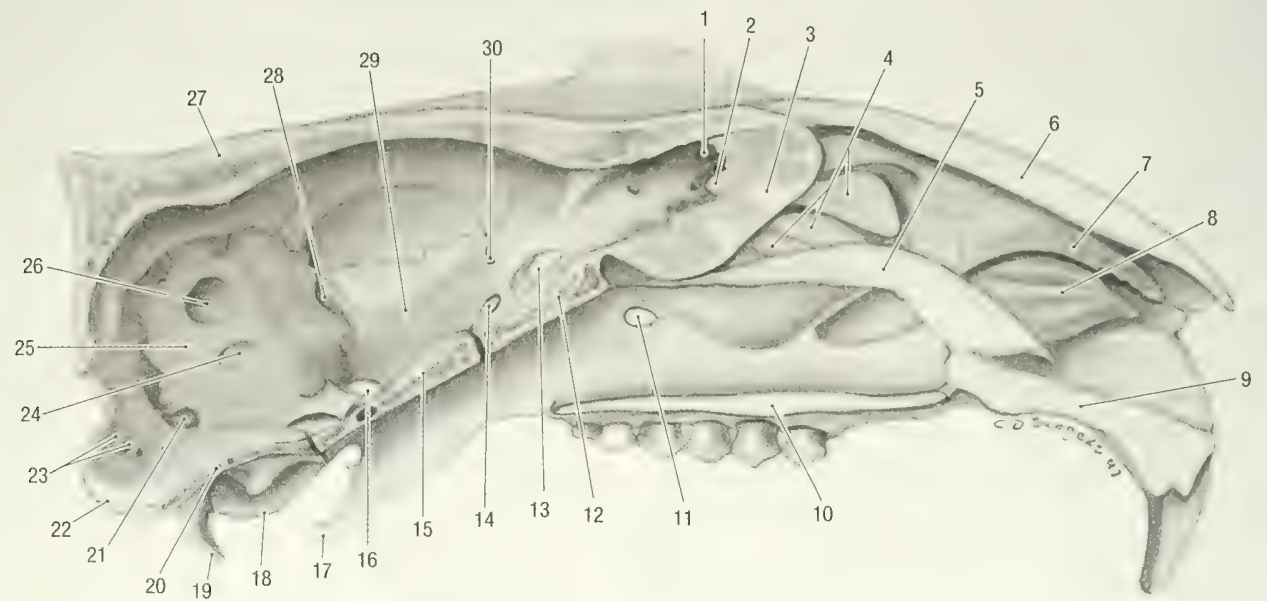

Fig. 2-11. Skull, median section, medial view. 1 cribriform plate of ethmoid bone, 2 crista galli, 3 perpendicular plate of ethmoid bone, 4 ethmoid turbinate bones, 5 vomer, 6 nasal bone, 7 dorsal nasal turbinate bone (ethmo-turbinate I), 8 ventral nasal turbinate bone, 9 incisive bone, 10 palatine process of maxilla, 11 sphenopalatine foramen, 12 presphenoid bone, 13 optic canal, 14 orbital fissure, 15 basisphenoid bone, 16 oval foramen, 17 hamulus of pterygoid bone, 18 tympanic bulla, 19 paracondylar process, 20 basioccipital bone, 21 jugular foramen, 22 exoccipital condyle, 23 canals for hypoglossal nerve, 24 internal acoustic meatus, 25 petrous temporal bone, 26 cerebellar fossa, 27 parietal bone, 28 foramen for stapedial artery, 29 groove for stapedial artery, 30 spheno-frontal foramen.

olfactory nerves pass, separates the nasal cavity from the cranial cavity. The ventral conchae, also known as the ethmoturbinal labyrinth, labyrinthus ethmoidales, fill the nasal cavity. A median nasal septum, the perpendicular plate or mesethmoid, separates the right and left nasal cavities. Attached to the ventral surface of this ethmoid complex is the vomer that thus forms part of the roof of the nasopharyngeal canal.

\section{BONES OF THE FACE, OSSA FACIEI}

The nasal bone, os nasale (Figs. 2-5 and 2-7), is paired and forms the dorsum of the nose. It is slightly convex and expanded rostrally to form two short nasal processes, one medially and one laterally. The left and right nasal bones fuse along the midline. They fuse with the frontal bones caudally, and with the incisive bones laterally. The dorsal nasal concha attaches to the inner, rostrolateral surface of the nasal bone.

The lacrimal bone, os lacrimale, is paired and wedged between the frontal, zygomatic and maxillary bones (Figs. 2-5 and 2-7). It forms the rostromedial part of the bony orbit and has a large lacrimal foramen for the passage of the lacrimal duct.

The maxillary bone, maxilla (Figs. $2-5,2-7$ to 2 9 ), is paired, each with a body and frontal, palatine, zygomatic and alveolar processes. The rostrolateral surface of the maxilla is concave, forming a long, wide groove extending from the alveolar border to the dorsolateral surface of the nose. The groove contains part of the masseter muscle. The maxilla encloses the maxillary paranasal sinus and serves for attachment of the ventral nasal concha internally.

The body of the maxilla, corpus maxillae, completes the medial wall of the bony orbit. The infraorbital canal, canalis infraorbitalis, traverses the body of the maxilla. The canal extends from the maxillary foramen, foramen maxillare, caudally, to the infra-orbital foramen, foramen infraorbitale, rostrally (Figs. 2-7 and 2-9). The ventrolateral wall of the infraorbital foramen is enlarged to form a prominent facial tubercle, tuber faciale (masseteric tubercle of Bryant, 1945). Along the medial wall of the bony orbit the body of the maxilla fuses with the palatine, frontal and lacrimal bones. Two sphenopalatine foramina, foramen sphenopalatinus, are present along the latter fissures, one rostral in the fissure between the lacrimal and maxillary bones and one caudal in the fissure between the maxillary and palatine bones (Fig. 2-7). The body of the maxilla fuses rostrally with the incisive bone (in front of the bony orbit).

The zygomatic process, processus zygomaticus, is flat and grooved to accommodate the zygomatic bone (Figs. 2-5, 2-7 and 2-8). It projects caudolaterally from the body of the maxilla and fuses with the zygomatic bone to complete the rostral part of the zygomatic arch. The short frontal process, processus frontalis, extends dorsorostrally from the body of the maxilla. It fuses with the frontal and incisive bones (Figs. 2-5 and 2-7).

The alveolar process, processus alveolaris, extends ventrally from the body of the maxilla. It has sock- 


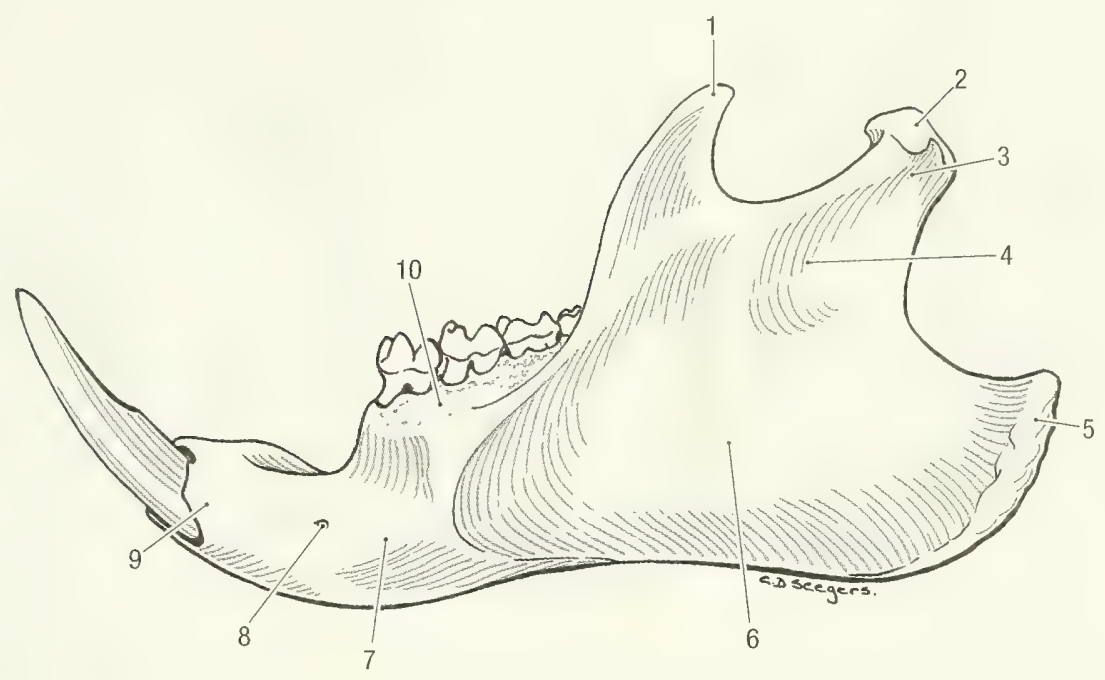

Fig. 2-12. Left mandible, lateral view. 1 coronoid process, 2 articular condyle, 3 condylar process, 4 mandibular ramus, 5 angular process, 6 masseteric fossa, 7 mandibular body, 8 mental foramen, 9 incisive part, 10 molar part.

ets, alveoli dentales, for the upper premolar and molar teeth (Fig. 2-7).

The palatine process, processus palatinus, projects medially from the body of the maxilla (Fig. 2-8). It fuses with the incisive bone rostrally, with the palatine bone caudally and laterally, and on the midline with the palatine process of the opposite side to form the middle section of the hard palate.

The lateral (superficial) surfaces of the body, zygomatic process and frontal process form a deep, wide groove (zygomatic plate) to accommodate part of the deep masseter muscle.

The incisive bone, os incisivum, (premaxilla) consists of a body, a caudodorsally directed nasal process, a medially directed palatine process, and an alveolar process. It forms the rostrolateral part of the face and the rostral part of the hard palate. The nasal process, processus nasalis, extends dorsally and caudally from the body of the incisive bone. It fuses with the nasal, frontal and maxillary bones (Figs. 2-5 and 2-7).

The palatine process projects medially from the body of the incisive bone. It fuses with the palatine process of the opposite side along the midline of the palate and helps to form the large, elongated palatine fissure, fissura palatina.

The alveolar process bears the socket for the large, curved upper incisor tooth. The tooth is embedded along the entire length of the incisive bone.

The rostral part of the ventral concha is attached along the medial (nasal) surface of the incisive bone, and the vomer is attached to a groove formed by the fusion of the right and left palatine processes of the incisive bones.

The palatine bone, os palatinum, consists of horizontal and perpendicular parts. The caudal part of the hard palate is formed by the horizontal laminae of the palatine bone (Fig. 2-8) that projects medially to meet on the midline. The caudal palatine foramen, foramen palatinum caudale, perforates or notches the caudolateral border of the horizontal lamina, just caudal to the last molar tooth (Fig. 28). The small major palatine foramen, foramen palatinum majus, is present on the rostral part of the horizontal lamina. The lamina fuses rostrally and laterally with the palatine and alveolar processes of the maxilla respectively. The perpendicular lamina of the palatine bone projects dorsally and caudally. It forms the ventromedial wall of the bony orbit and the medial wall of the rostral alar foramen and orbital fissure (the lateral walls are formed by the wing of the basisphenoid bone). The caudal sphenopalatine foramen lies along the fissure between the palatine, maxillary and frontal bones. The perpendicular lamina meets rostrally with the maxilla, dorsally with the frontal and presphenoid bones, and caudally with the basisphenoid bone.

The zygomatic bone, os zygomaticum (Figs. 2-5, 2-7 and 2-10), is also known as the jugal or malar bone. It is paired and spans the space between the zygomatic process of the maxilla and the zygomatic process of the temporal bone to complete the $\mathrm{zy}$ gomatic arch. The rostral end of the zygomatic bone joins the maxilla and lacrimal bones, and the caudal end joins the temporal bone.

The mandible, mandibula (Figs. 2-12 to 2-14), consists of a body and a ramus. The body, corpus mandibulae, has incisive and molar parts. The left and right incisive parts fuse rostrally to form a loose symphysis (the woodchuck can abduct the lower incisors when threatened). The symphysis is so placed that the two halves of the mandible diverge 


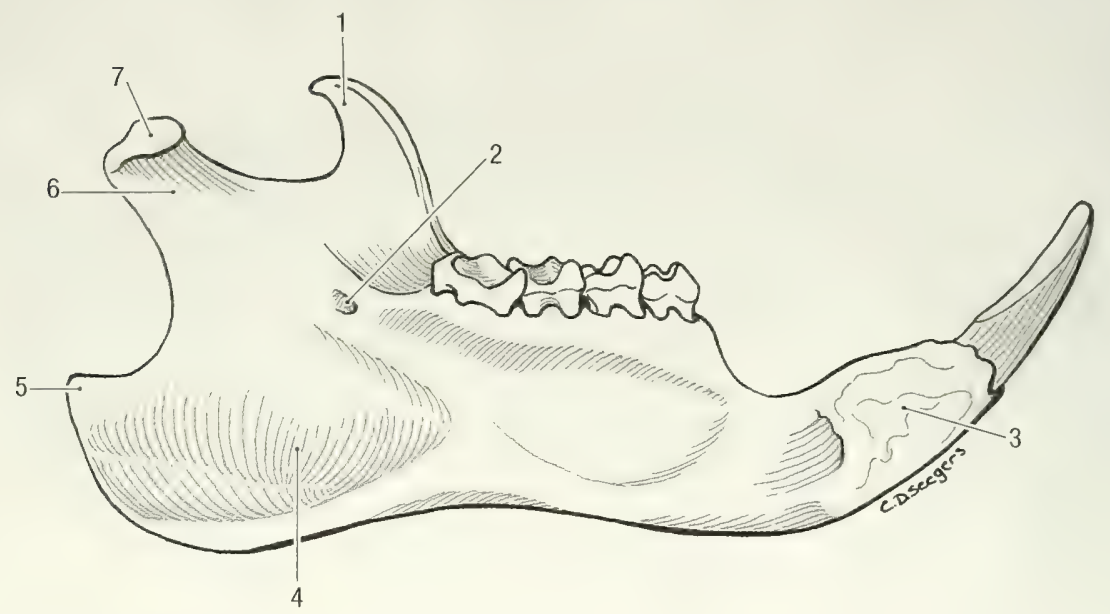

Fig. 2-13. Left mandible, medial view. 1 coronoid process, 2 mandibular foramen, 3 symphysis, 4 pterygoid fossa, 5 angular process, 6 pterygoid fovea, 7 articular condyle.

from each other caudally. The body of the mandible carries the alveolus for the lower incisor tooth. The latter is embedded along the length of the ventral part of the body. Between the incisor tooth and the first premolar tooth is a large interalveolar space (diastema). The mental foramen, foramen mentale, is located along the lateral border of the incisive part, just rostral to the roots of the first premolar tooth. The molar part carries the sockets for the molar and premolar teeth. Although the left and

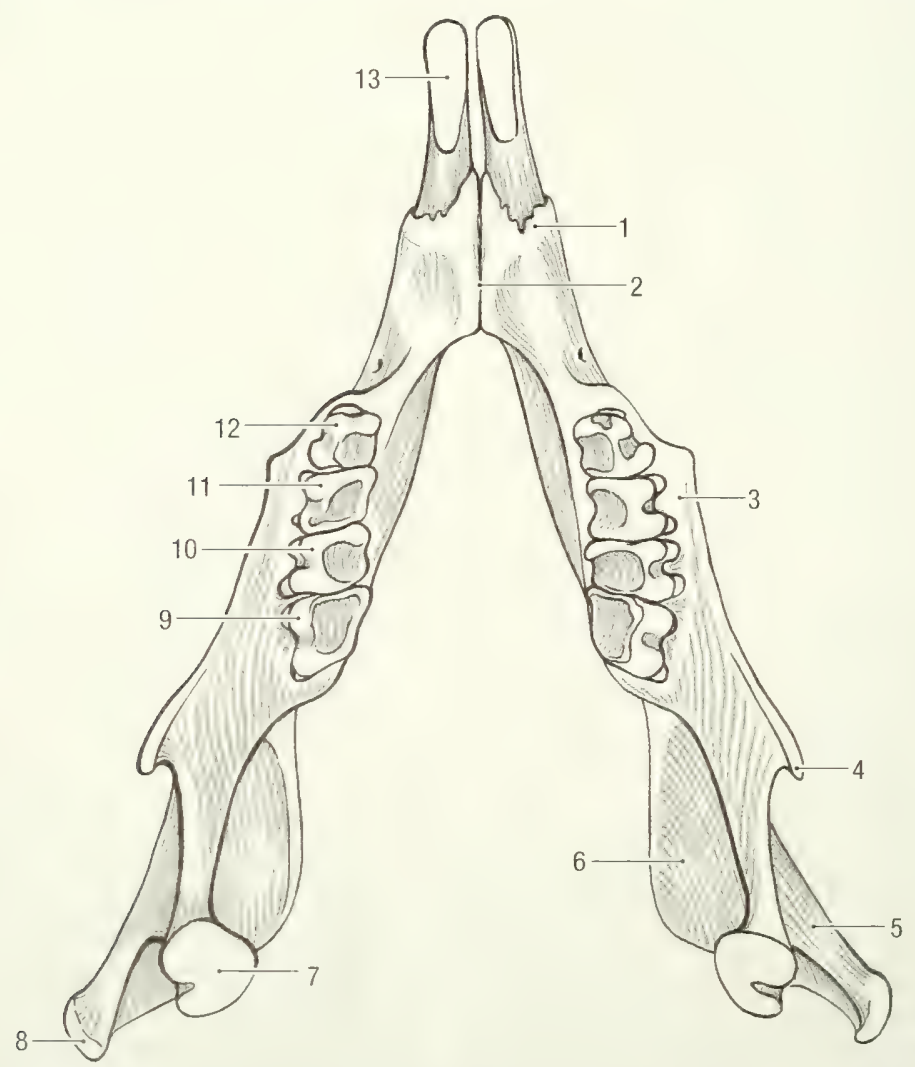

Fig. 2-14. Mandible, dorsal view. 1 incisive part of mandibular body, 2 symphysis, 3 molar part of mandibular body, 4 coronoid process, 5 masseteric fossa, 6 pterygoid fossa, 7 articular condyle, 8 angular process, 9 lower molar III, 10 lower molar II, 11 lower molar l, 12 lower premolar 4, 13 lower incisor. 


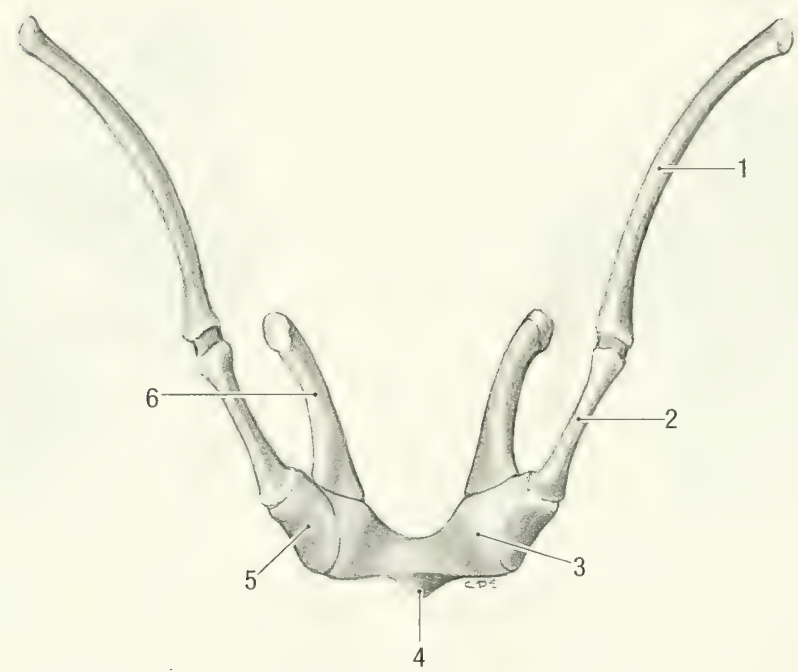

Fig. 2-15. Hyoid apparatus, rostral view. 1 stylohyoid, 2 epihyoid, 3 basihyoid, 4 lingual process, 5 ceratohyoid, 6 thyrohyoid.

right halves of the mandible diverge caudally, the molar parts are so placed that the two rows of cheek teeth are parallel to each other. The ramus of the mandible, ramus mandibulae, projects caudally and dorsally. Its lateral surface bears a large masseteric fossa, fossa masseterica (Fig. 2-12), for the attachment of the masseter muscle. Its medial surface bears a smaller but more distinct pterygoid fossa, fossa pterygoidea (Fig. 2-13), for the attachment of the medial pterygoid muscle. The caudal border is convex and thickened to form a large angular process, processus angularis. The dorsal border bears coronoid and articular processes. The coronoid process, processus coronoideus, inclines caudally and gives attachment to the temporal muscle. The condylar process, processus condylaris, lies caudal to the coronoid process and bears the rounded articular condyle, caput mandibulae, for articulation with the mandibular fossa of the squamous temporal bone. The mandibular foramen, foramen mandibulae (Fig. $2-13$ ), lies dorsal to the pterygoid fossa and caudal to the last molar tooth. A mandibular canal, canalis mandibulae, connects the mandibular and mental foramina.

Teeth, dentes, are discussed with the digestive system.

The hyoid apparatus, apparatus hyoideus (Fig. 215), consists of paired stylohyoid, epihyoid, ceratohyoid and thyrohyoid bones and a median basihyoid bone which bears a very small lingual process. The stylohyoid bone is attached to the temporal bone distal to the stylomastoid foramen.

\section{VERTEBRAL COLUMN, COLUMNA VERTEBRALIS}

The vertebral column (Fig. 2-1) is short and stout. It consists of seven cervical, twelve or thirteen thoracic, six or seven lumbar, four sacral and nineteen caudal vertebrae (7C, 12-13T, 6-7L, 4S, 19CD, total number of vertebrae 55-60).

A vertebra (Fig. 2-18) consists of a body, corpus vertebrae, with paired transverse processes and a dorsal vertebral arch, arcus vertebrae, that carries a spinous processes. The body has cranial and caudal articulating surfaces that are separated from each other by fibrous intervertebral discs, discus intervertebralis, which allow for some mobility.

The vertebral arch is composed of two pedicles, pediculus arcus vertebrae, and two laminae, lamina arcus vertebrae, that completes the arch dorsally, forming a vertebral foramen, foramen vertebrale. Successive vertebral foramina form the vertebral canal, canalis vertebralis, which contains the spinal cord. The vertebral arch has two transverse processes, processus transversus, and a single spinous process, processus spinosus. Two cranial and two caudal articular processes, processus articularis cranialis et caudalis, bears facets by which successive vertebrae articulate with each other. Each vertebral arch bears cranial and caudal vertebral incisures, incisura vertebralis cranialis, and incisura vertebralis caudalis. Together, the caudal incisure of one vertebra and the cranial incisure of the following vertebra form an intervertebral foramen, foramen intervertebrale. Intervertebral foramina are therefore present between the vertebral arches of successive vertebrae, allowing for the passage of nerves and blood vessels.

Atlas. The first cervical vertebra, C1 or atlas (Fig. 2-16), is highly modified. It consists of two lateral wings, a dorsal arch and a ventral body also called the "ventral arch". (This ventral portion of 


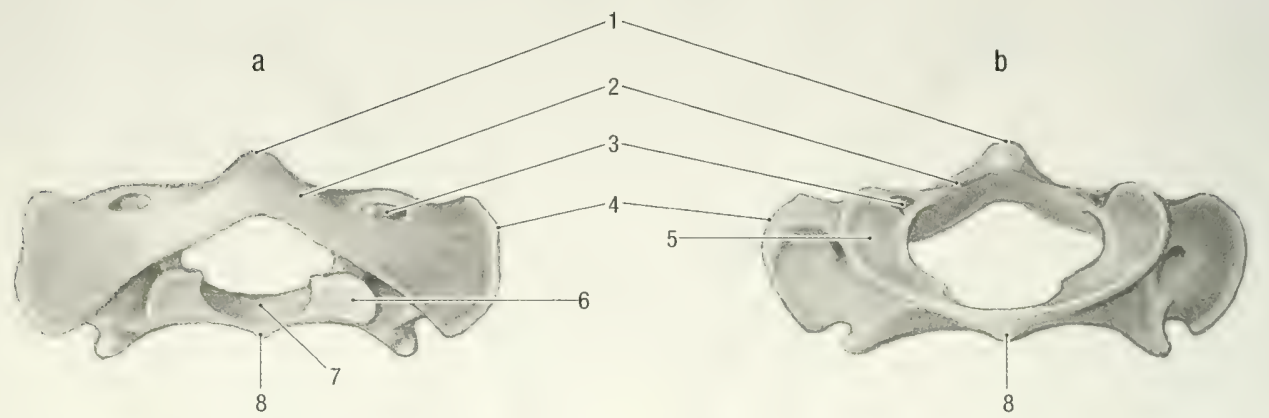

Fig. 2-16a, Atlas, caudal view, Fig. 2-16b, Atlas, cranial view. 1 dorsal tubercle, 2 dorsal arch, 3 alar foramen, 4 wing, 5 cranial articular surface, 6 caudal articular surface, 7 articular surface for the dens of the axis, 8 ventral arch or body.

the atlas is only half of the embryological centrum since the other half becomes the dens of the axis.) The cranial and caudal extremities of the atlas are modified to form articular surfaces, fovea articularis cranialis and fovea articularis caudalis, for articulation with the occipital condyles and axis respectively. The wing of the atlas, ala atlantis, bears the alar foramen, foramen alare, close to its cranial border. A small tubercle is present between the wing and the caudal articular process. The body has an articular fovea, fovea dentis, for articulation with the axis, while the dorsal arch bears a distinct dorsal tubercle. A fairly large interarcual space is present dorsally between the skull and the atlas.

Axis. The second cervical vertebra, $2 \mathrm{C}$ or axis (Fig. 2-17), is composed of several embryological elements not present in the atlas. The cranial extremity of the body of the axis projects into the atlas and bears a pointed projection or dens which represents one half of the embryological body of the atlas. The transverse process has a foramen, fo-

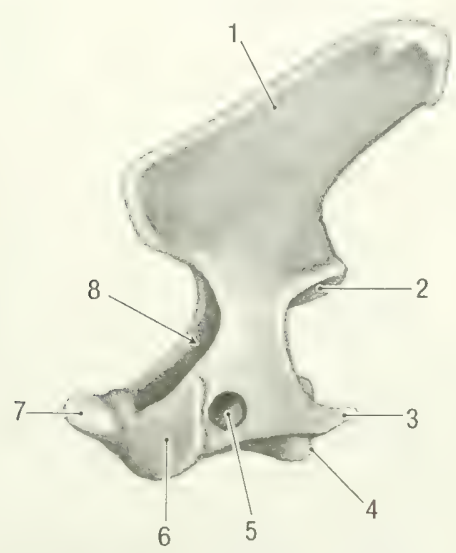

Fig. 2-17. Axis, left lateral view. 1 spinous process, 2 caudal articular facet, 3 transverse process, 4 caudal extremity, 5 transverse foramen, 6 cranial extremity, 7 dens, 8 vertebral foramen. ramen transversarium, and the long spinous process inclines caudally.

3-7C. The bodies of the third to seventh cervical vertebrae (Fig. 2-18) are similar to each other. They are compressed and the spinous processes are reduced to tubercles. The transverse processes of $3 \mathrm{C}$ to $6 \mathrm{C}$ have transverse foramina. Furthermore, the transverse process of $6 \mathrm{C}$ is expanded to form a ventral lamina, lamina ventralis. The caudal extremity of $7 \mathrm{C}$ bears articular foveae for the heads of the first ribs.

Thoracic Vertebrae. The thoracic vertebrae, vertebrae thoracicae (Fig. 2-19), are modified to carry ribs. The spinous processes are high and caudally inclined, the transverse processes are reduced and carry articulation facets, fovea costalis processus transversi, for articulation with the tubercles of the ribs. The bodies have cranial and caudal articular foveae, fovea costalis cranialis, and fovea costalis cauaalis, for articulation with the heads of the ribs. The tenth thoracic vertebra $(10 \mathrm{~T})$ is the anticlinal vertebra with its spine directed cranially. An accessory processes, processus accessorius, is present between the transverse and caudal articular process of 10-

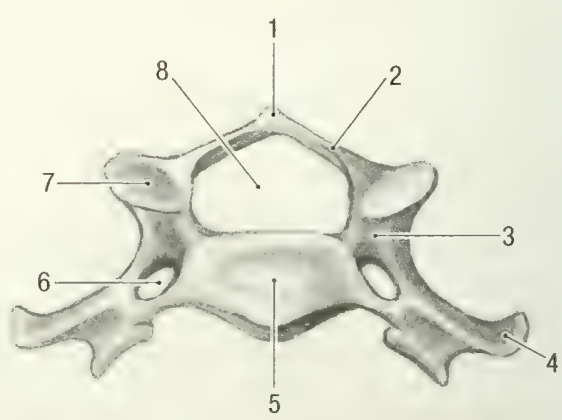

Fig. 2-18. Cervical vertebra, cranial view. 1 spinous process, 2 lamina, 3 pedicle, 4 transverse process, 5 cranial extremity, 6 transverse foramen, 7 cranial articular facet, 8 vertebral foramen. 


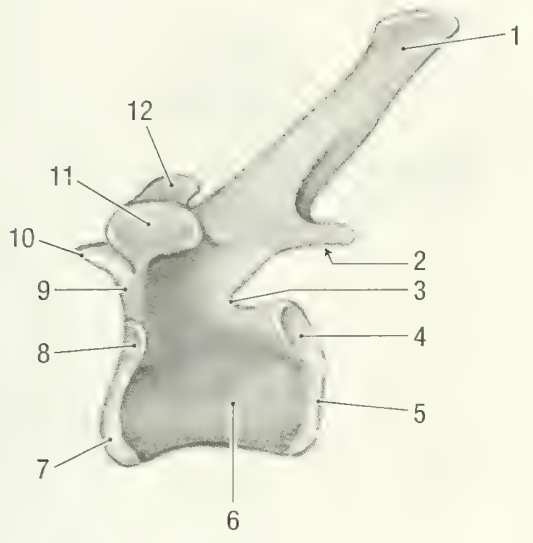

Fig. 2-19. Thoracic vertebra, left lateral view. 1 spinous process, 2 caudal articular facet, 3 caudal vertebral incisure, 4 caudal costal fovea, 5 caudal extremity, 6 body, 7 cranial extremity, 8 cranial costal fovea, 9 cranial vertebral incisure, 10 cranial articular facet, 11 costal fovea on the transverse process, 12 mamillary process.

13T, and a large mammillary process, processus mamillaris (Fig. 2-19), is present between the cranial articular and transverse processes of $6-12 \mathrm{~T}$.

Lumbar Vertebrae. Lumbar vertebrae, vertebrae lumbales (Fig. 2-20), are large and robust compared with other vertebrae. They have well-developed spinous processes that increase in length from cranial to caudal. The strong transverse processes incline ventrocranially, and they get larger from cranial to caudal in the series. Accessory and mammillary processes are present on 1-6L. The articular processes of the lumbar vertebrae are placed sagittally to allow maximum flexion and extension of the vertebral column.

Sacral Vertebrae. Sacral vertebrae, four in number, are fused as the sacrum or os sacrale (Figs. 2-21 to 2-23). This compound bone is triangular in shape. The base, basis ossis sacri, is cranial and the apex, apex ossis sacri, is caudal. There are dorsal and pelvic surfaces. The dorsal surface is characterized by fusion of the various processes. In older animals the tips of the spinous processes fuse to form a median sacral crest, crista sacralis mediana (Fig. 221). The transverse processes fuse to form the large lateral part of the sacrum. The lateral part carries the wing, ala sacralis, which is formed by the fused transverse processes of the first two sacral vertebrae. The dorsolateral part of the sacral wing is roughened and forms the auricular face, facies auricularis, (Fig. 2-21) which articulates with the wing of the ilium. The articular processes fuse to form an intermediate crest, crista sacralis intermedia. Due to the extensive fusion of the vertebrae, the three pairs of intervertebral foramina separate into dorsal fo-

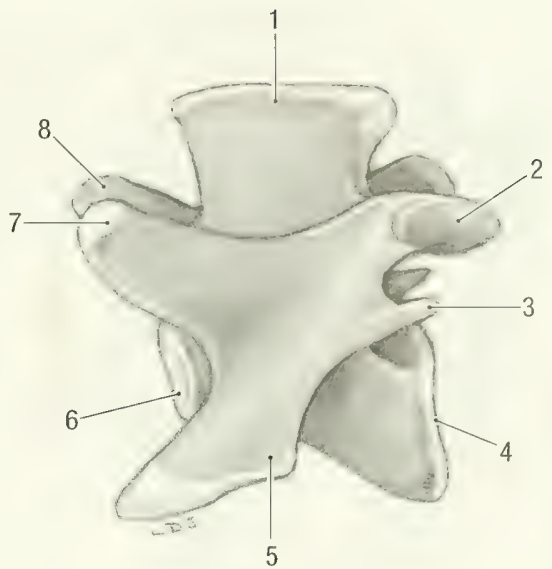

Fig. 2-20. Lumbar vertebra, left lateral view. 1 spinous process, 2 caudal articular facet, 3 accessory process, 4 caudal extremity, 5 transverse process, 6 cranial extremity, 7 mamillary process, 8 cranial articular facet.

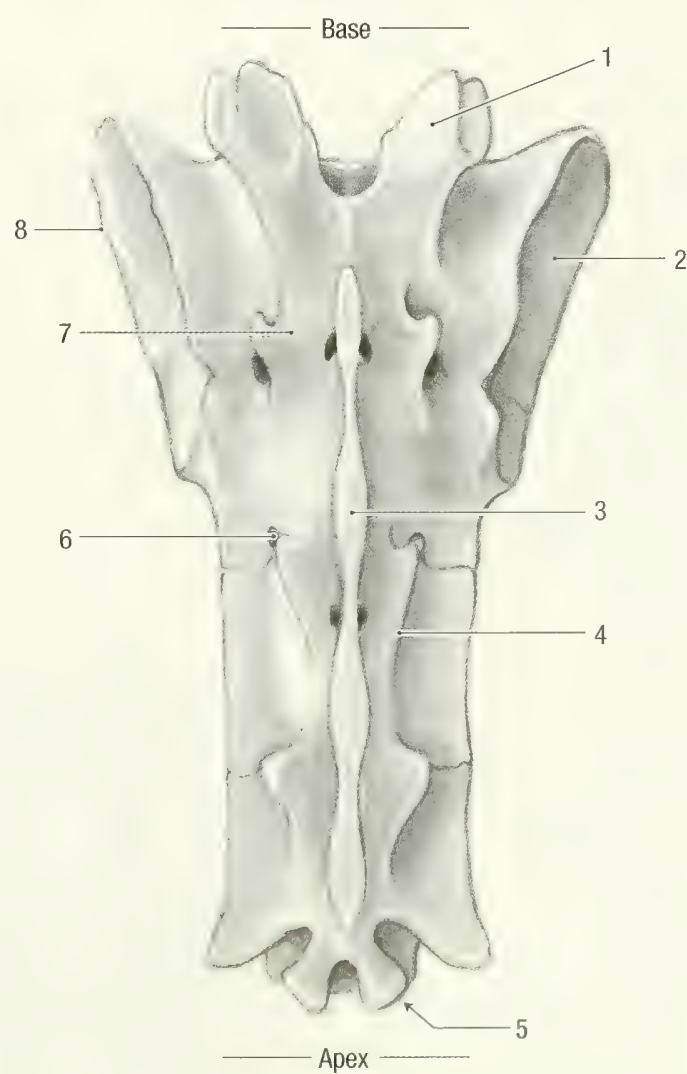

Fig. 2-21. Sacrum, dorsal view. 1 cranial articular facet, 2 auricular face, 3 median sacral crest, 4 intermediate sacral crest, 5 caudal articular facet, 6 dorsal sacral foramen, 7 lateral part, 8 wing. 


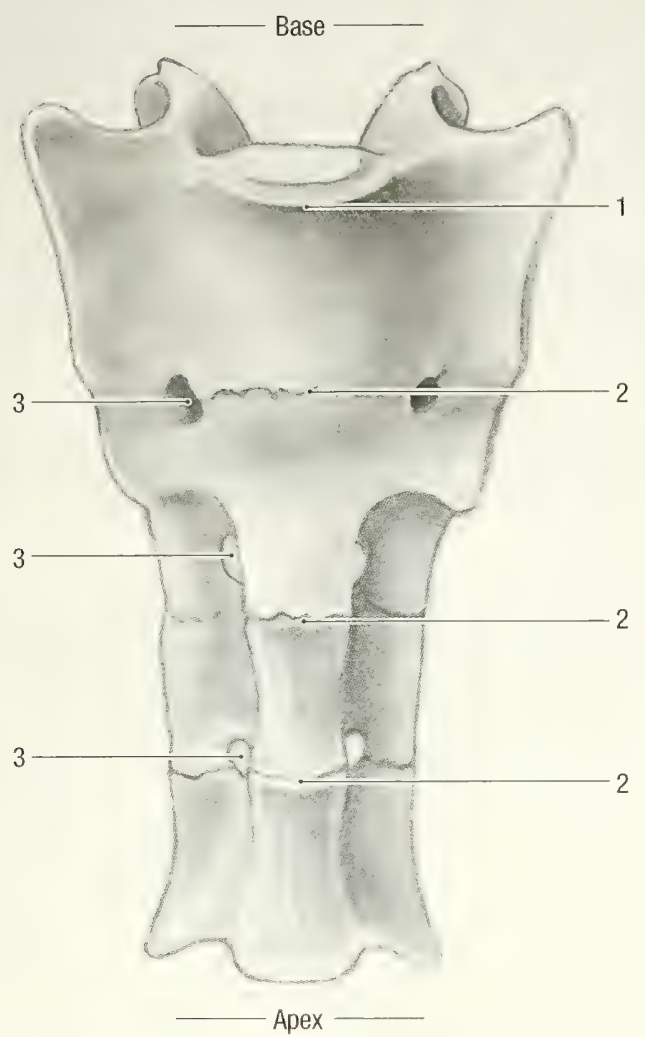

Fig. 2-22. Sacrum, ventral view. 1 promontory, 2 transverse lines, 3 ventral sacral foramina.

ramina, foramina sacralia dorsalia, on the dorsal surface, and ventral foramina, foramina sacralia ventralia, on the pelvic surface (Figs. 2-21 and 222). The bodies of the sacral vertebrae fuse and form three transverse lines, lineae transversae (Figs. 2-22 and 2-23) on the ventral (pelvic) surface. The cranioventral edge of the first sacral vertebra forms the sacral promontory, promontorium, lies at the level of the acetabulum.

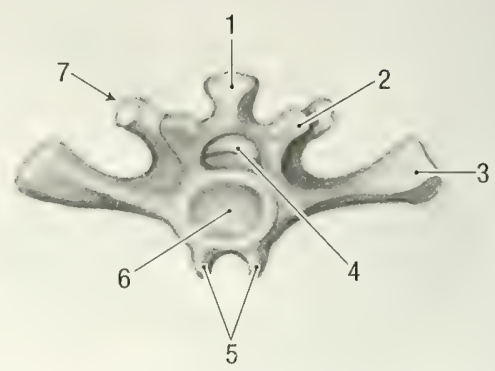

Fig. 2-24. First caudal vertebra, caudal view. 1 spinous process, 2 caudal articular process, 3 transverse process, 4 vertebral foramen, 5 ventral hemal processes, 6 caudal extremity, 7 cranial articular process.

Caudal Vertebrae. The caudal vertebrae, vertebrae caudales (Fig. 2-24), of the tail may vary in number, but usually 19 are present. The first few are typical vertebrae, but gradually lose their features until only the bodies remain as rounded rods. 1-4Cd has ventral hemal processes, processus hemalis.

\section{STERNUM}

The sternum (Fig. 2-25) consists of six bony segments or sternebrae that are joined by cartilage as synchondroses sternales. The first sternebra or manubrium sterni, is the largest. It is rod-shaped with a laterally expanded cranial part for articulation with the clavicle and the first rib. The manubrium lacks a manubrial cartilage. Succeeding sternebrae are also rod shaped but get progressively shorter caudally. The last sternebra has a xiphoid process, processus xiphoideus, which bears an anchor- or paddle-shaped xiphoid cartilage, cartilago xiphoidea. The costal cartilages of the first seven ribs articulate with the sternum at the level of the intersternebral cartilages.

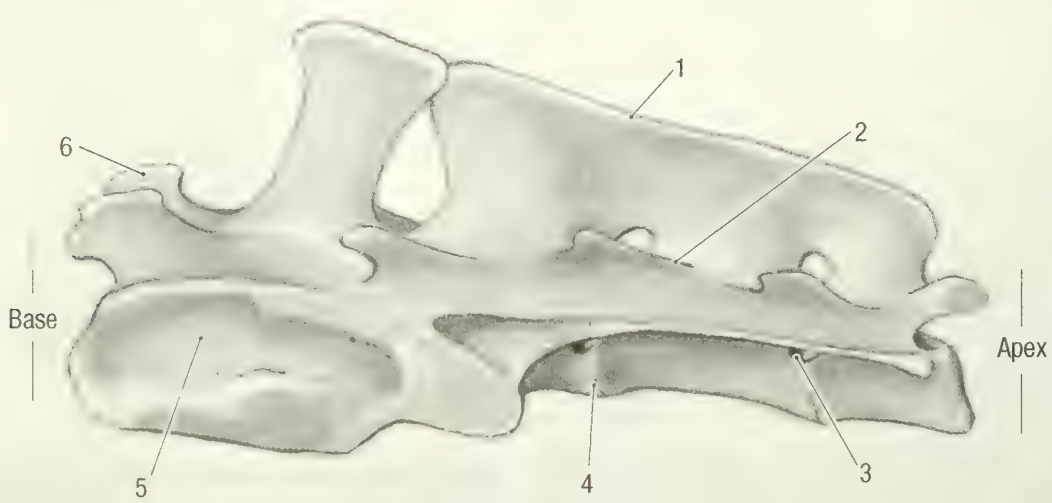

Fig. 2-23. Sacrum, left lateral view. 1 median sacral crest, 2 intermediate sacral crest, 3 ventral sacral foramen, 4 transverse line, 5 auricular face, 6 cranial articular facet. 


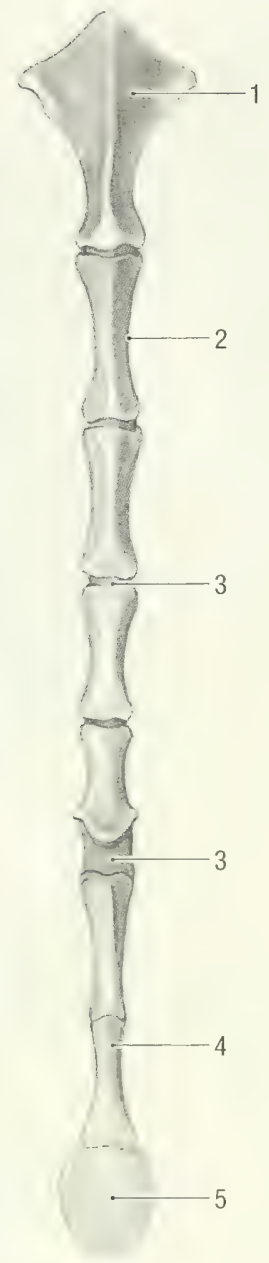

Fig. 2-25. Sternum, ventral view. 1 manubrium, 2 sternabra, 3 sternal synchondrosis, 4 xiphoid process, 5 xiphoid cartilage.

\section{RIBS}

There are 12 or 13 pairs of thoracic ribs, costae (Figs. 2-1 and 2-26). Each of the first 7 pairs articulate with the sternum by means of a costal cartilage, cartilago costalis. They are true ribs, costae verae, also called sternal ribs, costae sternales. The cartilages of the last five pairs of ribs attach to each other and to the cartilage of the seventh rib to form a costal arch, arcus costalis. They are false ribs, costae spuriae, also known as asternal ribs, costae asternales. The cartilages of the last pair of ribs often do not reach the costal arch and therefore are floating ribs, costae fluctuantes. The first rib is short and stout. The ribs get progressively longer caudally, reaching their maximum length at the seventh pair. The ribs and costal cartilages are of equal length.

A typical rib consists of a head, caput costae, a neck, collum costae, and a body, corpus costae. The head is rounded and articulates with two successive vertebral bodies. The neck is short and attaches the head to the body of the rib. The tubercle of the rib, tuberculum costae, projects dorsally from the junction of the neck and body, and articulates with the transverse process of the corresponding thoracic vertebra. The necks of the ribs get progressively shorter caudally, and the heads and tubercles move closer to each other. The last two pairs of ribs do not have tubercles. The body of the rib is rounded and the caudal surface of the proximal two thirds has a groove, sulcus costae, for the intercostal nerve and blood vessels. Distally the rib attaches to the costal cartilage, which forms a distinct bend, genu costae, before it articulates with the sternum. All the costochondral junctions that are caudal to the shoulder joint lie on the same horizontal plane (about $15 \mathrm{~mm}$ ventral to the shoulder joint).

\section{APPENDICULAR SKELETON}

The appendicular skeleton includes the bones of the limbs and the limb girdles.

Shoulder Girdle. The shoulder girdle, cingulum membri thoracici, consists of the scapula and a well developed clavicle. The scapula is attached to the trunk by muscles whereas the clavicle articulates with the manubrium of the sternum and with the acromion of the scapula (Fig. 2-1).

Scapula. The scapula, or shoulder blade, is roughly triangular in shape. It has lateral and costal surfaces, dorsal, caudal and cranial margins, and caudal, ventral and cranial angles.

The lateral surface, facies lateralis (Fig. 2-27), is divided by the spine of the scapula, spina scapulae, into a larger supraspinous fossa, fossa supraspinata, and a smaller infraspinous fossa, fossa infraspinata. The scapular spine extends over the entire length of the scapula. Distally, the free border of the spine curves caudally and expands to form an acromion, acromion. The proximal part of the acromion projects caudally and the distal part projects cranioventrally.

The costal surface, facies costalis (Fig. 2-28), consists of a small, serrated face, facies serrata, along the dorsal border and a large subscapular fossa, fossa subscapularis. Two longitudinal ridges divide the latter into three approximately equal parts. Distally (at the ventral angle) the scapula narrows to form a neck and then expands to form a shallow, hollow, articular surface, the glenoid cavity, cavitas glenoidalis. Cranial to the glenoid cavity is a large supraglenoid tubercle, tuberculum supraglenoidale, which bears the medio-ventrally directed coracoid process, processus coracoideus. The articular surface of the glenoid cavity encroaches unto and is enlarged by the supraglenoid tubercle. 


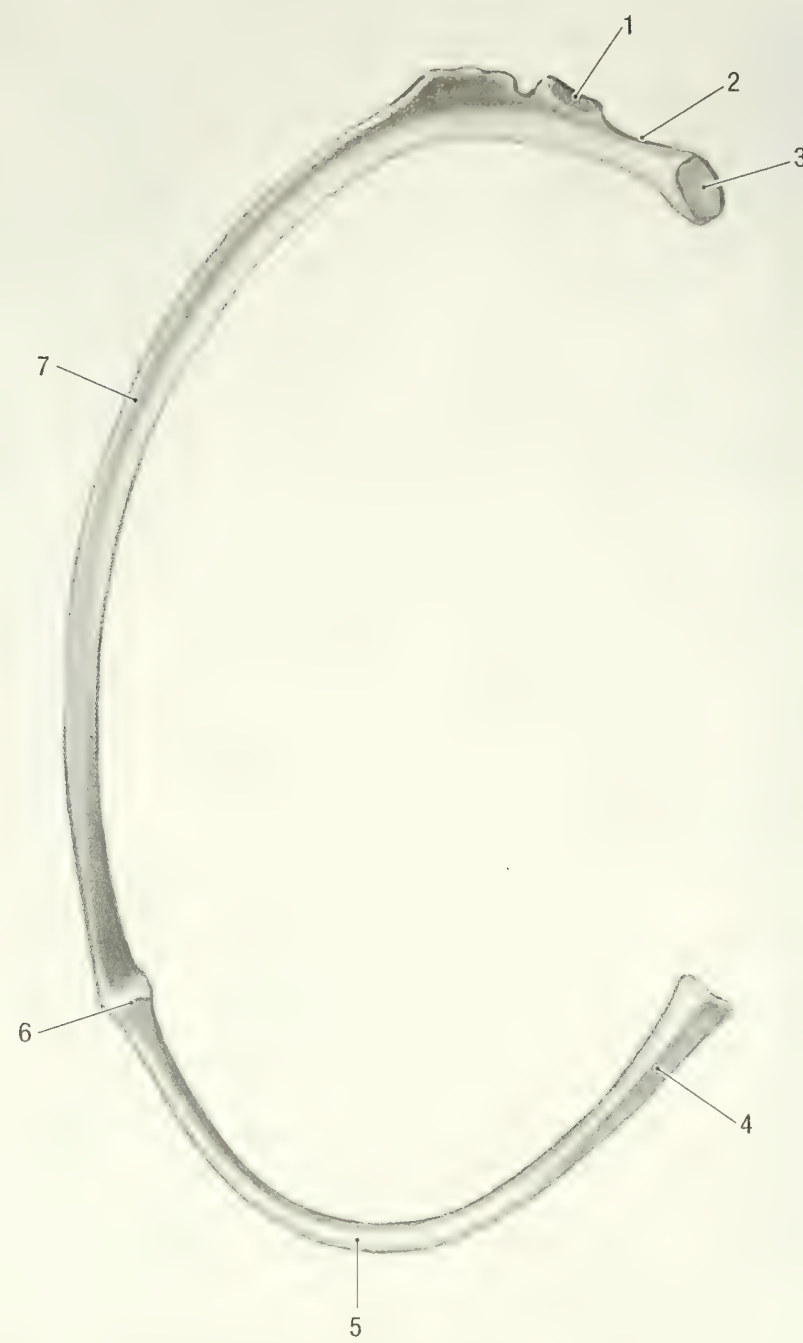

Fig. 2-26. Left rib, caudal view. 1 costal tubercle, 2 neck, 3 head, 4 costal cartilage, 5 genu, 6 costochondral junction, 7 costal groove.

Clavicula. The clavicle is a stout, somewhat bent bone (Figs. 2-1 and 2-29). Both ends are enlarged for articulation with the manubrium of the sternum and with the acromion of the scapula.

Thoracic Limb. The skeleton of the thoracic limb consists of the humerus, radius and ulna, carpals, metacarpals, and digital bones.

Humerus. The humerus, humerus (Figs. 2-30 and 2-31), is the bone of the brachium or upper arm. It articulates proximally with the scapula at the shoulder joint, and distally with the radius and ulna at the elbow joint. The humerus consists of two extremities and a body or shaft.

The proximal extremity consists of a head, caput humeri, which bears a large, oval articular surface, and two tubercles. The major tubercle, tuberculum majus, large and lies craniolateral to the head. The minor tubercle, tuberculum minus, is small and lies medial to the head. The two tubercles are separated cranially from each other by a shallow intertubercular groove, sulcus intertubercularis (Fig. 2-30), for the passage of the tendon of the biceps muscle.

The body of the humerus, corpus humeri, is rounded and has cranial, caudal, medial and lateral surfaces. It bears a large deltoid tubercle, tuberositas deltoidea, on the proximal cranial surface, and a small teres tubercle, tuberositas teres major, on the medial surface. A distinct ridge, crista humeri, extends along the caudal surface from the minor tubercle to the lateral epicondyle. This ridges spirals from medial to lateral, forming a distinct groove, sulcus $m$. brachialis, for the brachial muscle. The distal part of the shaft widens laterally to form a 


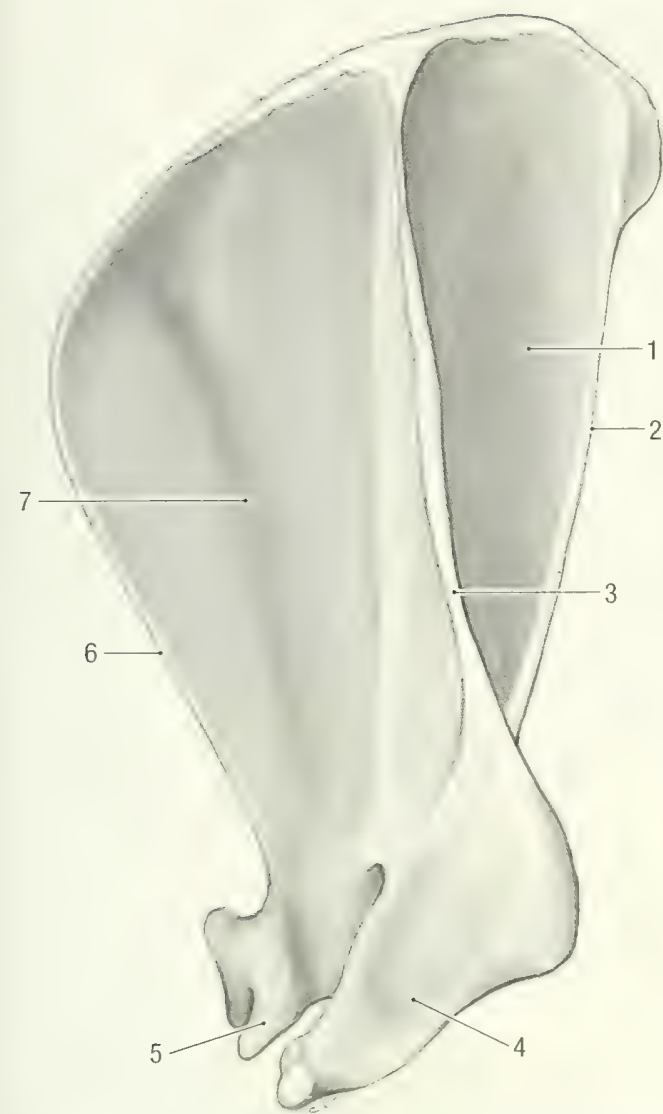

Fig. 2-27. Left scapula, lateral view. 1 infraspinous fossa, 2 caudal border, 3 scapular spine, 4 acromion, 5 supraglenoid tubercle, 6 cranial border, 7 supraspinous fossa, 8 dorsal border.

flat shelf of bone, crista epicondylus lateralis, which extends distally onto the lateral epicondyle.

The distal extremity consists of a condyle, condylus humeri, which bear the articular surface, and two epicondyles, epicondylus lateralis and epicondylus medialis (Fig. 2-31). The condyle, which consists of a trochlea and a capitulum, articulates with the radius and ulna. There is, however, no clear distinction between the trochlea and capitulum. Proximal to the condyle the caudal surface bears an olecranon fossa, fossa olecrani, and the cranial surface a radial fossa, fossa radialis (Fig. 2-30). A thin plate of bone separates the radial and olecranon fossae from each other. The latter is incomplete in some animals, in which case it bears a supratrochlear foramen, foramen supratrochleare. The medial epicondyle is large and projects medial to the condyle. In some animals it bears a supracondylar foramen, foramen supracondylare, for the passage of blood vessels and nerves.

The radius and ulna form the bones of the antebrachium or forearm. They articulate at the elbow

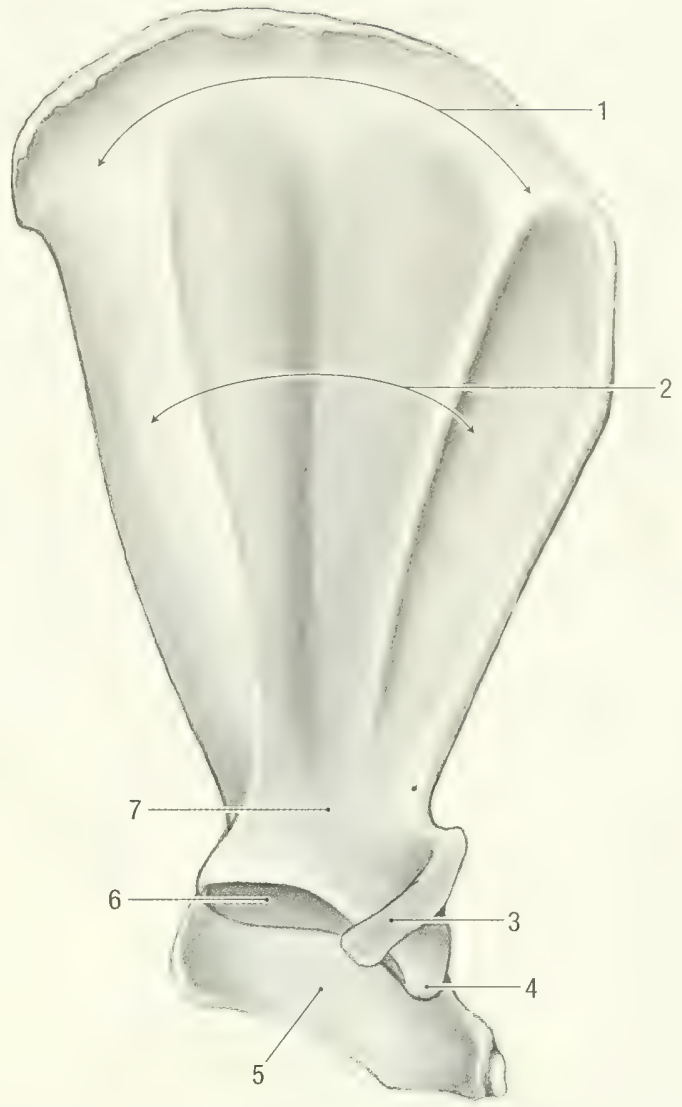

Fig. 2-28. Left scapula, medial view. 1 serrated face, 2 subscapular fossa, 3 coracoid process, 4 supraglenoid tubercle, 5 acromion, 6 glenoid cavity, 7 neck.

with the humerus and with each other. At the carpus the articulate with each other and with the proximal carpal bones.

Radius. The radius (Figs. 2-32 and 2-33) is shorter and smaller than the ulna. It has two extremities and a shaft, with cranial, caudal, lateral and medial margins and surfaces. The proximal extremity of the radius consists of a small, rounded head, caput radii, which bears an articular surface, fovea capitis radii, for the condyle of the humerus, and an articular circumference, circumferentia articularis, along its caudal surface for articulation with the ulna. A short, indistinct neck, collum radii, attaches the head to the shaft. The neck bears a small radial tuberosity, tuberositas radii, on its caudomedial surface. The shaft of the radius, corpus radii, is round and widens distally. The distal extremity consists of a radial trochlea, trochlea radii. The trochlea has a single, undivided articular surface, facies articularis carpea, for articulation with the intermedioradial carpal bone, a small articulation facet for the ulna on its lateral surface, and a styloid 


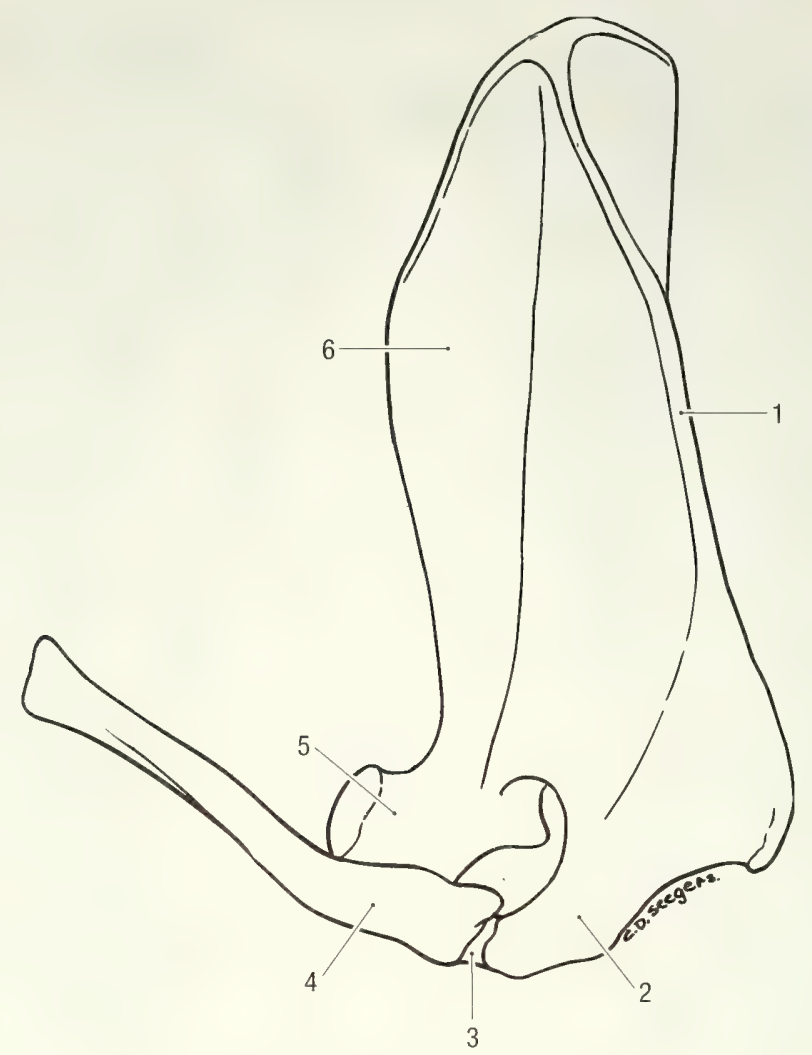

Fig. 2-29. Left scapula and clavicle, craniolateral view. 1 scapular spine, 2 acromion, 3 acromio-clavicular ligament, 4 clavicle, 5 supraglenoid tubercle, 6 supraspinous fossa.

process, processus styloideus, on the medial surface. It has a shallow groove dorsally for the extensor tendons.

Ulna. The ulna (Fig. 2-34) is the longest bone of the thoracic limb. It consists of an olecranon, a shaft, and a head, with lateral, medial, caudal and cranial surfaces and margins.

The olecranon is the proximal extremity of the ulna. Its cranial margin has a trochlear incisure, incisura trochlearis, for articulation with the trochlea of the humerus. The trochlear incisure ends proximally on the anconeal process, processus anconeus, and distally on the large medial coronoid and smaller lateral coronoid processes, processus coronoideus medialis, and processus coronoideus lateralis, respectively. The radial incisure, incisura radialis, for articulation with the head of the radius lies between the lateral and medial coronoid processes. The proximal end of the olecranon is expanded to form the olecranon tuber, tuber olecrani. The shaft of the ulna, corpus ulnae, is slightly curved and heavier than that of the radius.

The head of the ulna, caput ulnae, is the distal extremity of the ulna. It carries an articular facet for the radius medially and a styloid process, dis- tally, that articulates with the ulnar and accessory carpal bones.

Carpus. The nine carpal bones, ossa carpi (Figs. 2-35 and 2-36), of the carpus are arranged in three rows. The proximal row consists of the intermedioradial, ulnar and accessory carpal bones, the middle row of the central carpal bone, and the distal row of four carpal bones. The intermedioradial carpal bone (scapholunatum) represents the fused radial, os carpi radiale (os scaphoidum), and intermediate, os carpi intermedium (os lunatum), carpal bones. It is the largest bone in the proximal row, and lies medially, opposite the first three digits. It articulates proximally with the radius, laterally with the ulnar and accessory carpal bones, and distally with the central and first three carpal bones. The ulnar carpal bone, os carpi ulnare (os triquetrum), articulates palmarly with the accessory carpal bone, medially with the intermedioradial carpal bone, and distally with the fifth carpal bone. The accessory carpal bone, os carpi accessorium (os pisiforme) (Fig. 2-36), lies caudal to the other carpal bones. It articulates with the ulnar and radial carpal bones.

The central carpal bone, os carpi centrale, is a small, triangular bone between the proximal and 


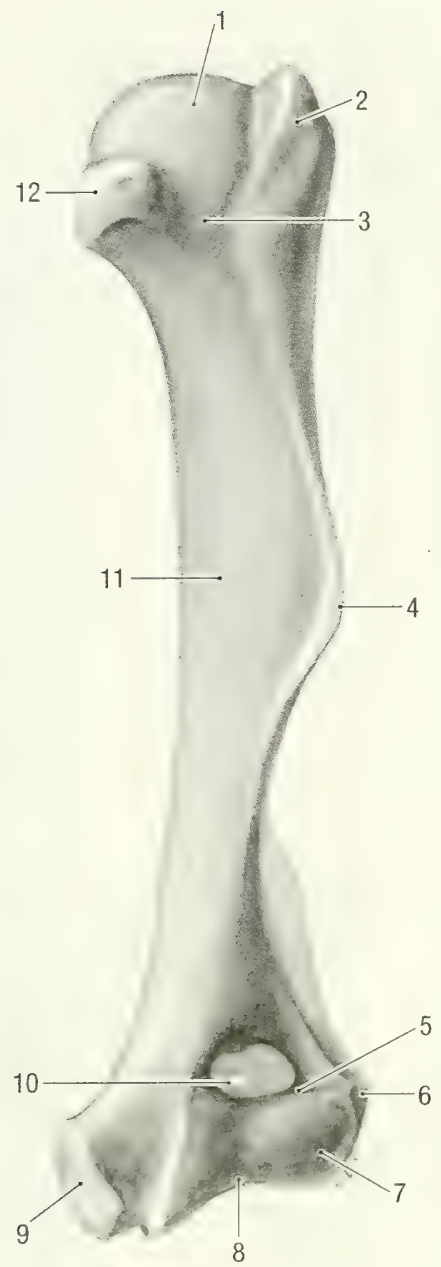

Fig. 2-30. Left humerus, cranial view. 1 head, 2 major tubercle, 3 intertubercular groove, 4 deltoid tuberosity, 5 radial fossa, 6 lateral epicondyle, 7 trochlea, 8 medial epicondyle, 9 supratrochlear foramen, 10 shaft, 11 minor tubercle.

distal rows of carpal bones. It articulates proximally with the intermedioradial carpal bone, medially with the second carpal and second metacarpal bones and laterally with the third carpal bone. The fourth carpal is the largest bone in the distal row, and the second carpal bone is the smallest.

The first carpal bone, os carpale 1 (os trapezium), is elongated and articulates proximally with the intermedioradial carpal bone, medially with the carpal sesamoid bone and laterally with the second carpal and metacarpal bones.

The second carpal bone, os carpale II (os trapezoideum), articulates distally with the second metacarpal bone, medially with the first carpal bone, proximally with the intermedioradial carpal bone, and proximolaterally with the central carpal bone.

The third carpal bone, os carpale III (os capitatum), articulates distally with the third metacarpal

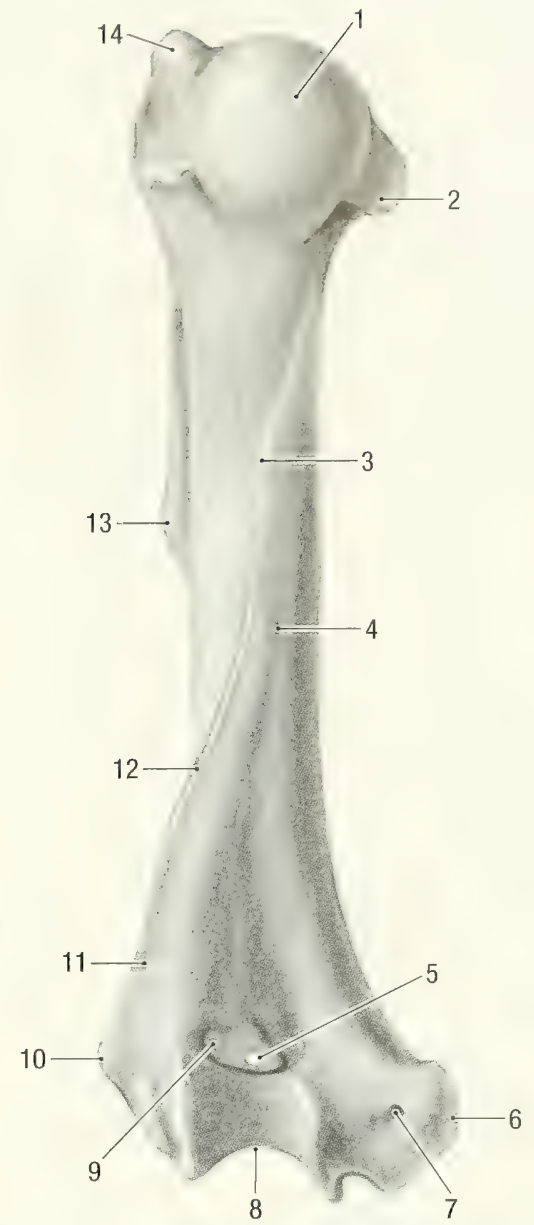

Fig. 2-31. Left humerus, caudal view. 1 head, 2 minor tubercle, 3 major teres tubercle, 4 shaft, 5 supratrochlear foramen, 6 medial epicondyle, 7 supracondylar foramen, 8 trochlea, 9 olecranon fossa, 10 lateral epicondyle, 11 lateral epicondylar crest, 12 humeral crest, 13 deltoid tuberosity, 14 major tubercle.

bone, proximo-medially with the central carpal bone, and laterally with the fourth metacarpal bone.

The fourth carpal bone, os carpale IV (os hamatum), is the largest and most lateral of the carpal bones in the distal row. It articulates distally with the fourth and fifth metacarpal bones, medially with the third carpal bone, and proximally with the ulnar carpal bone.

A large carpal sesamoid bone (radial sesamoid) is present in the medial carpal pad on the palmar aspect of the carpus (Fig. 2-36). It articulates distally with the first metacarpal bone, and proximally with the intermedioradial carpal bone. Its caudal, free end curves laterally to form the medial and caudal borders of the carpal canal.

Metacarpus. The five metacarpal bones, ossa metacarpalia $I-V$ (Figs. 2-35 and 2-36), in the ma- 


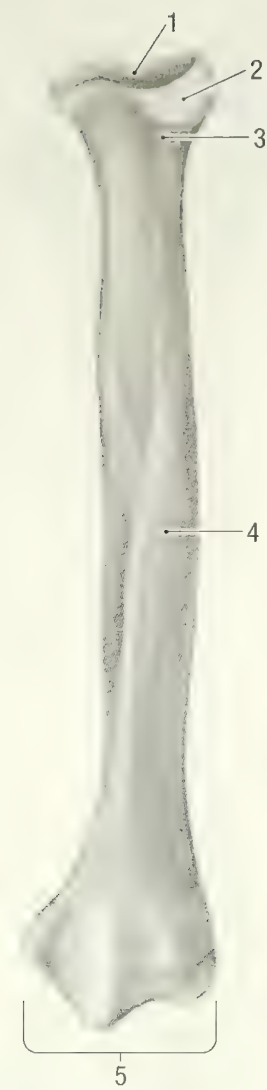

Fig. 2-32. Left radius, cranial view. 1 carpal articular surface, 2 head, 3 neck, 4 shaft, 5 trochlea.

nus all have a base, basis, that articulates with the carpal bones, an elongated and slightly flattened body, corpus, and a head, caput, that articulates with the first phalanges and with the proximal sesamoid bones. The first metacarpal bone is very short and almost square in outline. The third is the longest, the second and fourth are almost equal in length, and the fifth is slightly shorter than the previous two.

Digits. The phalanges, ossa digitorum manus (Figs. 2-35 and 2-38), form the five digits in the manus. Each digit has three phalanges, except the first digit that only has two. The phalanges are proximal, phalanx proximalis, middle, phalanx media, and distal, phalanx distalis. The proximal phalanx of each digit is the longest, and the distal phalanx the shortest. The first and second phalanges of digits $2-5$ consist of a base proximally, a body and a head distally. The proximal end of the distal phalanx bears a small extensor process, processus extensorius, dorsally, and a large flexor tubercle, tuberculum flexoria, on the palmar surface. The unguicular process, processus unguicularis, carries the nail or claw. A bony crest, crista unguicularis,

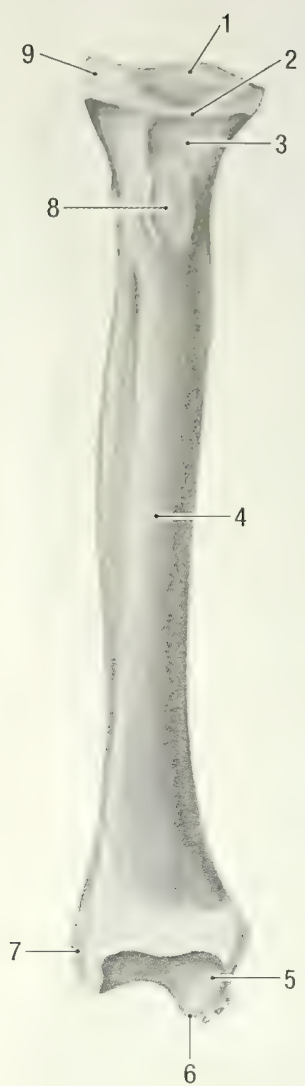

Fig. 2-33. Left radius, caudal view. 1 articular surface of head, 2 articular circumference, 3 neck, 4 shaft, 5 carpal articular surface, 6 medial styloid process, 7 articular surface for the ulna, 8 radial tuberosity, 9 head.

forms a groove, sulcus unguicularis, between the articular surface and the unguicular process. The groove houses the base of the nail. The flexor tubercle has axial and abaxial solear foramina for blood vessels and nerves.

Sesamoid Bones. Two proximal sesamoid bones, ossa sesamoidea proximalia, are present on the palmar surface of the metacarpophalangeal joints of digits 2-5 (Fig. 2-36). A distal sesamoid bone, os sesamoideum distale, is present on the palmar surfaces of the distal interphalangeal joints of digits $2-5$. The first digit does not have palmar sesamoid bones. However, a large sesamoid bone is sometimes present on the dorsal aspect of the interphalangeal joint.

Pelvic Girdle (Fig. 2-37). The pelvic girdle consists of the ilium, ischium, pubis and acetabular bone. The four bones fuse to form a hip bone, os coxae. Left and right ossa coxae fuse on the midline to form the pelvis. The pelvic girdle articulates medially with the wing of the sacrum and laterally with 


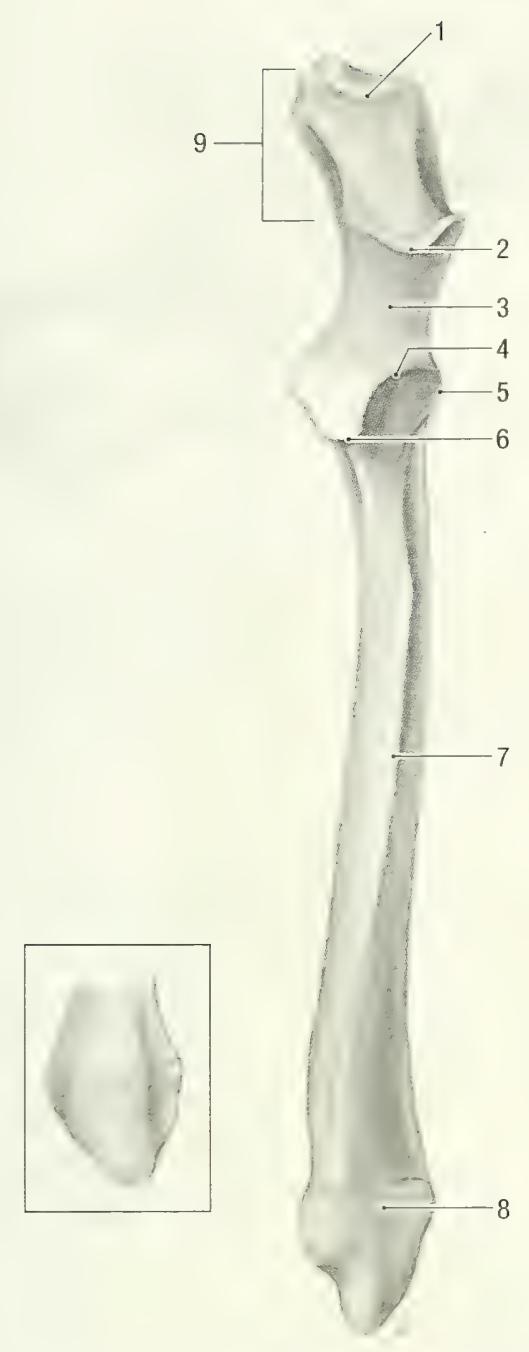

Fig. 2-34. Left ulna, cranial view. Insert, ulnar styloid process, caudal view. 1 olecranon tuber, 2 anconeal process, 3 trochlear incisure, 4 radial incisure, 5 lateral coronoid process, 6 medial coronoid process, 7 shaft, 8 head, 9 olecranon.

the head of the femur. The acetabulum, acetabulum, is a deep hollow on the lateral aspect of the os coxae for articulation with the head of the femur. The ilium, ischium and acetabular bones contribute to the formation of the acetabulum. A cartilaginous lip attached to the rim of the hollow deepens the acetabulum. The articular surface, $f a-$ cies lunata, is almost circular and caudoventrally incomplete. In the depth of the acetabulum is a small, roughened hollow, the acetabular fossa, fossa acetabuli, for the attachment of the ligament of the femoral head, ligamentum capitis ossis femoris. The caudoventral rim of the acetabulum bears a deep notch, the acetabular incisure, incisura acetabuli.

The acetabular bone, os acetabuli, is a small, tri-

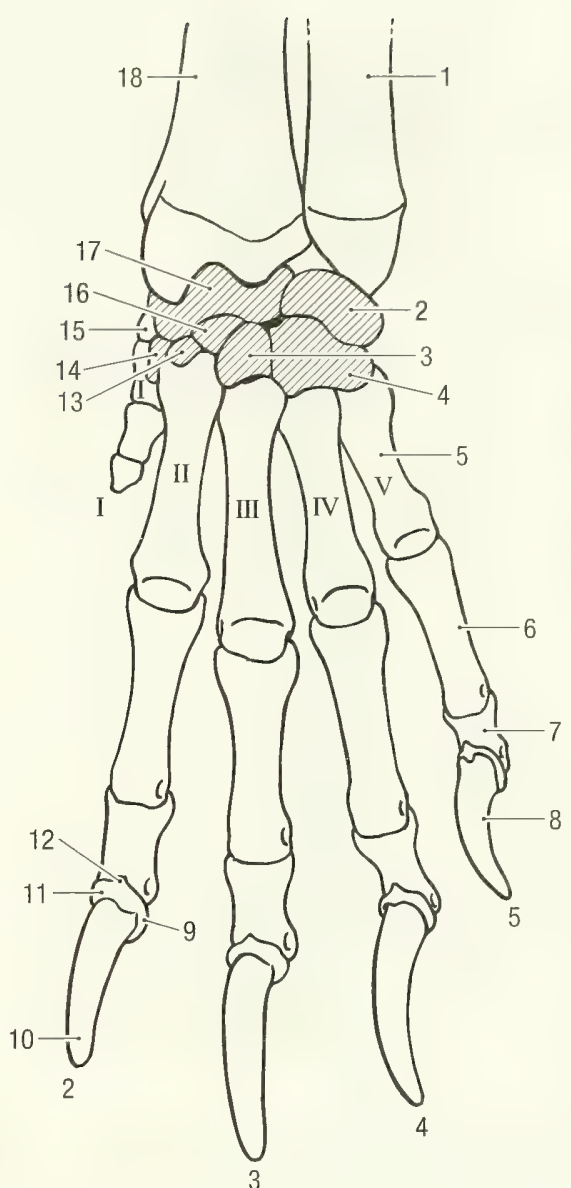

Fig. 2-35. Left forepaw, dorsal view. $1-\mathrm{V}=$ metacarpals, 1-5 = digits. 1 ulna, 2 ulnar carpal bone (triquetrum), 3 third carpal bone (capitatum), 4 fourth carpal bone (hamatum), 5 fifth metacarpal bone, 6 proximal phalanx of fifth digit, 7 middle phalanx of fifth digit, 8 distal phalanx of fifth digit, 9 unguicular crest, 10 unguicular process, 11 unguicular groove, 12 extensor process, 13 second carpal bone (trapezoideum), 14 first carpal bone (trapezium), 15 radial sesamoid, 16 central carpal bone, 17 intermedioradial carpal bone (scapholunatum), 18 radius.

angular bone located in the floor or ventral surface of the acetabulum. It fuses to the other bones of the os coxae to such an extent that it is only identifiable in a young animal.

The pubis, os pubis, consists of a body, corpus ossis pubis, with cranial and caudal rami. The cranial branch, ramus cranialis ossis pubis, extends dorsolaterally from the body to the acetabulum where it fuses with the other bones of the os coxae, without contributing to the formation of the acetabulum. The cranial ramus forms the cranial border or pecten, pecten ossis pubis, of the pelvis and bears a small iliopubic eminence, eminentia iliopubica, ventromedial to the acetabulum. The caudal branches of the left and right sides fuse in the midline to form the pelvic symphysis. 


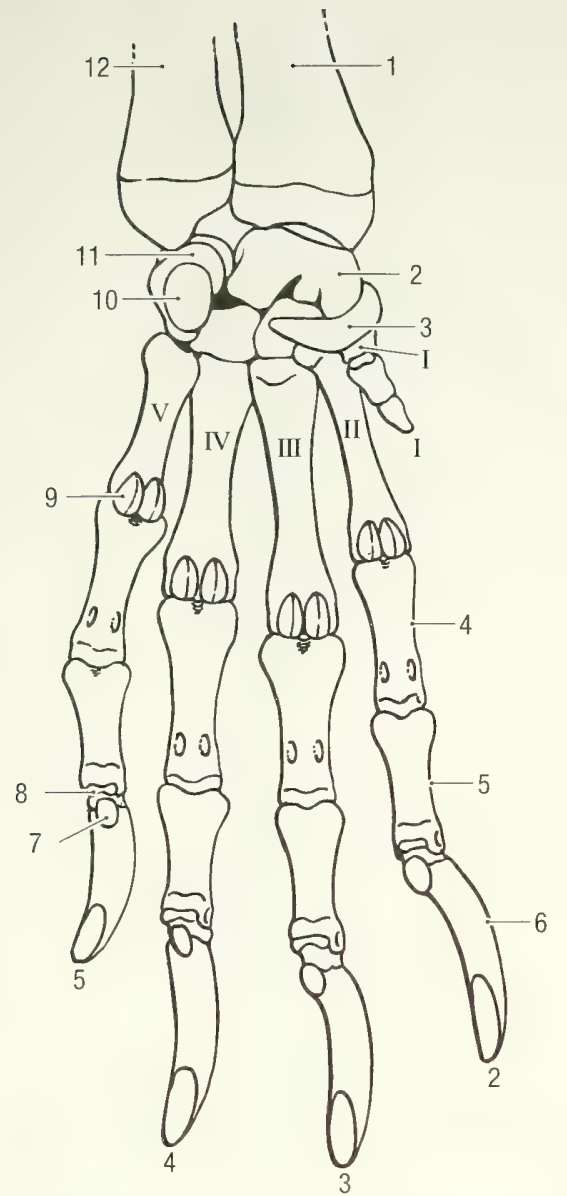

Fig. 2-36. Left forepaw, palmar view. $\mathrm{I}-\mathrm{V}=$ metacarpals, $1-5=$ digits. 1 radius, 2 intermedioradial carpal bone (scapholunatum), 3 radial sesamoid, 4 proximal phalanx of second digit, 5 middle phalanx of second digit, 6 distal phalanx of second digit, 7 flexor tubercle, 8 distal sesamoid, 9 proximal sesamoid, 10 accessory carpal bone (pisiform), 11 ulnar carpal bone (triquetrum), 12 ulna.

The pubic bone forms the cranial and medial borders of the obturator foramen, foramen obturatum.

The ischium, os ischii, forms the caudal part of the os coxa, and consists of a body and a ramus. It forms the caudal and lateral borders of the obturator foramen. The body of the ischium, corpus ossis ischii, fuses with the ilium, acetabular bone and pubis at the acetabulum. It has a low ischiatic spine, spina ischiadica, and caudal to that a lesser ischiatic notch, incisura ischiadica minor, for the tendon of the internal obturator muscle. The caudal, flattened part of the ischium forms the tabula, tabula ossis ischii. The latter bears the ischial tuberosity, tuber ischiadicum, for the attachment of the caudal thigh muscles. The ischial ramus, ramus ossis ischii, projects ventromedially from the caudal part of ischial body and fuses with the caudal branch of the pubic

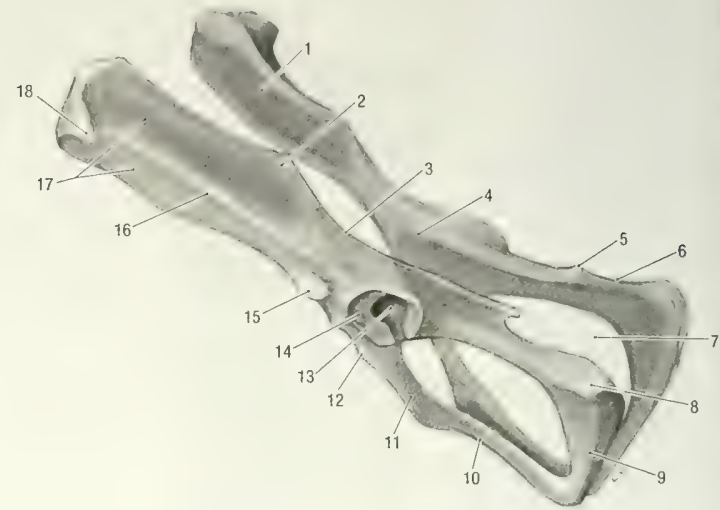

Fig. 2-37. Pelvis, oblique caudodorsal view. 1 auricular face, 2 sacral tuber, 3 major ischiadic incisure, 4 body of ilium, 5 ischiadic spine, 6 minor ischiadic notch, 7 obturator foramen, 8 ischial tuberosity, 9 ischial ramus, 10 caudal ramus of pubis, 11 cranial ramus of pubis, 12 iliopubic eminence, 13 acetabular fossa, 14 articular surface, 15 tubercle for the rectus femoris muscle, 16 gluteal line, 17 gluteal surfaces, 18 tuber coxae.

bone. It does not, however, contribute to the formation of the pelvic symphysis. The left and right ischial rami collectively form the ischial arch, arcus ischiadicus. The angle of the ischial arch of the male pelvis is more acute than that of the female pelvis.

The ilium, os ilium, forms the cranial part of the pelvis and articulates with the wing of the sacrum. It consists of a body and a wing.

The body, corpus ossis ilii, fuses with the pubis, ischium and acetabular bone. It bears a large tubercle dorsocranial to the acetabulum for the attachment of the rectus femoris muscle and a small tubercle along its ventral border for the minor psoas muscle.

The wing of the ilium, ala ossis ilii, is long, narrow and concave. A longitudinal ridge, the gluteal line, linea glutea, divides the lateral or gluteal surface, facies glutea, into dorsal and ventral gluteal surfaces. Cranially, the gluteal line turns laterally to end on the prominent tuber coxae, tuber coxae. The dorsal border of the wing bears a small sacral tuber, tuber sacrale. Caudal to the sacral tuber the dorsal border of the ilium is concave to form the major ischiadic incisure, incisura ischiadica major. The medial or pelvic surface has a long, narrow and roughened area, facies auricularis, for articulation with the sacrum.

Pelvic Canal. The pelvic canal is the bony passageway through the pelvis. The pelvic inlet is bounded dorsally by the promontorium of the sacrum, laterally by the wings of the ilium, and ventrally by the pecten of the pubis. The pelvic inlet is large, oval and placed obliquely (the promontorium lies cranial to the symphysis). The pelvic canal is 


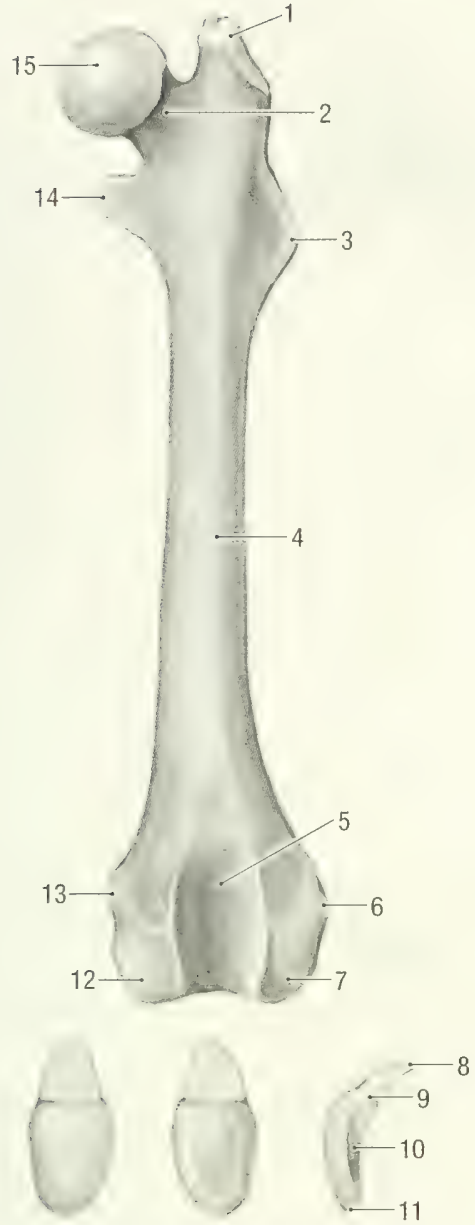

Fig. 2-38. Left femur, cranial view and cranial, caudal and lateral views of the patella. 1 major trochanter, 2 neck, 3 third trochanter, 4 shaft, 5 trochlea, 6 lateral epicondyle, 7 lateral condyle, 8 patellar cartilage, 9 base of patella, 10 articular surface of patella, 11 apex of patella, 12 medial condyle, 13 medial epicondyle, 14 minor trochanter, 15 femoral head.

formed laterally by the ischium, ventrally by the pubis, and dorsally by the sacrum and first three to four caudal vertebrae. The outlet of the pelvic canal is formed ventrally by the pubis, laterally by the ischiatic arch, and dorsally by the third or fourth caudal vertebra.

Pelvic Limb. The bones of the pelvic limb are the femur, patella, tibia, fibula, tarsals, metatarsals and phalanges.

The femur, os femoris (Figs. 2-38 and 2-39), is the bone of the thigh. It has a head and neck proximally, a body, and two condyles distally. The femur articulates proximally with the pelvis and distally with the tibia and patella.

The rounded head, caput ossis femoris, connects to the body by a distinct neck, collum ossis femoris.

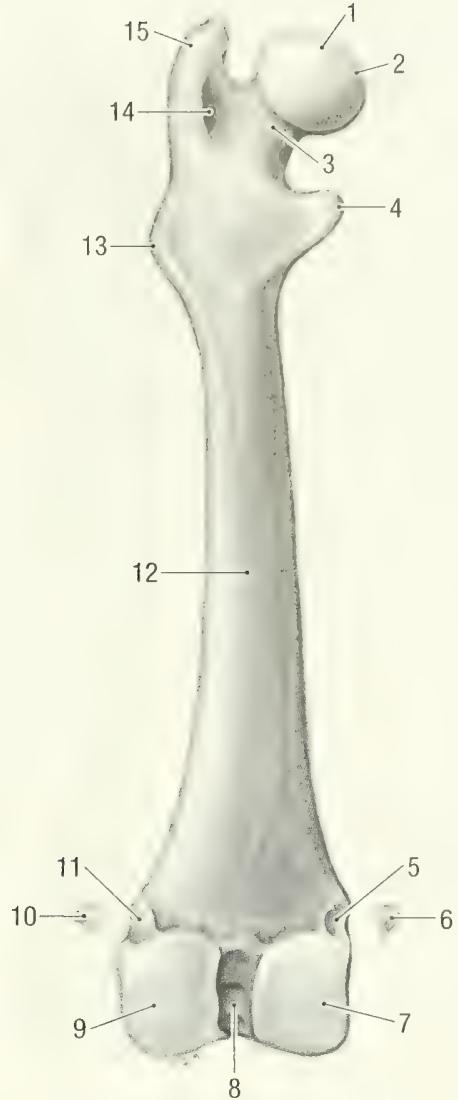

Fig. 2-39. Left femur, caudal view and fabellae. 1 head, 2 fovea of the head, 3 neck, 4 minor trochanter, 5 articular facet for medial fabella, 6 medial fabella, 7 medial condyle, 8 intercondylar fossa, 9 lateral condyle, 10 lateral fabella, 11 articular facet for lateral fabella, 12 shaft, 13 third trochanter, 14 intertrochanteric fossa, 15 major trochanter.

The head has a small, indented area, fovea capitis, for the attachment of its ligament. The proximal end of the body is flattened craniocaudally. Laterally, it bears a large major trochanter, trochantor major, medially a smaller minor trochanter, trochantor minor, and a small third trochanter, trochanter tertius, just distal to the major trochanter. The caudal surface of the major trochanter has a deep fossa, fossa trochanterica. Distally the shaft widens to form the two femoral condyles, condylus medialis and condylus lateralis, and a trochlear groove, trochlea ossis femoris. The femoral condyles articulate with the condyles of the tibia and the patella rides in the trochlear groove. The two condyles are separated caudally and distally by a deep intercondylar fossa, fossa intercondylaris. Dorsocaudal to each condyle is a small, rounded facet, facies articularis sesamoidea lateralis/medialis, for articulation with the sesamoid bones (fabellae) of the gastrocnemius muscle. The epicondyles, epicondylus lateralis/medialis, lie dorsal to the condyles. They 

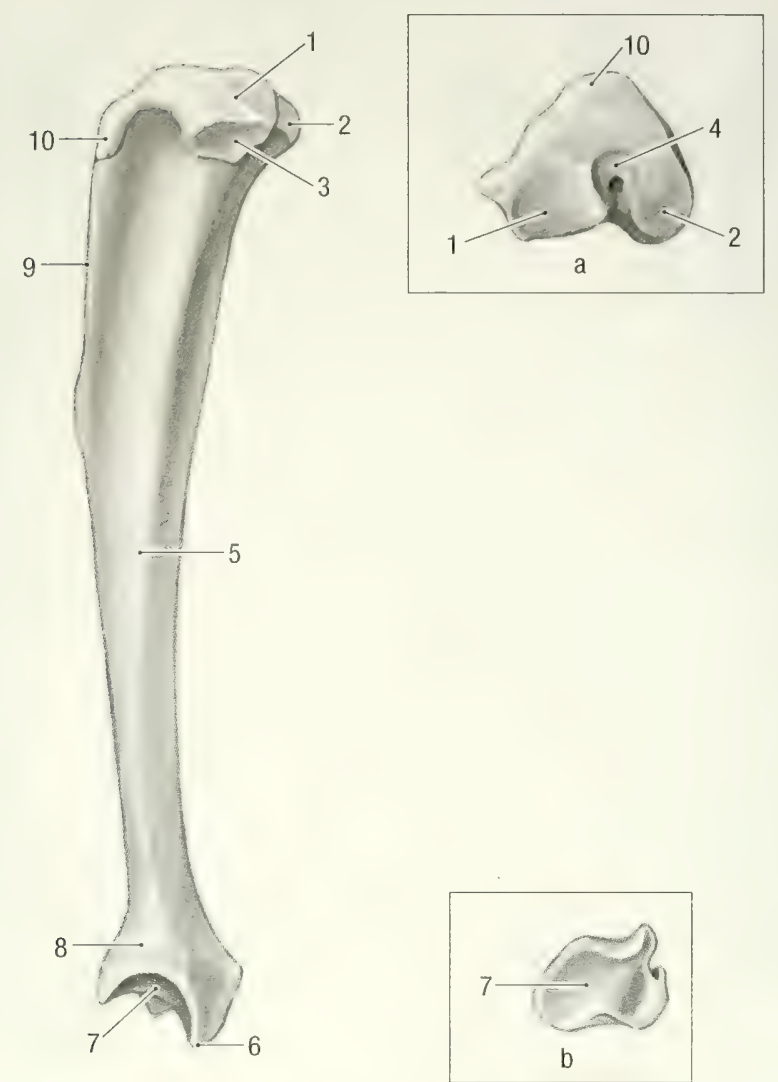

Fig. 2-40. Left tibia, lateral view and a, proximal end, b, distal end. 1 lateral condyle, 2 medial condyle, 3 articular facet for the head of the fibula, 4 intercondylar area, 5 shaft, 6 medial malleolus, 7 articular surface or cochlea, 8 distal end, 9 cranial tibial margin, 10 tibial tuberosity.

are separated cranially from each other by the trochlea, and caudally by the intercondylar fossa.

The patella (Fig. 2-38) is the largest sesamoid bone in the body. It is oval in shape and curved to articulate with the trochlea of the femur. The base of the patella, basis patellae, faces proximally and bears a large patellar cartilage (the cartilage is almost as large as the patella). The cartilage enlarges the articular surface and the area for the attachment of the quadriceps muscle. The apex of the patella, apex patellae, faces distally and is slightly pointed. The patellar ligament (the term for the distal tendon of the quadriceps muscle) extends between the apex and the tibial tuberosity.

The tibia (Fig 2.40) is a stout bone with two condyles, a body, and a cochlea. It articulates proximally with the femur and distally with the tarsal bones. The tibia is almost the same length as the femur and is slightly curved. Its proximal end consists of lateral and medial condyles, condylus lateralis/medialis. The articular surfaces of the condyles are separated from each other by cranial, central, and caudal intercondylar areas, area intercondylaris cranialis/centralis/caudalis. The lateral condyle has a large, oval facet, facies articularis fibularis, for articulation with the head of the fibula.

The proximal part of the tibial shaft is triangular in transverse section, with a prominent cranial tibial margin, margo cranialis. Proximally, the cranial tibial margin ends in a small tibial tuberosity, $t u b$ erositas tibiae. Distally the shaft widens to form a cochlea. The cochlea, cochlea tibiae, articulates with the talus. The medial border of the cochlea forms a small medial malleolus, malleolus medialis, while the lateral border has an articular facet for articulation with the fibula.

The fibula (Fig. 2-41) is a long, slender bone lateral to the tibia. It consists of a head, caput fibulae, which articulates with the lateral condyle of the tibia, a body, corpus fibulae, and a malleolus, malleolus lateralis, that articulates with the cochlea of the tibia and the talus of the tarsus.

The seven tarsal bones, ossa tarsi (Figs. 2-42 and $2-43$ ), in an adult animal are arranged in three rows. The proximal row of tarsal bones consists of the calcaneus and the talus.

The talus is cuboidal and lies between the tibia and the central tarsal bone. It has a proximal troch- 


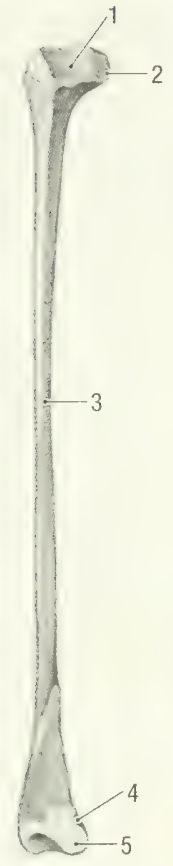

Fig. 2-41. Left Fibula, caudal view. 1 articular facet, 2 head, 3 shaft, 4 lateral malleolus, 5 articular facet.

lea, trochlea tali, that articulates with the cochlea of the tibia, and two plantar articular facets for articulation with the calcaneus and proximal tarsal sesamoid bone. Distally it has a rounded facet for articulation with the central tarsal bone.

The calcaneus is the largest of the tarsal bones and lies caudolaterally on the plantar aspect of the tarsus. It has a large, caudodorsally directed tuber, tuber calcanei, for the attachment of the extensor muscles of the ankle. In addition a medially directed sustentaculum tali provides a gliding surface for the tendon of the deep digital flexor muscle. Distally, the calcaneus has articular facets for the talus and fourth tarsal bone.

The middle row of tarsal bones consists of the central tarsal bone, os tarsi centrale (os naviculare). It articulates proximally with the talus, medially with the first tarsal bone, laterally with the fourth tarsal bone, and distally with the first, second, and third tarsal bones.

The distal row of tarsal bones consists of four bones, numbered from medial to lateral.

The first tarsal bone, os tarsale I (os cuneiforme mediale), is elongated and forms the greater part of the medial border of the tarsus. It articulates proximally with the central tarsal bone, medially with the second tarsal bone, and distally with the first metatarsal bone.

The second tarsal bone, os tarsale II (os cuneiforme intermedium), is small and cuboidal in shape. It articulates proximally with the central tarsal bone,

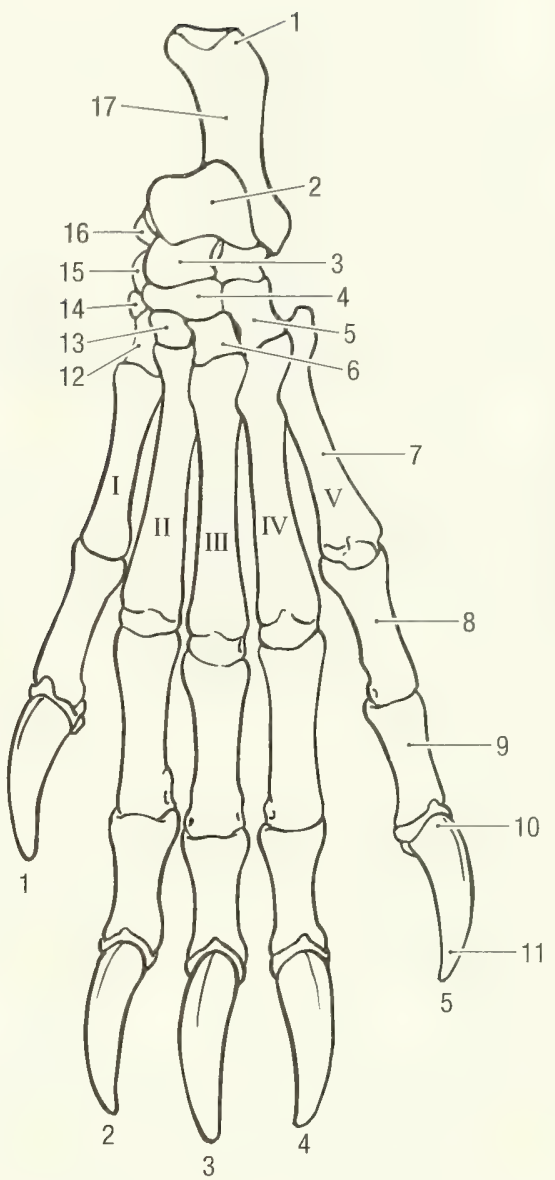

Fig. 2 42. Hind paw, dorsal view. $I-V=$ metatarsals, $1-5$ = digits. 1 calcaneal tuber, 2 trochlea of the talus, 3 talus, 4 central tarsal bone (navicular), 5 fourth tarsal bone (cuboideum), 6 third tarsal bone (lateral cuneiform), 7 fifth metatarsal, 8 proximal phalanx of fifth digit, 9 middle phalanx of fifth digit, 10 distal phalanx of fifth digit, 11 unguicular process, 12 first tarsal bone (medial cuneiform), 13 second tarsal bone (intermediate cuneiform), 14 distal tarsal sesamoid, 15 proximal tarsal sesamoid, 16 sustentaculum tali, 17 calcaneus.

medially with the first tarsal bone, laterally with the third tarsal bone, and distally with the second metatarsal bone.

The third tarsal bone, os tarsale III (os cuneiforme laterale), is cuboidal in shape and slightly larger than the second tarsal bone. It articulates proximally with the central tarsal bone, medially with the second tarsal and second metatarsal bones, laterally with the fourth tarsal bone, and distally with the third metatarsal bone.

The fourth tarsal bone, os tarsale IV (os cuboideum), is cuboidal in shape and is the largest of the distal row of tarsal bones. It articulates proximally with the calcaneus, medially with the central and third tarsal bones, and distally with the fourth and fifth metatarsal bones. 


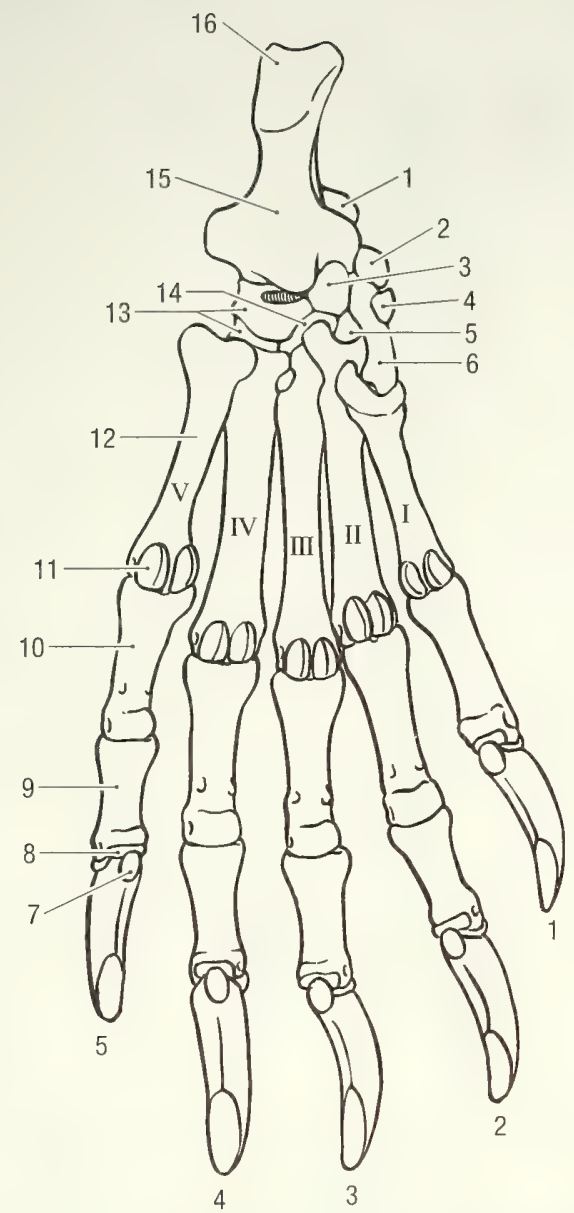

Fig. 2-43. Hind paw, plantar view. I-V = metatarsals, $1-$ 5 = digits. 1 talus, 2 proximal tarsal sesamoid, 3 central tarsal (navicular), 4 distal tarsal sesamoid, 5 second tarsal bone (intermediate cuneiform), 6 first tarsal bone (medial cuneiform), 7 flexor tubercle, 8 distal sesamoid, 9 middle phalanx of fifth digit, 10 proximal phalanx of fifth digit, 11 proximal sesamoid, 12 fifth metatarsal, 13 fourth tarsal cuboideum), 14 third tarsal bone (lateral cuneiform), $15 \mathrm{cal}-$ caneus, 16 calcaneal tuber.
The metatarsal and digital bones, ossa metatarsalia $1-V$ and ossa digitorum pedis (Fig. 2.42), are similar to those of the thoracic limb, except that the first metatarsal and first digit of the hind foot are well developed. All the metacarpal and metatarsal bones have distal epiphyseal growth lines, except for the first metatarsal, in which the growth line is proximal.

Sesamoid Bones of the Pelvic Limb. There are four sesamoid bones in the pelvic limb. Two of these, the lateral and medial fabellae, ossa sesamoidea m. gastrocnemii (Fig. 2-39), are located caudal to the knee in the tendons of origin of the gastrocnemius muscle. They are cuboidal and articulate with the condyles of the femur.

The third sesamoid, os sesamoideum m. poplitei, is located in the tendon of origin of the popliteal muscle. It is small, oval in shape, and articulates with the lateral condyle of the tibia. The fourth sesamoid bone lies in the cranial part of the medial meniscus of the knee. It is wedge-shaped and articulates with the medial condyles of the femur and the tibia. It is larger than the other sesamoid bones of the knee.

There are two sesamoid bones on the medial aspect of the tarsus (Figs. 2-42 and 2-43). The proximal tarsal sesamoid bone lies on the medial aspect of the talus. It is about $5 \mathrm{~mm}$ in diameter and articulates medially with the talus and distally with the first tarsal bone. The distal tarsal sesamoid bone lies on the medial aspect of the first tarsal bone. It is slightly elongated and is $5 \mathrm{~mm}$ long and $2 \mathrm{~mm}$ wide.

The sesamoid bones of the digits are similar to those of the thoracic limb. Each metatarso-phalangeal joint has two proximal plantar sesamoid bones, and each distal interphalangeal joint has one distal plantar sesamoid bone. 


\section{Chapter 3. Joints and Ligaments}

Joints are formed when two or more bones are united by fibrous, elastic, or cartilaginous tissue. Ligaments attach bone to bone, spanning at least one joint. Three main groups of joints are recognized and named according to their most characteristic structural features. These are fibrous joints, cartilaginous joints, and synovial joints. Synovial joints of the limbs permit the greatest degree of movement. They have a joint cavity, a joint capsule and articular surfaces covered by articular cartilage. The joint cavity is filled with synovial fluid. The fibrous portion of a joint capsule may thicken in specific areas to form ligaments. A meniscus is a complete or partial fibrocartilagenous plate which divides a joint cavity into proximal and distal compartments.

\section{SKULL}

The temporomandibular joint, art. temporomandibularis, for jaw articulation is between the temporal bone and the mandible. The joint is incongruent and allows for considerable sliding movement. A thin articular disc, discus articularis, lies between the two bones and aids movement. The disc is thickest caudally and thinnest centrally. It divides the temporomandibular joint into two separate compartments, one proximal and one distal. The lateral edge of the disc is firmly attached to the mandible.

The mandibular symphysis, art. intermandibularis, unites the right and left mandibular bodies by fibrous connective tissue, forming a fibrous joint. The mandibular symphysis of the woodchuck allows for restricted movement.

The bones of the skull are connected to each other by fibrous connective tissue or cartilage to form sutures, suturae capitis. Sutures formed by fibrous connective tissue are syndesmoses, those formed by cartilage are synchondroses. Many of the joints ossify as the animals age and cannot be identified. The sutures of the skull are named after the bones forming the suture.

Joints of the Hyoid Apparatus. The tympanohyoid articulates with the mastoid part of the temporal bone adjacent to the stylomastoid foramen, forming the temporohyoid joint, art. temporohyoidea. Distally the thyrohyoid articulates with the thyroid cartilage of the larynx. There are small synovial joints between all parts of the hyoid apparatus.

\section{VERTEBRAL COLUMN}

The atlanto-occipital joint, art. atlanto-occipitalis, is formed between the condyles of the occipital bone and corresponding concavities of the atlas.

The dorsal atlanto-occipital membrane, membrana atlanto-occipitalis dorsalis, extends between the cranial border of the dorsal arch of the atlas and the dorsal edge of the foramen magnum. Laterally it blends with the lateral atlanto-occipital ligaments.

The ventral atlanto-occipital membrane, membrana atlanto-occipitalis ventralis, extends between the ventral border of the foramen magnum and the cranial border of the ventral arch of the atlas. It is attached to the apical ligament of the dens of the axis.

The lateral atlanto-occipital ligament, lig. laterale, extends between the cranial border of the wing of the atlas and the paracondylar processes of the occipital bone. Medially the ligament blends with the dorsal membrane of the atlanto-occipital joint.

The atlantoaxial joint, art. atlantoaxialis, allows the head and atlas to rotate around a longitudinal axis.

The dorsal atlantoaxial membrane, membrana atlantoaxialis dorsalis, extends between the caudal border of the dorsal arch of the atlas and the cranial border of the neural arch of the axis.

The dorsal atlantoaxial ligament, lig. atlantoaxiale dorsale, extends between the cranial point of the spinous process of the axis and the dorsal arch of the atlas.

The apical ligament of the dens, lig. apicis dentis, extends from the tip of the dens to the ventral border of the foramen magnum. It is attached to the ventral atlanto-occipital membrane.

The transverse atlantal ligament, lig. transversum atlantis, extends between the two sides of the ventral arch of the atlas. It crosses dorsal to the dens of the axis and serves to hold the dens against the ventral arch of the atlas.

The supraspinous ligament, lig. supraspinale, extends between the apices of consecutive spinous processes, from $2 \mathrm{C}$ to $7-8 \mathrm{Cd}$.

The ventral longitudinal ligament, lig. longitudinale ventrale, extends from the first cervical to the 
third caudal vertebra. It lies on the ventral surfaces of the vertebral bodies, attaching one to the other.

The dorsal longitudinal ligament, lig. longitudinale dorsale, extends from the axis to the first few caudal vertebrae. It lies on the dorsal surfaces of the vertebral bodies. In the cervical region it fuses with the duramater.

The interspinous ligaments, ligg. interspinalia, connect adjacent spinous processes to each other. They are best developed in the caudal lumbar region.

The intertransverse ligaments, ligg. intertransversaria, connect adjacent transverse processes of lumbar vertebra.

An intervertebral disc, discus intervertebralis, is interposed at every intervertebral space, except that between the atlas and axis. They unite the vertebral bodies of the adjacent vertebrae. The discs are thickest in the lumbar region and thinnest in cervical and caudal regions. Each disc consists of an outer laminated fibrous ring, anulus fibrosus, and a central, gelatinous nucleus, nucleus pulposus. The intervertebral discs are attached to the dorsal and ventral longitudinal ligaments.

\section{RIBS}

Each typical rib articulates with the vertebral column by two synovial joints and with the sternum by one synovial joint. A costovertebral joint, art. costovertebralis, consists of an articulation between the head of the rib and the costal facets of the vertebral bodies, art. capitis costae, and an articulation between the tuberculum of the rib and the transverse process of the vertebra, art. costotransversaria. In the caudal thoracic region the two joints lie closer to each other. The last two pairs of ribs generally have only one articulation each. The costal cartilages of the first seven ribs articulate with the sternum by means of sternocostal joints, art. sternocostales.

Costovertebral Ligaments. The ligament of the rib head, lig. capitis costae radiatum, is a small band of fibrous connective tissue that attaches the head of the rib to the lateral part of the intervertebral disc. The last two capital ligaments attach to the vertebral bodies rather than to the intervertebral discs.

An intercapital ligament, lig. intercapitale, extends between the dorsal aspects of the heads of one pair of ribs, attaching them to each other. It runs from the head of one rib dorsal to the dorsal part of the intervertebral disc, but ventral to the dorsal longitudinal ligament of the vertebral column, to the head of the opposite rib.

The ligament of the rib tubercle, lig. costotrans- versarium, attaches the tubercle of the rib to the transverse process of the corresponding vertebra.

\section{THORACIC LIMB}

Clavicular Joints. The clavicle articulates with the sternum at the sternoclavicular joint, and with the scapula at the scapuloclavicular joint. The sternoclavicular joint is a synovial joint, whereas the scapuloclavicular joint is fibrous. The sternal articular surface of the clavicle is round and the scapular articular surface is flattened craniocaudally.

Clavicular Ligaments. The sternoclavicular ligament attaches the clavicle to the sternum. It extends from the ventral border of the articular surface of the clavicle to the cranial border of the manubrium of the sternum.

An acromioclavicular ligament attaches the clavicle to the acromion. It extends from the lateral border of the articular surface of the clavicle to the distal end (hamate process) of the scapular acromion:

The coracoclavicular ligament attaches the clavicle to the coracoid process of the scapula. It extends from the medial surface of the clavicle to the coracoid process.

Shoulder Joint, art. humeri. The shoulder joint is a shallow ball and socket joint between the glenoid cavity of the scapula and the head of the humerus. It allows movement in any direction, but its main movements are flexion and extension. The depth of the glenoid cavity is somewhat enlarged by a cartilaginous lip, labrum glenoidale, around its periphery. There are no true ligaments around the shoulder joint. However, a thickening of the fibrous capsule is present in the medial wall of the joint capsule. It extends from the supraglenoid tubercle to the caudomedial aspect of the minor tuberositas of the humerus. Furthermore, the subscapular, infraspinatus and supraspinatus muscles act as stabilizers of the joint.

Elbow Joint, art. cubiti. The elbow is a composite joint formed by the humeral condyle with the head of the radius and the trochlear notch of the ulna. Movement is restricted to flexion and extension.

Ligaments of the Elbow Joint. The lateral collateral ligament, lig. collaterale cubiti laterale, extends from the lateral epicondyle of the humerus to the head of the radius. Its attachment to the radius lies $2-3 \mathrm{~mm}$ distal to the articular surface.

The medial collateral ligament, lig. collaterale $\mathrm{cu}$ biti mediale, extends from the medial condyle of the 
humerus to the radial tuberosity and ulna. It attaches to the radius and ulna just proximal to the insertion of the biceps muscle.

The olecranon ligament, lig. olecrani, extends from the medial condyle of the humerus to the medial aspect of the anconeal process.

Radioulnar Joints. The radius and ulna form two joints, one proximal and one distal. The proximal radioulnar joint, art. radioulnaris proximalis, is formed between the articular circumference of the radial head and the radial incisure of the ulna. The joint is closely related to the elbow joint. It allows rotation (pronation and supination) of the manus. The two bones are held in position by two ligaments. The distal radioulnar joint, art. radioulnaris distalis, is formed between the head of the ulna and the trochlea of the radius.

Ligaments of the Radioulnar Joints. The lateral radioulnar ligament, lig. radioulnare laterale, extends from the lateral coronoid process of the ulna to the head of the radius. Its attachment to the radius is on the caudal rim of the articular fovea.

The medial radioulnar ligament, lig. radioulnare mediale, extends from the medial coronoid process of the ulna to the head of the radius. Its attachment to the radius is $2 \mathrm{~mm}$ distal to the medial border of the articular fovea (between the head and neck of the radius).

A distal radioulnar joint is formed between the ulnar notch of the radius and the styloid process of the ulna. A delicate interosseus membrane, membrana interossea antebrahii, attaches the two bones to each other.

The interosseus ligament of the antebrachium, lig. interosseum antebrachii, extends between the shaft of the radius and the shaft of the ulna. The ligament is approximately $1 \mathrm{~cm}$ wide and lies in the middle of the antebrachium.

Carpal Joint, art. carpi. The carpal joint includes proximal, middle, distal and intercarpal joint surfaces. The antebrachiocarpal joint, art. antebrachiocarpea, is located between the distal part of the radius and ulna and the proximal row of carpal bones. The middle (inter) carpal joints, art. intercarpeae, are located between the three rows of carpal bones. The carpometacarpal joints, art. carpometacarpeae, are located between the distal row of carpal bones and the metacarpal bones. Joints between the individual carpal bones constitute the intercarpal joints. The carpus as a whole permits flexion and extension.
Ligaments of the Carpal Joints. The individual carpal bones are attached to each other by short intercarpal ligaments. These can be dorsal, ligg. intercarpea dorsalia, palmar, ligg. intercarpea palmaria, or between the individual carpal bones, lig. intercarpea interossea.

The medial carpal collateral ligament, lig. collaterale carpi mediale, extends from the styloid process of the radius to the medial surface of the intermedioradial carpal bone. It also attaches to a sesamoid bone in the carpal pad.

The lateral carpal collateral ligament, lig. collaterale carpi laterale, extends from the styloid process of the ulna to the ulnar and accessory carpal bones.

Long collateral ligaments that extend from the radius/ulna to the metacarpal bones are absent.

Short ligaments attach the accessory carpal bone to the ulna, ulnar carpal, intermedioradial, fifth carpal and fifth metacarpal bones. These are the ligg. accessorioulnare, accessoriocarpoulnare and accessoriometacarpeum.

The palmar carpal ligament extends between the caudal surface of the radius and ulna proximally, and the palmar surfaces of the metacarpal bones distally. It attaches to all the individual carpal and metacarpal bones and represents the fused palmar ligaments of the carpus. The palmar ligament serves as a gliding surface for the tendons of the digital flexor muscles and supports the carpus when it bears weight.

A band of fibrocartilage extends between the free ends of the accessory carpal bone and the sesamoid bone of the carpal pad. Together with the palmar ligament it forms a fibro-osseus carpal canal, canalis carpi, on the palmar surface of the carpus for the flexor tendons, blood vessels, and nerves.

Metacarpal Joints and Ligaments. There are three groups of metacarpal joints. These are the carpometacarpal joints of the carpus, the intermetacarpal joints, and metacarpophalangeal joints.

The intermetacarpal joints, art. intermetacarpeae, are close-fitting joints between the proximal ends of adjacent metacarpal bones. The bones are united for variable distances by interosseus metacarpal ligaments, ligg. metacarpea interossea.

The metacarpophalangeal joints, art. metacarpophalangeae, are formed by the distal ends of the metacarpal bones and the proximal ends of the proximal phalanges, as well as the proximal palmar sesamoid bones of each joint. Each metacarpophalangeal joint has axial and abaxial collateral ligaments, ligg. collateralia, which unite the metacarpal bone and proximal phalanx.

Ligaments of the Proximal Sesamoid Bones. Each pair of proximal sesamoid bones is 
attached to their specific metacarpal bone and proximal phalanx by axial and abaxial collateral ligaments, ligg. sesamoidea collateralia.

Each pair of proximal palmar sesamoid bones is joined together by an intersesamoidean or palmar ligament, ligg. palmaria.

Phalangosesamoidean ligaments, ligg. phalangosesamoidea, extend from the proximal sesamoid bones to the proximal ends of the proximal phalanges.

Ligaments of Phalangeal Joints. The proximal interphalangeal joints, art. interphalangeae proximales, are formed by the heads of the proximal phalanges and the bases of the middle phalanges. The bones of each joint are attached to each other by axial and abaxial collateral ligaments, ligg. collateralia. The distal interphalangeal joints, art. interphalangeae distales, are formed by the heads of the middle phalanges and the distal phalanges, as well as the distal palmar sesamoid bones. The bones of each joint are attached to each other by axial and abaxial collateral ligaments, ligg. collateralia.

\section{PELVIC LIMB}

In young animals the left and right os coxae are united midventrally by cartilage to form the pelvic symphysis, symphysis pelvina. The symphysis ossifies in the adult.

The sacroiliac joint, art. sacroiliaca, connects the opposed auricular surfaces of the sacrum and the wing of the ilium are to each other by fibrocartilage, forming a fibrocartilagenous joint.

The dorsal sacroiliac ligaments, ligg. sacroiliaca dorsalia, extend from the caudodorsal surfaces of the ilia to the sacrum. The ligaments cover the caudodorsal surfaces of the sacroiliac joints.

Ventral sacroiliac ligaments, ligg. sacroiliaca ventralia, extend from the ventral surface of the sacrum to the ilia. They cover the ventral surfaces of the sacroiliac joints.

The hip joint, art. coxae, is formed between the head of the femur and the acetabulum of the pelvis. Although flexion and extension are the main movements of the joint, its construction allows for a wide range of movement. A cartilaginous lip, labrum acetabulare, deepens the acetabulum. The lip spans the acetabular notch as a transverse ligament, lig. transversum acetabuli. The lip is thickest dorsally, and thinnest cranioventrally.

The ligament of the femoral head, lig. capitis ossis femoris, holds the head of the femur in the acetabulum. It extends from the fovea of the head of the femur to the acetabular fossa.

The knee joint, art. genus, formed by the rounded condyles of the femur, the flattened condyles of the tibia, and the patella. In addition to these, a small triangular bone is present cranially between the medial condyles of the femur and tibia. It represents the ossified cranial part of the medial meniscus. Two menisci are present in the knee joint. They divide the femorotibial joint into proximal and distal compartments. A large pad of adipose tissue is present between the patellar ligament and the femoropatellar joint.

The menisci are semilunar fibrocartilaginous discs situated between the condyles of the femur and the tibia. The medial meniscus, meniscus medialis, lies between the medial condyle of the femur and the medial condyle of the tibia. The lateral meniscus, meniscus lateralis, lies between the lateral condyle of the femur and the lateral condyle of the tibia. They have sharp, thin, concave axial borders, and thick, convex abaxial borders. The medial meniscus is larger and thicker than the lateral meniscus. Its cranial part is ossified and forms a triangular bone that articulates with both the femur and tibia. Both menisci are attached to the tibia along their cranial and caudal borders by meniscotibial ligaments. The medial meniscus is attached to the medial collateral ligament, while the tendon of origin of the popliteus muscle lies between the lateral meniscus and the lateral collateral ligament. The lateral meniscus is also attached to the femur by means of a meniscofemoral ligament, lig. meniscofemorale.

The femoropatellar joint, art. femoropatellaris, is formed between the patella and the trochlea of the femur. It communicates with the femorotibial joint. The patella has two ligaments attaching it to the femur and one ligament attaching it to the tibia.

The lateral femoropatellar ligament, lig. femoropatellare laterale, extends between the patella and the femur. It consists of a broad band of connective tissue between the lateral border of the patella and the lateral epicondyle of the femur.

The medial femoropatellar ligament, lig. femoropatellare mediale, consists of a broad band of connective tissue which extends from the medial border of the patella to the medial epicondyle of the femur.

The patellar ligament, lig. patellare, extends between the patella and the tibia. Proximally it attaches to the apex of the patella and distally it attaches to the tibial tuberosity and cranial tibial margin (the patellar ligament is homologous to the distal tendon of the quadriceps femoris muscle).

The femorotibial joint, art. femorotibialis, consists of medial and compartments that communicate with each other and with the femoropatellar joint. Each compartment is formed between a femoral condyle and its corresponding tibial condyle.

The lateral collateral ligament, lig. collateralis laterale, extends from the lateral epicondyle of the femur to the head of the fibula. It lies caudal to the tendon of origin of the long digital extensor muscle 
and is separated from the lateral meniscus by the tendon of origin of the popliteus muscle.

The medial collateral ligament, lig. collaterale mediale, The extends from the medial epicondyle of the femur to the medial aspect of the shaft of the tibia, $1.5 \mathrm{~cm}$ distal to the tibial condyle.

The cranial cruciate ligament, lig. cruciatum craniale, extends between the caudomedial part of the lateral condyle (in the intercondylar fossa) of the femur and the cranial intercondylar area of the tibia. Its attachment to the tibia lies caudal to the cranial attachment of the lateral meniscus.

The caudal cruciate ligament, lig. cruciatum caudale, extends between the cranial border of the intercondylar fossa of the femur and the medial edge of the popliteal notch of the tibia.

The cranial meniscotibial ligament of the medial meniscus extends from cranial, ossified part of the meniscus to the cranial intercondylar area of the tibia. It is the most cranial of all the ligaments that attach to the intercondylar area. The caudal meniscotibial ligament attaches the caudal edge of the medial meniscus to the cranial edge of the popliteal notch of the tibia (between the lateral and medial condyles).

The cranial tibial ligament of the lateral meniscus attaches the cranial border of the meniscus to the intercondylar area of the tibia, immediately caudal to the attachment of the cranial tibial ligament of the medial meniscus. The caudal tibial ligament of the lateral meniscus attaches the caudal edge of the lateral meniscus to the caudolateral edge of the popliteal notch. The ligament is poorly developed and is the weakest of all the meniscal ligaments. Additionally, the caudal edge of the lateral meniscus is attached to the intercondylar fossa (on the medial condyle) of the femur by the femoromeniscal ligament, lig. meniscofemorale.

The fibula articulates with the tibia at each end, forming proximal and distal tibiofibular joints.

The proximal tibiofibular joint, art. tibiofibularis proximalis, is formed between the head of the fibula and the lateral condyle of the tibia. The two bones attach to each other by means of the lateral collateral ligament of the knee.

The distal tibiofibular joint, art. tibiofibularis distalis, is formed between the malleolus of the fibula and the distal lateral surface of the tibia. The two bones are attached to each other by cranial and caudal tibiofibular ligaments, lig. tibiofibulare craniale and lig. tibiofibulare caudale, respectively.

A distal crural interosseus ligament, lig. interosseus cruris, attaches the fibula to the tibia. It is about $2 \mathrm{~mm}$ wide and lies $1 \mathrm{~cm}$ proximal to the malleolus of the fibula. The interosseus membrane, membrana interossea cruris, spans the space between the two bones. It extends from the proximal to the distal tibiofibular joints.

The tarsal joints, art. tarsi, include the talocrural, proximal intertarsal, distal intertarsal and tarsometatarsal joints.

The tarsocrural joint, art. tarsocruralis, is formed between the cochlea of the tibia, the fibula, and the trochlea of the talus. It permits the greatest degree of movement in the tarsus.

The proximal intertarsal joint, art. talocalcaneocentralis, is formed between the talus and calcaneus, and between the talus and central tarsal bone. Some rotation, as well as flexion and extension, is possible as the rounded, distal end of the talus fits into the glenoid articular surface of the central tarsal bone.

The distal intertarsal joint, art. centrodistalis, is formed by the calcaneus and central tarsal bone proximally and the distal row of tarsal bones. They are stable joints that allow a minimum amount of movement. The tarsometatarsal joints, art. tarsometatarseae, are formed between the distal row of tarsal bones and the proximal ends of the metatarsal bones. The articular surfaces allow a minimum amount of movement.

The lateral collateral ligament, lig. collateralis tarsi laterale, consists of a poorly developed sheet of connective tissue that extends from the malleolus of the fibula to the proximal end of the fifth metatarsal bone. The deep part of the ligament, lig. collateralis laterale breve, attaches to the tarsal bones which it crosses. One short ligament, the calcaneofibular ligament, pars calcaneofibularis, extends between the malleolus of the fibula and the tuber calcis. The ligament prevents excessive flexion of the tibiotarsal joint.

The medial collateral ligament, lig. collateralis tarsi mediale, is well developed and extends between the malleolus of the tibia and the proximal end of the first metatarsal bone. The deep part of the ligament, lig. collateralis mediale breve, attaches to the tarsal bones which it crosses.

The plantar talofibular ligament, lig. talofibulare plantare, is a short, well developed ligament which extends from the malleolus of the fibula to the lateral aspect of the talus.

The plantar tibiotalar ligament, lig. tibiotalare plantare, extends between the malleolus of the tibia and the medial border of the talus.

The medial talocalcanean ligament, lig. talocalcaneum mediale, extends from the dorsal edge of the sustentaculum tali to the caudodorsal border of the trochlea of the talus.

The calcaneoquartal ligament, lig. calcaneoquartale, lies on the plantar surface of the tarsus and extends between the distomedial border of the calcaneus and the fourth tarsal bone.

Apart from the above mentioned ligaments, the 
various tarsal bones are attached to each other by dorsal and plantar intertarsal ligaments, ligg. tarsi dorsalia and ligg. tarsi plantaria. They extend from one tarsal bone to another. The distal row of tarsal bones is attached to the proximal ends of the meta- tarsal bones by dorsal and plantar tarsometatarsal ligaments.

The metatarsal and phalangeal joints and ligaments are similar to comparable joints and ligaments of the forepaw. 


\section{Chapter 4. Muscular System}

Bryant (1945) studied the phylogeny of Neartic Sciuridae, making reference to the muscular system of the yellow-bellied marmot, M. flaviventris. Alternate terms used by him, and other authors, are cited in the text where they differ from that of the Nomina Anatomica Veterinaria (1994).

\section{HEAD}

The muscles of the head are grouped on the basis of their embryonic origin and innervation. They are: the facial muscles innervated by the facial nerve; the masticatory muscles innervated by the mandibular division of the trigeminal nerve; the tongue muscles innervated by the hypoglossal nerve; the pharyngeal muscles innervated by the glossopharyngeal and vagus nerves; the laryngeal muscles innervated by the vagus nerve; and the eye muscles innervated by the oculomotor, trochlear and abducent nerves.

Cutaneous Muscles of the Head. The head has two cutaneous muscles that form part of the superficial musculature of the head. These muscles and their derivatives in the European marmot (Marmota marmota) were studied in detail by Meinertz (1943) and are the same in the woodchuck.

The platysma, platysma (Figs. 4-1 and 4-2), is a large, well-developed cutaneous muscle of the head and neck. It consists of facial and cervical parts. The facial part, $m$. cutaneus faciei, (dorsal division of platysma of Bryant, 1945) extends from the superficial fascia of the neck, and the lateral aspect of the shoulder, arm and antebrachium, to the face. It consists of a superficial layer that takes origin from the lateral aspect of the neck, shoulder, and thoracic limb and a deep layer that takes origin from the craniodorsal cervical region. The thick, dorsal border of the superficial layer passes ventrolaterally and curves around the base of the ear to insert in the fascia of the skin ventral to the medial canthus of the eye, and the lateral aspect of the nose, upper lip and the angle of the mouth. The deep layer passes ventrolaterally around the base of the ear where its fibers blend with those of the superficial part. The cervical part, $m$. colli ventralis, (ventral division of the platysma of Bryant) is a very extensive, fanshaped muscle, forming the ventral border of the neck. It is the most superficial muscle along the ventral neck region.
Origin: From the first four sternebrae.

Insertion: On the fascia over the masseter muscle, from the ear to the upper lip. It blends ventrally with the acromiomandibular muscle to insert on the angle of the mouth, lower lip and the chin.

Action: Retracts the angle of the mouth, the lateral nasal region, and the area below the eye.

Innervation: $\mathrm{N}$. facialis.

The superficial sphincter of the neck, $m$. sphincter colli superficialis, consists of dorsal and ventral parts. The dorsal part takes origin from the fascia ventral to the eye. It passes superficial to the platysma and inserts on the lateral aspect of the acromiomandibular muscle. The isolated fibers of the ventral part pass transversely over the inter-mandibular and the laryngeal areas.

Innervation: $\mathrm{N}$. facialis.

The muscles of the face are derived from the platysma. They include the muscles of the lips and cheek, nose, eyelids and ear.

Muscles of the Lip, Nose and Cheek. The levator of the nose and upper lip, $m$. levator nasolabialis (Fig. 4-3; nasolabialis of Bryant, 1945), takes origin from the dorsum of the nose and inserts on the fascia of the upper lip and nostril. It elevates and retracts the upper lip.

Innervation: $\mathrm{N}$. facialis.

The zygomatic muscle, $m$. zygomaticus (auriculolabialis of Bryant, 1945), is a thin, strap like muscle that takes origin from the temporal fascia, caudalateral to the lateral canthus of the eye, and inserts on the fascia at the angle of the mouth. It retracts and elevates the angle of the mouth.

Innervation: $\mathrm{N}$. facialis.

The orbicularis oris muscle, $m$. orbicularis oris, forms a muscular ring around the mouth within the lips. It can be divided into a maxillary part that lies in the upper lip and a mandibular part that lies within the lower lip. The muscle covers the large scent gland associated with the angle of the mouth.

Innervation: $\mathrm{N}$. facialis.

The buccinator muscle, $m$. buccinator (Fig. 4-3), forms a muscular sheet in the cheek, caudal to the orbicularis oris muscle. It is a complex muscle consisting of buccal and molar parts. The buccal part blends with the $\mathrm{m}$. orbicularis oris.

Innervation: $\mathrm{N}$. facialis.

The dilator of the nose, $m$. dilatator naris apicalis 


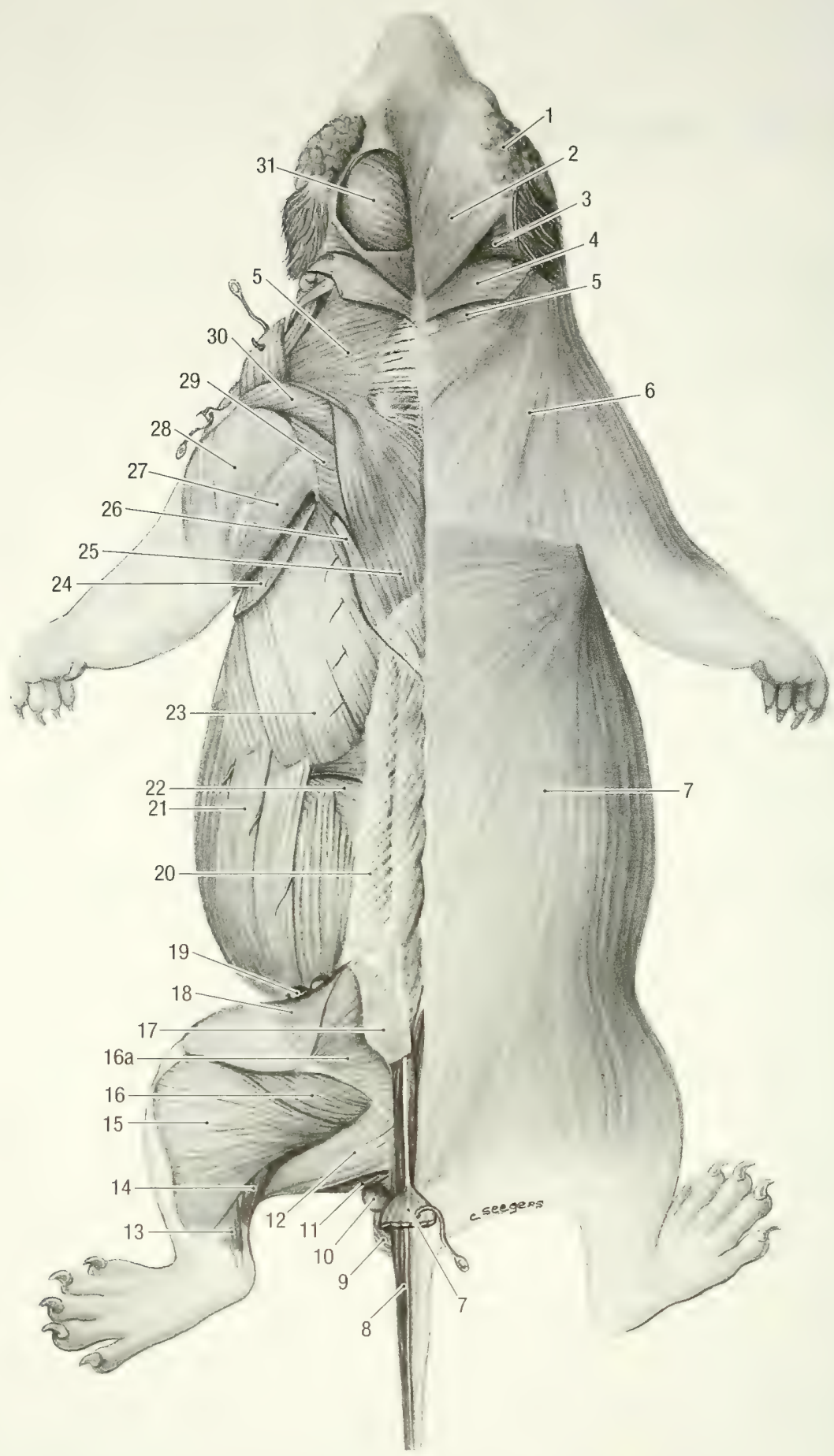

Fig. 4-1. Muscles of the trunk, dorsal view. 1 temporal gland, 2 superficial cervicoauricular m., 3 middle cervicoauricular m., 4 parietoauricular m., 5 cervical trapezius m., 6 platysma (cutaneus muscle of the face), 7 cutaneus $m$. of the trunk, 8 dorso-medial sacrocaudal $m$., 9 external anal sphincter, 10 anal sac, 11 semimembranous $\mathrm{m}$., 12 semitendinous m., 13 lateral digital flexor m., 14 gastrocnemius m. (lateral head), 15 biceps femoris m., 16 caudofemoral m., 16a middle gluteal m., 17 superficial giuteal m., 18 tensor fascia lata m., 19 subiliac lymph nodes, 20 thoracolumbar fascia, 21 external oblique abdominal $\mathrm{m}$., 22 internal oblique abdominal $\mathrm{m}$., 23 serratus ventralis $\mathrm{m}$., 24 cutaneus trunci m., 25 trapezius m., 26 latissimus dorsi m., 27 long head of the triceps brachii m., 28 lateral head of the triceps brachii m., 29 scapular part of the deltoid $\mathrm{m}$., 30 acromial part of the deltoid $\mathrm{m} ., 31$ temporalis $\mathrm{m}$. 


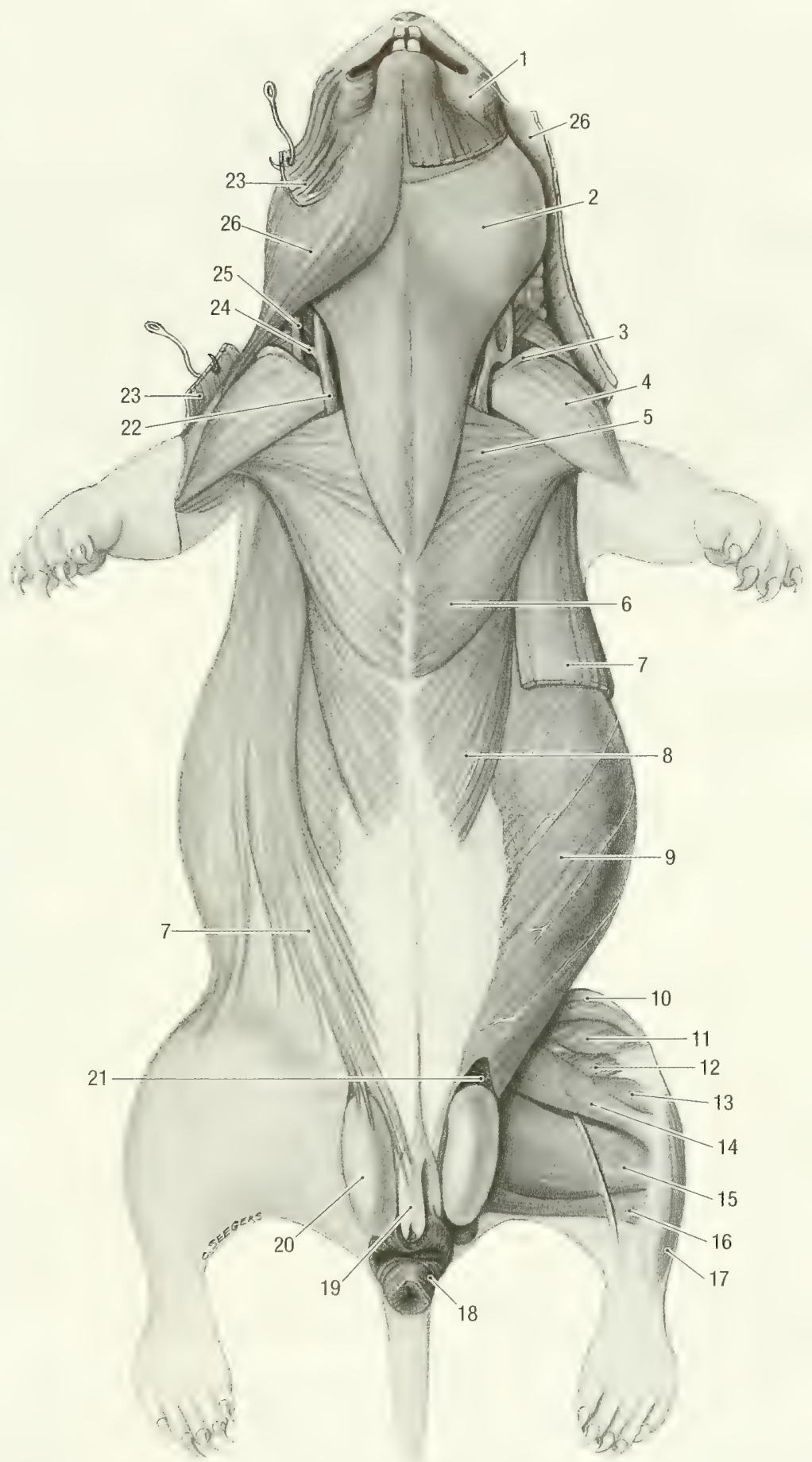

Fig. 4-2. Muscles of the trunk, ventral view. 1 orbicularis oris $\mathrm{m}$., 2 cutaneus colli $\mathrm{m} ., 3$ clavicle, 4 cleidobrachialis m., 5 descending pectoral m., 6 transverse pectoral m., 7 cutaneus trunci m., 8 deep pectoral m., 9 external oblique abdominal m., 10 tensor fasciae latae m., 11 medial vastus m., 12 long adductor m., 13 short adductor m., 14 gracilis m., 15 semimembranosus m., 16 semitendinosus m., 17 cranial tibial m., 18 external anal sphincter m., 19 prepuce and penis, 20 testicle, 21 transverse abdominal m., 22 continuation of the linguofacial vein, 23 platysma, 24 connection between the linguofacial and maxillary veins, 25 maxillary vein, 26 acromiomandibular $\mathrm{m}$. 


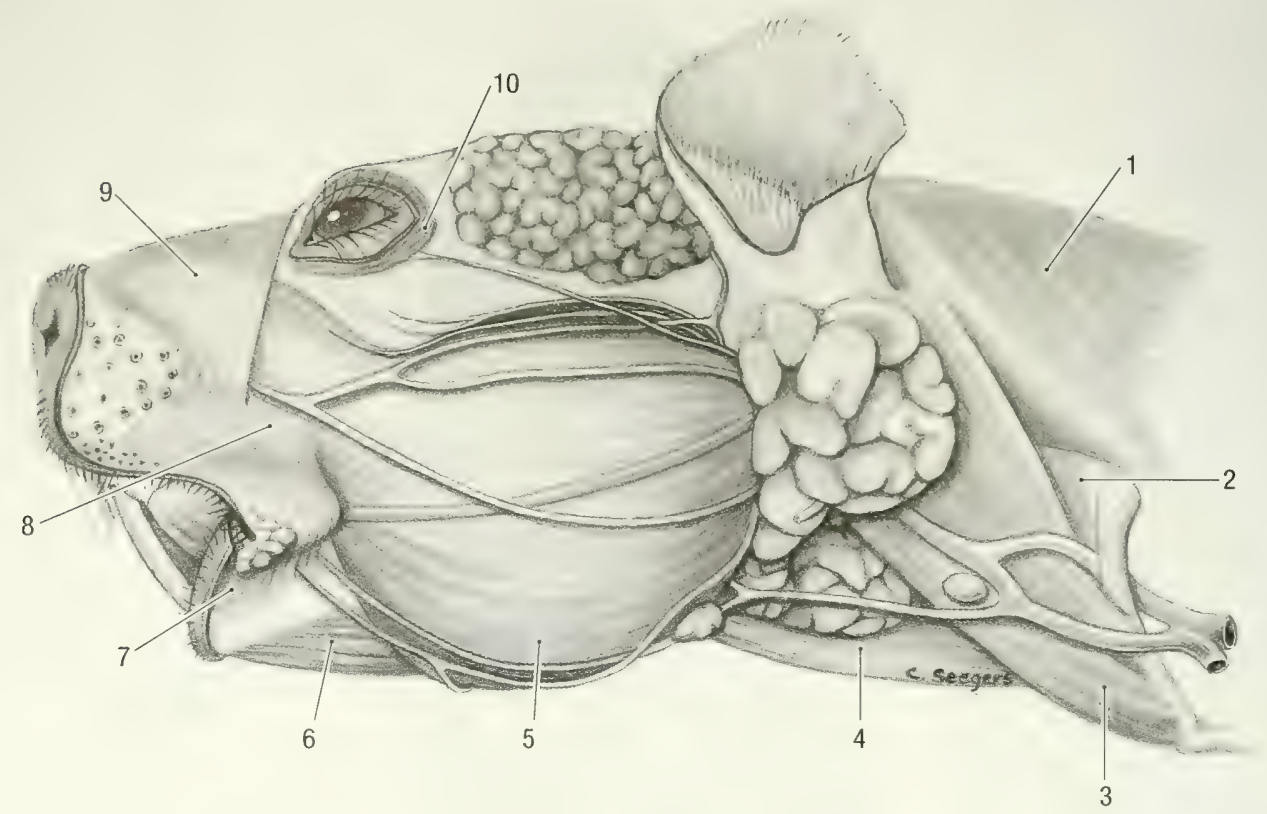

Fig. 4-3. Head and neck, left lateral view. 1 cervical trapezius m., 2 sternocephalic m., 3 cleidocephalic m. (combined mastoid and occipital parts), 4 cutaneus colli m., 5 masseter m., 6 digastric m. (rostral belly, cutaneus colli removed), 7 orbicularis oris $m ., 8$ buccinator $m$., 9 levator nasolabialis $m ., 10$ orbicularis oculi $m$.

(maxillonasalis of Bryant, 1945), takes origin from the fascia of the masseter muscle ventral to the medial canthus of the eye. It has a long, flat tendon of insertion that passes to the dorsum of the nose where it inserts, in common with the muscle from the opposite side, into the fascia in the apex of the nose. It lies deep to the levator naso-labialis muscle.

Innervation: $\mathrm{N}$. facialis.

The frontal muscle, $m$. frontalis (preauricularis of Bryant, 1945), is a small muscle that takes origin from the supraorbital margin and fans out laterally to insert on the fascia deep to the temporal cutaneous glands. The latter is situated between the lateral canthus of the eye and the ear.

Innervation: $\mathrm{N}$. facialis.

Muscles of the Eyelids. The orbicularis oculi muscle, $m$. orbicularis oculi, is a thin muscle that surrounds the eye. It lies within the eyelids and can be divided into superior (upper) and inferior (lower) parts. It serves to close the eyelids.

Innervation: $\mathrm{N}$. facialis.

The malar muscle, $m$. malaris, is a thin muscular sheet that extends from the lower eyelid into the fascia below the eye. It pulls the lower eyelid ventrally.

Innervation: $\mathrm{N}$. facialis.

The retractor of the lateral angle of the eye, $m$. retractor anguli oculi lateralis (Bryant, 1945, considers this muscle part of the orbicularis oculi), is a flat muscle band that extends caudoventrally from the lateral canthus of the eye to the temporal fascia and the zygomatic arch. It retracts the lateral canthus of the eye.

Innervation: N. facialis.

The levator of the upper eyelid, $m$. levator palpebrae superioris (Bryant, 1945 considers this muscle part of the orbicularis oculi), is a thin sheet of muscle which lies superficial to the dorsal straight muscle of the eye. It takes origin from the same area as the dorsal straight muscle and inserts on the fascia of the upper eyelid.

Innervation: N. oculomotorius.

The levator of the medial angle of the eye, $m$. levator anguli oculi medialis (Bryant, 1945 considers the muscle part of the oribicularis oculi), is a fan shaped muscle that extends from the medial canthus of the eye to the supraorbital region.

Innervation: N. facialis.

Muscles of the Ear. Meinertz (1943) made an extensive study of the muscles of the ear of Marmota marmota. Alternate terms used by him are cited in the text where they differ from that of the NAV. The muscles of the ear are grouped into rostral, ventral, caudal and dorsal ear muscles. All the muscles of the ear are innervated by the facial nerve.

The rostral muscles of the ear, $\mathrm{mm}$. auriculares rostrales, rotate the ear inwards, and are innervated by the rostral auricular branch of the facial nerve. The $m$. zygomaticoauricularis ( $m$. frontalis pars au- 
ricularis of Meinertz, 1943) takes origin from the temporal fascia and zygomatic arch, and inserts on the distomedial part of the pinna.

The $m$. scutuloauricularis superficialis ( $m$. auricularis anterior superficialis of Meinertz, 1943) is the auricular part of the frontal muscle, and attaches to the mediodistal part of the pinna.

The scutuloauricularis profundus (m. cervico-auricularis of Meinertz, 1943) extends from the deep face of the scutiform cartilage to the medial border of the pinna. It is the deepest of the inward rotators of the ear.

The ventral muscles of the ear, $\mathrm{mm}$. auriculares ventrales, are represented by the $m$. parotidoauricularis (pars oris m. sphincter colli profundus of Meinertz, 1943). This thin muscular slip takes origin from the ventrolateral part of the ear cartilage and inserts on the deep face of the sterno-mandibular muscle. It depresses the ear and is innervated by the ramus colli of the facial nerve.

The caudal muscles of the ear, $\mathrm{mm}$. auriculares caudales, rotate the ear outward and are innervated by the caudal auricular branch of the facial nerve.

The cervicoauricularis superficialis ( $m$. cervico-auricularis anterior medius of Meinertz, 1943) is a broad, flat muscle that takes origin from the median raphe of the neck and inserts on the rostromedial border of the ear cartilage. A large part of the muscle extends over the temporal muscle to the frontal region.

The $\mathrm{mm}$. cervicoauricularis medius ( $m$. cervico-auricularis posterior medius of Meinertz, 1943) is a small muscle that lies deep to the superficial cervico-auricular muscle. It takes origin from the median raphe of the neck and inserts on the caudolateral part of the ear cartilage.

The $m$. cervicoauricularis profundus ( $m$. cervicoauricularis posterior profundus of Meinertz, 1943) takes origin from the temporal fascia caudal to the ear and insert on the caudomedial aspect of the ear cartilage.

The dorsal ear muscles, $\mathrm{mm}$. auriculares dorsales, pull the pinna medially, and are innervated by the caudal and rostral auricular branches of the facial nerve.

The $m$. interscutularis is a thin muscular strand that extends from one scutiform cartilage to the other. It is part of the frontal muscle and is innervated by the rostral auricular branch of the facial nerve.

The $m$. parietoscutularis ( $m$. cervico-occipitalis of Meinertz, 1943) extends from the parietal region to the scutiform cartilage, and is innervated by the caudal auricular branch of the facial nerve.

The $m$. parietoauricularis ( $m$. cervico-anterior profundus of Meinertz, 1943) extends from the occipital and parietal regions to the pinna, and is inner- vated by the caudal auricular branch of the facial nerve.

Apart from the above-mentioned muscles, a series of short, intrinsic muscles ( $\mathrm{mm}$. auriculares) alter the shape and position of the ear cartilage and ear canal.

Muscles of the Eye. The seven muscles of the eye are arranged in such a way that they can move or rotate the eyeball around three axes: an anteroposterior axis, a dorso-ventral axis, and a craniocaudal axis. There are four straight muscles, two oblique muscles, and one retractor muscle. All of the muscles lie deep to the periorbita.

The lateral straight muscle, $m$. rectus lateralis, lies along the lateral surface of the eyeball.

Origin: Lateral to the optic canal.

Insertion: Sclera on the lateral aspect of the eyeball, just caudal to the limbus of the eye.

Action: Rotates the eyeball laterally around a vertical axis through the equator of the eye.

Innervation: $\mathrm{N}$. abducens.

The dorsal straight muscle, $m$. rectus dorsalis, lies along the dorsal border of the eyeball, deep to the levator of the upper eyelid.

Origin: Dorsal to the optic canal.

Insertion: Sclera on the dorsal aspect of the eyeball, caudal to the limbus of the eye.

Action: Rotate the eyeball dorsally around a horizontal axis through the equator of the eye.

Innervation: $\mathrm{N}$. oculomotorius.

The medial straight muscle, $m$. rectus medialis, lies along the medial border of the eyeball, ventral to the dorsal oblique muscle of the eye.

Origin: Rostral to the optic canal.

Insertion: Sclera on the medial aspect of the eye, caudal to the limbus.

Action: Rotates the eyeball medially around a vertical axis through the equator of the eye.

Innervation: N. oculomotorius.

The ventral straight muscle, $m$. rectus ventralis, lies along the ventral border of the eyeball.

Origin: Rostroventral to the optic canal.

Insertion: Sclera on the ventral aspect of the eye, caudal to the limbus.

Action: Rotates the eyeball ventrally around a horizontal axis.

Innervation: $\mathrm{N}$. oculomotorius.

The dorsal oblique muscle, $m$. obliquus dorsalis, lies along the medial border of the eyeball, dorsal to the medial straight muscle of the eye. Its tendon of insertion hooks around a small cartilaginous trochlea where it turns dorsolaterally to reach the dorsal aspect of the eyeball.

Origin: Rostral to the optic canal.

Insertion: On the sclera along the dorsal surface of the eyeball. 
Action: Medial rotation of the eyeball around an axis through the poles of the eye.

Innervation: $\mathrm{N}$. trochlearis.

The ventral oblique muscle, $m$. obliquus ventralis, lies transversely across the ventral aspect of the eyeball.

Origin: Lacrimal and palatine bones.

Insertion: Sclera on the ventral aspect of the eyeball.

Action: Rotates the eyeball ventrally around an axis through the poles of the eye.

Innervation: $\mathrm{N}$. oculomotorius.

The retractor of the bulb, $m$. retractor bulbi, is a cone shaped muscle consisting of four fascicles arranged around the optic nerve.

Origin: Deep to the rectus muscles around the optic canal.

Insertion: On the sclera around the equator of the eyeball, caudal to the rectus muscles.

Action: Retracts the eyeball.

Innervation: N. abducens.

Muscles of Mastication. The muscles of mastication consist of four muscles that adduct or close the jaw, and one muscle which abducts or opens the jaw. All the adductors of the jaw are innervated by branches of the mandibular division of the trigeminal nerve, whereas the abductor is innervated by branches of the mandibular and facial nerves. Thorington and Darrow (1996) reviewed and illustrated the jaw muscles of old world sciurids. They provided descriptions of the jaw muscles for twelve genera and discussed the functional significance of the differences. Stark and Wehrli (1935) described and illustrated the jaw muscles of M. marmota.

The masseter muscle, $m$. masseter (Fig. 4-3), is large and powerful. It lies on the lateral aspect of the mandible, ventral to the zygomatic arch. Hill (1937), Bryant (1945) and Ryan (1989) describe five layers of the masseter muscle in the pocket gopher (Geomyidae), in members of the Sciuridae, and in members of Heteromyidae, respectively. The masseter of the woodchuck is similar to that of other rodents and the terminology used for the following description is based on that of Ryan (1989).

The superficial masseter, $m$. masseter superficialis, fills the ventral half of the space between the $\mathrm{zy}$ gomatic arch and ventral border of the mandible. Its rostral part covers the rostral part of the deep lateral masseter muscle, while the caudal part is intimately fused to the caudal part of the deep lateral masseter muscle.

Origin: By means of a narrow tendon from the facial tuberosity of the maxilla, ventrolateral to the infraorbital foramen. Its fibers run caudally and caudoventrally.
Insertion: On the lateral aspect of the angular process and on the ventral margin of the mandible.

Action: Pulls the jaw forward.

The lateral deep masseter muscle, rostral part, $m$. masseter lateralis profundus pars rostralis, fills the groove (zygomatic plate) between the zygomatic arch and the facial tuberosity.

Origin: From the zygomatic plate and zygomatic arch of the maxilla. Its fibers pass caudoventrally.

Insertion: Laterally on the body of the mandible, deep to the insertion of the superficial masseter muscle and along the rostral border of the masseteric fossa.

Action: Close the jaw and pull it forward.

The lateral deep masseter muscle, caudal part, $m$. masseter lateralis profundus pars caudalis, is intimately fused at its origin to the superfical masseter muscle, making it almost impossible to separate the two muscles.

Origin: From the zygomatic arch. Its fibers pass ventrocaudally.

Insertion: In the masseteric fossa and on the lateral aspect of the angular process of the mandible.

Action: Close the jaw.

The medial masseter muscle, rostral part, $m$. masseter medialis pars rostralis, lies deep to the superficial masseter and rostral to the coronoid process of the mandible.

Origin: From the rostromedial aspect of the zygomatic arch.

Insertion: Masseteric fossa and rostral margin of the coronoid process.

Action: Close the jaw.

The medial masseter muscle, caudal part, $m$. masseter medialis pars caudalis, lies deep to the superficial masseter and caudal to the coronoid process of the mandible.

Origin: From the caudomedial aspect of the zygomatic arch.

Insertion: Caudal aspect of the coronoid process and the notch between the coronoid and condylar processes.

Innervation: N. massetericus.

The temporal muscle, $m$. temporalis (Figs. 4-1 and $5 \mathrm{~b}$ ), is a large and powerful muscle in the temporal fossa of the skull.

Origin: From the temporal line, nuchal crest and a small part from the zygomatic arch.

Insertion: On the lateral and medial aspects of the coronoid process of the mandible.

Action: Close the jaw.

Innervation: $\mathrm{Nn}$. temporales profundi.

The medial pterygoid muscle, $m$. pterygoideus medialis (pterygoideus internus of Bryant, 1945), fills the pterygoid fossa on the medial surface of the ramus of the mandible.

Origin: From the pterygoid crest and the ptery- 


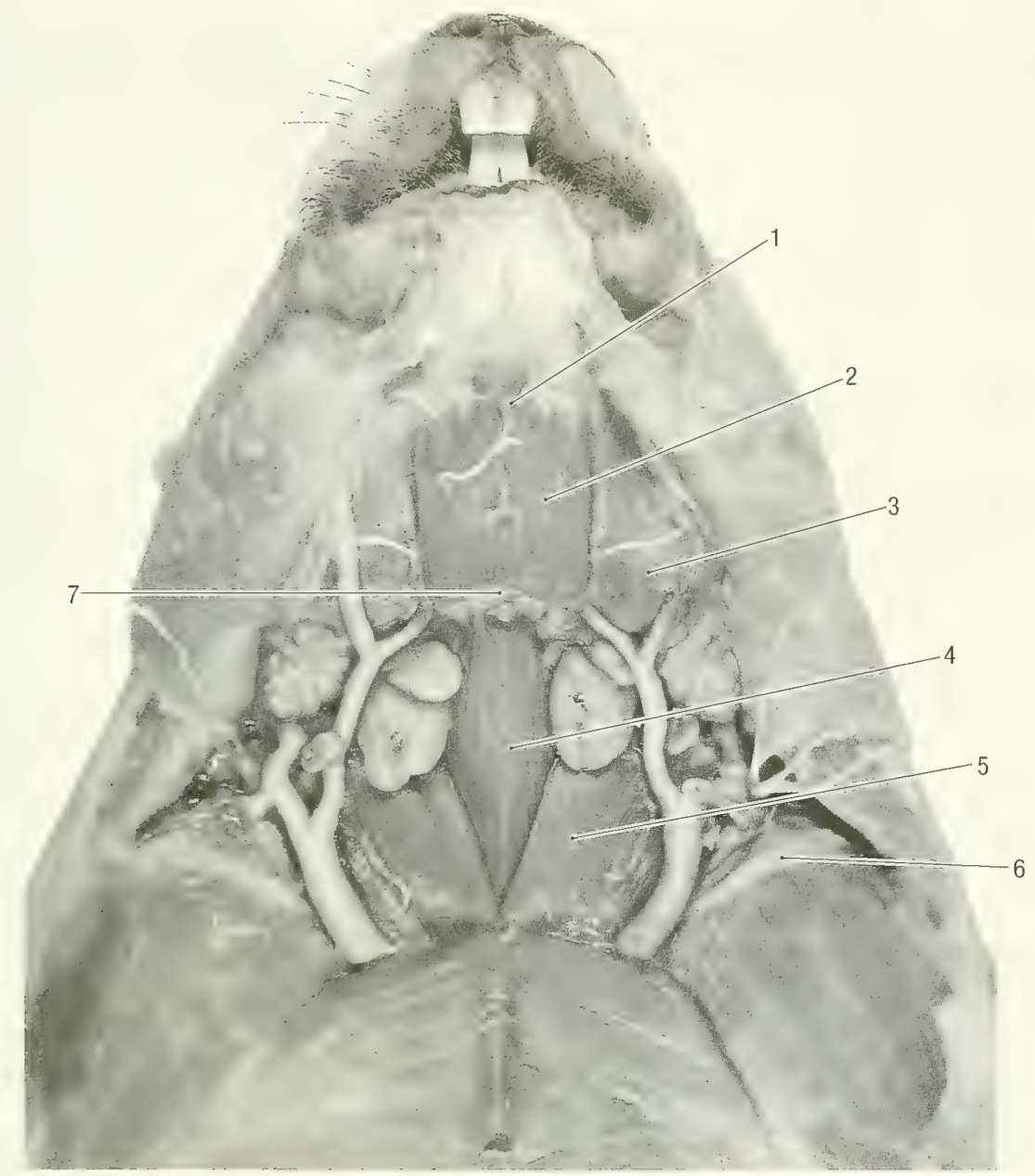

Fig. 4-4. Middle muscle layer of the ventral neck, ventral view. 1 mylohyoid m., 2 rostral belly of the digastric m., 3 masseter m., 4 sternohyoid m., 5 sternocephalic m., 6 left clavicle, 7 fibrous intersection between the rostral and caudal bellies of the digastric $m$.

goid process of the basisphenoid bone, and from the pterygoid bone.

Insertion: On the fossa pterygoidea and on the medial aspect of the angular process of the mandible.

Action: Acting together they close the jaws, and acting unilaterally they close the jaws and pull the mandible to the contralateral side.

Innervation: N. pterygoideus medialis.

The lateral pterygoid muscle, $m$. pterygoideus lateralis (pterygoideus externus of Bryant, 1945), lies medial to the condylar process of the mandible, and caudolateral to the medial pterygoid muscle.

Origin: Wing and pterygoid crest of the basisphenoid bone, lateral to the origin of the medial pterygoid muscle.

Insertion: On the articular meniscus and ventral to the articular surface on the medial aspect of the condylar process.
Action: Protracts the jaw.

Innervation: N. pterygoideus lateralis.

The digastric muscle, $m$. digastricus, consists of two large bellies, one caudal and the other rostral. The caudal belly, venter caudalis (Fig. 4-5), lies lateral to the pharynx. It is a pear shaped muscle that extends between the paracondylar process of the occipital bone and the basihyoid bone.

Origin: Paracondylar process.

Insertion: The tendon of insertion divides into medial and lateral parts. The medial part inserts on the basihyoid bone and the lateral part joins the tendon of the rostral belly.

Action: Together with the rostral belly they open the jaw and pull it caudally.

Innervation: N. facialis.

The rostral belly, venter rostralis (Fig. 4-4), lies in the intermandibular space, deep to the cutaneous muscle and superficial to the mylohyoid muscle. 
The left and right muscles are in contact with each other in the midline. Muscle fibers from one muscle may cross the midline to join the muscle from the opposite side.

Origin: Mandible, just caudal to the symphysis. Insertion: On the tendon of the caudal belly.

Action: Together with the caudal belly it opens the jaw and pulls it caudally.

Innervation: N. mylohyoideus.

Muscles of the Tongue. Three extrinsic muscles and one intrinsic muscle are present in the tongue.

The styloglossal muscle, $m$. styloglossus, is the most lateral of the tongue muscles. It extends rostrally from the distal part of the stylohyoid bone into the tongue.

Origin: Stylohyoid bone.

Insertion: Tongue.

Action: Draws the tongue caudally.

Innervation: N. hypoglossus.

The hyoglossal muscle, $m$. hyoglossus, lies medial to the styloglossal muscle. It extends rostrally from the thyrohyoid bone into the tongue.

Origin: From the thyrohyoid bone.

Insertion: Tongue.

Action: Pulls the tongue down and caudally.

Innervation: N. hypoglossus.

The genioglossal muscle, $m$. genioglossus (Fig. 46 ), is the most medial of the tongue muscles. It radiates caudally from the symphysis and from the body of the mandible into the tongue.

Origin: From the symphysis and body of the mandible.

Insertion: Tongue.

Action: Draws the tongue forward.

Innervation: N. hypoglossus.

The intrinsic muscle of the tongue, $m$. lingualis proprius, consists of a mass of muscle fibers that traverse the tongue in all directions. They alter the shape of the tongue and are innervated by the hypoglossal nerves.

Muscles of the Hyoid Apparatus. The omohyoid muscle, $m$. omohyoideus (Fig. 4-7), is a thin, strap-like muscle that extends from the shoulder to the hyoid bone.

Origin: From the cranial border of the neck of the scapula, proximal to the coracoid process. It emerges between the subscapular and supraspinatus muscles.

Insertion: On the basihyoid bone, lateral to the sternohyoid muscle.

Action: Pulls the hyoid bone caudally.

Innervation: Ventral branches of the cervical spinal nerves.

The sternohyoid muscle, m. sternohyoideus (Fig.
4-4), extends between the sternum and the hyoid bone. It lies deep to the sternocephalic muscle and is superficial to the sternothyroid muscle along the ventral aspect of the neck.

Origin: Manubrium of the sternum.

Insertion: On the basihyoid bone.

Action: Pulls the hyoid bone caudally.

Innervation: Ventral branches of the first two cervical spinal nerves.

The thyrohyoid muscle, $m$. thyrohyoideus (Fig. 4$5 a)$ extends from the thyroid cartilage of the larynx to the hyoid bone.

Origin: Lamina of the thyroid cartilage.

Insertion: Thyrohyoid and basihyoid bones.

Action: Draws the hyoid apparatus caudally if the larynx is fixed, or the larynx rostrally if the hyoid bone is fixed.

Innervation: Ventral branches of the first two cervical spinal nerves.

The stylohyoid muscle, m. stylohyoideus (Fig. 4 $5 b)$ lies medial to the caudal belly of the digastric muscle on the lateral aspect of the pharynx.

Origin: By means of a long tendon from the stylomastoid process of the temporal bone.

Insertion: It has a fleshy insertion on the epihyoid, ceratohyoid and basihyoid bones.

Action: Lifts the hyoid apparatus.

Innervation: N. facialis.

The ceratohyoid muscle, $m$. ceratohyoideus, lies in the angle between the thyrohyoid and ceratohyoid bones.

Origin: From the tip of the thyrohyoid bone.

Insertion: Ceratohyoid bone and the tip of the epihyoid bone.

Action: Pulls the thyrohyoid bone rostrally.

Innervation: N. vagus.

The geniohyoid muscle, m. geniohyoideus (Fig. 46) lies deep to the mylohyoid muscle in the intermandibular space.

Origin: Symphysis of the mandible.

Insertion: On the basihyoid bone.

Action: Draws the hyoid apparatus rostrally.

Innervation: $\mathrm{N}$. hypoglossus.

The mylohyoid muscle, $m$. mylohyoideus (Figs. 4$5 a$ and $4-6$ ), forms a sling for the tongue between the bodies of the mandibles. It lies deep to the rostral belly of the digastric muscle.

Origin: From the mylohyoid line on the medial surface of the mandible.

Insertion: Basihyoid bone. The greater part of the muscle inserts on a median raphe in the intermandibular space in common with the muscle of the opposite side.

Action: Raises the floor of the mouth and draws the hyoid apparatus rostrally.

Innervation: N. mylohyoideus. 


\section{Chapter 4-Muscular System}

a

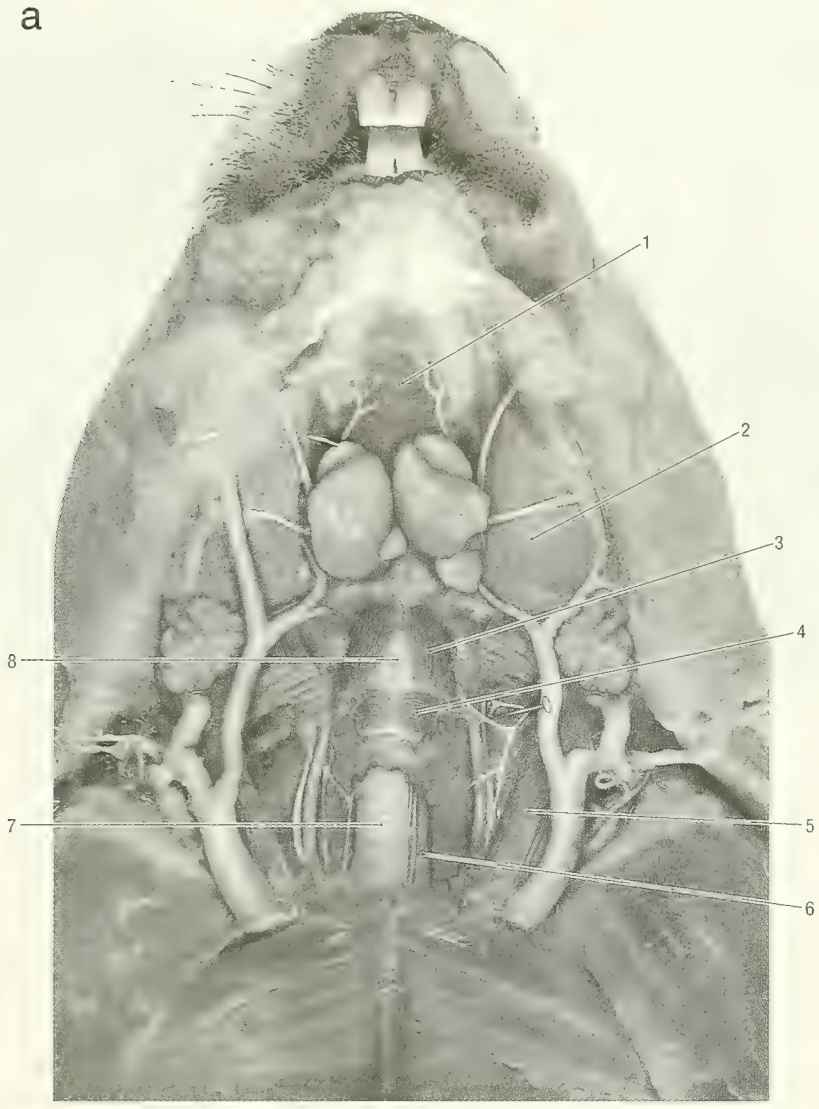

b

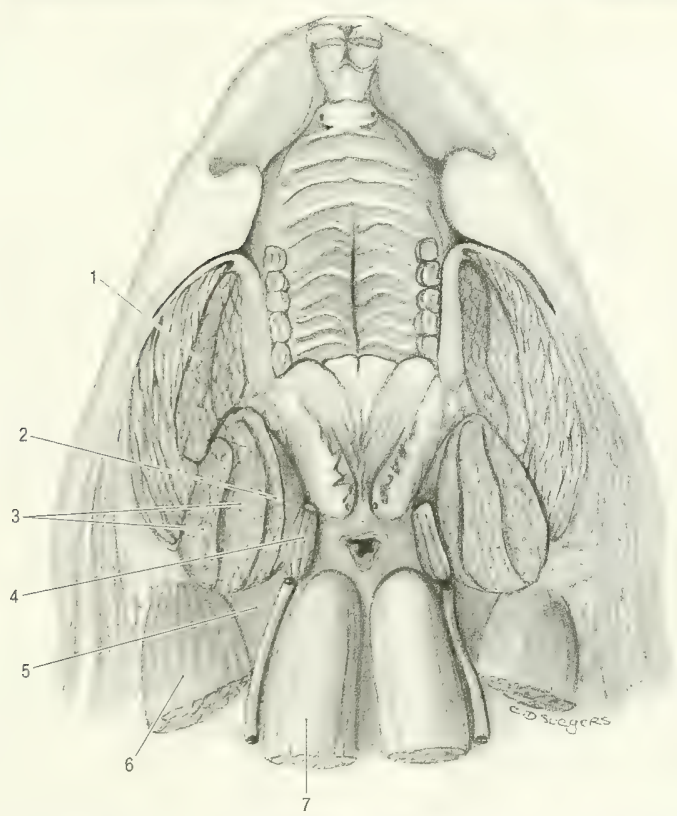

Fig. 4-5. a) Deep muscles of the ventral neck, ventral view. 1 mylohyoid m., 2 masseter m., 3 thyrohyoid m., 4 cricothyroid m., sternomastoid m., 6 sternothyroid m., 7 trachea, 8 larynx. b) Horizontal section through the mouth and muscles of mastication, ventral view. 1 masseter m., 2 medial pterygoid m., 3 temporal m., 4 stylohyoid m., 5 caudal belly of the digastric m., 6 sternomastoid m., 7 longus capitis $m$. 


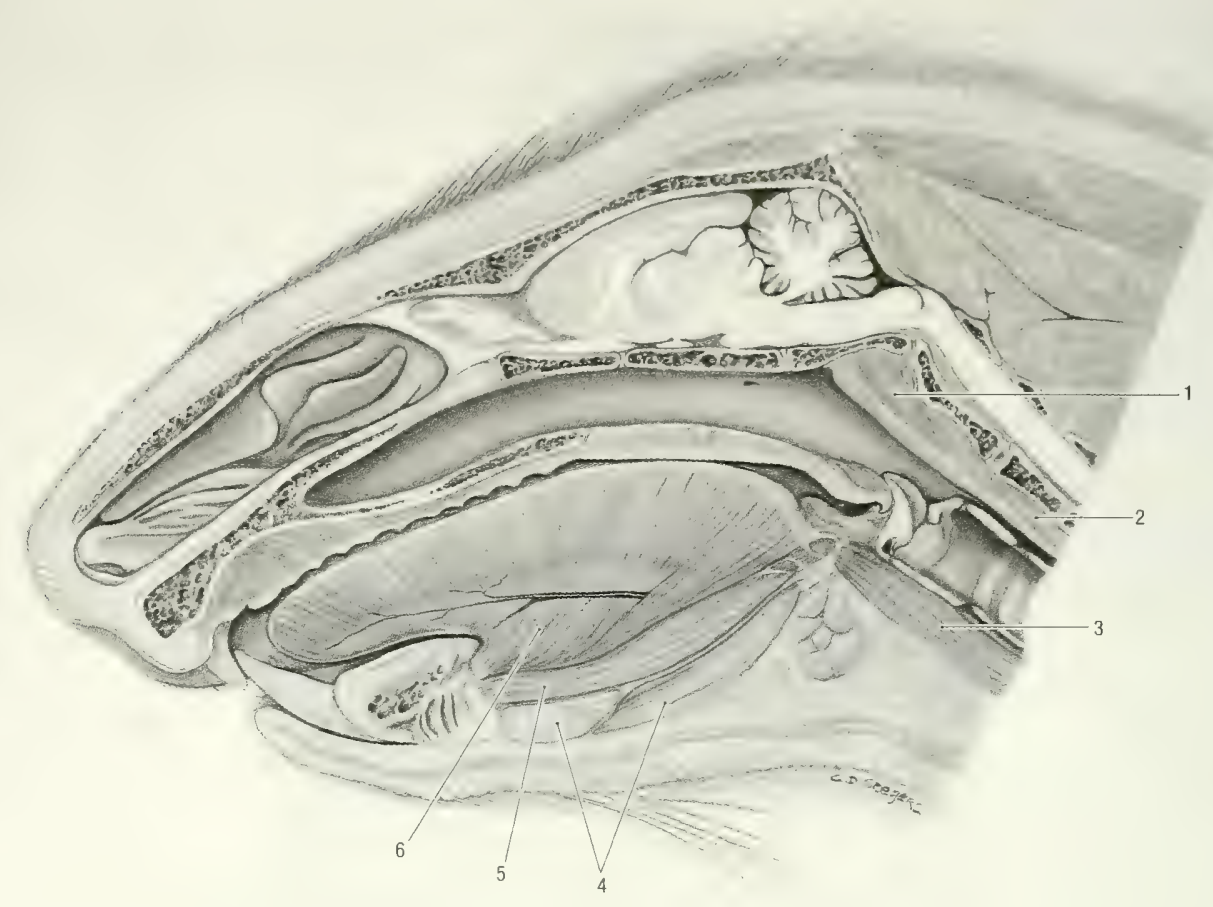

Fig. 4-6. Sagittal section of the head, medial view. 1 longus capitis m., 2 longus colli m., 3 sternohyoid m., 4 mylohyoid $\mathrm{m} ., 5$ geniohyoid $\mathrm{m}$., 6 genioglossus $\mathrm{m}$.

Muscles of the Soft Palate. The soft palate is raised and tensed by two muscles.

The tensor of the soft palate, $m$. tensor veli palatini, extends between the base of the skull and the lateral part of the soft palate.

Origin: From the muscular process of the basioccipital bone and the tympano-occipital suture (between the tympanic bulla and the basi-occipital bone).

Insertion: The tendon of the muscle passes lateral to the pterygoid bone and then turns medially around the hamulus to insert on the soft palate.

Action: It tenses the soft palate between the pterygoid bones.

Innervation: N. mandibularis.

The levator of the soft palate, $m$. levator veli palatini, extends between the base of the skull and the caudal part of the soft palate.

Origin: Caudal to the tensor of the soft palate, from the muscular process and tympano-occipital suture.

Insertion: It passes rostro-ventrally to insert on the soft palate.

Action: Raises the soft palate.

Innervation: $\mathrm{N}$. mandibularis.

The pharyngeal muscles are arranged in such a way that they act as constrictors of the pharynx. They can be grouped into rostral, middle and caudal constrictors. The pharynx has only one dilator.
Rostral Constrictors. The pterygopharyngeal muscle, $m$. pterygopharyngeus, radiates from the pterygoid bone into the pharynx.

Origin: From the hamulus of the pterygoid bone. Insertion: The muscle passes caudally into the pharynx and around the intrapharyngeal opening.

Action: Constricts the pharynx and closes the intrapharyngeal opening.

Innervation: $\mathrm{N}$. vagus.

The palatopharyngeal muscle, $m$. palatopharyngeus, extends caudally from the caudal border of the hard palate into the soft palate.

Origin: Caudal border of the hard palate.

Insertion: Rostral part of the pharynx.

Action: Draws the roof of the pharynx rostrally and ventrally.

Innervation: $\mathrm{N}$. vagus.

Middle Constrictor. The hyopharyngeal muscle, $m$. hyopharyngeus, extends from the hyoid bone into the roof of the pharynx.

Origin: Tip of the thyrohyoid bone.

Insertion: The fibers pass dorsomedially to insert on the median dorsal raphe of the pharynx.

Action: Constricts the middle of the pharynx.

Innervation: $\mathrm{N}$. vagus.

Caudal Constrictors. The thyropharyngeal muscle, $m$. thyropharyngeus, extends dorsally from 
the thyroid cartilage of the larynx into the roof of the pharynx.

Origin: From the lateral aspect of the thyroid lamina.

Insertion: On the median dorsal raphe of the pharynx.

Action: Constricts the caudal part of the pharynx.

Innervation: N. vagus.

The cricopharyngeal muscle, $m$. cricopharyngeus, is the most caudal of the pharyngeal muscles. It extends dorsally from the cricoid cartilage of the larynx into the roof of the pharynx. It is continuous with the circular muscle of the esophagus.

Origin: From the arch of the cricoid cartilage.

Insertion: On the median dorsal raphe of the pharynx.

Action: Constricts the caudal part of the pharynx.

Innervation: $\mathrm{N}$. vagus.

Dilator of the Pharynx. The caudal stylopharyneal muscle, $m$. stylopharyngeus caudalis, extends ventrally from the stylohyoid bone into the roof of the pharynx.

Origin: From the proximal part of the stylohyoid bone.

Insertion: On the roof of the pharynx.

Action: Lifts and opens the pharynx.

Innervation: N. glossopharygeus.

The muscles of the larynx are grouped into extrinsic muscles that move the larynx in relation to the body, and intrinsic muscles that move the laryngeal cartilages in relation to each other.

Extrinsic Muscles of the Larynx. The sternothyroid muscle, m. sternothyroideus, (Fig. 4-5a) extends along the ventro-lateral border of the trachea, from the sternum to the thyroid lamina of the larynx.

Origin: From the second sternebra and second costal cartilage.

Insertion: Thyroid cartilage.

Action: Pulls the larynx caudally.

Innervation: $\mathrm{N}$. accessorius and ventral branches of cervical spinal nerves.

The thyrohyoid muscle, $m$. thyrohyoideus (Fig. 4$5 a)$, extends rostrally from the thyroid cartilage to the hyoid bone.

Origin: Thyroid cartilage.

Insertion: Basihyoid bone.

Action: Pulls the larynx rostrally.

Innervation: Ventral branches of cervical spinal nerves.

Intrinsic Muscles of the Larynx. The dorsal cricoarytenoid muscle, $m$ cricoarytenoideus dorsalis, lies dorsally on the lamina of cricoid cartilage. It extends between the cricoid and arytenoid cartilages.

Origin: From the dorsolateral surface of the lamina of the cricoid cartilage.

Insertion: The fibers converge to insert on the muscular process of the arytenoid cartilage.

Action: Abducts the vocal process, thereby opening the glottis.

Innervation: $\mathrm{N}$. vagus.

The lateral cricoarytenoid muscle, $m$. cricoarytenoideus lateralis, lies laterally on the larynx, between the thyroid and cricoid cartilages.

Origin: From the lateral and cranial surface of the arch of the cricoid cartilage.

Insertion: On the muscular process of the arytenoid cartilage.

Action: Adducts the vocal process, thereby closing the rima glottis.

Innervation: $\mathrm{N}$. vagus.

The thyroarytenoid muscle, $m$. thyroarytenoideus, extends from the thyroid to the arytenoid cartilages. It lies medial to the thyroid cartilage and rostral to the lateral cricoarytenoid muscle.

Origin: Along the internal midline of the thyroid cartilage.

Insertion: On the muscular process of the arytenoid cartilage.

Action: It relaxes the vocal cord and constricts the glottis.

Innervation: $\mathrm{N}$. vagus.

The vocal muscle, $m$. vocalis, extends between the floor of the larynx and the vocal process of the arytenoid cartilage.

Origin: From the internal midline of the thyroid cartilage.

Insertion: On the vocal process of the arytenoid cartilage.

Action: To draw the arytenoid cartilage downward, thus relaxing the vocal cord.

Innervation: $\mathrm{N}$. vagus.

The cricothyroid muscle, $m$. cricothyroideus (Fig. 4-5a), lies on the ventral aspect of the larynx. It extends between the cricoid and thyroid cartilages.

Origin: From the ventrolateral surface of the arch of the cricoid cartilage.

Insertion: On the caudal margin and medial surface of the thyroid cartilage.

Action: To pivot the cricoid cartilage on its thyroid articulation, thus tensing the vocal cords.

Innervation: $\mathrm{N}$. vagus.

The hyoepiglottic muscle, $m$. hyoepiglotticus, is the only muscle associated with the epiglottis. It extends from the base of the epiglottis to the hyoid bone.

Origin: From the lingual surface of the epiglottis. Insertion: On the basihyoid bone. 


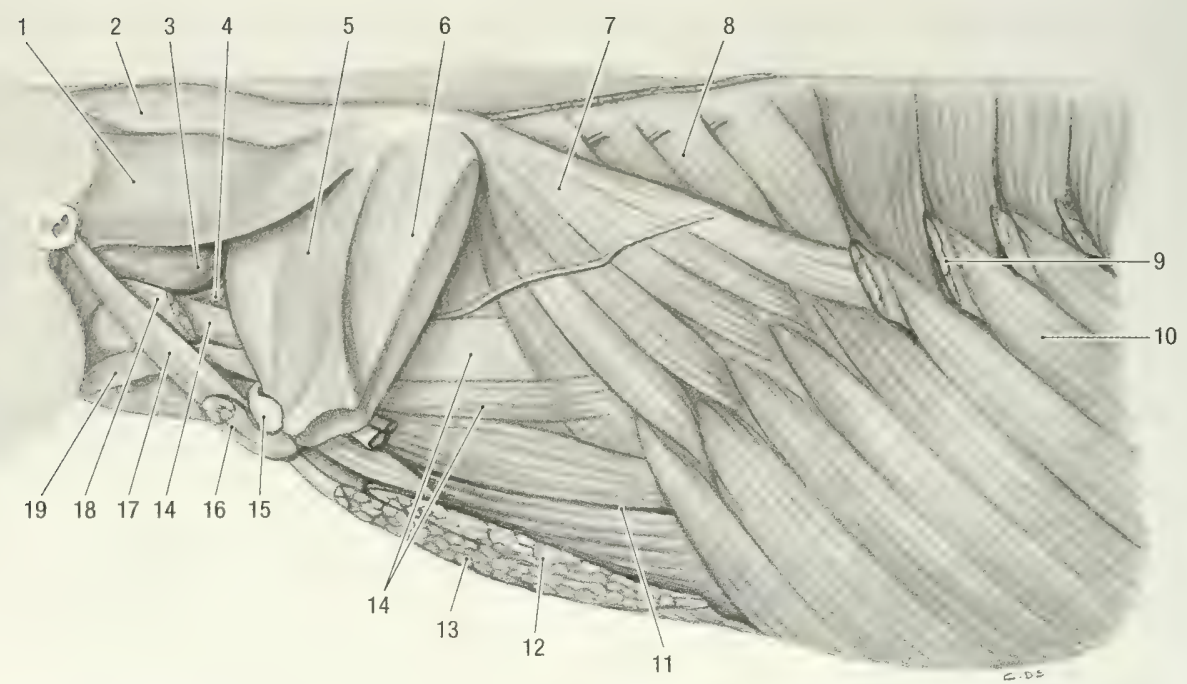

Fig. 4-7. Neck and thorax, left lateral view. 1 rhomboid m., capital part, 2 rhomboid m., cervical part, 3 splenius m., 4 serratus ventralis m., cervical part, 5 supraspinatus m., 6 infraspinatus m., 7 serratus ventralis m., thoracic part, 8 serratus dorsalis $\mathrm{m}$., 9 latissimus dorsi $\mathrm{m}$. (cut), 10 external oblique abdominal $\mathrm{m}$., 11 rectus abdominis $\mathrm{m}$., 12 deep pectoral $\mathrm{m}$., 13 superficial pectoral m., 14 dorsal scalene m., 15 clavicle, 16 cleido-occipital m., 17 cleidomastoid m., 18 omotransversarius $\mathrm{m}$., 19 omohyoid $\mathrm{m}$.

Action: Draws the epiglottis ventrally and rostrally.

Innervation: $\mathrm{N}$, vagus.

\section{THORACIC LIMB}

Gilbert (1940) and Bryant (1945) studied the thoracic limb muscles of M. monax.

\section{Muscles that Attach the Thoracic Limb to} the Body. The muscles that attach the thoracic limb to the body are arranged around the scapula in four layers, two lateral and two medial to the scapula. They all belong to the muscles of the thorax, but are discussed here because their primary action is on the thoracic limb.

The acromiomandibular muscle, $m$. acromiomandibularis (Figs. 4-1 and 4-2) (acromial part of the platysma of Gilbert, 1940; platysma of Bryant, 1945), is the most superficial of all the muscles that attach the thoracic limb to the body. Knowledge of its homology in the trunk musculature is unsure. At its origin it lies deep to the platysma and superficial to the cervical trapezius muscles. It extends to the face over the same area as the $\mathrm{m}$. platysma, but deep to it.

Origin: From the distal part of the acromion.

Insertion: On a wide area covering the lateral aspect of the face. It inserts ventral to the eye, lateral aspect of the nose, the upper lip, the lower lip and the mandible.

Action: When the arm is fixed it retracts the upper and lower lips and moves the head laterally. If the head is fixed it pulls the limb forward.
Innervation: Ventral branches of the cervical spinal nerves (mainly ventral branches of the third cervical spinal nerve).

The trapezius muscle, $m$. trapezius (Figs. 4-1 and 4-7), forms a flat, muscular sheet on the lateral aspect of the shoulder. It extends from the dorsal midline of the thorax and neck and converges on the spine of the scapula. The muscle can be divided into thoracic and cervical parts.

The thoracic part, m. trapezius pars thoracica, (spinotrapezius of Gilbert, 1940; and Bryant, 1945).

Origin: Muscular origin from the spinous processes of the first six thoracic vertebrae and by means of an aponeurosis from the following two thoracic vertebrae.

Insertion: On the proximal part of the scapular spine.

Action: Lifts the scapula and pulls it caudally.

The cervical part, $m$. trapezius pars cervicalis (acromio- and clavotrapezius of Gilbert, 1940; acromiotrapezius of Bryant, 1945).

Origin: Muscular from the occiput, atlas and axis, and by means of an aponeurosis from the spinous processes of the rest of the cervical vertebrae and the median raphe of the neck.

Insertion: On the distal two thirds of the scapular spine and the acromion.

Action: Lifts the scapula and pulls it cranially.

Innervation: $\mathrm{N}$. accessorius.

The latissimus dorsi muscle, $m$. latissimus dorsi (Figs. 4-1, 4-7 and 4-8), is a fan shaped muscle that lies caudal to the scapula, deep to the thoracic part of the trapezius muscle. 


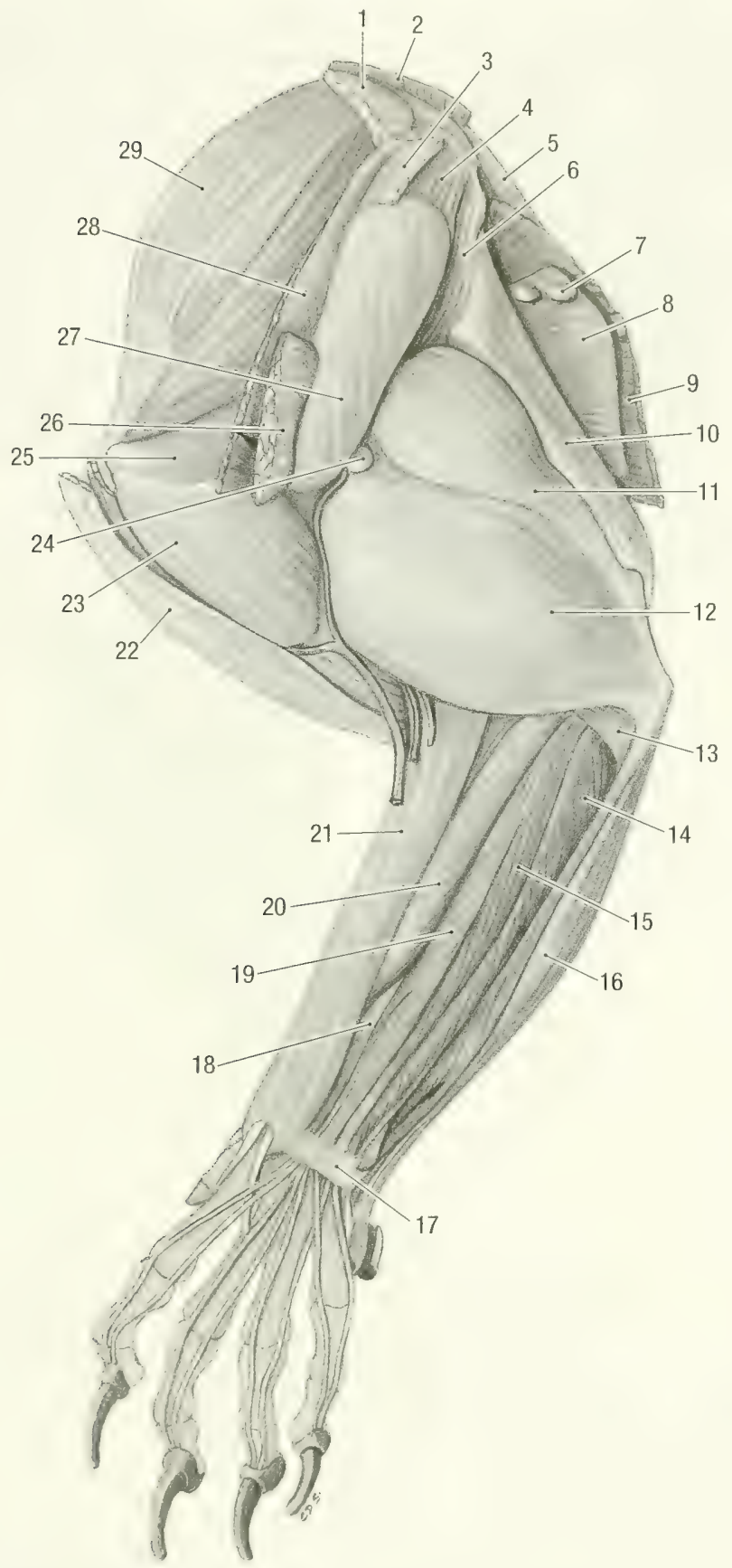

Fig. 4-8. Left thoracic limb, lateral view. 1 rhomboid m., capital part, 2 rhomboid m., cervical part, 3 m. trapezius, thoracic part, $4 \mathrm{~m}$. infraspinatus, $5 \mathrm{~m}$. serratus ventralis, thoracic part, 6 teres major $\mathrm{m}$., 7 accessory axillary In., 8 cutaneus trunci m., 9 latissimus dorsi m., 10 tensor fasciae antebrachii m., 11 triceps brachii m., long head, 12 triceps brachii m., lateral head, 13 anconeus $\mathrm{m}$., 14 extensor carpi ulnaris $\mathrm{m}$., 15 lateral digital extensor m., 16 flexor carpi ulnaris m., 17 extensor retinaculum, 18 long abductor $\mathrm{m}$. of digit I, 19 common digital extensor $\mathrm{m}$., 20 extensor carpi radialis $\mathrm{m}$., 21 brachioradialis m., 22 cleidobrachialis m., 23 deltoid m., acromial part, 24 infraspinatus In., 25 infraspinatus m., 26 acromiomandibularis m., 27 deltoid m., scapular part, 28 trapezius m., cervical part, 29 supraspinatus m. 
Origin: From the spinous processes of all the thoracic vertebrae, as well as from the last four ribs.

Insertion: In common with the deep pectoral muscle on the major tubercle of the humerus and on the fascia along the caudal border of the teres major muscle.

Action: Retracts the non-weight-bearing limb, or extends the shoulder joint during weight bearing, thus propelling the body forward.

Innervation: $\mathrm{N}$. thoracodorsalis.

The rhomboid muscle, $m$. rhomboideus (Fig. 47 ), is very robust muscle that can be divided into thoracic, cervical and capital parts. It lies deep to the trapezius muscle.

Thoracic part, $m$. rhomboideus thoracis (rhomboideus of Bryant, 1945).

Origin: Muscular from the spinous process of the first thoracic vertebra.

Insertion: Laterally and medially on the caudal part of the dorsal border of the scapula.

Action: Pulls the thoracic limb forward and lifts the scapula.

Innervation: Ventral branches of cervical and thoracic spinal nerves.

Cervical part, $m$. rhomboideus cervicis (rhomboideus of Bryant, 1945).

Origin: Muscular from the spinous processes of all the cervical vertebrae.

Insertion: Laterally and medially on the cranial part of the dorsal border of the scapula.

Capital part, $m$. rhomboideus capitis (occipitoscapularis of Gilbert, 1940; and of Bryant, 1945).

Origin: Muscular from the nuchal crest and the mastoid process.

Insertion: In common with the cervical part on the scapula.

The omotransversarius muscle, $m$. omotransversarius (Fig. 4-7), lies deep to the cervical trapezius muscle, and consists of two distinct parts. Gilbert (1940) identified the ventral part of the muscle and described the dorsal part, but did not name it.

Ventral part (atlantoscapularis of Gilbert, 1940; atlantoscapularis dorsalis of Bryant, 1945).

Origin: Ventral aspect of the wing and ventral arch of the atlas.

Insertion: Caudally on the distal part of the acromion.

Dorsal part (unidentified muscle of Gilbert, 1940, atlantoscapularis ventralis of Bryant, 1945).

Origin: Ventral aspect of the wing of the atlas.

Insertion: In common with the ventral part on the acromion.

Action: Pulls the thoracic limb cranially.

Innervation: $\mathrm{N}$. accessorius.

The superficial pectoral muscle, $m$. pectoralis superficialis (Figs. 4-2 and 4-7) (pectoralis major of Gilbert, 1940), forms the superficial, medial attach- ment of the thoracic limb to the body. It is partly covered by the sternomandibular muscle and can be divided into transverse and descending parts. Division and separation of the two parts, however, is difficult.

Descending part, $m$. pectoralis descendens, (ectopectoralis of Bryant, 1945).

Origin: From the manubrium of the sternum.

Transverse part, $m$. pectoralis transversus (ectopectoralis of Bryant, 1945).

Origin: From all the sternebrae.

Insertion: Both parts insert with a common aponeurosis on the humeral crest distal to the deltoid tubercle.

Action: Adducts and retracts the limb.

Innervation: $\mathrm{N}$. pectoralis cranialis.

The deep pectoral muscle, $m$. pectoralis profundus (Figs. 4-2, and 4-7) (pectoralis profundus and pectoralis abdominis of Gilbert, 1940; pectoralis minor and pectoralis abdominis of Bryant, 1945). The muscle can be divided into a cranial part that lies deep to (covered by) the superficial pectoral muscle, and a caudal part that lies caudal to the superficial pectoral muscle.

Cranial part

Origin: From the third sternebra to the xiphoid cartilage of the sternum.

Caudal part

Origin: From the xiphoid cartilage and the linea alba.

Insertion: Both parts insert on the major tubercle and the crest between the major and deltoid tubercles of the humerus.

Action: Adducts and retracts the limb.

Innervation: $\mathrm{N}$. pectoralis caudalis.

Note: The latissimus and cutaneus trunci muscles attach and blend distally with the caudal part of the deep pectoral muscle.

The serratus ventralis muscle, $m$. serratus ventralis (Figs, 4-7, 4-7 and 4-9) (serratus magnus of Gilbert, 1940; levator scapulae and serratus anterior of Bryant, 1945), suspends the trunk between the thoracic limbs. It can be divided into thoracic and cervical parts. The thoracic part lies deep to the latissimus dorsi muscle, and the cervical part lies deep to the omotransversarius and the trapezius muscles.

Origin: The thoracic part takes origin from the distal parts of the first nine ribs, and the cervical part takes origin from the transverse processes of the last three cervical vertebrae.

Insertion: On the facies serrata of scapula.

Action: Suspends and elevates the trunk.

Innervation: N. thoracicus longus (thoracic part) and the ventral branches of the last three cervical spinal nerves (cervical part).

The subclavian muscle, $m$. subclavius, is fairly 


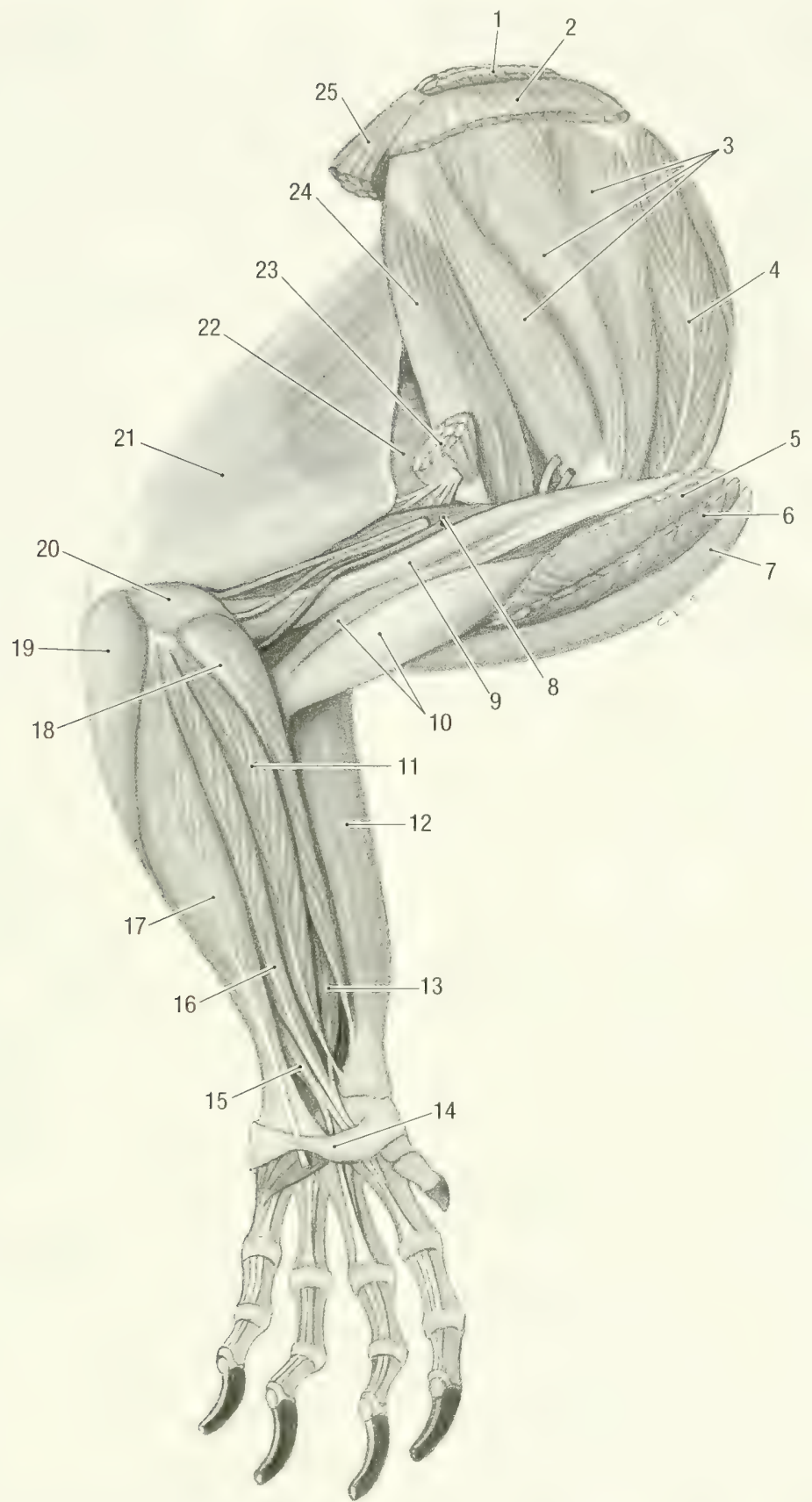

Fig. 4-9. Left thoracic limb, medial view. 1 rhomboideus $m$., 2 serratus ventralis $\mathrm{m}$., cervical part, 3 subscapular m., 4 supraspinatus $m ., 5$ deep pectoral $m ., 6$ superficial pectoral $m ., 7$ cleidobrachialis $m ., 8$ triceps brachii m., medial head, 9 coracobrachialis $\mathrm{m}$., 10 biceps brachii $\mathrm{m}$., 11 flexor carpi radialis $\mathrm{m}$., 12 brachioradialis $\mathrm{m}$., 13 deep digital flexor $\mathrm{m}$., radial head, 14 flexor retinaculum, 15 superficial digital flexor m., 16 deep digital flexor m., humeral head, 17 flexor carpi ulnaris $\mathrm{m}$., 18 pronator teres $\mathrm{m}$., 19 superficial digital flexor $\mathrm{m}$., epicondylar head, 20 anconeus m., 21 tensor fasciae antebrachii $\mathrm{m}$., 22 triceps brachii $\mathrm{m}$., long head, 23 latissimus dorsi $\mathrm{m}$. (cut), 24 teres major m., 25 serratus ventralis $\mathrm{m}$., thoracic part. 
well developed and lies deep to the superficial pectoral muscle. It extends from the first rib to the clavicle.

Origin: From the costal cartilage of the first rib. Insertion: On the medial aspect of the lateral two-thirds of the clavicle.

Action: Pulls the clavicle caudally.

Innervation: $\mathrm{N}$. subclavius.

Muscles of the Shoulder. The muscles of the shoulder are arranged along the lateral and medial surfaces of the scapula. They stabilize the shoulder and act as extensors and flexors of the shoulder joint.

The supraspinous muscle, $m$. supraspinatus (Figs. 4-7 and 4-8), lies in the supraspinous fossa of the scapula.

Origin: Fossa supraspinata.

Insertion: Laterally on the major tubercle of the humerus.

Action: Extension of the shoulder joint.

Innervation: $\mathrm{N}$. suprascapularis.

The infraspinous muscle, $m$. infraspinatus (Figs. 4-7 and 4-8) lies in the infraspinous fossa of the scapula. A thick layer of fascia and the scapular head of the deltoid muscle cover the muscle laterally.

Origin: Fossa infraspinata.

Insertion: Laterally on the distal part of the major tubercle of the humerus by means of a strong tendon.

Action: Lateral rotation of the humerus and flexion of the shoulder joint.

Innervation: $\mathrm{N}$. suprascapularis.

The subscapular muscle, $m$. subscapularis (Fig. 49), lies in the subscapular fossa of the scapula, distal to the insertion of the serratus ventralis muscle. A large synovial bursa is present between the distal part of the muscle and the coracoid process of the scapula.

Origin: Subscapular fossa.

Insertion: On the minor tubercle of the humerus.

Action: Extends the shoulder joint.

Innervation: $\mathrm{N}$. subscapularis.

The deltoid muscle, $m$. deltoideus (Figs. 4-1 and $4-8$ ), is a triangular muscle lateral to the shoulder, and consists of clavicular, acromial and scapular heads.

Clavicular head, $m$. deltoideus pars clavicularis (clavodeltoideus of Bryant, 1945).

Origin: Lateral two-thirds of the clavicle, deep to the cleido-brachialis muscle.

Acromial head, $m$, deltoideus pars acromialis (acromiodeltoid of Gilbert, 1940, acromiodeltoideus of Bryant, 1945).

Origin: Acromion of the scapula.

Scapular head, $m$. deltoideus pars scapularis (spi- nodeltoid of Gilbert, 1940; spinodeltoideus of Bryant, 1945).

Origin: From the fascia covering the infraspinous muscle and the spine of the scapula.

Insertion: All three parts insert on the deltoid tubercle of the humerus.

Action: Flexion of the shoulder joint.

Innervation: $\mathrm{N}$. axillaris.

The coracobrachial muscle, $m$. coracobrachialis (Fig. 4-9), lies on the medial aspect of the shoulder joint where it is closely associated with the joint capsule. Gilbert, 1940 divided the muscle into three parts.

Origin: By means of a long tendon from the coracoid process of the scapula. A synovial sheath surrounds the tendon.

Insertion: Muscular on the distal half of the caudomedial border of the humerus.

Action: Extension of the shoulder.

Innervation: $\mathrm{N}$. musculocutaneus.

The teres major muscle, $m$. teres major (Fig. 4 9 ), is the most caudal of the shoulder muscles. It lies medial to the deltoid and long head of the triceps muscles.

Origin: From the caudal angle of the scapula.

Insertion: On the teres major tubercle of the humerus.

Action: Flexion of the shoulder.

Innervation: $\mathrm{N}$. axillaris.

The teres minor muscle, $m$. teres minor, is a small muscle caudal to the shoulder joint. It is covered laterally by the deltoid muscle and is closely attached to the caudal border of the infraspinous muscle.

Origin: From the distal part of the caudal scapular margin, medial to the origin of the long head of the triceps muscle.

Insertion: On the tricipital line of the humerus, immediately distal to the insertion of the infraspinous muscle.

Action: A lateral rotator of the humerus and flexor the shoulder joint.

Innervation: $\mathrm{N}$. axillaris.

Muscles of the Elbow. The muscles of the elbow are reduced to flexors and extensors.

Flexors of the Elbow. The biceps muscle, $m$. biceps brachii (Fig. 4-9), lies cranially on the arm and has two heads. It is covered laterally by the deltoid and cleidobrachialis muscles.

Origin: The one head takes origin from the supraglenoid tubercle by means of a long tendon that is surrounded by a synovial sheath. The second head has a muscular origin from the tendon of the coracobrachialis muscle.

Insertion: On the radial tubercle along the caudo-medial border of the radius. 
Action: Flexion of the elbow.

Innervation: $\mathrm{N}$. musculocutaneus.

The brachial muscle, $m$. brachialis, lies along the caudal border of the humerus and spirals from caudal to lateral in the brachial groove under cover of (deep to) the lateral head of the triceps muscle.

Origin: Caudally on the humerus, just distal to the head.

Insertion: On the tubercle along the craniomedial border of the ulna, just distal to the medial coronoid process.

Action: Flexion of the elbow.

Innervation: N. musculocutaneus.

The cleidobrachial muscle, $m$. cleidobrachialis (Figs. 4-8 and 4-9) (clavobrachialis of Gilbert, 1940; clavodeltoideus of Bryant, 1945), forms the craniomedial muscular border of the arm.

Origin: From the lateral two-thirds of the clavicle.

Insertion: In common with the brachial muscle on the ulna.

Action: Draws the limb forward and flexes the elbow.

Innervation: N. musculocutaneus.

Extensors of the Elbow. The triceps muscle, m. triceps brachii (Figs. 4-8 and 4-9), is a triangular muscle mass which fills the space caudal to the shoulder joint. It is a very powerful extensor of the elbow and consists of a long head, caput longum, a lateral head, caput laterale, a medial head, caput mediale, and an accessory head, caput accessorium (Neither Gilbert, 1940 nor Bryant, 1945 recognized an accessory head).

Origin: Long head. Caudal border of the distal third of the scapula.

Lateral head. By means of a broad, flat tendon from the crest that runs from the major tubercle to the deltoid tubercle of the humerus (tricipital line).

Medial head. Along the caudomedial border of the humeral shaft.

Accessory head. From the caudolateral aspect of the humerus. It is fused to the medial head over most of its length.

Insertion: Olecranon.

Action: Extends the elbow.

Innervation: N. radialis.

The tensor of the antebrachial fascia, $m$. tensor fasciae antebrachii (Fig. 4-9) (epitrochlearis of Gilbert, 1940; dorsoepitrochlearis of Bryant, 1945), is the most caudal of the arm muscles. It lies along the caudomedial border of the triceps muscle.

Origin: From the caudal border of the teres major muscle and the lateral aspect of the latissimus dorsi muscle, close to the attachment of the latter on the teres major muscle.

Insertion: Distally on the tendon of the long head of the triceps muscle and on the caudomedial antebrachial fascia.

Action: Extends the elbow and tenses the antebrachial fascia.

Innervation: $\mathrm{N}$. radialis.

The anconeus muscle, $m$. anconeus (Fig. 4-8) (epitrochleo-anconeus of Bryant, 1945), covers the lateral and medial aspects of the elbow joint.

Origin: The lateral part takes origin from the lateral epicondyle and the medial part originates from the medial epicondyle of the humerus. Gilbert, 1940, as well as Bryant, 1945, only identified the medial part of the muscle.

Insertion: The lateral part inserts laterally and the medial part medially on the olecranon.

Action: Extends the elbow.

Innervation: $\mathrm{N}$, radialis.

Muscles of the Antebrachium. The flexors and extensors of the carpus and digits are arranged around the antebrachium. A thick layer of antebrachial fascia covers all the muscles. The extensors are arranged craniolaterally, and the flexors are arranged caudomedially on the antebrachium. The rotators of the manus lie deep to the flexors and extensors.

Extensors of the Carpus. The extensor carpi radialis, $m$. extensor carpi radialis (Fig. 4-8) (extensor carpi radialis longus and brevis of Gilbert, 1940; and of Bryant, 1945), is the most medial of the extensor muscles, consists of two distinct parts each with its own tendon. Both tendons are held in position over the carpus by the extensor retinaculum and have separate synovial sheaths.

Origin: From the lateral epicondyle of the humerus. The lateral head of the triceps covers its origin.

Insertion: The tendon of the medial part inserts proximomedially on the second metacarpal bone, and the lateral part inserts on the proximomedial aspect of the third metacarpal bone.

Action: Extends the carpus.

Innervation: $N$. radialis.

The extensor carpi ulnaris, $m$. extensor carpi $u l$ naris (Fig. 4-8), lies caudolaterally on the antebrachium, lateral to the lateral extensor of the digits.

Origin: Lateral condyle of the humerus.

Insertion: Proximally on the fifth metacarpal bone.

Action: Extends the carpus.

Innervation: $\mathrm{N}$. radialis.

Flexors of the Carpus. The flexor carpi radialis, $m$. flexor carpi radialis (Fig. 4-9), lies caudomedial on the shaft of the radius. Its proximal part lies caudal to the pronator teres muscle. 
Origin: From the medial condyle of the humerus.

Insertion: On caudal surface of the proximal end of the second and third metacarpal bones.

Action: Flexes the carpus.

Innervation: $N$. medianus.

The flexor carpi ulnaris, $m$. flexor carpi ulnaris (Fig. 4-8), lies caudolaterally on the shaft of the ulna.

Origin: From the olecranon and the shaft of the ulna, and from the medial epicondyle of the humerus (in common with the deep digital flexor muscle).

Insertion: Accessory carpal bone. It often has a tendinous attachment to the lateral carpal pad.

Innervation: N. medianus.

Extensors of the Digits. The common digital extensor, $m$. extensor digitorum communis (Fig. 4-8), lies lateral to the radial extensor of the carpus. The tendon divides into four. Proximal to the carpus the four tendons have a common synovial sheath, and are held in position by the extensor retinaculum.

Origin: Lateral condyle of the humerus.

Insertion: Extensor processes of the distal phalanges of digits two to five.

Action: Extends the toes (digits).

Innervation: $\mathrm{N}$. radialis.

The lateral digital extensor, $m$. extensor digitorum lateralis (Fig. 4-8) (extensor digiti quinti of Gilbert, 1940; extensor digiti quinti proprius of Bryant, 1945). The muscle lies lateral to the common digital extensor. At the carpus the tendon divides into two and is held in position by the extensor retinaculum.

Origin: Lateral condyle of the humerus.

Insertion: In common with the common digital extensor on the extensor processes of the distal phalanges of digits four and five. The tendons are also attached to the fascia of the second phalanges.

Action: Extends the outer two toes (digits IV and V).

Innervation: $\mathrm{N}$. radialis.

The extensor of the third digit, $m$. extensor digiti III, lies on the cranial surface of the shaft of the radius and is covered by the common digital extensor.

Origin: Lateral condyle of the humerus, distal to the origin of the common digital extensor.

Insertion: In common with the common digital extensor to the third digit. It also gives off a small branch to the common digital extensor at the level of the carpus.

Action: Extends the third digit.

Innervation: $\mathrm{N}$. radialis.

The extensor of the second digit, $m$. extensor digiti II (extensor indicis of Gilbert, 1940; and of Bryant, 1945), lies deep to the common extensor.
Origin: From the dorsal aspect of the shaft of the radius.

Insertion: Together with the common digital extensor on digit two.

Action: Extends the second digit.

Innervation: $\mathrm{N}$. radialis.

The long abductor of the first digit, $m$. abductor digiti I longus (Fig. 4-8) (extensor metacarpi pollicis of Gilbert, 1940; abductor pollicis longus of Bryant, 1945), appears distally between the extensor carpi radialis and the common digital extensor muscles. Its tendon of insertion crosses from lateral to medial over the tendon of the extensor carpi radialis and is surrounded by a synovial sheath.

Origin: Laterally from the proximal half of the shaft of the ulna (from the lateral coronoid process distally).

Insertion: Proximally on the first metacarpal bone.

Action: Abducts digit I.

Innervation: $\mathrm{N}$. radialis.

Flexors of the Digits. The superficial digital flexor, $m$. flexor digitorum superficialis (Fig. 4-9) (flexor digitorum sublimis of Bryant, 1945), has two heads, one from the humeral epicondyle (palmaris longus of Gilbert, 1940) and one from the humeral condyle (flexor digitorum sublimis of Gilbert, 1940). The head from the epicondyle forms the most caudal muscle of the antebrachium. Both tendons pass deep to the palmar sesamoid bone and are held in position by palmar fascia. At the metacarpo-phalangeal joints each of the tendons to digits two to five divide to form a sleeve or manicum flexorium for the passage of the deep digital flexor tendons.

Origin: Medial condyle and epicondyle of the humerus.

Insertion: The head from the medial condyle inserts mainly on the palmar fascia and palmar pads but also gives off branches to the tendons of the epicondylar head. At the carpus, the tendon of the epicondylar head divides into four to insert on the proximal part of phalanx two of digits two to five.

Action: Flexion of the toes.

Innervation: $\mathrm{N}$. medianus.

The deep digital flexor, $m$. flexor digitorum profundus (Fig. 4-9), lies caudal to the flexor carpi radialis and deep to the superficial and ulnar flexors. Its three heads fuse to form a wide tendon which passes through the carpal canal, deep to the tendon of the superficial digital flexor, and then divides into four. The four tendons are held in position by the palmar annular ligaments as they pass over the metacarpo-phalangeal joints. They then pass through the superficial tendons and are bound 
down to the digits by proximal and distal digital annular ligaments.

Origin: Ulnar head-From the olecranon and the proximal half of the shaft of the ulna.

Humeral head-from the medial condyle of the humerus.

Radial head-from the proximal half of the caudomedial border of the radius.

Insertion: On the flexor surface of the distal phalanges of digits two to five.

Action: Flexion of the toes.

Innervation: By both the $\mathrm{n}$. medianus and $\mathrm{n}$. ulnaris.

Rotators of the Manus. The manus has four rotators; two pronators and two supinators.

The pronator teres, $m$. pronator teres (Fig. 4-9), lies medial on the proximal part of the radius. It is not covered by any of the antebrachial muscles.

Origin: Medial condyle of the humerus.

Insertion: Distal half of the medial border of the radius.

Action: Pronation of the manus.

Innervation: $\mathrm{N}$. medianus.

The pronator quadratus, $m$. pronator quadratus, runs transversely in the distal half of the interosseus space between the radius and ulna.

Origin: Distal half of the ulna.

Insertion: Distal half of the radius.

Action: Pronation of the manus.

Innervation: N. medianus.

The brachioradialis, $m$. brachioradialis (Figs. 4-8 and 4-9), is a very large and well-developed muscle of the arm. It lies craniomedially on the antebrachium, medial to the radial carpal extensor.

Origin: From the lateral epicondyle of the humerus, proximal to the origin of the $\mathrm{m}$. extensor carpi radialis.

Insertion: On the fascia over the dorsomedial aspect of the carpus and the styloid process of the radius.

Action: Supination of the antebrachium.

Innervation: $\mathrm{N}$. radialis.

The supinator, $m$. supinator, lies deep to the lateral antebrachial muscles in the proximal part of the forearm.

Origin: From the lateral condyle of the humerus.

Insertion: On the craniomedial border of the proximal part of the radius.

Action: Supination of the manus.

Innervation: $\mathrm{N}$. radialis.

Muscles of the Manus. Apart from the muscles of the digits arranged around the antebrachium, several short muscles of the digits are arranged on the palmar aspect of the manus. They function

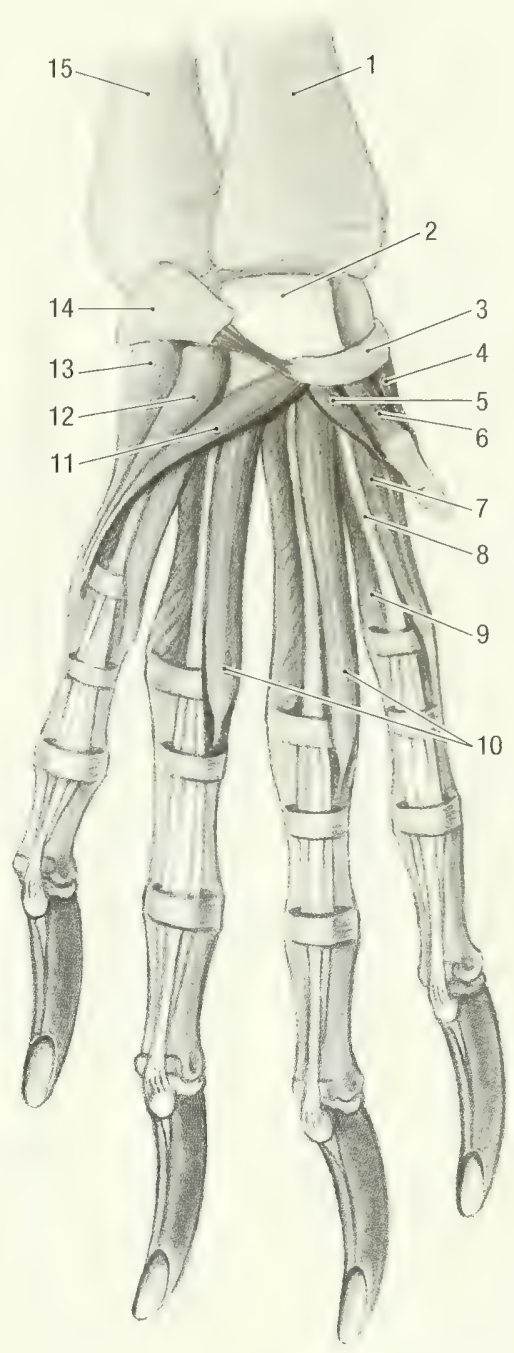

Fig. 4-10. Left manus, superficial palmar view. 1 radius, 2 tendon of superficial digital flexor m., 3 radial sesamoid bone, 4 abductor $\mathrm{m}$. of first digit, 5 adductor $\mathrm{m}$. of first digit, 6 short flexor $\mathrm{m}$. of first digiti, 7 abductor $\mathrm{m}$. of second digit, 8 tendon of superficial digital flexor m., 9 interosseus $\mathrm{mm}$., 10 lumbricales $\mathrm{mm}$., 11 opponens $\mathrm{m}$. of the fifth digit, 12 palmaris brevis $m ., 13$ abductor $m$. of the fifth digit, 14 accessory carpal bone, 15 ulna.

primarily as flexors, adductors and abductors of the digits.

The lumbricales, $\mathrm{mm}$. lumbricales (Figs. 4-10 and 4-11) consist of three thin muscle bundles between the branches of the deep digital flexor tendon. The first lies between the tendons of digits two and three, the second lies between the tendons of digits three and four, and the third lies between the tendons of digits four and five.

Origin: Tendons of the deep digital flexor.

Insertion: On the medial aspects of digits three to five. The first one goes to digit three, the second to digit four and the third to digit five. 


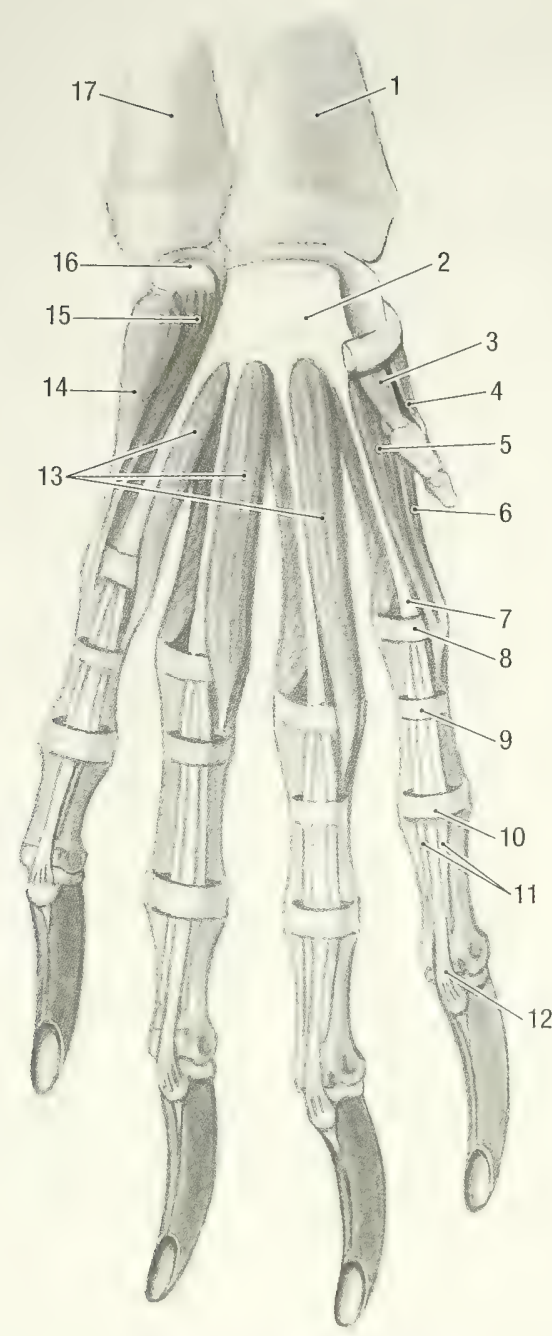

Fig. 4-11. Left manus, middle palmar view. 1 radius, 2 tendon of superficial digital flexor m., 3 short flexor $\mathrm{m}$. of first digit, 4 abductor $\mathrm{m}$. of first digit, 5 flexor $\mathrm{m}$. of second digit, 6 abductor $\mathrm{m}$. of second digit, 7 manicum flexorium, 8 palmar annular ligament, 9 proximal digital annular ligament, 10 distal digital annular ligament, 11 insertions of superficial digital flexor $\mathrm{m}$. tendon, 12 insertion of the deep flexor $\mathrm{m}$. tendon, 13 lumbricales $\mathrm{mm}$., 14 abductor $\mathrm{m}$. of the fifth digit, 15 flexor $\mathrm{m}$. of the fifth digit, 16 accessory carpal bone, 17 ulna.

Action: Flexion of the metacarpophalangeal joint.

Innervation: N. ulnaris.

The four interosseus muscles, $\mathrm{mm}$. interossei (Figs. 4-10 and 4-12), form the bulk of the muscle mass on the palmar surface of the manus. Each of the four muscles divides distally to insert on the proximal sesamoid bone. Some authors, including Bryant, 1945, regard them as separate muscles, giving a total of eight interosseus muscles.

Origin: Proximal ends of metacarpals II-V.

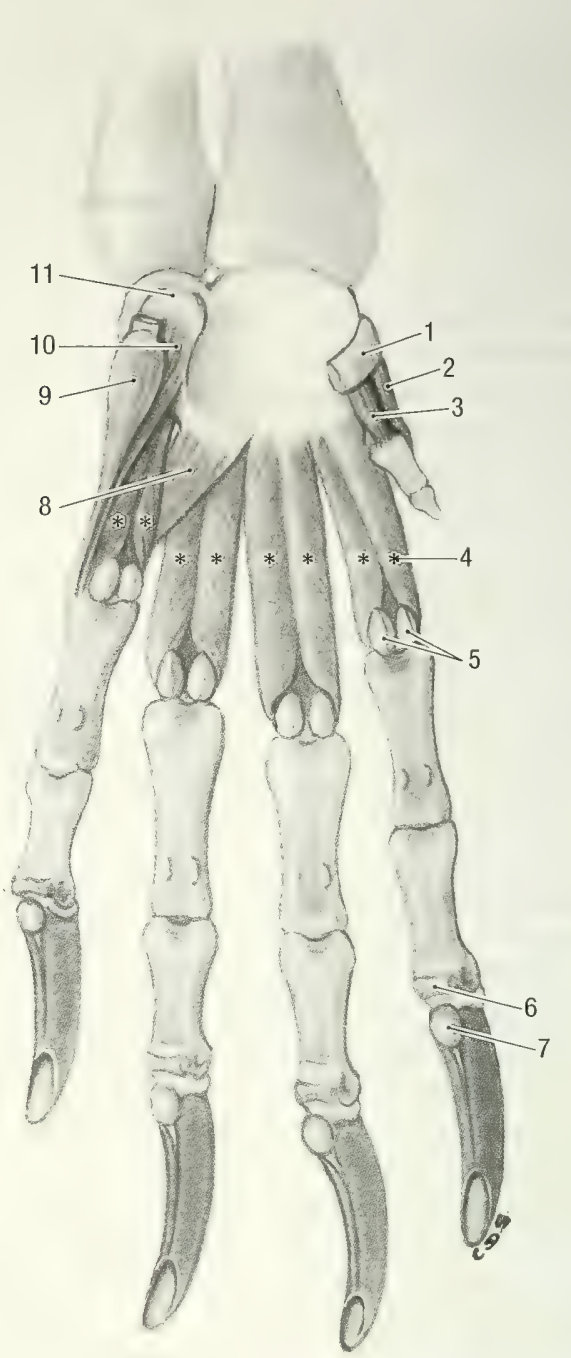

Fig. 4-12. Left manus, deep palmar view. 1 radial sesamoid bone, 2 abductor $\mathrm{m}$. of first digit, 3 short flexor $\mathrm{m}$. of first digit, 4 interosseus mm., 5 proximal sesamoid bones, 6 distal sesamoid bone, 7 flexor tubercle of distal phalanx, 8 adductor $\mathrm{m}$. of fifth digit, 9 abductor $\mathrm{m}$. of fifth digit, 10 flexor $\mathrm{m}$. of fifth digit, 11 accessory carpal bone.

Insertion: Proximal sesamoid bones of digits II-V.

Action: Flexion of the metacarpophalangeal joints.

Innervation: N. ulnaris.

The short flexor of the first digit, $m$. flexor digiti I brevis (Figs. 4-10 and 4-11), lies between the abductor and adductor of the first digit.

Origin: From the distal palmar surface of the first carpal bone.

Insertion: Phalanx I of the first digit, distal to the insertion of the abductor.

Action: Flexion of the first digit.

Innervation: N. ulnaris. 
The adductor of the first digit, $m$. adductor digiti $I$ (Fig. 4-10), lies lateral and deep to the short flexor of the first digit.

Origin: Inner surface of the first carpal bone.

Insertion: Caudolateral border of the first phalanx of digit I.

Action: Adducts the first digit.

Innervation: $\mathrm{N}$. ulnaris.

The short abductor of the first digit, $m$. abductor digiti I brevis (Fig. 4-11), is the most medial of the short muscles of the first digit.

Origin: Caudal, free end of the first carpal bone. Insertion: Base of the proximal phalanx of digit I. Action: Abducts the first phalanx.

Innervation: $\mathrm{N}$, ulnaris.

The short flexor of the second digit, $m$. flexor digiti II (Fig. 4-10), lies medial to the lumbricalis muscle of the same digit.

Origin: Carpal ligament.

Insertion: Medially on the proximal phalanx of digit II.

Action: Flexion of the second digit.

Innervation: N. ulnaris.

The abductor of the second digit, $m$. abductor digiti II is the most medial of the short muscles of the second digit.

Origin: Carpal ligament.

Insertion: In common with the short flexor on the proximal phalanx of digit II.

Action: Abduction of the second digit.

Innervation: $\mathrm{N}$. ulnaris.

The abductor of the fifth digit, $m$. abductor digiti $V$ (Figs. 4-10 and 4-12), is the most lateral of the short muscles of the manus.

Origin: Accessory carpal bone (cartilage of the lateral metacarpal pad) and the palmar retinaculum.

Insertion: By means of a fascial attachment to the lateral surface of the short flexor of digit $\mathrm{V}$.

Action: Abducts the fifth digit.

Innervation: $\mathrm{N}$. ulnaris.

The flexor of the fifth digit, $m$. flexor digiti $V$ (Figs. 4-11 and 4-12), lies deep to the abductor of the same digit.

Origin: Accessory carpal bone.

Insertion: On the lateral surface of the outer (abaxial) proximal sesamoid bone of digit $\mathrm{V}$.

Action: Flexion of the fifth digit.

Innervation: $\mathrm{N}$. ulnaris.

The opposing muscle of the fifth digit, $m$. opponens digiti $V$ (Fig. 4-10), lies medial to the adductor of the fifth digit. It takes origin from the accessory carpal bone and inserts on the lateral aspect of the fifth digit.

Action: Adducts the fifth digit.

Innervation: $\mathrm{N}$. ulnaris.

The adductor of the fifth digit, $m$. adductor digiti
$V$ (Fig. 4-12), lies between the third and fourth interosseus muscles.

Origin: Carpal ligament.

Insertion: On the medial aspect of the proximal phalanx of digit $\mathrm{V}$.

Action: Adducts the fifth digit.

Innervation: N. ulnaris.

The opposing muscle of the carpal pads, $m$. palmaris brevis (Fig. 4-10), is a well-developed muscle that lies within the lateral and medial carpal pads. It consists of two bellies that radiate from the carpal sesamoid bone and medial carpal pad to the lateral carpal pad and lateral surface of the fifth digit. A cartilage attached to the accessory carpal bone supports the lateral carpal pad. The carpal sesamoid bone supports the medial carpal pad (see osteology, carpal bones). The medial carpal pad is mobile, the lateral one is fairly fixed in position. Therefore, the muscle adducts the fifth digit and draws the medial carpal pad towards the lateral carpal pad.

Innervation: N. ulnaris.

\section{TRUNK}

The trunk muscles are divided topographically into the muscles of the cervical, thoracic, and lumbar vertebrae, the lateral and ventral thoracic wall, including the diaphragm, the abdomen, and the tail. Apart from these muscles, a very extensive cutaneous muscle covers most of the trunk.

Cutaneous Muscle of the Trunk. The cutaneus muscle of the trunk, $m$. cutaneus trunci (Figs. 4-1 and 4-2) (cutaneous maximus of Bryant, 1945), is a very extensive muscle that covers the thorax, abdomen, pelvis and thighs. It extends from the dorsal sacral region, the lateral aspects of the pelvis and thighs, and the ventral abdominal region, to the medial aspect of the arm. The left and right muscles meet along the dorsal midline and are attached to the lateral sacral crest. Caudoventrally the muscle gives off two muscular slips, one attaches to the scrotum, and the other attaches to the lateral aspect of the preputium, forming the cranial preputial muscle, $m$. preputialis cranialis.

Origin: From the lateral sacral crest and the thoracolumbar, lateral femoral, and ventral abdominal fascia.

Insertion: On the $\mathrm{m}$. latissimus dorsi, medial to the arm.

Action: The muscle functions to protract the scrotum and preputium, and retract the arm. It also moves the skin of the trunk.

Innervation: $\mathrm{N}$. thoracicus lateralis.

Muscles of the Vertebral Column. The muscles of the vertebral column are grouped into five layers that lie beside and above each other. Of 


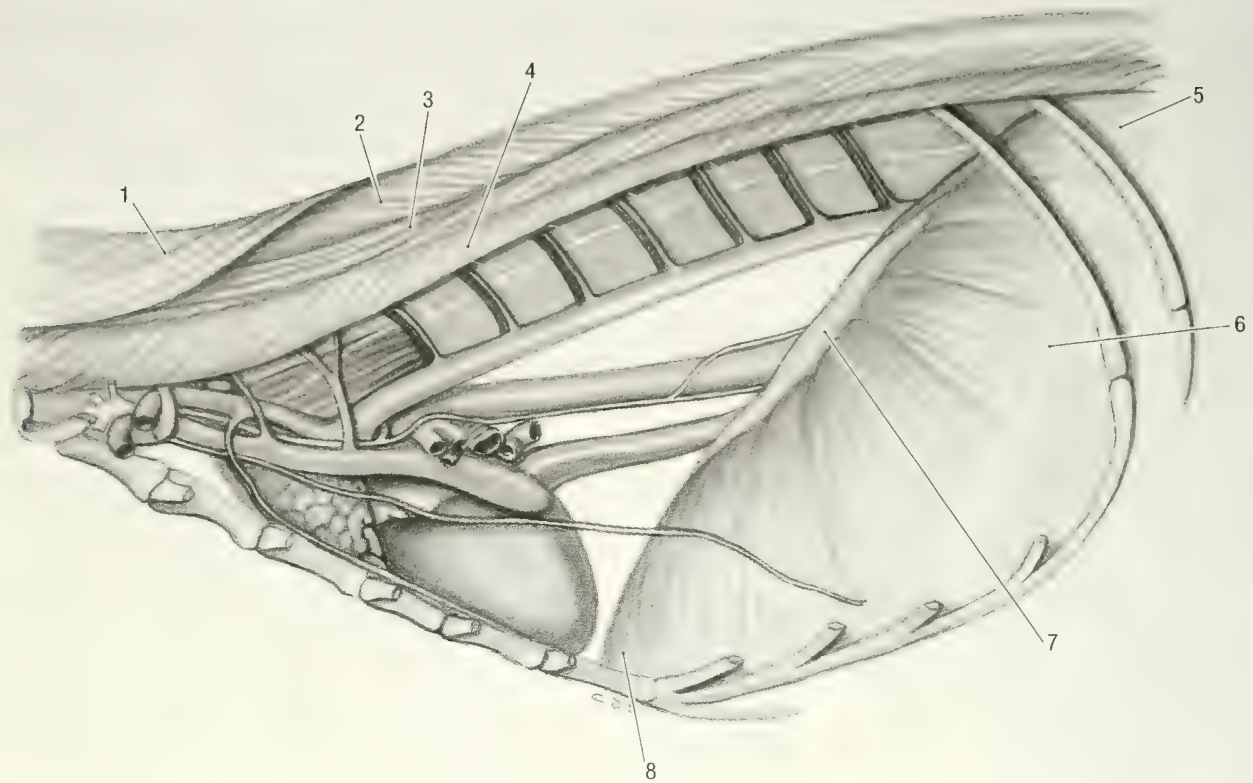

Fig. 4-13. Thorax, left lateral view. 1 splenius $m ., 2$ spinalis et semispinalis $m ., 3$ longissimus m., thoracic part, 4 iliocostalis m., thoracic part, 5 diaphragm, left crus, 6 diaphragm, costal part, 7 diaphragm, tendinous center, 8 diaphragm, sternal part.

these five the two superficial layers and part of the third layer attach the thoracic limb to the body.

The muscles of the first layer are the m. trapezius and $\mathrm{m}$. omotransversarius. Those of the second layer are the $\mathrm{m}$. latissimus dorsi and $\mathrm{m}$. rhomboideus. Those of the third layer are the $m$. serratus ventralis, $\mathrm{m}$. serratus dorsalis and $\mathrm{m}$ splenius. Those of the fourth layer are the $\mathrm{m}$. iliocostalis, $\mathrm{m}$. longissimus and $\mathrm{m}$. spinalis et semispinalis, and those of the fifth layer are the $\mathrm{m}$. multifidus, $\mathrm{m}$. interspinales, $\mathrm{m}$. intertransversarii, and the dorsal muscles of the atlanto-occipital and axio-atlantal joints.

Functionally the iliocostalis, longissimus, and transversospinalis muscle groups belong to the $\mathrm{M}$. erector spinae.

The muscles that attach the thoracic limb to the body are discussed under the muscles of the thoracic limb.

The dorsal serratus muscle, $m$. serratus dorsalis (Fig. 4-7), consists of cranial and caudal parts.

The cranial part, $m$. serratus dorsalis cranialis (serratus posterior superior of Bryant, 1945), lies deep to the serratus ventralis muscle, and its fibers run dorsocranially.

Origin: By means of a wide aponeurosis from the spinous processes of $2 \mathrm{C}$, from the dorsal median raphae of the neck, and from the spinous processes of $1 \mathrm{~T}-12 \mathrm{~T}$.

Insertion: Muscular on ribs 4 to 10 , just lateral to the iliocostalis muscle.
Action: Pulls the ribs cranially during inspiration.

The caudal part, $m$. serratus dorsalis caudalis (serratus posterior inferior of Bryant, 1945), lies deep to the latissimus dorsi muscle. Its fibers run caudoventrally and overlap (lies superficial to) the caudal fascicles of the cranial part.

Origin: By means of a wide aponeurosis from the spinous processes of $12 \mathrm{~T}-1 \mathrm{~L}$.

Insertion: Muscular on the last four ribs.

Action: Pulls the last four ribs caudally for expiration.

Innervation of both parts: Nn. intercostales.

The splenius, $m$. splenius (Figs. 4-7 and 4-13), is a very large and powerful muscle of the neck that lies deep to the rhomboideus.

Origin: Muscular from the median raphae of the neck (from 1-7C) and by means of an aponeurosis from the spinous processes of $1 \mathrm{~T}-8 \mathrm{~T}$.

Insertion: On the mastoid process and the nuchal crest.

Action: Bilateral contraction extends and raises the head. Unilateral action draws the head and neck laterally.

Innervation: Cervical spinal nerves.

Epaxial Muscles. The epaxial muscles (Fig. 412a) belong to the fourth layer of trunk muscles. They are divided into three longitudinal muscle masses, each consisting of many overlapping fasci- 
cles, and are arranged above the level of the transverse processes of the vertebrae. Dorsal branches of spinal nerves innervate the muscle group.

The iliocostal muscle, $m$. iliocostalis, is the most lateral of the epaxial muscles. It extends from the wing of the ilium to the sixth cervical vertebra. The muscle consists of a lumbar part, $m$. iliocostalis lumborum, a thoracic part, $m$. iliocostalis thoracis and a cervical part, $m$. iliocostalis cervicis.

Origin: From the tuber coxae, the transverse processes of the lumbar vertebrae, the lumbar fascia, and the ribs.

Insertion: Angle of the ribs and the transverse processes of $6 \mathrm{C}$ and $7 \mathrm{C}$.

Action: Fixation of the vertebral column during bilateral contraction, or lateral flexion during unilateral contraction of the muscle.

Innervation: Dorsal branches of the thoracic and lumbar spinal nerves.

Note: The lumbar part takes origin from the tuber coxae and inserts on the transverse processes of the lumbar vertebrae and the last rib. The thoracic part takes origin from the ribs, spans a few ribs and then inserts on the angle of the ribs. The cervical part comes from the ribs and inserts on the transverse processes of the last two cervical vertebrae.

The longissimus, $m$. longissimus, lies medial to the iliocostalis muscle and is covered by the serratus dorsalis and splenius muscles. Its overlapping fascicles extend from the ilium to the head and are divided into lumbar, $m$. longissimus lumborum, thoracic, $m$. longissimus thoracis, cervical, $m$. longissimus cervicis, and capital, m. longissimus capitis, parts.

Origin: From the wing of the ilium and the spinous processes of the lumbar and thoracic vertebrae and from the cervical transverse processes.

Insertion: The muscle bundles run craniolaterally to insert on the accessory, articular and mammillary processes, and on the ribs. The capital part inserts on the mastoid process of the temporal bone.

Action: Fixation of the vertebral column during bilateral contraction, or lateral flexion with unilateral contraction. The capital part extends the atlanto-occipital joint.

Innervation: Dorsal branches of the lumbar, thoracic and cervical spinal nerves.

The spinalis and semispinalis muscles, $m$. spinalis et semispinalis (Fig. 4-13), are the most medial and deepest of the epaxial muscle mass. The spinalis and semispinalis components fuse to such an extent in the thoracic and cervical regions that it is very difficult to separate and identify them individually. The muscle bundles extend craniolaterally from the thoracic region to the head and can be divided into thoracic, cervical, and capital parts. Its thoracic part is covered by the $\mathrm{m}$. serratus dorsalis, the cervical part is covered by the $\mathrm{m}$. splenius and $\mathrm{m}$. longissimus cervicis. The capital part, $m$. semispinalis capitis, consists of a dorsal biventer, $m$. biventer cervicis and a ventral complexus, $m$. complexus.

Origin: From the fascia covering the lumbar part of the longissimus muscle, the spinous and mammillary processes of the thoracic vertebrae (thoracic part), the transverse and spinous processes of the first few thoracic vertebrae, and the caudal articular processes of the last few cervical vertebrae (capital part).

Insertion: The thoracic part inserts on the spinous processes of the thoracic vertebrae, the cervical part inserts on the spinous processes of $2-7 \mathrm{C}$ and on the median fibrous raphe of the neck, and the capital part inserts on the nuchal crest by means of a wide aponeurosis.

Action: Bilateral contraction fixes the thoracic vertebral column and raises the neck (thoracic and cervical parts), and raises the head and neck (capital part). With unilateral contraction the head, neck and thorax are flexed laterally.

Innervation: Dorsal branches of the cervical and thoracic spinal nerves.

The longissimus, spinalis and semispinalis muscles cover the deepest (fifth) muscle layer of the vertebral column. The muscle layer consists of short muscle bundles that extend between from one spinous process to the next ( $\mathrm{mm}$. interspinales), from a transverse process to a preceding spinous process ( $\mathrm{mm}$. rotatores brevis), from a transverse process to a preceding spinous process, but skipping one vertebra $(\mathrm{mm}$. rotatores longi), or from a transverse, mammillary, or articular process to a preceding spinous process, skipping at least two vertebrae $(\mathrm{mm}$. multifidi). The interspinal muscles are the deepest and the multifidi muscles the most superficial (lateral) of the short muscles. In addition to the above mentioned, the $m m$. intertransversarii are a series of muscle bundles between the transverse processes of most of the vertebrae. They are considered to be deep segments split off from the longissimus muscles and are divided into dorsal, intermediate, and ventral $\mathrm{mm}$. intertransversarii of the cervical, thoracic, lumbar, and caudal regions. All the muscles are not present along the whole length of the vertebral column, and within a group, they are only present on some segments of the spine. Some of the groups extend caudally unto the tail, forming the dorsal muscles of the tail. Their collective functions include extension, rotation, or lateral flexion of the vertebral column.

In the cranial cervical region, some of the deep muscles specialize in attaching the head and neck. They include the straight and oblique muscles of the head and are all situated deep to the $\mathrm{m}$. semispinalis capitis. 


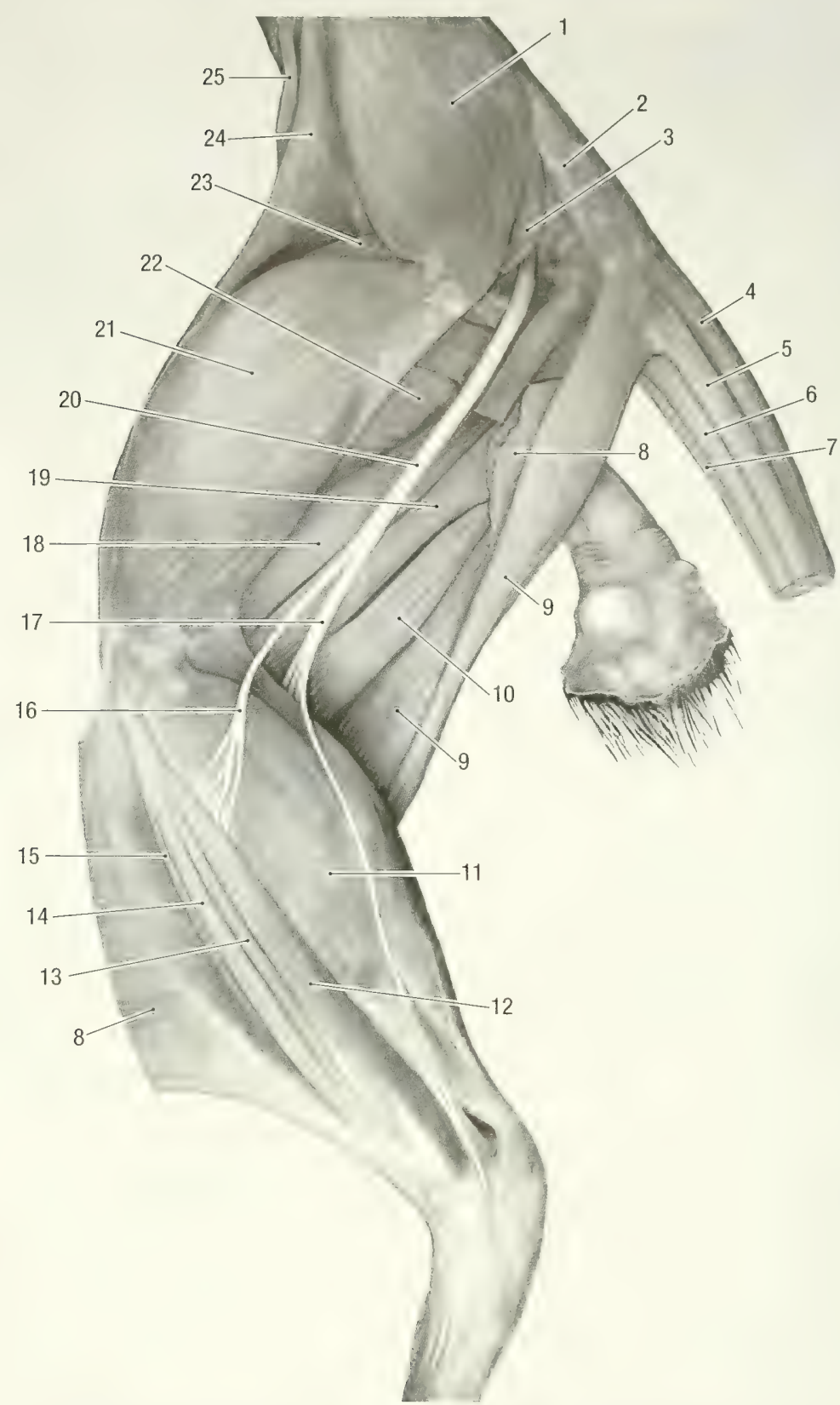

Fig. 4-14. Pelvic limb of female, lateral view. 1 middle gluteal m., 2 superficial gluteus m. (cut), 3 piriform m., 4 dorsomedial sacrococaudal m., 5 dorsolateral sacrococaudal m., 6 ventrolateal sacrocaudal m., 7 ventromedial sacrocaudalis $\mathrm{m}$., 8 biceps femoris $\mathrm{m}$. (cut), 9 semitendinosus $\mathrm{m}$., 10 semimembranosus $\mathrm{m}$., 11 gastrocnemius m., 12 lateral head of deep digital flexor m., 13 long digital extensor m., 14 peroneus longus $\mathrm{m}$., 15 cranial tibial m., 16 peroneal $n$., 17 tibial n., 18 proper adductor m., 19 femoral part of the adductor brevis m., 20 ischiadic n., 21 vastus lateralis m., 22 femorococcygeus $m ., 23$ rectus femoris m., 24 tensor fasciae latae m., 25 iliopsoas $m$.

The dorsal straight muscle of the head, $m$. rectus capitis dorsalis, extends from the axis to the occiput. It is the most superficial of the short muscles of the head.

Origin: Muscular from the spinous process 2C. Insertion: The muscle has a very extensive mus- cular insertion on the occiput below the nuchal crest.

Action: Extends the atlanto-occipital joint.

Innervation: Dorsal branch of the first cervical spinal nerve.

The lateral straight muscle of the head, $m$. rectus 
capitis lateralis, extends from the atlas to the occipital bone.

Origin: Wing of the atlas.

Insertion: On the paracondylar process.

Action: Flexion of the atlanto-occipital joint.

Innervation: Ventral branches of cervical spinal nerves.

The caudal oblique muscle of the head, $m$. obliquus capitis caudalis, lies deep to the dorsal straight muscle. It extends craniolaterally between the axis and atlas.

Origin: Spine of the axis.

Insertion: Wing of the atlas.

Action: Unilateral contraction rotates the atlas and thus the head on the axis.

Innervation: Dorsal branches of the first two cervical spinal nerves.

The cranial oblique muscle of the head, $m$. obliquus capitis cranialis, lies deep to the dorsal straight muscle and extends between the atlas and the occiput.

Origin: Wing of the atlas.

Insertion: Occiput, ventral to the insertion of the $\mathrm{m}$. rectus capitis dorsalis.

Action: Extension of the atlanto-occipital joint.

Innervation: Dorsal branch of the first cervical spinal nerve.

Hypaxial Muscles of the Trunk. These muscles lie ventrally along the vertebral axis and are innervated by ventral branches of the spinal nerves. They include the longus colli, longus capitis (see deep muscles of the ventral neck), and psoas (see pelvic limb) muscles.

Muscles of the Ventral Neck Region. The muscles of the ventral neck region can be grouped by relation to the trachea and esophagus (superficial muscles) and by relation to the cervical vertebrae (deep muscles).

The ventral cutaneous muscles of the neck are continuous with the platysma and the cutaneous muscles of the head.

The sternomastoideus, $m$. sternomastoideus (Figs. 4-4 and 4-5b), extends from the sternum to the head, deep to the $\mathrm{m}$. colli ventralis.

Origin: From the manubrium of the sternum.

Insertion: On the mastoid process of the temporal bone.

Action: Flexes the neck.

Innervation: $\mathrm{N}$. accessorius and ventral branches of the cervical spinal nerves.

The cleido-occipital, m. cleido-occipitalis (Fig. 47) (clavotrapezius of Gilbert, 1940), extends from the clavicle to the head, deep to the platysma.

Origin: From the ventral surface of the clavicle.

Insertion: On the nuchal crest of the skull.
Innervation: Ventral branches of the cervical spinal nerves.

The cleidomastoid, m. cleidomastoideus (Fig. 47), extends from the clavicle to the skull, deep to the cleido-occipital muscle.

Origin: From the medial third of the clavicle.

Insertion: On the mastoid process.

Innervation: Ventral branches of the cervical spinal nerves.

Together the cleido-occipital and cleidomastoid muscles can be regarded as the cleidocephalic muscle, $m$. cleidocephalicus.

The scalene muscles extend from the ribs to the cervical vertebrae. They are well developed and consist of the dorsal scalene, $m$. scalenus dorsalis, and the middle scalenus, $m$. scalenus medius, muscles.

Origin: The dorsal scalene muscle has a muscular origin from the proximal parts of the fourth and fifth ribs. The muscle fascicles penetrate (pass through) the ventral border of the $\mathrm{m}$. serratus ventralis. The middle scalene muscle takes origin from the proximal end of the first rib. It lies dorsal to the brachial plexus and deep to the dorsal scalene muscle.

Insertion: The dorsal scalene inserts on the wing of the atlas, and the middle scalene inserts on the transverse processes of $2-7 \mathrm{C}$.

Action: Unilateral contraction draws the neck sideways, bilateral contraction draws the neck ventrally. When the neck is fixed the dorsal part will draw the fourth and fifth ribs cranially, aiding inspiration.

Innervation: Ventral branches of the cervical and thoracic spinal nerves.

The longus colli, $m$. longus colli (Fig. 4-6), lies along the bodies of the cervical and cranial thoracic vertebrae. It consists of a series of diverging fascicles along the ventral surface of the first 5 thoracic vertebrae and converging fascicles along the ventral aspect of the cervical vertebrae.

Origin: The thoracic part takes origin from the ventral ridge of the first five thoracic vertebral bodies, whereas those in the cervical region take origin from the transverse processes of cervical vertebrae.

Insertion: The fascicles of the thoracic part diverge craniolaterally to insert on the ventral aspect of the transverse processes, whereas those of the cervical part converge craniomedially to insert on the ventral crest of the vertebral bodies.

Action: Flexion of the neck when contracted bilaterally; bending of the neck to one side when contracted unilaterally.

Innervation: Ventral branches of the cervical spinal nerves.

The longus capitis, $m$. longus capitis (Fig. 4-6), 
lies lateral to the $\mathrm{m}$. longus colli along the ventrolateral aspect of the cervical vertebral bodies.

Origin: Transverse processes of the cervical vertebrae.

Insertion: On the muscular tubercle of the basioccipital bone.

Action: Flexion of the neck and atlanto-occipital joint.

Innervation: Ventral branches of the cervical spinal nerves.

Muscles of the Tail. The muscles of the tail are continuations of the muscles of the vertebral column. They are divided into dorsal, lateral and ventral tail muscles. The dorsal and ventral groups consist of lateral and medial muscles.

The dorsomedial muscle of the tail, $m$. sacrocaudalis dorsalis medialis (extensor caudae medialis of Bryant, 1945), is a direct continuation of the multifidus system.

Origin: From the dorsolateral tubercles on the sacrum and the caudal vertebrae.

Insertion: Spinous processes or median tubercles of the sacrum and the caudal vertebrae.

Action: Extension and lateral flexion of the tail.

Innervation: Dorsal branches of the sacral and the caudal spinal nerves.

The dorsolateral muscle of the tail, $m$. sacrocaudalis dorsalis lateralis (extensor caudae lateralis of Bryant, 1945), is the largest of the dorsal tail muscles and is a direct continuation of the longissimus system.

Origin: Wing and shaft of the ilium, and the articular processes of the sacrum and caudal vertebrae.

Insertion: Spinous processes or dorsal tubercles of the caudal vertebrae.

Action: Extension or lifting of the tail.

Innervation: Dorsal branches of the sacral and caudal spinal nerves.

The ventrolateral muscle of the tail, $m$. sacrocaudalis ventralis lateralis (flexor caudae lateralis of Bryant, 1945), lies along the ventrolateral aspect of the caudal vertebrae.

Origin: From the ventral surface of the sacrum and the transverse processes of the caudal vertebrae.

Insertion: On the hemal processes or ventral tubercles of the caudal vertebrae.

Action: Depresses the tail.

Innervation: Ventral branches of the sacral and caudal vertebrae.

The ventromedial muscle of the tail, $m$. sacrocaudalis ventralis medialis (flexor caudae medialis of Bryant, 1945), lies medial to the ventrolateral tail muscle along the ventral midline of the tail.

Origin: Ventral surface of the sacrum and caudal vertebral bodies.
Insertion: In common with the lateral muscle on the hemal processes of the caudal vertebrae.

Action: Depresses the tail.

Innervation: Ventral branches of the caudal spinal nerves.

The intertransverse muscles of the tail, $\mathrm{mm}$. intertransversarii caudae, are continuations of the intertransversarii of the trunk. They are divided into the dorsal and ventral intertransversarius muscles of the tail.

Origin: Transverse processes of the caudal vertebrae.

Insertion: Transverse process of the preceding caudal vertebra.

Action: Lateral flexion of the tail.

Innervation: Ventral branches of the caudal spinal nerves.

Muscles of the Thoracic Wall. The rectus thoracis muscle, $m$. rectus thoracis, is a small muscle that lies ventral to the dorsal scalenus muscle on the cranioventral aspect of the thorax, deep to the m. serratus ventralis.

Origin: Upper half of the first rib.

Insertion: By means of an aponeurosis on the last three sternebrae and their costal cartilages.

Action: Draws the rib cartilages cranially during inspiration.

Innervation: Nn. intercostales.

The intercostal muscles, $m m$. intercostales, are divided into external, $\mathrm{mm}$. intercostales externi, and internal, $\mathrm{mm}$. intercostales interni, layers, arranged at right angles to each other. The fibers of the external layer run caudoventrally, and those of the internal layer run cranioventrally. During inspiration the ribs are pulled forward and outward, thus expanding the thoracic cavity. On expiration the ribs are pulled caudally and medially, therefore decreasing the thoracic space. Elevation and depression of the ribs is a result of the position of the articular surfaces of the heads and the tubercles of the ribs.

The external and internal intercostal muscles arise and insert on the margins of adjacent ribs.

Action: When the intercostal contract, they pull the ribs together. When the scalenes contract and fix the rib cage cranially, the action of both intercostal muscles aids inspiration. When the rib cage is fixed caudally the intercostals aid expiration.

Innervation: Nn. intercostales (ventral branches of the thoracic spinal nerves).

The transverse thoracic muscle, $m$. transversus thoracis, lies on the dorsal (inner) surface of the sternum and on the costal cartilages on the floor of the thorax.

Origin: Dorsal surface of the sternum.

Insertion: Costal cartilages. 
Action: They draw the rib cartilages caudally during expiration.

Innervation: $\mathrm{Nn}$. intercostales.

The diaphragm, diaphragma (Fig. 4-13), separates the thoracic cavity from the abdominal cavity. It looks like a muscular fan attached on the inner surface of the ribs, sternum, and abdomen. The fibrous central portion, centrum tendineum, lacks muscle. The muscular part is divided into lumbar, costal, and sternal parts.

The lumbar part, pars lumbalis, consists of right and left crura, crus dextrum et sinistrum, that are tendinous at their origins and become muscular as they fan out. From their origins on the ventral surface of lumbar vertebrae the crura extend craniolaterally and ventrally, forming a lumbocostal arch, arcus lumbocostalis, between the cranial lumbar and caudal costal regions. Dorsal to the arch (between the last rib and the dorsal border of the diaphragm) is a small, triangular area free of muscle. Pleura and peritoneum forms the only separation between the thorax and abdomen in the latter area. The abdominal aorta passes between the two crura through the aortic hiatus, hiatus aorticus. The esophagus passes through the esophageal hiatus, hiatus esophageus in the right crus.

The costal part, pars costalis, originates from the costal arch, and the sternal part, pars sternalis, originates from the xiphoid.

The muscle fibers of the three parts converge to form a large fibrous tendinous center, centrum tendineum. The caudal vena cava passes through an opening, foramen venae cavae, in the tendinous center.

Origin: The lumbar part has a tendinous origin from the vertebral bodies of the first and second lumbar vertebrae. The costal part has a muscular origin from the costal arch and costal cartilages. The sternal part has a muscular origin from the last sternebra and xiphoid cartilage.

Insertion: On the centrum tendineum.

Action: Inspiration.

Innervation: N. phrenicus.

Muscles of the Abdominal Wall. The external oblique abdominal muscle, $m$. obliquus externus abdominis (Figs. 4-1 and 4-2), forms the outer muscular layer of the abdominal wall. Its muscle fibers extend caudoventrally from the thorax to the pelvis and to the floor of the abdomen. The muscle inserts by means of a broad aponeurosis. The caudal border of the aponeurosis (between the tuber coxae and the pubis) is thickened to form a strong inguinal ligament. Just cranial to the pubis, the muscular part separates from the aponeurosis to form a longitudinal slit (approximately $3 \mathrm{~cm}$ long). The slit represents the external or superficial ring of the inguinal canal (the lateral border of the ring is muscular, the medial wall aponeurotic).

Origin: Muscular from the last nine ribs, from the first six fascicles interdigitating with those of the $\mathrm{m}$. serratus ventralis, and from the last three fascicles interdigitating with those of the $\mathrm{m}$. serratus dorsalis, and by means of an aponeurosis from the lumbar fascia.

Insertion: By means of a wide aponeurosis on the linea alba (superficial to the $\mathrm{m}$. rectus abdominis), the xiphoid cartilage, the pubis (prepubic tendon), and the tuber coxae.

Action: Compresses the abdomen and supports the abdominal viscera.

Innervation: Ventral branches of the caudal thoracic and the lumbar spinal nerves.

The internal oblique abdominal muscle, $m . o b$ liquus internus abdominis, lies deep to the external oblique. Its fibers extend cranioventrally from the tuber coxae to the thorax and the abdominal floor. Its aponeurosis passes superficial to the $\mathrm{m}$. rectus abdominis, except for the cranial quarter where it passes deep to it.

Origin: Muscular from the tuber coxae, the inguinal ligament, and the lumbar fascia.

Insertion: By means of an aponeurosis on the linea alba and the costal arch. It also has a muscular insertion on the last rib and on the proximal part of the costal arch.

Action: Compresses the abdomen and supports the abdominal viscera.

Innervation: Ventral branches of the caudal thoracic and the lumbar spinal nerves.

The transverse abdominal muscle, $m$. transversus abdominis, is an extensive muscle that lies deep to the internal oblique. Its fibers extend transversely between the lumbar region and the floor of the abdomen. Cranial to the pubis the caudal border of the muscle extends onto the tunica vaginalis of the testis (Fig. 4-2). It forms a loop around the tunic that is the external cremaster muscle.

Origin: Muscular from the inguinal ligament, tuber coxae, and by means of an aponeurosis from the lumbar fascia.

Insertion: By means of an aponeurosis on the linea alba, and muscular on the costal arch and the cartilage of the seventh rib. The cranial three quarters of the aponeurosis passes deep to the $\mathrm{m}$. rectus abdominis, whereas the caudal quarter passes superficial to the rectus muscle.

Action: Compresses the abdomen and supports the abdominal viscera.

Innervation: Ventral branches of the caudal thoracic and the lumbar spinal nerves.

The straight abdominal muscle, $m$. rectus abdominis, extends from the pubis to the sternum along the floor of the abdomen. It is divided into seg- 
ments by transverse fibrous bands or intersections. The external surface of the cranial three-quarters of the muscle is covered by the aponeuroses of the oblique abdominal muscles. The caudal quarter is covered by the aponeuroses of the oblique and transverse abdominal muscles.

Origin: Muscular from the first rib, the costal cartilage, and the manubrium of the sternum.

Insertion: Muscular on the pecten of the pubis and on the prepubic tendon.

Action: Compresses the abdomen and supports the abdominal viscera.

Innervation: Ventral branches of the thoracic and lumbar spinal nerves.

The inguinal canal, canalis inguinalis, is a connective-tissue-filled space between the caudal parts of the oblique abdominal muscles. The canal lies cranial to the pubis and lateral to the rectus abdominis muscle. It serves as the canal for the passage of the vaginal process and the testicle of the male animal. In the female animal it contains the external pudendal vessels and nerves. The canal has a deep inguinal ring, anulus inguinalis profundus, and a superficial inguinal ring, anulus inguinalis $s u$ perficialis.

The inguinal ligament forms the lateral and caudal borders of the deep inguinal ring, the caudal border of the internal oblique abdominal muscle forms the cranial border, and the rectus abdominis muscle forms the medial border. Furthermore, the transverse abdominal muscle forms a loop around the proximal part of the tunica vaginalis.

The superficial ring or opening is a longitudinal slit, approximately $3 \mathrm{~cm}$ long, along the junction of the external oblique abdominal muscle and its aponeurosis (Fig. 4-2). The caudal part of the superficial opening lies directly over the deep opening.

\section{PERINEUM}

The pelvic diaphragm is the vertical, muscular closure of the pelvic outlet through which the rectum and urogenital tract passes. The coccygeus and levator ani muscles form the lateral walls of the pelvic diaphragm. The rest of the perineal muscles are associated with the terminal portions of the digestive and the urogenital tracts.

The coccygeal muscle, $m$. coccygeus, is fan shaped, narrow at its origin and wide at its insertion. It lies deep to the gluteal and caudal thigh muscles.

Origin: From the caudal, pointed area on the iliac crest.

Insertion: Ventrolaterally on the transverse processes of the first three caudal vertebrae.

Action: Depresses the tail.

Innervation: N. sacralis.
The levator ani muscle, $m$. levator ani, extends from the floor of the pelvis to the tail. It lies medial to the $\mathrm{m}$. coccygeus and consists of the iliocaudalis muscle, $m$. iliocaudalis, and the pubocaudalis muscle, $m$. pubocaudalis. The obturator nerve passes through the fleshy body of the $\mathrm{m}$. pubocaudalis.

Origin: The $\mathrm{m}$. iliocaudalis takes origin from the ischial arch, medial to the origin of the coccygeus. The $\mathrm{m}$. pubocaudalis takes origin from the pecten of the pubis, dorsal to the internal obturator muscle.

Insertion: The $\mathrm{m}$. iliocaudalis muscle has a fascial attachment on the $\mathrm{m}$. sacrocaudalis medialis at the level of the seventh caudal vertebra, the m. pubocaudalis inserts on the hemal process of the fourth caudal vertebra.

Action: Depresses the tail.

Innervation: Ramus musculi levatoris ani.

The external anal sphincter, $m$. sphincter ani externus (Figs. 4-2 and 4-15), is a circular, striated muscle encircling the terminal two to three $\mathrm{cm}$ of the digestive tract. It does not have any skeletal attachments. The cranial part of the muscle passes ventrally, deep to the $m$. bulbospongiosus, to attach on the fascia of the bulb of the penis. It gives rise to the retractor penis muscle.

Innervation: $\mathrm{N}$. rectalis caudalis.

The bulbospongiosus muscle, $m$. bulbospongiosus (Fig. 4-2), is represented by muscle bundles that pass laterally over the slip of the external anal sphincter that inserts on the bulb of the penis. It is not present in the female.

Origin: Dorsolaterally from the fascia of the bulbus penis.

Insertion: Caudoventrally on the bulb of the penis, in common with the muscle from the opposite side.

Action: Compresses the penile bulb.

Innervation: $N$. perinealis profundus.

The ischiocavernous muscle, $m$. ischiocavernosus, is a large, well-developed muscle that covers the crura of the penis in the male. In females the muscle covers the crura of the clitoris, but it is very small.

Origin: Caudomedial on the tuber ischii.

Insertion: On the tunica albuginea of the crus penis.

Action: Compresses the crus penis.

Innervation: $\mathrm{N}$. perinealis profundus.

The retractor penis muscle, $m$. retractor penis, extends from the anal sphincter to the flexure of the penis.

Origin: Ventral margin of the anal sphincter muscle.

Insertion: On the tunica albuginea of the penis. Action: Retracts the penis.

Innervation: N. perinealis profundus. 


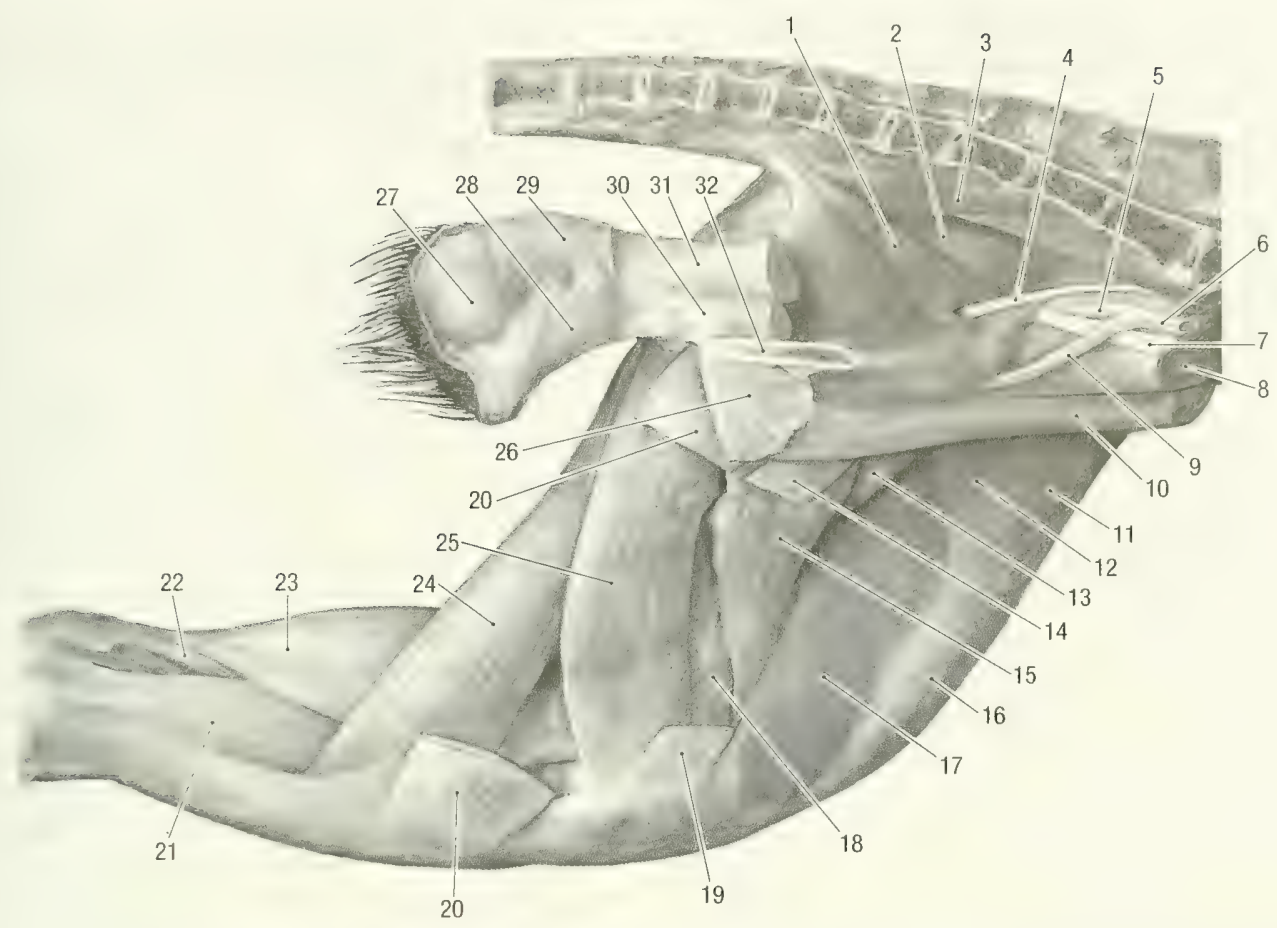

Fig. 4-15. Pelvic limb of female, medial view. 1 iliocaudalis $\mathrm{m} ., 2$ pubocaudalis $\mathrm{m}$., 3 ventromedial sacrocaudalis $\mathrm{m}$., 4 obturator n., 5 internal iliac a., 6 common iliac a., 7 tendon of psoas minor m., 8 psoas major m., 9 external iliac a., 10 rectus abdominis $\mathrm{m}$., 11 tensor fasciae latae $\mathrm{m}$., 12 sartorius $\mathrm{m}$., 13 pectineus $\mathrm{m}$., 14 gracilis $\mathrm{m}$. (cut), 15 adductor longus m., 16 rectus femoris m., 17 vastus medialis m., 18 adductor brevis m., 19 sartorius m. (cut), 20 gracillis m. (distal cut end), 21 medial head of the deep digital flexor m., 22 tendon of superficial digital flexor m., 23 gastrocnemius m., 24 semitendinosus $\mathrm{m}$., 25 semimembranosus $\mathrm{m}$., 26 insertion of the $\mathrm{m}$. internal oblique abdominal $\mathrm{m}$. onto symphysis of pelvis, 27 anal sinus, 28 constrictor vestibuli m., 29 external anal sphincter $\mathrm{m}$., 30 vagina, 31 rectum, 32 pelvic symphysis (cut).

The constrictor vestibuli muscle, $m$. constrictor vestibuli, extends from the ventral surface of the anal sphincter, around the lateral border of the vaginal vestibule of the female.

Origin: Ventral border of the external anal sphincter muscle.

Insertion: The muscles from the left and right sides meet ventral to the vestibule and insert on the surrounding fascia.

Action: Constricts and elevates the vaginal vestibule.

Innervation: N. perinealis profundus.

\section{PELVIC LIMB}

Sublumbar Muscles. The sublumbar muscles function as flexors of the hip joint. They extend from the sublumbar region to the medial aspect of the thigh.

The major psoas muscle, m. psoas major (Fig. 415 ), extends from the sublumbar region to the medial aspect of the thigh.

Origin: From the ventrolateral aspect of all the lumbar vertebral bodies.
Insertion: On the trochanter minor of the femur. Action: Flexion of the hip and lateral rotation of the femur.

Innervation: Ventral branches of the lumbar spinal nerves.

The iliacus muscle, $m$. iliacus, lies on the wing and the shaft of the ilium, and joins the major psoas muscle to form a common iliopsoas muscle.

Origin: From the wing and cranioventral border of the shaft of the ilium.

Insertion: In common with the psoas major muscle on the trochanter minor of the femur.

Action: Flexion of the hip and medial rotation of the femur.

Innervation: Ventral branches of the lumbar spinal nerves.

The minor psoas muscle, m. psoas minor (Fig. 414), lies medial to the m. psoas major in the sublumbar region.

Origin: From the ventromedial aspect of the vertebral bodies of 10T-7L.

Insertion: On the psoas tubercle of the ilium.

Action: Flexes the lumbar part of the vertebral column and the sacro-pelvic joint. 
Innervation: Ventral branches of the lumbar spinal nerves.

Muscles of the Hip. The hip muscles act as extensors, rotators, or abductors of the hip joint.

The superficial gluteal muscle, $m$. gluteus superficialis (Fig. 4-1 and 4-14) (gluteus maximus of Bryant, 1945), is the most superficial muscle of the gluteal region. The muscle extends from the pelvis and sacrum to the lateral aspect of the thigh, covering the middle and deep gluteal muscles.

Origin: From the tuber coxae, gluteal fascia and the sacrum, and from the transverse processes of the caudal vertebrae.

Insertion: Trochanter tertius of the femur.

Action: Extension of the hip joint.

Innervation: $\mathrm{N}$. gluteus caudalis.

The middle gluteal muscle, $m$. gluteus medius (Figs 4-1 and 4-14), is a large and powerful muscle mass that fills most of the area between the ilium, the sacrum and the hip. It lies deep to the superficial gluteal muscle. The origin of the muscle is extensive, and its caudal part curls laterally around the dorsolateral muscle of the tail to reach the lateral aspect of the sacrum.

Origin: From the area dorsal to the linea glutea on the wing of the ilium, gluteal fascia, as well as from the ventrolateral aspect of the sacrum.

Insertion: Trochanter major.

Action: Extends the hip joint.

Innervation: N. gluteus cranialis.

The deep gluteal muscle, $m$. gluteus profundus (gluteus minimus of Bryant, 1945), lies deep to the middle gluteal muscle and is the deepest of the gluteal muscles.

Origin: From the area ventral to the linea glutea on the wing of the ilium.

Insertion: Trochanter major, cranial and deep to the insertion of the gluteus medius muscle.

Action: Extends and abducts the hip.

Innervation: N. gluteus cranialis.

The piriform muscle, $m$. piriformis (Fig. 4-14), is fused to the caudal border of the middle gluteal muscle.

Origin: Via the caudal fascia from the median sacral spine.

Insertion: On the caudal border of the trochanter major.

Action: Extension of the hip.

Innervation: $\mathrm{N}$. gluteus caudalis.

The tensor fascia lata muscle, $m$. tensor fasciae latae (Figs. 4-1, 4-14 and 4-15) is a triangular muscle that forms the cranial, craniolateral and craniomedial borders of the proximal thigh.

Origin: Tuber coxae and the ventral margin of the wing of the ilium (between the $\mathrm{m}$. iliacus medially and the $\mathrm{m}$. gluteus medius laterally).
Insertion: On the femoral fascia cranially and laterally on the thigh, as well as on the trochanter tertius of the femur.

Action: Flexion of the hip and extension of the knee joints.

Innervation: $\mathrm{N}$. gluteus cranialis.

The gemelli muscles, $\mathrm{mm}$. gemelli, lie caudal to the deep gluteal muscle and deep to all the muscles that take origin from the sacrum and tail. Two muscles are present, one cranial (gemellus superior of Bryant, 1945) and one caudal (gemellus inferior of Bryant, 1945) to the tendon of the internal obturator muscle.

Origin: From the iliac crest and outer surface of the ramus of the ischium.

Insertion: Proximally in the trochanteric fossa.

Action: Outward rotation of the hip joint.

Innervation: $\mathrm{N}$. ischiadicus.

The internal obturator muscle, $m$. obturatorius internus, lies on the floor of the pelvis, internally covering the obturator foramen of the pelvis. It is covered by the pubic part of the $\mathrm{m}$. levator ani. Its tendon passes over the lessor sciatic notch, separating the two parts of the $\mathrm{m}$. gemelli superficially. A synovial bursa is present under the tendon where it passes over the lessor sciatic notch.

Origin: From the intrapelvic part of the symphysis of the pelvis.

Insertion: The tendon of insertion passes over the lesser ischiatic notch to insert in the trochanteric fossa, just proximal to the insertion of the $\mathrm{m}$. gemelli.

Action: Outward rotation of the hip joint.

Innervation: $\mathrm{N}$. ischiadicus.

The external obturator muscle, $m$. obturatorius externus, lies along the ventral (external) surface of the pelvis, deep to the pectineus and adductor muscles. It covers the obturator foramen externally.

Origin: Outer surfaces of the pubis and ischium around the obturator foramen.

Insertion: In the trochanteric fossa, distal to the insertion of the $\mathrm{m}$. gemelli.

Action: Outward rotation of the hip joint.

Innervation: N. obturatorius.

The quadratus femoris muscle, $m$. quadratus femoris, is the most caudal of the hip rotator muscles. It extends from the ischium to the femur, deep to the caudal thigh muscles.

Origin: Tuber ischii and the ramus of the ischium (from the arcus ischiadicus).

Insertion: Caudally on the area between the major and third trochanters.

Action: Extension and outward rotation of the hip.

Innervation: $\mathrm{N}$. ischiadicus.

Medial Thigh Muscles (Adductors of the Limb). The sartorius muscle, $m$. sartorius (Figs. 
4-2 and 4-15), is a very thin, strap-like muscle, on the craniomedial aspect of the thigh, superficially and covers the saphenous vessels and nerves.

Origin: From the fascia over the iliopsoas muscle and the inguinal ligament.

Insertion: On the fascia over the medial aspect of the tibial tuberosity and cranial tibial margin.

Action: Flexion of the hip and knee.

Innervation: N. femoralis.

The gracilis muscle, $m$. gracilis (Figs. 4-2 and 415), consists of a narrow cranial part and a broad caudal part. Together they form most of the superficial musculature on the medial aspect of the thigh.

Origin: The cranial belly takes origin from the cranial part of the pelvic symphysis, the caudal belly takes origin from the pelvic symphysis and ischial arch, caudal to the origin of the cranial belly.

Insertion: The cranial part inserts on the tibial tuberosity and the cranial tibial margin, whereas the caudal part inserts on the cranial tibial margin by means of a short aponeurosis.

Action: Adducts the limb and flexes the knee.

Innervation: $\mathrm{N}$. obturatorius.

The pectineus muscle, $m$. pectineus (Fig. 4-15), is small, triangular and is the most cranial of the medial thigh muscles.

Origin: Pecten of the pubis.

Insertion: On the caudomedial aspect of the proximal shaft of the femur.

Action: Adducts the thigh.

Innervation: N. obturatorius.

The adductor muscle, $m$. adductor, is a large, complex muscle that consists of five different parts. Most of the muscle is covered superficially by the $\mathrm{m}$. gracilis. The nomenclature used here is based on Hill (1937).

The long adductor muscle, $m$. adductor longus (Fig. 4-15), lies immediately caudal to the pectineus muscle.

Origin: Cranial edge of the ramus of the pubis and from the cranial part of the pelvic symphysis, just caudal to the origin of the $\mathrm{m}$. pectineus.

Insertion: On the caudomedial border of the shaft of the femur, distal to the insertion of the $\mathrm{m}$. pectineus.

Action: Adducts the limb.

Innervation: N. obturatorius.

The genicular part of the short adductor muscle, $m$. adductor brevis pars genicularis, lies caudal to the long adductor.

Origin: From the lateral aspect of the pelvic symphysis and the ischial arch ventral to the tuber ischii.

Insertion: On the caudomedial border of the distal half of the femur, including the medial epicondyle.

Action: Adducts the limb.
Innervation: $N$. obturatorius.

The small adductor muscle, $m$. adductor minimus, lies ventral to the quadratus femoris muscle and deep to the semitendinous and femorocaudalis muscles.

Origin: From the ventral aspect of the pelvic symphysis, lateral to the origin of the $\mathrm{m}$. gracilis.

Insertion: On the caudal part of and just distal to the trochanter tertius.

Action: Adducts the limb.

Innervation: $\mathrm{M}$. obturatorius.

The proper adductor muscle, $m$. adductor magnus proprius (Figs. 4-14 and 4-15), lies just distal to the adductor minimus, covered laterally by the semitendinous and femorocaudalis muscles.

Origin: From the angle between the pelvic symphysis and the ischial arch (ventral ischial tuberosity).

Insertion: Caudally on the shaft of the femur, from the trochanter tertius to just above the lateral epicondyle.

Action: Adducts the limb and rotates the hip inward.

Innervation: N. obturatorius.

The femoral part of the short adductor, m. adductor brevis pars femoralis (Fig. 4-15), lies just distal to the $\mathrm{m}$. adductor magnus proprius, covered laterally by the semitendinous and the femorocaudalis muscles.

Origin: From the lateral aspect of the pelvic symphysis, deep to the origin of the $m$. gracilis and superficial to the $\mathrm{m}$. adductor minimus and the adductor magnus proprius.

Insertion: Medial border of the shaft of the femur to the level of the medial condyle.

Action: Adducts the limb.

Innervation: $\mathrm{N}$. obturatorius.

Caudal Thigh Muscles. The caudal thigh muscles are very powerful and function as extensors of the hip (retractors of the limb) and of the knee joints when the foot is on the ground. They also act as flexors of the knee when the limb does not carry weight. From their origins, the muscles fan out distally so that they have a very extensive insertion on the lateral aspect of the thigh and crus.

The femoral biceps muscle, $m$. biceps femoris (Figs. 4-1 and 4-14), forms most of the caudolateral muscle mass of the thigh.

Origin: Laterally from the tuber ischii.

Insertion: Via a broad aponeurosis on the patella, the patellar ligament and on the craniomedial margin of the tibia to the ankle. It also attaches to the calcanean tendon and the tuber calcanei.

Action: Extends the hip, knee and ankle joints, and retracts the limb.

Innervation: $\mathrm{N}$. ischiadicus. 
The caudal abductor of the crus, $m$. abductor cruris caudalis (tenuissimus of Bryant, 1945), is a thin, strap-like muscle situated on the medial (deep) surface of the $\mathrm{m}$. biceps femoris. It extends from the base of the tail to the ankle.

Origin: From the fascia covering the dorsolateral muscle of the tail.

Insertion: On the fascia over the lateral aspect of the distal crus and ankle.

Action: Abducts the limb.

Innervation: $\mathrm{N}$. ischiadicus.

The femorococcygeal muscle, $m$. femorococcygeus (Fig. 4-14), lies deep to the superficial gluteal muscle cranially and the vertebral head of the m. semitendinosus caudally.

Origin: Muscular from the transverse processes of the first and second caudal vertebrae and via the gluteal fascia from the articular and spinous processes of the first caudal vertebra.

Insertion: Between the $\mathrm{m}$. vastus lateralis and the adductor on the distal caudomedial border of the shaft of the femur.

Action: Extends the hip joint and retracts the limb.

Innervation: $\mathrm{N}$. ischiadicus.

The femorocaudal muscle, $m$. femorocaudalis, extends from the pelvis to the femur, deep to the $\mathrm{m}$. femorococcygeus and $\mathrm{m}$. biceps femoris. It covers the lateral aspect of the adductor muscles.

Origin: Craniolaterally from the tuber ischii.

Insertion: It has a muscular insertion in common with, but deep to, the femorococcygeus muscle on the distal caudolateral border of the femur up to, but not including, the lateral epicondyle.

Action: Extends the hip joint and retracts the limb.

Innervation: $\mathrm{N}$. ischiadicus.

The semitendinous muscle, $m$. semitendinosus (Figs. 4-1, 4-14 and 4-15), consists of vertebral and pelvic heads. The vertebral head covers the caudal parts of the superficial gluteal and the femorococcygeal muscles. The muscle is the most superficial muscle on the caudal aspect of the gluteal region.

Origin: The vertebral head takes origin from the gluteal fascia and from the spinous processes of the sacral and the caudal vertebrae. The pelvic head takes origin from the lateral aspect of the tuber ischii, just caudal to the origin of the biceps femoris muscle.

Insertion: By means of an aponeurosis on the medial aspect of the cranial tibial margin, deep to the insertion of the $\mathrm{m}$. gracilis.

Action: Extension of the hip, knee and ankle joints, or flexion of the joints when the limb is not bearing weight.

Innervation: N. ischiadicus.

The semimembranous muscle, $m$. semimembra- nosus (Figs. 4-1, 4-14 and 4-15), lies between the semitendinous and the adductor muscles on the caudal aspect of the thigh.

Origin: From the tuber ischii and the ischial arch.

Insertion: By means of a short, flat tendon on the medial aspect of the tibial tuberosity.

Action: Extension of the hip and knee joints. It is a large and powerful retractor of the limb.

Innervation: $\mathrm{N}$. ischiadicus.

Cranial Thigh Muscles. The cranial thigh muscles extend from the pelvis and femur to the patella and function as extensors of the knee joint.

The quadriceps femoris muscle, $m$. quadriceps femoris (Figs. 4-14 and 4-15), lies cranial on the shaft of the femur. It consists of the three vastus muscles and the rectus femoris muscle that are largely fused to each other. The muscle mass converges to form a single patellar ligament that inserts on the tibial tuberosity and the cranial tibial margin. The patella is interposed in the ligament. The quadriceps muscle is a powerful extensor of the knee joint.

The vastus lateralis muscle, $m$. vastus lateralis (Fig. 4-14), is the most lateral of the quadriceps muscles. It lies deep to the $\mathrm{m}$. biceps femoris and is partly covered by the thick fascia lata of the femoral region.

Origin: Proximal part of the femur (the area bounded laterally by the neck, trochanter major and trochanter tertius).

Insertion: On the tibia.

Action: Extends the knee joint.

Innervation: $\mathrm{N}$. femoralis.

The vastus intermedius muscle, $m$. vastus intermedius, lies cranially on the shaft of the femur and is the deepest of the cranial thigh muscles.

Origin: Cranial aspect of the shaft of the femur. Insertion: On the tibia.

Action: Extends the knee joint.

Innervation: N. femoralis.

The vastus medialis muscle, $m$. vastus medialis (Fig. 4-15), is bordered cranially by the $\mathrm{m}$. rectus femoris and caudally by the adductor muscle. It is also covered by the thin $\mathrm{m}$. sartorius.

Origin: Caudomedial border of the shaft of the femur.

Insertion: On the tibia.

Action: Extends the knee joint.

Innervation: $\mathrm{N}$. femoralis.

The rectus femoris muscle, $m$. rectus femoris (Fig. 4-14), extends from the pelvis to the patella. It lies between the lateral and medial vastus muscles and superficial to the $\mathrm{m}$. vastus intermedius. Its proximal part is covered by the $\mathrm{m}$. tensor fasciae latae.

Origin: From the rectus femoral muscle area of the ilium.

Insertion: On the tibia. 
Action: Extends the knee joint.

Innervation: $\mathrm{N}$. femoralis.

Muscles of the Crus. All the muscles of the ankle and digits are arranged around the crus. They are covered by a very thick layer of fascia, the fascia cruris, which gives off sheets of fascia between the muscles, separating them from each other. The extensors of the ankle and flexors of the digits lie caudally and the flexors of the ankle and extensors of the digits lie cranially on the crus. The term fibularis is used in preference to peroneus to describe the muscles associated with the lateral or fibular aspect of the crus.

The popliteal muscle, $m$. popliteus, lies deep to the medial head of the gastrocnemius muscle on the caudal aspect of the crus. Its tendon of origin passes between the lateral collateral ligament of the knee and the lateral meniscus and has a synovial sheath.

Origin: From the popliteal fossa on the lateral condyle of the femur.

Insertion: On the proximal caudal surface of the tibia.

Action: Limited extension of the knee jont and inward rotation of the leg.

Innervation: N. tibialis.

Extensors of the Ankle Joint. The gastrocnemius muscle, $m$. gastrocnemius (Figs. 4-1 and 414 ), extends from the distal femur to the calcaneus. It consists of two muscle bellies that take origin from the corresponding condyles of the femur. The fabellae or sesamoid bones lie within the tendons of origin of the muscles and articulate with the femoral condyles. The medial head has a lateral and a medial part.

Origin: The lateral part of the medial head takes origin from the tendon of origin of the superficial digital flexor muscle as well as from the lateral head of the gastrocnemius muscle. The medial part takes origin from the medial femoral condyle.

Lateral head takes origin from the lateral femoral condyle.

Insertion: On the calcaneal tuber. A small bursa is present between the tendon and the tuber.

Action: Extension of the ankle joint.

Innervation: $\mathrm{N}$. tibialis.

The caudal tibial muscle, $m$. tibialis caudalis, lies cranial to the deep digital flexor and distally to the popliteus muscles along the caudomedial border of the tibia. Its tendon of insertion passes caudally around the malleolus of the tibia, cranial to that of the medial digital flexor muscle and then along the medial aspect of the plantar sesamoid bone to the metatarsus.
Origin: From the head and caudomedial aspect of the proximal third of the fibula.

Insertion: Medial on the proximal end of the first metatarsal bone.

Action: Flexion of the ankle joint.

Innervation: $\mathrm{N}$. tibialis.

The soleus muscle, $m$. soleus, lies deep to the lateral head of the gastrocnemius muscle and is not visible proximally. Distally it lies deep to the tendon of the lateral head of the gastrocnemius muscle. The muscular belly extends to the tuber calcis.

Origin: Head of the fibula.

Insertion: Via the tendon of the lateral head of the gastrocnemius muscle on the calcaneal tuber.

Action: Extends the ankle joint.

Innervation: $\mathrm{N}$. tibialis.

Flexors of the Ankle Joint. The cranial tibial muscle, $m$. tibialis cranialis (Figs. 4-2 and 4-14), is a broad, flat muscle that forms the cranial muscular margin of the crus. The muscle belly extends from the proximal tibia to the ankle where it abruptly becomes tendinous. At this point the tendon is held in position by an annular ligament. From the annular ligament the tendon passes distomedially to insert on the metatarsus.

Origin: From the head of the fibula, the lateral condyle of the tibia and the cranial tibial margin.

Insertion: On the medioplantar aspect of the first metatarsal bone.

Action: Flexion of the ankle joint and lateral rotation of the pes.

Innervation: N. fibularis.

The long peroneal (fibular) muscle, $m$. peroneus (fibularis) longus (Fig. 4-14), extends from the knee to the ankle. Proximally it lies caudal to the $\mathrm{m}$. tibialis cranialis and distally caudal to the m. fibularis brevis.

Origin: Head and proximal half of the shaft of the fibula.

Insertion: The tendon passes through the malleolar groove of the fibula, then through a groove between the fifth tarsal and metatarsal bones where it turns medially and passes transversely, deep to the deep digital flexor muscle, to insert on the first tarsal bone.

Action: Medial rotation of the hind paw so that the plantar surface faces laterally.

Innervation: $\mathrm{N}$. fibularis.

The short peroneal (fibular) muscle, $m$. peroneus (fibularis) brevis, lies laterally on the leg and is only visible in the distal half of the crus, caudal to the long digital extensor muscle. It takes origin lateral to the origin of the extensor muscle of the first digit.

Origin: It has a fascial origin from the fibular 
head and a muscular origin from the whole cranial border of the shaft of the fibula.

Insertion: The tendon passes through the groove of the fibular malleolus, deep to the tendon of the $\mathrm{m}$. fibularis longus, to insert on the proximal lateral aspect of the fifth metatarsal bone.

Action: Flexion of the ankle joint.

Innervation: N. fibularis.

Extensors of the Digits. The long extensor of the digits, $m$. extensor digitorum longus (Fig. 414 ), lies caudolateral to the $\mathrm{m}$. tibialis cranialis. Proximally it is covered by the tibialis muscle and is only visible in the distal part of the crus. It extends from the knee to the digits. At the ankle the tendon is held in position by the annular ligament of the cranial tibial muscle. The tendon of the muscle divides into lateral and medial branches. The lateral branch divides to insert on digits III-V, and the medial branch divides to insert on digits II-III.

Origin: By means of a tendon from the extensor fossa on the lateral condyle of the femur.

Insertion: On the extensor processes of the distal phalanges of digits II-V.

Action: Extension of digits II-V.

Innervation: $\mathrm{N}$. fibularis.

The extensor of the fifth digit, $m$. extensor digiti $V$ (extensor digitorum brevis of the fifth digit of Bryant, 1945), lies caudal to the m. fibularis brevis, deep to the $\mathrm{m}$. fibularis longus and proximal to the extensor of the fourth digit. Its tendon of insertion passes around the fibular malleolus, deep to the tendon of the long digital extensor. At the malleolus the tendon lies lateral to the tendon of the $\mathrm{m}$. extensor digiti IV.

Origin: Proximal quarter of the fibula.

Insertion: On the lateral aspect of the extensor process of the third phalanx of digit five.

Action: Extends the fifth digit.

Innervation: N. fibularis.

The extensor of the fourth digit, $m$. extensor digiti $I V$ (extensor digitorum brevis of the fourth digit of Bryant, 1945), is a long, slender muscle that lies caudal and deep to the m. fibularis brevis. Its tendon of insertion passes through the groove of the fibular malleolus, deep to the tendon of the m. fibularis brevis.

Origin: From the distal fibular shaft (third quarter), caudal to the origin of the $\mathrm{m}$. fibularis brevis.

Insertion: On the lateral aspect of the extensor process of the third phalanx of digit four.

Action: Extends the fourth digit.

Innervation: N. fibularis.

The extensor of the first digit, $m$. extensor digiti I (extensor hallucis longus of Bryant, 1945), is an independent muscle and is not part of the long digital extensor. It lies deep to the $\mathrm{m}$. tibialis cranialis.
Distally the muscle belly is held down by the annular ligament of the cranial tibial muscle.

Origin: From the cranial aspect of the middle third of the fibula.

Insertion: On the dorsal aspect of the second phalanx of the first digit.

Action: Extension of the first digit.

Innervation: N. fibularis.

Flexors of the Digits. The superficial digital flexor muscle, $m$. flexor digitorum superficialis (Fig. $4-15$ ), lies caudal to the lateral head of the m. gastrocnemius. Its medial border lies deep to the medial head of the $\mathrm{m}$. gastrocnemius. The tendon of the superficial digital flexor muscle twists from medial to lateral around the tendon of the $\mathrm{m}$. gastrocnemius, and forms part of the common calcaneal tendon. A large synovial bursa is present between the tendon and the tuber calcis. Distal to the calcaneus the tendon divides into a superficial part that extends to the level of the metatarsus, and a deep part that extends to the middle phalanges of the digits. At the metatarso-phalangeal joints the tendons divide and form synovial sheaths (manicum flexorium) around the deep digital flexor tendons.

Origin: In common with the lateral head of the gastrocnemius muscle from the caudal aspect of the femur.

Insertion: The tendon attaches laterally and medially on the tuber calcis as it passes over the ankle and then divides into superficial and deep parts. The superficial part fans out into the tarsal and metatarsal pads. The deep part divides into four tendons. Each of the four tendons inserts on the proximal part of the second phalanx of digits II-V.

Action: Flexion of the proximal interdigital joints of digits II-V. Due to its attachment on the calcaneus it also functions as an extensor of the ankle.

Innervation: $\mathrm{N}$. tibialis.

The deep digital flexor muscles, $m m$. flexores digitorum profundi, consist of medial and lateral digital flexor muscles. The caudal tibial muscle does not form part of the deep digital flexor muscles.

The medial digital flexor muscle, $m$. flexor digitorum medialis (Fig. 4-15) (flexor digitorum tibialis of Bryant, 1945), lies along the medial border of the tibia, cranial to the medial head of the m. gastrocnemius and distal to the m. popliteus. The tendon of the muscle passes through a groove on the caudal surface of the tibial malleolus where it is held in position by a band of connective tissue. The muscle does not function as a flexor of the toes, but rather as an extensor of the ankle.

Origin: From the caudomedial aspect of the middle third of the tibia.

Insertion: On the plantar sesamoid bone and the medial aspect of the first tarsal bone. 
Action: Extension of the ankle joint.

Innervation: $\mathrm{N}$. tibialis.

The lateral digital flexor muscle, $m$. flexor digitorum lateralis (Fig. 4-14) (flexor digitorum fibularis of Bryant, 1945), is the largest of the digital flexor muscles. It is the only part of the deep digital flexor muscle that reaches the toes. It lies cranially to the muscles that insert on the calcaneal tuber and has an extensive origin from the caudal aspect of the tibia distal to the popliteus muscle. The tendon of the muscle passes over the sustentaculum tali and then divides into five branches, one branch for each toe. At the metatarso-phalangeal joints the tendons are held in position by the plantar ligaments. They pierce the tendons of the superficial digital flexor muscle, and are held in position on the proximal phalanges by proximal and distal digital annular ligaments.

Origin: From the caudal aspect of the shaft of the tibia, and the head and shaft of the fibula.

Insertion: On the flexor surfaces of the distal phalanges of digits $\mathrm{I}-\mathrm{V}$.

Action: Flexion of the digits.

Innervation: $\mathrm{N}$. tibialis.

Muscles of the Pes. All but one of the muscles of the pes are arranged on the plantar aspect of the foot. They function as short extensors, flexors, adductors or abductors of the digits.

The four lumbricales muscles, $\mathrm{mm}$. lumbricales, lie between the tendons of the deep digital flexor muscle.

Origin: Deep digital flexor tendon.

Insertion: On the medial aspects of the proximal phalanges of digits II-V.

Action: Flexion of the digits.

Innervation: $\mathrm{N}$. tibialis.

The single interflexorius muscle, $m$. interflexorius (flexores digitorum brevis of Bryant, 1945), extends between the tendons of the deep digital flexor and the superficial digital flexor muscles.

Origin: Tendon of the deep digital flexor muscle. Insertion: On the superficial digital flexor tendon of the second digit.

Action: Flexion of the second digit.

Innervation: $\mathrm{N}$. tibialis.

The four interosseus muscles, $\mathrm{mm}$. interossei, extend from the proximal metatarsal region to the proximal sesamoid bones of digits II-V. Some authors, including Bryant, 1945, regard them as separate muscles, giving a total of eight.

Origin: From the proximal ends of the five metatarsal bones.

Insertion: On the proximal sesamoid bones.

Action: Flexion of the metatarso-phalangeal joints.

Innervation: N. tibialis.
The abductor of the first digit, $m$. abductor digiti I (abductor hallucis of Bryant, 1945), is the most medial of the short muscles of the pes.

Origin: From the plantar aspect on the distal end of the second tarsal bone.

Insertion: On the abaxial sesamoid bone of the first digit.

Action: Abduction of the first digit.

Innervation: $\mathrm{N}$. tibialis.

The adductor of the first digit, $m$. adductor digiti I (adductor hallucis of Bryant, 1945), extends between the tarsus and the first digit.

Origin: From the plantar ligament.

Insertion: On the axial aspect of the first digit.

Action: Adduction of the first digit.

Innervation: $\mathrm{N}$. tibialis.

The flexor of the fifth digit, $m$. flexor digiti $V$ (adductor digiti quinti of Bryant, 1945), extends between the tarsus and the fifth digit.

Origin: From the plantar aspect of the calcaneus.

Insertion: By means of a long, thin tendon on the plantarolateral aspect of the proximal phalanx of the fifth digit.

Action: Flexion of the fifth digit.

Innervation: $\mathrm{N}$. tibialis.

The adductor of the fifth digit, $m$. adductor digiti $V$ (abductor ossis metacarpi quinti of Bryant, 1945), lies laterally to the short flexor of the fifth digit.

Origin: From the plantar aspect of the calcaneus, lateral to the short flexor of the fifth digit.

Insertion: Proximally on the lateral aspect of the fifth metatarsal bone.

Action: Adduction of the fifth metatarsal bone and digit.

Innervation: N. tibialis.

The abductor of the fifth digit, $m$. abductor digiti $V$ (abductor digiti quinti of Bryant, 1945), extends between the tarsus and the fifth digit.

Origin: From the tarsal ligament.

Insertion: On the axial surface of the proximal phalanx of fifth digit.

Action: Abduction of the fifth digit.

Innervation: $\mathrm{N}$. tibialis.

The quadratus plantae muscle, $m$. quadratus plantae, is a broad, muscle in the plantar surface of the pes. Its fibers run distomedially and merge into a broad, flat tendon. The tendon is as wide and as long as the muscle belly.

Origin: From the tubercle on the distal, lateral aspect of the calcaneus.

Insertion: On the deep flexor tendon, just proximal to the origin of the interflexorius muscle.

Action: Flexion of the digits.

Innervation: $\mathrm{N}$. tibialis.

The short extensor of the digits, $m$. extensor digitorum brevis, is the only muscle on the dorsal aspect of the pes. It has two muscle bellies, their ten- 
dons dividing to go to the second, third and fourth digits. (Bryant (1945) considers each muscle belly a separate muscle and names them accordingly.) Origin: Laterally on the fifth tarsal bone.
Insertion: On the abaxial surfaces of the second, third and fourth digits.

Action: Extension of the second to fourth digits. Innervation: $\mathrm{N}$. fibularis. 


\section{Chapter 5. Digestive System}

The digestive system consists of the oral cavity, pharynx, alimentary, canal and accessory digestive organs. The accessory organs include the teeth, tongue, salivary glands, pancreas, liver and gall bladder.

Mouth and Associated Structures. The mouth designates the oral cavity and its contents. The oral cavity is divided into the vestibule and the oral cavity proper.

The oral vestibule, vestibulum oris, is the space bounded laterally by the lips and cheeks and medially by the gums and teeth. A shallow cheek pouch is present caudal to the angle of the mouth. It lies opposite the diastema (space between the teeth) and extends to the level of the first molar tooth.

The lips, labia oris, form the rostral and most of the lateral boundaries of the vestibule. The upper and lower lips meet opposite the first premolar teeth to form the angles of the mouth. The lips bound the oral fissure, rima oris, which is the opening of the oral cavity. Their outer surfaces are covered by skin and their inner surfaces are lined by the oral mucosa. The upper lips pass caudoventrally and laterally. At the level of the first premolar teeth they turn acutely medially to join the lower lips. The oral scent glands are associated with the angle of the mouth. The secretory ducts of the glands open on the mucocutaneous junction at the angle of the mouth. The philtrum of the upper lip is shaped like an inverted V. It extends through the total thickness of the lip, dividing the latter into left and right halves and exposes the upper incisor teeth. The lower lip is shorter than the upper lip.

The cheek, bucca, is narrow and shallow, except for the cheek pouch. The duct of the parotid salivary gland opens on the parotid papilla, papilla parotidea, opposite the first upper premolar, while the duct of the zygomatic gland opens opposite the last cheek tooth.

The oral cavity proper, cavum oris proprium, is bounded dorsally by the palate, laterally and rostrally by the teeth and gums and ventrally by the tongue and the reflected mucous membrane below it. It extends from the incisor teeth rostrally to the isthmus faucium caudally. When the mouth is closed, the tongue fills the cavity. The ducts of the mandibular and monostomatic sublingual salivary glands open on the sublingual papilla, caruncula sublingualis, under the tongue. The papilla lies on the floor of the mouth, just caudal to the lower incisor teeth. The gums and lower cheek are separated from the tongue by a deep sublingual groove, recessus sublingualis lateralis. The ducts of the polystomatic sublingual salivary glands open into the floor of the recess.

The hard palate, palatum durum, and the rostral part of the soft palate, palatum molle, (Figs. 5-1 and 5-2) form the roof of the oral cavity. The hard palate presents 12 to 14 transverse ridges, rugae palatinae, and is largely pigmented. The second ridge is triangular in outline rostrally, with the apex rostrally and the base caudally (the first ridge lies directly behind the upper incisor teeth). The oral surface of the soft palate is smooth and, like the rest of the oral mucosa, unpigmented. A small incisive papilla, papilla incisiva, is present just caudal to the upper incisor teeth. It carries the openings of the incisive ducts from the nasal cavity and vomeronasal organ.

The tongue, lingua (Fig. 5-3), consists primarily of skeletal muscle covered by a thick mucosa. It feels soft and velvety.

The tongue has an apex, body and a base. The tip of the tongue, apex linguae, is rounded and somewhat bifid in appearance. The body, corpus linguae, forms the bulk of the tongue. It is attached rostrally to the floor of the mouth by a short, median mucosal fold, the frenulum linguae. The latter is extensive enough to permit good mobility of the tongue. The caudal part of the body is slightly raised and is continuous with the root of the tongue. The root, radix linguae, is that part of the tongue caudal to the vallate papillae and is attached to the hyoid bone.

The epithelium on the dorsal and dorsolateral surfaces of the tongue form many lingual papillae. The filiform papillae, papillae filiformes, are the smallest and the most numerous of the lingual papillae. They are short, thin threads of non-keratinized epithelium and have a mechanical function. The filiform papillae are located over most of the dorsal and dorsolateral surfaces of the tongue. They increase in size towards the root of the tongue.

The fungiform papillae, papillae fungiformes, are mushroom-shaped. They appear as small, white beads on the dorsal surface and lateral margins of 


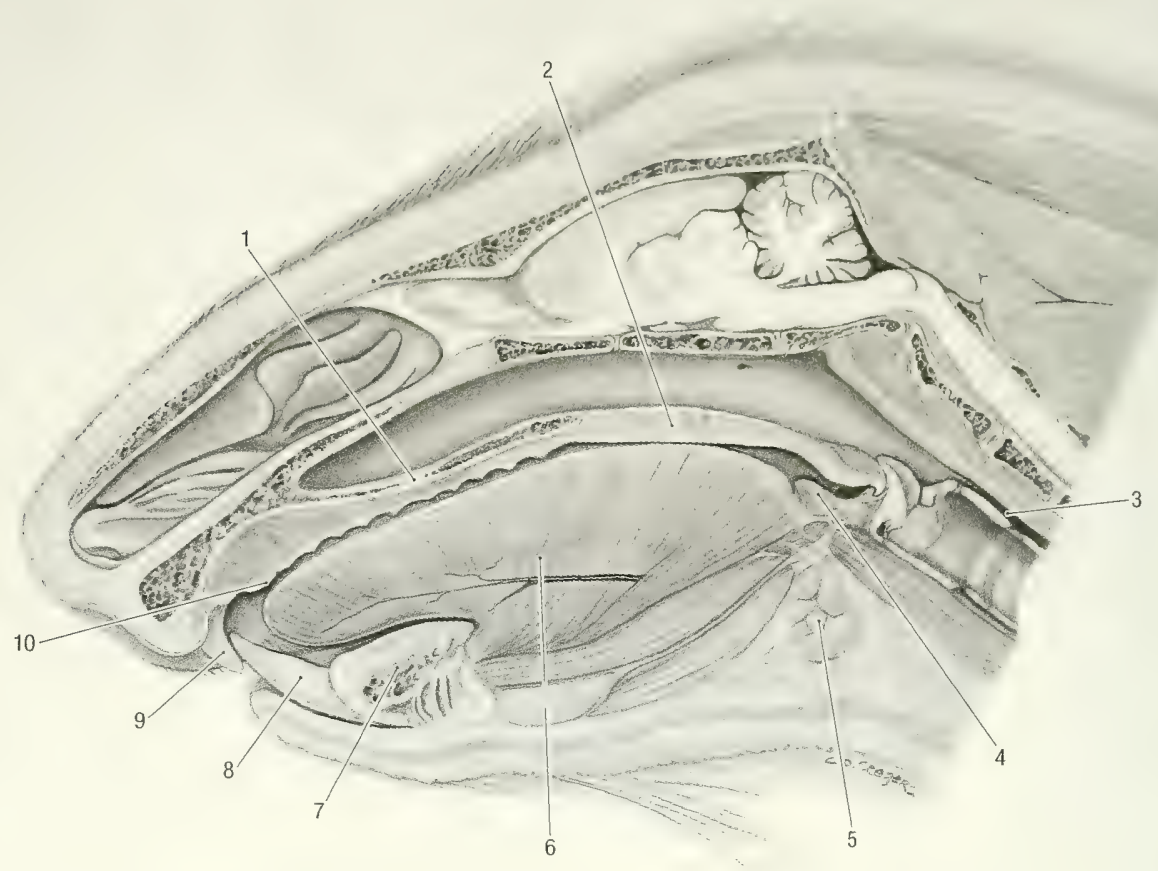

Fig. 5-1. Sagital section of the head, medial view. 1 hard palate, 2 soft palate, 3 esophagus, 4 palatine tonsil, 5 mandibular salivary gland, 6 tongue, 7 mandibular symphysis, 8 lower incisor tooth, 9 upper incisor tooth, 10 incisive papilla.

the tongue. Fungiform papillae carry taste buds. The vallate papillae, papillae valatae, are the most prominent of the lingual papillae. They measure approximately $6 \mathrm{~mm}$ in diameter and consist of a central, raised part, surrounded by a moat. There are 3 to 5 vallate papillae on the caudal third of the dorsum of the tongue. They mark the boundary between the body and root of the tongue. Vallate papillae carry taste buds. There are two groups of foliate papillae, papillae foliatae. Each group is 8 to $10 \mathrm{~mm}$ long and contains 14 to 18 leaf-like papillae. They are located on the lateral margin of the tongue, immediately rostral to the palatoglossal fold. Foliate papillae carry taste buds.

Blood supply: The lingual artery. The veins of the tongue are satellites of the arteries.

Innervation: The tongue is innervated by the chorda tympani and the lingual, glossopharyngeal and hypoglossal nerves.

The lingual nerve is the principal sensory nerve to the tongue. It innervates the tongue rostral to the vallate papillae. The lingual branch of the glossopharyngeal nerve supplies sensory fibers to the root of the tongue. It also innervates the taste buds on the vallate and foliate papillae. The chorda tympani innervates the taste buds rostral to the vallate papillae. The hypoglossal nerve innervates the muscles of the tongue.
Lymph drainage: By the retropharyngeal lymph nodes.

The gums, gingiva, are composed of dense fibrous connective tissue covered by a smooth mucosa. The connective tissue extends around the necks of the teeth and down into the alveoli. It is continuous with the alveolar periosteum. The gums are externally continuous with the mucosa of the vestibule and internally with the floor of the oral cavity proper or the hard palate.

The teeth, dentes, (Figs. 2-8, 2-13, and 5-2) are not as robust as in other rodents of similar size. They serve to cut and crush plant materials rather than grind it. The incisors can also be used for defense. Each tooth consists of a crown, corona dentis, a neck, cervix dentis, and a root or roots, radix dentis.

The surface of a tooth that faces the lip or cheek is the vestibular surface, facies vestibularis. The lingual surface, facies lingualis, faces the tongue. The surfaces adjacent to other teeth in the dental arch are the contact surfaces, facies contactus. For all teeth the contact surfaces are mesial and distal. The mesial surfaces are towards the median plane, the distal surfaces are away from the median plane. The surface that faces the opposite dental arch is the occlusal surface, facies occlusalis.

The incisor teeth, dentes incisivi, are long, curved 


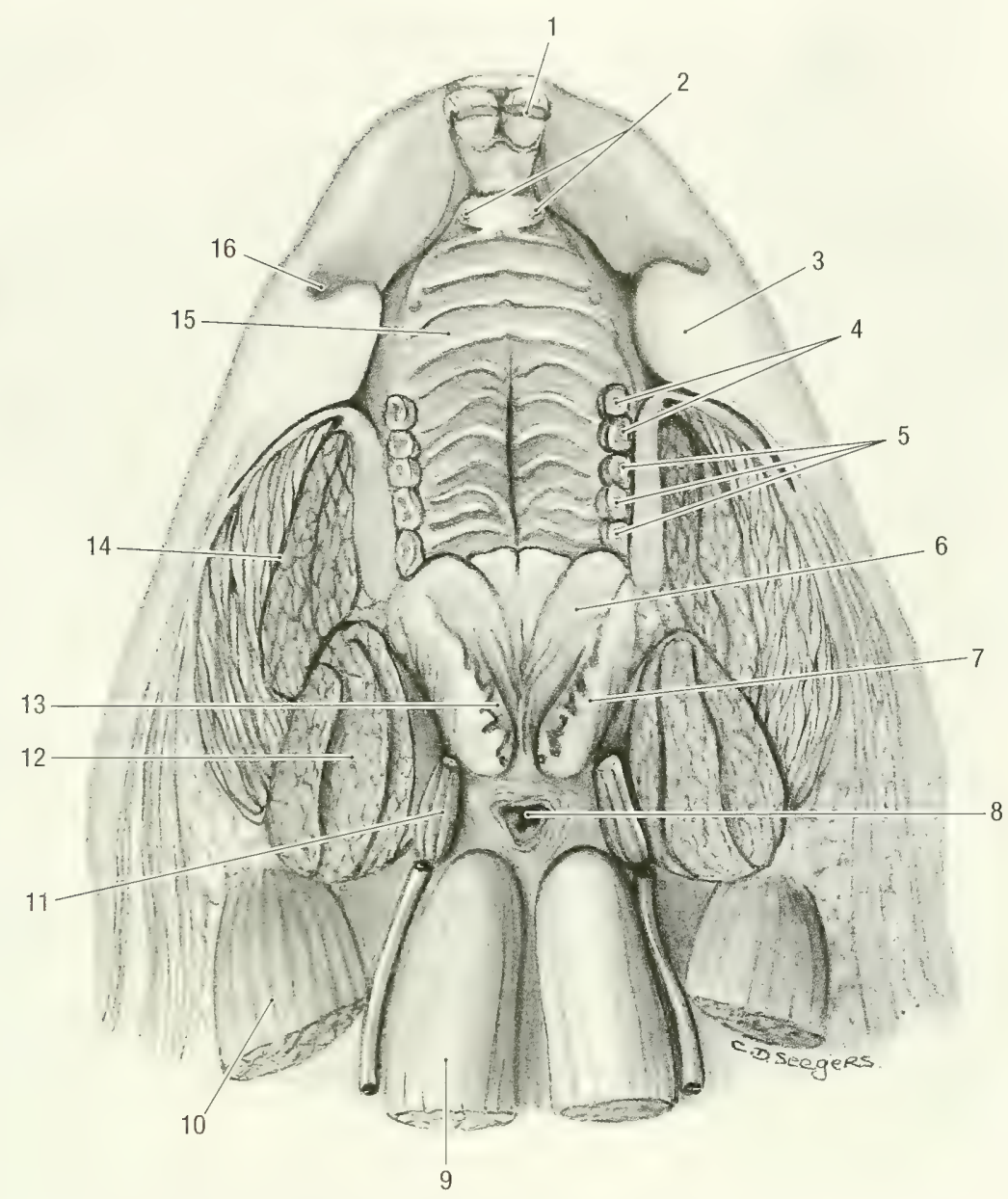

Fig. 5-2. Horizontal section of the head through the oral cavity, ventral view. 1 upper incisor tooth, 2 openings of incisive ducts on each side of the incisive papilia, 3 buccal fold, 4 premolar teeth, 5 molar teeth, 6 soft palate, 7 cut lateral wall of the oropharynx, 8 esophagus, 9 longus capitis m., 10 sternomastoid m., 11 stylohoid bone and muscle, 12 temporal m., 13 palatine tonsil, 14 masseter m., 15 hard palate, 16 cheek pouch.

and have a beveled cutting edge (see osteology for the alveolus). In the young animal they are white, but become brownish as the animals age. The lower incisors protrude from the mandibles further than do the upper incisors from the maxillae. The enamel on the labial side of the incisor is not pigmented as in many other rodents.

The cheek teeth, dentes premolares and dentes molares, are typical, each with a crown that protrudes above the gum, a neck and root. The enamel of the crowns form ridges and tubercles on the occlusal surfaces. The neck is a slight constriction of the tooth located at the gum line, the enamel of the neck is covered by cement. The root is the portion below the gum and does not have an enamel layer. For the most part it is embedded in the alveolus of the jaw.

There are both deciduous and permanent teeth. The deciduous teeth, dentes decidui, are replaced by the larger, permanent teeth, dentes permanentes, when the animals are 3 to 4 months old. The teeth are arranged as upper and lower dental arches. The upper arch is longer than the lower arch. The teeth are anchored in sockets, or alveoli, of the upper and lower jaws. They are so placed that the upper incisors lie rostral to the lower ones, while the premolars and molars form a tuberculate crushing surface.

The incisor teeth, dentes incisivi, are permanent teeth, and are characterized by permanent growth that compensates for the strong wear. They therefore have long crowns, short neck and roots and retain the same length. If they do not wear down, e.g., with malocclusion, they keep on growing until finally they prevent the mouth from closing (Fig. 1-3). They have a thick layer of enamel on their vestibular surfaces and a very thin layer on their lingual surfaces. Since the enamel on the vestibular 


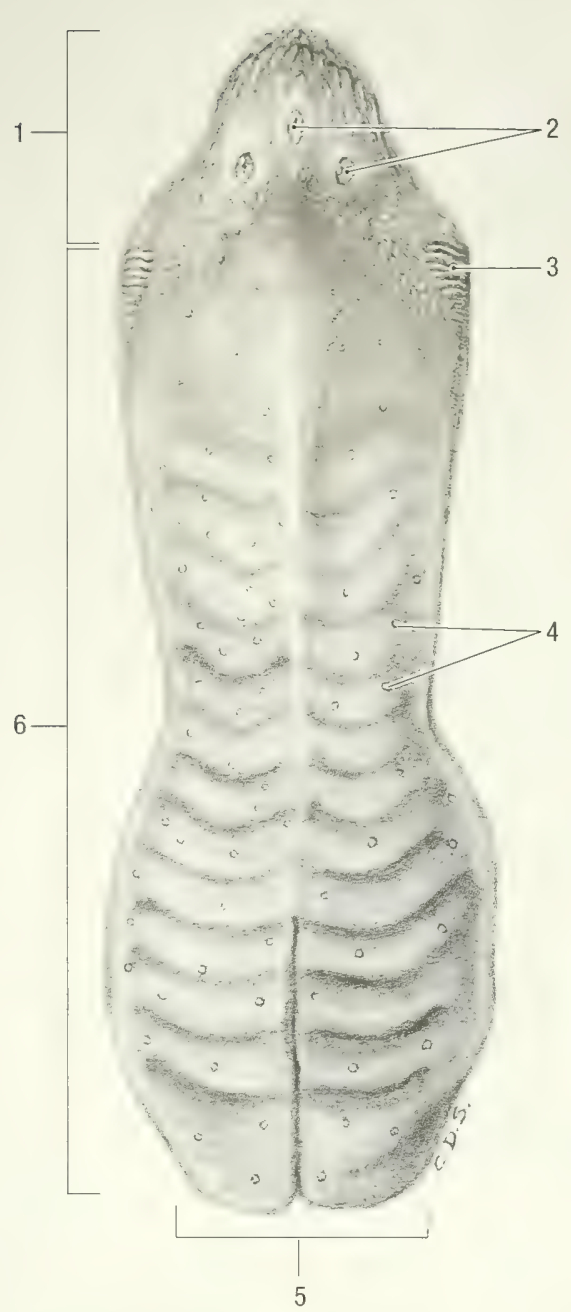

Fig. 5-3. Tongue, dorsal view. 1 root of the tongue, 2 vallate papillae, 3 foliate papillae, 4 fungiform papillae, 5 apex of the tongue, 6 body of the tongue.

surface wears more slowly than that of the lingual surface, it maintains a beveled cutting edge along the lingual surface. The incisors are embedded in deep alveoli. The upper alveoli describe a larger arc of a smaller circle and the lower ones a smaller arc of a larger circle. The alveolus of an upper incisor extends to a point dorsally to the infraorbital foramen and that of a lower incisor to a point ventrally to the last molar tooth. The upper incisors are embedded in the alveoli of the incisive bones and are placed rostral to the lower incisors. They are about $5 \mathrm{~cm}$ long and strongly curved. The mesial surface is flattened and the distal surface is rounded. Approximately $1 \mathrm{~cm}$ of an upper incisor is visible at any given time. The lower incisors are embedded in the alveoli of the mandible and are placed behind the upper ones. They are about $6 \mathrm{~cm}$ long and slightly curved. The mesial surface is flat- tened and the distal surface is rounded. About 1.5 $\mathrm{cm}$ of the lower incisors is visible at any given time. The lower incisors erupt when the animal is approximately 10 to 20 days old, the upper pair erupt about 14 days later.

The premolar teeth, dentes premolares, are represented by the third and fourth premolars in the upper jaw and by the fourth premolar in the lower jaw. The deciduous premolars erupt when the animals are 35 to 38 days old. They are replaced by the permanent premolars when the animals are about 3 to 4 months old. The third upper premolar is a small, conical tooth with one root. Its contact surface has one tubercle. The fourth upper premolar has three roots, one on the lingual side and two on the vestibular side. The contact surface has three transverse enamel ridges, separated from each other by two transverse grooves. One large tubercle is present on the lingual side and three smaller ones on the vestibular side of the contact surface. The fourth lower premolar has two roots, one on the mesial and one on the distal side. Both roots are grooved. The contact surface has two transverse ridges. They are separated from each other by a hollow into which the lingual tubercle of the fourth upper premolar fits.

The molar teeth, dentes molares, are represented by the first, second and third molar teeth of the upper and lower jaws. They erupt when the animals are 4 to 5 weeks old. The upper and lower molar teeth have three roots each, one on the lingual and two on the vestibular side. The contact surfaces of all the molar teeth are similar in structure. Each has three transverse ridges, separated from each other by two transverse grooves. Four tubercles are present, one large tubercle on the lingual side and three smaller ones on the vestibular side. The transverse ridges of the lower molar teeth wear down quickly. They are replaced by one large hollow surrounded by three tubercles. The contact surfaces of the upper teeth seem to be more resistant to wear.

Dental formula: The woodchuck has 6 deciduous and 22 permanent teeth. Since the teeth are grouped according to position and form, it is possible to express their arrangement as a dental formula. The abbreviation representing the particular teeth (i, incisor; c, canine; $\mathrm{p}$, premolar; $\mathrm{m}$, molar) are followed by the number of such teeth on one side of the upper and lower jaw.

The dental formula for the deciduous dentition is: $\mathrm{i} 0 / 0, \mathrm{c} 0 / 0, \mathrm{p} 2 / 1, \mathrm{~m} 0 / 0$, total 6

The dental formula for the permanent dentition is: i $1 / 1, \mathrm{c} 0 / 0, \mathrm{p} 2 / 1, \mathrm{~m} \mathrm{3/2}$, total 22

Blood supply: The infraorbital artery supplies the upper teeth, the inferior alveolar artery supplies the lower teeth. The veins are satellites of the arteries. 


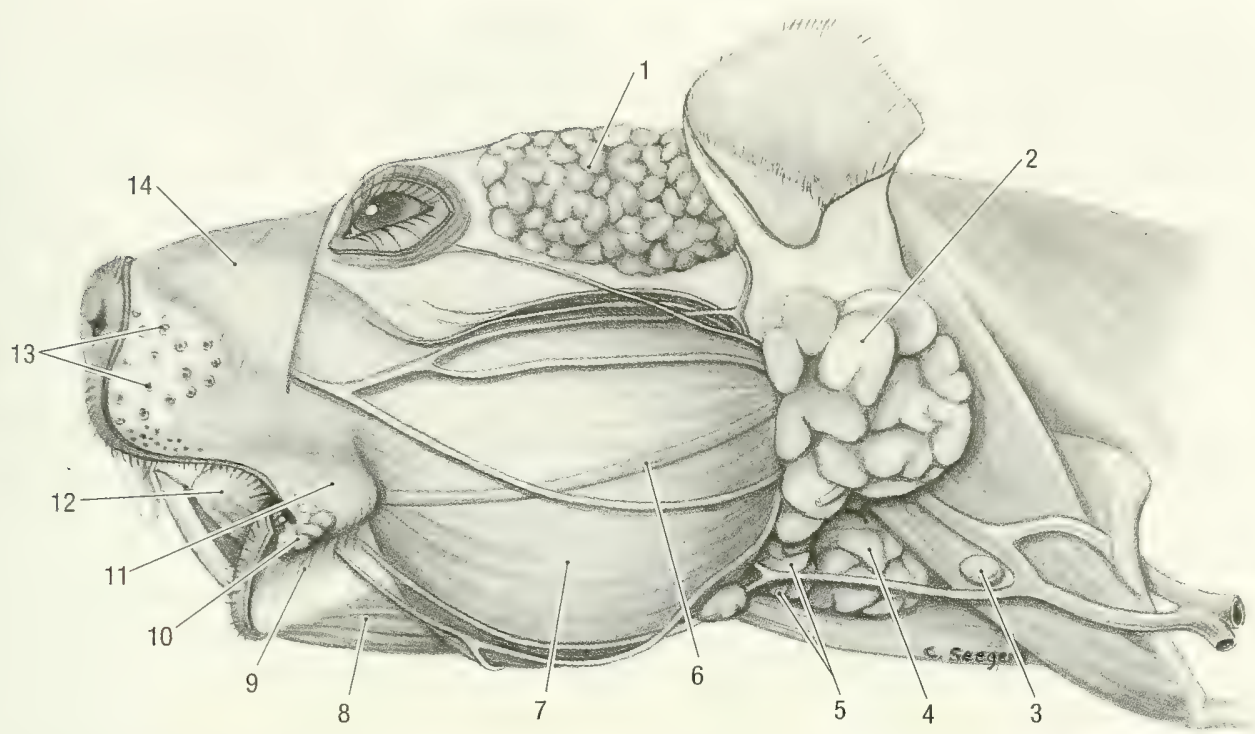

Fig. 5-4. Head, lateral view. 1 temporal gland, 2 parotid salivary gland, 3 mandibular lymph node, 4 mandibular salivary gland, 5 monostomatic sublingual salivary gland, 6 parotid salivary duct, 7 masseter m., 8 digastric m., 9 orbicularis oris m., 10 oral scent gland, 11 buccinator m., 12 tongue, 13 tactile hair follicles, 14 nasolabial m.

Innervation: Upper teeth by the maxillary nerve and lower teeth by the mandibular nerve.

Salivary Glands. The salivary glands are all empty their secretions into the oral cavity. They are the parotid, mandibular, sublingual, buccal and zygomatic salivary glands. In addition to these major groupings, lesser glands are also present under the mucosa of the lips and in the tongue. The secretions of the glands can be serous, mucoserous or mucous in nature.

The parotid salivary gland, gl. parotis (Fig. 5-4), has an irregular rectangular shape. It is pinkish brown and lobulated. The gland is about 3 to $4 \mathrm{~cm}$ long, 1.5 to $2 \mathrm{~cm}$ wide and $1 \mathrm{~cm}$ thick. The lobules are loosely connected to each other by delicate connective tissue. The gland wraps around the base of the ear between the caudal border of the mandible and the neck. It is caudal to the masseter and medial pterygoid muscles, ventral to the base of the ear, lateral to the external ear canal and the sternomastoid muscle, and dorsal to the mandibular salivary gland and linguofacial vein. The acromiomandibularis muscle covers its ventrolateral surface. The parotid duct, ductus parotideus, passes across the middle of the masseter muscle, dorsal to middle buccal branch of the facial nerve. It turns medially around the rostral border of the masseter muscle and passes between the latter and the pouch of the cheek. The duct opens on the parotid papilla, papilla parotidea. The latter lies opposite the third upper premolar (first cheek tooth) in the oral vestibule on the dorsal edge of the cheek pouch.
Blood supply: By branches from the superficial temporal artery.

Innervation: Parasympathetic innervation is from the glossopharyngeal nerve via the otic ganglion.

Lymph drainage: By the deep parotid lymph nodes.

The mandibular salivary gland, $g l$. mandibularis (Figs. 5-1 and 5-4), lies at the ventral end of the parotid salivary gland, medial to the lingual vein and angle of the mandible. The gland is caudal to the masseter and medial pterygoid muscles, medial to the lingual vein, lateral to the sternohyoid muscle, and ventral to the sternomastoid muscle. The gland is pinkish brown, is lobulated and is more compact than the parotid salivary gland. It is about $1.5 \mathrm{~cm}$ long and $1 \mathrm{~cm}$ wide. The duct of the gland crosses the lateral surface of the tendon between the two bellies of the digastric muscle and then passes rostrally along the deep face of the mylohyoid muscle. It opens into the floor of the oral cavity on a small sublingual caruncula just caudal to the symphysis of the mandible.

Blood supply: From the facial and lingual arteries.

Innervation: Parasympathetic innervation from the chorda tympani of the facial nerve via the mandibular ganglion.

Lymph drainage: By the mandibular lymph node.

The zygomatic salivary gland, gl. zygomatica, lies in the ventral part of the bony orbit. It is, lobulated, pinkish brown and is about $3 \mathrm{~cm}$ in diameter. The 
gland is lateral to the maxillary blood vessels and nerves, and ventral to the eye and periorbita. It frequently consists of a smaller, medial gland and a larger lateral gland. The duct of the gland opens into the oral vestibule, opposite the last cheek tooth.

Blood supply: By branches from the malar and maxillary arteries.

Innervation: Parasympathetic innervation is from the glossopharyngeal nerve via the otic ganglion.

The buccal salivary gland, gl. buccalis, lies caudolateral to the last molar tooth, and extends medially onto the hard palate. It is loosely lobulated, pale pink and measures about $2 \mathrm{~cm}$ in length and $0.5 \mathrm{~cm}$ in width. The lobules of the gland open into the oral cavity by means of many small ducts.

Blood supply: By branches from the buccal and major palatine arteries.

Innervation: Parasympathetic innervation is from the glossopharyngeal nerve via the otic ganglion.

The sublingual salivary glands, gll. sublinguales, consist of two distinct parts. The monostomatic part is associated with the mandibular salivary gland, while the polystomatic part is associated with the sublingual recess. The monostomatic part, $g l$. sublingualis monostomatica (Figs. 5-4 and 9-5), lies against the rostral pole of the mandibular salivary gland. The two glands share a common capsule. The gland is lobulated, pale pink (as opposed to the pinkish brown of the mandibular salivary gland) and measures $1 \mathrm{~cm}$ in diameter. The duct of the gland joins the duct of the mandibular salivary gland or remains separate but accompanies the latter duct to the sublingual caruncule. The polystomatic part of the sublingual salivary gland, $g l$. sublingualis polystomatica, lies under the mucosa of the sublingual recess. It is related laterally to the mandible, medially to the geniohyoid muscle and ventrally to the mylohyoid muscle. It is pink, lobulated and 2.5 to $3 \mathrm{~cm}$ long, $0.2 \mathrm{~cm}$ wide, and 1.5 $\mathrm{cm}$ thick. The lobules are small and tightly attached to each other by connective tissue. The ducts of the gland open into the sublingual recess.

Blood supply: By branches from the sublingual arteries.

Innervation: Parasympathetic innervation is from the chorda tympani of the facial nerve via small sublingual ganglia.

Abdomen and Peritoneum. The abdomen, abdomen, is that part of the trunk which extends from the diaphragm to the pelvis. The abdominal cavity within this portion of the trunk contains the abdominal viscera. The abdominal cavity is continuous with the pelvic cavity. The diaphragm bounds the abdomen cranially, the sublumbar muscles and the crura bound it dorsally, the abdominal muscles bound it laterally and ventrally, and the pelvic inlet caudally. The latter is attached to the abdominal and pelvic walls by an intervening layer of connective tissue, the fascia transversalis. During the summer large amounts of fat accumulate in the transverse fascia between the two peritoneal layers of the falciform ligament of the liver, the broad ligament of the uterus/mesorchium and beneath the peritoneum medial to the attachment of the broad ligament/mesorchium to the body wall. The fat serves as an important source of energy during hibernation and when the animals emerge in the spring (Davis 1967).

The peritoneum, peritoneum, lines the abdominal and pelvic cavities. The vaginal process on each side is an extra-abdominal extension of the peritoneum through the inguinal canal of both male and female animals. All the abdominal and pelvic viscera are covered and sometimes suspended by folds of peritoneum. The peritoneum may be divided into the parietal peritoneum that lines the abdominal and pelvic cavities and the visceral peritoneum that covers and suspends the abdominal and pelvic viscera. A double sheet of peritoneum connects the parietal and visceral parts. These connecting sheets are referred to as mesenteries that suspend the intestine, the lesser and greater omentum that suspends the stomach, hepatic ligaments that suspend the liver, the mesorchium of the testis and broad ligament that suspends the female genital systems, and the suspensory ligaments of the urinary bladder. The peritoneum extends into the pelvic cavity for some distance and is then reflected from the pelvic viscera and pelvic walls. It thus forms pouches or excavations between the pelvic wall and pelvic organs, as well as between the different pelvic organs. The excavation between the dorsolateral walls of the pelvis and rectum is the pararectal fossa, fossa pararectalis, and the excavation between the rectum and genital tract is the rectogenital excavation, $e x-$ cavatio rectogenitalis. Both excavations extend caudally as far as the ischial arch. The excavation between the genital tract and the urinary bladder is the vesicogenital excavation, excavatio vesicogenitalis. It is shallow and lies at the level of the pelvic inlet. The peritoneum is reflected directly from the bladder to the abdominal floor at the pelvic brim, therefore a pubovesical excavation, excavatio pubovesicalis, normally does not exist. A fold of peritoneum, the falciform ligament, lig. falciforme hepatis, extends between the umbilicus and the liver. It contains the round ligament of the liver.

The greater omentum, omentum majus (Fig. 55 ), is an extensive fold of peritoneum that extends from the dorsal body wall to the greater curvature 


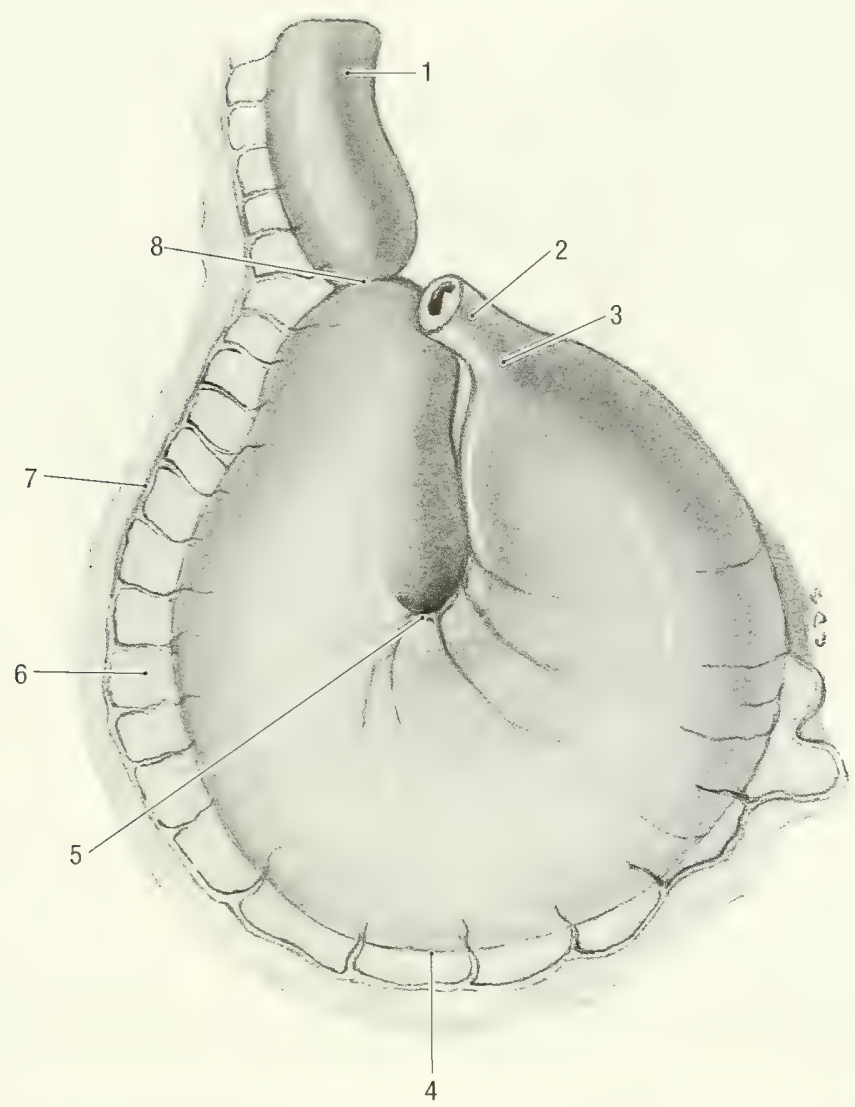

Fig. 5-5. Stomach, parietal surface. 1 duodenum, 2 esophagus, 3 cardiac part of the stomach, 4 major curvature of the stomach, 5 minor curvature of the stomach, 6 greater omentum, 7 right gastroepiploic a., 8 pyloric part of stomach.

of the stomach. It hangs as a fat filled, lacy apron from the stomach. Its caudal, free border is very mobile and can reach as far caudally as the pelvic inlet. In all the animals examined, it was wedged in between the stomach and the intestinal mass, covering only the caudal (visceral) surface of the stomach. The omentum is longer than the distance between the dorsal body wall and the stomach. It therefore doubles on itself, forming deep and superficial layers or parts. The space between the two layers is the omental bursa, bursa omentalis. The omental bursa has only one opening, the epiploic foramen, into the general peritoneal cavity. The epiploic foramen, foramen epiploicum, is a narrow passage that lies to the right of the median plane and ventromedial to the caudate process of the liver. It is bounded dorsally by the caudal vena cava, ventrally by the portal vein and cranially by the caudate process of the liver. The foramen leads into the vestibule of the omental bursa, cranial to stomach. Access to the omental bursa from the general peritoneal cavity is through the epiploic foramen (into the omental vestibule) and then caudally over the lesser curvature of the stomach into the main part of the bursa.

The lesser omentum, omentum minus, is a remnant of the ventral mesentery that extends between the liver and the terminal esophagus, stomach and duodenum. (For details see the ligaments of the liver)

Alimentary Canal. The esophagus, esophagus (Figs. 5-1, 5-2, 5-5 to 5-7), extends from the limen of the esophageal vestibule to the cardia of the stomach. In the mature animal it is approximately $15 \mathrm{~cm}$ long. It can be divided into cervical, thoracic and abdominal parts. The cervical part, pars cervicalis, begins at the level of the caudal border of the cricoid cartilage. The mucous membrane of the esophagus contains longitudinal folds and is clearly demarcated from the esophageal vestibule of the laryngopharynx. The cervical esophagus is approximately $4 \mathrm{~cm}$ long and is related dorsally to the left longus colli and longus capitis muscles and ventrally to the trachea. It is slightly longer than the neck itself, inclining to the left of the trachea. At 


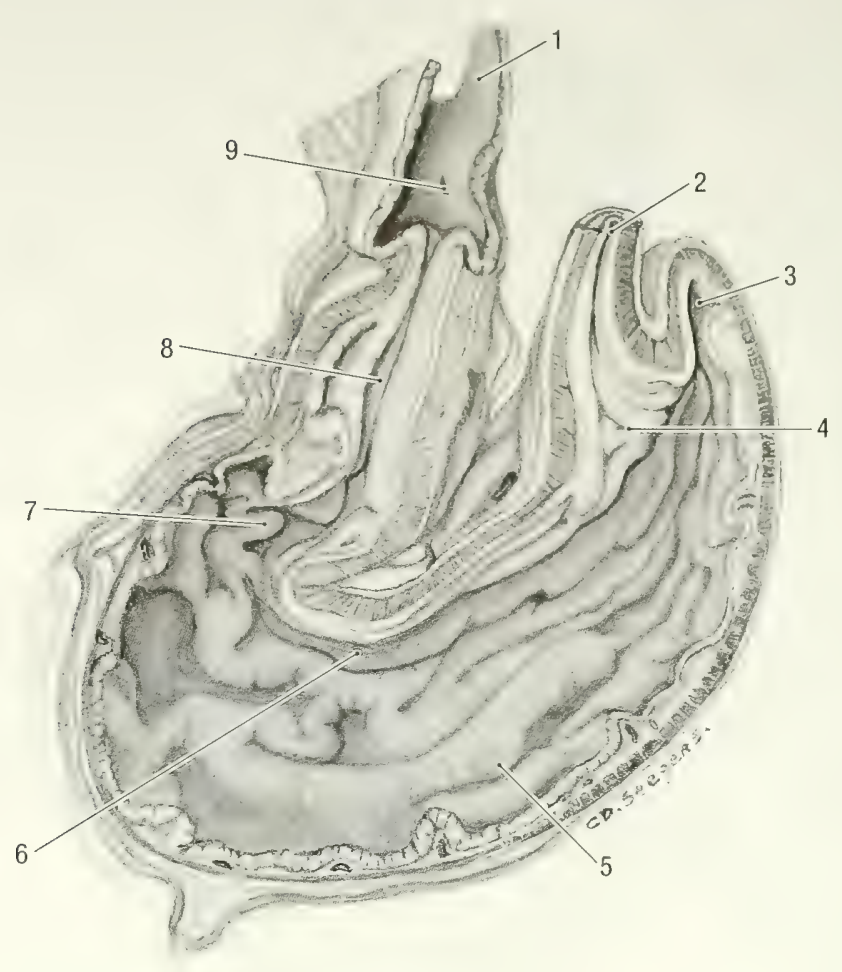

Fig. 5-6. Stomach, longitudinal section. 1 duodenum, 2 esophagus, 3 fundus of the stomach, 4 cardiac part of the stomach, 5 body of the stomach, 6 ventricular groove, 7 pyloric antrum, 8 pyloric canal, 9 major duodenal papilla.

the middle of the neck it lies lateral to the trachea. The cervical esophagus enters the thorax dorsal to the trachea. On the left side the left common carotid artery, vagosympathetic trunk, internal jugular vein and tracheal duct, lies in the angle between the esophagus and longus capitis muscle.

The thoracic part, pars thoracica, extends from the thoracic inlet to the esophageal hiatus of the diaphragm. It is about $9 \mathrm{~cm}$ long and lies in the mediastinum. Cranial to the heart it passes dorsal to the trachea and thymus and to the right of the aortic arch. Caudal to the heart the esophagus lies ventral to the descending thoracic aorta and is related to the infracardiac bursa (see mediastinal pleura under the respiratory system). It passes through the esophageal hiatus. The abdominal part, pars abdominalis, is approximately $2 \mathrm{~cm}$ long along its ventral border and $1 \mathrm{~cm}$ long along its dorsal border. It is attached to the diaphragm along its dorsal border by the greater omentum and to the papillary lobe of the liver along its ventral border by the lesser omentum.

Blood supply: Branches of the thyroid arteries supply the cervical part, the broncho-esophageal artery supplies the thoracic part and the left gastric artery supplies the abdominal part.

Innervation: The cervical part is innervated by fibers from the pharyngoesophageal and recurrent laryngeal branches of the vagal nerves, and the thoracic part by the dorsal and ventral vagal trunks.

The stomach, ventriculus (Figs. 5-5, 5-7, 5-10, 511 and 5-14), lies transversly on the median plane. Its size and shape varies greatly, depending on the quantity of food it contains. When it is empty it measures $11 \mathrm{~cm}$ along its greater curvature and 7 $\mathrm{cm}$ along its lesser curvature. The major divisions of the stomach are the cardia, fundus, body, and the pyloris. It has visceral and parietal surfaces and lesser and greater curvatures.

The esophagus enters the stomach at the cardia, pars cardiaca. The different layers of the esophagus are continuous with and blend with those of the stomach. It only differs from the esophagus in the type of glands present in the mucosa.

The gastric fundus, fundus ventriculi, is the rather large, blind expansion of the stomach to the left and dorsal to the cardia.

The body, corpus ventriculi, is the middle portion of the stomach and extends from the fundus to the pyloric portion.

The pyloric part, pars pylorica, is approximately the distal third of the stomach as measured along the lesser curvature. Its initial part, the pyloric antrum, antrum pyloricum (Fig. 5-4), is somewhat di- 


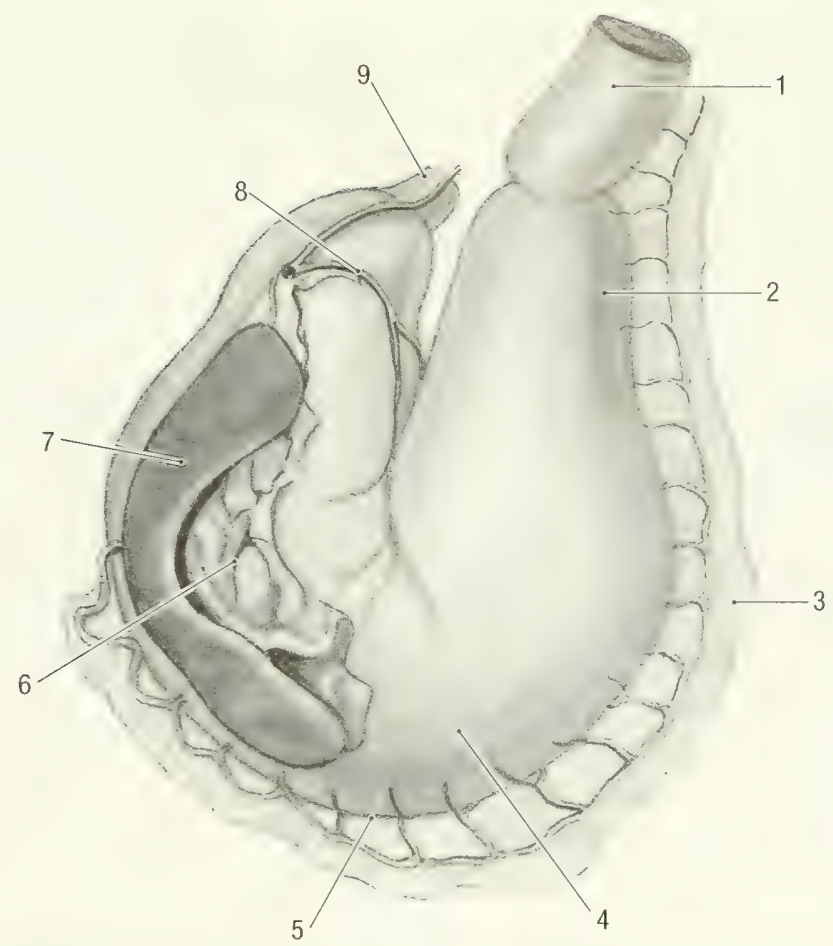

Fig. 5-7. Stomach and spleen, visceral surface. 1 duodenum, 2 pyloric part of the stomach, 3 major omentum, 4 body of the stomach, 5 major curvature of the stomach, 6 gastrosplenic lig., 7 spleen, 8 left gastric a., 9 esophagus.

lated and narrows to form the pyloric canal, canalis pyloricus.

A muscular sphincter closes the opening between the pyloric part and duodenum.

The greater curvature, curvatura ventriculi major, of the stomach is convex and extends from the cardia to the pylorus. It is the greatest distance between the two parts. The superficial layer of the greater omentum attaches to the greater curvature (Fig. 6-5). The spleen, which lies in the superficial layer of the greater omentum, is attached to the stomach by the gastrosplenic ligament, lig. gastrolienale).

The lesser curvature, curvatura ventriculi minor (Fig. 5-5), extends from the cardia to the pylorus. It is the shortest distance between the two parts. The shortest path that ingesta can take in passing from the cardia to the pyloric portion of the stomach is known as the gastric groove, sulcus ventriculi. This path follows the lesser curvature. The cardia lies approximately $2 \mathrm{~cm}$ to the left of the pylorus, resulting in an acutely concave lesser curvature. The papillary process of the liver lies in the lesser curvature, with the body of the stomach to the left and the pylorus to the right of the papillary process (Fig. 6-6). The caudal edge of the lesser omentum attaches to the lesser curvature.

The parietal surface of the stomach, facies par- ietalis, faces ventrally and cranially. It lies against the dorsal part of the visceral surface of the left lateral lobe of the liver. The empty stomach does not extend beyond the edges of the liver. However, when it is distended, it projects dorsally and laterally beyond the borders of the liver and lies in contact with the diaphragm and left dorsal body wall.

The visceral surface of the stomach, facies visceralis (Fig. 5-7), faces caudodorsally. It lies in direct contact with part of the papillary process of the liver and the left lobe of the pancreas that is enclosed in the visceral layer of the greater omentum. Furthermore, the left part of the visceral surface is related to the spleen and is separated from the intestinal mass and left kidney by the superficial layer of the greater omentum.

Topography: The stomach lies in the cranial abdomen within the thoracic cage, to the left of the midline. When the stomach is moderately full, the cranial border lies on a transverse plane through the last thoracic vertebra and the caudal border on a transverse plane through the third lumbar vertebra (ventromedial to the caudal pole of the left kidney). As the stomach fills the cranial border does not change, but the caudal border shifts caudally.

Blood supply: The main arteries to the stomach are the left gastric artery (Fig. 5-7) along its lesser curvature and the left and right gastroepiploic ar- 


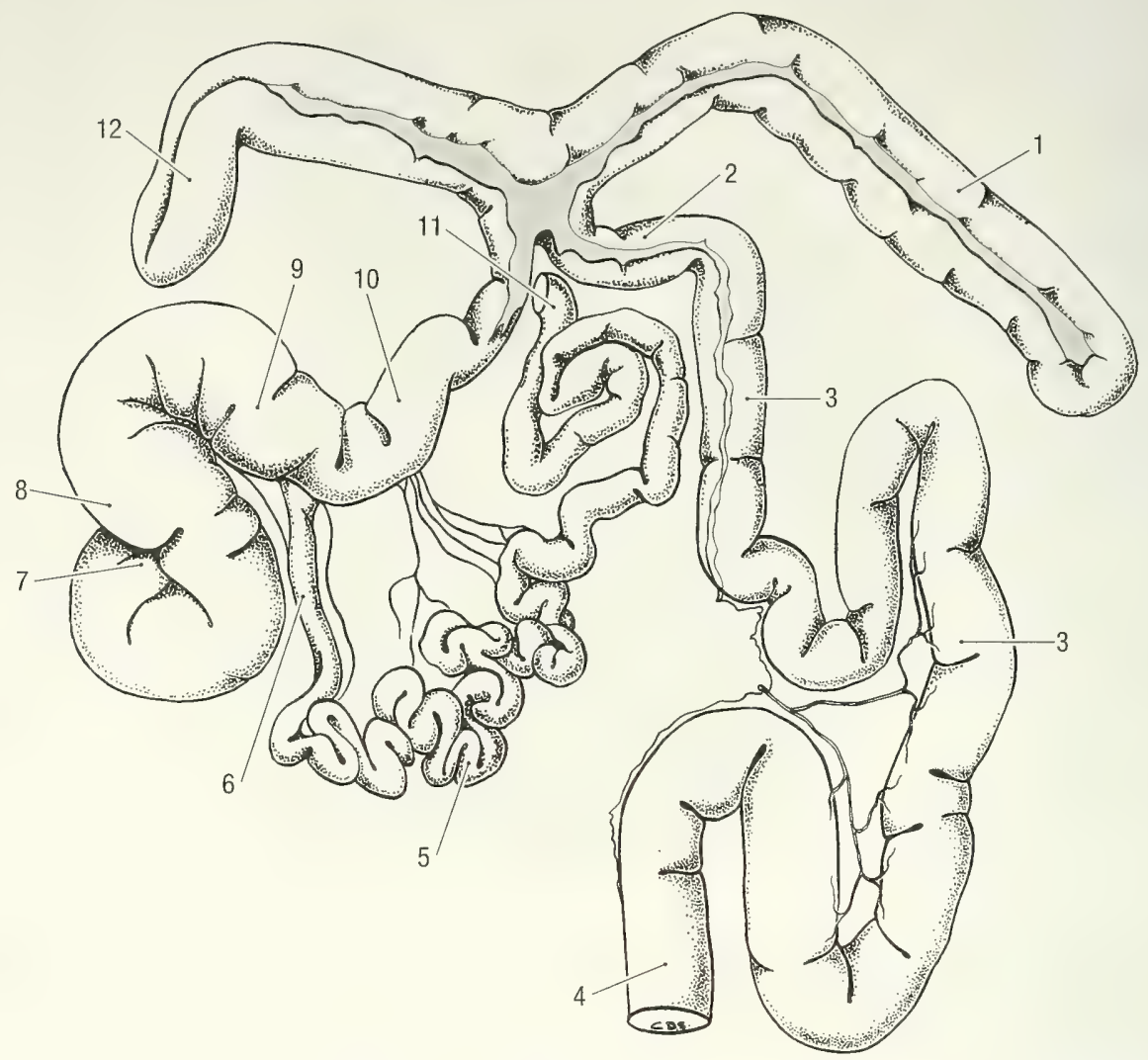

Fig. 5-8. Isolated intestinal track. 1 second loop of ascending colon, 2 transverse colon, 3 descending colon, 4 rectum, 5 jejunum, 6 ileum, 7 apex of the cecum, 8 body of the cecum, 9 base of the cecum, 10 ascending colon, 11 duodenum, 12 first loop of the ascending colon.

teries (Fig. 5-5) along its greater curvature. The left gastric artery is a branch of the celiac artery. The right gastric and right gastroepiploic arteries are branches of the hepatic artery and the left gastroepiploic artery is a branch of the splenic artery. The gastroesophageal artery arises from the terminal part of the hepatic artery and supplies the abdominal esophagus and cardia of the stomach.

The veins of the stomach parallel the arteries. They drain into the portal vein (see chapter 9 for the detail of the portal vein). The gastroduodenal vein also drains into the left branch of the portal vein.

Innervation: The stomach is supplied by the vagus nerve and receives sympathetic fibers from the celiac plexus. The ventral vagal trunk supplies the lesser curvature, pylorus and visceral surface of the stomach. The dorsal vagal trunk supplies the greater curvature and parietal surface of the stomach.

Lymph drainage: Lymph is drained mainly by the gastric lymph node, situated in the lesser omentum.

Garon and Pierard (1972) made a gross comparison of the stomach of Marmota monax with that of the muskrat, Ondatra zibethicus, and present a table of weights and capacities for each species. Whereas the digestive glands of the muskrat were concentrated in a well-circumscribed area of the body of the stomach, the same was not true of the woodchuck.

The small intestine, intestinum tenue, extends from the pylorus of the stomach to the ileocolic orifice leading into the large intestine. In adult animals it is 200 to $250 \mathrm{~cm}$ long. The small intestine consists of the relatively fixed and short duodenum, the freely movable jejunum, and a very short terminal part, the ileum. There are no clear anatomical differences between the three parts. The transition point of the one into the other is therefore very arbitrary and not always constant.

The duodenum, duodenum (Figs. 5-8 and 5-10), is the first and most fixed part of the small intestine. It consists of a cranial part and a loop with descending and ascending limbs. It is suspended from the dorsal body wall by a short mesoduodenum.

The cranial part, pars cranialis, begins at the py- 
lorus in the upper half of the abdomen, to the right of the median plane, on a transverse plane through the last thoracic vertebra. It is approximately $5 \mathrm{~cm}$ long. The major duodenal papilla, papilla duodeni major, lies approximately $1 \mathrm{~cm}$ from the pylorus and contains the opening of the bile duct. From the pylorus the cranial part passes to the right and slightly dorsally. It crosses the visceral surface of the liver between the gall bladder ventrally and the right kidney dorsally. It is related caudoventrally to the second loop of the ascending colon, to which it is attached by a short peritoneal fold. It reaches the dorsal body wall just caudal to the last rib. It then turns caudally, forming the cranial duodenal flexure, flexura duodeni cranialis, and continues as the descending duodenum. The descending duodenum, pars descendens, is approximately $12 \mathrm{~cm}$ long. It passes caudomedially along the dorsal body wall, lateral to the right kidney and medial to the attachment of the mesorchium or broad ligament of the uterus. Its lateral border is related to the first loop of the ascending colon, but is separated from it by the aforementioned ligaments. Its medial border is related to the right kidney and right lobe of the pancreas. The descending duodenum reaches the dorsal midline at the level of 6 or 7L. It then turns to the left and cranially, forming the caudal duodenal flexure, flexure duodeni caudalis. From the caudal flexure it passes cranially as the ascending duodenum. The ascending duodenum, pars ascendens, is about $10 \mathrm{~cm}$ long. It passes cranially along the dorsal body wall, just to the left of the midline. Its initial part is attached to the initial portion of the ascending colon by a short fold of peritoneum, the duodenocolic fold, plica duodenocolica. The ascending duodenum reaches the terminal end of the ascending colon and the transverse colon at the level of 2 to $3 \mathrm{~L}$, where it turns ventrally and to the right to continue as the jejunum. The ascending duodenum contains the lesser duodenal papilla, $p a-$ pilla duodeni minor, onto which the accessory pancreatic duct opens, approximately $20 \mathrm{~cm}$ from the pylorus.

The jejunum, jejunum (Figs. 5-8 and 5-9), is the longest part of the small intestine and measures approximately $200 \mathrm{~cm}$ in length. It forms a coiled mass of intestine that is attached to the dorsal body wall by the mesojejunum. The mesojejunum is very long, resulting in a very mobile jejunum that can vary greatly in position. It is most commonly found in the right dorsal part of the abdomen. The jejunum joins the ileum without a clear demarcation between the two parts.

The ileum, ileum (Fig. 5-8), is the terminal part of the small intestine. It is 4 to $5 \mathrm{~cm}$ long and opens into the large intestine at the ileocolic orifice, ostium ileale. The ileum is attached to the dorsal body wall by the meso-ileum (which is essentially a part of the mesojejunum) and to the cecum by the ileocecal ligament.

Blood supply to the small intestine: The cranial pancreaticoduodenal artery from the hepatic artery, and the caudal pancreaticoduodenal artery from the cranial mesenteric artery, supply the duodenum. Jejunal and ileal branches from the cranial mesenteric artery supply the jejunum and ileum. Venous blood drains into the portal vein (see the portal vein for detail).

Lymph drainage: Lymph is drained through the mesenteric lymph nodes situated in the root of the mesentery.

Innervation: Parasympathetic innervation is from the vagus nerves and sympathetic innervation from the splanchnic nerves via the celiac and cranial mesenteric plexuses.

The large intestine, intestinum crassum, is about $110 \mathrm{~cm}$ long. Its diameter varies greatly, depending on the amount of ingesta present. This part of the intestine is highly specialized for microbial breakdown of plant material. It consists of a large cecum, a complex ascending colon, a short transverse colon and a long descending colon.

The cecum, cecum (Figs. 5-8 and 5-9), is large. It is approximately 8 to $10 \mathrm{~cm}$ long and 2 to $3 \mathrm{~cm}$ in diameter. Its shape varies according to the amount of ingesta present. When empty, it is " $\mathrm{C}$ " shaped and when full comma shaped. The cecum has a base, basis ceci, into which the ileum opens, a body, corpus ceci, and a blind ending apex, apex ceci. It lies transversely, to the left of the midline, with its base and body in contact with the ventral abdominal wall. The base lies close to the midline caudally and the apex lies dorsocranially and to the right. Its cranial limit is on a transverse plane through 3L, and its caudal limit on a transverse plane through $7 \mathrm{~L}$. The coils of the small intestine lie dorsal to the cecum.

The ascending colon, colon ascendens (Figs. 5-8 and 5-9), consists of a short, straight segment and two loops. One loop lies along the right abdominal wall and the other loop lies transversely caudal to the liver. The initial portion of the ascending colon is closely attached to the dorsal body wall by the mesocolon. Folds of peritoneum attach the limbs of the loops to each other. The two loops lie free in the abdomen, with the result that their topographical positions in the abdomen can vary. The following description gives the most commonly found positions.

From its origin at the ileocecal junction, the straight portion of the ascending colon passes cranially along the right dorsal body wall, between the two limbs of the duodenum. Caudal to the pylorus it turns to the right to form the first loop. This loop 


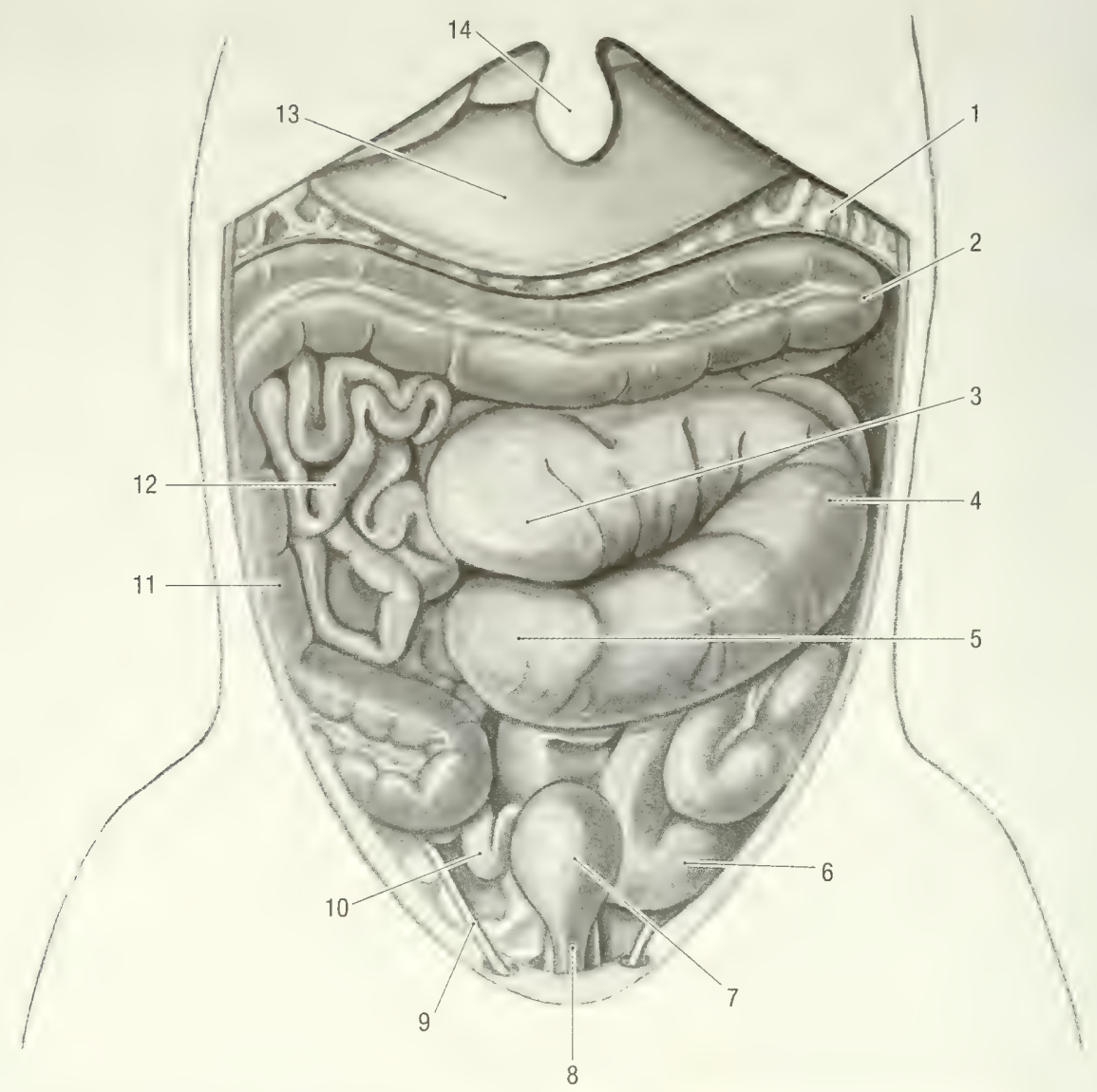

Fig. 5-9. Abdomen, superficial ventral view. 1 major omentum, 2 second loop of the ascending colon, 3 apex of the cecum, 4 body of the cecum, 5 base of the cecum, 6 descending colon, 7 urinary bladder, 8 urethra, 9 round lig. of uterus, 10 uterus, 11 first loop of the ascending colon, 12 jejunum, 13 left lateral liver lobe, 14 xiphoid cartilage of sternum.

lies along the right dorsolateral abdominal wall. It can extend caudally as far as the pelvic inlet. The terminal end of its ascending limb turns to the left, caudal to the liver, to form the second loop.

The second loop lies transversely across the abdominal floor caudal to the stomach and liver. It extends to the left paralumbar fossa and is commonly separated from the stomach by the greater omentum. The terminal end of the second loop continues as the transverse colon.

The transverse colon, colon transversum (Figs. 58 and 5-10), is short and passes transversely, cranial to the cranial mesenteric artery. It turns caudally to continue as the descending colon.

The descending colon, colon descendens (Figs. 58 and 5-10), is about $35 \mathrm{~cm}$ long and thinner than the other parts of the colon. Its mesocolon is long, resulting in considerable coiling of the terminal part of the large intestine. The descending colon passes caudally, to the left of the midline and me- dial to the left kidney. It enters the pelvic cavity and continues as the rectum.

Blood supply: The ascending, transverse and initial part of the descending colon is supplied by branches of the cranial mesenteric artery. The caudal mesenteric artery supplies the rest of the descending colon. The veins parallel the arteries and drain into the portal vein.

Lymph drainage: Lymph is drained by the colic lymph nodes situated in the mesocolon.

Innervation: The vagal nerves supply the initial part of the large intestine, while the pelvic nerves supply the last part of the descending colon.

The rectum, rectum (Fig. 4-14), is that part of the large intestine that lies in the pelvic cavity. It is approximately $4 \mathrm{~cm}$ long. Its initial part is suspended from the roof of the pelvis by the mesorectum. The greater part however, lies retroperitoneally. The terminal part of the rectum lies caudal to the pelvis, faces ventrally, and is surrounded by the external anal sphincter and perianal fat. 


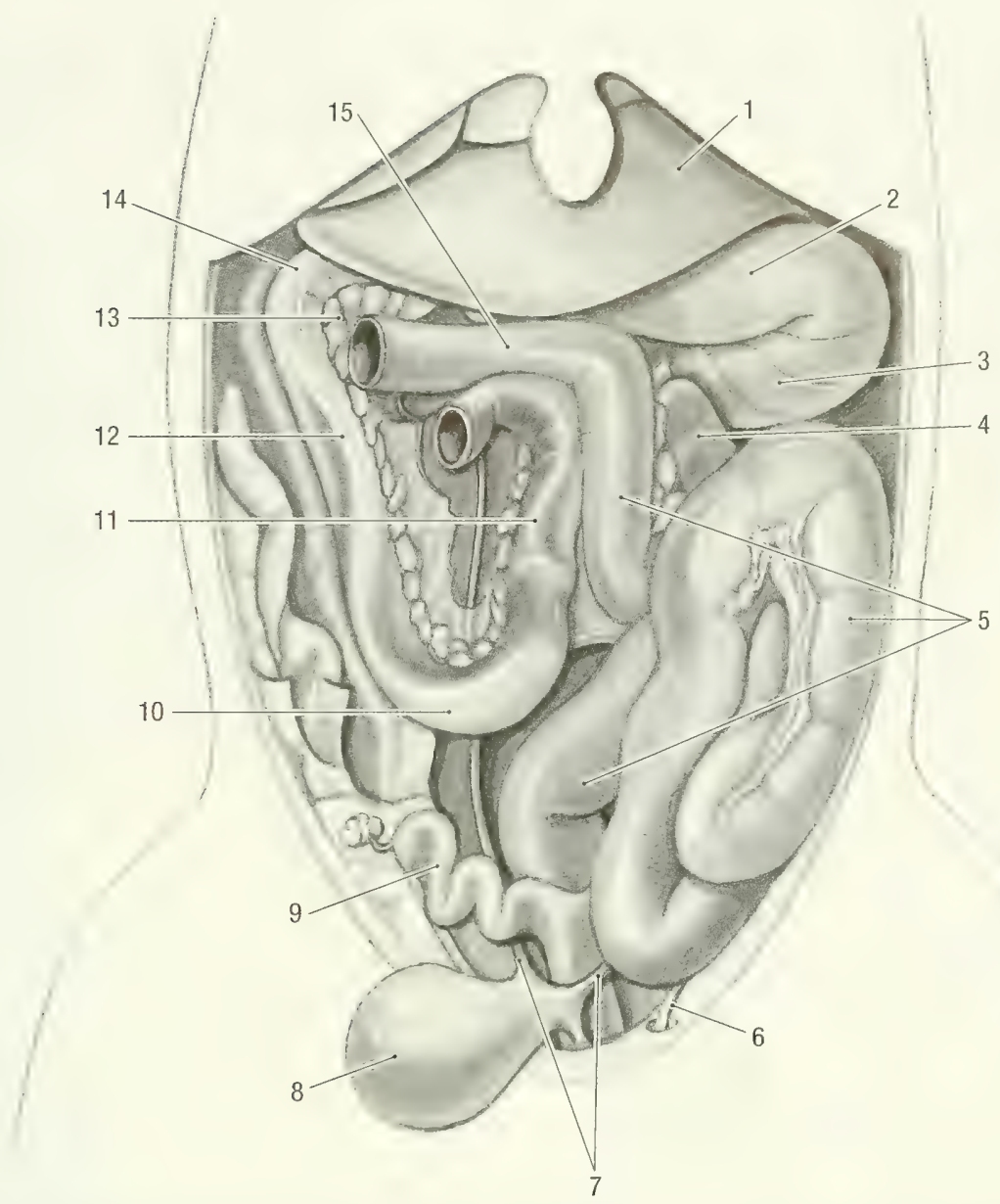

Fig. 5-10. Abdomen with cecum, ascending colon and jejunum removed. 1 liver, 2 pyloric part of the stomach, 3 body of the stomach, 4 spleen, 5 descending colon, 6 round lig. of the uterus, 7 ureters, 8 urinary bladder, 9 uterus, 10 caudal duodenal flexure, 11 ascending duodenum, 12 descending duodenum, 13 pancreas, 14 cranial flexure of the duodenum, 15 transverse colon.

The anal canal, canalis analis, forms the terminal part of the digestive tract. It is about $1 \mathrm{~cm}$ long and is surrounded by the caudal part of the external anal sphincter. The mucous membrane of the anal canal consists of three distinct zones. The columnar zone is the continuation of the rectum and is characterized by longitudinal folds of mucous membrane. The short intermediate zone separates the columnar zone from the cutaneous zone. The cutaneous zone is continuous with the skin and contains the openings of the three para-anal sacs, saccus paranalis (Fig. 4-14). One sac lies on the ventral midline, the other two on either side of it. The woodchuck everts the openings of the sacs when threatened or frightened.

Blood supply: Five vessels supply the rectum and anal canal. The cranial rectal artery lies on the dorsal surface of the rectum and anal canal. The middle rectal artery lies along the ventrolateral border of the retroperitoneal part of the rectum. The two caudal rectal arteries supply the ventrolateral part of the terminal rectum and anal canal. Satellite veins drain the rectum and anal canal. All the veins drain into the caudal vena cava, and do not contribute to the formation of the portal vein.

Innervation: By parasympathetic fibers from the pelvic plexus (Nn. pelvini).

Lymph drainage: Lymph drains into the caudal mesenteric lymph nodes that are arranged around the origin of the caudal mesenteric artery.

Liver, Hepar (Figs. 5-9, 5-12 and 5-14). The average weight of the liver of over a hundred adult animals weighing $2.6 \mathrm{~kg}$ was $42 \mathrm{gm}$. Compared to its body weight this is a relatively small liver. The liver constitutes $1.6 \%$ of the body mass, compared to $4.3 \%$ in the ferret and $3.4 \%$ in the dog. The fresh liver is deep red, firm but friable, and has six lobes. These are the left lateral and medial lobes, the quadrate and caudate lobes, and the 


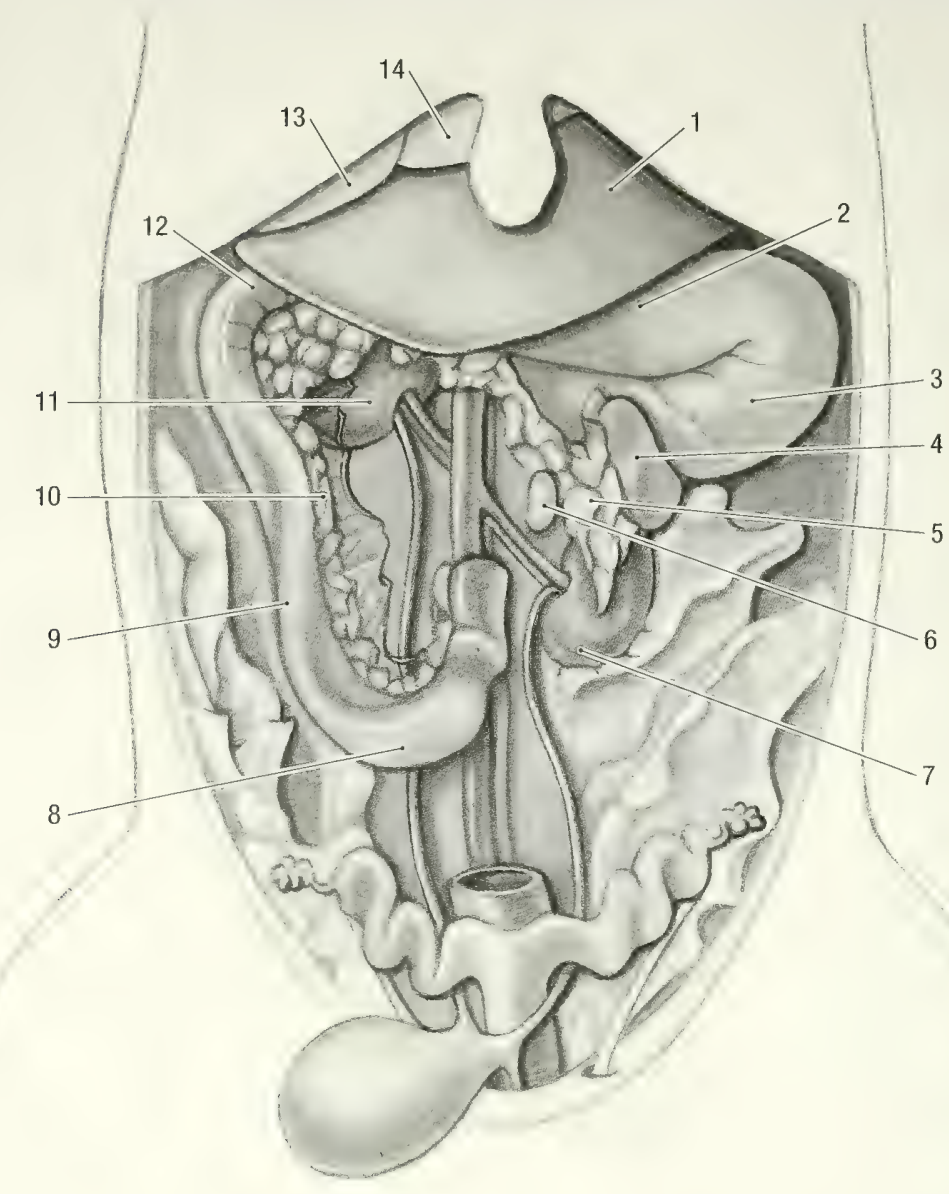

Fig. 5-11. Abdomen, deep ventral view. 1 left lateral liver lobe, 2 pyloric part of the stomach, 3 body of the stomach, 4 spleen, 5 left lobe of the pancreas, 6 left adrenal gland, 7 left kidney, 8 caudal duodenal flexure, 9 descending duodenum, 10 right lobe of the pancreas, 11 right kidney, 12 cranial flexure of the duodenum, 13 quadrate liver lobe, 14 left medial liver lobe.

right lateral and medial lobes. The caudate lobe presents papillary and caudate processes. Interlobar fissures separate the caudate and papillary processes, as well as the left lateral and right lateral lobes, from each other and from the rest of the liver. The right medial, left medial and quadrate lobes are fused to a lesser or greater extent.

The liver presents diaphragmatic and visceral surfaces and dorsal, ventral, left and right borders. The diaphragmatic surface, facies diaphragmatica, faces cranioventrally. It is strongly convex in all directions and lies against the diaphragm and the cranial part of the abdominal floor. The ligaments of the liver are attached to the diaphragmatic surface (see below). The visceral surface, facies visceralis, is irregularly concave, facing mainly caudodorsally and is in contact with various abdominal organs. Some parts of the liver lobes overlap each other, forming interlobar surfaces between the overlapping parts. All lobes of the liver, with the exception of the left medial lobe, contribute to the visceral surface. Various abdominal organs are in contact with the visceral surface, leaving their impressions that account in part for its irregular shape. The dorsal border, margo dorsalis, and the left and right borders, margo sinister et dexter, lie within the rib cage and thus are not palpable. The ventral border, margo ventralis, projects beyond the costal arch and sternum by 4 to $5 \mathrm{~cm}$ and is palpable. The dorsal border is deeply grooved by the caudal vena cava, sulcus venae cavae, and notched by the esophagus, impressio esophagea. Furthermore, the ventral border of the fused right medial and quadrate lobes is notched for the gall bladder, fossa vesicae felleae, allowing the gall bladder to contact the abdominal floor. The hepatic vessels, nerves, lymphatics and bile duct enter or leave the liver through the porta, porta hepatica. Together the hepatic porta and its contents form the hilus. It is situated close to the dorsal border of the liver, ventral to the caudate 
lobe and caudal vena cava. The portal vein enters the porta dorsally, the bile duct leaves it ventrally and the hepatic artery and nerves enter between them.

\section{Lobes of the Liver and their Relations.} The left lateral lobe, lobus hepatis sinister lateralis, is the largest of the six lobes. In the average adult animal it weighs $23.5 \mathrm{gm}$ and represents $57.25 \%$ of the total weight of liver. It is approximately $8 \mathrm{~cm}$ wide and $8 \mathrm{~cm}$ long. A deep interlobar fissure separates it from the left medial and quadrate lobes. The diaphragmatic surface of the lobe can be divided into roughly three equal parts; one third of the area is in contact with the diaphragm, one third overlaps the left medial and quadrate lobes cranially and the other third lies on the abdominal floor. The visceral surface of the left lateral lobe makes up at least three-quarters of the visceral surface of the liver. The right ventral part is in contact with the gall bladder and the ventral 1 to $2 \mathrm{~cm}$ is in contact with the ascending colon. The rest of the visceral surface lies against the parietal surface of the stomach, with the pylorus against the medial border of the left medial lobe and gall bladder. The central, dorsal part of the visceral surface of the lobe lies against the papillary process of the caudate lobe.

The left medial lobe, lobus hepatis sinister medialis, is a fairly small lobe, measuring 4 by $6 \mathrm{~cm}$ and weighing $3.7 \mathrm{gm}$, representing $9 \%$ of the total weight of the liver. It is separated from the left lateral lobe by a deep interlobar fissure. Medially it is fused to the quadrate lobe. However, a shallow interlobar fissure along its visceral surface and ventral border and the attachment of the falciform ligament along its diaphragmatic surface indicates the medial border or limit of the lobe. The diaphragmatic surface of the lobe lies mainly against the diaphragm. Approximately $1 \mathrm{~cm}$ of the ventral border projects beyond the costal arch and xiphoid cartilage of the sternum, just to the right of the midline. This is the only part of the left medial lobe that lies on the abdominal floor. The left lateral lobe overlaps the greater part of the visceral surface of the left medial lobe. Only a narrow area along the medial border is in contact with the pylorus of the stomach.

The quadrate lobe, lobus quadratus, is the second largest of the liver lobes. It measures 5 by $7 \mathrm{~cm}$ and weighs $5.3 \mathrm{gm}$, representing $12.8 \%$ of the total weight of the liver. On the left it is fused to the left medial lobe, except along its visceral surface where a shallow interlobar fissure separates the two. On the right it is fused to the right medial lobe, separated only by a shallow groove on its visceral surface for the cystic duct and the fossa for the gall bladder. The diaphragmatic surface lies against the diaphragm. Approximately $1 \mathrm{~cm}$ of the lobe projects beyond the costal arch and xiphoid cartilage of the sternum, just to the right of the midline. This is the only part of the lobe that lies on the abdominal floor. Its visceral surface lies against the gall bladder and the pylorus of the stomach.

The right medial lobe, lobus hepatis dexter medialis, is fairly small, measuring approximately 4 by $7 \mathrm{~cm}$. It weighs $3.5 \mathrm{gm}$ and represents $8.5 \%$ of the total weight of the liver. It is largely fused to the quadrate lobe except on the visceral surface where a shallow groove separates the two. A deep interlobar fissure separates the right medial and lateral lobes. The diaphragmatic surface of the lobe lies against the diaphragm and does not project beyond the costal arch. The visceral surface is related to the gall bladder, cystic duct, and the ascending colon. The right lateral lobe overlaps a large part of the visceral surface.

The right lateral lobe, lobus hepatis dexter lateralis is cone shaped, with the apex of the cone at the hilus and pointing cranially. It is approximately 5 $\mathrm{cm}$ long and $3 \mathrm{~cm}$ wide. The lobe weighs $3.25 \mathrm{gm}$ and represents $8 \%$ of the total weight of the liver. It is separated from the right medial lobe and caudate process by deep interlobar fissures. The medial half of the diaphragmatic surface lies against the diaphragm, the lateral half overlaps the right medial lobe. The dorsal part of the visceral surface overlaps the caudate process and in some specimens also lies against the right kidney. The ventral part of the visceral surface lies against the ascending colon. The caudal vena cava grooves and is attached to the medial border of the lobe.

The caudate lobe, lobus caudatus, consists of the caudate and papillary processes and lies dorsal to the porta. It weighs $2 \mathrm{gm}$ and represents $4.8 \%$ of the total weight of the liver. The caudate process, processus caudatus, lies caudal to the right lateral lobe. It is roughly pyramidal in shape, with the base and one side forming the visceral surface. It is approximately $4 \mathrm{~cm}$ long and $3 \mathrm{~cm}$ wide and weighs $0.8 \mathrm{gm}$, representing $42 \%$ of the weight of the caudate lobe. The diaphragmatic surface of the lobe consists of a small triangular area that lies against the diaphragm and a large area that lies against the right medial lobe. The caudodorsal part of the visceral surface carries the renal impression, impressio renalis, for the right kidney. The rest of the visceral surface lies against the ascending colon. The caudal vena cava separates the caudate and papillary process and is attached to the medial border of the caudate lobe and to the lateral border of the papillary process. The papillary process, processus papillaris, is irregularly V-shaped. The hepatic vein that drains each limb of the $\mathrm{V}$ separates them from each other. It weighs $1.1 \mathrm{gm}$ and represents $58 \%$ of the 


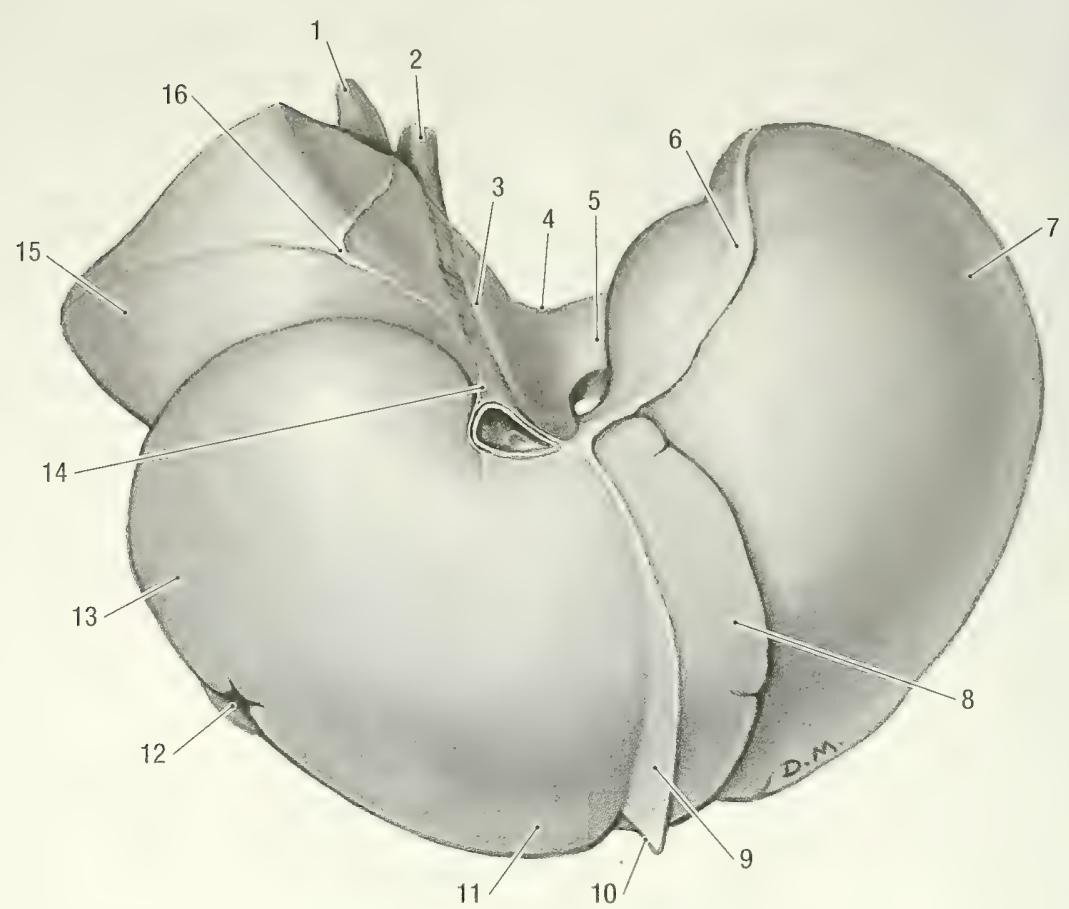

Fig. 5-12. Liver, parietal surface. 1 caudate processus, 2 caudal vena cava, 3 groove for the caudal vena cava, 4 esophageal impression, 5 caudate lobe, 6 left triangular lig., 7 left lateral lobe, 8 left medial lobe, 9 falciforme lig., 10 round lig., 11 quadrate lobe, 12 gall bladder, 13 right medial lobe, 14 coronary lig., 15 right lateral lobe, 16 right triangular lig.

weight of the caudate lobe. The cranial surface of the lobe lies against the left lateral lobe, while its visceral surface is related to the stomach and duodenum. The lobe fits into the lesser curvature of the stomach (Fig. 5-6). The result is that the cranial part of the process is related to the esophagus and parietal surface of the gastric fundus, while the caudal part crosses the lesser curvature of the stomach and is therefore related to the visceral surface of the pylorus.

Ligaments of the Liver (Fig. 5-12). The liver is covered by visceral peritoneum that forms its serous coat. The serous coat is fused to the underlying fibrous capsule. The only parietal attachment of the liver to the diaphragm is by means of continuations of the serous and fibrous coats from the diaphragmatic surface, forming coronary, triangular and falciform hepatic ligaments.

The falciform ligament, lig. falciforme hepatis, is a remnant of the embryonic ventral mesentery. It extends from the liver and diaphragm to the ventral body wall cranial to the umbilicus. The round ligament, ligamentum teres hepatis (Fig. 5-12), of the liver, which is the remnant of the fetal umbilical vein, lies in the free border of the falciform ligament. In mature animals it is very thin and sometimes impossible to distinguish. The round liga- ment enters the ventral border of the liver between the left medial and quadrate lobes. The part of the falciform ligament that attaches the liver to the diaphragm is thin and does not contain any adipose tissue. It attaches to the parietal surface of the liver between the left medial and quadrate lobes, from the ventral border to the caudal vena cava. Its attachment to the diaphragm lies nearly in the median plane, from the sternum to the caudal vena cava.

The coronary ligament, lig. coronarium hepatis, attaches the parietal surface of the liver around the caudal vena cava, to the diaphragm. It is formed by the continuations of the falciform ligament around the vein. In the woodchuck the falciform ligament attaches to the caudal vena cava, about $3 \mathrm{~mm}$ from the liver, and not to the liver itself. (In the majority of specimens examined, the ligament did not attach to the liver and therefore, in the narrow sense of the definition of a coronary ligament, does not exist).

Two triangular ligaments attach the liver to the diaphragm. Both are continuations of the coronary ligament. Their attachments to the liver extend from the caudal vena cava to the left and right lobes of the liver. The left triangular ligament, lig. triangulare sinistrum, extends laterally (to the left) and 


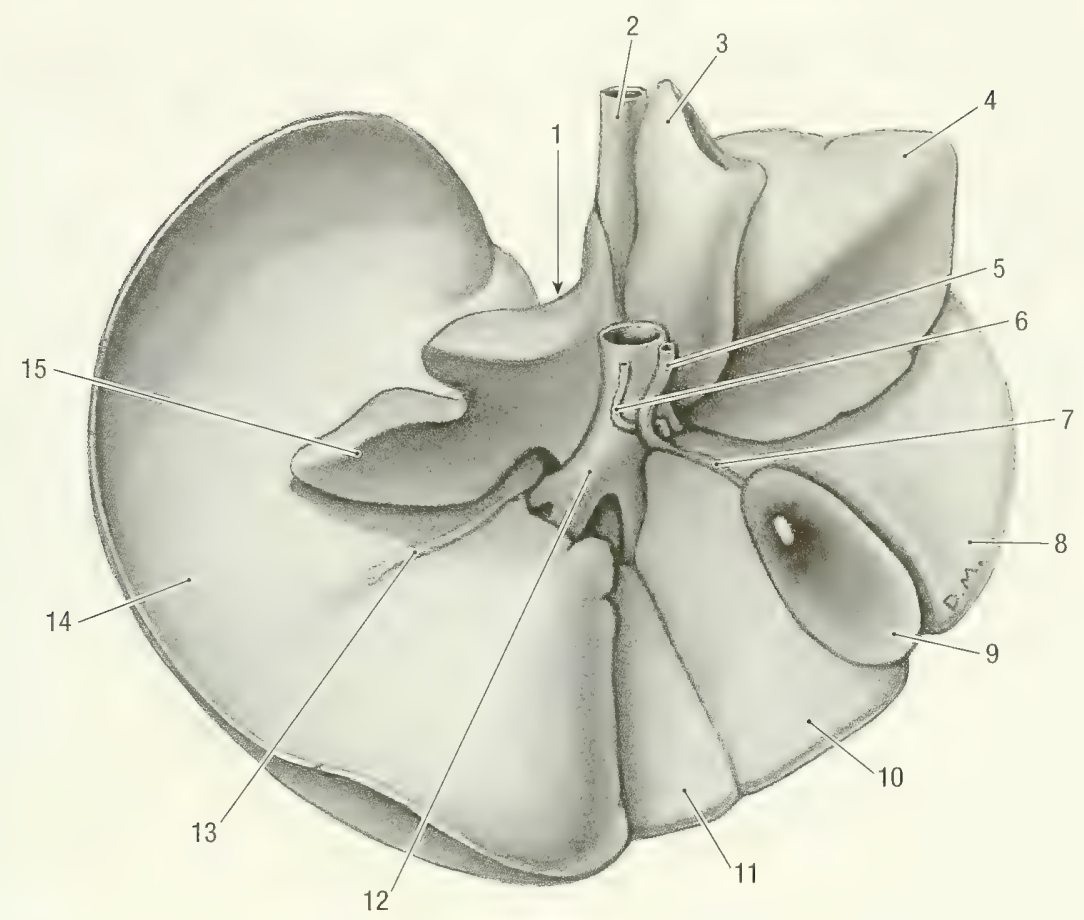

Fig. 5-13. Liver, visceral surface. 1 esophageal impression, 2 caudal vena cava, 3 caudate process, 4 right lateral lobe, 5 bile duct, 6 hepatic artery, 7 cystic duct, 8 right medial lobe, 9 gall bladder, 10 quadrate lobe, 11 left medial lobe, 12 hepatic portal v., 13 lesser omentum, 14 left lateral lobe, 15 papillary process.

caudally along the dorsal border of the left lateral lobe, attaching the latter to the centrum tendineum and left costal part of the diaphragm. There are no attachments to the left medial lobe. The right triangular ligament, lig. triangulare dextrum, extends caudolaterally (to the right) along the dorsal border of the right lateral lobe, attaching the latter to the right crus of the diaphragm. It does not have an attachment to the right medial lobe. In some specimens the ligament divides so that it has a V-shaped attachment to the liver.

Apart from these parietal attachments, the liver is attached to stomach and duodenum by the lesser omentum.

The lesser omentum, omentum minus (Fig. 5-13), is a fold of peritoneum that extends from the visceral surface of the liver to the terminal esophagus, stomach and duodenum. It can be divided into the hepatogastric ligament, lig. hepatogastricum, and the hepatoduodenal ligament, lig. hepatoduodenale. Its attachment to the liver, about $5 \mathrm{~cm}$ long, extends ventrally from the ventrolateral surface of the caudal vena cava to the ventral third of the left lateral lobe. At the porta hepatis it gives off a branch that attaches to the left and right borders of the papillary process. The part of the lesser omentum that attaches the liver to the lesser curvature of the stomach is the hepatogastric ligament, and the part that goes to the cranial part of the duodenum is the hepatoduodenal ligament. The hepatogastric ligament contains the hepatic lymph node and hepatic artery, as well as the portal vein, while the bile duct lies in the hepatoduodenal ligament.

Bile Ducts and Gall Bladder. Bile, secreted by the liver cells into bile canaliculi, is collected by small intralobar bile ductules. These in turn unite and leave the lobes as interlobar bile ductules. The interlobar ductules that drain the left lateral and medial lobes, as well as the quadrate lobe unite to form the left hepatic duct, ductus hepaticus sinister. Bile from the caudate and right lateral lobes is drained by the right hepatic duct, ductus hepaticus dexter. Both left and right ducts open separately into the cystic duct, ductus cysticus. Bile from the right medial lobe drains into the cystic duct by means of an accessory bile duct. The latter duct opens into the cystic duct, halfway between the gall bladder and the opening of the left hepatic duct. There is no common bile duct. Variations in the pattern of the bile duct system are common.

The gall bladder, vesica fellea (Fig. 5-13), is pear shaped and averages $4 \mathrm{~cm}$ in length and $1 \mathrm{~cm}$ in width. Its volume is 5 to $6 \mathrm{ml}$. The gall bladder is located in a depression on the visceral surface of the liver between the right medial lobe on the right 
and the quadrate and left lateral lobes on the left. The cranial surface is attached to the quadrate and right medial lobes by connective tissue, while the caudal or free surface lies against the ascending colon. When the gall bladder is distended it projects 1 to $1.5 \mathrm{~cm}$ beyond the ventral border of the liver to contact the abdominal floor. Proximally the gall bladder narrows to form a neck that continues as the cystic duct. The latter is approximately $2 \mathrm{~cm}$ long, embedded in connective tissue and occupies a shallow groove between the quadrate and right medial lobes of the liver. The cystic duct is joined by the three hepatic ducts. Distally to the last duct which joins it, the cystic duct continues as the bile duct or ductus choledochus. The bile duct is about $3 \mathrm{~cm}$ long and lies in the hepatoduodenal ligament. It opens into the lumen of the duodenum on a small duodenal papilla $1 \mathrm{~cm}$ distal to the pyloric sphincter.

Blood supply: The arterial blood supply to the liver and gall bladder is from the hepatic artery, a branch of the celiac artery. At the porta hepatis the hepatic artery (Fig. 5-13) divides into a right and left hepatic branches. The right branch gives rise to caudally and dorsally directed vessels. The former enters the right medial lobe at the porta, but before doing so gives off the cystic artery to the cystic duct and gall bladder. The latter passes along the medial border of the right lateral lobe (in the groove between the lobe and the portal vein) and divides into two branches: one for the caudate process and the other for the right lateral lobe. The left branch is the continuation of the hepatic artery. At its origin it gives off a small vessel to the papillary lobe. The left branch then enters the left part of the porta where it divides: one branch enters the fused quadrate and left medial lobes, the other the left lateral lobe. Variations to this pattern of supply are common.

The portal vein, vena portae (Figs. 5-13 and 95), drains the blood from the digestive tract (for details of its tributaries (see portal vein, chapter 10). It passes along the floor (ventral border) of the epiploic foramen, dorsal to the junction of the pylorus and duodenum. (Note that the caudal vena cava lies dorsal to the portal vein and forms the roof of the epiploic foramen. These are handy landmarks for access to both veins). As the vein approaches the right part of the hepatic porta, it divides into right and left branches. The right branch, ramus dexter, immediately divides again into a ventral branch to the right medial lobe, a medial branch to the papillary process and a lateral branch to the right lateral lobe and the caudate process. The left branch, ramus sinister, passes ventrolaterally and divides into two branches, one to the fused left medial and quadrate lobes, the other to the left lateral lobe. (The branching of the portal vein very closely follows that of the hepatic artery). Variations to this pattern of branching are common.

Venous drainage: Blood from the central veins of the liver lobules collect in small interlobular veins that join to form intralobar veins. These unite to form right, middle, and left hepatic veins that enter the caudal vena cava. The hepatic vein that drains the caudate process is the first or most caudal to enter the caval vein. The vein from the right lateral lobe opens independently and just cranial to the latter, while the vein from the right medial lobe forms the middle hepatic vein and opens into the floor of the caudal vena cava. The left hepatic vein drains the left lateral and medial lobes, as well as the quadrate lobe and papillary process and opens into the caudal vena cava, just to the left of the middle hepatic vein. (Note: Corrosion casts of the hepatic vasculature showed that the hepatic artery and its larger branches remained close to the visceral surface of the liver. In contrast to the arteries, the portal and hepatic veins branched in the depth of the organ.)

Innervation: The liver is supplied by both afferent and efferent fibers from the vagus nerves, and by sympathetic fibers from the celiac plexus.

Lymph drainage is via the hepatic lymph nodes.

The pancreas (Figs. 5-10 and 5-11) is V shaped, pinkish gray and lobulated. It consists of a body and left and right lobes.

The body, corpus pancreatis, forms the bottom of the $\mathrm{V}$ and lies in the mesoduodenum, close to the pylorus.

The right lobe, lobus pancreatis dexter, is long and narrow. It lies in the mesoduodenum, close to the duodenum itself. The lobe extends caudally from the pylorus to the caudal flexure along the descending duodenum and then cranially along the ascending duodenum for 2 to $3 \mathrm{~cm}$. Its distal extremity is also attached to the base of the cecum.

The left lobe, lobus pancreatis sinister, is shorter and broader than the right lobe. It crosses the median plane and lies mainly against the dorsal body wall between the transverse colon and the stomach. The initial part of the lobe lies in the attachment of the greater omentum to the dorsal body wall, while the distal part lies within the greater omentum.

The pancreatic secretion drains by many intraand interlobular ducts into a large pancreatic duct or into an accessory pancreatic duct. The pancreatic duct, ductus pancreaticus, begins in the distal end of the left lobe of the pancreas, passes through the left lobe and joins the accessory pancreatic duct. The accessory pancreatic duct, ductus pancreaticus accessorius, drains the right lobe of the 


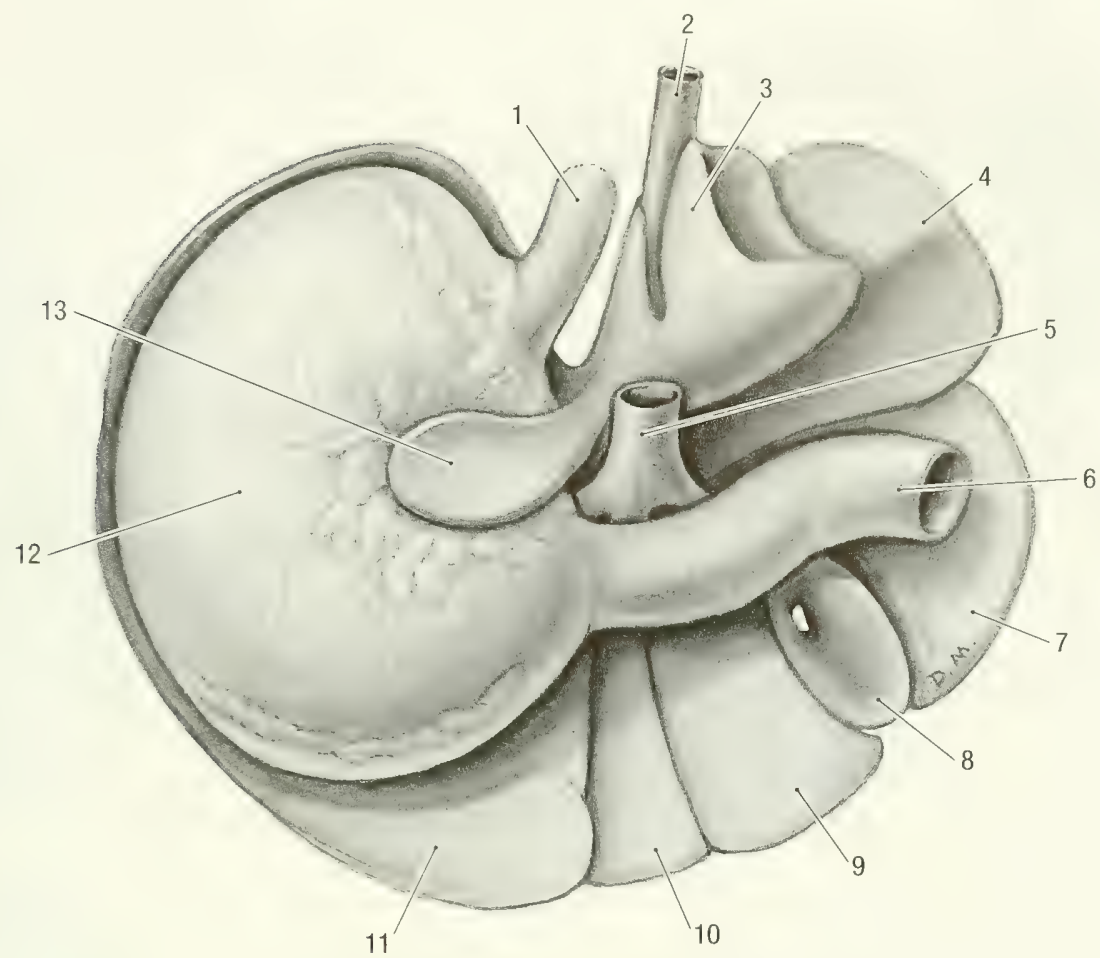

Fig. 5-14. Visceral surface of liver and stomach. 1 esophagus, 2 caudal vena cava, 3 caudate process, 4 right lateral lobe, 5 hepatic portal v., 6 duodenum, 7 right medial lobe, 8 gall bladder, 9 quadrate lobe, 10 left medial lobe, 11 left lateral lobe, 12 stomach, 13 papillary process.

pancreas and opens into the ascending duodenum at the lesser duodenal papilla, about $20 \mathrm{~cm}$ from the pylorus.

Blood supply: The cranial and caudal pancreaticoduodenal arteries are the main supply to the right lobe, while the pancreatic branch of the splenic artery supplies the left lobe.
Nerve supply: Sympathetic fibers from the celiac and cranial mesenteric plexuses reach the gland via the cranial and caudal pancreaticoduodenal arteries.

Lymph drainage: The lymphatics from the pancreas drain into the surounding duodenal, hepatic, splenic and mesenteric lymph nodes. 


\section{Chapter 6. Respiratory System}

The respiratory system consists of the nose and nasal cavity, pharynx, larynx, trachea, bronchi and the lungs.

Nose, Nasal Cavity and Paranasal Sinuses. The external nose, nasus externus, consists of a fixed bony case and a moveable cartilaginous framework. The walls of the bony portion of the nose are formed by parts of the incisive, maxillary and nasal bones (Fig. 1-5). They form the bony nasal aperture rostrally, to which the nasal cartilages, cartilagines nasi externi, attach. The cartilages consist of dorsolateral, ventrolateral and alar parts. The cartilaginous portion of the nose is moveable by virtue of several skeletal muscles associated with it.

The apical portion of the nose or nasal plane, planum nasale, is rounded and contains the nostrils. The skin of this area is pigmented and covered with fine, short hair. A narrow strip around the nostrils and the area between the nostrils are bare. A vertical groove divides the nasal plane into right and left halves. The groove extends downward and is continued on the upper lip by the philtrum.

The nasal cavity, carum nasi (Fig. 6-1), is the facial portion of the respiratory pathway. It extends from the nostrils to the choanae and is divided into right and left halves by the nasal septum. The nasal septum, septum nasi, consists of bony, cartilaginous and membranous parts. The bony part is formed by the perpendicular lamina of the ethmoid bone and the vomer, the cartilaginous part by the nasal cartilage, cartilago septi nasi, and the membranous part, pars membranacea, by connective tissue.

The nostril, naris, opens into the nasal vestibule, vestibulum nasi. The latter is lined by skin and contains the opening of the nasolacrimal duct, ostium nasolacrimale, on the medial surface of the alar fold. There is an abrupt transition from the skin of the vestibule to the mucous membrane of the nasal cavity proper.

The nasal cavity, cavum nasi, is surprisingly short and narrow. The rostral border of the choanae lies $1.5 \mathrm{~cm}$ from the nostrils. The roof of the nasal cavity forms a fundus that extends caudally as far as the cribriform plate of the ethmoid bone. The nasal cavity is laterally compressed by the alveoli of the large upper incisor teeth.

The nasal conchae are ossified scrolls that occupy the major portion of the nasal cavity. They include the dorsal nasal concha, concha nasalis dorsalis, middle nasal concha, concha nasalis media and the ventral nasal concha, concha nasalis ventralis. The nasal conchae divide the nasal cavity into nasal passages or nasal meatuses. The dorsal nasal meatus, meatus nasi dorsalis, is between the dorsal nasal concha and the nasal bone. The middle nasal meatus, meatus nasi medius, is between the dorsal and ventral nasal conchae, and the ventral nasal meatus, meatus nasi ventralis, is between the ventral nasal concha and the floor of the nasal cavity. The common nasal meatus, meatus nasi communis, is a narrow vertical space on either side of the nasal septum. It communicates freely with the other nasal meatuses.

The choanae or caudal nares, choanae, are the openings of the two nasal passages into the nasopharynx. They are about $1 \mathrm{~cm}$ long and face caudoventrally. The rostral border of the choanae lies on a transverse plane just caudal to the upper incisors, the caudal border lies on a transverse plane just rostral to the first premolar teeth.

Blood supply: Branches of the ethmoidal and sphenopalatine arteries supply the nasal cavity. Blood from the fundus of the nasal cavity drains into the cavernous sinus, while satellite veins drain the rest of the cavity.

Innervation: The sensory innervation of the nasal cavity is by branches of the trigeminal and olfactory nerves. For the detail of the nerves, see the olfactory nerve and the ophthalmic and maxillary divisions of the trigeminal nerve.

Lymph drainage: Lymph is drained by the mandibular and retropharyngeal lymph nodes.

The paranasal sinuses, sinus paranasalis, consist of two large cavities, one on each side of the median plane. They pneumatisize the frontal, maxillary and lacrimal bones. The two cavities (left and right) are separated from each other by a median bony septum. The frontal, maxillary and lacrimal bones bound the sinus laterally, the frontal and maxillary bones bound it medially. A thin plate of bone separates the rostral part of its medial wall from the nasal cavity. The nasomaxillary opening, apertura nasomaxillaris, is very small. It lies at the lowest point of the sinus and opens into the middle meatus of the nasal cavity, lateral to the middle nasal concha. 


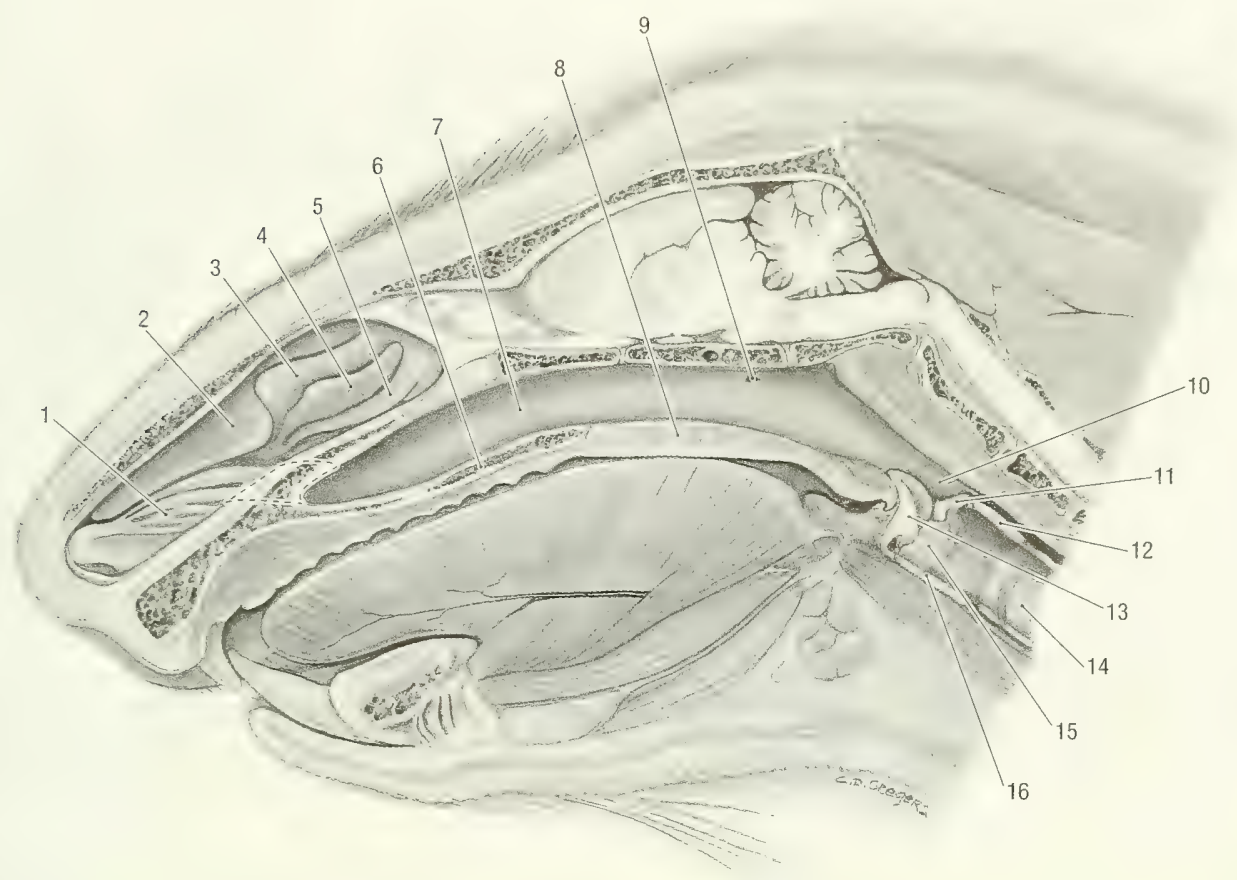

Fig. 6-1. Sagittal section of the head, medial view. 1 ventral nasal concha, 2 dorsal nasal concha, 3 middle nasal concha, 2-5 ethmoid conchae, 6 hard palate, 7 nasopharynx, 8 soft palate, 9 pharyngeal opening of the auditory tube, 10 palatopharyngeal arch, 11 aryepiglottic fold, 12 lamina of the cricoid cartilage, 13 epiglottis, 14 trachea, 15 vocal fold, 16 thyroid cartilage.

Pharynx. The pharynx (Fig. 6-1) connects the nasal cavity to the larynx and the oral cavity to the esophagus. It is divided into nasal, oral and laryngeal parts. The respiratory and digestive pathways cross each other in the laryngeal segment of the pharynx.

The nasopharynx, pars nasalis pharyngis (Fig. 61 ), connects the nasal cavity with the laryngopharynx. It extends from the choanae to the intrapharyngeal opening. The nasopharynx is bounded ventrally by the hard and soft palates, laterally by the maxillary and palatine bones and dorsally by the vomer and base of the skull. Due to the relatively rostral position of the choanae the nasopharynx is exceptionally long and tube-like. The opening of the auditory (Eustachian) tube, ostium pharyngeum tubae auditivae, lies in its lateral wall of the nasopharynx, about $1 \mathrm{~cm}$ from the intrapharyngeal.

The palatopharyngeal arch, arcus palatopharyngeus, is a mucosal fold along the lateral wall of the pharynx. It extends from the lateral end of the free border of the soft palate to the caudal wall of the pharynx. The left and right arches are continuous caudally to form the intrapharyngeal opening, ostium intrapharyngeum. The latter is the caudal limit of the nasopharynx.
The oropharynx, pars oralis pharyngis, connects the oral cavity with the laryngopharynx. It extends from the isthmus faucium rostrally to the base of the epiglottis caudally and is bounded dorsally by the soft palate, ventrally by the root of the tongue and laterally by the fauces. The isthmus faucium is the orifice between the oral cavity and the oropharynx. It is bounded ventrally by the root of the tongue, dorsally by the soft palate and laterally the palatoglossal arch, arcus palatoglossus. The latter are two mucosal folds that extend from the lateral surface of the tongue to the soft palate. The fauces are the lateral walls of the oropharynx. They contain the palatine tonsils, tonsilla palatina. The latter is an accumulation of lymphoid tissue in the mucosa of the oropharynx. It is a rounded body, about 3 $\mathrm{mm}$ in diameter, located in the fauces just rostral to the epiglottis. It lies in a depression, the tonsillar fossa, fossa tonsillaris. The tonsil is partially obscured by a mucosal fold.

The laryngopharynx, pars laryngea pharyngis, is that portion of the pharynx that lies dorsal and lateral to the larynx. It extends from the epiglottis rostrally to the limen of the esophagus. Its caudal limit, therefore, lies on a transverse plane through the caudal border of the cricoid cartilage of the larynx. Dorsally the laryngopharynx communicates 


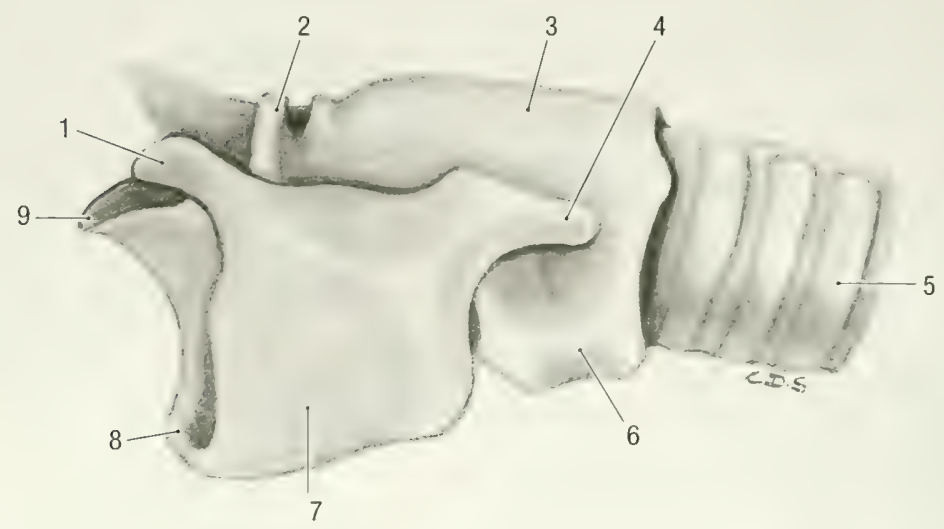

Fig. 6-2. Laryngeal cartilages and trachea, left lateral view. 1 rostral horn of the cricoid cartilage, 2 corniculate process, 3 lamina of cricoid cartilage, 4 caudal horn of the of thyroid cartilage, 5 tracheal cartilage, 6 arch of the cricoid cartilage, 7 thyroid cartilage, 8 base of epiglottis, 9 apex of epiglottis.

with the nasopharynx through the intrapharyngeal opening. The laryngopharynx is divided into the piriform recess and the vestibule of the esophagus. The piriform recess, recessus piriformis, lies on either side of the larynx. The esophageal vestibule, vestibulum esophagi, lies caudal to the piriform re-

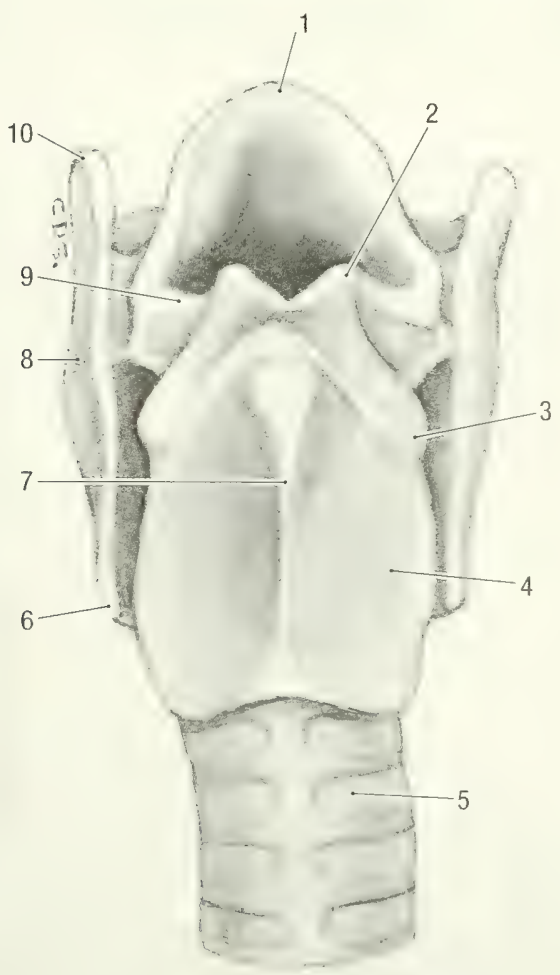

Fig. 6-3. Laryngeal cartilages and trachea, dorsal view. 1 apex of the epiglottis, 2 corniculate process, 3 muscular process, 4 lamina of cricoid cartilage, 5 tracheal cartilage, 6 caudal horn of thyroid cartilage, 7 median crest, 8 thyroid cartilage, 9 aryepiglottic fold, 10 rostral horn of thyroid cartilage. cesses and forms the entrance to the esophagus (Fig. 6-1). A transverse mucosal fold, the limen pharyngoesophageum, marks the pharyngoesophageal junction.

Blood supply: Branches of the maxillary and linguofacial arteries supply the pharynx. The veins are satellites of the arteries. For the detail of the vessels, see the maxillary and linguofacial arteries.

Innervation: Branches of the trigeminal, glossopharyngeal and vagus nerves innervate the pharynx. For the detail of the nerves, see the maxillary division of the trigeminal nerve, and the glossopharyngeal and vagus nerves.

Lymph drainage: Lymph is drained by the mandibular and retropharyngeal lymph nodes.

Larynx. The larynx (Figs. 6-1 to 6-5) consists of laryngeal cartilages, intrinsic laryngeal muscles and a mucosal lining. It aids in vocalization. The larynx lies ventral to the atlas, directly caudal to the root of the tongue and the soft palate. It is approximately $1.8 \mathrm{~cm}$ long, $1.5 \mathrm{~cm}$ wide and $1.3 \mathrm{~cm}$ deep.

The five laryngeal cartilages, cartilagines laryngis, are the cricoid, thyroid and arytenoid cartilages and the epiglottis.

The epiglottis, epiglottis, is the most rostral of the laryngeal cartilages. It is roughly triangular, with an attached base, basis, and a free apex, apex. The dorsal or laryngeal surface, facies laryngea, is concave dorsally. Its lateral borders are attached to the corniculate processes of the arytenoid cartilages by the aryepiglottic folds, plica aryepiglottica. It is attached ventrally to the basihyoid bone by the hyoepiglottic muscles. It is attached to the thyroid cartilage caudally.

The paired arytenoid cartilages, cartilago arytenoidea, are irregular in shape with a base and artic- 


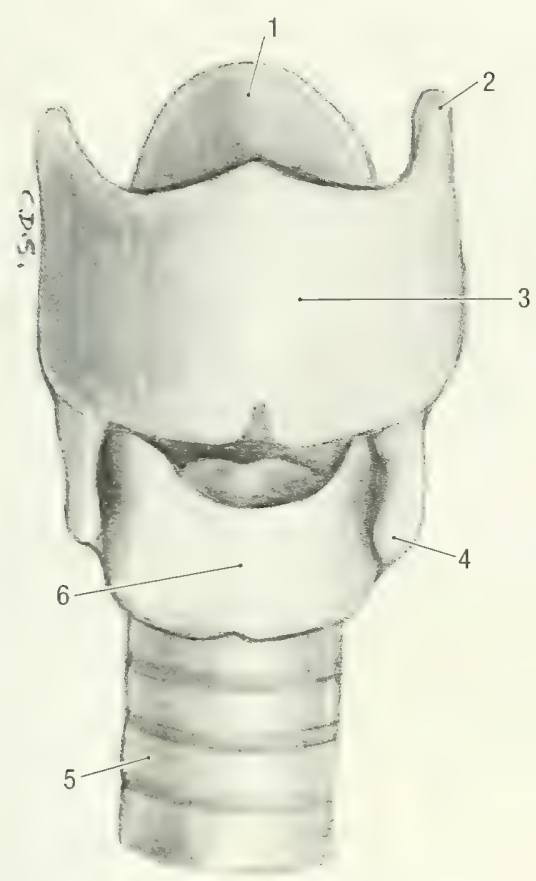

Fig. 6-4. Laryngeal cartilages and trachea, ventral view. 1 apex of epiglottis, 2 rostral horn of thyroid cartilage, 3 lamina of thyroid cartilage, 4 caudal horn of thyroid cartilage, 5 tracheal cartilage, 6 arch of the cricoid cartilage.

ular, dorsal, lateral and medial faces, and articulate with the rostrodorsal border of the cricoid cartilage. They have corniculate, muscular and vocal processes. The muscular process, processus muscularis, projects laterally from the lateral face and serves for the attachment of the cricoarytenoid and thyroarytenoid muscles. The vocal process, processus vocalis, projects ventrally from the base and serves for the attachment of the vocal muscle and vocal ligament. The corniculate process, processus corniculatus, projects rostrodorsally from the dorsal face. It is attached to the epiglottis by the aryepiglottic fold. The articular face lies dorsomedially and bears an articular facet for articulation with the cricoid cartilage.

The thyroid cartilage, cartilago thyroidea, is the largest of the laryngeal cartilages. It forms the middle portion of the larynx and is open dorsally. The thyroid cartilage consists of two laminae, lamina dextra et sinistra, which are united ventrally to form a deep trough. Each lamina is expanded dorsally to form rostral and caudal horns, cornu rostrale and cornu caudale, respectively. Rostral and caudal thyroid notches, incisura rostrale and incisura caudale, separate the respective processes from the lamina. The rostral cornu articulates with the thyrohyoid bone.

The cricoid cartilage, cartilago cricoidea, consists of an arch and a lamina to form a complete ring.

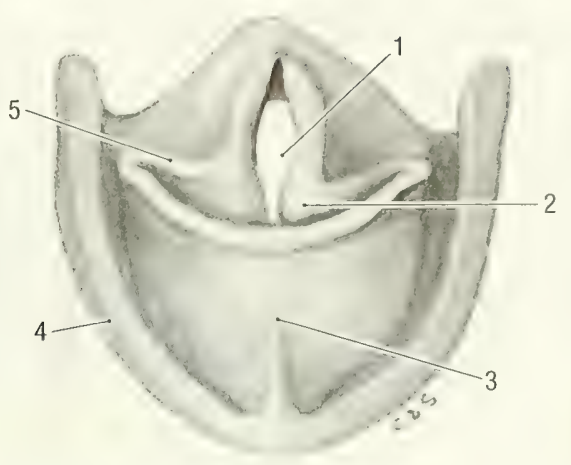

Fig. 6-5. Laryngeal cartilages, cranial view. 1 glottic slit, 2 corniculate process, 3 epiglottis, 4 thyroid cartilage, 5 aryepiglottic fold.

The expanded dorsal part is the lamina, lamina cartilaginis cricoideae. It bears a longitudinal ridge, crista mediana, along its dorsal surface. The arch, arcus cartilaginis cricoideae, extends ventrally from the lamina to complete the ring.

The laryngeal cavity, cavum laryngis, is divided into three transverse segments. From rostral to caudal they are the laryngeal vestibule, glottis and infraglottic cavity. The epiglottis, aryepiglottic folds and the corniculate processes bound the laryngeal opening, aditus laryngis. The opening leads into the vestibule of the larynx. The vestibule of the larynx, vestibulum laryngis, is the portion of the laryngeal cavity rostral to the glottis. The glottis is the middle, narrow portion of the laryngeal cavity. It consists of paired vocal folds with the slit between the folds. The vocal process of an arytenoid cartilage and its associated muscle, ligament and the mucous membrane covering it forms a vocal fold, plica vocalis. Between the walls of the glottis is a narrow passageway or glottic slit, the rima glottis. The slit leads into the caudal part of the laryngeal cavity. The infraglottic cavity, cavum infraglotticum, extends from the rima glottis to the cavity of the trachea. The thyroid and cricoid cartilages bound the infraglottic cavity.

Blood supply: The laryngeal artery supplies the larynx. The laryngeal veins are satellites of the arteries.

Innervation: The larynx is innervated by the cranial and caudal (recurrent) laryngeal branches of the vagus nerves (see the vagus nerve for the detail of the branches).

Lymph drainage: Lymph is drained by the retropharyngeal lymph node.

Trachea. The trachea (Figs. 6-1 to 6-5) extends between the larynx and the lungs and is topographically divided into cervical and thoracic segments. It lies along the ventral border of the cervical and cranial thoracic vertebral bodies and con- 
sists of approximately 23 , dorsally incomplete cartilaginous tracheal rings. The rings are irregular in width and the last one is almost triangular in shape. The tracheal rings are closed dorsally by the tracheal muscle. The trachea divides dorsal to the base of the heart, at the level of the second intercostal space, into the left and right primary bronchi. Each primary bronchus supplies a lung.

The left primary bronchus, bronchus principalis sinister, divides into two secondary or lobar bronchi, bronchi lobares, before it reaches the hilus of the lung.

The right primary bronchus, bronchus principalis dexter, divides into four lobar bronchi. The first one comes off laterally and supplies the caudal lobe of the lung and the second one comes off ventrally and supplies the accessory lobe. The last branch divides into two, one each for the middle and cranial lobes.

Blood supply: The cervical trachea is supplied by branches from the common carotid arteries, while the thoracic part of the trachea and bronchial tree are supplied by the bronchial branches of the bronchoesophageal artery (for the origin and course of the vessels, see the subclavian artery and its branches). Venous blood from the bronchial tree and lung tissue is drained by the bronchial veins that enter the azygos vein directly, or via an intercostal vein.

Innervation: By tracheo-bronchal branches from the sympathetic trunk and vagus nerves.

Lymph drainage: Lymph is drained by the mediastinal lymph nodes.

Thoracic Cavity and Pleurae. In the wider sense, the muscles and bones of the thoracic wall bound the thoracic cavity, cavum thoracis. A thin layer of subserous connective tissue, the endothoracic fascia, fascia endothoracica, covers the inner surface of the thoracic wall. It attaches the pleura to the thoracic wall. This thin layer of endothoracic fascia constitutes the true thoracic cavity. Dorsally the thoracic wall is formed by the thoracic vertebral bodies and the intra-thoracic part of the longus colli muscles, laterally by the ribs and intercostal muscles, and ventrally by the sternum, costal cartilages and the transverse thoracic and intercostal muscles. The thoracic cavity is separated from the abdominal cavity caudally by the dome-shaped diaphragm. The thoracic inlet is bounded cranially by the first ribs and their costal cartilages, the longus colli muscles and the sternothyroid muscles. The trachea, esophagus, blood vessels and nerves and the connective tissue surrounding the structures (deep fascia of the neck) traverses the thoracic inlet. The deep fascia of the neck is continuous with the endothoracic fascia.

Cranially the thorax is narrow. It widens caudal- ly, making the thorax roughly cone-shaped. The diaphragm extends craniomedially from its origin on the inner surfaces of the ribs and costal cartilages, forming a narrow recess (see below).

The thoracic cavity contains the trachea and lungs, esophagus, heart, thymus, lymph nodes, blood vessels and nerves.

The pleurae are the serous membranes that line the walls of the thorax and cover the lungs, heart and structures in the mediastinum. The pleura forms two complete sacs, one on either side, which are known as the pleural cavities. The left pleural cavity extends from the first rib to the diaphragm, the right pleural cavity extends from the second rib to the diaphragm. Each pleural cavity is only a potential cavity, containing a capillary film of serous fluid that moistens its surface. For the purpose of description, the pleura are designated as the parietal and pulmonary pleura. Except for the intervening capillary fluid, the pulmonary pleura lie in contact with the parietal pleura.

The parietal pleura, pleura parietalis, forms the walls of the pleural cavities. It is subdivided into costal pleura, pleura costalis, that lines the walls of the thorax, mediastinal pleura, pleura mediastinalis, that lines the mediastinum, and the diaphragmatic pleura, pleura diaphragmatica, that lines the thoracic surface of the diaphragm. At the attachment of the diaphragm to the lateral thoracic wall the diaphragmatic pleura reflects acutely forward onto the thoracic wall to form the costal pleura. The resulting narrow space between the diaphragmatic and costal pleura is the costodiaphragmatic recess, recessus costodiaphragmaticus. The line of pleural reflection passes from the seventh sternocostal junction, through the genu of the eighth rib and then almost vertically to the middle of the last rib. (It crosses the seventh rib $2 \mathrm{~cm}$, the eighth rib $1.5 \mathrm{~cm}$ and the ninth $1 \mathrm{~cm}$ ventral to the respective costochondral junctions, and the tenth and eleventh ribs at the costochondral junctions). With maximum inspiration the lungs can theoretically expand to the lines of pleural reflection, but in practice it probably never happens. To the right of the midline and mediastinum a fold of parietal pleura, the plica venae cavae, attaches the caudal vena cava to the sternum and diaphragm. The resulting space between the plica vena cava and the mediasinum is the mediastinal recess, recessus mediastini. It contains the accessory lobe of the right lung.

The mediastinal pleura can be topographically subdivided into the cranial mediastinal, pericardial, and caudal mediastinal pleura. The caudal mediastinum is displaced to the left by the large accessory lobe of the right lung. It is therefore attached to the diaphragm to the left of the midline.

The pulmonary pleura, pleura pulmonalis, ad- 


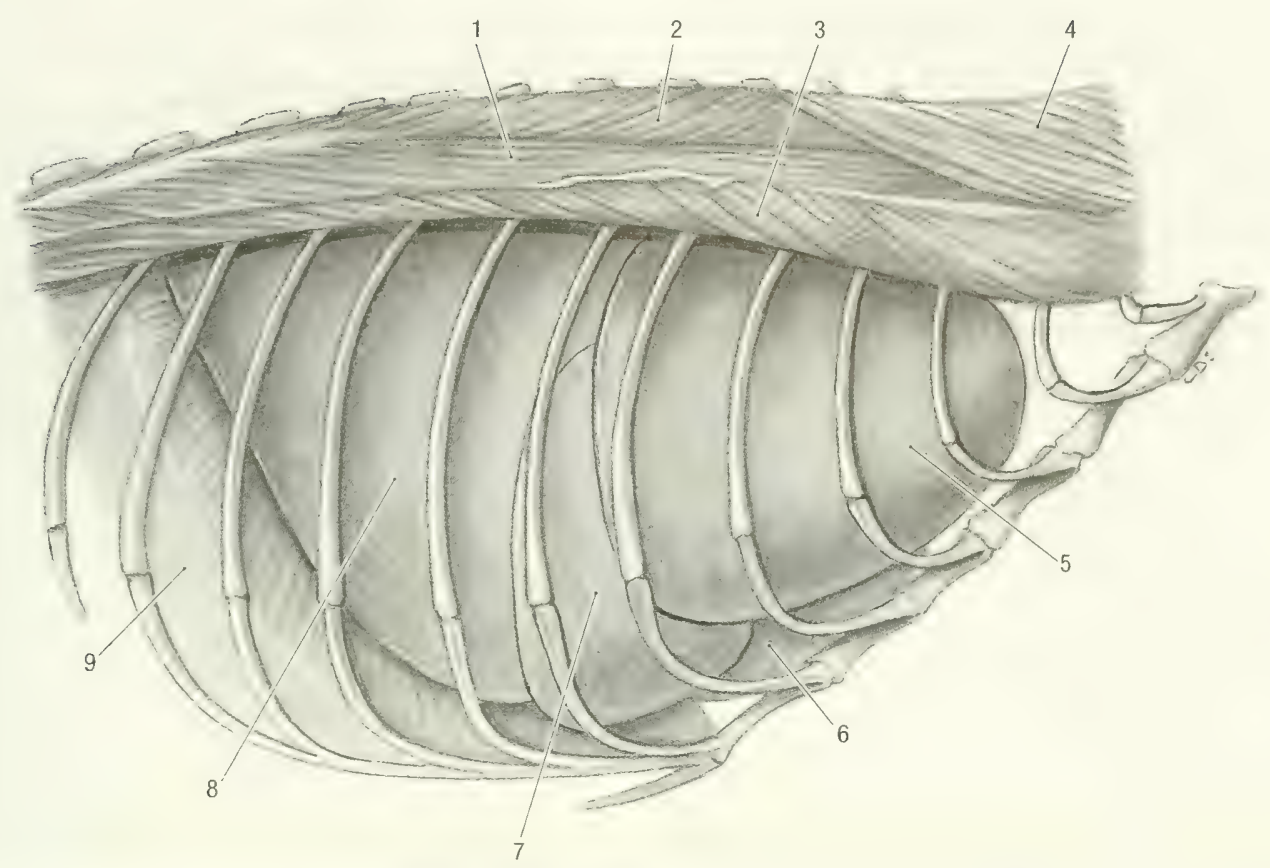

Fig. 6-6. Thorax, right lateral view (intercostal muscles removed). 1 thoracic part of longissimus m., 2 thoracic part of semispinalis m., 3 iliocostalis m., 4 splenius m., 5 cranial lobe of lung, 6 heart, 7 middle lobe of lung, 8 caudal lobe of lung, 9 diaphragm.

heres tightly to the surface of the lung and follows its outline. The pulmonary ligaments, ligg. pulmonales, are folds of pulmonary pleura that leaves the dorsomedial surface of each lung caudal to its hilus. They attach the caudal lobes of the lungs along their dorsomedial borders to the mediastinum. A separate fold of pulmonary pleura extends from the dorsal surface of the accessory lobe of the right lung to the caudal mediastinum (It attaches to the right wall of the infracardiac bursa).

The mediastinum is the endothoracic fascia and mediastinal pleura along the midline of the thorax. It encloses the thymus, thoracic duct and lymph nodes, heart and blood vessels, trachea and bronchi, and the vagus, phrenic and sympathetic nerves. The heart divides the mediastinum into cranial, middle and caudal divisions. The cranial mediastinum lies in front of the heart, the middle mediastinum contains the heart and the caudal mediastinum lies caudal to the heart. The infracardiac bursa, cavum mediastini serosum, is a closed serous cavity to the right of the esophagus in the caudal mediastinum. The cavity extends from the diaphragm to the heart and can communicate with the peritoneal cavity through the hiatus esophageus of the diaphragm.

Lungs. The lungs, pulmo (Figs. 6-6 to 6-8), extend laterally from the mediastinum. They reduce the pleural cavity to a potential space. The lungs are pyramidal in shape with a narrow apex, apex pulmonis, and a broad base, basis pulmonis, which rests on the diaphragm. Each lung is bounded dorsally by the thoracic vertebral bodies, laterally by the ribs and costal cartilages, medially by the mediastinum, ventrally by the sternum and caudally by the diaphragm. They therefore have dorsal, ventral (sternal) and basal (diaphragmatic) borders and costal, diaphragmatic and mediastinal surfaces. The bronchi, blood vessels and nerves enter the lung at the hilus, hilus pulmonis. The latter lies on the mediastinal surface of the lung.

The left lung, pulmo sinister, consists of one large, undivided lobe. Internally, according to the branches of the bronchus, it can be divided into cranial and caudal lobes.

The right lung, pulmo dexter, consists of cranial, middle, caudal and accessory lobes. Interlobar fissures separate the lobes from each other. The fissures extend to the hilus, except for a small area along the dorsal border where the lung tissue of the cranial and caudal lobes is continues. In the embalmed specimen the cranial lobe, lobus cranialis, extends from the second rib to the fourth costal cartilage along its ventral border and to the sixth rib along its dorsal border. It is separated from the middle lobe by the cranial interlobar fissure, fissura interlobaris cranialis.

The middle lobe, lobus medius, lies caudoven- 


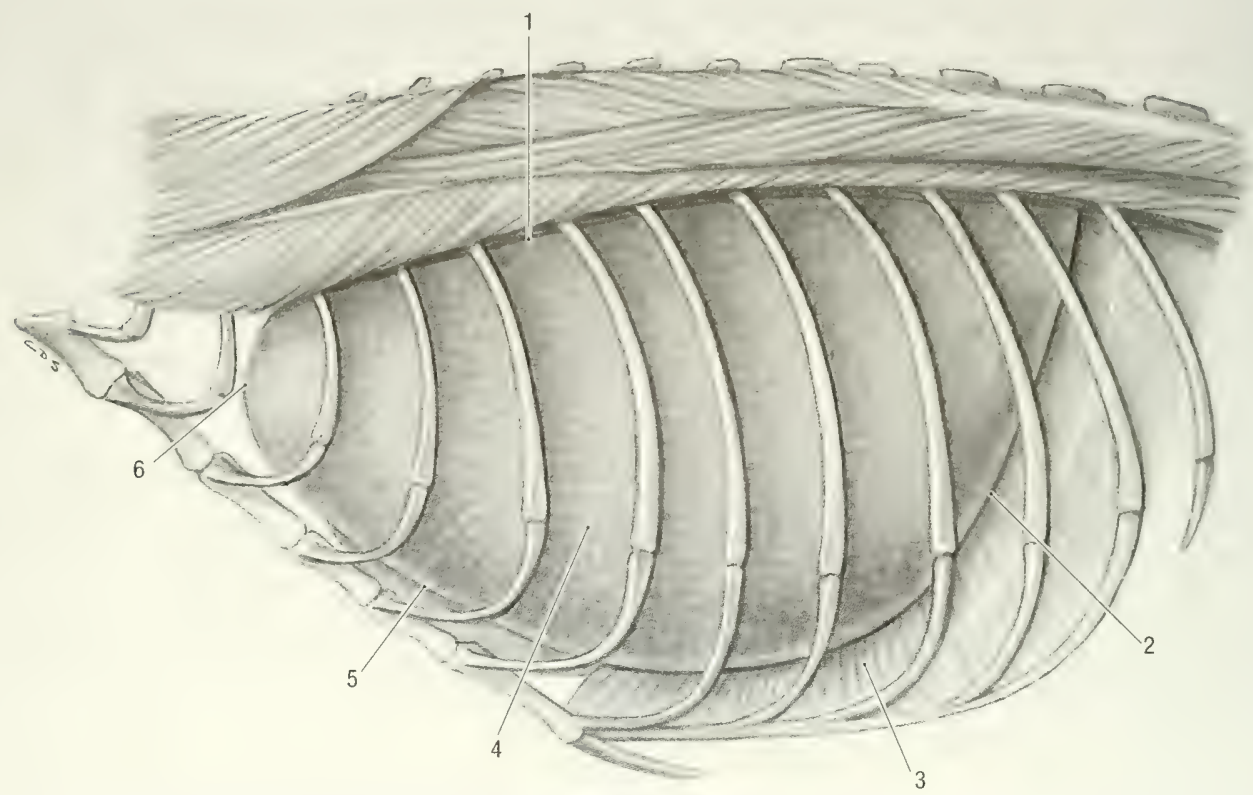

Fig. 6-7. Thorax, left lateral view, intercostal muscles removed. 1 dorsal margin of left lung, 2 basal margin of left lung, 3 diaphragm, 4 undivided left lung, 5 ventral margin of left lung, 6 apex of lung.

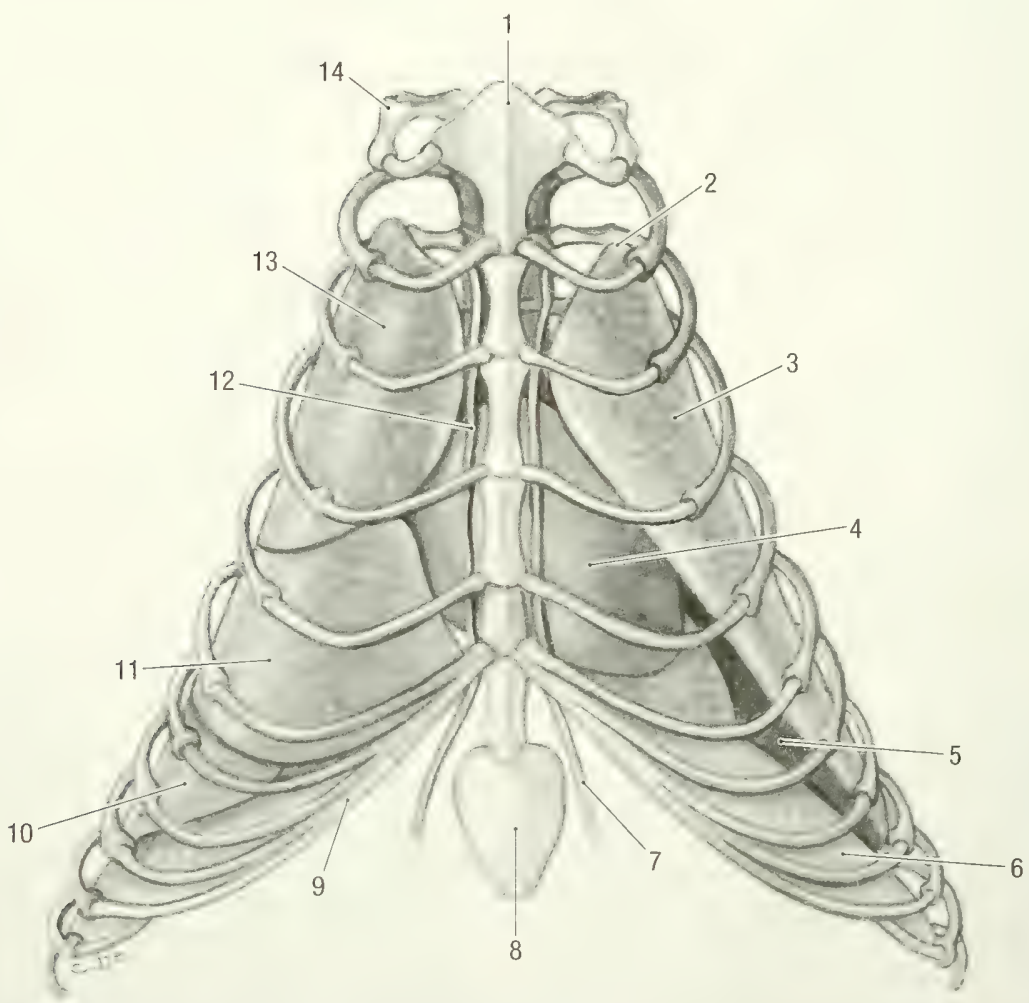

Fig. 6-8. Thorax, ventral view, intercostal muscles removed. 1 manubrium, 2 apex of lung, 3 undivided left lung, 4 heart, 5 diaphragmatic surface on the base of lung, 6 diaphragm, 7 cranial epigastric a., 8 xiphoid cartilage, 9 costal arch, 10 caudal lobe of right lung, 11 middle lobe of right lung, 12 internal thoracica a., 13 cranial lobe of right lung, 14 first rib. 
trally to the cranial lobe. It extends from the fourth rib to the fifth intercostal space along its ventral border and from the fourth to the sixth ribs along its dorsal border. It is separated from the caudal lobe by the caudal interlobar fissure, fissura interlobaris caudalis. The caudal lobe, lobus caudalis, extends from the sixth to the eleventh ribs along its dorsal border. The accessory lobe, lobus accessorius, lies in the mediastinal recess. It is separated from the caudal and middle lobes by the plica vena cava and from the caudal lobe of the left lung by the caudal mediastinum.
Blood supply: Bronchial branches of the bronchoesophageal artery supply the lung tissue. The pulmonary arteries divide along the bronchial tree to form the capillary network around the alveoli of the lung for oxygenation of the blood. Oxygenated venous blood returns to the heart by means of the pulmonary veins.

Innervation: Pulmonary branches from the sympathetic trunks and vagus nerves innervate the lungs.

Lymph drainage: Lymph is drained by the tracheobroncheal lymph nodes. 


\section{Chapter 7. Urogenital Apparatus}

The urogenital apparatus consists of the urinary and reproductive organs.

The urinary organs include the kidneys, ureters, urinary bladder and urethra.

Kidney. The kidney, ren (Figs. 7-1 and 7-2), is bean-shaped, reddish-brown and weighs about 4 gm. Both the right and left kidneys are $3 \mathrm{~cm}$ long, $2 \mathrm{~cm}$ wide and $1.5 \mathrm{~cm}$ thick. Each kidney has cranial and caudal poles, medial and lateral borders and dorsal and ventral surfaces. The lateral border, margo lateralis, is convex and smooth. The medial border, margo medialis, is concave and indented by the cavity of the kidney, the renal sinus, sinus renalis. The entrance into the renal sinus is the renal hilus, hilus renalis. Blood vessels, nerves, lymphatics and the ureter enter or leave the kidney through the hilus. The renal sinus contains a variable amount of adipose tissue.

Both kidneys are invested with a thin, fibrous capsule. The capsule dips inward at the hilus to line the walls of the renal sinus and to form the adventitia of the renal pelvis. The dorsal surface, facies dorsalis, lies against the sublumbar muscles.

Both kidneys are retroperitoneal in the sublumbar region, one on either side of the aorta and caudal vena cava (Fig. 7-3). They are embedded in perirenal fat, except along the ventral surface which is covered by peritoneum. The amount of perirenal fat determines the extent to which the kidney is related to other abdominal organs.

The left kidney extends from the caudal border of the second to the middle of the fourth lumbar vertebra. It lies slightly obliquely, with the caudal pole, extremitas caudalis, closer to the midline than the cranial pole, extremitas cranialis. The craniolateral surface is medial to the spleen, the caudolateral surface lies medial to the stomach and greater omentum, and the craniomedial surface lies lateral the pancreas. The second loop of the ascending colon lies against the caudoventral surface of the kidney. The left adrenal gland lies cranial to the renal blood vessels, between the cranial pole of the kidney and the aorta.

The right kidney extends from the caudal border of the first to the caudal border of the third lumbar vertebra. Its cranial pole lies in the renal impression of the liver, the lateral surface lies against the descending duodenum and the ventral surface lies against the first loop of the ascending colon. The right adrenal gland lies cranial to the renal blood vessels, between the cranial pole of the kidney and the caudal vena cava.

Internal features: The parynchema of the kidney consists of an outer cortex, cortex renis, containing the renal corpuscles and an inner medulla, medulla renis, made up of the renal tubules. The medulla forms four renal pyramids, pyramides renales. Each pyramid consists of a base and an apex or papilla, papilla renalis. The urinary secretory ducts or papillary ducts open on the papillae. Each papilla is surrounded by a space, the calyx, calices renalis, that receives the urine. The calices are continous with the renal pelvis. Outside the kidney the renal pelvis continues as the ureter.

Blood supply: Renal arteries supply the kidneys. Both the renal arteries divide into two or more branches before they enter the hilus of the kidneys. Renal veins drain the kidneys.

Innervation: Sympathetic and parasympathetic nerves form a dense network around the renal vessels and enter the kidney with the vessels.

Lymph drainage: Through the renal or lumbar lymph nodes.

Ureter. The ureter (Figs. 7-1 to 7-4) is a thin, fibromuscular tube that carries urine from the kidneys to the urinary bladder. It begins at the renal pelvis and passes caudoventrally and medially towards the bladder. Both ureters are embedded in the retroperitoneal fat on either side of the abdominal aorta and caudal vena cava (Fig. 5-10). They are bounded dorsally by the psoas muscles. Along their course they lie dorsal to the testicular/ovarian blood vessels, pass ventral to the deep circumflex iliac and external iliac arteries and veins and finally cross the dorsal surfaces of the ductus deferens of the male or the uterine horns of the female. The ureters reach the dorsolateral aspect of the bladder caudal to the line of peritoneal reflection on the bladder. They therefore do not enter or pass in the lateral ligaments of the bladder, but remain retroperitoneally. Each ureter opens into the bladder by means of a slitlike orifice, ostium ureteris.

Blood supply: Ureteral branches from the renal and umbilical arteries. The veins are satellites of the arteries.

Innervation: The autonomic nerves to the ureters come from the celiac and pelvic plexus. 


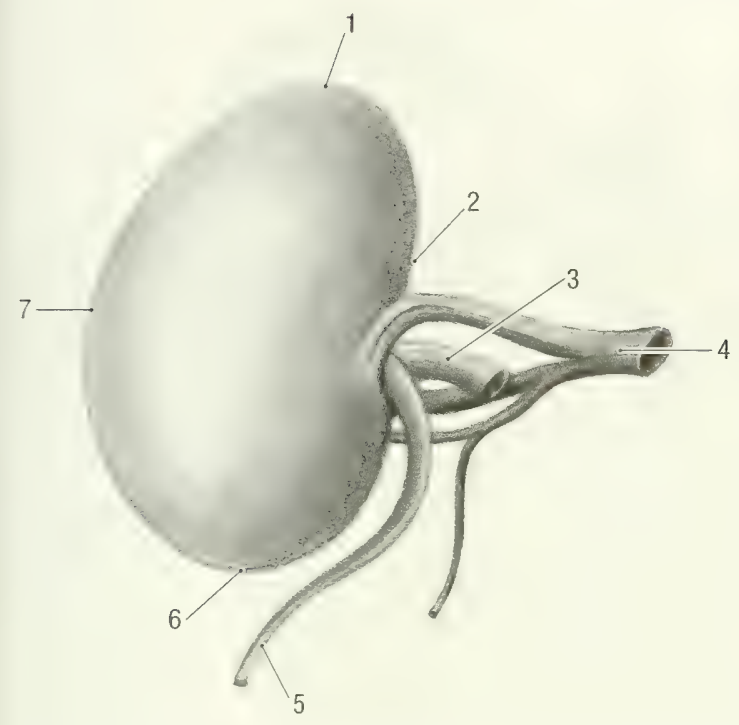

Fig. 7-1. Right kidiney, visceral surface. 1 cranial extremity, 2 medial margin, 3 renal a., 4 renal v., 5 ureter, 6 caudal extremity, 7 lateral margin.
Urinary bladder. The urinary bladder, vesica urinaria (Figs. 7-3 and 7-4), varies in size, shape and position, depending on the amount of urine it contains. It receives urine from the kidneys, through the ureters, and stores it until it is disposed of through the urethra. The entire urinary bladder lies cranial to the pelvic inlet and is therefore abdominal in position (Fig. 5-8). When empty and contracted it is $2 \mathrm{~cm}$ long and $1 \mathrm{~cm}$ wide. The holding capacity is about 35 mil.

The urinary bladder consists of a body, corpus vesicae, and a neck, cervix vesicae, which is connected to the urethra. It is topographically related to the descending duodenum, cecum and small intestine, as well as to the uterine horns of the female animal or the deferent ducts of the male. The extent of contact with the abdominal viscera is dependent on the degree of filling of the bladder. In the male the deferent ducts lie dorsal to the neck of bladder, in the female the fused uterine horns and cervices are in contact with the dorsal surface of the bladder.

The body of the urinary bladder is held in position by three ligaments. These are reflections of the peritoneum onto the bladder. The median lig-

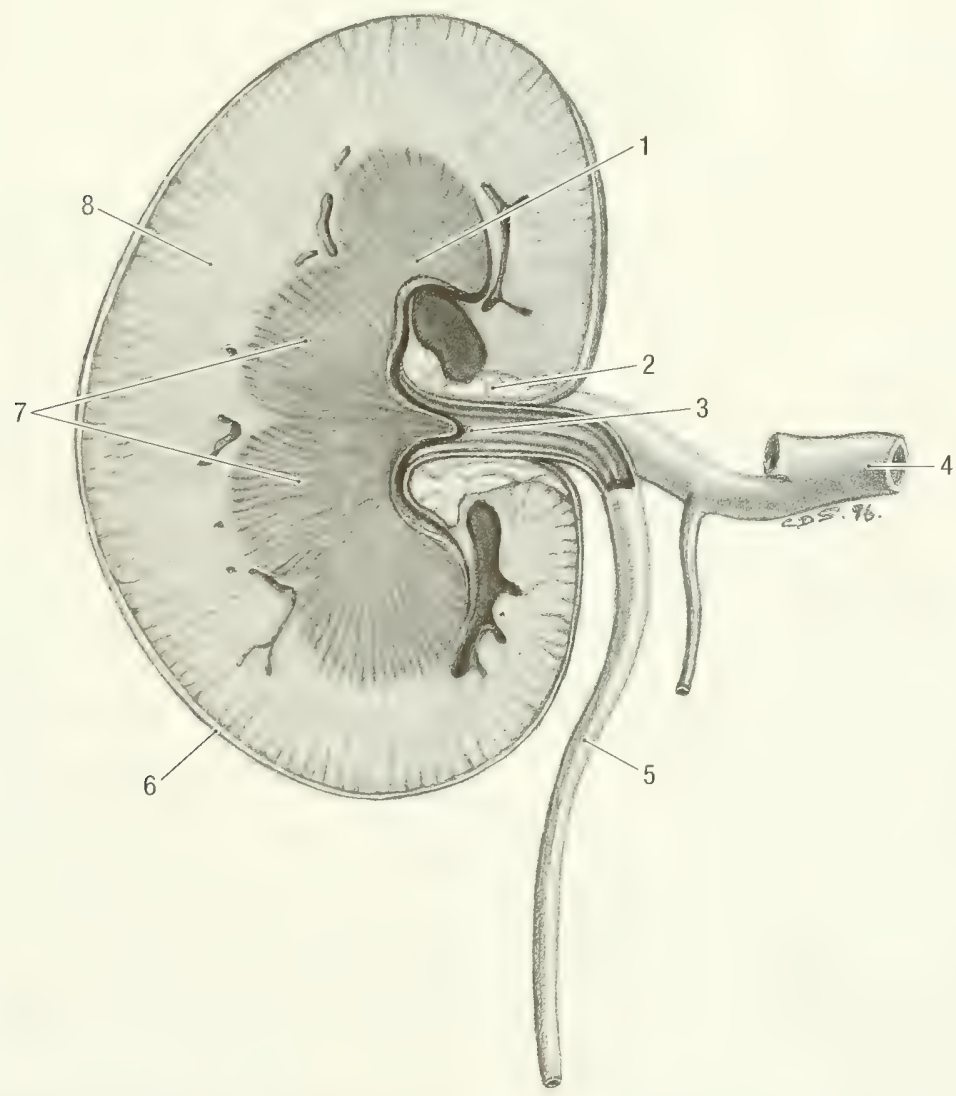

Fig. 7-2. Kidney, longitudinal section. 1 renal papilla, 2 renai sinus, 3 renal pelvis, 4 renal v., 5 ureter, 6 fibrous capsule, 7 renal pyramid (medulla), 8 renal cortex. 


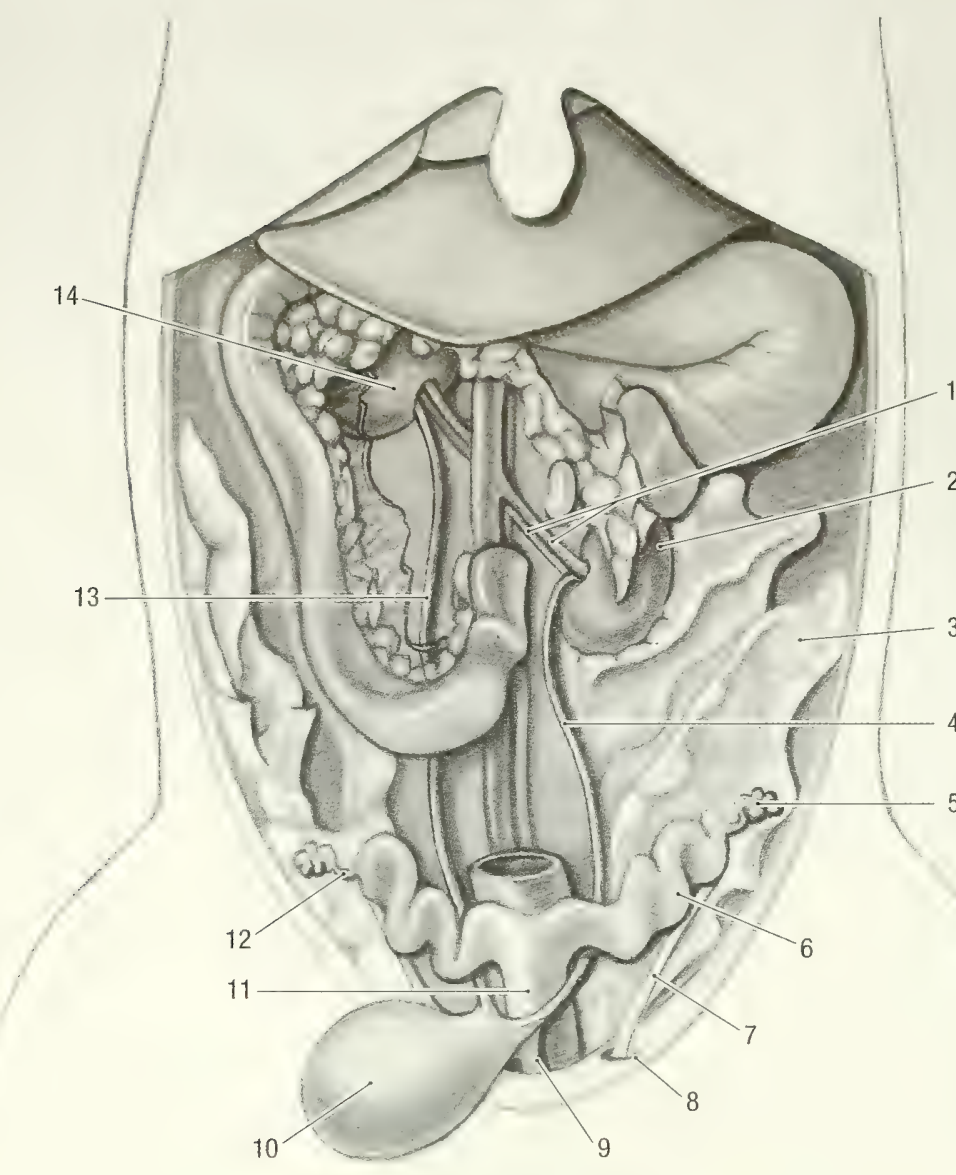

Fig. 7-3. Female urogenital tract, vental view. 1 left renal a. and v., 2 left kidney, 3 mesometrium, 4 left ureter, 5 ovary, 6 uterine horn, 7 round lig. of uterus, 8 vaginal ring, 9 urethra, 10 urinary bladder, 11 body of uterus, 12 uterine tube, 13 right ureter, 14 right kidney.

ament of the bladder, lig. vesicae medianum, is reflected from the ventral surface of the bladder to the linea alba as far cranial as the umbilicus. The lateral ligaments of the bladder, lig. vesicae laterale, connect the lateral surfaces of the bladder to the lateral abdominal walls. They are not directly attached to the body wall, but blend with the broad ligament of the uterus in the female, or with the mesorchium in the male. The neck of the bladder receives the ureters. It lies caudal to the line of the peritoneum reflection onto the bladder and is therefore retroperitoneal in position.

Internally, each ureter forms a ridge or column, columna ureterica, under the mucous membrane of the bladder as they pass through the wall (Fig. 7 4). Their openings, ostium ureteris, lie close to each other in the neck of the bladder. A triangular area, trigonum vesicae, is formed between the columns. An indistinct, broad fold of mucous membrane, plica ureterica, continues distally from the openings. The two folds meet and are continuous with a dor- sal median ridge in the urethra (see urethra). The neck of the bladder continues through the internal urethral opening, ostium urethrale internum, into the urethra.

Blood supply: The cranial part of the bladder is supplied by the cranial vesical artery (a branch of the umbilical artery), and the neck by the caudal vesical artery (a branch of the prostatic/vaginal artery). Venous blood is drained into the caudal vena cava.

Innervation: Parasympathetic fibers from the pelvic nerves and sympathetic fibers from the hypogastric nerves innervate the pelvis.

Lymph drainage: By the hypogastric and lumbar lymph nodes.

Urethra. The urethra (Fig. 7-4) originates at from the neck of the urinary bladder just cranial to the pelvic inlet. A short portion of the pelvic urethra therefore lies in the abdomen.

In the female the urethra extends caudally to 


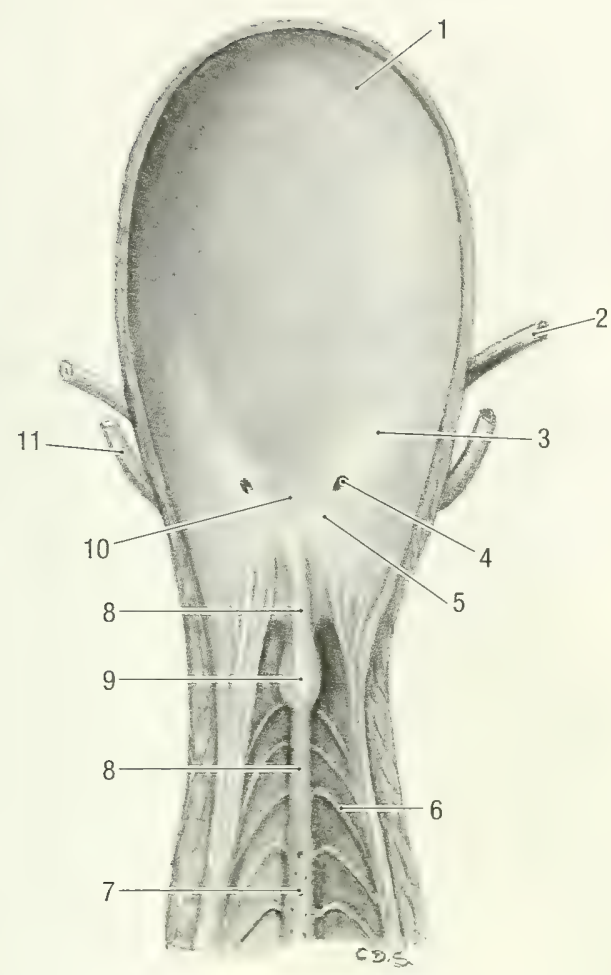

Fig. 7-4. Male urinary bladder, opened ventrally. 1 urinary bladder, 2 ureter, 3 ureteric column, 4 ureteric opening, 5 ureteric fold, 6 mucosal folds of urethra, 7 openings of prostate gland, 8 urethral crest, 9 seminal colliculus, 10 urinary bladder trigone, 11 deferent duct.

meet the genital tract at the vagino-vestibular junction (Fig. 7-5). Its dorsal wall is in close apposition to the ventral wall of the vagina. The female urethra is about $20 \mathrm{~mm}$ long and opens into the floor of the vagina at the urethral orifice, ostium urethrae externum.

In the male the urethra carries both urine and semen to the distal end of the penis. It is 80 to 90 $\mathrm{mm}$ long and is divided into pelvic and penile parts. The pelvic part, pars pelvina, consists of a very short $(7 \mathrm{~mm})$ preprostatic portion, pars preprostatica, and a longer prostatic portion, pars prostatica (Fig. 7-6). The prostatic part is approximately $35 \mathrm{~mm}$ long and $7 \mathrm{~mm}$ wide and extends from the prostate gland to the bulb of the penis. Proximally it has a longitudinal crest, crista urethralis, along its roof. The crest is approximately $35 \mathrm{~mm}$ long and carries a slight enlargement, the colliculus seminalis, colliculus seminalis, approximately $10 \mathrm{~mm}$ from its beginning. The deferent ducts and the ducts of the seminal vesicles open into the urethra on the dorsolateral surfaces of the colliculus seminalis, while the ducts from the prostate open more caudally along the urethral crest. Caudally, at the ischial arch, the bulbourethral glands open by means of prominent ducts into the distal part of the pelvic urethra (Fig. 7-4). The mucosa of the pelvic urethra has a large number of folds that are arranged in a herringbone pattern.

The penile part, pars penina, of the urethra extends from the ischial arch to the tip of the penis. It is approximately $45 \mathrm{~mm}$ long, $3 \mathrm{~mm}$ wide and lies in a groove on the penis. A thin vascular layer, the corpus spongiosum penis, surrounds the penile portion. The corpus spongiosum has two enlargements; the first is the bulb of the penis, bulbus penis, which lies between the two crura of the penis, the second is the glans, glans penis, which lies at the tip of the penis. The bulb of the penis is 1.3 $\mathrm{cm}$ wide, $0.7 \mathrm{~cm}$ long and $0.6 \mathrm{~cm}$ thick. The bulbospongiosus muscle covers the distal half of the penile bulb. The glans is about $0.1 \mathrm{~cm}$ in diameter.

Blood supply: By urethral branches from the prostatic/vaginal and internal pudendal arteries. The artery of the penile bulb supplies the penile portion. The urethral veins are satellites of the arteries.

Innervation: By nerves derived from the pelvic plexus.

Male Genital Organs. The male genital organs consist of the testes and scrotum, the epididymis, the deferent ducts, the accessory genital glands, the penis and the urethra.

The scrotum is a pouch of skin located between the pelvic limbs, ventral to the abdomen and pelvis. It lies craniolateral to the penis and is divided into two compartments. During the breeding season each compartment contains a vaginal tunic and a testis with its associated structures. However, during the non-breeding season the testis and vaginal tunic are abdominal in position and the scrotum is empty. The wall of the scrotum consists of an outer layer of skin and an inner layer of smooth muscle, the tunica dartos.

Blood supply: The principal blood vessel to the scrotum is the scrotal branch of the external pudendal artery, with minor contributions from the internal pudendal artery. The veins are satellites of the arteries.

Innervation: The scrotum is innervated by the genitofemoral and superficial perineal nerves.

The testes (Figs. 7-6 and 7-7) are located in the abdominal cavity during the non-breeding season and in the scrotum when the animal is sexually active. They are oval in shape and vary in size, depending on the season. During the non-breeding season they are about $17 \mathrm{~mm}$ long, $10 \mathrm{~mm}$ wide and $15 \mathrm{~mm}$ thick. During the breeding season they are $25 \mathrm{~mm}$ long, $15 \mathrm{~mm}$ wide and $15 \mathrm{~mm}$ thick. They have capital and caudal extremities, lateral and medial surfaces and free and epididymal borders. The head of the epididymis is very loosely 


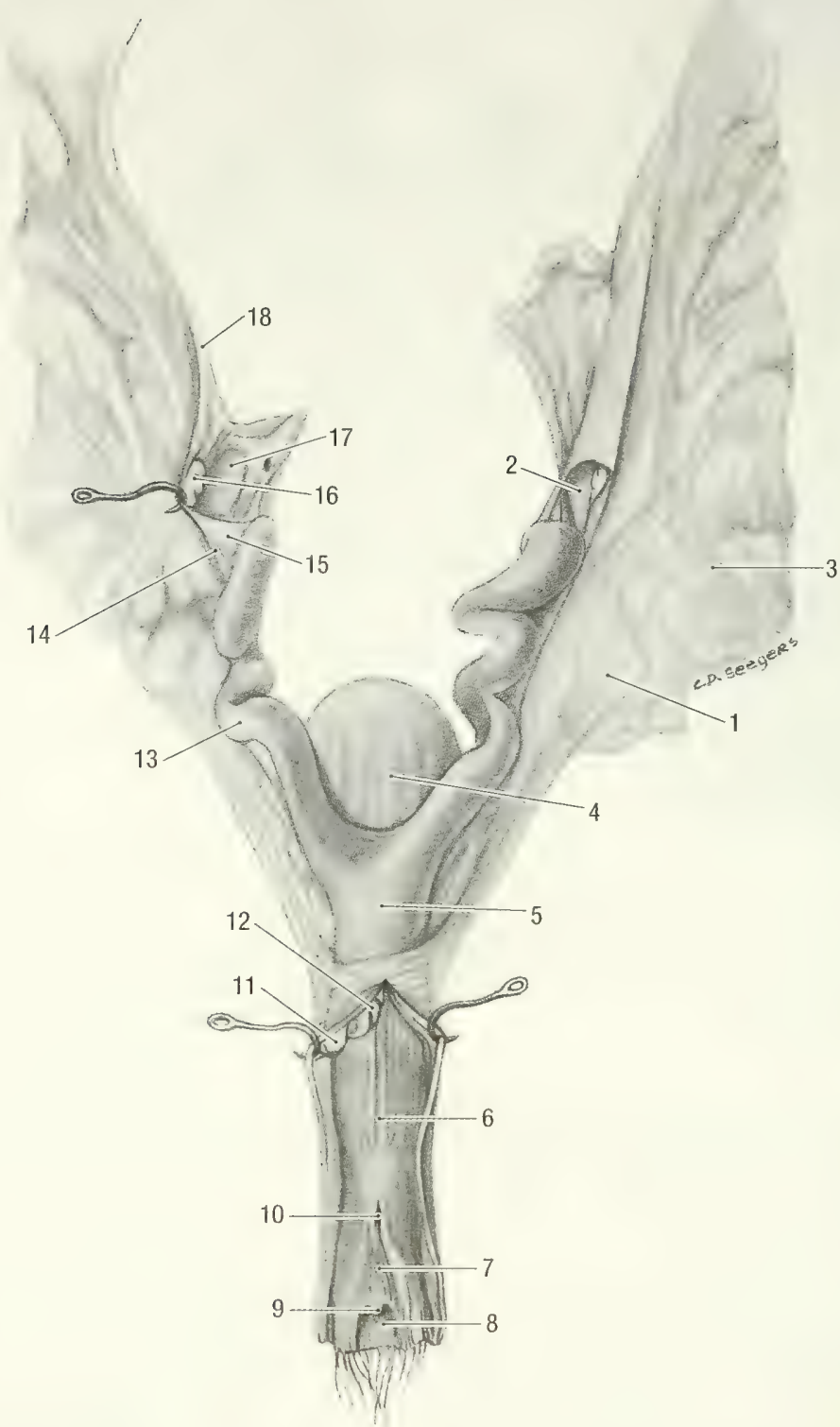

Fig. 7-5. Isolated female genital tract, vagina opened dorsally. 1 mesometrium, 2 ovary, 3 ovarian blood vessels, 4 urinary bladder, 5 uterine body, 6 vagina, 7 vaginal vestibule, 8 clitoral fossa, 9 clitoris, 10 external urethral opening, 11 right cervix, 12 left cervix, 13 uterine horn, 14 uterine tube, 15 mesosalpinx, 16 fimbria of uterine tube, 17 ovary, 18 suspensory lig. of ovary.

attached to the capital extremity, extremitas capitata, of the testis, while the caudal extremity, extremitas caudata, is more closely associated with the tail of the epididymis. The free border, margo liber, lies ventral and the epididymal border, margo epididymalis, dorsolateral. In the scrotal position the testes are orientated longitudinally with the capital extremity facing cranially.

A thick, fibrous capsule, the tunica albuginea covers the parenchyma of the testis. Efferent ductules, ductuli efferentes, pass from the craniomedial pole of the testis to the head of the epididymis.

The vaginal tunic, tunica vaginalis, is a pouch of peritoneum that extends into the scrotum. It contains the vaginal cavity, cavum vaginale, which freely communicates with the peritoneal cavity. When the testis is descended, the parietal layer, lamina parietalis, lines the inside of the scrotum and is reflected unto the testis and associated structures as the visceral layer, lamina visceralis. 


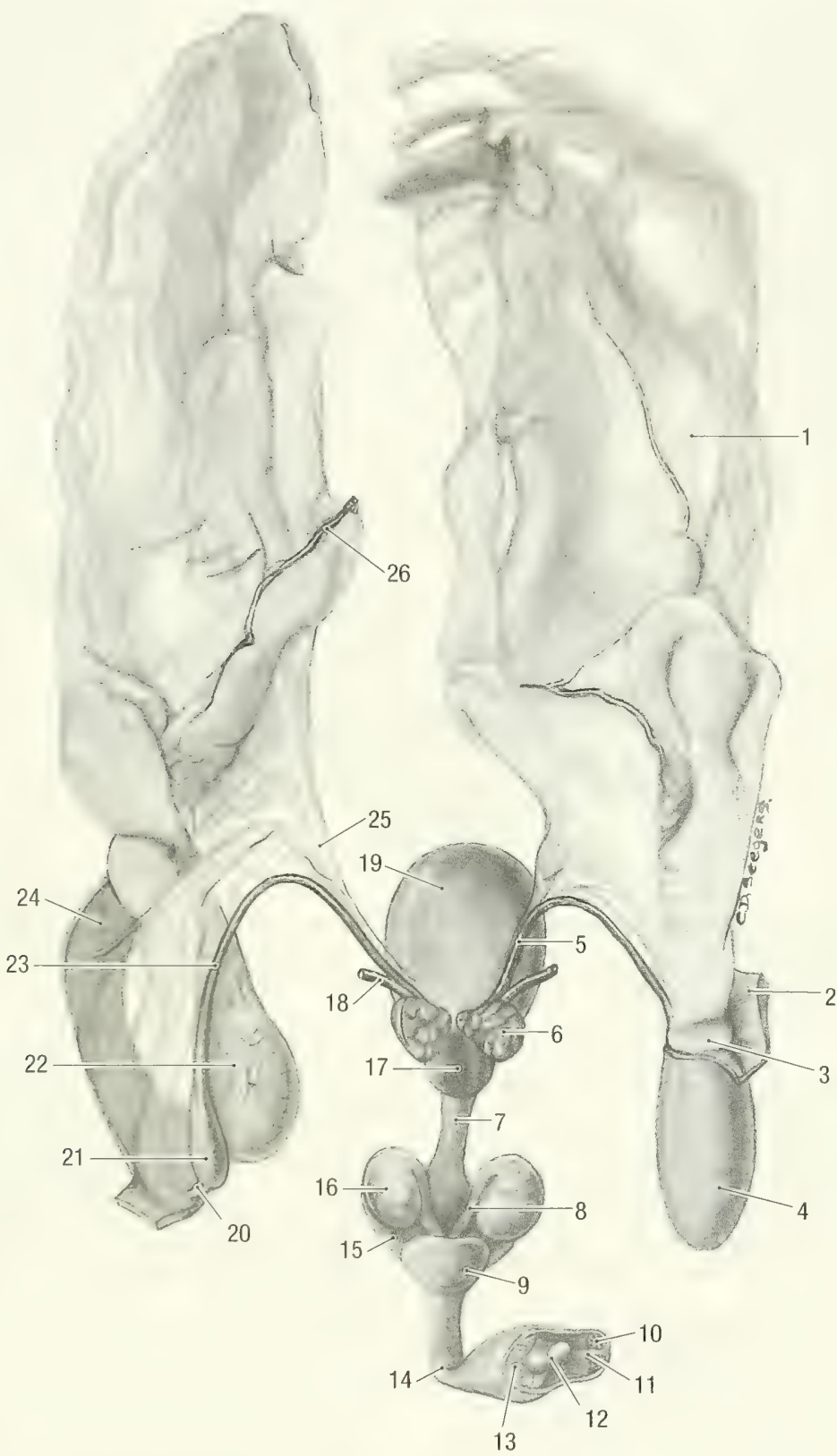

Fig. 7-6. Isolated male genital tract, dorsal view. 1 mesorchium, 2 body wall at the inguinal canal (cut), 3 peritoneum forming the vaginal ring, 4 vaginal tunic, 5 deferent duct, 6 vesicular gland, 7 urethra, 8 duct of bulbourethral gland, 9 bulb of penis, 10 internal lamina of preputium, 11 frenulum of preputium, 12 glans of penis, 13 free part of penis, 14 penis, 15 crus of penis, 16 bulbourethral gland, 17 prostate, 18 ureter, 19 urinary bladder, 20 ligament of the tail of epididymis, 21 tail of epididymidis, 22 testis, 23 deferent duct, 24 parietal layer of vaginal tunic, 25 mesoductus deferens, 26 testicular a.

The visceral layer invests the testis, epididymus and deferent duct. All the viscera of the scrotum are therefore suspended from the scrotal and lateral body walls by a double fold of peritoneum, the mesorchium, mesorchium, that extends from the parietal layer lining the scrotal wall to the visceral layer that invests the organs. The attachment and structure of the mesorchium is similar to that of the broad ligament of the uterus (see below). During the non-breeding season the testis is retracted into the abdomen and the parietal layer of the vaginal tunic is inverted into the peritoneal cavity. It forms a loosely folded area of peritoneum at the deep inguinal ring. 


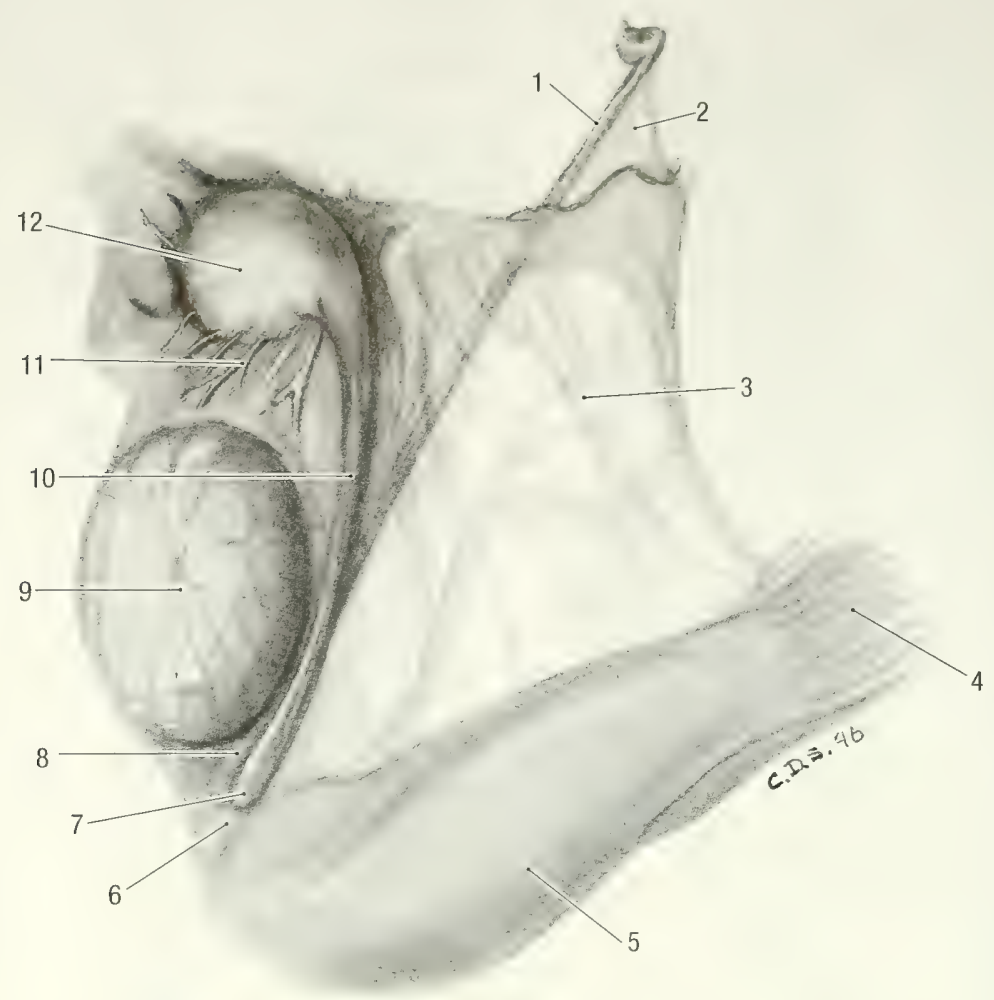

Fig. 7-7. Isolated testis, vaginal tunic opened. 1 deferent duct, 2 mesoductus deferens, 3 mesorchium, 4 cremaster m., 5 parietal layer of vaginal tunic, 6 ligament of the tail of epididymis, 7 tail of epididymis, 8 proper ligament of testis, 9 testis, 10 body of epididymis, 11 efferent ductules, 12 head of epididymis.

The external cremaster muscle inserts on the external surface of the vaginal tunic.

Blood supply: The testis is supplied by the testicular artery and drained by the testicular vein. The tortuous vessels are visible between the peritoneal covering of the testis and the tunica albuginea, medial to the epididymis.

Innervation: The testis is innervated by the sympathetic nervous system. The nerve fibers accompany the testicular blood vessels.

Lymph drainage: Lymphatics accompany the blood vessels and drain into the renal or lumbar lymph nodes.

The epididymis (Figs. 7-6 and 7-7) is composed of a long, convoluted tube, ductus epididymidis. It lies along the dorsolateral border of the testis and is divided into a head, a body and a tail. The head of the epididymis, caput epididymidis, begins on the medial surface of the testis, and curves around the cranial extremity to attain a dorsolateral position. It is very loosely attached to the cranial extremity of the testis by the visceral layer of the vaginal tunic, blood vessels and efferent ductules. The blood vessels and nerves of the testis lie medial to the head of the epididymis. The body of the epididymis, corpus epididymidis, passes along the dorsolat- eral border of the testis, and is about $20 \mathrm{~mm}$ long and $2 \mathrm{~mm}$ wide. It is attached to the testis by a fold of vaginal tunic, the mesepididymis. The tail of the epididymis, cauda epididymidis, is loosely attached to the caudal extremity of the testis by the proper ligament of the testis, lig. testis proprium, and to the parietal layer of the vaginal tunic by the ligament of the tail of the epididymis, lig. caudae epididymidis. It turns dorsally and cranially to continue as the ductus deferens.

The ductus deferens (Figs. 7-4, 7-6 and 7-7) is the continuation of the duct of the epididymis. It extends from the tail of the epididymis to the colliculus seminalis of the pelvic urethra. Initially it is convoluted, but gradually straightens out. Beginning at the tail of the epididymis, it passes cranially along the medial border of the testis and enters the abdominal cavity through the inguinal canal. In the abdomen it lies in a fold of peritoneum, the mesoductus deferens. The duct passes dorsally, crossing the ventral surface of the ureter to reach the dorsal aspect of the neck of the bladder. It continues caudally between the urethra and the seminal vesicles and penetrates the prostate gland to open into the urethra on the colliculus seminalis.

Blood supply: The ductus deferens and the epi- 
didymis is supplied by the $a$. ductus deferentis, a branch of the umbilical artery. It accompanies the ductus deferens to the epididymis and anastomoses with the testicular artery. The veins are satellites of the arteries. The testicular veins drain into the caudal vena cava. In some animals the left testicular vein drains into the left renal vein.

Innervation: The nerves to the ductus deferens are autonomic, arising from the pelvic plexus. The fibers are distributed with the blood vessels.

Lymph drainage: Lymph is drained through the medial iliac lymph nodes.

Accessory Genital Glands. The paired vesicular glands, gll. vesiculares (Fig. 7-6), are elongated, lobular glands situated dorsal to the ductus deferens and the neck of the bladder. They are about 1 $\mathrm{cm}$ long and $0.3 \mathrm{~cm}$ wide. The ducts of the glands open into the ductus deferens.

The prostate gland, prostata (Fig. 7-6), lies caudal to the vesicular glands and is attached to the dorsal aspect of the urethra. It consists of two lobes that fuse to form a dorsoventrally flattened, circular mass. The prostate gland is about $0.7 \mathrm{~cm}$ in diameter and $0.3 \mathrm{~cm}$ thick. The ducts of the glands open into the prostatic part of the urethra, lateral to the colliculus seminalis.

The paired bulbourethral glands, gll. bulbourethrales (Fig. 7-6), are two circular, laterally compressed glands situated at the level of the ischial arch. They lie dorsal to the urethra and just cranial to the penile bulb. The glands are about $0.7 \mathrm{~cm}$ in diameter and $0.3 \mathrm{~cm}$ wide. Their ducts open into the terminal part of the pelvic urethra.

Blood supply: Branches from the prostatic artery supply the vesicular and prostate glands. Branches off the internal pudendal artery supply the bulbourethral glands. The veins are satellites of the arteries.

Innervation: Fibers from the pelvic and hypogastric nerves reach the accessory genital glands via the pelvic plexus and blood vessels.

Lymph drainage: Lymph is drained through the medial iliac lymph nodes.

The penis (Figs. 4-2 and 7-6) has three principle divisions: root, body and glans.

The root of the penis, radix penis, is formed by two crura, crus penis, which are attached to the ischial tuberosities. A crus consists of vascular spaces, the corpus cavernosum penis, enclosed by a tough, fibrous coat, the tunica albuginea corporum cavernosorum. Each crus is covered by an ischiocavernosus muscle. As the two crura pass ventrally and cranially they converge and fuse with each other to form the body of the penis. The other components of the penile root are the urethra and penile bulb. The penile bulb is an enlargement of the vascular spaces around the urethra that continues distally as the corpus spongiosum penis.

The body of the penis, corpus penis, is continuous with the root. It passes cranioventrally for about 30 $\mathrm{mm}$ and then makes an acute ventrocaudal flexure. The flexure lies between the two halves of the scrotum. The body of the penis continues caudally for another $25 \mathrm{~mm}$ and ends at the glans penis. The penile urethra lies in a groove, $0.3 \mathrm{~cm}$ wide, on the caudal (before the flexure) and dorsal (after the flexure) surfaces of the penile body. The last $5 \mathrm{~mm}$ of the penis lies free in the preputial cavity and is the pars libera penis. The corpus cavernosum of the free part of the penis is replaced by bone, forming an os penis or baculum.

The glans penis is a small, rounded enlargement of the corpus spongiosum at the tip of the penis. It measures about $1 \mathrm{~mm}$ in diameter. The external opening of the urethra lies on the glans penis.

Blood supply: The crura and corpus cavernosum are supplied by the deep penile arteries, the bulb of the penis, corpus spongiosum and glans penis are supplied by the artery of the penile bulb and the surrounding tissues (adnexa) are supplied by the dorsal penile artery. The dorsal penile vein drains the penis.

Innervation: The penis is supplied by the pudendal nerve and receives autonomic fibers from the pelvic plexus.

Lymph drainage: Lymph is drained by the superficial inguinal lymph nodes.

The prepuce, preputium (Fig. 7-6), is a fold of skin reflected over the penis. It is composed of two layers, a lamina externa consisting of skin covered with hair, and a lamina interna consisting of stratified squamous epithelium that lines the preputial cavity. The external lamina is continuous with the internal lamina at the preputial orifice, ostium preputiale, that is the entrance into the preputial cavity, cavum preputiale. The lamina interna is reflected onto and attached to the free part of the penis. A thin fold of the internal lamina, the frenulum preputii, extends from the ventral surface of the penis to the preputial orifice. The preputial cavity is about $15 \mathrm{~mm}$ deep. It is obliterated when the penis is extruded. The preputial orifice lies 30 to $40 \mathrm{~mm}$ cranioventral to the anus.

Blood supply: Branches from the external pudendal artery supply the preputium. The preputial veins are satellites of the arteries.

Innervation: By branches of the genitofemoral nerve.

Lymph drainage: Lymph is drained through the superficial inguinal lymph nodes.

Female Genital Organs. The female genital organs consist of the ovaries, uterine tubes, uterus, 
vagina, vulva, and clitoris. The ovaries, oviducts and uterus are abdominal in position. They are suspended from the dorsolateral body walls by the broad ligament of the uterus.

The broad ligament, lig. latum uteri, is a double fold of peritoneum that suspends the female genital organs from the dorsolateral abdominal wall and pelvic cavity. It also contains vessels and nerves to the genitalia and serves as an important fat depot. The attachment of the broad ligament extends from the last rib to the pelvic inlet along a line about 10 $\mathrm{mm}$ from the dorsal margin of the transverse abdominal muscle. In the pelvic cavity the ligament is reflected off the uterus onto the rectum dorsally, ventrally onto the urinary bladder and laterally onto the wall of the pelvic cavity. A peritoneal fold that extends from the ovary to the inguinal canal arises from the ventral surface of the broad ligament. It contains the round ligament, lig. teres uteri, of the uterus. The peritoneal extension through the inguinal canal is the vaginal tunic. Its development varies from animal to animal. Following the structure that it suspends, the broad ligament is divided into the mesovarium, the mesosalpinx and the mesometrium.

The mesovarium, mesovarium, is the part of the broad ligament that attaches the ovary to the abdominal wall.

The mesosalpinx, mesosalpinx (Fig. 7-5), extends laterally from the mesovarium to suspend the uterine tube. The space between the mesovarium and mesosalpinx is the ovarian bursa, bursa ovarica. The bursa is wide open ventromedially and does not enclose the ovary.

The mesometrium, mesometrium (Fig. 7-5), suspends the uterine horns. It is continuous cranially with the mesovarium. It begins at the cranial edge of the uterine horn and extends caudally to the point where the peritoneum of the broad ligament reflects onto the urinary bladder and the colon.

The ovary, ovarium (Figs. 7-3 and 7-5), lies in the abdominal cavity caudal to each kidney. They lie against the abdominal wall, opposite the bifurcation of the abdominal aorta on a transverse plane through the sixth lumbar vertebra. In the inactive state they are approximately $4 \mathrm{~mm}$ long, $1.6 \mathrm{~mm}$ wide and weighs $25 \mathrm{mg}$. However, during estrous they are 9 to $10 \mathrm{~mm}$ long, 3 to $9 \mathrm{~mm}$ wide and weighs 50 to $150 \mathrm{mg}$. During estrous up to 13 to 15 follicles can develop and ovulate, resulting in multiple corpora lutea. They have medial and lateral surfaces, free and mesovarian borders and uterine and tubal extremities. The ovary is attached to the last rib by its suspensory ligament, lig. suspensorium ovarii, and to the tip of the uterine horn by its proper ligament, lig. ovarii proprium. Both ligaments lie within the broad ligament.
Blood supply: The ovaries are supplied by the ovarian arteries and drained by the ovarian veins.

Innervation: Sympathetic fibers reach the ovaries from the renal and aortic plexuses.

Lymph drainage: By means of the ovarian lymph nodes.

The uterine tube, tuba uterina (Figs. 7-3 and 75 ), extends from the ovary to the cranial end of the uterine horn, embedded between the two peritoneal layers of the mesosalpinx. It averages $15 \mathrm{~mm}$ in length and $2 \mathrm{~mm}$ in diameter. The infundibulum, infundibulum tubae uterinae, is the funnel shaped origin of the tube with an abdominal opening, ostium abdominale tubae uterinae, into the peritoneal cavity. The infundibulum lies at the cranial pole of the ovary and its abdominal opening is fringed by poorly developed fimbria, fimbriae tubae. The uterine tube joins the tip of the uterine horn and opens into the latter by means of a small opening, the ostium uterinum tubae.

The uterus, uterus (Figs. 7-3 and 7-5) has two long horns, a short, partially fused body and two cervices. The uterine horn, cornu uteri, is slightly convoluted and extends from the uterine tube to the cervix. In anestrus they are approximately 50 to $60 \mathrm{~mm}$ long and 1 to $2 \mathrm{~mm}$ in diameter. During estrus they increase to 100 to $120 \mathrm{~mm}$ in length and $5 \mathrm{~mm}$ in diameter. The uterine horns present two borders, a mesometrial border along the attached surface and an antimesometrial free border. Caudally, the two horns fuse superficially to form a uterine body, corpus uteri, while they remain as two separate tubes internally. Externally, a shallow groove indicates the borders of the fused horns. Each tube communicates with the vagina through a cervix, cervix uteri. The cervical canal, canalis cervicis, extends from the internal opening in the uterine horn, ostium uteri internum, to the external opening in the vagina, ostium uteri externum. The caudal part of the cervix protrudes into the vagina to form a small portio vaginalils uteri. In anestrous the cervix is approximately $3 \mathrm{~mm}$ long and increases to $5 \mathrm{~mm}$ in length during estrus.

Blood supply: By the uterine and ovarian arteries. The two vessels anastomose near the tip of the uterine horn. The veins are satellites of the arteries.

Innervation: By sympathetic fibers from the hypogastric plexus and parasympathetic fibers from the pelvic nerves.

Lymph drainage: By the lumbar and hypogastric lymph nodes.

The vagina (Fig. 7-5) extends from the cervix to the opening of the urethra in the floor of the tract. The vagina lies in the retroperitoneal space on the floor of the pelvic canal and is separated from the rectum by a sheet of connective tissue. In the mature animal it is approximately $22 \mathrm{~mm}$ long and 
$7.5 \mathrm{~mm}$ in external diameter. The two cervices open separately into the cranial part of the vagina without forming a distinct fornix. The vagina ends at the level of the urethral opening. In the live animal it is demarcated from the vestibule by a low, transverse mucosal ridge.

The vaginal vestibule, vestibulum vaginae (Fig. 75 ), is the terminal part of the female genital tract. It extends from the urethral opening to the vulva. It is approximately $20 \mathrm{~mm}$ long and $7.5 \mathrm{~mm}$ in external diameter.

Blood supply: By vaginal branches from the uterine and vaginal arteries. The veins are satellites of the arteries.

Innervation: The vagina and vestibule are innervated by sympathetic and parasympathetic fibers from the pelvic plexus.

Lymph drainage: Both the vagina and vestibule are drained by the medial iliac lymph nodes.

The vulva, pudendum femininum (Fig. 7-5), is the external genitalia of the female. It consists of two labia, labium pudendi, and the clitoris, clitoris. The two labia border the slit-like opening of the genital tract to the exterior and are covered by hair. They unite dorsally and ventrally, forming dorsal and ventral commissures. The clitoris is well developed. It consists of two vascular bodies or crura, crura clitorides, which are attached to the ischial arch, a body, corpus clitoridis, and a glans, glans clitoridis. The glans is located in a small depression of the vestibule, fossa clitoridis, just cranial to the ventral commissure. In the glans the corpus cavernosum of the clitoris is sometimes replaced by cartilage or bone, forming an os clitoridis.

Blood supply: Branches of the vaginal and external pudendal arteries supply the labia, deep and dorsal clitoridal branches of the internal pudendal artery supply the clitoris. The veins are satellites of the arteries.

Innervation: Somatic nerves, which innervate the external genitalia, arise from the pudendal and genital nerves. The autonomic innervation of the external genitalia is via the hypogastric and pelvic nerves.

Lymph drainage: The vulva is drained by the superficial lymph nodes. 


\section{Chapter 8. Endocrine Organs}

Adrenal Glands. The adrenal glands, glandula adrenalis (Fig. 5-11), are near the cranial poles of the kidneys, embedded in the perirenal fat. They are approximately $10 \mathrm{~mm}$ long, $5 \mathrm{~mm}$ wide and 5 mm thick. The right adrenal gland lies between the cranial pole of the right kidney and the caudal vena cava, cranial to the renal blood vessels. The left adrenal gland lies between the cranial pole of the left kidney and the abdominal aorta, cranial to the renal blood vessels.

Blood supply: The adrenal glands are supplied by the adrenal arteries that arise from the abdominal aorta, or occasionally from the renal arteries. The veins are satellites of the arteries.

Innervation: By a dense sympathetic nerve plexus.

Thyroid Gland. The thyroid gland, gl. thyroidea (Fig. 4-5a), consists of two thin, elongated lobes, connected by an isthmus. Each lobe is approximately $15 \mathrm{~mm}$ long and $5 \mathrm{~mm}$ wide. The isthmus is $0.5 \mathrm{~mm}$ wide. On average the total mass of the thyroid is $28 \mathrm{mg}$. Both lobes lie dorsolateral to the larynx and trachea and are covered laterally by the sternothyroid muscle. The isthmus lies ventral to the second tracheal cartilage. The color of the gland varies from light to dark brown.

Blood supply: By thyroid arteries.
Innervation: By autonomic fibers from the cranial cervical ganglion that accompanies the arteries to the glands.

Parathyroid Gland. The parathyroid gland, gl. parathyroidea, is mostly embedded in the craniomedial part of the thyroid lobes. When visible they appear darker than the thyroid gland. In some animals they lie in the connective tissue medial to the thyroid gland. They are approximately $1 \mathrm{~mm}$ in diameter.

Pineal Gland. The pineal gland, gl. pinealis, is associated with the diencephalon and lies in the roof of the third ventricle. It is approximately $1 \mathrm{~mm}$ in diameter. The pineal recess of the third ventricle extends into the gland

Hypophysis. The hypophysis (Fig. 10-4) lies in the hypophyseal fossa of the sella tursica on the floor of the cranial cavity. It is attached to the diencephalon by the hypophyseal stalk and is approximately $5 \mathrm{~mm}$ in diameter and $2 \mathrm{~mm}$ thick.

Blood supply: By the hypophyseal arteries from the cerebral arterial circle.

There are other organs that have endocrine components e.g., the gonads, pancreas and the gut. These components however, do not form macroscopically identifiable structures. 


\section{Chapter 9. Cardiovascular System}

\section{HEART AND PERICARDIUM}

The pericardium, pericardium, is the fibro-serous envelope of the heart. It consists of an outer fibrous pericardium, pericardium fibrosum, attached to the large blood vessels at the base of the heart, and an inner serous pericardium, pericardium serosum. The serous pericardium consists of a parietal layer attached to the inner surface of the fibrous pericardium, and a visceral layer that is attached to the surface of the heart to form the epicardium. The pericardial cavity, cavum pericardii, is the space between the two serous layers. It contains a small amount of serous pericardial fluid. The heart and pericardium lie in the mediastinum and is therefore covered by mediastinal or pericardial pleura. A variable amount of adipose tissue is present between the pleura and pericardium.

Heart, Cor (Figs. 9-3 and 9-4). The heart is cone-shaped and obliquely placed in the thorax (Fig. 9-1). It consists of two atria that receive returning venous blood and two ventricles that function as muscular pumps to circulate the blood through the body and lungs. The heart has a broad base, basis cordis, and narrow apex, apex cordis, with a left face, facies auricularis, and a right face, facies atrialis.

The heart of a woodchuck with a body mass of $2.7 \mathrm{~kg}$ is about $35 \mathrm{~mm}$ long, $25 \mathrm{~mm}$ wide and weighs $7 \mathrm{gm}$.

\section{Orientation and Topography (Figs. 9-1 and} 9-2). The heart extends from the second intercostal space to the cranial border of the fifth rib and fills the ventral halve of the thoracic cavity. A longitudinal axis through the heart lies at an angle of approximately $45^{\circ}$ with the apex caudal and displaced to the left. The base of the heart faces craniolaterally (to the right) and lies to the right of the median plane. The right ventricle lies against the sternum and costal cartilages. It is covered by the left lung and the accessory lobe of the right lung. The left ventricle faces dorsally and is related to the accessory and middle lobes of the right lung and the tendinous center of the diaphragm. Thus the heart is ventral and medial to the lungs, cranial to the diaphragm, caudal to the thymus and dorsal to the sternum and costal cartilages.
Surface Anatomy (Figs. 9-3 and 9-4). Superficially, the atria and ventricles are separated from each other by the coronary groove, sulcus coronarius. The coronary groove encircles the heart, except cranioventrally, where the conus arteriosus and pulmonary trunk intervenes. The groove is filled with adipose tissue and contains the coronary arteries. The interventricular grooves are distinct surface markings, indicating the position of the interventricular septum. They extend between the base and the apex of the heart and contain branches of the coronary arteries and small amounts of adipose tissue. The left or paraconal interventricular groove, sulcus paraconalis interventricularis, begins to the left of the pulmonary trunk and passes along the cranioventral border of the ventricles to the apex of the heart. The right or subsinuosal interventricular groove, sulcus interventricularis subsinuosus, begins ventral to the caudal vena cava and passes along the caudodorsal border of the ventricles to the apex of the heart.

Atria. The left and right atria and the associated blood vessels form the base of the heart. Internally the atria are separated from each other by the interatrial septum.

The right atrium, atrium dextrum, lies dorsal to the right ventricle. It consists of a fibro-muscular cavity and a muscular auricle. The fibrous part, sinus venarum cavarum, receives venous blood from the body by means of three caval veins and from the heart by means of the cardiac veins. It is separated from the left atrium by the interartrial septum, septum interatriale. Externally the right cranial vena cava is separated from the right auricle by the sulcus terminalis, sulcus terminalis. A muscular terminal crest, crista terminalis, represents the sulcus internally. A transverse muscular ridge, tuberculum intervenosum, situated internally between the caudal vena cava and the right cranial vena cava diverts the incoming blood from the veins through the right atrioventricular opening. The fossa ovalis, fossa ovalis, is a shallow depression in the interatrial septum caudal to the tuberculum intervenosum. It represents the foramen ovale of the unborn animal.

The caudal vena cava opens into the caudal part of the right atrium. The left cranial vena cava passes between the left atrium and left ventricle to open into the coronary sinus, sinus coronarius. The latter 


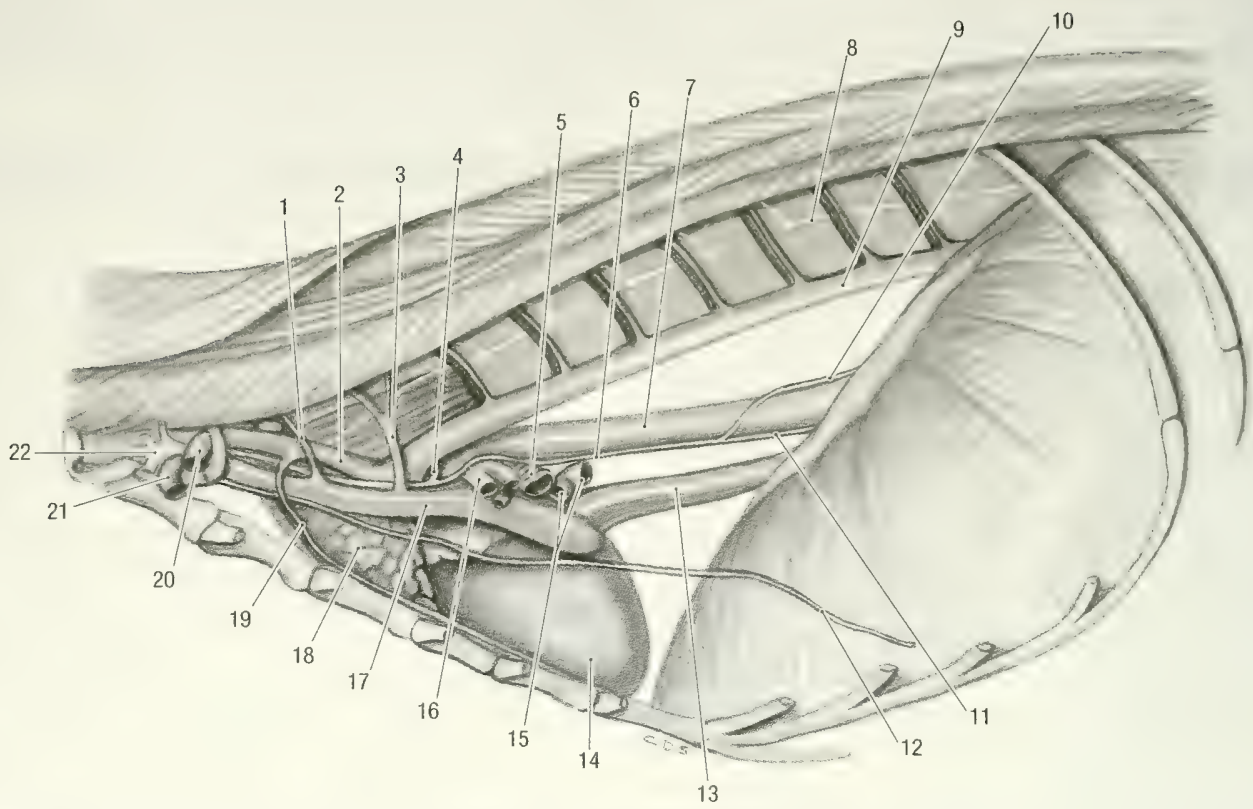

Fig. 9-1. Thoracic cavity, left lateral view. 1 vertebral v., 2 left subclavian a., 3 costocervical v., 4 recurrent laryngeal n., 5 right bronchus, 6 vagal n., 7 esophagus, 8 sympathic trunk, 9 thoracic aorta, 10 dorsal vagal trunk, 11 ventral vagal trunk, 12 phrenic n., 13 caudal v. cava, 14 heart, 15 pulmonary vv., 16 left pulmonary a., 17 left cranial vena cava, 18 thymus, 19 internal thoracic a., 20 axillary a., 21 axillary v., 22 brachial plexus.

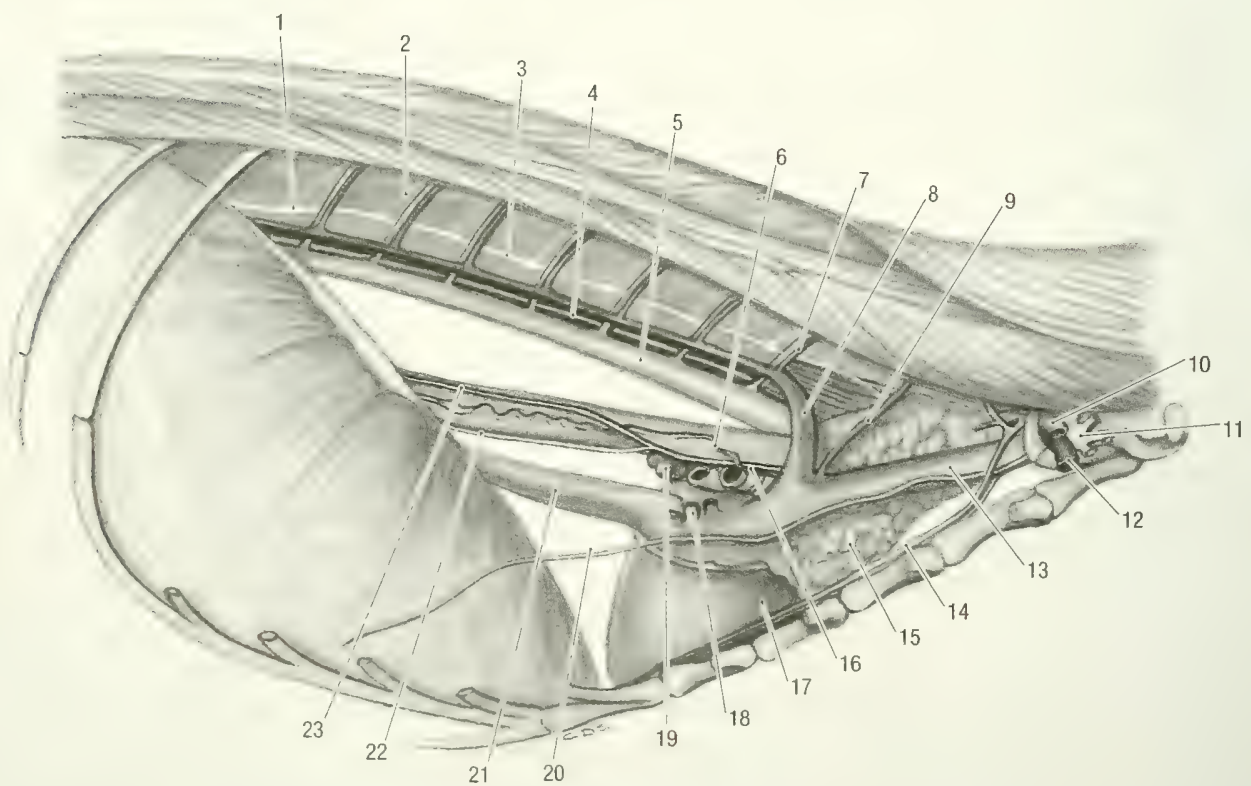

Fig. 9-2. Thoracic cavity, right lateral view. 1 sympathic trunk ganglion, 2 communicating branch, 3 sympathic trunk, 4 thoracic duct, 5 thoracic aorta, 6 broncho-esophageal a., 7 dorsal intercostal a. and v., 8 right azygos v., 9 costocervical v., 10 axillary a., 11 brachial plexus, 12 axillary v., 13 right cranial v. cava, 14 internal thoracic a., 15 thymus, 16 vagal n., 17 heart, 18 pulmonary v., 19 middle tracheobroncheal In., 20 phrenic n., 21 caudal v. cava, 22 ventral vagal trunk, 23 dorsal vagal trunk. 


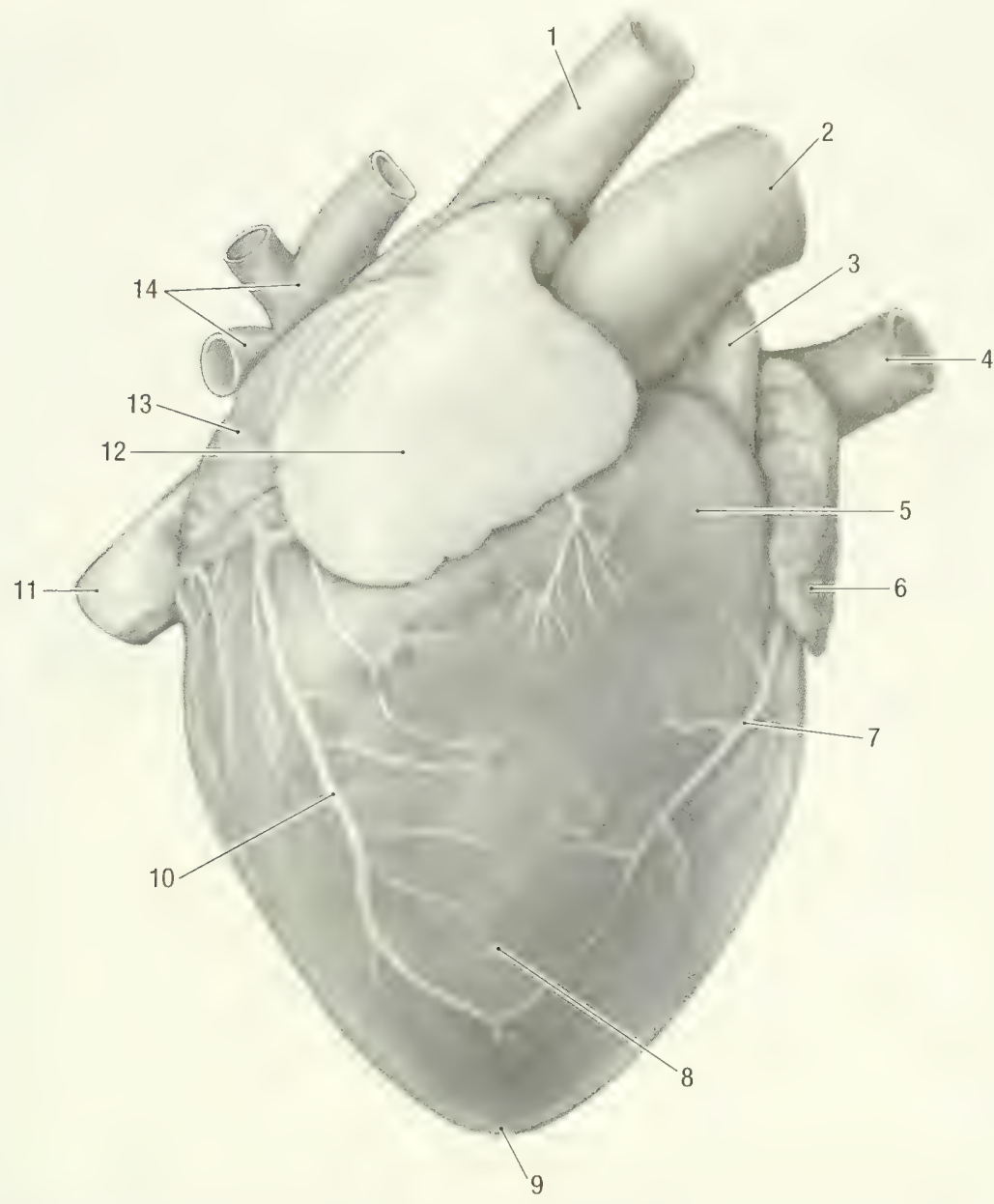

Fig. 9-3. Heart, cranial view. 1 right cranial vena cava, 2 aorta, 3 pulmonary trunk, 4 left cranial vena cava, 5 conus arteriosus, 6 left auricle, 7 paraconal interventricular branch, 8 right ventricle, 9 apex of heart, 10 right cardiac v., 11 caudal vena cava, 12 right auricle, 13 right atrium, 14 pulmonary $\mathrm{v}$.

sinus lies ventrally to the opening of the caudal vena cava and also receives the veins draining the heart. The right cranial vena cava opens into the cranial part of the right atrium. The right atrioventricular opening leads from the right atrium to the right ventricle.

The left atrium, atrium sinistrum, lies dorsal to the left ventricle. It receives all the pulmonary veins. The left auricle lies caudal to the right auricle and is separated from the latter by the pulmonary trunk and aorta. The left atrioventricular opening leads from the left atrium to the left ventricle.

Ventricles. The two ventricles form the bulk of the muscular heart. The wall of the right ventricle is thin, that of the left ventricle is thick. Internally the ventricles are separated from each other by a muscular septum, septum interventriculare. Externally the position of the interventricular septum is indicated by the interventricular grooves.
The right ventricle, ventriculus dexter, lies against the sternum and costal cartilages and to the left of the midline. Its large right atrioventricular opening, ostium atrioventriculare, is guarded by the tricuspic or right atrioventricular valve, valva atrioventricularis dextra. The valve consists of three leaflets or cusps. The parietal cusp, cuspis parietalis, is attached to the lateral border of the opening, the septal cusp, cuspis septalis, to the septal or medial border of the opening and the angular cusp, cuspis angularis, to the cranial border of the opening.

Fibrous cords, chordae tendineae, attach the free ends of the cusps to papillary muscles, $\mathrm{mm}$. papillares. The outflow track of the right ventricle forms the arterial cone, conus arteriosus, that leads to the opening of the pulmonary trunk, ostium trunci pulmonalis. The latter opening is guarded by the pulmonary valve, valva trunci pulmonalis, which consists of three semilunar valvules, the valvula semilunaris dextra, intermedia et sinistra. 


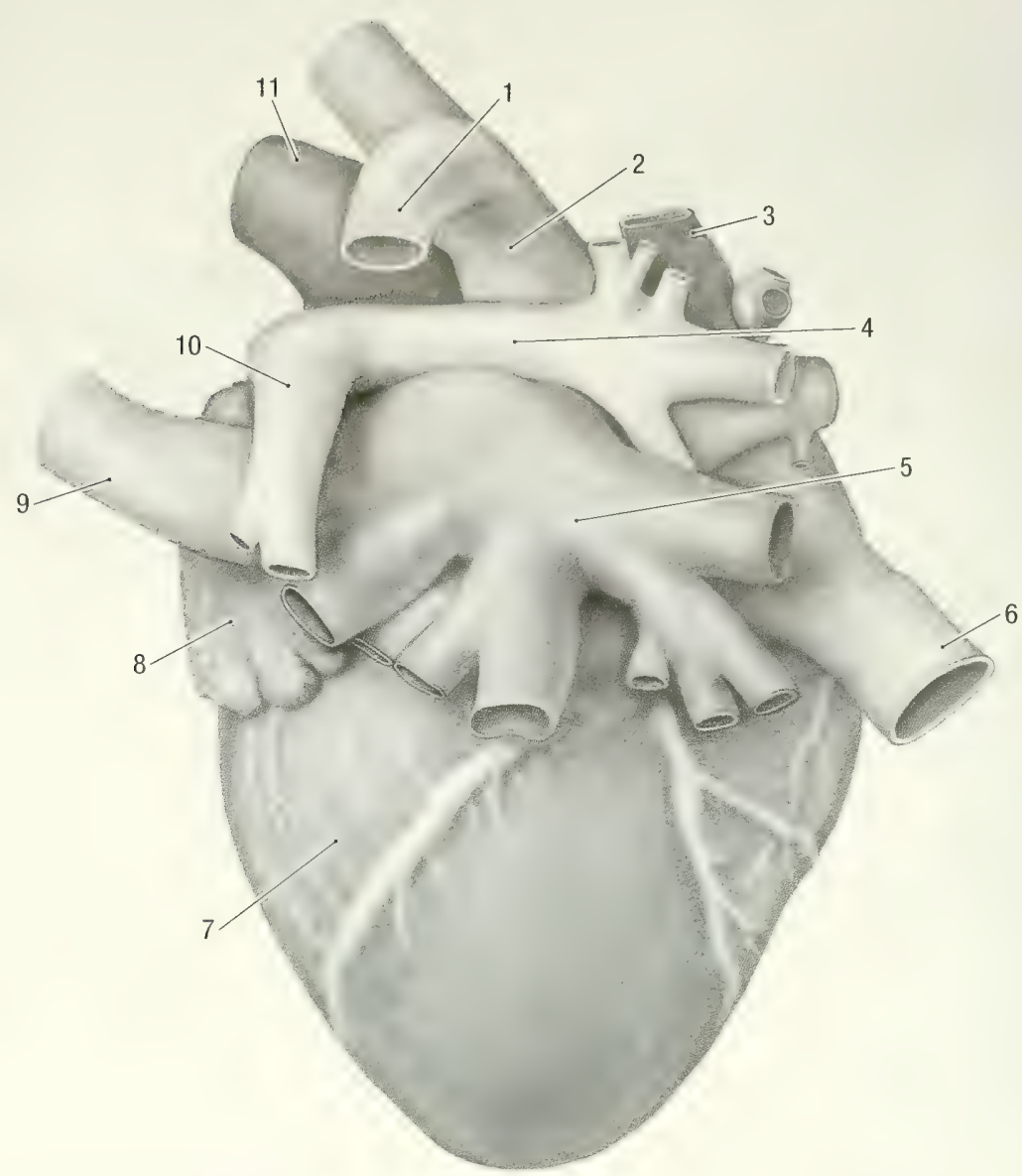

Fig. 9-4. Heart, caudal view. 1 right azygos v., 2 right cranial vena cava, 3 pulmonary v., 4 right pulmonary a., 5 pulmonary vv., 6 caudal vena cava, 7 left ventricle, 8 left auricle, 9 left cranial vena cava, 10 right pulmonary a., 11 aorta.

The left ventricle, ventriculus sinister, lies caudodorsally to the right ventricle and to the left of the midline. Its atrioventricular opening, ostium atrioventriculare sinistrum, is guarded by the bicuspid or left atrioventricular valve, valva atrioventricularis sinistra. The valve consists of the septal cusp cuspis septalis which is attached to the septal or medial aspect of the opening and the parietal cusp cuspis parietalis which is attached to the lateral aspect of the opening. Chordae tendineae attach the free edges of the cusps to papillary muscles. The outflow track of the left ventricle leads to the opening of the aorta, ostium aortae. The opening is guarded by three semilunar valvules: the valvula semilunaris dextra, septalis et sinistra.

Innervation: The heart is innervated by sympathetic fibres from the sympathetic trunk, cervicothoracic ganglion and middle cervical ganglion and by branches from the vagus nerves.

Vascular System of the Heart. Heart muscle is supplied by the coronary arteries. The right coronary artery, a. coronaria dextra, leaves the right caudolateral aspect of the aorta. The right auricle covers the aorta at its origin. The artery passes craniolaterally to reach the coronary groove, turns caudally as the right circumflex branch, $r$. circumflexus, and enters the subsinuosal interventricular groove as the subsinuosal interventricular branch, $r$. interventricularis subsinuosus. It supplies atrial and ventricular branches.

The left coronary artery, a. coronaria sinistra, leaves the left caudolateral aspect of the aorta. It passes to the left, caudal to the pulmonary trunk, to reach the coronary groove. At the coronary groove it gives off the paraconal interventricular branch, $r$. interventricularis paraconalis, and then continues caudally in the coronary groove as the left circumflex branch, $r$. circumflexus. Close to its origin the coronary artery gives off one or two septal branches to the interventricular septum and supplies ventricular and atrial branches as it passes along the paraconal interventricular and coronary grooves.

Cardiac veins drain the heart. The large cardiac vein, vena cordis magna, drains the area supplied by 
the left coronary artery. It accompanies the artery, but in opposite directions. The right coronary vein, vena cordis dextra, drains the right atrium and ventricle and accompanies the circumflex branch of the right artery. The middle cardiac vein, vena cordis media, drains both ventricles in the vicinity of the subsinuosal interventricular groove. It accompanies the subsinuosal branch of the right coronary artery. All the main cardiac veins drain into the coronary sinus. Some of the minor right cardiac veins drain directly into the right atrium.

\section{ARTERIAL SYSTEM}

Aorta and its Branches (Figs. 9-1 and 9-

2 ). The aorta leaves the left ventricle at the level of the third costal cartilage, just dorsal to the sternum. It passes cranially for a short distance as the ascending aorta, then arches dorsally to form the aortic arch and finally passes caudally as the descending aorta.

The ascending aorta, aorta ascendens, passes cranially for approximately $10 \mathrm{~mm}$ before it turns to the left and dorsally to form the aortic arch. It has a slight dilatation, the aortic bulb, bulbus aortae, immediately distal to the aortic valve. Both coronary arteries arise from the aortic bulb. The right coronary artery passes between the aorta and right auricle to reach the coronary groove. The left coronary artery passes between the pulmonary trunk and left auricle to reach the coronary groove. For the detail of their distribution, see the vascular system of the heart.

The aortic arch, arcus aortae, lies opposite the left second costal cartilage. The first vessel that comes off the aortic arch is the brachiocephalic trunk, truncus brachiocephalicus. The brachiocephalic trunk passes cranially in the cranial mediastinum where it gives rise to the left and right common carotid arteries to the head (occasionally the two vessels have a common origin from the aortic arch). The brachiocephalic trunk then turns to the right as the right subclavian artery (for the detail of the distribution of the carotid arteries, see the arteries of the head and neck). The next vessel to come off the aortic arch is the left subclavian artery, a. subclavia sinister (Fig. 10-1). It arises just distal to the origin of the brachiocephalic trunk.

The descending aorta, aorta descendens, passes caudally along the left ventrolateral border of the vertebral column. In the thorax it forms the thoracic aorta, in the abdomen the abdominal aorta. The thoracic aorta, aorta thoracica, supplies dorsal intercostal arteries, aa. intercostales dorsales, to the third to twelfth pair of ribs (for the detail of their distribution see the arteries of the trunk). The tho- racic aorta passes from the thorax to the abdomen between the two crura of the diaphragm.

The abdominal aorta, aorta abdominalis, passes along the ventral border of the sublumbar muscles, to the left and dorsal to the caudal vena cava. Branches of the abdominal aorta supply the abdominal viscera and body wall.

The lumbar arteries, aa. lumbales, leave the dorsal aspect of the abdominal aorta at each lumbar vertebra. Each lumbar artery divides into a dorsal branch, ramus dorsalis, which supplies the surrounding muscles and a spinal branch, ramus spinalis, which enters the intervertebral foramen to supply the meninges and spinal cord.

The celiac artery, a. celiaca, leaves the ventral surface of the abdominal aorta at the level of the first lumbar vertebra. It divides into the hepatic, left gastric and splenic arteries.

The hepatic artery, a. hepatica, leaves the cranial surface of the celiac artery. Ten to $20 \mathrm{~mm}$ from its origin it gives off the gastroduodenal artery and continues to the liver over the lesser curvature of the stomach (between the caudal vena cava to the right, and the papillary process of the liver to the left). It enters the right part of the porta hepatis where it divides into left and right hepatic branches. The terminal part of the left branch gives off a small gastroesophageal artery that supplies the abdominal esophagus and cardia of the stomach. For the detail of the branches to the lobes of the liver, see the blood supply to the liver.

The gastroduodenal artery, a. gastroduodenalis, passes to the right, dorsally to the pylorus of the stomach. It divides into the cranial pancreaticoduodenal and right gastroepiploic arteries.

The cranial pancreaticoduodenal artery, a. pancreaticoduodenalis cranialis, passes along the dorsal border of the duodenum to supply the right lobe of the pancreas and the descending duodenum.

The right gastroepiploic artery, a. gastroepiploica dextra, crosses the visceral surface of the pylorus and then turns to the right along the greater curvature of the stomach. It anastomoses with the left gastroepiploic artery.

The left gastric artery, a. gastrica sinistra, divides into three branches which supply the lesser curvature of the stomach. The first branch supplies the visceral surface, the second branch the parietal surface and the third branch the pylorus of the stomach.

The splenic artery, a. lienalis, comes off the caudal aspect of the celiac artery. It passes ventrally in the superficial leaf of the greater omentum, gives off a few branches to the spleen and continues along the greater curvature of the stomach as the left gastroepiploic artery, a. gastroepiploica sinistra. The latter anastomoses with the right gastroepiploic 
artery and supplies the greater curvature of the stomach.

The cranial mesenteric artery, a. mesenterica cranialis, leaves the ventral surface of the abdominal aorta at the level of the second lumbar vertebra. It passes ventrally in the mesentery and forms the blood supply to the intestine. Close to its origin it divides into many branches, each branch supplying a specific part of the intestine. From proximal to disal the following branches are given off:

The middle colic artery, a. colica media, supplies the transverse colon and the second loop of the ascending colon.

The right colic artery, a. colica dextra, supplies the first loop of the ascending colon.

The caudal pancreaticoduodenal artery, a. pancreaticoduodenalis caudalis, supplies the ascending duodenum and the left lobe of the pancreas.

A colic branch, ramus colicus, supplies the initial (straight) part of the ascending colon.

The next four or five branches are jejunal arteries, aa. jejunales, that supply the jejunum.

The continuation of the cranial mesenteric artery is the ileocolic artery, a.ileocolica, that supplies the ileum and cecum.

The left and right cranial abdominal arteries, $a a$. abdominales craniales, come off the abdominal aorta between the second and third lumbar vertebrae. They pass dorsal to the cranial poles of the kidneys and supply the cranial part of the dorsolateral abdominal wall. For the detail of the vessels, see the arteries of the body wall.

The adrenal artery, $a$. adrenalis, arises just cranial to the renal artery of the same side. It supplies the adrenal glands.

The renal arteries, aa. renales (Fig. 7-3), are paired. The right renal artery arises from the abdominal aorta at the level of the third lumbar vertebra and the left renal artery just caudal to it. Both renal arteries gives rise to caudolaterally directed vessels which pass ventral to the caudal poles of the kidneys. These branches supply the mesorchium in the male animal, or the broad ligament of the uterus in the female animal. The caudal phrenic artery, a. phrenica caudalis, arises from the right renal artery or directly from the abdominal aorta. It supplies the crura of the diaphragm. Both renal arteries divide into two or more branches before they enter the hilus of the kidneys.

The caudal mesenteric artery, a. mesenterica caudalis, arises from the ventral surface of the abdominal aorta at the level of the sixth lumbar vertebra. The vessel lies within the mesocolon and divides into left colic and cranial rectal arteries. The left colic artery, a. colica sinistra, supplies the descending colon. The cranial rectal artery, a. rectalis cranialis, passes along the dorsal surface of the rectum, from the pelvic inlet to the anus. It supplies most of the rectum and its terminal branches supply the peri-rectal and peri-anal adipose tissue between the tail and anus.

Deep circumflex iliac artery, a. circumflexa ilium profunda, arises from the aorta on each side between the sixth and seventh lumbar vertebrae. The vessels pass laterally and are joined by the lateral cutaneous femoral nerves before they pass through the body wall, just ventral to the tuber coxa. For the detail of the area of supply of the vessel, see the arteries of the body wall.

The testicular/ovarian artery, a. testicularis/ovarica, leaves the ventral surface of the abdominal aorta between the caudal mesenteric and deep circumflex iliac arteries. Each passes distally in the mesorchium of the male or in the broad ligament of the uterus in the female. Before they reach the gonad each vessel gives off a branch that passes cranially to supply the mesorchium/broad ligament. The main vessel continues to the testis or ovary. It becomes very tortuous and is closely associated with the testicular/ovarian vein to form a pampiniform plexus. In the female the ovarian artery also gives off a branch that supplies the uterine tubes and cranial part of the uterine horns.

At the level of the seventh lumbar vertebra the abdominal aorta divides into three, forming the two common iliac arteries and the median sacral artery. For the detail of their distribution, see the arteries of the pelvis and pelvic limb.

\section{Brachiocephalic Trunk and Subclavian} Arteries. The brachiocephalic trunk and subclavian arteries give rise to the vessels that supply the cranial and ventral parts of the thorax, the head, neck and the thoracic limbs. For the origin of the vessels, see the aortic arch.

From its origin the left subclavian artery, $a$. subclavia sinistra (Fig. 9-1), passes craniodorsally, crossing the lateral surface of the intra-thoracic part of the longus colli muscle. At the level of the first intercostal space it gives off the left internal thoracic artery, then the left costocervical trunk and medial to the first rib, the left vertebral and left dorsal scapular arteries. It leaves the thorax cranial to the first rib and continues in the thoracic limb as the right axillary artery.

The right subclavian artery, a. subclavia dextra, is the continuation of the brachiocephalic trunk after the common carotid arteries are given off. The first branch to leave the right subclavian artery is the right costocervical trunk, then the right internal thoracic, right dorsal scapular and right vertebral arteries. The right costocervical trunk gives off a large bronchoesophageal artery, $a$. broncho-esophagea (Fig. 9-2) close to its origin from the right sub- 
clavian artery. The broncho-esophageal artery passes caudally in the mediastinum along the dorsolateral aspect of the trachea (between the trachea and right cranial vena cava). It gives off branches to the mediastinum, trachea and esophagus. At the bifurcation of the trachea it divides into two branches which continue to the lungs along the dorsal aspects of the bronchi.

See the blood supply to the body wall for the detail of the arteries that originate from subclavian arteries.

Arteries of the Head and Neck. The head and neck are supplied by two major vessels, the common carotid and vertebral arteries. For a detailed comparative description of the arteries of the head in sciurids see Tandler 1899.

The common carotid artery, a. carotis communis, forms the main blood supply to the head, excluding the brain. In all sciurids the internal carotid artery obliterates after the origin of the stapedial artery, and the vertebral arteries supply the brain (Bugge 1971; Guthrie 1963). At the thoracic inlet the left and right arteries lie between the longus capitis and cleidomastoideus muscles. In the neck they are contained in a fibrous carotid sheath, accompanied by the internal jugular veins and vagosympathetic trunks, in a muscular groove between the sternothyroid and the longus capitis muscles. They are therefore separated from the trachea and esophagus by muscle.

Above the larynx the common carotid artery gives off a common stem for the internal carotid, occipital and condylar arteries. After a short course the internal carotid artery gives off the stapedial artery, a. stapedia. In some of the specimens examined the stapedial artery left the common carotid artery independently of the internal carotid artery.

The stapedial artery, a. stapedia, is the first branch of either the common carotid artery or the internal carotid artery. It passes rostrodorsally and enters the caudal part of petro-occipital fissure. Within the fissure it enters the petrous temporal bone, passes rostrally along the roof of the middle ear cavity and enters the cranial cavity through a foramen situated rostral to the petrous crest of the petrous temporal bone (see Fig. 2-11 for the foramen). It gives off the middle meningeal artery, $a$. meningea media, and continues rostrally along the ventrolateral aspect of the cranial cavity. Along its intracranial course the vessel receives an anastomotic branch from the maxillary artery through the petrotympanic fissure (Tandler 1899). The stapedial artery leaves the cranial cavity rostrally through the sphenofrontal foramen that opens into the caudal aspect of the bony orbit (see Fig. 2-7). As the vessel emerges from the foramen, it gives rise to the supraorbital artery, the external ethmoidal and external ophthalmic arteries. For a detailed description of the stapedial artery and its extracranial branches, see Bugge 1971.

The supraorbital artery, a.supraorbitalis, passes rostrodorsally along the dorsal wall of the bony orbit and then through the supraorbital incisure to ramify in the muscle and skin above the eye.

The external ethmoidal artery, a. ethmoidalis externa, passes dorsally for a short distance and then enters the ethmoid foramen to reach the ethmoidal fossa of the cranial cavity. It anastomoses with the internal ethmoidal branch of the rostral cerebral artery and then passes through the cribriform plate of the ethmoid bone. Its terminal branches ramify in the caudal part of the nasal cavity.

The external ophthalmic artery, a. ophthalmica externa, supplies the muscles, sclera and surrounding tissues of the eye.

The occipital artery, a. occipitalis, arises from a common stem for the occipital, condylar and internal carotid arteries. It passes dorsally, crossing the caudal surface of the paracondylar process and ramifies in the muscles of the cranial cervical and occipital regions.

The condylar artery, a. condylaris, passes through the condylar canal of the occipital bone and supplies the periosteum and dura mater along the floor of the foramen magnum and basi-occipital bone.

The internal carotid artery, a. carotis interna, comes off a common stem for the occipital, condylar and internal carotid arteries, or directly from the common carotid artery. In most animals the internal carotid artery is obliterated after the origin of the stapedial artery. In some animals a very small vessel remains that enters the petro-occipital fissure, passes rostrally and enters the cranial cavity through the carotid canal. It joins the cerebral arterial circle, but contributes minimally to the blood supply of the brain.

The internal carotid nerve accompanies the artery.

The external carotid artery, a. carotis externa, is the continuation of the common carotid artery beyond the origin of the common trunk for the internal carotid, occipital and condylar arteries. Close to its origin the external carotid artery gives off the cranial thyroid and cranial laryngeal arteries.

The cranial thyroidal artery, a. thyroidea cranialis, leaves the ventral aspect of the common carotid artery and enters the cranial pole of the thyroid gland. It passes along the deep face of the gland, giving off small branches that enter and supply the glandular tissue.

The cranial laryngeal artery, a. laryngea cranialis, leaves the ventral aspect of the common carotid ar- 
tery and enters the larynx through the cranial laryngeal incisure. It supplies the mucosa, cartilages and muscles of the larynx.

The external carotid artery continues rostrally along the dorsolateral surface of the pharynx. It gives off branches to the pharynx, passes medial to the stylohyoid bone and stylohyoid muscle, gives off the superficial temporal artery and then divides into the maxillary (internal maxillary artery of Tandler 1899) and linguofacial arteries.

The superficial temporal artery, a. temporalis superficialis, passes dorsolaterally, crossing the caudal surface of the tympanic bulla. Just ventral to the external acoustic meatus it gives off the caudal auricular artery (posterior auricular artery of Tandler 1899) and then it turns rostrally, crossing the base of the ear, deep to the parotid salivary gland.

The caudal auricular artery, a. auricularis caudalis, passes dorsally along the caudal surface of the external ear canal. It supplies the pinna by means of three vessels, one along the lateral border, one along the medial border, and one along the middle of the caudal surface of the pinna.

The superficial temporal artery continues rostrally, deep to the parotid salivary gland. It gives off numerous branches to the parotid gland, gives rise to the masseteric artery, a. masseterica, to the masseter muscle, the transverse facial and rostral auricular arteries (anterior auricular artery of Tandler 1899), and finally ramifies in the skin and muscle of the temporal region.

The rostral auricular artery, a. auricularis rostralis, supplies the ear canal and some of the muscle and skin along the rostromedial part of the pinna.

The transverse facial artery, a. transversa faciei, passes rostrally along the ventral border of the zygomatic arch, accompanied by the transverse facial nerve and vein. It supplies the surrounding tissues, including the lateral canthus of the eye.

The linguofacial artery, a. linguofacialis, gives rise to the lingual and facial arteries. In $50 \%$ of animals the two vessels arise separately from the external carotid artery. At the origin of the lingual artery a large pharyngeal branch comes off either from the lingual or maxillary artery.

The lingual artery, a. lingualis, forms the blood supply of the tongue. It passes medial to the digastric muscle and enters the root of the tongue deep to the hyoglossal muscle and hyoid bone. The vessel is joined by the hypoglossal nerve as it passes rostrally along the deep face of the genioglossus muscle. Along its course from the root to the apex of the tongue it gives off dorsally directed branches which supply the tongue. A pharyngeal branch, $r$. pharyngealis, leaves the lingual or maxillary artery, ramifies in the rostral part of the pharynx and supplies the palatine tonsil.
The facial artery, a. facialis (external maxillary artery of Tandler 1899), passes lateral to the digastric muscle, gives off the sublingual artery (submental artery of Tandler 1899) and emerges superficially in the groove between the rostral belly of the digastric muscle and the ventral border of the masseter muscle. It curves dorsally along the rostral border of the masseter muscle and gives off branches to the lower lip, angle of the mouth and the caudal part of the upper lip, medial canthus of the eye and lateral nasal region.

The sublingual artery, a. sublingualis, passes rostrally along the deep face of the mylohyoid muscle. It supplies the floor of the mouth. Either the left or the right sublingual artery continues to the tip of the tongue, the other emerges superficially between the two bellies of the digastric muscle to supply the chin.

The maxillary artery, a. maxillaris (internal maxillary artery of Tandler 1899), turns dorsally at its origin from the external carotid artery. It gives off the inferior alveolar artery as well as branches to the roof of the pharynx and pterygoid muscles before it enters the caudal alar foramen. The vessel passes through the alar canal and emerges at the rostral alar foramen where it gives off small branches to the muscles of the eye, the lacrimal artery and then the large buccal and small minor palatine arteries. It continues rostrally along the ventral border of the periorbita, giving off the sphenopalatine, major palatine and malar arteries before it enters the maxillary foramen to become the infraorbital artery.

The inferior alveolar artery, a. alveolaris inferior, enters the mandibular foramen, passes rostrally in the alveolar canal and emerges at the mental foramen to supply part of the lower lip. Along its course in the alveolar canal it gives off branches to the teeth of the lower jaw.

The buccal artery, a. buccalis (buccolabial artery of Tandler 1899), turns rostrolaterally, gives off branches to the temporal and masseter muscles and then ramifies in and supplies the tissues of the buccal region.

The minor palatine artery, a. palatina minor, passes ventrally through the caudal palatine foramen. It supplies the soft palate.

The major palatine artery, a. palatina major, passes through the palatine canal and then rostrally along the ventral surface of the hard palate. It supplies the hard palate and surrounding tissues.

The sphenopalatine artery, a. sphenopalatina, passes through the sphenopalatine foramen and then rostrally along the lateral wall of the nasopharynx. It supplies the floor and walls of the nasopharynx as well as the choanae.

The malar artery, a. malaris, passes dorsally along 
the rostral border of the bony orbit. It ramifies in the lower eye-lid and skin below the eye.

The infraorbital artery, a. infraorbitalis, passes through the infraorbital canal and emerges at the infraorbital foramen. Within the canal it gives off branches to the upper teeth. Its terminal branches supply the lateral aspect of the nose and upper lip.

The vertebral artery, $a$. vertebralis, arises from the subclavian artery and runs dorsocranially, leaving the thorax medial to the first rib. It passes medial to the transverse process of the seventh cervical vertebra and enters the transverse foramen of the sixth cervical vertebra. The vessel continues cranially through the transverse foramina of successive cervical vertebrae. At each cervical vertebra it gives off branches to the surrounding muscle, as well as spinal branches which enter the vertebral canal through the intervertebral foramina. The spinal branches join the ventral spinal artery.

The vertebral artery passes through the lateral vertebral foramen of the atlas to enter the vertebral canal. It courses ventrally and is joined by the smaller ventral spinal artery. The left and right vertebral arteries anastomose on the ventral surface of the myelencephalon and continue rostrally as the basilar artery. The vertebral arteries form the main blood supply to the brain.

Arteries of the Brain and Spinal Cord (Fig. 9-6). The ventral spinal artery, a. spinalis ventralis, is formed by contributions from the spinal branches of the vertebral, intercostal, lumbar and sacral arteries. It lies in the ventral median fissure of the spinal cord and gives off branches that penetrate and supply the spinal cord. At the level of the atlas the ventral spinal artery joins the vertebral arteries.

The basilar artery, a. basilaris, continues rostrally along the ventral surface of the myelencephalon. It gives off branches to the myelencephalon, inner ear, pons and cerebellum. At the rostral border of the pons the basilar artery divides into two caudal communicating arteries, a. communicans caudalis. The vessels pass on either side of the hypophysis and form the caudal part of the cerebral arterial circle, circulus arteriosus cerebri. Each gives rise to a caudal cerebral artery, a. cerebri caudalis, which supplies the medial part of the cerebral hemisphere and is joined by the internal carotid artery. The latter vessel is minute and its contribution to the arterial supply of the brain is minimal. A small choroidal artery, a. choriodea rostralis, accompannies each optic nerve to supply the retina of the eye. The next vessel to leave the arterial circle is the middle cerebral artery, a. cerebri media, which supplies the lateral part of the cerebral hemisphere. The arterial circle continues as the rostral cerebral artery, a. cer- ebri rostralis, which supplies the rostromedial aspect of the cerebral hemisphere and the olfactory bulb. They anastomose with the ethmoidal arteries (see the branches of the maxillary artery). Rostral to the optic chiasma the cerebral arterial circle is completed by the rostral communicating artery, a. communicans rostralis. The latter is a small vessel that connects the left and right rostral cerebral arteries.

Arteries of the Trunk. The costocervical trunk, truncus costocervicalis, is a branch of the subclavian artery. From its origin the costocervical trunk passes dorsally, gives off the dorsal intercostal arteries to the first two ribs and continues as the deep cervical artery. The deep cervical artery, a. cervicalis profunda, leaves the thorax between the heads of the second and third ribs. It supplies the deep (epaxial) muscles of the caudal cervical and cranial thoracic regions.

The dorsal intercostal arteries, aa. intercostales dorsales (Fig. 9-2), are a series of paired, segmentally arranged vessels to the thoracic wall. The first two pairs arise from the costocervical trunks and the rest from the thoracic aorta. All the vessels pass dorsolaterally to reach the caudal aspect of the ribs. From there they follow the arch of the ribs, accompanied by the intercostal nerves and veins. They anastomose with the ventral intercostal arteries and supply the intercostal muscles and surrounding tissues, including the parietal pleura.

The internal thoracic artery, a. thoracica interna (Figs. 9-1 and 9-2), arises from the ventral aspect of the subclavian artery at the level of the first intercostal space. It passes caudoventrally in the cranial mediastinum and gives off branches to the thymus and surrounding tissue. It continues caudally, deep to the transverse thoracic muscle and approximately $10 \mathrm{~mm}$ from the midline, giving off the ventral intercostal arteries to the ribs.

The ventral intercostal arteries, aa. intercostales ventrales, cross the medial aspect of the costal cartilages to reach the caudal aspect of the ribs. They anastomose with the dorsal intercostal vessels.

Small perforating vessels arise from the internal thoracic artery. They supply the deep pectoral muscles and the medial aspect of the thoracic mammary glands.

At the level of the seventh intercostal space the internal thoracic artery divides into the musculophrenic and cranial epigastrica arteries. The musculophrenic artery, a. musculophrenica, passes dorsolaterally between the diaphragm and the costal arch. It supplies the costal part of the diaphragm and the surrounding intercostal muscles.

The cranial epigastric artery, a. epigastrica cranialis, passes between the costal arch and the xiphoid cartilage to gain the deep face of the rectus abdom- 
inis muscle (between the rectus and transverse abdominal muscles). It supplies both muscles and anastomoses with the caudal epigastric artery.

The dorsal scapular artery, a. scapularis dorsalis, is a branch of the subclavian artery that leaves the thorax medial to the first rib and reaches the medial aspect of the shoulder where it divides into cranial, middle and caudal branches. All the branches lie on the lateral surface of the serratus ventralis muscle. The cranial and caudal branches accompany the dorsal thoracic vein and long thoracic nerve. The dorsal branch passes dorsocaudally to the insertion of the serratus ventralis muscle. It supplies the serratus ventralis and rhomboideus muscles at their insertions on the scapula.

The cranial abdominal artery, a. abdominalis cranialis, is a branch of the abdominal aorta. Form its origin it passes laterally, crosses the dorsal aspect of the cranial pole of the kidney, and is joined by the iliohypogastric nerve before it passes through the abdominal wall. The vessel accompanies the nerve and supplies the cranial part of the dorsolateral wall of the abdomen.

The deep circumflex iliac artery, a. circumflexa ilium profunda, is a branch of the abdominal aorta that appears on the surface of the abdomen just ventral to the tuber coxae. It lies deep to the accompaning vein and branches to supply the skin over the cranial gluteal, lateral femoral and caudal abdominal regions. One branch passes distally along the craniolateral aspect of the thigh. It accompanies the lateral cutaneous femoral nerve to the knee.

The external pudendal artery, a. pudenda externa, is a branch of the external iliac artery that emerges at the caudal angle of the superficial inguinal ring. In the male it lies deep to the tunica vaginalis of the testis. The vessel gives rise to the preputial/ mammary, ventral scrotalllabial and caudal superficial epigastric arteries.

In the male the preputial branch, ramus preputialis, supplies the preputium, and the ventral scrotal branch, ramus scrotalis ventralis, supplies the scrotum.

In the female the mammary branch, ramus mammarius, supplies the inguinal mammary gland and the ventral labial artery, ramus labialis ventralis, supplies the vulva.

The caudal superficial epigastric artery, a. epigastrica caudalis superficialis, passes craniolaterally to supply the ventral abdominal region. At its origin the vessel lies $10 \mathrm{~mm}$ from the ventral midline, covered by three or four superficial inguinal lymph nodes. In the female it forms part of the arterial supply to the inguinal and abdominal mammary glands.
Arteries of the Thoracic Limb. The subclavian artery leaves the thorax cranial to the first rib and enters the medial aspect of the axilla. It continues as the axillary artery that forms the blood supply to the thoracic limb.

The large axillary artery $a$. axillaris, passes distally along the medial aspect of the axilla. It gives rise to the superficial cervical, external thoracic, lateral thoracic, thoracodorsal and subscapular arteries.

The superficial cervical artery, a. cervicalis superficialis, is the first branch of the axillary artery (in some specimens it comes from the subclavian artery). It leaves the cranial border of the axillary artery, ventral to the brachial plexus. The vessel gives off the suprascapular artery and continues cranially, deep to the maxillary vein, to supply the tissues at the base of the neck, including the superficial cervical lymph node. The suprascapular artery, a. suprascapularis, accompanies the suprascapular nerve around the cranial aspect of the scapular neck to supply the supra- and infraspinatus muscles.

The external thoracic artery, a. thoracica externa, leaves the craniomedial border of the axillary artery. It supplies the superficial pectoral muscles.

The lateral thoracic artery $a$. thoracica lateralis, leaves the caudomedial border of the axillary artery under cover of the brown adipose tissue of the axilla. It passes caudally along the deep face of the deep pectoral muscle, accompanied by the external thoracic vein and lateral thoracic nerve. The axillary lymph nodes are arranged along the initial part of the artery. The vessel branches profusely in the surrounding adipose tissue and supplies branches to the deep pectoral muscles. It appears superficially along the caudolateral border of the deep pectoral muscle and continues caudally on the abdominal muscles, supplying the surrounding tissues, including the two thoracic mammary glands. It finally anastomosis with the caudal superficial epigastric artery.

The thoracodorsal artery, a. thoracodorsalis, leaves the caudal border of the axillary artery, distal to the origin of the lateral thoracic artery. It passes dorsocaudally in the groove between the latissimus dorsi and subscapular muscles, supplying branches to the surrounding muscles. The terminal branches of the artery emerge superficially behind the long head of the triceps muscle where it ramifies in the surrounding tissues, including the accessory axillary lymph node.

The subscapular artery, a. subscapularis, leaves the caudal border of the axillary artery. It is the largest branch of the axillary artery and is accompanied by the axillobrachial vein. It gives rise to the collateral radial artery and passes laterally across the caudal border of the shoulder joint, between the long head of the triceps and the teres major muscles 
and ramifies in the muscles caudolateral to the shoulder. The collateral radial artery, a. collateralis radialis, arises from the subscapular artery caudal to the shoulder joint. It accompanies the radial nerve as the latter pass caudodistally in the arm between the triceps and brachial muscles. Along its course it supplies the triceps muscles. The artery appears superficially in the cubital fossa from underneath the lateral head of the triceps muscle to supply the skin of the cranial antebrachial region.

Distal to the origin of the subscapular artery the axillary artery continues as the brachial artery.

The brachial artery, a. brachialis, is the main artery of the brachium. It passes distally along the medial aspect of the arm, in the groove between the medial head of the triceps and biceps muscles. The ulnar and median nerves, and the brachial vein accompany the artery. Along its course it gives rise to the deep brachial, bicipital, collateral ulnar and transverse cubital arteries.

The deep brachial artery, a. profunda brachii, leaves the caudal aspect of the brachial artery to supply the triceps muscles.

The bicipital artery, a. bicipitalis, leaves the cranial aspect of the brachial artery to supply the distal part of the biceps brachii muscle.

The collateral ulnar artery, a. collateralis ulnaris, comes off the distal part of the brachial artery. It passes caudally along the medial aspect of the elbow and then turns distally on the caudal aspect of the antebrachium to supply the skin of the surrounding area.

The transverse cubital artery, a. transversa cubiti, leaves the brachial artery at the level of the elbow. It passes from medial to lateral across the cubital fossa, deep to the brachialis and biceps brachii muscles. Along its course it gives off the superficial cranial antebrachial artery and then turns distally, under cover of the $\mathrm{m}$. extensor carpi radialis, to supply all the extensors of the antebrachium. The superficial cranial antebrachial artery, a. antebrachialis superficialis cranialis, appears from underneath the lateral head of the triceps muscle and passes distally along the craniomedial aspect of the antebrachium, medial to the cephalic vein, to the carpus and manus. On the manus it divides to form the dorsal superficial supply to the digits. The vessels to the first, fourth and fifth toes are reinforced by branches from the palmar arteries (see radial artery).

The brachial artery continues in the antebrachium as the median artery, a. mediana. The vessel passes through the supracondylar foramen or cranially to the medial condyle of the humerus when the supracondylar foramen is absent. It crosses the craniomedial aspect of the elbow joint and passes between the humerus and the pronator teres muscle (between the pronator teres and the insertion of the biceps brachii muscles). It continues distally in the antebrachium, deep to the radial carpal flexor muscle. Proximally it gives off a small cranial interosseus artery, a large ulnar artery and the deep antebrachial artery. In the middle of the forearm it gives off the radial artery. Above the carpus it forms a sigmoid flexure and joins the median nerve on the caudomedial aspect of the deep digital flexor muscle. The median artery passes medial to the accessory carpal bone, between the digital flexor tendons and the carpal pads and ends by forming the superficial palmar arterial arch from which the vessels that supply the manus arise.

The cranial interosseus artery, $a$. interossea cranialis, passes distally in the interosseus space between the radius and ulna. It supplies the pronator quadratus muscle.

The ulnar artery, a. ulnaris, passes caudodistally between the ulnar and humeral heads of the deep digital flexor muscle to join and accompany the ulnar nerve. It supplies the surrounding muscles and anastomoses with the superficial palmar arch to reinforce the supply to the digits.

The deep antebrachial artery, a. profunda antebrachii, leaves the caudal surface of the median artery. It supplies the flexor muscles of the antebrachium.

The radial artery, $a$. radialis, passes craniodistally in the forearm, deep to the flexor carpi radialis muscle and appears superficially on the medial aspect of the distal antebrachium. It gives off an anastomosing branch to the superficial cranial antebrachial artery that reinforces the dorsal superficial supply to the manus and then divides into dorsal and palmar branches. The dorsal branch forms a poorly developed dorsal carpal rete, rete carpi dorsalis, on the dorsal aspect of the carpus. The palmar branch joins the small deep palmar arch. The contribution to the blood supply of the manus from the dorsal rete and deep palmar arch is minimal.

Arteries of the Manus. The manus have superficial and deep dorsal and palmar vessels. Both dorsal and deep palmar vessels are poorly developed and contribute little to the arterial supply of the digits. The continuation of the median artery, the superficial palmar arch, forms the main supply to the digits.

The superficial dorsal arteries of the digits are derived from the superficial cranial antebrachial artery. At the carpus the vessel divides to supply a branch to each toe. Branches from the radial and collateral ulnar arteries reinforce the branches to the first and fifth toes.

The deep dorsal arteries of the digits are derived from the dorsal carpal rete. The rete gives rise to small vessels that supply the proximal part of the 
manus. The vessels do not reach the metacarpophalangeal joints.

The palmar arteries of the digits are derived from the superficial and deep palmar arches.

The superficial palmar arch, arcus palmaris superficialis, is the largest and is formed by the median artery. It supplies a palmar digital artery to each toe.

The palmar branch of the radial artery forms the deep palmar arch, arcus palmaris profundus. The arch gives rise to branches to each toe. The branches supply the short muscles of the manus and anastomose with the superficial palmar digital arteries at the level of the phalangodigital joints.

Arteries of the Pelvis and Tail. The abdominal aorta divides at the level of the last lumbar vertebra into the two common iliac and the median sacral arteries. The three vessels supply the pelvis and its contents, the tail and the pelvic limb. The origin of the vessels that supply a specific area is very variable. In the following descriptions the origins most commonly found are used.

The median sacral artery, a. sacralis mediana, passes caudally along the median pelvic surface of the sacrum. It gives rise to dorsally directed segmental sacral branches, rami sacrales, which enter the ventral sacral foramina. They supply the contents of the sacral canal. The median sacral artery continues along the ventral aspect of the tail as the median artery of the tail.

The median artery of the tail, a. caudalis mediana, gives off dorsally directed vessels which anastomose along the lateral aspect of the tail to form the left and right ventrolateral arteries of the tail, a. caudalis ventrolateralis. Small branches leave the median and lateral arteries to supply the muscles and surrounding tissues of the tail.

From its origin off the abdominal aorta the common iliac artery, a. iliaca communis (Fig. 4-14), passes ventrolaterally, crossing the abdominal surface of the iliopsoas muscles. It gives rise to the cranial gluteal and iliacofemoral arteries before it divides into the external and internal iliac arteries.

The cranial gluteal artery, a. glutea cranialis, leaves the dorsal aspect of the common iliac artery. It passes along the caudodorsal wall of the pelvic cavity and through the greater ischiadic foramen to supply the deep and middle gluteal muscles.

The iliacofemoral artery, a. itiacofemoralis, leaves the dorsal aspect of the common iliac artery. It passes caudolaterally, between the iliopsoas muscle and the shaft of the ilium (approximately $1 \mathrm{~cm}$ dorsal to the acetabulum) to reach the deep face of the tensor fascia lata muscle. The artery supplies the iliopsoas and tensor fascia lata muscles, as well as the surrounding tissues.
Close to its origin (approximately $10 \mathrm{~mm}$ ) the internal iliac artery, a. iliaca interna, gives rise to the umbilical and caudal gluteal arteries and continues in the pelvis as the internal pudendal artery.

The umbilical artery, a. umbilicalis, leaves the ventral surface of the internal iliac artery. From its origin it passes caudomedially in the cranial part of the lateral ligament of the urinary bladder. It gives rise to an ureteric branch which supplies the ureter, a branch to the ductus deferens of the male animal and the cranial vesicle artery to the bladder.

The caudal gluteal artery, a. glutea caudalis, is a large vessel that leaves the dorsal aspect of the internal iliac artery. It passes caudodorsally along the lateral wall of the pelvic cavity, gives rise to the obturator and the first ventral sacral arteries and then it leaves the pelvic cavity through the lesser ischiadic foramen. The artery ramifies in the muscles on the lateral aspect of the pelvis.

The obturator artery, a. obturatoria, supplies the intra pelvic muscles and and then passes through the obturator foramen to supply most of the medial thigh muscles (adductor group).

The internal pudendal artery, $a$. pudenda interna, passes caudally into the pelvic cavity. It is closely attached to the dorsolateral border of the urethra in the male or the vagina in the female. It gives rise to the prostatic/vaginal artery, caudal rectal artery and the artery of the penis/clitoris. In the male the prostatic artery, a. prostatica, forms the main blood supply to the pelvic viscera of the male. It gives off the caudal vesical artery, a. vesicalis caudalis, to the neck and caudal part of the urinary bladder, the urethral artery, a. urethralis, to the urethra and branches to the prostate and seminal vesicles. As in the male, the vaginal artery, a. vaginalis, of the female gives off arteries to the bladder and urethra, as well as the uterine artery. The uterine artery, $a$. uterina, is the main branch of the vaginal artery. It enters the mesometrium at the level of the cervix, supplies a branch to the vagina and passes cranially along the mesometrial border of the uterine horn. It supplies the uterus and anastomoses with the uterine branch of the ovarian artery.

The prostatic/vaginal artery continues caudally along the ventrolateral aspect of the urethra/vagina. At the pelvic outlet it gives off the middle rectal artery, a. rectalis media, to the ventrolateral aspect of the rectum and the ventral perineal artery, $a$. perinealis ventralis, to the perineal region.

The caudal rectal artery, a. rectalis caudalis, leaves the internal pudendal artery and enters the cranial border of the external anal sphincter. It is accompanied by the caudal rectal nerve and supplies the rectum and anus.

In the male, the artery of the penis, $a$. penis, supplies three branches to the penis, namely the deep 
artery of the penis, the artery of the penile bulb and the dorsal penile artery. The deep artery of the penis, a. profunda penis, supplies the crura (corpus cavernosum) of the penis. The artery of the penile bulb, a. bulbi penis, supplies the penile bulb and corpus spongiosum of the penis. The dorsal artery of the penis, a. penis dorsalis, passes along the dorsal aspect of the penis to supply the tissues surrounding the penis. The equivelant vessels in the female are the artery of the clitoris, $a$. clitoridis, giving off the artery of the vestibular bulb, a. bulbi vestibuli, the deep clitoridal artery, a. profunda clitoridis and the dorsal clitoridal artery, $a$. dorsalis clitoridis.

Arteries of the Pelvic Limb. The external iliac artery and its branches supply the pelvic limb.

The external iliac artery, a. iliaca externa passes distally along the cranial border of the body of the ilium. The external iliac vein and the femoral nerve accompany the artery. It gives off the medial circumflex femoral artery and the pudendoepigastric trunk and then continuous as the femoral artery.

The medial circumflex femoral artery, a. circumflexa femoris medialis, is the first branch of the external iliac artery. It passes caudally, crossing the medial surface of the iliopsoas and vastus medialis muscles. It supplies the medial thigh muscles.

The pudendoepigastric trunk, truncus pudendoepigastricus, is the second branch of the external iliac artery that comes off opposite the medial circumflex femoral artery and is accompanied by the femoro-inguinal nerve. The vessel passes laterally and divides into the caudal epigastric and external pudendal arteries.

The caudal epigastric artery, a. epigastrica caudalis, passes cranially along the deep face of the rectus abdominis muscle. It supplies the latter muscle and the surrounding peritoneum.

The external pudendal artery, a. pudenda externa, passes through the inguinal canal to supply the skin of the external genitalia. For the detail of its distribution, see the arteries of the trunk.

The continuation of the external iliac artery in the pelvic limb is the femoral artery. The latter passes through the vascular lacuna to reach the medial aspect of the thigh. Initially it lies superficially, but passes progressively deeper between the femur and adductor muscles. It reaches the caudal aspect of the femur just proximal to the knee.

Along its course the femoral artery, a. femoralis, gives rise to the lateral circumflex femoral, saphenous, descending genicular, and caudal femoral arteries.

The lateral circumflex femoral artery, a. circumflexa femoris lateralis, leaves the femoral artery just distal to the vascular lacuna. It enters the quadriceps muscle proximally between the rectus femoris and vastus medialis muscles. The vessel is accompanied by the femoral nerve and supplies the quadriceps muscle. Its terminal branches anastomose with branches from the medial circumflex femoral artery.

The saphenous artery, a. saphena, leaves the medial surface of the femoral artery at the middle of the thigh. It is accompanied by the saphenous nerve and vein and forms the blood supply to the pes and toes. The sartorius muscle initially covers the vessels and nerve. They appear superficially along the caudal border of the muscle in the distal third of the thigh. In this position the nerve lies cranial to the artery and the vein. As the artery passes distally along the medial aspect of the crus, it gives off small vessels to the surrounding tissues. In the middle of the crus it divides into cranial and caudal branches.

The cranial branch divides into a larger lateral branch and a smaller medial branch. The medial branch remains with the saphenous vein and nerve, and anastomoses with the dorsal pedal arch. The lateral branch passes distolaterally between the tibia and the tendon of the long digital flexor muscle. It forms a sigmoid curve just above the ankle. At the sigmoid curve it anatomoses with the cranial tibial artery and a branch from the caudal femoral artery. The vessel continues over the flexor surface of the ankle, lateral to the tendon of the cranial tibial muscle and deep to the tendon of the long digital extensor muscle, as the dorsal pedal artery, a. pedis dorsalis. The dorsal pedal artery gives off small vessels to the ankle and a small dorsal pedal arch from which the dorsal digital arteries to the pes arise. The main vessel continues distally, between the second and third metacarpal bones and deep to the extensor tendons. At the middle of the pes it passes from the dorsal to the plantar surface between the second and third metacarpal bones to anastomose with the superficial plantar arch (see below).

The caudal branch of the saphenous artery passes over the plantar surface of the ankle, medial to the calcaneus and superficial to the deep flexor tendon. It continues distally in the pes, deep to the superficial flexor tendons, where it forms the superficial plantar arch. The dorsal pedal artery reinforces the plantar system by anastomosing with the superficial plantar arch. The plantar arteries to the pes arise from the superficial plantar arch.

At the metatarsophalangeal joints the dorsal digital arteries anastomose with their equivalent plantar digital arteries before they divide to form the proper digital arteries.

The descending genicular artery, a. genus descendens, of the knee leaves the cranial aspect of the femoral artery distal to the origin of the saphenous artery. It passes distally in the groove between the 
vastus medialis and adductor muscles and supplies the craniomedial aspect of the thigh and knee.

There are four or five caudal femoral arteries, $a a$. caudales femores. They arise from the femoral artery at the level of the knee joint to supply the caudal thigh and gastrocnemius muscles. One branch supplies the popliteal lymph node and accompanies the caudal sural nerve in the crus. It anastomoses with the sigmoid flexure of the saphenous artery above the ankle. The continuation of the femoral artery beyond the origin of the most distal caudal femoral artery is the popliteal artery.

The popliteal artery, a. poplitea, passes between the two condyles of the femur and over the flexor surface of knee joint. It supplies branches to the knee and divides into the cranial and caudal tibial arteries.

The caudal tibial artery, a. tibialis caudalis, is the larger of the two vessels. It passes distally in the crus on the caudal surface of the popliteal and deep digital flexor muscles. The vessel accompanies the tibial nerve and supplies the surrounding muscles.

The cranial tibial artery, a. tibialis cranialis, passes deep to the popliteus muscle, then between the tibia and fibula to reach the cranial aspect of the tibia. It continues distally in the crus along the cranial border of the tibia, deep to the peroneus brevis muscle. The deep peroneal nerve accompanies the artery. The vessel emerges distally from under the muscles and joins the sigmoid flexure of the saphenous artery above the ankle.

Arteries of the Pes. The cranial and caudal branches of the saphenous artery supply the foot (see above). They form dorsal and plantar pedal arches from which the vessels to the digits arise. The dorsal pedal arch gives rise to the dorsal digital arteries and the plantar pedal arch gives rise to the plantar digital arteries. The latter are large and form the main arterial supply to the digits. In addition to the plantar digital arteries, the plantar pedal arch also gives rise to deep digital vessels that supply the short flexor muscles of the pes.

\section{VENOUS SYSTEM}

The venous drainage of the body generally follows the pattern of arterial supply (the veins are satellites of the arteries). In addition to these vessels, a superficial venous system is often present.

Two cranial caval veins and one caudal caval vein return venous blood from the body to the right atrium of the heart. In addition to the caval veins, pulmonary veins drain the lungs and cardiac veins drain the heart.

Pulmonary Veins. Four or five pulmonary veins, vw. pulmonales (Figs. 9-3 and 9-4), drain the lungs. They leave the lungs at the hilus and open directly into the dorsal aspect of the left atrium.

Cardiac Veins. Cardiac veins drain the heart. See "Blood vessels of the heart" for the detail of the vessels.

Cranial Vena Cava. Two cranial caval veins are present in the woodchuck. They drain the head, neck, thoracic limbs and thoracic wall.

The left cranial vena cava, $v$. cava cranialis sinistra (Fig. 9-1), is the continuation of the left brachiocephalic vein towards the heart after receiving the left costocervical vein. The left cranial vena cava crosses the lateral surface of the aortic arch and passes between the hilus of the lung and the left auricle. The vein enters the coronary groove caudal to the left auricle and opens into the coronary sinus of the right atrium.

The right cranial vena cava, $v$. cava cranialis dextra (Fig. 9-2), is the continuation of the right brachiocephalic vein towards the heart after receiving the azygos vein. The cranial vena cava passes dorsal and lateral to the ascending aorta and drains into the cranial part of the right atrium.

Veins of the Head and Neck. The linguofacial and maxillary veins drain the head. The linguofacial vein drains the rostral part of the head and the maxillary vein the caudal part of the head. Many of the veins of the head drain into or originate from the ophthalmic and pterygoid plexuses.

An ophthalmic plexus, plexus ophthalmicus, lies caudal to the eyeball in the bony orbit. It drains the structures of the orbit and anastomoses with the supraorbital and superficial temporal veins. It also receives a small vein from the temporal muscle.

The ophthalmic plexus drains into the cavernous sinus (see venous drainage of the central nervous system) through the orbital fissure and gives rise to the external ophthalmic vein. The ophthalmic vein is not a satellite vein of the external ophthalmic artery. It drains directly into the maxillary vein.

The pterygoid plexus, plexus pterygoideus, is a large venous plexus between the mandible and the medial pterygoid muscle. Many of the veins of the head drain into the plexus or anastomose with it. The plexus is connected with the cavernous sinus and also drains into the maxillary vein.

The linguofacial vein, $v$. linguofacialis (Figs. 9-5 and 9-8), is formed by the confluence of the lingual and facial veins. They are satellites of the linguofacial artery and its branches, and drain the areas supplied by the homologous arteries.

The facial vein, $v$. facialis, drains the face and is formed by the supraorbital, dorsal nasal, buccal, in- 


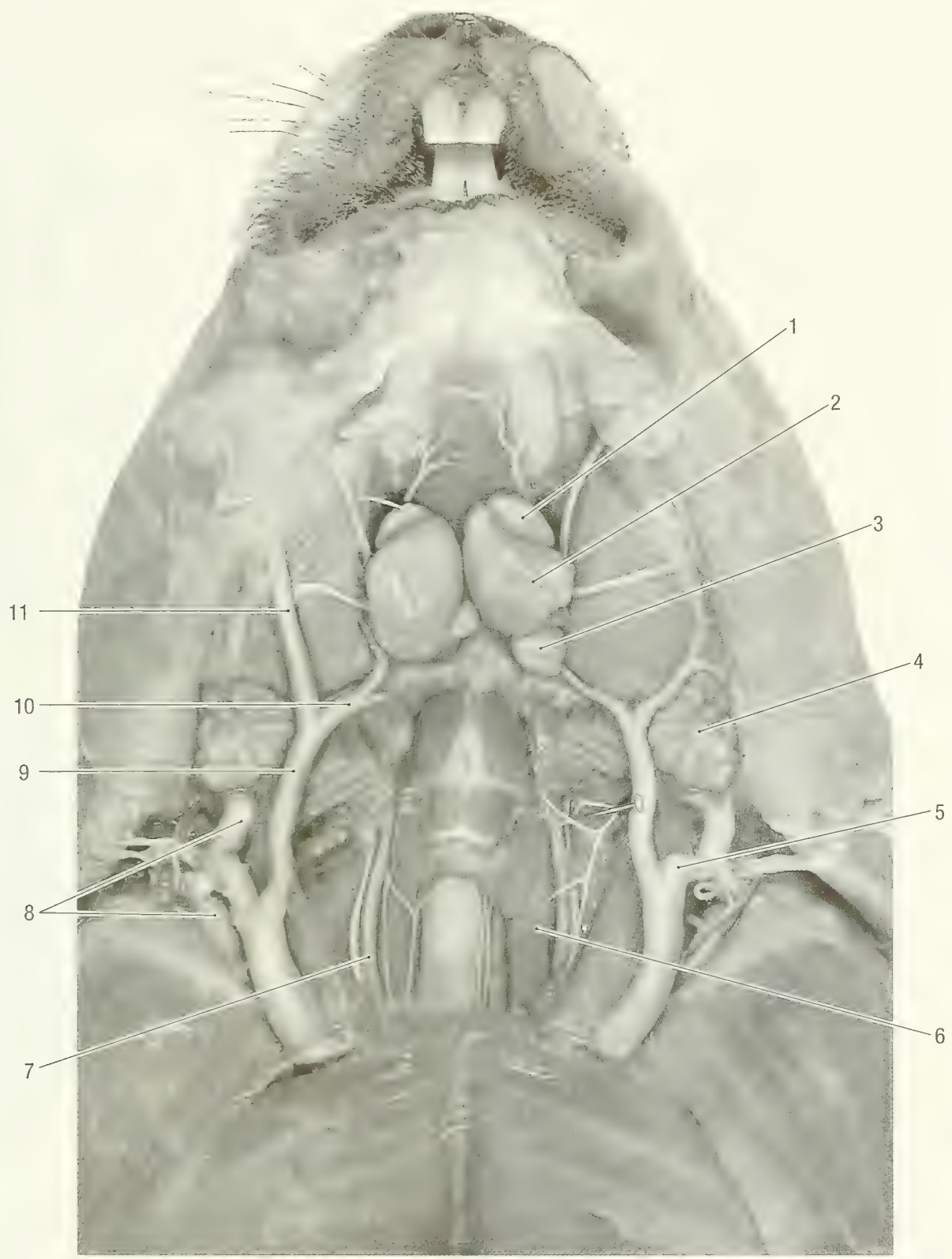

Fig. 9-5. Blood vessels of the ventral neck region. 1 monostomatic sublingual salivary gland, 2 mandibular salilvary gland, 3 mandibular In., 4 parotid salivary gland, 5 anastomosis between linguofacial and maxillary veins, 6 thyroid gland, 7 common carotid a., 8 maxillary v., 9 linguofacial v., 10 lingual v., 11 facial v.

ferior and superior labial veins, as well as the veins of the lower eyelid and angle of the eye.

The supraorbital vein, $v$. supraorbitalis, drains the area medial to the medial canthus of the eye. It is connected rostrally to the dorsal nasal vein, caudally it passes through the supraorbital incisure to anastomose with the ophthalmic plexus.

The dorsal nasal vein, $v$. dorsalis nasi, drains the apex and dorsum of the nose. It anastomoses rostrally with the veins of the nasal cavity (see the "Ve- nous drainage of the central nervous system, dorsal sagittal sinus"), caudally it is connected to the supraorbital vein.

The vein of the angle of the eye, $v$. angularis oculi, drains the medial canthus and the lower lid of the eye.

The buccal vein, v. buccalis, drains the buccal region. It lies lateral to the mandible and medial to the buccal and masseter muscles. It is also connected to the pterygoid plexus caudally. 


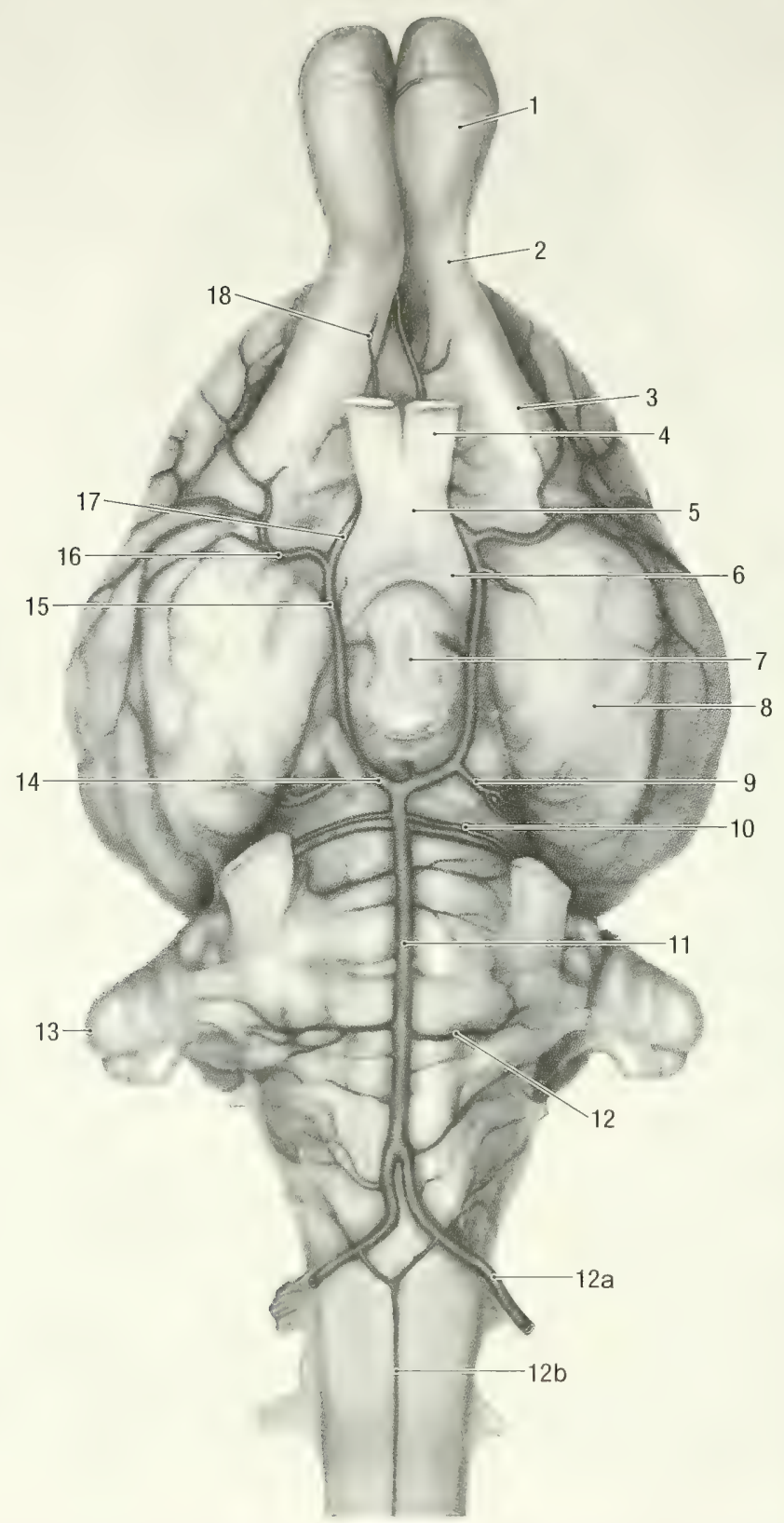

Fig. 9-6. Arteries of the brain, ventral view. 1 olfactory bulb, 2 olfactory peduncle, 3 lateral olfactory tract, 4 optic nerve, 5 optic chiasma, 6 optic tract, 7 hypophysis, 8 olfactory peduncle, 9 caudal cerebral a., 10 rostral cerebellar a., 11 basilar a., 12 caudal cerebellar a., 12 a vertebral a., 12 b ventral spinal a., 13 paraflocculus, 14 caudal communicating a., 15 cerebral arterial circle, 16 middle cerebral a., 17 and 18 rostral cerebral a.

The vein of the upper lip, $v$. labialis superior, drains the ventral aspect of the upper lip and the angle of the mouth.

The vein of the lower lip, v. labialis inferior, drains the angle of the mouth and the lower lip.

Note: The infraorbital vein drains the greater part of the upper lip.

The facial vein passes caudally over the ventro- lateral surface of the masseter muscle and joins the lingual vein caudoventral to the angle of the mandible.

The lingual vein, v. lingualis (Figs. 9-5 and 9-8), drains the tongue and the floor of the mouth by means of the deep lingual and sublingual alveolar veins. It passes between the parotid and mandibular salivary glands and is closely associated with the 


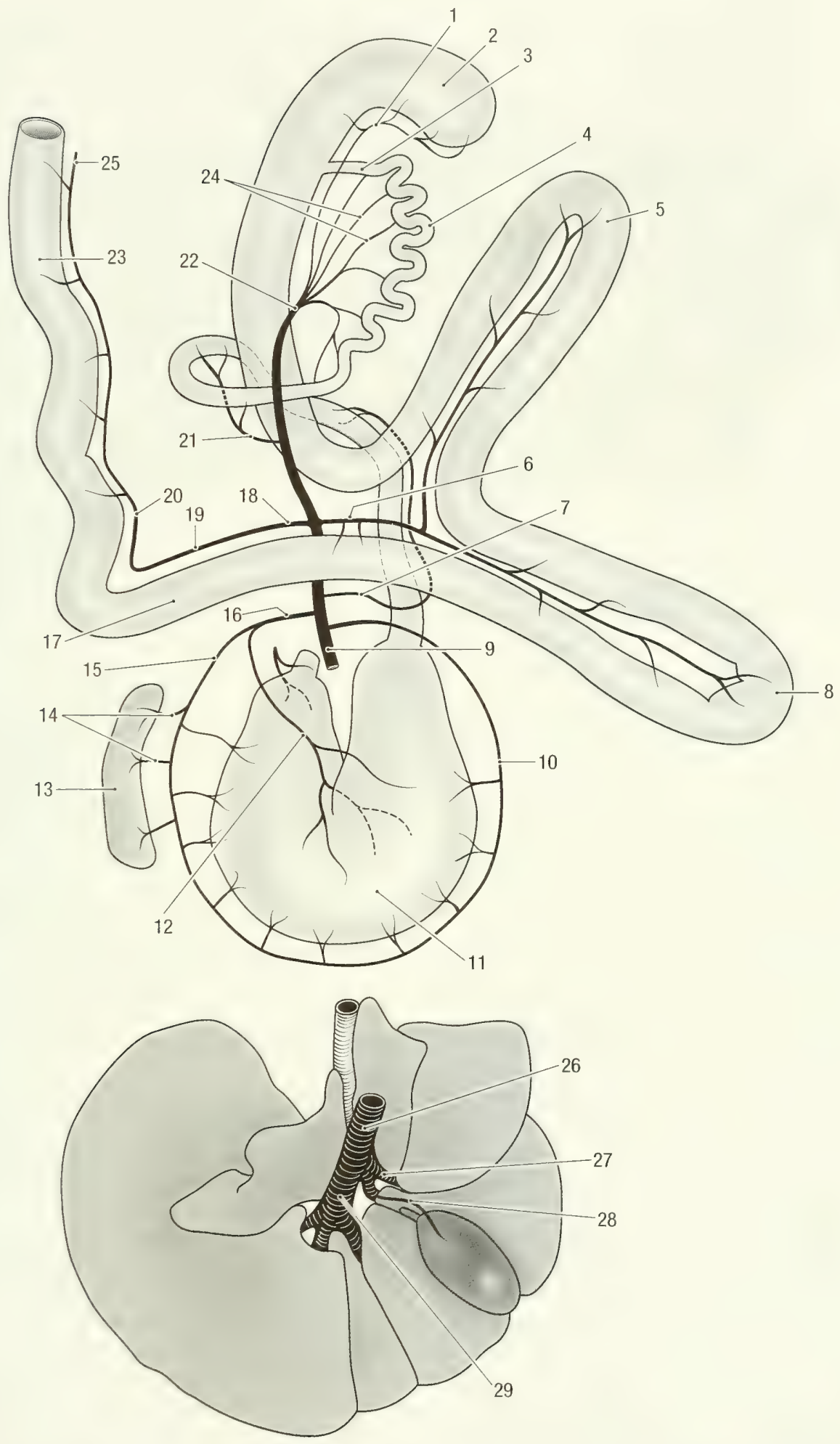

Fig. 9-7. Schematic dorsal view of the hepatic portal vein. 1 cecal v., 2 cecum, 3 ileum, 4 jejunum, 5 first loop of ascending colon, 6 right colic v., 7 cranial pancreaticoduodenal v., 8 second loop of ascending colon, 9 portal v., 10 right gastroepiploic v., 11 stomach, 12 left gastric v., 13 spleen, 14 splenic branches, 15 left gastroepiploic v., 16 splenic v., 17 transverse colon, 18 caudal mesenteric v., 19 middle colic v., 20 left colic v., 21 caudal pancreaticoduodenal v., 22 cranial mesenteric v., 23 rectum, 24 jejunal vv., 25 cranial rectal v., 26 hepatic portal v., 27 right branch of hepatic portal v., 28 cystic v., 29 left branch of hepatic portal $v$. 


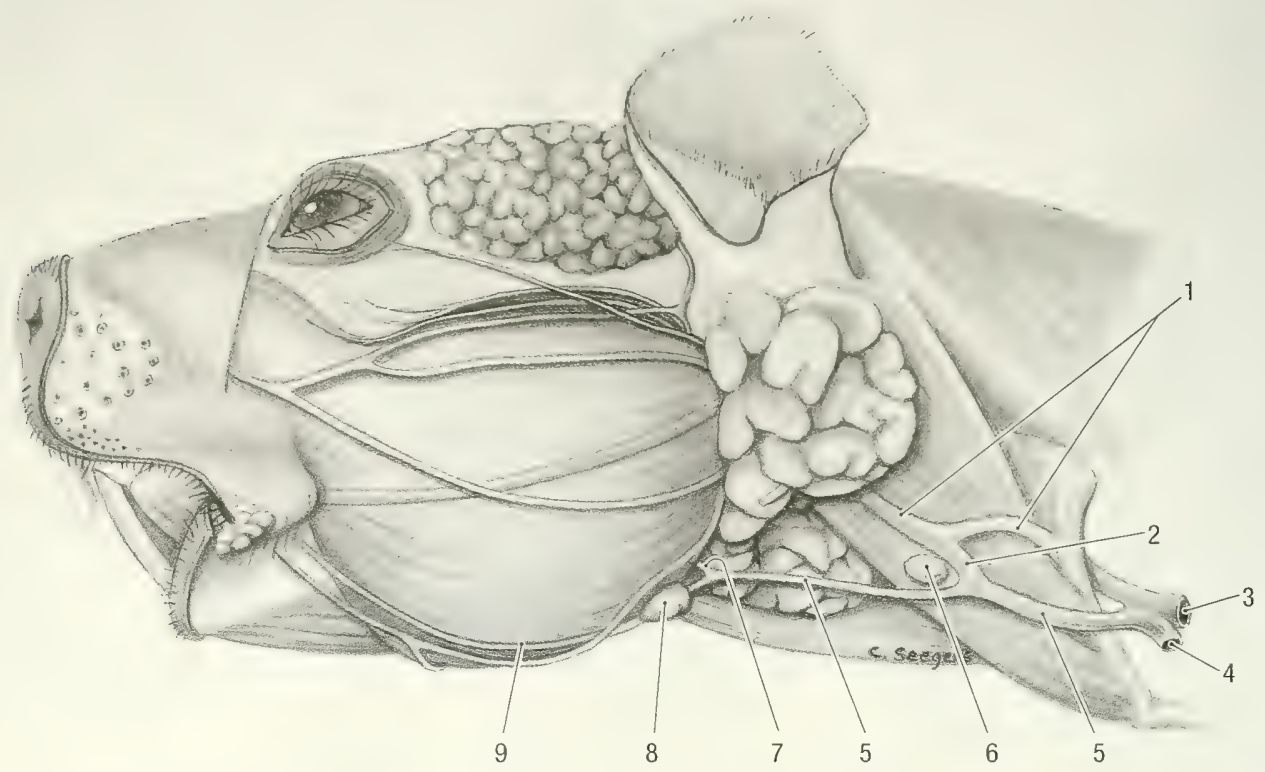

Fig. 9-8. Veins and lymph nodes of the head, lateral view. 1 maxillary v., 2 anastomoses between maxillary and facial veins, 3 brachiocephalic trunk, 4 axillary v., 5 linguofacial v., 6 superficial cervical In., 7 lingual v., 8 mandibular In., 9 facial $v$.

mandibular lymph nodes. At the angle of the jaw the lingual vein has a large anastomoses with the pterygoid plexus.

The deep lingual veins, $v v$. profundae linguae, drain the tongue. They arise from a dorsal lingual plexus. The vessels pass ventrally and unite along the ventral border of the tongue to form the lingual vein.

The sublingual vein, $v$. sublingualis, drains the floor of the mouth and the intermandibular area. It passes caudally and drains into the lingual vein. The left and right sublingual veins anastomose with each other across the intermandibular space by means of a small hyoid arch, arcus hyoideus.

The linguofacial vein passes caudally along the ventral border of the sternocephalic muscle, deep to the ventral cutaneous muscle of the neck. In the mid-cervical region it anastomoses with the maxillary vein (Figs. 9-5 and 9-8). The linguofacial vein passes ventral (external) to the clavicle and enters the thorax medial to the first rib. It joins the maxillary and axillary veins to form the brachiocephalic vein. The latter continues as the cranial caval vein.

Note: Hill (1937) considered the anastomoses between the maxillary and linguofacial veins to be the continuation of the maxillary vein. He described a venous loop off the external jugular vein around the clavicle that is considered here to be the continuation of the maxillary vein.

The maxillary vein, $v$. maxillaris, drains the caudal and deep parts of the head, including the brain. Blood flows by means of the transverse facial, su- perficial temporal, infraorbital, inferior alveolar, palatine, deep temporal, masseteric, caudal auricular, vertebral, internal jugular and retroarticular emissary veins. The maxillary vein originates from the pterygoid plexus caudal to the temporomandibular joint and passes caudoventrally under cover of the parotid salivary gland.

The transverse facial vein, $y$. transversa faciei, lies ventral to the zygomatic arch. It drains the lateral canthus of the eye, the lateral part of the lower lid of the eye and the area immediately ventral to the zygomatic arch. The vein drains into the superficial temporal vein caudal to the temporomandibular joint.

The superficial temporal vein, v. temporalis superficialis, drains the upper lid of the eye and the superficial temporal region. It anastomoses rostrally with the ophthalmic plexus and caudally it drains into the maxillary vein.

The infraorbital vein, $v$. infraorbitalis, drains the upper lip, lateral nasal region and the rostral part of the hard palate. It anastomoses with the facial vein, passes through the infraorbital canal and drains into the pterygoid plexus.

The inferior alveolar vein, v. alveolaris inferior, drains the lower jaw and teeth. The vessel leaves the inferior alveolar canal and drains into the pterygoid plexus.

The palatine veins, $v v$. palatinae, drain the soft palate and the caudal part of the hard palate. They open into the pterygoid plexus.

The deep temporal vein, v. temporalis profunda, 
drains the temporal muscle and opens into the pterygoid plexus.

The masseteric vein, $v$. masseterica, drains the caudal part of the masseter muscle. It passes caudally and drains into the maxillary vein.

The caudal auricular vein, v. auricularis caudalis, drains the pinna and caudal muscles of the ear. It is formed by the confluence of the lateral, intermediate and medial veins of the ear. The vein passes along the caudolateral border of the ear and drains into the maxillary vein.

The retroarticular emissary vein, v. emissaria foraminis retroarticularis, connects the transverse sinus of the brain with the maxillary vein. It leaves the retro-articular foramen and passes ventrally to the external acoustic meatus where it drains into the maxillary vein (see the venous drainage of the brain),

The caudal continuation of the maxillary vein in the neck passes along the dorsomedial border of the sternocephalic muscle. It receives the anastomosing branch from the linguofacial vein and enters the thorax dorsal to the clavicle and medial to the first rib. It joins the axillary and linguofacial veins to form the brachiocephalic vein.

The internal jugular vein, v. jugularis interna, has a course independent of the linguofacial or maxillary veins. It is formed by the confluence of the sigmoid and ventral petrosal sinuses of the cranial cavity. It leaves the petro-occipital fissure and receives veins from the pharynx, larynx, esophageal plexus, thyroid gland and veins from the occipital region. The vein accompanies the common carotid artery in the neck and enters the thorax where it drains into the brachiocephalic vein.

\section{Venous Drainage of the Central Nervous} System. Dorsal and ventral cerebral and cerebellar veins drain the brain. The veins open into sinuses that lie between the dura mater and periosteum of the cranial cavity. The sinuses of the cranial cavity drain by means of emissary veins, vv. emissariae, into the veins of the head and are connected to the ventral venous sinuses of the vertebral canal.

The sinuses of the dura mater are grouped into dorsal and ventral systems. The dorsal system consists of the dorsal sagittal, straight, transverse, temporal and dorsal petrosal sinuses. The ventral system consists of the cavernous, ventral petrosal and basilar sinuses. The sigmoid sinus connects the two systems to each other.

The veins of the spinal cord drain into the internal vertebral venous plexus. The latter lies in the periosteum along the floor of the vertebral canal. It anastomoses with systemic veins by means of intervertebral, intercostal and lumbar veins.
Dorsal System of Sinuses. The dorsal sagittal sinus, sinus sagittalis dorsalis, lies in the falx cerebri. It begins by the confluence of the veins from the nasal cavity and the olfactory bulbs. The sinus receives the dorsal cerebral veins and the straight sinus. It divides caudally to form the two transverse sinuses.

The straight sinus, sinus rectus, drains the corpus callosum and the medial aspect of the cerebral hemispheres. It opens into the dorsal sagittal sinus at the point where the falx cerebri joins the tentorium cerebelli membranaceum.

The transverse sinus, sinus transversus, passes laterally in the tentorium cerebelli membranaceum. It receives the middle meningeal vein and the dorsal petrosal sinus. The transverse sinus connects with the sigmoid sinus before it enters the temporal meatus to form the temporal sinus, sinus temporalis. The latter continues through the temporal canal and emerges at the retroarticular foramen as the retroarticular emissary vein, v. emissaria foraminis retroarticularis. The vein finally drains into the maxillary vein.

The dorsal petrosal sinus, sinus petrosus dorsalis, lies in the tentorium cerebelli membranaceum. It receives cerebral and trigeminal veins and drains into the transverse sinus.

The sigmoid sinus, sinus sigmoideus, connects the transverse sinus with the ventral petrosal sinus. It passes dorsally and caudally to the cerebellar fossa of the petrous temporal bone, receives the ventral petrosal sinus and leaves the cranial cavity through the jugular foramen. The sinus is also connected to the condylar vein. The latter passes through the condyloid canal and continues along the ventrolateral floor of the caudal cranial cavity as the basilar sinus. The emissary vein of the jugular foramen, $v$. emissaria foraminis jugularis, continues as the internal jugular vein (see above).

Ventral System of Sinuses. The paired cavernous sinus, sinus cavernosus, lies on the floor of the cranial cavity. They extend from the orbital fissures to the petrobasilar canal and are connected to each other. They communicate rostrally through the orbital fissures with the ophthalmic and pterygoid plexuses. Laterally they give off emissary veins through the round, oval and external carotid foramina and continue caudally as the ventral petrosal sinuses.

The ventral petrosal sinus, sinus petrosus ventralis, lies in the petrobasilar canal. It extends between the cavernous sinus rostrally and the ventral end of the sigmoid sinus caudally.

The basilar sinus, sinus basilaris, is the connection between the sigmoid sinus in the condyloid 
canal and the ventral internal vertebral plexus through the foramen magnum.

Veins of the Thoracic Limb. Superficial and deep veins drain the thoracic limb. The superficial veins accompany the cranial superficial antebrachial artery and its branches. The deep veins accompany the axillary artery and its branches (excluding the superficial antebrachial artery).

Superficial Veins. The cephalic vein, v. cephalica, forms the superficial venous drainage of the thoracic limb. Veins from the palmar aspect of the manus drain into a superficial palmar venous arch, arcus palmaris superficialis. The cephalic vein originates from the medial part of arch. It passes proximally along the medial border of the carpus and turns cranially around the medial border of the antebrachium.

Above the carpus the accessory cephalic vein drains into the cephalic.

The veins on the dorsal aspect of the digits and metacarpus drain into a superficial network of vessels, rete carpi dorsale. The accessory cephalic vein drains or originates from the network. Proximal to the carpus it drains into the cephalic vein.

The cephalic vein passes proximally along the craniomedial aspect of the antebrachium. The superficial antebrachial artery and the cutaneous branches of the radial nerve accompany the vein. It crosses the flexor surface of the elbow and continues proximally in the groove between the lateral head of the triceps and the acromial part of the deltoid muscles. Just distal to the shoulder the cephalic vein gives off the axillobrachial vein, passes deep to the cleidobrachial and acromiomandibular muscles and finally drains into linguofacial vein at the base of the neck.

The axillobrachial vein, $v$. axillobrachialis passes dorsocaudally in the groove between the lateral head of the triceps and deltoid muscles. It turns medially to pass between the teres major and subscapular muscles and drains directly into the axillary vein. In some specimens it also anastomoses with the collateral radial vein.

Deep Veins. The deep veins of the thoracic limb accompany the arteries. They drain the areas supplied by their homologous arteries. For the detail of the branching pattern of the veins, see the arterial supply to the thoracic limb. The main venous channels are the median vein in the forearm, the brachial vein in the upper-arm and the axillary vein in the shoulder region.

At the antebrachium the median, radial, deep antebrachial and ulnar veins represent the deep venous system.
The radial vein, $v$. radialis, receives small vessels from the dorsal and palmar aspects of the carpus as well as the deep palmar digital veins. It joins the median vein in the middle of the antebrachium.

The ulnar vein, $v$. ulnaris, drains some of the antebrachial muscles and is joined by the deep antebrachial vein before it opens into the median vein.

A large anastomosing branch connects the ulnar and the collateral ulnar veins.

The deep antebrachial vein, v. profunda antebrachii, drains the muscles of the forearm and joins the ulnar vein before the latter opens into the median vein.

The median vein, $v$. mediana, receives the radial vein in the middle of the forearm, and distal to the elbow it receives the ulnar vein. It passes over the medial surface of the elbow joint and continues in the brachial region as the brachial vein.

The brachial vein, $v$. brachialis, is the main vein of the upper arm. It receives the transverse cubital, collateral ulnar, bicipital and deep brachial veins, and drains into the axillary vein.

The transverse cubital vein, $v$. transversa cubiti, anastomoses with the cephalic and collateral radial veins and drains into the distal part of the brachial vein.

The collateral ulnar vein, $v$. collateralis ulnaris, anastomoses with the ulnar vein and drains into the distal part of the brachial vein.

The bicipital vein, $v$. bicipitalis, drains the biceps brachii muscle and opens into the distal part of the brachial vein.

The deep brachial vein, $v$. profunda brachii, drains the triceps muscle. It is the largest of the veins draining the brachium and joins the brachial vein in the middle of the arm.

The brachial vein continues as the axillary vein beyond the deep brachial vein.

The axillary vein, $v$. axillaris, accompanies the axillary artery along the medial aspect of the shoulder. It receives all the veins that drain the shoulder region, as well as the large axillobrachial vein.

The collateral radial vein, $v$. collateralis radialis, receives anastomosing branches from the transverse cubital and axillobrachial veins and anastomoses with the deep brachial vein. It drains into the axillary vein at the level of the shoulder joint.

The subscapular vein, $v$. subscapularis, drains into the linguofacial vein at the base of the neck.

The thoracodorsal vein, $v$. thoracodorsalis, receives veins from the area caudal to the shoulder and drains into the axillary vein.

The lateral thoracic vein, $v$. thoracica lateralis, drains the deep pectoral muscles and opens into the axillary vein.

The external thoracic vein, v. thoracica externa, 
drains the superficial pectoral muscle and opens into the axillary vein.

The suprascapular vein, v. suprascapularis, drains the supra- and infraspinatus muscles. It is closely associated with the suprascapular artery and opens into the axillary vein.

The dorsoscapular vein, v. scapularis dorsalis, accompanies the dorsoscapular artery and drains the area supplied by the satelite artery. It drains into the axillary vein.

The superficial cervical vein, $v$. cervicalis superficialis, drains the structures at the base of the neck, as well as the serratus ventralis muscles and the tissues of the axilla. It opens into the proximal end of the axillary vein.

The axillary vein passes around the cranial border of the first rib, ventral to the axillary artery. The internal jugular, linguofacial and maxillary veins from the head join the axillary vein at this point to form the brachiocephalic vein. A true subclavian vein is therefore not present in the woodchuck.

The brachiocephalic vein, $v$. brachiocephalica (Fig. 9-8), is formed by the confluence of the internal jugular, axillary, linguofacial and maxillary veins. It receives the internal thoracic, vertebral and costocervical veins and continues as the cranial caval vein.

The internal thoracic vein, $v$. thoracica interna, is a satellite of the internal thoracic artery. It drains the ventral abdominal wall, thoracic mammae, the diaphragm, ventral intercostal area and the cranial mediastinum. It receives the thymic veins before it drains into the brachiocephalic vein.

The vertebral vein, $v$. vertebralis, originates from the same area as the internal jugular vein. It accompanies the vertebral artery in the neck and has numerous connections with the ventral internal vertebral plexus.

The costocervical vein, $v$. costocervicalis, drains the area supplied by the costocervical artery, including the first two intercostal spaces. The vein passes medial to the proximal end of the first rib and enters the thorax. In some specimens the right costocervical vein drains into the right azygos vein instead of the right brachiocephalic vein.

The right azygos vein, v. azygos dextra (Figs. 9-2 and 9-4), drains the cranial abdominal and thoracic walls. It begins ventral to the second lumbar vertebra, between the psoas minor muscles, as a single trunk formed by the confluence of the second lumbar veins. The vein passes cranially, accompanied by the thoracic duct and flanked by the crura of the diaphragm and the psoas minor muscles. In the thorax it appears at the cranial border of the psoas muscles and inclines to the right where it lies between the vertebral bodies and the aorta. At the level of the sixth thoracic vertebra it turns ventrally to pass around the hilus of the right lung. The vein drains into the terminal part of the right cranial vena cava. The right azygos vein receives all dorsal intercostal veins, except the first two or three intercostal veins on the left. In some specimens it also receives the right costocervical vein.

The dorsal intercostal veins, $v v$. intercostales dorsales (Fig. 9-2), drain the area supplied by dorsal intercostal arteries, including the internal vertebral plexus of the vertebral canal. The open into the azygos vein or the costocervical vein.

The caudal vena cava, v. cava caudalis (Figs. 9-1 to 9-4), drains the pelvis and tail, pelvic limbs and abdominal wall and some of the abdominal viscera. It begins ventral to the seventh lumbar vertebra by the confluence of the two common iliac veins and the middle sacral vein. The common iliac veins are formed by the confluence of the internal and external iliac veins.

The internal iliac vein, $v$. iliaca interna, drains the pelvic viscera by means of the internal pudendal and prostatic/vaginal veins, and part of the pelvic wall by means of the caudal gluteal vein.

The internal pudendal vein, v. pudenda interna, drains the anus and penis/clitoris by means of the caudal rectal vein and the vein of the penis/clitoris respectively.

The vein of the penis/clitoris, v. penis/clitoridis, in the drains the penis/clitoris and its adnexa.

The caudal rectal vein, $v$. rectalis caudalis, drains the anus and caudal part of the rectum.

The prostatic/vaginal vein, v. prostatica/vaginalis, drains the viscera of the pelvis. The vein and its tributaries accompany the artery. In the female it drains the genital tract, (excluding the ovaries), middle part of the rectum, ureters, urinary bladder and pelvic urethra. In the male it drains the ductus deferens, middle part of the rectum, ureters, urinary bladder and pelvic urethra. The internal iliac vein is formed by the confluence of the prostatic/ vaginal and internal pudendal veins.

The caudal gluteal vein, v. glutea caudalis, accompanies the homologous artery through the lesser ischiatic foramen. It drains the proximal parts of the caudal thigh muscles, the superficial gluteal muscles and the sacral region. It receives the obturator and lateral tail veins.

The lateral vein of the tail, v. caudalis lateralis, accompanies the homologous artery in the tail. At the base of the tail it passes laterally to drain into the caudal gluteal vein.

The obturator vein, v. obturatoria, accompanies the obturator artery along the lateral wall of the pelvic cavity. It passes through the obturator foramen and drains the medial thigh (adductor) and intra pelvic muscles. 
Veins of the Pelvic Limb. Superficial and deep veins drain the pelvic limb. Both the deep and superficial veins follow the arteries.

The medial saphenous vein, $v$. saphena medialis, forms the superficial venous system of the pelvic limb and almost exclusively drains the pes. It accompanies the saphenous artery and its branches and has cranial and caudal branches. The veins of the toes and pes drain into a superficial dorsal arch that lies transversely across the dorsum of the pes, distal to the tarsus. The dorsal branch of the medial saphenous vein arises from the medial part of the arch. It passes proximally along the craniomedial border of the tarsus where it is joined by the small caudal. The vessel continues proximally along the medial aspect of crus and thigh. It passes deep to the sartorius muscle and joins the femoral vein in the proximal femoral region.

The cranial tibial vein, $v$. tibialis cranialis, originates proximal to the ankle. It accompanies the cranial tibial artery and drains into the popliteal vein.

The caudal tibial vein, $v$. tibialis caudalis, accompanies the caudal tibial artery and the tibial nerve. At the knee it gives off an anastomosing branch to the cranial tibial vein and receives the descending vein of the knee. It continues proximally to drain into the femoral vein, opposite the junction of the latter and the saphenous vein. The descending vein of the knee $v$. genus descendens, accompanies the descending artery of the knee on the medial aspect of the knee.

The popliteal vein, v. poplitea, is formed by the confluence of the cranial tibial vein and the anastomosing branch from the caudal tibial vein. It is a short vessel that accompanies the popliteal artery on the caudal surface of the knee. It receives the caudal femoral vein and continues proximally as the femoral vein.

The femoral vein, $v$. femoris, accompanies the femoral artery along the medial surface of the thigh. It receives the caudal femoral, saphenous and lateral circumflex femoral veins.

The caudal femoral vein, v. caudalis femoris, accompanies the caudal femoral artery. It drains the popliteal lymph node and the muscles caudal to the knee.

The lateral circumflex femoral vein, v. circumflexa femoris lateralis, is a large vessel that drains the quadriceps femoris muscle. It accompanies the circumflex femoral artery and femoral nerve and drains into the proximal part of the femoral vein.

The femoral vein passes through the vascular lacuna and continues in the caudal abdomen as the external iliac vein. The external iliac vein accompanies the external iliac artery and receives the pudendoepigastric and medial circumflex femoral veins.
The pudendoepigastric vein, v. pudendoepigastri$c a$, drains the caudal abdominal region, in the male the preputium and scrotum, in the female the labia and inguinal mammary gland. It accompanies the pudendoepigastric artery though the inguinal canal.

The medial circumflex femoral vein, v. circumflexa femoris medialis, drains the medial thigh muscles. It accompanies the medial circumflex femoral artery.

The common iliac vein, v. iliaca communis, receives the cranial gluteal, iliacofemoral and umbilical veins.

The cranial gluteal vein, $v$ glutea cranialis, drains the middle and deep gluteal muscles and accompanies the cranial gluteal artery through the greater ischiatic foramen.

The iliacofemoral vein, v. iliacofemoralis, accompanies the iliacofemoral artery. It drains the iliopsoas and tensor fascia lata muscles. The vein drains into the common iliac vein at the confluence of the external and internal iliac veins.

The umbilical vein, v. umbilicalis, drains the cranial part of the urinary bladder, the ureter and part of the ductus deferens. It accompanies the umbilical artery.

The median sacral vein, $v$. sacralis mediana, is the cranial continuation of the median vein of the tail, v. caudalis mediana. The latter accompanies the median artery of the tail along the ventral midline of the tail and has many anastomoses with the lateral veins of the tail. The median sacral artery passes cranially along the ventral surface of the sacrum. It accompanies the median artery of the sacrum.

The abdominal portion of the caudal vena cava lies in the groove between the left and psoas muscles, ventral to the lumbar vertebral bodies and to the right of the abdominal aorta. As it passes cranially it gradually inclines ventrally and to the right until it reaches the medial border of the caudate lobe of the liver. It deeply grooves or tunnels the caudate lobe before it reaches the diaphragm. The vein passes through the foramen vena cava of the diaphragm. The thoracic portion of the vein lies in the plica vena cava and is asociated with the right phrenic nerve. It passes between the acessory and caudal lobes of the right lung and opens into the caudal part of the right atrium. Along its course it receives the lumbar, the deep circumflex iliac, renal and right testicular/ovarian, cranial abdominal and hepatic veins.

The last five pairs of lumbar veins, $v v$. lumbales, drain into the caudal vena cava. They accompany the lumbar arteries and are connected to the ventral internal vertebral plexus.

The deep circumflex iliac vein, $v$. circumflexa ilium profunda, drains the abdominal wall and surrounding tissue ventral to the tuber coxa. It accom- 
panies the artery and the lateral cutaneous femoral nerve.

The renal veins, $v v$. renales (Figs. 7-1 to 7-3), drain the kidney and adrenal gland. In most cases the left renal vein also receives the left testicular/ ovarian vein.

The testicular/ovarian veins, v.testicularis/ovarica, drain the testicle/ovary, The right vein drains directly into the caudal vena cava, the left vein generally drains into the left renal vein.

Hepatic veins, vv. hepaticae. As the caudal vena cava passes through the caudate lobe of the liver and receives four veins. The hepatic veins from the caudate process and right lateral lobes open independently into the caudal vena cava, the right hepatic vein drains the right medial lobe and the left hepatic vein drains the quadrate lobe, papillary process and the left lobes of the liver.

The portal vein, v. portae (Fig. 9-7), and its tributaries from the digestive tract forms the hepatic portal system. The vein arises from a capillary bed in the gall bladder, spleen, pancreas and all of the gastrointestinal tract, except the anal canal. Variations exist in the pattern of formation and division of the portal vein. The cranial mesenteric and splenic veins are the two main tributaries of the hepatic portal vein.

The gastroduodenal vein, v. gastroduodenalis, is formed by the confluence of the right gastroepiploic and cranial pancreaticoduodenal veins. In some animals the two veins remain separate.

The right gastroepiploic vein, v. gastroepiploica dextra, drains the right part of the greater curvature of the stomach.

The cranial pancreaticoduodenal vein, v. pancreaticoduodenalis cranialis, drains the descending duodenum and the right lobe of the pancreas. In $50 \%$ of the animals it drains directly into the portal vein.

The splenic vein, $v$. lienalis, is formed by the confluence of the left gastric and left gastroepiploic veins. The splenic branches drain the spleen.

The left gastric vein, $v$. gastrica sinistra, drains the parietal and visceral surfaces of the lesser curvature of the stomach, the cardia and the abdomen part of the esophagus. In $20 \%$ of animals the branch from the cardia and esophagus drains directly into the left branch of the portal vein.

The left gastroepiploic vein, $v$. gastroepiploica sinistra, drains the left part of the greater curvature of the stomach, spleen and greater omentum.

The splenic vein joins the portal vein close to or in common with the caudal mesenteric vein.

The caudal mesenteric vein, $v$. mesenterica caudalis, drains part of the large intestine and the cranial part of the rectum.

The cranial rectal vein, $v$. rectalis cranialis, drains most of the rectum and is continuous with the left colic vein.

The left colic vein, $v$. colica sinistra, drains the entire descending colon and is continuous with the middle colic vein.

The middle colic vein, $v$. colica media, drains the left part of the transverse colon.

The caudal mesenteric vein joins the portal vein opposite the right colic vein.

The right colic vein, $v$. colica dextra, drains the two loops of the ascending colon as well as the right part of the transverse colon. The vein is formed by the confluence of two veins, one for each loop. The vein that drains the second loop also drains the right part of the transverse colon. In some animals the veins that drain the transverse colon opens independently into the right colic vein. In $12 \%$ of the animals the two branches of the right colic vein open separately into the portal vein.

The cranial mesenteric vein, $v$. mesenterica cranialis, is the largest of the tributaries of the portal vein. It is formed by the confluence of the ileocolic, ileal, jejunal and caudal pancreaticoduodenal veins.

The ileocolic vein, v. ileocolica, drains the cecum and initial part of the ascending colon.

Ileal veins, $v v$. ilei, drain the ileum and jejunal veins, $v v$. jejunales, drain the jejunum.

The caudal pancreaticoduodenal vein, $v$. pancreaticoduodenalis caudalis, drains the ascending duodenum and the left lobe of the pancreas.

The cystic vein, $v$. cystica, drains the gall bladder and opens directly into the right branch of the portal vein.

The portal vein enters the porta of the liver. Close to the liver it divides into left and right branches.

The right branch is very short and almost immediately divides into a branch to the right lateral lobe and caudate process and a branch to the right medial lobe.

The left branch of the portal vein is longer. It passes deep to the right branch of the hepatic artery and bile duct and then divides into a branch that enters the quadrate and left medial lobes and a branch to the left lateral lobe.

There are small anastomoses between branches of the portal vein and branches of the caudal vena cava. The cranial rectal vein always anastomoses with the middle rectal vein. Anastomoses between the branches of the cranial mesenteric and renal veins are present in $10 \%$ of the animals. The connections between the portal venous system and the systemic veins are generally very small.

\section{LYMPHATIC SYSTEM}

The lymphatic system, systema lymphaticum, consists of a network of permeable lymphatic cap- 
illaries, collecting vessels, a filtering system in the form of lymph nodes and conducting channels which return the lymph to the circulatory system via the large veins cranial to the heart. The lymph nodes are grouped into lymph centra. A lymph centrum is a lymph node or a group of lymph nodes that occur in the same region of the body and receives afferent vessels from approximately the same region.

The parotid lymph center, lymphocentrum parotideum, contains the deep parotid lymph nodes, $\ln$. parotideum profundi, which lie at the caudal border of the mandible, deep to the parotid salivary gland. They consist of 3 to 4 small nodes, approximately $5 \mathrm{~mm}$ in diameter.

The mandibular lymph center, lymphocentrum mandibula, contains the mandibular lymph nodes, Inn. Mandibulares (Fig. 9-5). They lie rostral to the mandibular salivary gland and caudal to the ramus of the mandible and are arranged along the linguofacial vein. The medial node is the largest and measures approximately $15 \mathrm{~mm}$ in length and 5 $\mathrm{mm}$ in width. The lateral node is about $5 \mathrm{~mm}$ in diameter. Both nodes are covered by the ventral cutaneous muscle of the neck and are therefore difficult to palpate.

The retropharyngeal lymph center, lymphocentrum retropharyngeum, contains the lateral and medial retropharyngeal lymph nodes.

The lateral retropharyngeal lymph node, $l n$. retropharyngeus lateralis, lies deep to the caudal border of the parotid salivary gland, ventral to the wing of the atlas and lateral to the sternomastoid muscle. It measures about $15 \mathrm{~mm}$ in length and $5 \mathrm{~mm}$ in width.

The medial retropharyngeal lymph node, $\ln$. retropharyngeus medialis, lies dorsally on the laryngopharynx, lateral to the omohyoid muscle and caudal to the caudal belly of the digastric muscle. The node is covered laterally by the sternomastoid muscle.

The superficial cervical lymph center, lymphocentrum cervicale superficiale, of the neck contains the ventral superficial cervical lymph node, $\ln$. cervicalis superficialis ventralis (Fig. 9-8) at the junction of the linguofacial and maxillary veins. It lies caudal to the distal border of the parotid salivary gland and is covered by the ventral cutaneous muscle of the neck. The node measures about $2 \mathrm{~mm}$ in diameter and is not palpable.

The axillary lymph center, lymphocentrum axillare, contains the axillary, accessory axillary and infraspinous lymph nodes.

The axillary lymph nodes, Inn. axillares, are six to eight in number. They are embedded in the brown adipose tissue along the course of the lateral thoracic artery, deep to the lateral border of the ascending pectoral muscle. Each node is about 5 $\mathrm{mm}$ in diameter.

The accessory axillary lymph node, $\ln$. axillaris accessorius, lies on the lateral surface of the tensor fascia antebrachii or cutaneous trunci muscle, in the angle between the triceps and thoracic trapezius muscles. The node(s) is about $8 \mathrm{~mm}$ long and 3 mm wide.

The infraspinous lymph node, $\ln$. infraspinatus, is lateral to the shoulder at the distal end of the scapular part of the deltoid muscle. It lies on the proximal end of the lateral head of the triceps muscle and is covered by the platysma. The node is about $10 \mathrm{~mm}$ long and $3 \mathrm{~mm}$ wide.

The ventral thoracic lymph center, lymphocentrum thoracicum ventrale, consists of the cranial sternal lymph node $l n$. sternalis cranialis. The lymph node lies cranial to the heart along the caudal border of the internal thoracic artery, ventral to the cranial vena cava. The node is very small, measuring about $2 \mathrm{~mm}$ in diameter.

The mediastinal lymph center, lymphocentrum mediastinale, contains the cranial mediastinal lymph nodes $\ln n$. mediastinales craniales, which lie in the cranial mediastinum, dorsal to the cranial caval veins. One to four nodes are present on the left and right. They vary from 1 to $10 \mathrm{~mm}$ in length and are approximately 3 to $5 \mathrm{~mm}$ wide.

The bronchial lymph center, lymphocentrum bronchale, is represented by left, right and middle tracheobronchial lymph nodes. The right and middie nodes are very small and often absent.

The left tracheobronchial lymph node, $\ln$. tracheobronchalis sinsiter, is approximately $1 \mathrm{~mm}$ in diameter. It lies cranial to the left bronchus on the caudal surface of the aortic arch.

The right tracheobronchial lymph node, $\ln$. tracheobronchalis dexter, lies at the tracheal bifurcation, to the right of the right bronchus.

The middle tracheobronchial lymph node, $\ln$. tracheobronchalis medius (Fig. 9-2), lies dorsally on the trachea at the bifurcation.

The lumbar lymph center, lymphocentrum lumbale, contains the aortic and testicular/ovarian lymph nodes.

The lumbar aortic lymph nodes, Inn. lumbales aortici, consist of a series of small nodes arranged along the dorsal aspect of the abdominal aorta and caudal vena cava. They are closely associated with the lumbar lymphatic ducts. The nodes are about $1 \mathrm{~mm}$ in diameter.

The testicular/ovarian lymph nodes, $\ln$. testicularis/ovaricus, lie along the ventral aspect of the aorta at the origin of the testicular/ovarian artery. The nodes are 3 to $8 \mathrm{~mm}$ long and 1 to $3 \mathrm{~mm}$ wide.

The celiac lymph center, lymphocentrum celiacum, consists of a series of nodes arranged along 
the branches of the celiac artery. They vary greatly in size and number. The nodes drain the stomach, pancreas, duodenum, spleen and the liver. They are named according to the organ that they drain.

Two or three splenic lymph nodes, Inn. lienales, are associated with the spleen. They lie in the gastrosplenic ligament.

The gastric lymph nodes, Inn. gastrici, are associated with the lesser curvature of the stomach. From two to ten nodes are present in the hepatogastric ligament.

The hepatic lymph nodes, $\ln n$. hepatici, are generally present in the connective tissue at the porta hepatica. They lie opposite the papillary lobe, between the hepatic artery, portal vein and caudal vena cava.

The cranial mesenteric lymph center, lymphocentrum mesentericum craniale, consists of a series of nodes in the root of the mesentery, arranged around the branches of the cranial mesenteric artery. They are named according to the part of the intestine that they drain. It consists of cranial mesenteric, jejunal, cecal and colic lymph nodes.

The cranial mesenteric lymph nodes, $\operatorname{lnn}$. mesenterici craniales, drain the duodenum and pancreas, jejunal lymph nodes, $\ln n$. jejunales, drain the jejunum and part of the ileum and cecal lymph nodes, Inn. cecales, drain part of the ileum and the cecum. The colic lymph nodes, Inn. colici, drain the ascending, transverse and initial part of the descending colon.

The caudal mesenteric lymph center, lymphocentrum mesentericum caudale, consists of one or more caudal mesenteric lymph nodes, Inn. mesenterici caudales, arranged around the origin of the caudal mesenteric artery. They drain the terminal part of the descending colon and part of the rectum.

The iliosacral lymph center, lymphocentrum iliosacrale, consists of medial iliac, sacral and hypogastric lymph nodes.

The medial iliac lymph nodes, Inn. iliaci mediales, lie lateral to the common iliac blood vessels. They are large and measure approximately $10 \mathrm{~mm}$ in length and $5 \mathrm{~mm}$ in width.

The sacral lymph node, $\ln$. sacralis, lies between the common iliac blood vessels, on the ventral surface of the middle sacral artery. It measures about $5 \mathrm{~mm}$ in diameter.

The hypogastric lymph node, In. hypogastricus, lies in the angle between the obturator and cranial gluteal arteries. It is very small and measures about $1 \mathrm{~mm}$ in diameter.

The inguinofemoral lymph center, lymphocentrum inguinofemorale, consists of superficial inguinal and subiliac lymph nodes.

The superficial inguinal lymph nodes, Inn. inguinales superficiales, are represented by the scrotal lymph nodes, $\operatorname{lnn}$. scrotales, of the male and the mammary lymph nodes, Inn. mammarii, of the female. They consist of three or four nodes arranged along the caudal superficial epigastric artery, just cranial to the inguinal canal. In the female they lie deep to the inguinal and abdominal mammary glands. The nodes are 3 to $8 \mathrm{~mm}$ long and 1 to 3 mm wide.

The subiliac lymph nodes, Inn. subiliaci, one or two in number, are situated ventral to the tuber coxae. They lie superficial to the deep circumflex iliac blood vessels and are embedded in the mass of adipose tissue found in this region. They are about $6 \mathrm{~mm}$ in diameter.

The popliteal lymph center, lymphocentrum popliteum, contains the popliteal lymph nodes, Inn. poplitei, that lie caudal to the knee at the origin of the gastrocnemius muscles. A smaller lymph node is constantly present cranial to the main node. It is closely associated with the large blood vessels and nerves of the region. The nodes are 3 to $10 \mathrm{~mm}$ in diameter.

The thoracic duct, ductus thoracicus, is the main channel for the return of the lymph from the body. Its origin, course and termination vary greatly. It begins between the crura of the diaphragm as the cranial continuation of the cisterna chyli. The cistern is the dilated portion of the lymph channel and is formed by the lumbar lymph trunks that are the cranial continuations of the lymph vessels from the iliac lymph nodes. The cistern also receives the lymph vessels from the intestine. In the thorax the thoracic duct passes cranially, between the aorta and the right azygos vein. At the level of the sixth thoracic vertebra it inclines to the left, passes between the aorta and the azygos vein and enters the cranial mediastinum. It passes cranioventrally in the cranial mediastinum and opens into the left brachiocephalic trunk, or into one of the large veins at the thoracic inlet.

The tracheal trunk, truncus trachealis, is formed by the left and right tracheal ducts which arise from the retropharyngeal lymph nodes and pass caudally in the neck along the internal jugular veins. The left duct commonly drains into the thoracic duct, or into the large veins at the base of the neck. The right duct drains into the large veins at the base of the neck.

The spleen, lien (Fig. 5-7), is a relatively small strap-like organ approximately $7 \mathrm{~cm}$ long, $15 \mathrm{~mm}$ wide and $0.5 \mathrm{~cm}$ thick. It has a thin capsule and is reddish-brown. The spleen has a parietal or free surface and a visceral or attached surface, dorsal and ventral extremities, and cranial and caudal margins. The visceral surface is divided into two sub-equal parts by the gastrosplenic ligament. The visceral surface is attached to the greater curvature 
of the stomach by the gastrosplenic ligament and its dorsal extremity is attached to the left kidney by the phrenicosplenic ligament. The hilus extends over the whole length of the visceral surface. The spleen is innervated by nerves from the celiac plexus, and receives its blood supply from the splenic artery. The vessel lies in the gastrolienal ligament. It gives off branches to the spleen along the entire length of the hilus. Venous drainage is via the splenic vein. Lymph is drained through the splenic lymph nodes.

The thymus, thymus (Figs. 9-1 and 9-2), is a lymphoid organ that is developed best in immature an- imals. It undergoes involution and in the adult is mostly replaced by fat and connective tissue. The thymus of the woodchuck consists of left and right lobes that lie in the cranial mediastinum. It extends between the first rib and the heart. In the newborn animal it extends for a short distance beyond the first rib into the base of the neck. The left lobe is more extensive than the right and extends to the left of the heart. It is attached to the pericardium.

The thymus is supplied with blood by thymic branches from the internal thoracic arteries. Venous blood drains into brachiocephalic veins. Sympathetic and vagal branches innervate the thymus. 


\section{Chapter 10. Central Nervous System}

\section{MENINGES}

The central nervous system is well protected within the cranium and vertebral canal. Three membranes, the dura mater, arachnoid and pia mater envelop the central nervous system.

The dura mater, dura mater, is the most superficial of the meninges. It forms a tough, fibrous sheath around the brain and spinal cord. The two parts are continuous through the foramen magnum. The dura mater of the brain and spinal cord has slightly different relationships and are described separately.

The spinal dura mater, dura mater spinalis, surrounds the spinal cord. It attaches to the foramen magnum of the skul and tapers to a slender sheath, filum durae matris spinalis, which is attached to the third or fourth caudal vertebra.

In the sacral, lumbar and thoracic parts of the vertebral canal it is separated from the periosteum of the vertebrae by a wide epidural space, cavum epidurale. The latter is filled with fat. In the cervical region the duramater fuses with the periosteum of the cervical vertebrae along the floor of the vertebral canal, thereby obliterating the epidural space ventrally. Within the vertebral canal the dura mater surrounds the spinal nerves and attaches to the intervertebral foramina.

The cranial dura mater, dura mater encephali, fuses with the periosteum of the cranial cavity. It is firmly attached to the crista galli, crista petrosa, dorsum sellae, around the foramina of the cranial cavity and at the foramen magnum. A fold of dura mater, the falx cerebri, falx cerebri, lies in the dorsal longitudinal fissure between the two cerebral hemispheres. A transverse sheet of dura mater, the membranous tentorium cerebelli, tentorium cerebelli membranaceum, lies in the transverse fissure separating the cerebral hemispheres and cerebellum. On the floor of the cranial cavity it spans the hypophyseal fossa, forming the diaphragma sellae through which the stalk of the hypophysis passes.

The arachnoid, arachnoid is the middle layer of the meninges and consists of spinal and cranial parts, arachnoidea spinalis/encephali. Together with the pia mater it forms the leptomeninges. The arachnoid is separated from the dura mater by a potential subdural space and from the pia mater by the subarachnoid space, cavum subarachnoideale. Its superficial surface lies against the dura mater, held in position by cerebrospinal fluid pressure. The inner surface of the arachnoid is attached to the pia mater by long, delicate strands of connective tissue, the arachnoid trabeculae. The trabeculae traverse the subarachnoid space. The latter is filled with cerebrospinal fluid. The depth of the subarachnoid space is variable since the arachnoid lies against the dura mater and the pia mater follows every irregularity of the brain surface. At certain sites the subarachnoid space forms enlargements or cisternae. The largest of the cisternae is the cisterna cerebellomedullaris, or cisterna magna, located between the caudal surface of the cerebellum and the dorsal surface of the myelencephalon.

The pia mater, pia mater, is the innermost layer of the meninges. It is a very delicate layer and is generally one cell layer thick. The pia consists of spinal and cranial parts, pia mater spinallis/encephali, and covers the entire external surface of the central nervous system. It continues into the depth of various sulci, fissures and perivascular channels. It is very vascular since all blood vessels to and from the central nervous system must travel in the arachnoid trabeculae and pia mater. The pia mater is thickened along each side of the spinal cord, forming the denticulate ligament, lig. denticulatum. The denticulate ligament has lateral extensions which traverse the subarachnoid space to attach to the dura mater between the entrances of successive spinal nerve roots into the dural sheath. It therefore suspends the spinal cord within the dural sheath.

The leptomeninges (arachnoid and pia) of the brain are reflected onto the cranial nerves and those of the spinal cord are reflected onto the roots of the spinal nerves.

\section{BRAIN, ENCEPHALON}

The brain develops from the rostral (cephalic) portion of the neural tube, where, at first, three vesicles develop. These vesicles give rise to the forebrain or prosencephalon, the midbrain or mesencephalon, and the hindbrain or rhombencephalon. With further development the forebrain gives rise to the telencephalon and diencephalon, the midbrain remains undivided and the hind brain gives rise to the metencephalon (cerebellum and pons) and myelencephalon (medulla oblongata).

Collectively, the diencephalon, mesencephalon, pons and myelencephalon form the brain stem. 


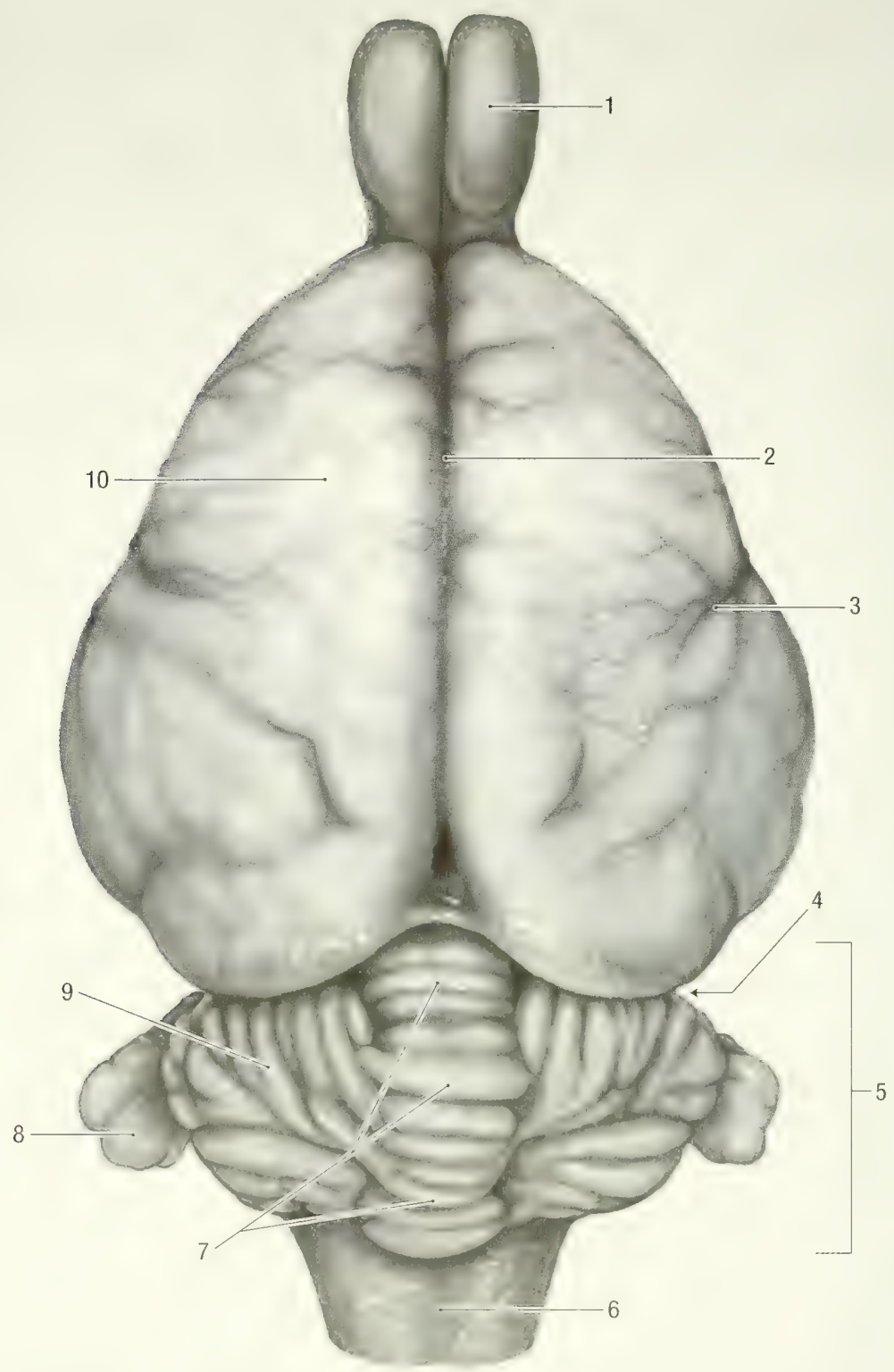

Fig. 10-1. Brain, dorsal view. 1 olfactory bulb, 2 median fissure, 3 pseudosylvian fissure, 4 transverse fissure, 5 metencephalon (cerebellum), 6 myelencephalon, 7 vermis, 8 paraflocculus, 9 cerebellar hemisphere, 10 cerebrum.

Meier (1983) studied the relative brain size within American sciurids, and Doetsh and Towe (1981) reported on the pyramidal tracts. The woodchuck's brain is approximately $45 \mathrm{~mm}$ long, $30 \mathrm{~mm}$ wide and $20 \mathrm{~mm}$ thick. It weighs approximately 11 grams.

Viewed from above (Fig. 10-1) it has an almost triangular, smooth, forebrain rostrally and a corrugated cerebellum caudally. The olfactory bulbs project $10 \mathrm{~mm}$ beyond the rostral border of the cerebral hemispheres and caudally the myelencephalon projects $5 \mathrm{~mm}$ beyond the caudal border of the cerebellum. The paraflocculi of the cerebellum are about $5 \mathrm{~mm}$ long and $5 \mathrm{~mm}$ wide and project laterally from the cerebellar hemispheres.

The two cerebral hemispheres are separated from each other by the longitudinal fissure, fissura longitudinalis cerebri, which contains the falx cerebri and from the cerebellum by the transverse fissure, fissura transversa cerebri, which contains the tentorium cerebelli membranaceum.

Viewed laterally (Fig. 10-2), the cerebrum is somewhat wedge-shaped, with the widest part caudally. The olfactory peduncle, pedunculus olfactorius, lateral olfactory tract, tractus olfactorius lateralis, and piriform lobe, lobus piriformis, are 


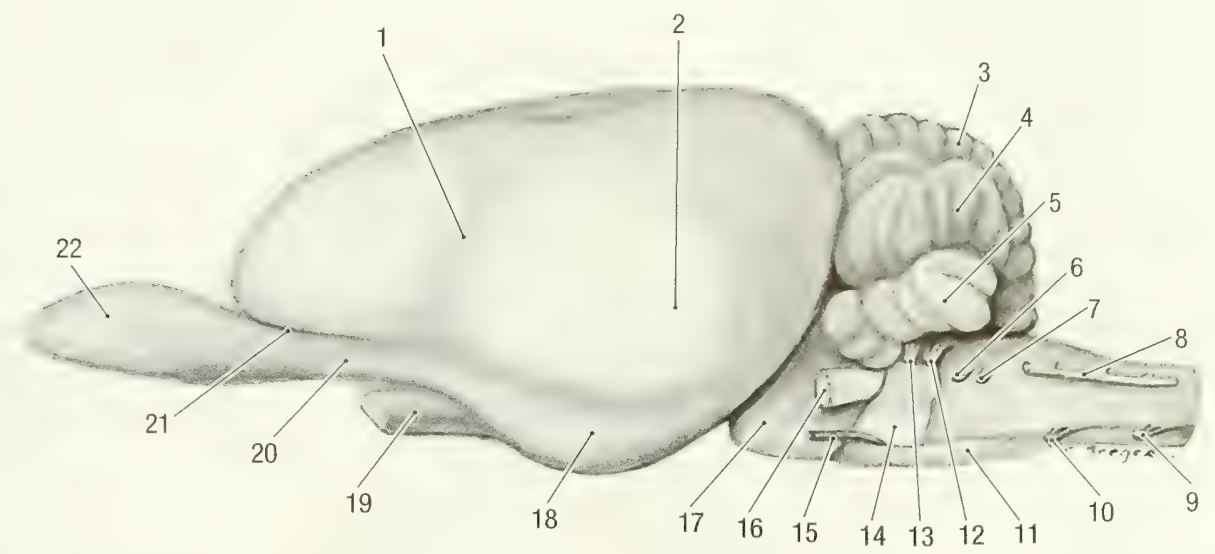

Fig. 10-2. Brain, lateral view. 1 Pseudosylvian fissure, 2 temporal lobe, 3 vermis, 4 cerebellar hemisphere, 5 parafloccular lobe, 6 glossopharyngeal n., 7 vagus n., 8 accessory n., 9 first cervical spinal n., 10 hypoglossal n., 11 myelencephalon, 12 vestibulocochlear n., 13 facial n., 14 pons, 15 abducent n., 16 trigeminal n., 17 mesencephalon, 18 piriform lobe, 19 optic n., 20 lateral olfactory tract, 21 lateral olfactory groove, 22 olfactory bulb.

separated from the rest of the cerebral hemisphere by a distinct lateral olfactory sulcus, sulcus rhinalis lateralis. The optic chiasma, chiasma opticum, lies ventromedial to the junction of the lateral olfactory tract and piriform lobe and rostral to the stalk of the hypophysis. The hypophysis, hypophysis, lies medial and ventral to the lowest part of the piriform lobe. The diencephalon is obscured from view by the piriform lobe.

Only a small, triangular portion of the mesencephalon (crus cerebri) and its oculomotor nerve is visible between the hypophysis and the pons. The trochlear nerve emerges between the caudal border of the piriform lobe and the pons and crosses the lateral surface crus cerebri.

The cerebellum, cerebellum, pons, pons, and myelencephalon, myelencephalon, lie caudal to the fore- and midbrain. The cerebellum lies dorsal to the myelencephalon. It is approximately $12 \mathrm{~mm}$ wide and $12 \mathrm{~mm}$ high. The pons consists separates the mesencephalon and myelencephalon. The myelencephalon lies caudal to the pons and ventral to the cerebellum. It gives rise to cranial nerves $\mathrm{X}$ to $\mathrm{X} 11$ and continues caudally as the cervical spinal cord.

Viewed ventrally (Fig. 10-3), the olfactory bulbs, olfactory peduncles and piriform lobes form the medial part of the cerebral hemispheres. The optic nerves converge at the optic chiasma and then diverge as the optic tracts, tractus opticus. The latter pass caudolaterally, between the hypophysis and piriform lobes, where they are concealed from view. The tuber cinereum, tuber cinereum, is a round, slightly elevated area of gray matter caudal to the optic chiasma. It encircles the stalk of the hypophysis and is only visible when the hypophysis is removed. Attached to the caudal border of the tuber cinereum is a small, elevated area of white matter, the mamillary body, corpus mamillare. The crus cerebri consists of two elevated tracts of nerve fibers along the floor of the mesencephalon. They converge somewhat and pass deep to the pons. Between the two crura, caudal to the hypophysis, is a deep depression, the fossa interpeduncularis. The oculomotor nerves appear along the lateral borders of the two crura.

The pons forms a prominent transverse band across the brain stem. It bears a deep, median groove, the sulcus basilaris. The latter contains the basilar artery. The parafloccular lobes, paraflocculus, of the cerebellum form two prominent projections laterally to the pons.

The ventral surface of the myelencephalon is visible caudal to the pons. It is divided by a longitudinal groove, fissura mediana, which contains the basilar artery. Lateral to the median fissure are two longitudinal tracts of nerve fibers, the pyrimids, pyramis. Caudal to the pons is a transverse bundle of fibers, the trapezoid body, corpus trapezoideum. Lateral to the pyramis and caudal to the trapezoid body is an oval elevation, the olive, oliva. Cranial nerves $\mathrm{V}$ to $\mathrm{X} 11$ emerge from the ventral and lateral surfaces of the myelencephalon.

A sagittal section (Fig. 10-4) between the two cerebral hemispheres and through the cerebellum and brain stem allows a median view of the cerebral hemispheres. It reveals some of the structures obscured from view in the intact brain.

The two cerebral hemispheres are connected by a broad band of nerve fibers, the corpus callosum. From rostral to caudal, the corpus callosum consists of a genu, genu corporis callosi, a body, truncus corporis callosi, and a splenium, splenium corporis callosi. The genu bends ventrally to form the ros- 


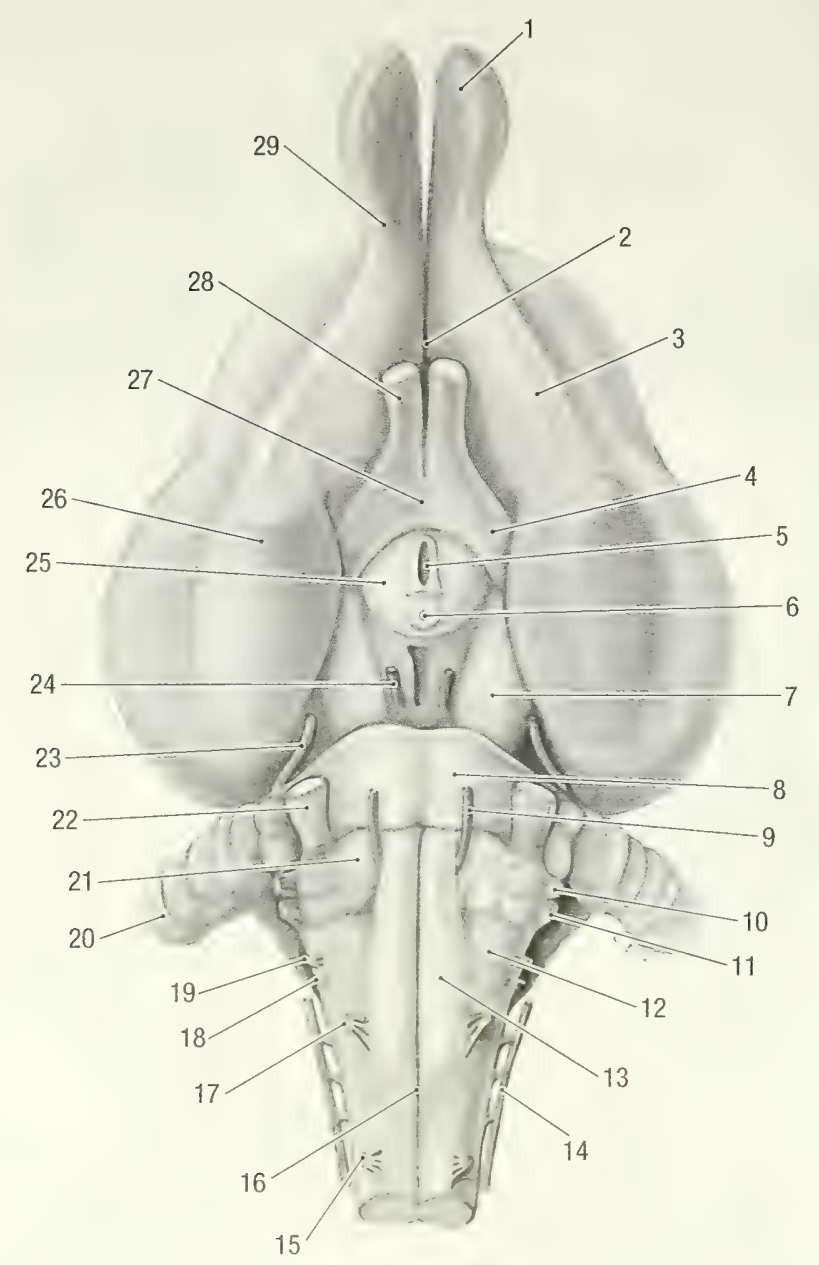

Fig. 10-3. Brain, ventral view. 1 olfactory bulb, 2 medial olfactory tract, 3 lateral olfactory tract, 4 optic tract, 5 neurohypophyseal recess within the stalk of the hypophysis (removed), 6 mammillary body, 7 cerebral crus, 8 pons, 9 abducent $\mathrm{n} ., 10$ facial $\mathrm{n}$., 11 vestibulocochlear $\mathrm{n} ., 12$ olive, 13 pyramid, 14 accessory n., 15 first cervical spinal $\mathrm{n}$., 16 median fissure, 17 hypoglossal n., 18 vagus n. 19 glossopharyngel n., 20 parafloccular lobe of cerebellum, 21 trapezoid body 22 trigeminal n., 23 trochlear n., 24 oculomotor n., 25 tuber cinereum, 26 piriform lobe, 27 optic chiasma, 28 optic n., 29 olfactory peduncle.

trum corporis callosi. The latter continues as the lamina terminalis. The rostral commissure, commissura rostralis, is a prominent, round bundle of transverse nerve fibers in the lamina terminalis.

Ventral to the corpus callosum is another prominent band of fibers, the fornix. The corpus callosum and fornix are connected by a mass of septal nuclei known as the telencephalic septum, septum telencephali (formerly the septum pellucidum). The latter separates the two lateral ventricles, ventriculus lateralis, from each other. Ventral to the fornix is the cavity of the third ventricle, ventriculus tertius. The third ventricle encircles the interthalamic adhesion, adhesio interthalamica, which connects the left and right halves of the diencephalon. The lateral and third ventricles communicate with each other through the interventricular foramen, fora- men interventriculare. The latter is visible in the rostral part of the third ventricle, just lateral to the point where the fornix and interthalamic adhesion join. The third ventricle forms various recesses. These are the suprapineal recess, recessus suprapinealis, that extends dorsal to the pineal gland, the pineal recess, recessus pinealis, in the peduncle of the pineal gland, an optic recess, recessus opticus, dorsal to the optic chiasma and the neurohypophyseal recess, recessus neurohypophysialis, that extends into the hypophyseal stalk. The pineal gland, $g l$. $p i-$ nealis, lies in the roof of the third ventricle, caudoventral to the splenium of the corpus callosum. The third ventricle continues caudally into the mesencephalon as the mesencephalic aqueduct, aqueductus mesencephali.

The large rostral colliculus, colliculus rostralis, 


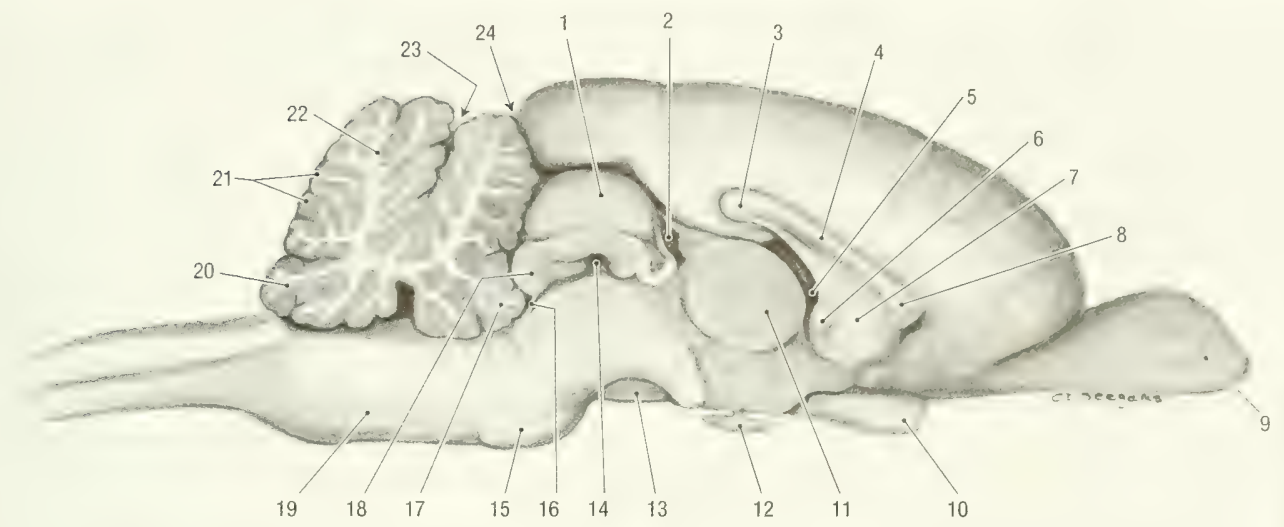

Fig. 10-4. Brain, median section. 1 rostral colliculus, 2 pineal recess, 3 splenium of the corpus callosum, 4 body of the corpus callosum, 5 interventricular for., 6 rostral commissure, 7 telencephalic septum, 8 genu of the corpus callosum, 9 olfactory bulb, 10 optic chiasma, 11 interthalamic adhesion, 12 hypophysis, 13 cerebral crus, 14 mesencephalic aqueduct, 15 pons, 16 rostral medullary velum, 17 lingula, 18 caudal colliculus, 19 myelencephalon, 20 nodulus, 21 folia, 22 arbor vitae, 23 primary fissure, 24 transverse fissure.

and the smaller caudal colliculus, colliculus caudalis, of the mesencephalon lie dorsally to the aqueduct. The mesencephalic peduncle, pedunculus cerebri, lies ventral to the aqueduct.

The cerebellar cortex is arranged as thin leaves or folia, folia cerebelli. The folia consist of centrally located white matter, arbor vitae, covered by gray matter.

The mesencephalic aqueduct continues caudally into the rhombencephalon as the fourth ventricle, ventriculus quartus. The roof of the fourth ventricle is formed by a rostral and a caudal medullary velum, velum medulare rostrale and velum medulare caudale. The fourth ventricle continues caudally as the central canal of the spinal cord, canalis centralis.

Subdivisions of the Brain. The telencephalon (cerebrum) is the largest, most rostral part of the brain. It is approximately $30 \mathrm{~mm}$ long, $30 \mathrm{~mm}$ wide and $20 \mathrm{~mm}$ thick. Along the midline, the cerebrum is divided into two cerebral hemispheres by the longitudinal fissure. The cerebral hemispheres are separated caudally from the cerebellum by the transverse fissure. Each cerebral hemisphere consists of frontal, parietal, occipital and temporal lobes. The lobes are topographical areas and their boundaries are quite arbitrary. The frontal lobe comprises the rostral part of the cerebral hemisphere, the occipital lobe the caudal part, the temporal lobe the ventrolateral part and the parietal lobe the remaining dorsal part of the cerebral hemisphere.

From without inwards the cerebrum consists of a layer of gray matter or cerebral cortex, white matter beneath the cortex, centrally located basal nuclei and the rhinencephalon at the base of the brain. Inside each cerebral hemisphere is a cavity, the lat- eral ventricle. The two ventricles are separated from each other by the telencephalic septum. The latter is often double, with a cavity between the two layers. The two cerebral hemispheres are connected to each other by the corpus callosum.

The cerebral cortex, cortex cerebri (Figs. 10-1 to 10-4), consists of three phylogenetically different parts, namely the neocortex, the palleocortex and the archicortex. The neocortex is laterally separated from the paleocortex by the lateral rhinal sulcus and medially from the archicortex by the very indistinct splenial sulcus. It forms the greater part of the cerebral cortex that is visible on the surface of the brain. Together the archicortex and paleocortex form the rhinencephalon.

The surfaces of the neocortex, neopallium, are mostly smooth. The gyri are generally poorly defined and the sulci very shallow. Furthermore, the development of the sulci and the gyri varies between animals and often between the left and the right sides. The pseudosylvian fissure, fissura pseudosylvia, is indicated by a shallow depression on the lateral surface of the cerebral hemisphere. It contains the middle cerebral artery or one of its branches. The marginal sulcus, sulcus marginalis, is a very shallow, longitudinal groove or depression along the dorsal surface of the cerebral hemisphere. It varies greatly in length. On some specimens a cruciate sulcus, sulcus cruciatus, is present on the rostrodorsal surface.

A very faint and shallow splenial sulcus, sulcus splenialis, is present on the medial surface of the cerebral hemisphere. It generally contains the caudal cerebral artery. The callosal sulcus, sulcus corporis callosi, bounds the corpus callosum rostrally, dorsally and caudally. At the caudal end of the cor- 
pus callosum, the callosal sulcus blends with the hippocampal sulcus, sulcus hippocampi, which passes ventrorostral to the temporal lobe.

The gyri of the cerebral cortex are separated from each other by the various sulci and are generally named after an adjacent sulcus. However, very few gyri are identifiable on the surface of the cerebral cortex of the woodchuck. On the lateral surface, the marginal gyrus, gyrus marginalis, lies between the marginal and splenial sulci. It extends along the dorsal and caudal margin of the cerebral hemisphere and is continues with the occipital gyrus, gyrus occipitalis. On the medial surface, the cingulate gyrus, gyrus cinguli, surrounds the corpus callosum rostrally, dorsally and caudally. It lies between the splenial and callosal sulci.

The dentate gyrus, gyrus dentatus, is partly involuted in the depth of the hippocampal sulcus and extends from the temporal lobe to the splenium of the corpus callosum. Its free border is visible along the concave lateral side of the hippocampal sulcus.

The rhinencephalon consists of basal, septal and limbic parts.

Basal part, pars basalis rhinencephali: The olfactory nerves from the olfactory mucosa enter the olfactory bulbs. The latter lie in the ethmoidal fossae of the ethmoid bone. The olfactory bulbs are about $10 \mathrm{~mm}$ long, $5 \mathrm{~mm}$ wide and $5 \mathrm{~mm}$ deep. They are connected to the cerebral hemispheres by the olfactory peduncles, pedunculus olfactorius. The peduncles consist of lateral and medial olfactory tracts. The lateral olfactory tract, tractus olfactorius lateralis, is the larger of the two tracts. It connects the olfactory bulb with the caudal part of the piriform lobe and is separated from the neocortex by the lateral rhinal sulcus, sulcus rhinalis lateralis.

The lateral rhinal sulcus is the most prominent and constant groove on the surface of the brain and extends along the entire length of the cerebrum. It separates the dorsally situated neocortex from the paleocortex. The sulcus is divided into rostral and caudal parts by the pseudosylvian fissure. The lowest point of the piriform lobe is the tuberculum hippocampi.

The medial olfactory tract, tractus olfactorius medialis, curves around the medial aspect of the cerebral hemisphere and connects the olfactory bulb with the septal part of the rhinencephalon. Between the two tracts is a slightly elevated, oval area, the olfactory tubercle, tuberculum olfactorium. The latter represents the rostral part of the piriform lobe.

Septal part, pars septalis rhinencephali: This part of the rhinencephalon lies on the medial surface of the cerebral hemisphere, rostroventral to the corpus callosum, and includes the interventricular septum.

Limbic part, pars limbica rhinencephali: This part of the rhinencephalon consists of the indusium griseum, hippocampus, and fornix. The indusium griseum, indusium griseum, is a thin layer of gray matter that lies on the corpus callosum. It bends ventrally over the caudal point of the corpus callosum and blends with the dentate gyrus. The latter joins the hippocampus and follows it to the tuberculum hippocampi. The hippocampus, hippocampus, is a C-shaped mass of cerebral cortex in the caudal floor of the third ventricle. It extends from the tuberculum hippocampi, first caudally, then dorsomedially and finally rostromedially, around the thalamus. The ventricular surface of the hippocampus is covered by a thin layer of white matter, the alveus, alveus hippocampi. The major part of the fibers from the alveus gathers along the concave (rostral) edge of the hippocampus to form the fimbria of the hippocampus, fimbria hippocampi. The fimbria continues medially and rostrally along the rostral edge of the hippocampus to form the crus of the fornix, crus fornicis. The left and right crura join ventrally to the splenium of the corpus callosum to form the body of the fornix, corpus fornicis. Just caudal to the point where the two crura meet, some of the fibers decussate to form the commissure of the fornix, commissura fornicis. The fornix continues rostrally as two columns, columna fornicis. At the level of the rostrum of the corpus calosum the columns bend ventrally and end in the mammillary body. The interventricular foramen lies caudal to the columns of the fornix.

The basal nuclei (subcortical gray matter) are a group of nuclei located deep in the frontal, parietal and temporal lobes of the cerebral hemispheres. They consist of the corpus striatum, the claustrum and the amygdala.

The corpus striatum, corpus striatum, belongs to the neocortex and is the largest of the basal nuclei. It is subdivided into the caudate and the lentiform nuclei. The caudate nucleus, nucleus caudatus, is an oval mass that protrudes into the third ventricle, rostral to the hippocampus. It is separated from the thalamus by the stria terminalis, and from the lentiform nucleus by the internal capsule.

Fiber Tracts. The white matter of the telencephalon consists of two nerve fiber systems. These are the corticocortical fibers and the projection fibers.

The corticocortical fibers have both their origins and terminations in the cerebral cortex. They connect different cortical areas of the same cerebral hemisphere (association fibers), or homologous areas on opposite sides of the cerebrum (commissural fibers).

Projection fibers originate in the cerebral cortex and descend to lower centers, or originate in lower centers and ascend to the cerebral cortex. The pro- 
jection fibers of the neocortex form the internal capsule. The fibers pass through the diencephalon and form the peduncles of the mesencephalon.

Lateral Ventricles. Each cerebral hemisphere contains a lateral ventricle. A ventricle consists of a rostral horn, cornu rostrale, a central part, pars centralis, and a temporal horn, cornu temporale. The telencephalic septum separates the ventricles from each other.

The rostral horn extends into the olfactory peduncle and bulb. Its medial wall is formed by the telencephalic septum and the head of the caudate nucleus lies in the lateral wall.

In the central part, the fornix lies in the medial wall and the rest of the caudate nucleus in the lateral wall. The hippocampus and its fimbria projects into the temporal horn. The latter extends into the tuberculum hippocampi.

The choroid plexus of the lateral ventricle, plexus choroideus ventriculi lateralis, extends along the fimbria and fornix. On each side an interventricular foramen connects the lateral ventricle with the third ventricle of the diencephalon.

Diencephalon (Figs. 10-3 and 10-4). This is the most rostral portion of the brain stem. Most of it is obscured from view by the piriform lobes of the cerebrum, but is visible if the latter is lifted and pulled rostrally. The structures of the diencephalon are arranged around the third ventricle. During early embryonal development the lateral walls of the diencephalon thicken and protrude into the cavity of the third ventricle. The thickened areas fuse in the midline to form the interthalamic adhesion, adhesio interthalamica. The adhesion transforms the third ventricle into a circular cavity.

The subdivisions of the diencephalon are the epithalamus (pineal) along the dorsal midline, the thalamus in the center, the hypothalamus along the midline at the base of the diencephalon, the metathalamus dorsolateral and subthalamus caudoventral to the thalamus and caudolateral to the hypothalamus.

The thalamus, thalamus, is the largest part of the diencephalon. It forms the dorsomedial part of the diencephalon. Except for the area of the adhesion along the midline of the thalami, the third ventricle separates the two sides. The dorsal surface of the thalamus forms a slightly elevated, triangular area of grey matter. Viewed from above the thalamus is separated from the caudate nucleus by the stria terminalis and bounded medially by the habenular stria, stria habenularis thalami. The roof of the third ventricle is attached to the habenular stria along the tenia thalami.

The metathalamus, metathalamus, consists of a large lateral and smaller medial geniculate bodies. The lateral geniculate body, corpus geniculatum laterale, is located dorsally at the caudolateral angle of the thalamus. It receives the fibers from the optic tract. The medial geniculate body, corpus geniculatum mediale, lies caudoventral to the lateral geniculate body. It forms the caudalmost part of the diencephalon and receives fibers from the vestibulocochlear nerves.

The epithalamus, epithalamus, is a narrow area along the dorsal midline of the diencephalon. It consists of the pineal gland and habenula. The pineal gland, glandula pinealis, lies in the roof of the third ventricle, caudo-ventral to the splenium of the corpus callosum. It is about $1 \mathrm{~mm}$ in diameter and is attached to the ventricular wall by a peduncle. The habenula, habenula, is a small mass of gray matter located under the rostral limb of the pineal peduncle.

On either side of the midline the hypothalamus, hypothalamus, lies at the base of the diencephalon. It lies medial to the subthalamus and lateral to the third ventricle. Major landmarks on its ventral surface are the optic chiasma, the tuber cinereum and the mammillary bodies. The relationship of the optic chiasma to the hypothalamus is largely topographical.

The subthalamus, subthalamus, is a transitional area between the thalamus and the mesencephalon and lies caudal and lateral to the hypothalamus.

Third Ventricle, Ventriculus Tertius. This is the cavity of the third ventricle. It lies in the median plane and encircles the interthalamic adhesion. It connects rostrodorsally with the lateral ventricles by way of the interventricular foramen and caudally with the aqueduct of the midbrain. The choroid plexus of the third ventricle protrudes from the dorsal aspect of the third ventricle into its lumen and is continuous with the choroid plexus of the lateral ventricles through the interventricular foramen. The third ventricle has suprapineal, pineal, optic and neurohypophyseal recesses.

\section{Midbrain, Mesencephalon (Figs. 10-2 to 10-} 4). The midbrain lies between the diencephalon cranially and the metencephalon caudally, and is divided by the aqueduct, aqueductus mesencephali, into the tectum dorsally and peduncles ventrally.

The tectum, tectum mesencephali, lies dorsal to the aqueduct. It consists of four rounded elevations, two rostral colliculi and two caudal colliculi. Each colliculus is connected to the brain stem by a fiber tract.

The rostral colliculi, colliculi rostrales, are approximately $7 \mathrm{~mm}$ in diameter and $4 \mathrm{~mm}$ high. Each rostral colliculus is connected to a lateral ge- 
niculate body of the metathalamus by a band of fibres, the brachium colliculi rostralis.

The caudal colliculi, colliculi caudales, are small. They are approximately $5 \mathrm{~mm}$ wide, $2 \mathrm{~mm}$ long and $2 \mathrm{~mm}$ high. Each caudal colliculus is connected to a medial geniculate body of the metathalamus by a fiber tract, the brachium colliculi caudalis.

The mesencephalic peduncles, pedunculus cerebri, consist of two crura ventrally and a tegmentum dorsally, separated from each other by the substantia nigra. Each crus, crus cerebri, is composed of a large group of superficially located nerve fibers along the ventrolateral surface of the midbrain. The crus extends from the optic tract to the pons. The crura emerge from the caudal aspect of the optic tracts, halfway between the optic chiasma and the lateral geniculate body. They converge caudally and are separated by a deep depression, the fossa interpeduncularis. The tegmentum, tegmentum, lies between the crura ventrally and the aquaduct dorsally. It consists of various nuclei.

The oculomotor (III) and trochlear (IV) nerves originate in the mesencephalon.

The aqueduct of the mesencephalon connects the third ventricle of the diencephalon with the fourth ventricle of the rhombencephalon. It is tube-like in shape and shows two dorsal enlargements that extend into the rostral colliculi.

Pons (Figs. 10-2 to 10-4). The pons is a bridge of fibers that externally separates the metencephalon from the midbrain and the myelencephalon. It consists of a superficial band of transverse nerve fibers and deeper nuclei. The pons is connected on both sides to the cerebellum by the middle cerebellar peduncles, pedunculus cerebellaris medius.

Cerebellum (Figs. 10-1 to 10-4). The cerebellum is about $15 \mathrm{~mm}$ long and $30 \mathrm{~mm}$ wide. It lies dorsal to the fourth ventricle and is attached to the brain stem on each side by nerve fibers forming rostral, middle and caudal cerebellar peduncles. The rostral cerebellar peduncle, pedunculus cerebellaris rostralis, connects the cerebellum to the midbrain, the middle cerebellar peduncle, pedunculus cerebellaris medius, connects the cerebellum to the pons. A caudal cerebellar peduncle, pedunculus cerebellaris caudalis, connects the cerebellum to the dorsal funiculus of the spinal cord. The surface the cerebellum is deeply fissured, dividing it into folia or leaves. The primary fissure, fissura prima, divides the cerebellum into rostral and caudal lobes. In spite of its considerable depth, the fissura prima is not easily detectable among the large number of similar sulci unless the cerebellum is hemisected. Topographically the cerebellum is divided into a median portion, the vermis, vermis, and a cerebellar hemisphere, hemispherium cerebelli, on each side. The vermis and hemispheres are divided into a number of lobules that are designated by the Roman numerals I to X. (For the classification of the lobules see Larsell 1953).

The rostral lobule of the vermis is the lingula, lingula cerebelli, and the caudal lobule the nodulus, nodulus.

On the ventral surface of each hemisphere, just lateral to the middle peduncle of the cerebellum, is a small lobule, the flocculus, flocculus. Immediately dorsal to the flocculus lies the prominent parafloccular lobe, paraflocculus.

Myelencephalon (Figs. 10-2 to 10-4). The myelencephalon is the most caudal portion of the brain stem. It extends from the caudal edge of the pons to the beginning of the spinal cord and contains the nuclei of some of the cranial nerves, ascending and descending fiber bundles with associated nuclei, and the fourth ventricle.

On the ventral surface of each side of the median fissure lies the pyramids, pyramis. According to Doetsch and Towe (1981), the pyramids consist of fibers that originate entirely within the rostral half of the cerebral hemispheres. They follow a course that is typical for rodents.

Caudal to the pons a bundle of transverse fibers form the trapezoid body, corpus trapezoideum.

Lateral to the pyramids, just caudal to the trapezoid body, is a round, elevated area, the olive, oliva.

On the dorsal surface the myelencephalon is a hollow, the rhomboid fossa, fossa rhomboidea, that forms the floor of the fourth ventricle. The fossa is separated into two halves by the median sulcus, sulcus medianus, and each half is divided into dorsal and ventral segments by the sulcus limitans. Rostrolateral to the rhomboid fossa is a prominent swelling, the acoustic tubercle, tuberculum acusticum. The cuneate nucleus, nucleus cuneatus, forms a prominent swelling along the caudolateral border of the rhomboid fossa.

Fourth Ventricle, Ventriculus Quartus. The fourth ventricle is the cavity of the rhombencephalon. It lies between the cerebellum dorsally and the pons and myelencephalon ventrally. It opens rostrally into the aqueduct of the midbrain and it continues caudally as the central canal of the spinal cord. It has two lateral recesses, recessus lateralis ventriculi quarti, and communicates with the subarachnoid space through two lateral openings, apertura laterales ventriculi quarti.

The roof of the fourth ventricle is mostly a thin membrane. The rostral part, or rostral medullary 
vellum, velum medullare rostrale, lies between the rostral cerebellar peduncles. The vellum is attached rostrally to the midbrain and caudally to the cerebellum. The caudal part of the ventricular roof, or caudal medullary vellum, velum medullare caudale, lies between the two caudal cerebellar peduncles. It extends from the central canal of the spinal cord to the cerebellum. The lateral apertures lie in the caudal part of the roof of the fourth ventricle.

The choroid plexus of the fourth ventricle is attached to the roof of the ventricle. Part of the plexus protrudes through the Iateral apertures into the subarachnoid space.

Circulation of Cerebrospinal Fluid. The pia mater is closely applied to the surface of the brain and contains a rich network of blood vessels. In the lateral and fourth ventricles the neural tube thins, leaving only the pia mater and the epithelial lining of the ventricles, the ependyma, to form a thin sheet of non-nervous tissue, the tela choroidea to serve as a roof to the brain cavity. The blood vessels in the tela choroidea branch profusely, forming a choroid plexus. The choroid plexus secretes cerebrospinal fluid into the ventricles. From the lateral ventricles the fluid passes through the interventricular foramina into the third ventricle, and from there through the aquaduct of the midbrain into the fourth ventricle. From the fourth ventricle cerebrospinal fluid circulate down the central canal of the spinal cord, as well as through the lateral apertures of the fourth ventricle into the subarachnoid space surrounding the brain and spinal cord. Cerebrospinal fluid is absorbed back into the blood from the subarachnoid space, mostly at arachnoid villi, located along the lateral sides of the dorsal sagittal sinus.

\section{SPINAL CORD, MEDULLA SPINALIS}

This division of the central nervous system is enclosed within the vertebral canal. It develops from the neural tube caudal to the three brain vesicles and extends from the foramen magnum to the level of the first lumbar vertebra (Figs. 10-5 and 10-6).

The spinal cord has two enlargements, one in the cervicothoracic area and the other at the thoracolumbar junction. The cervical enlargement, intumescentia cervicalis, lies at the cervicothoracic junc-

Fig. 10-5. Brain and spinal cord in situ, schematic dorsal view. 1 cerebrum, 2 wing of atlas, 3 transverse process of first thoracic vertebra, 4 lumbar intumescence, 5 terminal filum in the cauda equina, 6 first sacral vertebra, 7 first caudal vertebra, 8 first lumbar vertebra, 9 spinal ganglia, 10 cervical intumescence, 11 cerebellum.

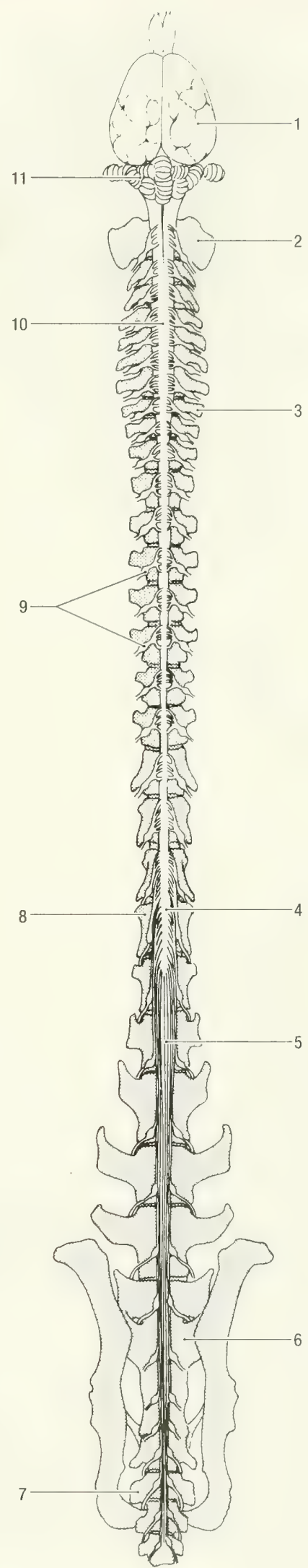




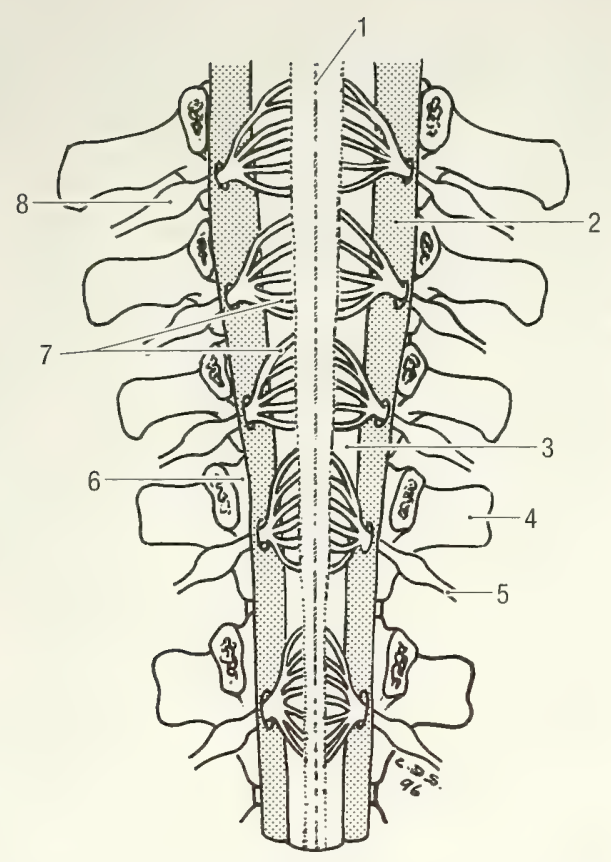

Fig. 10-6. The cervico-thoracic spinal cord exposed in dorsal view. 1 median groove, 2 dura mater, opened and reflected, 3 subarachnoid space, 4 transverse process, 5 spinal n., 6 epidural space, 7 dorsal roots of spinal n., 8 spinal ganglion.

tion. It indicates the area from which the nerves to the thoracic limb arise. The lumbar enlargement, intumescentia lumbalis, lies at the thoracolumbar junction. It indicates the origin of the nerves to the pelvic limbs. Caudal to the lumbar enlargement the spinal cord tapers to a cone, the conus medullaris. The spinal cord ends as a thin strand of tissue, the filum terminale. Within the vertebral canal, sacral and caudal spinal roots pass caudally toward the intervertebral foramina where they exit. Collectively, these roots form the cauda equina.

A ventral median fissure, fissura mediana, and a dorsal median groove, sulcus medianus, divide the spinal cord into symmetrical halves. Dorsolateral and ventrolateral grooves, sulcus lateralis dorsalis and sulcus lateralis ventralis, further subdivides each halve of the spinal cord. The dorsal and ventral roots of the spinal nerves emerge at the dorsolateral and ventrolateral grooves respectively. The median fissure contains the ventral spinal artery.

The substance of the spinal cord is arranged around the central canal, canalis centralis, with the gray matter centrally and the white matter peripherally. The white matter consists of tracts; bundles of axons and since these are myelinated, the overall color is white. Gray matter consists of aggregations of nerve cell bodies showing little myelin and so the overall appearance is gray.
In transverse section the gray matter is butterflyshaped. Each wing of gray matter is divided into dorsal and ventral horns, cornu dorsale and cornu ventrale. An intermediate zone or lateral horn, cor$n u$ laterale, is only visible in the thoracolumbar section of the spinal cord.

The white matter is divided into longitudinal bundles or funiculi. The dorsal funiculus, funiculus dorsalis, lies medial to the dorsal roots of the spinal nerves. The lateral funiculus, funiculus lateralis, lies between the dorsal and ventral roots and the ventral funiculus, funiculus ventralis, lies ventral to the ventral roots.

\section{PERIPHERAL NERVOUS SYSTEM, CRANIAL NERVES}

General Considerations. There are twelve pairs of cranial nerves. These are the olfactory, optic, oculomotor, trochlear, trigeminal, abducent, facial, vestibulochochlear, glossopharyngeal, vagus, accessory and hypoglossal nerves (Fig. 10-3). Seven categories of fibers are present in the cranial nerves. These are somatic afferent, special somatic afferent, somatic efferent, visceral afferent, special visceral afferent, visceral efferent and special visceral efferent fibres.

Somatic afferent fibers transmit impulses from the skin, muscles and mucous membranes to the brain stem. These fibers have their cell bodies in sensory ganglia associated with the roots of the cranial nerves outside the central nervous system.

Special somatic afferent fibers come from the special sensory organs of vision, hearing and balance.

Somatic efferent fibers arise from nuclei within the brain stem. They innervate the striated muscles of the eye and tongue.

Visceral afferent fibers conduct sensory impulses from the internal organs, not only from the head, but also from some of the thoracic and abdominal viscera. These fibers have their cell bodies in sensory ganglia associated with the roots of the cranial nerves outside the central nervous system.

Special visceral afferent fibers are those which come from the visceral sensory organs of taste and olfaction.

Visceral efferent fibers have their cell bodies in nuclei in the brain stem. They are preganglionic fibers and belong to the parasympathetic division of the autonomic nervous system.

Special visceral efferent or branchial motor fibers supply striated muscles of branchial origin. They are found in the trigeminal, facial, glossopharyngeal, vagus and accessory nerve. The nerves supply the muscles of mastication, the muscles of the face, 
pharynx, soft palate and muscles of the neck and thoracic limb that are of branchial origin.

Cranial Nerve I, Olfactory Nerves, $\boldsymbol{n}$. olfactorii. The first cranial nerve consists of many small bundles of nerve fibers that pass from the olfactory mucosa, through the cribriform plate of the ethmoid bone, to the olfactory bulb (Fig. 103). The cell bodies of olfactory neurons are located in the olfactory epithelium of the nasal cavity. The vomeronasal nerve transmits from the epithelium of the vomeronasal organ via the lateral olfactory tract to the amygdala.

\section{Cranial Nerve II, Optic Nerve, n. opticus} (Fig. 10-3). This second cranial nerve is composed of the central processes of the ganglion cells of the retina. The fibers from the retina leave the caudal pole of the eye along a horizontal line approximately $10 \mathrm{~mm}$ long and $1 \mathrm{~mm}$ wide. They converge to form the optic nerve that enters the cranial cavity through the optic canal. Some of the fibres decussate, forming the optic chiasma, before they enter the lateral geniculate body. The optic nerve is surrounded by extensions of the cranial meninges over most of its course. The dura mater, however, does not reach the eye, but ends approximately $1 \mathrm{~mm}$ from the sclera. The fibers of the optic nerve are classified as special somatic afferent.

\section{Cranial Nerve III, Oculomotor Nerve, $\boldsymbol{n}$.} oculomotorius (Fig. 10-3). The third cranial nerve contains general somatic efferent fibers (motor) to the striated muscles of the eye and general visceral efferent (parasympathetic) fibers to the ciliary ganglion.

It leaves the cranial cavity through the orbital fissure. The nerve enters the periorbita and immediately divides into dorsal and ventral branches.

The ventral branch gives off branches to the medial rectus, ventral rectus and ventral oblique muscles of the eye. Parasympathetic fibers pass in the ventral branch and synapse in the ciliary ganglion, ganglion ciliare. Postganglionic fibers innervate the muscles of the ciliary body (which regulates the curvature of the lens) and the sphincter muscle of the iris (which reduces the diameter of the pupil).

The dorsal branch innervates the dorsal rectus muscle of the eye, as well as the levator of the upper eyelid.

Cranial Nerve IV, Trochlear Nerve, $\boldsymbol{n}$. trochlearis (Fig. 10-3). The fourth cranial nerve leaves the cranial cavity through the orbital fissure. As it emerges from the fissure, it turns dorsomedially and enters the dorsal oblique muscle of the eye. The latter is the only muscle supplied by the trochlear nerve. Its fibers are classified as general somatic efferent.

Cranial Nerve V, Trigeminal Nerve, $\boldsymbol{n}$. trigeminus (Fig. 10-3). The fifth cranial nerve is the largest of the cranial nerves. Its sensory fibers (general somatic afferent) receive impulses from the skin and cutaneous muscles of the head as well as from the nasal and oral cavities. Its motor fibers (special visceral efferent) innervate the muscles of mastication. The trigeminal ganglion, ganglion trigeminale, is the sensory ganglion of the trigeminal nerve. It contains the neurons of the afferent fibers. The sensory fibers of all three divisions of the trigeminal nerve arise from the trigeminal ganglion. The three divisions are the ophthalmic, maxillary and mandibular nerves. All the fibers of the ophthalmic and maxillary nerves are sensory, while the mandibular nerve carries both sensory and motor fibers.

The ophthalmic nerve, $n$. ophthalmicus, is the main sensory nerve of the orbit. It leaves the cranial cavity through the orbital fissure and divides into its three primary branches, namely the frontal, lacrimal and nasociliary nerves.

The frontal nerve, $n$. frontalis, runs rostrodorsally, deep to the periorbita and dorsally to the dorsal rectus and levator palpebral muscles. It passes through the supraorbital incisure of the frontal bone and ramifies in the skin of the upper eyelid and supraorbital regions.

The lacrimal nerve, $n$. lacrimalis, passes dorsolaterally along the lateral edge of the lateral rectus muscle. It supplies the lacrimal gland and surrounding tissues, including a small area of the upper eyelid, as well as the frontal sinus.

The nasociliary nerve, $n$. nasociliaris, passes rostrally, ventral to the trochlear nerve, and gives off the ciliary, ethmoidal and infratrochlear nerves. The ciliary nerve, $n$. ciliaris, passes through the sclera of the eye to innervate the eyeball. The ethmoidal nerve, n. ethmoidalis, passes rostomedially, enters the cranial cavity through the ethmoidal foramen and then passes through the cribriform plate of the ethmoid bone to reach and innervate the part of the nasal cavity as far rostrally as the nasal vestibule. The infratrochlear nerve, $n$. infratrochlearis, runs rostrodorsally along the lateral edge of the dorsal oblique muscle. It passes ventral to the trochlea for the tendon of the dorsal oblique muscle and ramifies in the skin at the medial canthus of the eye.

The maxillary nerve, $n$. maxillaris, is the largest of the three trigeminal nerve divisions. It supplies the skin of the cheek, side of the nose, muzzle, mucuous membrane of the nasopharynx, soft and hard palates and the upper teeth and gingiva. The nerve 
leaves the cranial cavity through the round foramen, passes rostrally through the alar canal and then it divides into the zygomatic, pterygopalatine and infraorbital nerves.

The zygomatic nerve, $n$. zygomaticus, enters the apex of the periorbita and divides into zygomaticofacial and zygomaticotemporal branches. The zygomaticotemporal branch, ramus zygomaticotemporalis, is the dorsal of the two branches. It passes rostrally and dorsally along the lateral part of the periorbita to emerge at the lateral canthus of the eye. It ramifies in the skin of the lateral part of the upper eyelid and rostral temporal region. The zygomaticofacial branch, ramus zygomaticofacialis, parallels the zygomaticotemporal nerve within the periorbita. It appears on the surface ventral to the lateral canthus of the eye and ramifies in the lower eyelid and surrounding skin. In some animals the two branches pass together and only seperate from each other close to the point where they emerge.

The pterygopalatine nerve, $n$. pterygopalatinus, leaves the medial aspect of the maxillary nerve as the latter courses rostrally along the ventral border of the periorbita. It is intimately associated with the pterygopalatine ganglion of the autonomic nervous system. The nerve gives rise to the minor palatine, major palatine and caudal nasal nerves. The minor palatine nerve, $n$. palatinus minor, innervates the soft palate, while the major palatine nerve, n. palatinus major, enters the caudal palatine foramen to innervate the mucosa of the hard palate and surrounding gingiva. The caudal nasal nerve, $n$. nasalis caudalis, enters the nasal cavity through the sphenopalatine foramen. It innervates most of the mucous membrane of the nasal cavity.

The infraorbital nerve, $n$. infraorbitalis, is the continuation of the maxillary nerve. It enters the maxillary foramen in the pterygopalatine fossa, passes through the infraorbital canal and emerges at the infraorbital foramen. Within the infraorbital canal it gives rise to branches which innervate the teeth of the upper jaw. Within the infraorbital canal the nerve divides into a number of large fasciculi that are distributed to the skin and tactile hairs of the upper lip, muzzle and lateral nasal regions.

The mandibular nerve, $n$. mandibularis, carries both sensory and motor fibers. It leaves the cranial cavity through the oval foramen. The trunk of the mandibular nerve is short and immediately divides upon emerging from the oval foramen. It has eight branches. The lateral pterygoid, buccal and masticatory nerves leave the mandibular nerve as it emerges from the oval foramen. The three nerves pass rostrally to enter the alar canal through the caudal alar foramen, then dorsally to exit through the foramen alare parvum.

The lateral and medial pterygoid nerves, n. pter- ygoideus lateralis and $n$. pterygoideus medialis, innervate the lateral and medial pterygoid muscles respectively.

The masticatory nerve, $n$. masticatorius, emerges through the foramen alare parvum. It divides into the deep temporal and masseteric nerves. The deep temporal nerve, $n$. temporalis profundus, innervates the temporal muscle. The masseteric nerve, $n$. massetericus, passes laterally along the rostral border of the temporo-mandibular joint and then from medial to lateral over the mandibular notch to innervate the masseter muscle.

The auriculotemporal nerve, $n$. auriculotemporalis (Fig. 10-9), passes laterally along the caudal border of the temporomandibular joint. It emerges between the external auditory canal and the caudal border of the masseter muscle where it divides into rostral auricular and transverse facial branches. The rostral auricular branch, $n$. auricularis rostralis, crosses the zygomatic arch and innervates the skin on the outer surface of the ear canal, as well as the temporal and zygomatic regions of the head. The transverse facial branch, ramus transversus faciei, passes rostrally over the masseter muscle and joins the dorsal buccal branch of the facial nerve. It innervates the skin over the lateral facial region.

The lingual nerve, $n$. lingualis, passes rostroventrally between the lateral and medial pterygoid muscles. At the rostral border of the medial pterygoid muscle the lingual nerve is joined by the chorda tympani from the facial nerve. It turns ventrally around the rostral border of the medial pterygoid muscle, gives off a few branches to the isthmus faucium and then joins the lingual blood vessels and the mandibular duct. It accompanies the blood vessels and duct to supply the sensory innervation of the rostral part of the tongue.

The buccal nerve, $n$. buccalis, emerges through the foramen alare parvum. It passes dorsal to the lateral pterygoid muscle, crosses the lateral surface of the zygomatic salivary gland and ramifies in the buccal mucosa.

The mylohyoid nerve, n. mylohyoideus, is the most caudal of the mandibular branches. It leaves the ventral aspect of the mandibular nerve and passes rostroventrally between the lateral and medial pterygoid muscles. At the angle of the mandible it gives off a few nerves to the mylohyoid muscle and then divides into two branches. One branch continues rostrally along the deep face of the mylohyoid muscle, innervating the muscle along the way. The terminal end of the nerve emerges superficially between the rostral bellies of the digastric muscles to innervate the skin of the chin and rostral intermandibular region (Fig. 10-10). The second branch passes rostrally in the groove between the rostral belly of the digastric muscle and the mas- 


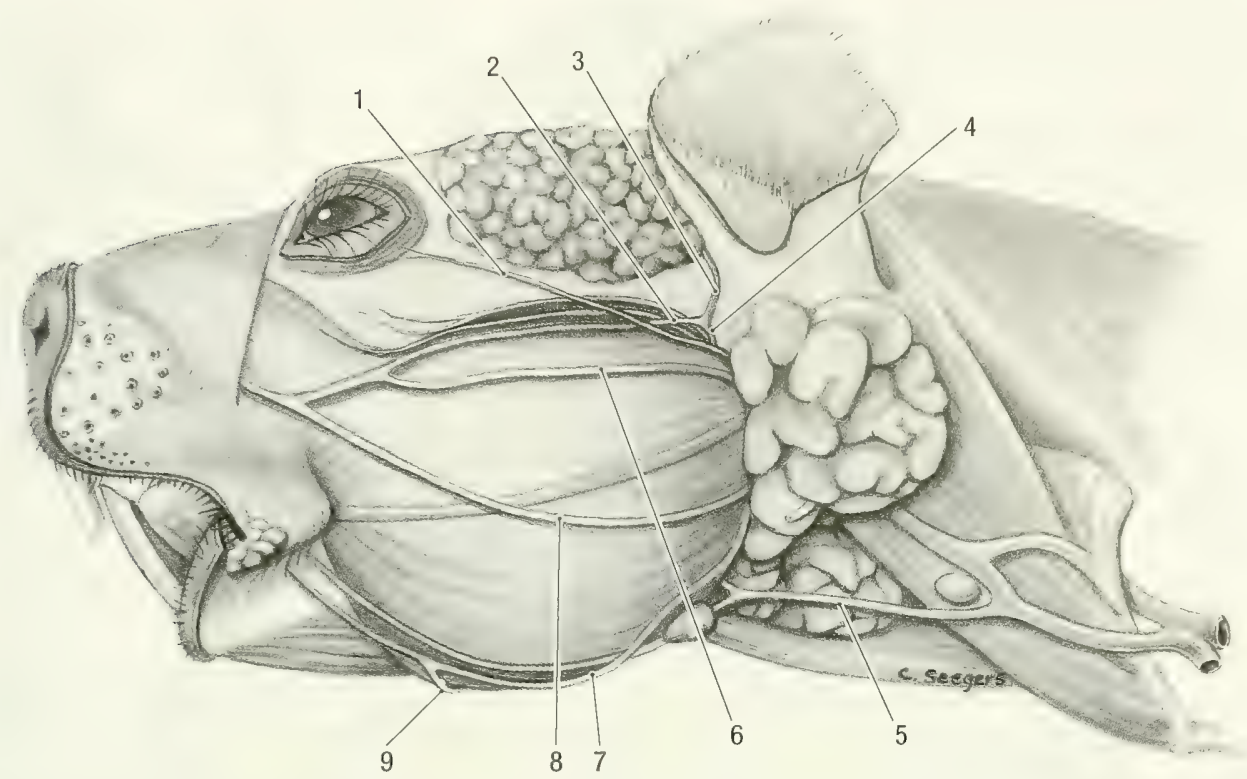

Fig. 10-7. Head, superficial left lateral view. 1 zygomatic branch of facial n., 2 transverse facial branch of mandibular n., 3 rostral auricular nerve, 4 auriculotemporal n., 5 branch of facial nerve to the platysma m., $6-8$ buccal branches of facial $\mathrm{n} ., 9$ mylohyoid $\mathrm{n}$.

seter muscle. It joins the facial blood vessels. At the rostral border of the masseter muscle the nerve turns dorsally, crossing the blood vessels, to join the ventral buccal branch of the facial nerve (Fig. 10-10). It innervates the area around the angle of the mouth.

The inferior alveolar nerve, $n$. alveolaris inferior, leaves the dorsal aspect of the mandibular nerve. It enters the mandibular canal through the mandibular foramen and innervates the teeth of the lower jaw. Some fibers leave the mandibular canal through the mental foramen to innervate the area of the lower lip.

Cranial Nerve VI, Abducent Nerve, $\boldsymbol{n}$. $\boldsymbol{a} \boldsymbol{b}$ ducens (Fig. 10-3). The sixth cranial nerve leaves the cranial cavity through the orbital fissure and enters the periorbita. It is the most ventral and medial of the cranial nerves which leave the cranial cavity through the orbital fissure. The nerve innervates the retractor bulbi and lateral rectus muscles of the eye. Its fibers are classified as general somatic efferent.

\section{Cranial Nerve VII, Facial Nerve, $\boldsymbol{n}$. facialis} (Figs. 10-3 and 10-9). The seventh cranial nerve contains special visceral efferent, special visceral afferent, general visceral efferent (parasympathetic) and general visceral afferent fibers. The special visceral efferent fibers constitute the largest portion of the facial nerve, innervating the platysma, auricular, palpebral and facial muscles. The general visceral efferent fibers are motor to the glands of the nasal cavity, mandibular and sublingual salivary glands and the lacrimal gland. All the afferent fibers have their cell bodies in the geniculate ganglion, ganglion geniculi. They carry impulses from taste buds on the tongue and visceral receptors in the epithelium of the nasal cavity, soft palate and nasopharynx.

The facial nerve accompanies the vestibulocochlear nerve into the internal acoustic meatus where it enters the facial canal. Within the canal it turns sharply, forming the genu of the facial nerve and gives off the chorda tympany and major petrosal nerves. The geniculate ganglion is located at the genu. Distal to the geniculate ganglion the facial nerve receives the auricular branch of the vagus nerve, then passes through the roof of the middle ear cavity and emerges from the canal at the stylomastoid foramen. It immediately divides into dorsal and ventral trunks. The dorsal trunk gives rise to the internal auricular, caudal auricular, auriculopalpebral and dorsal buccal nerves. The ventral trunk gives rise to the digastric, middle and ventral buccal nerves and the ramus colli.

The internal auricular branch, ramus auricularis internus, penetrates the caudal aspect of the auricular cartilage and supplies the sensory innervation of the skin on the inner surface of the ear.

The caudal auricular nerve, $n$. auricularis caudalis, passes dorsally to innervate the caudal ear muscles. 


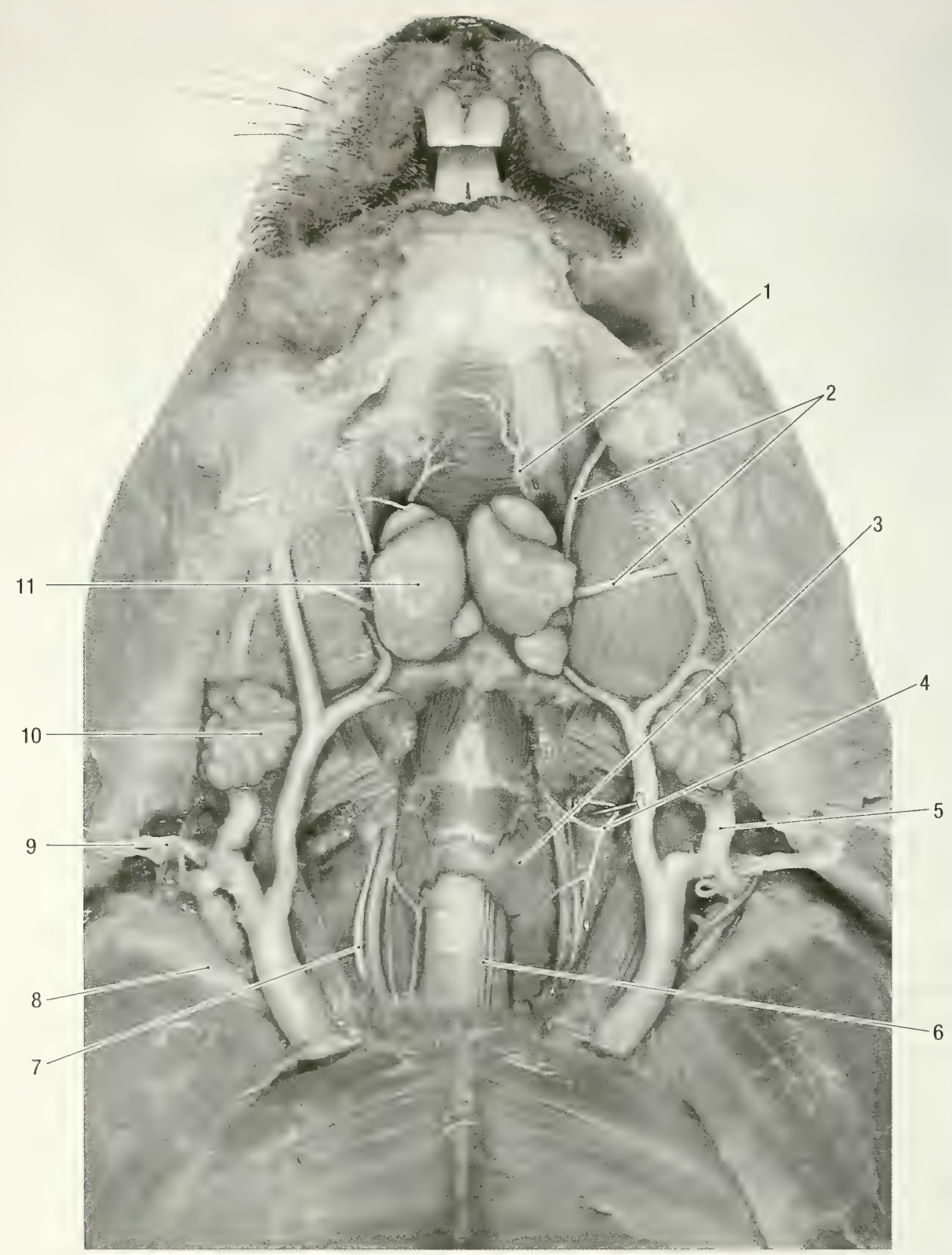

Fig. 10-8. Ventral neck region, ventral view. 1 cutaneous branch of mylohyoid n., 2 buccal branches of facial n., 3 thyroid gland, 4 pharyngeal branches of vagus n., 5 anastomoses between linguofacial and maxillary vv., 6 recurrent laryngeal n., 7 vagus n., 8 right clavicle, 9 ventral branches of third cervical spinal n., 10 parotid salivary gland, 11 mandibular salivary gland.

The auriculopalpebral nerve, $n$. auriculopalpebralis, passes dorsally and rostrally. It lies dorsal to the maxillary vein, crosses the zygomatic arch and divides into the rostral auricular and zygomatic branches. The rostral auricular branch, ramus auricularis rostralis, innervates the rostral ear muscles. The zygomatic branch, ramus zygomaticus, passes rostrally and medially to innervate the palpebral muscles, excluding the levator of the upper eyelid.

A digastric branch, ramus digastricus, passes ven- trally to innervate the caudal belly of the digastric muscle. It also gives off a branch to the stylohyoid muscle.

The ramus colli, ramus colli, is a large nerve. It passes ventrolaterally, gives off the ventral buccal branch and emerges through the distal part of the parotid salivary gland. It innervates the platysma muscle, but not the cutaneous muscle of the neck (see below).

There are three buccal branches, rami buccales. 


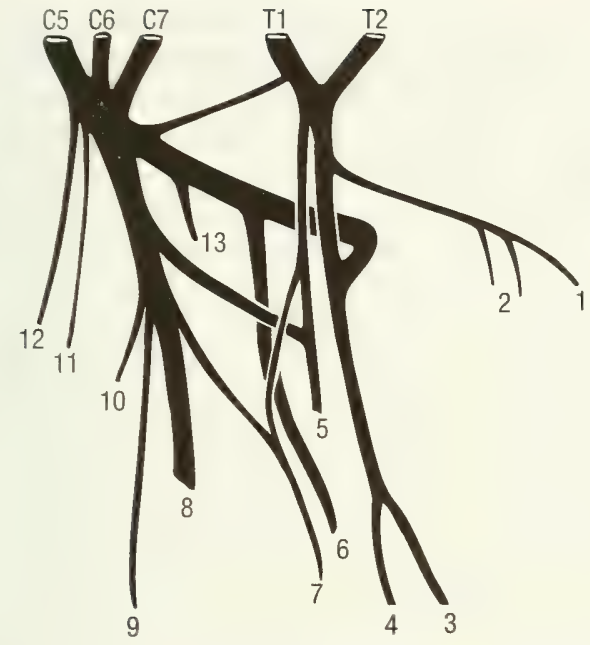

Fig. 10-9. Left brachial plexus, schematic lateral view. C5T2 ventral branches of last three cervical and first two thoracic spinal nerves, 1 lateral thoracic n., 2 caudal pectoral nn., 3 median n., 4 musculocutaneus n., 5 ulnar n., 6 radial n., 7 dorsal thoracic n., 8 axillary n., 9 branch of axillary $n$. to teres major m., 10 subscapular n., 11 long thoracic $n$., 12 suprascapular $n$.

The dorsal buccal branch passes dorsal to the maxillary blood vessels. It separates from the auriculopalpebral nerve and crosses the masseter muscle just ventral to the zygomatic arch. It is joined by branches of the transverse facial nerve and gives off small branches to some of the facial muscles before it joins the middle buccal branch.

The middle buccal branch passes ventral to the maxillary blood vessels, turns rostrally and emerges between the rostral border of the parotid salivary gland and the masseter muscle. It crosses the masseter muscle ventral to the parotid duct and joins the dorsal buccal branch at the rostral border of the masseter muscle. The dorsal and middle buccal branches innervate the muscles of the upper lip, cheek and nose.

The ventral buccal branch initially accompanies the ramus colli. It passes ventrally, deep to the parotid salivary gland and crosses the lateral surface of the rostral belly of the digastric muscle. It gives off a branch to the cutaneous muscle of the neck and is joined by a branch from the mylohyoid nerve before it ramifies in the muscles of the lower lip and cheek.

The chorda tympani, chorda tympani, carries preganglionic parasympathetic (efferent) fibers to the mandibular and sublingual salivary glands, as well as afferent fibers from the taste buds on the rostral part of the tongue. It leaves the facial nerve in the facial canal. The nerve passes through the middle ear, crosses the malleus and emerges superficially through the petrotympanic fissure. It joins the lingual branch of the mandibular nerve. The fibers to the salivary glands join the ducts of the glands. Ganglia are found scattered along their course to the glands.

The major petrosal nerve, n. petrosus major, leaves the facial nerve in the facial canal. It carries parasympathetic (efferent) fibers to the lacrimal and nasal glands. It passes through the petrous temporal bone where it is joined by the deep petrosal (sympathetic) nerve to form the nerve of the pterygoid canal. The latter passes through the pterygoid canal and runs with the maxillary division of the trigeminal nerve in the alar canal. The preganglionic parasympathetic fibers synapse in the pterygopalatine ganglion. The latter lies on or close to the pterygopalatine division of the maxillary nerve. From here postganglionic fibers innervate the lacrimal and nasal glands.

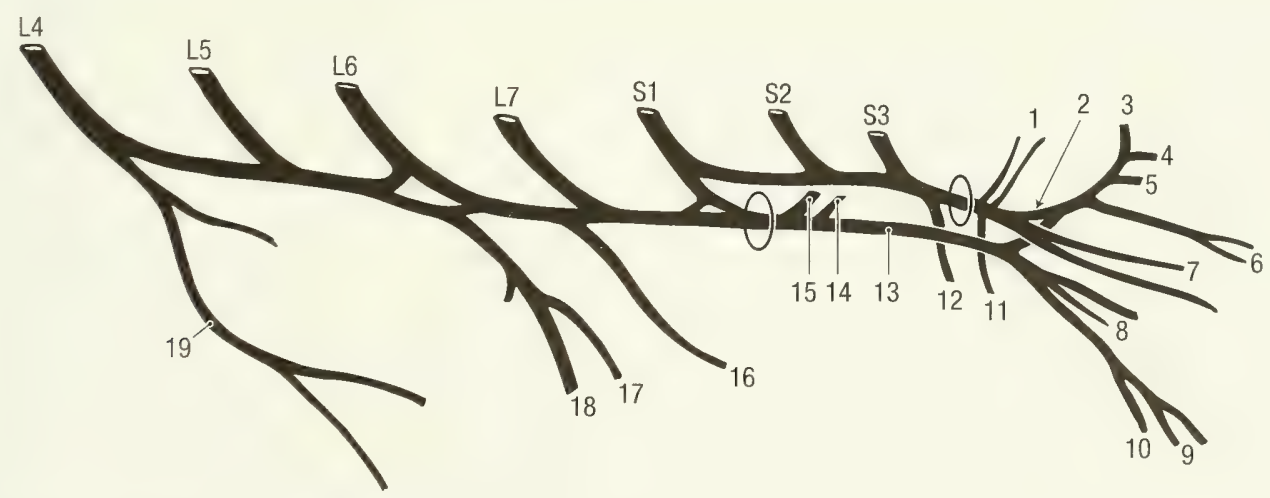

Fig. 10-10. Left lumbosacral plexus, schematic lateral view. L4-S3 ventral branches of lumbar spinal nerves 4 to 7 and sacral spinal nerves 1 to 3 . a) greater sciatic foramen, b) lesser sciatic foramen. $1 \mathrm{nn}$. to coccygeal and levator ani mm., 2 pudendal n., 3 caudal rectal n., 4 dorsal penile/clitoral n., 5 and 6 deep perineal nn., 7 superficial perineal nn., 8 muscular branches of ischiadic n., 9 peroneal (fibular) n., 10 tibial n., 11 caudal cutaneous femoral n., 12 pelvic n., 13 ischiadic n., 14 caudal gluteal n., 15 cranial gluteal n., 16 obturator n., 17 saphenous n., 18 femoral n., 19 lateral cutaneous femoral $n$. 
Cranial Nerve VIII, Vestibulo-Cochlear Nerve, n. vestibulo-cochlearis (Fig. 10-3). The vestibulocochlear nerve is composed of special somatic afferent fibers from the inner ear. The fibers are arranged in two bundles, the vestibular and cochlear parts. The vestibular part innervates the ampullae of the semicircular ducts of the inner ear and is involved with the sense of balance, the cochlear part innervates the cochlea of the inner ear and is responsible for the sense of hearing. They leave the inner ear through the internal acustic meatus.

\section{Cranial Nerve IX, Glossopharyngeal Nerve,} n. glossopharyngeus (Fig. 10-3). The glossopharyngeal nerve contains special visceral efferent fibers to the stylopharyngeus muscle, general visceral efferent (parasympathetic) fibers to the parotid, buccal and zygomatic salivary glands, special visceral afferent fibers from the taste buds on the caudal part of the tongue and general visceral afferent fibers from the tongue, pharynx and carotid sinus. It gives rise to the tympanic nerve before it leaves the cranial cavity through the jugular foramen and then divides into pharygeal and lingual branches.

The pharyngeal branch, ramus pharyngeus, passes rostrally and ramifies in the mucosa of the pharynx.

The lingual branch, ramus lingualis, passes ventrally along the caudomedial border of the stylohyoid bone. It gives off branches to the stylopharyngeus muscle and ramifies in the mucosa of the lateral pharyngeal wall, palatine tonsil and in the root of the tongue.

The tympanic nerve, $n$. tympanicus, enters the middle ear cavity to form a tympanic plexus, plexus tympanicus. The plexus is visible in the mucous membrane that covers the promontary of the middle ear cavity. Preganglionic parasympathetic fibers leave the tympanic plexus to form the minor petrosal nerve, $n$. petrosus minor. Fibers of the latter nerve synapse in the otic ganglion, ganglion oticum, at the origin of the mandibular division of the trigeminal nerve. Post-ganglionic fibers join the auriculotemporal nerve to innervate the parotid salivary gland and the buccal nerve to innervate the buccal and zygomatic salivary glands.

The cell bodies of the afferent fibers are located in the proximal ganglion, ganglion proximale, situated at the jugular foramen. They carry sensory fibers from the taste buds on the root of the tongue and pharynx.

Cranial Nerve $X$, Vagus Nerve, $n$. vagus (Fig. 10-8). This is the longest of the cranial nerves. It traverses the neck, thorax and abdomen and its components indicate its distribution and function. The components of the vagus nerves are:
General visceral efferent (parasympathetic) fibers to the heart, smooth muscle and glands of the thorax and abdominal viscera. Special visceral efferent fibers to the muscles of the pharynx (excluding the stylopharyngeus muscle), larynx and esophagus. General visceral afferent fibers from the pharynx, larynx, digestive tract, trachea, bronchi and lungs and heart.

General somatic afferent fibers from the skin of the external ear canal (internal auricular nerve).

The vagus nerve leaves the cranial cavity through the jugular foramen and appears on the external surface through the tympano-occipital fissure. Its proximal ganglion lies in the jugular foramen, the distal ganglion lies outside the tympano-occipital fissure. As the nerve leaves the tympano-occipital fissure it lies caudal to glossopharyngeal and internal carotid nerves and the blood vessels that pass through the tympano-occipital fissure.

From the tympano-occipital fissure the nerve passes caudoventrally, crossing the lateral surface of the longus capitis muscle, to reach a position dorsal to the common carotid artery. Close to the tympano-occipital fissure it gives rise to pharyngeal and cranial laryngeal branches before it joins the sympathetic trunk. The auricular branch, ramus auricularis, leaves the vagus nerve at the level of the jugular foramen. It passes through the petrous temporal bone to join the facial nerve within the facial canal. Its fibers form the internal auricular component of the facial nerve.

The pharyngeal branch, ramus pharyngeus (Fig. $10-8$ ), leaves the vagus nerve about $4 \mathrm{~mm}$ from the tympano-occipital fissure. It passes ventrally to ramify in the pharyngeal muscles and esophagus. The pharyngeal branch has extensive connections with the pharyngeal branch of the glossopharyngeal nerve, forming a pharyngeal plexus.

The cranial laryngeal nerve, $n$. laryngeus cranialis, leaves the vagus nerve and passes ventrally, between the thyropharyngeus and hyopharyngeus muscles, to enter the larynx. It supplies the cricothyroid muscle and ramifies in the mucous membrane of the larynx.

In the neck the vagus nerve runs alongside the sympathetic trunk, dorsal to the common carotid artery (Fig. 10-10). Just cranial to the first rib it separates from the sympathetic trunk and enters the thorax, passing between the subclavian artery and brachiocephalic vein (Fig. 9-1). The right vagus nerve gives off the right recurrent laryngeal nerve and continues caudally along the dorsomedial surface of the right cranial vena cava (between the vein and the lateral aspect of the trachea). It passes medial to the right azygos vein and then along the dorsal aspect of the trachea. At the bifurcation of the trachea it passes between the primary bronchus 
and the pulmonary vessels and then divides into dorsal and ventral branches. The ventral branch continues along the ventral aspect of the esophagus, while the dorsal branch obliquely crosses the lateral aspect of the esophagus to reach its dorsal border. Along its course it gives off branches to the heart, trachea and lungs.

The left vagus nerve continues along the dorsomedial aspect of the left cranial vena cava. Cranial to the aortic arch it gives off the left recurrent laryngeal nerve and continues caudally over the lateral aspect of the aortic arch, giving off cardiac and pulmonary branches. It passes dorsal to the pulmonary artery and then divides into dorsal and ventral branches. The ventral branch continues along the ventral aspect of the esophagus, often joining the right ventral branch to form a ventral vagal trunk, truncus vagalis ventralis. The dorsal branch obliquely crosses the esophagus and joins the right dorsal branch to form the dorsal vagal trunk, truncus vagalis dorsalis. Both trunks continue along the esophagus to the abdomen where they supply the abdominal viscera with general visceral efferent (parasympathetic) and visceral afferent (sensory) fibers.

The right recurrent laryngeal nerve turns cranially around the right subclavian artery, while the left recurrent laryngeal nerve turns cranially around the aortic arch. Both recurrent laryngeal nerves, $n$. laryngeus recurrens, give off branches to the heart, trachea and lungs and then continue cranially along the dorsolateral border of the trachea (Fig. 10-10). They pass deep to the thyroid gland and enter the larynx where they supply all the laryngeal muscles, excluding the cricothyroid muscle.

Cranial Nerve XI, Accessory Nerve, n. accessorius (Fig. 10-3). The accessory nerve has a spinal origin in addition to several rootlets from the brain stem. The spinal rootlets are located between the dorsal and ventral roots of the cervical spinal nerves. The spinal root enters the cranial cavity through the foramen magnum and is joined by the rootlets from the brain stem. The accessory nerve leaves the cranial cavity through the jugular foramen, caudal to the vagus nerve, and exits at the petro-occipital fissure. It turns caudally and passes between the stylohyoid and longus colli muscles and then divides into dorsal and ventral branches. The ventral branch innervates the sternocephalic muscle and forms part of the innervation of the sternohyoid, omohyoid, and thyrohyoid muscles. The dorsal branch passes caudally along the dorsal border of the omotransversarius muscle, deep to the sternocephalic and trapezius muscles. It innervates the omotransversarius and trapezius muscles.
Cranial Nerve XII, Hypoglossal Nerve, $\boldsymbol{n}$. hypoglossus (Fig. 10-3). The hypoglossal nerve leaves the cranial cavity through the hypoglossal canal. It lies medial to the accessory nerve and lateral to the vagus nerve and occipital and carotid arteries. The nerve curves rostroventrally on the lateral surface of the hyopharyngeus and hyoglossus muscles and deep to the stylohyoid, digastric and mylohyoideus muscles. It innervates all the muscles of the tongue.

\section{SPINAL NERVES, NERVI SPINALES}

The spinal nerves (Figs. 10-6, 10-9, and 10-10) have retained their embryological segmental characteristics, although the spinal cord from which they arise and the structures that they supply have largely lost this feature. Each spinal nerve is attached to its corresponding segment of the spinal cord by dorsal and ventral roots. The dorsal roots carry sensory or afferent impulses the ventral roots carry motor or efferent impulses. There are four categories of fibers in the spinal nerves. These are the somatic and visceral afferent fibers of the dorsal roots and the somatic and visceral efferent fibers of the ventral roots. The cell bodies of the afferent fibers are located in the spinal ganglia, situated along the dorsal roots, and those of the efferent fibers are situated in the ventral or lateral grey columns of the spinal cord.

The spinal nerves leave the vertebral canal through the intervertebral or lateral vertebral foramina. Each vertebral level has one pair of spinal nerves that are numbered according to the vertebra in front of it. The exceptions are the cervical spinal nerves that are numbered according to the vertebra behind them. This is because the first cervical spinal nerve leaves the vertebral canal in front of the atlas. Therefore there are eight cervical nerves but only seven cervical vertebrae. There are twelve or thirteen thoracic, six or seven lumbar, three sacral and nine or ten caudal pairs of spinal nerves. As a spinal nerve emerges from the intervertebral foramen it divides into dorsal and ventral branches.

The dorsal branches, rami dorsales, give rise to motor nerves which innervate the epaxial muscles and cutaneous branches which form the dorsal cutaneous nerves of the neck and trunk.

The cutaneous branches emerge close to the dorsal midline in the cervical and cranial thoracic (interscapular) regions and pass ventrolaterally to innervate the skin. In the thoracic region, caudal to the scapula, they emerge through the latissimus dorsi along the ventral border of the thoracic trapezius muscle. In the lumbar region they emerge between the iliocostalis and longissimus muscles, approximately 20 to $30 \mathrm{~mm}$ from the dorsal mid- 
line, to innervate the dorsal third of the abdominal wall. Over the sacral and caudal regions they again emerge close to the dorsal midline. The dorsal $\mathrm{cu}-$ taneous branch of $5 \mathrm{~L}$ passes over the tuber coxae onto the gluteal region, forming the first cluneal nerve. The cranial cluneal nerves, $n n$. clunium craniales, are thus formed by the dorsal branches of 5 to $7 \mathrm{~L}$, the middle cluneal nerves, $n$ n. clunium medii, by the dorsal branches of 1 to $3 \mathrm{~S}$ and the caudal cluneal nerves, nn. clunium caudales, by the ventral branch of the last sacral spinal nerve.

In the neck the ventral branches of the spinal nerves pass ventrolaterally, in the thorax they pass along the caudal border of the ribs as intercostal nerves. The ventral branch of the last thoracic spinal nerve is the costoabdominal nerve, $n$. costoabdominalis. Those of the first four lumbar spinal nerves are the cranial iliohypogastric nerve, $n$. iliohypogastricus cranialis, the caudal iliohypogastric nerve, $n$. iliohypogastricus caudalis, the ilioinguinal nerve, $n$. ilioinguinalis and the genitofemoral nerve, n. genitofemoralis. Animals with only six lumbar vertebrae lack a caudal iliohypogastric nerve. The five nerves supplying the abdominal wall pass distally, about $15 \mathrm{~mm}$ apart, between the transverse and external oblique abdominal muscles. They innervate the surrounding muscles and give rise to lateral and ventral cutaneous nerves to the skin.

In the cervical region the lateral cutaneous branches appear superficially at approximately the middle of the neck. In the thoracic and abdominal regions they appear superficially 50 to $60 \mathrm{~mm}$ from the dorsal midline. They then pass caudoventrally to innervate the middle third of the thoracic and abdominal walls. The lateral branch of the second or third intercostal nerve forms the intercostobrachial nerve, $n$. intercostobrachialis. It turns around the distal, caudal border of the long head of the triceps muscle to innervate the skin over the caudal scapular region.

The ventral cutaneous branches appear about 10 $\mathrm{mm}$ from the midline to innervate the ventral third of the body wall. In the female animal they innervate the thoracic and abdominal mammary glands. In the male animal they innervate the preputium and scrotum. The ventral branches terminate in the rectus abdominis muscle.

In addition to the superficial nerves, the ventral branches at the cervical, cervicothoracic, lumbosacral and sacral regions form the cervical, brachial, lumbosacral and sacral plexuses that innervate the neck, front and pelvic limbs and pelvis.

Cervical Plexus, plexus cervicalis. The cervical plexus is formed by the ventral branches of the second, third and fourth cervical spinal nerves. Some of the nerves that originate from the cervical plexus also receive branches from other cervical spinal nerves.

The second cervical spinal nerve is exceptionally large. It is connected to the third cervical spinal nerve and appears at the ventral borders of the cervical trapezius and cleido-occipital muscles. Appart from supplying the surrounding muscles and skin, it also gives rise to an auricular and a cervical nerve. The great auricular nerve $n$. auricularis magnus, passes dorsally and supplies sensory innervation to the caudal aspect of the ear.

The transverse nerve of the neck, $n$. transversus colli, passes cranioventrally and supplies sensory innervation to the cranioventral neck and the caudal intermandibular regions.

The third cervical spinal nerve gives rise to the supraclavicular nerve, $n$. supraclavicularis, which spreads out over the caudal neck and scapular regions to supply sensory innervation to the skin of the caudoventral neck and the cranial aspect of the shoulder regions.

The phrenic nerve, $n$. phrenicus (Fig. 9-1), to the diaphragm is formed by ventral branches from the fourth, fifth and sometimes sixth cervical spinal nerves. The main contribution to the nerve comes from the fourth cervical spinal nerve. At its origin it is closely related and attached to the first root of the brachial plexus.

The right phrenic nerve leaves the medial aspect of the brachial plexus cranial to the first rib. It passes ventral to the axillary artery (between the artery and the vein) and then enters the thorax medial to the first rib. In the thorax it passes along the ventral border of the right cranial vena cava, crosses the base of the heart between the right atrium and caval veins and continues to the diaphragm in the plica vena cava along the ventral border of the caudal vena cava. It supplies motor innervation to the right side of the diaphragm.

The left phrenic nerve passes medial to the brachial plexus and then enters the thorax medial to the first rib. It passes caudally along the ventral border of the left cranial vena cava, crosses the base of the heart and continues in the caudal mediastinum to reach and innervate the left side of the diaphragm.

Brachial Plexus, plexus brachialis (Fig. 109). The ventral branches of the last three cervical and first two thoracic spinal nerves form the brachial plexus. The five roots appear along the ventral border of the scalenus muscle, cranial to the first rib. The brachial plexus also receives fibres from the sympathetic trunk. At its origin the plexus is related to the linguofacial vein cranially, to the axillary and maxillary veins caudally and to the axillary artery ventrally. The nerve bundles pass ventrolaterally to 
reach the medial aspect of the thoracic limb at the level of the shoulder joint. The brachial plexus gives rise to the nerves of the thoracic limb.

The subclavian nerve, $n$. subclavius, arises from the cranial division of the brachial plexus. It supplies motor innervation to the subclavian muscle.

The suprascapular nerve, $n$. suprascapularis, is the most cranial of the nerves from the brachial plexus. It arises from the cranial division of the plexus and passes between the subscapular and supraspinatus muscles to reach the lateral aspect of the scapula. The nerve supplies motor innervation to the infraand supraspinatus muscles. It is accompanied by the suprascapular blood vessels.

The long thoracic nerve, $n$. thoracicus longus, arises from the sixth and seventh cervical spinal roots of the brachial plexus. It passes caudally along the lateral surface of the thoracic part of the serratus ventralis muscle. The nerve supplies motor innervation to the thoracic serratus ventralis.

The supscapular nerve, n. subscapularis, arises from the middle division of the brachial plexus. It consists of two or three branches that supply motor innervation to the subscapular muscle.

The axillary nerve, $n$. axillaris, arises from the middle division of the brachial plexus. It gives off a branch to the teres major muscle and then crosses the caudal aspect of the shoulder joint. The nerve passes from medial to lateral between the subscapular and teres major muscles to reach the lateral aspect of the shoulder. It supplies motor innervation to the teres minor and deltoid muscles and emerges superficially as the lateral cranial brachial cutaneous nerve, $n$. cutaneus brachii lateralis cranialis.

The lateral cranial brachial cutaneous nerve appears superficially in the groove between the lateral head of the triceps and the acromiodeltoid muscles, just distal to the shoulder joint. It accompanies the cephalic vein and supplies sensory innervation to the craniolateral aspect of the arm and forearm.

The cranial pectoral nerve, $n$. pectoralis cranialis, arises from the cranial division of the brachial plexus. It leaves the plexus in common with the musculocutaneous nerve, but separates from the latter to supply motor innervation to the superficial pectoral muscle.

The thoracodorsal nerve, $n$. thoracodorsalis, arises from the middle division of the brachial plexus and is associated with the radial nerve for a short distance. It passes caudally along the deep face of the latissimus dorsi muscle and is accompanied by the thoracodorsal blood vessels. The thoracodorsal nerve supplies motor innervation to the latissimus dorsi muscle.

The radial nerve, $n$. radialis, is the largest nerve of the thoracic limb. It arises from both the middle and caudal divisions of the brachial plexus. In the proximal part of the upper arm it lies caudal to the brachial vessels and ulnar nerve. It supplies motor innervation to the extensors of the elbow, carpus and digits, as well as sensory innervation to the cranial and dorsal aspect of the thoracic limb. From its origin the nerve passes distally along the medial aspect of the shoulder, giving off branches to the tensor fascia antebrachii and the long and medial heads of the triceps muscles. Just distal to the shoulder joint it turns laterally to pass between the long and medial heads of the triceps muscle. It joins the collateral radial blood vessels and gives off branches to the rest of the triceps muscle. The nerve follows the collateral radial blood vessels as they pass distolaterally, from medial to lateral, between the brachialis and triceps muscles. Distally it divides into superficial and deep branches.

The superficial branch, ramus superficialis, divides into two or three branches that appear superficially from under the distal border of the lateral head of the triceps muscle. One branch ramifies in the skin of the craniolateral forearm ( $n$. cutaneus antibrachii lateralis), the other accompanies the cephalic vein to the digits. It supplies the sensory innervation to the dorsal aspect of the metacarpus and digits I to IV.

The deep branch, ramus profundus, continues distally, passing between the distal end of the brachial muscle and the origin of the extensor carpi radialis muscle. It divides to supply the motor innervation to all extensor muscles of the carpus and digits, as well as to the supinator muscles of the forearm.

The musculocutaneous nerve, $n$. musculocutaneus, arises from the cranial division of the brachial plexus and is intially associated with the median nerve. It supplies motor innervation to the biceps, brachialis and coracobrachialis muscles and sensory innervation to the medial aspect of the forearm. At its origin the nerve passes lateral to the other nerves of the brachial plexus as well as the axillary blood vessels. A large connecting branch to the median nerve forms a loop, the ansa axillaris, around the brachial artery. Distal to the loop it gives off the proximal muscular branch, ramus muscularis proximalis, to the biceps and coracobrachialis muscles. It continues distally along the caudal, deep surface of the biceps muscle. Just above the elbow it gives rise to three branches. The first is a connecting branch to the median nerve, the second is the distal muscular branch, ramus muscularis distalis, to the brachialis muscle. The third branch is the medial antebrachial cutaneous nerve, $n$. cutaneus antebrachii medialis. The latter passes between the tendons of insertion of the brachialis and biceps muscles. At the elbow it becomes superficial between the cla- 
vicular head of the deltoid and extensor carpi radialis muscles. The nerve passes distally in the groove between the teres major and extensor carpi radialis muscles and supplies sensory innervation to the skin over the medial aspect of the forearm. A small artery accompanies the nerve.

The median nerve, $n$. medianus, comes from the cranial division of the brachial plexus. It receives the ansa subclavia from the musculocutaneous nerve and continues distally in the brachium along the cranial border of the brachial artery. It receives the distal connecting branch from the musculocutaneous nerve and passes through the supracondylar foramen when the latter is present, or cranial to the medial epicondyle of the humerus. Distal to the elbow it passes between the radius and the pronator teres muscle and continues in the antebrachium between the flexor carpi radialis and ulnar head of the deep digital flexor muscles. In the middle of the antebrachium it is joined by the median artery and divides into a medial and a lateral branch. The branches continue distally, on either side of the median artery, to the manus. Both branches pass medial to the accessory carpal bone, between the superficial and deep digital flexor tendons. They then divide to form the superficial sensory innervation of the palmar aspect of the metacarpus and the digits. The medial branch innervates the first and second digits, the lateral branch innervates the outer three digits (note that the outer two digits are also innervated by the ulnar nerve).

The ulnar nerve, $n$. ulnaris, comes from the caudal division of the brachial plexus. Initially it is associated with the median nerve, but separates from the latter in the middle of the arm. It crosses the medial aspect of the brachial vessels and passes distally along the caudal border of the blood vessels (in the groove between the medial head of the triceps muscle and the humerus). The cutaneous branch of the ulnar nerve, $n$. cutaneous antebrachii caudalis, comes off before the ulnar nerve separates from the median nerve. The cutaneous nerve emerges superficially in the cubital fossa to supply sensory innervation to the caudomedial aspect of the forearm.

The ulnar nerve passes caudal to the medial condyle of the humerus, then distally in the forearm between the superficial and deep digital flexor muscles. It gives off motor branches to the flexor carpi ulnaris and humeral head of the deep digital flexor, and is joined by the ulnar blood vessels. In the middle of the forearm it divides into dorsal and palmar branches. The dorsal branch, ramus dorsalis, appears superficially just proximal to the accessory carpal bone. It passes over the lateral aspect of the accessory carpal bone, then distally along the dorsolateral aspect of the paw. It forms the sensory innervation to the dorsal abaxial surfaces of the fifth digit. The palmar branch, ramus palmaris, divides into superficial and deep branches. Both branches pass through the carpal canal, medial to the accessory carpal bone and deep to the superficial digital flexor tendon. The superficial branch forms the sensory innervation to the palmar aspect of metacarpus and outer two digits. The deep branch supplies motor innervation to the palmar muscles of the manus (a small contribution to the sensory supply of the metacarpus and digits is made by the continuation of the deep branches).

The lateral thoracic nerve, $n$. thoracicus lateralis, arises from the caudal division of the brachial plexus. It leaves the medial aspect of the plexus and passes caudally along the deep face of the deep pectoral muscle. The nerve is accompanied by the lateral thoracic blood vessels. It supplies motor innervation to the cutaneous trunci muscle, including the cranial preputial muscle.

The caudal pectoral nerves, nn. pectorales caudales, arise from the caudal division of the brachial plexus. They accompany the lateral thoracic nerve and blood vessels along the dorsal border of the deep pectoral muscle. The nerves leave the lateral thoracic nerve to supply motor innervation to the deep pectoral muscle.

\section{Lumbosacral Plexus, plexus lumbosacralis} (Fig. 10-10). The lumbosacral plexus is derived from the ventral branches of the last four lumbar and the three sacral spinal nerves. It is divided into a lumbar and sacral divisions and gives rise to the nerves that supply the pelvis and pelvic limb. Some nerves originate from one division only, while others receive fibers from both.

The lumbar plexus lies deep to the iliopsoas muscles, the ventral tail muscles cover the sacral plexus. The lumbosacral plexus forms two main nerve trunks.

The first trunk comes from the lumbar segment with a contribution from 1S. It gives rise to the femoral and obturator nerves before it leaves the pelvic cavity through the cranial part of the greater ischiatic notch. The extra pelvic part of the trunk gives rise to the gluteal and sciatic nerves.

The second trunk comes from the sacral segment of the lumbosacral plexus. It leaves the pelvic cavity through the lesser ischiatic foramen and gives rise to the pudendal and caudal cutaneous femoral nerves, as well as to muscular branches to the coccygeus, levator ani and femorococcygeus muscles.

Pelvic Nerves, nn. Pelvini. These nerves form the parasympathetic innervation of the pelvic viscera and the descending colon. They arise from the last sacral segment of the sacral plexus. The 
nerves pass ventrally, forming an extensive plexus under the peritoneal lining of the pelvis. Fibers from the plexus pass directly to the viscera, or reach the organs via blood vessels or other nerves of the pelvis.

The pudendal nerve, $n$. pudendus, arises from the first two sacral segments of the sacral plexus. It leaves the dorsal aspect of the second trunk of the lumbosacral plexus and passes lateral to the coccygeus muscle. The pudendal nerve gives rise to one or two superficial perineal nerves and then turns medially around the caudal border of the coccygeus muscle. It gives rise to the deep perineal and caudal rectal nerves and continues along the dorsal surface of the penis or clitoris as the dorsal penile nerve, $n$. dorsalis penis, of the male or dorsal clitoridal nerve, $n$. dorsalis clitoridis, of the female. The dorsal penile and dorsal clitoridal nerves form the sensory innervation of the penis/clitoris and adnexa. They also carry the parasympathetic innervation to these organs.

One or two superficial perineal nerves, $n$. perinealis superficialis, leave the ventral aspect of the pudendal nerve in the ischiorectal fossa, lateral to the coccygeus muscle. They pass caudally and appear superficially at the caudal border of the femorococcygeus muscle (in the angle between the tail and the latter muscle). They pass through the adipose tissue of the ischiorectal fossa to form the sensory innervation of the perineal region.

The deep perineal nerve, $n$. perinealis profundus, originates from the pudendal nerve. They innervate all the muscles of the perineum, excluding the anal sphincter, coccygeus and levator ani muscles.

The caudal rectal nerve, $n$. rectalis caudalis, leaves the pudendal nerve distal to the superficial perineal nerves. It passes caudoventrally and enters the cranioventral aspect of the external anal sphincter muscle. It is accompanied by the caudal rectal blood vessels and forms the motor innervation to the external anal sphincter muscle.

Two muscular branches, $r$. musculi coccygei and r. musculi levatoris ani, leave the dorsal aspect of the caudal trunk of the lumbosacral plexus proximal to the origin of the pudendal nerve. They pass caudally and supply motor innervation to the coccygeus and levator ani muscles.

Nerves of the Pelvic Limb. The cranial gluteal nerve, $n$. gluteus cranialis, originates from the last lumbar and first sacral segments of the lumbosacral plexus. It branches from the plexus ventral to the second sacral vertebra and leaves the pelvis through the cranial border of the greater ischiadic foramen. The nerve passes between the deep and middle gluteal muscles where it gives off branches to supply motor innervation to the middle gluteal, deep gluteal and tensor fascia lata muscles.

The caudal gluteal nerve, $n$. gluteus caudalis, originates from the last lumbar and first sacral segments of the lumbosacral plexus. It leaves the cranial trunk of the plexus distal to the origin of the cranial gluteal nerve and passes around the caudal border of the middle gluteal muscle. The caudal gluteal nerve supplies motor innervation to the superficial gluteal and piriform muscles.

A lateral cutaneous femoral nerve, $n$. cutaneus femoris lateralis, arises from the ventral branch of $4 \mathrm{~L}$ and appears ventrally between the psoas minor and major muscles. It passes caudolaterally, covered by peritoneum, to join the deep circumflex iliac blood vessels. The nerve penetrates the abdominal wall and appears superficially just ventral to the tuber coxae. It accompanies the deep circumflex iliac artery along the craniolateral border of the quadriceps muscle to innervate the skin of the lateral thigh.

The caudal cutaneous femoral nerve, $n$. cutaneus femoris caudalis, leaves the caudal trunk of the lumbosacral plexus. It passes medially to the superficial gluteal muscle and emerges superficially between the femorococcygeus and biceps femoris muscles, just ventral to the tuber ischii. From there it passes distally on the thigh, forming the sensory innervation of the caudal aspect of the thigh.

The femoral nerve, $n$. femoralis, is derived from the ventral roots of $4 \mathrm{~L}$ and $5 \mathrm{~L}$, with a small contribution from 6L. It passes caudoventrally within the substance of the iliopsoas muscle, giving off motor branches to the muscle along the way. The nerve appears on the ventral surface of the iliopsoas muscle and joins the femoral vessels just before they pass through the vascular lacuna. It gives rise to the saphenous nerve and then enters the proximal part of the quadriceps muscle between the vastus medialis and rectus femoris muscles. The femoral nerve supplies motor innervation to the iliopsoas and quadriceps muscles.

The saphenous nerve, $n$. saphenus, is mainly (but not exclusively) sensory in function. It can therefore be regarded as the sensory component of the femoral nerve. Close to its separation from the femoral nerve it gives off a motor branch to the pectineus muscle. The saphenous nerve passes through the vascular lacuna and joins the saphenous blood vessels on the medial aspect of the thigh. Proximally it gives off one or two branches that innervate the skin on the medial aspect of the thigh. The remaining nerve divides into cranial and caudal branches. The caudal branch supplies the caudomedial aspect of the crus. The cranial branch continues distally to supply the craniomedial aspect of 
the crus and metatarsus, as well as the dorsal aspect of digits I and II.

The ischiadic nerve, (sciatic nerve) $n$. ischiadicus (Fig. 4-14), is the continuation of the cranial trunk of the lumbosacral plexus beyond the origins of the cranial and caudal gluteal nerves. It also receives a small contribution from the caudal trunk of the lumbosacral plexus. The nerve passes caudally, first along the deep face of the middle gluteal muscle medial to the greater trochanter, then on the dorsal surface of the gemelli and tendon of the internal obturator muscles. It gives off branches to the rotators of the hip (excluding the external obturator muscle) and the caudal thigh muscles and then turns ventrally. As it passes distally in the thigh it lies in succession on the lateral surface of the quadratus femoris, adductor and semimembranosus muscles. It is covered laterally first by the superficial gluteal muscle and then by the biceps femoris muscle. Furthermore, it lies in close association with the abductor cruris caudalis muscle. In the distal third of the thigh the nerve divides into the tibial and peroneal nerves.

The common peroneal nerve, $n$. peroneus communis, separates from the tibial nerve approximately $20 \mathrm{~mm}$ above the knee. It supplies all the flexor muscles of the ankle and the extensor muscles of the toes, as well as the sensory supply to the dorsal aspect of the pes. Proximally to the knee it gives off the lateral cutaneous sural nerve, $n$. cutaneus surae lateralis, which penetrates the distal part of the biceps femoris muscle and appears superficially on the lateral aspect of the distal thigh. It consists of one or more branches that innervate the lateral aspect of the crus and tarsus.

The common peroneal nerve passes laterally to the lateral head of the gastrocnemius muscle and the head of the fibula. It enters the groove between the gastrocnemius and long peroneal muscles where it divides into the superficial and deep peroneal nerves.

The superficial peroneal nerve, $n$. peroneus superficialis, gives off motor branches to the extensor muscles of digits I and II and the peroneus brevis muscle. It continues distally in the groove between the long peroneal muscle and extensor muscle of the fourth digit. The nerve appears superficially on the flexor surface of the ankle and ramifies in the skin over the dorsal aspect of the tarsus.

The deep peroneal nerve, $n$. peroneus profundus, supplies the flexors of the ankle and the extensors of the toes, excluding the ones supplied by the superficial peroneal nerve. Two branches, one lateral and the other medial, continues distally in the crus. In some animals the lateral branch comes off the superficial peroneal nerve. The lateral branch passes distally along the cranial border of the peroneus longus muscle. It emerges superficially on the dorsolateral aspect of the ankle. The branch continues distally, passing caudal to the lateral malleolus and then divides to form the dorsal sensory supply to digit $\mathrm{V}$ and the dorsal axial surface of digit IV. It also innervates the short extensors of the toes. The medial branch passes distally along the medial border of the peroneus brevis muscle. It lies on the tibia and is accompanied by the cranial tibial blood vessels. The branch emerges distally between the tendons of the tibialis cranialis and long digital extensor muscles. At the ankle it divides to supply the dorsal aspect of digit III and the dorsal abaxial surface of digit IV (note that the dorsal aspects of digits I and II are supplied by the cranial branch of the saphenous nerve).

The tibial nerve, $n$. tibialis, separates from the peroneal nerve in the popliteal fossa. It supplies all the extensor muscles of the ankle and the flexor muscles of the toes, as well as the sensory innervation to the caudomedial crus and plantar surface of the pes. Just above the gastrocnemius muscle it gives rise to the caudal cutaneous sural nerve, $n$. cutaneus surae caudalis, and then enters the gastrocnemius muscle between its medial head and the superficial digital flexor muscle. The caudal cutaneus sural nerve, passes distally in the groove between the superficial digital flexor and lateral head of the gastrocnemius muscles. Just above the ankle it divides into a lateral and a medial branch. The medial branch passes between the common calcanean tendon and the deep digital flexor muscle and then joins the deep branch of the tibial nerve. The lateral branch continues distally and supplies the plantar surface of digit $\mathrm{V}$ and the abaxial plantar surface of digit IV.

As the tibial nerve enters the muscles, it divides into muscular branches that innervate the extensor muscles of the ankle and the flexor muscles of the toes. One branch continues distally between the medial head of the gastrocnemius and superficial digital flexor muscles. It gradually separates into the lateral and medial plantar nerves. Both nerves emerge superficially along the distal border of the gastrocnemius muscle on the medial surface of the crus. The medial plantar nerve continues distally over the medial aspect of the ankle and on the plantar aspect of the pes, superficial to the superficial digital flexor tendons. It divides to supply the plantar aspect of digits I to III, as well as the plantar axial surface of digit IV.

Proximal to the ankle the lateral plantar nerve joins the medial branch of the caudal cutaneous sural nerve. The combined nerve continues distally over the ankle, medial to the calcaneus. Just distal to the ankle it turns laterally over the plantar surface of the pes and passes between the superficial 
and deep digital flexor tendons. It innervates the short flexor muscles of the pes.

\section{AUTONOMIC NERVOUS SYSTEM}

The autonomic nervous (general visceral efferent) system can be defined as that portion of the nervous system concerned with the motor innervation of smooth muscle, cardiac muscle, glands and organs. It is subdivided into sympathetic and parasympathetic divisions. The cell body of the first or preganglionic neuron of both systems is located in the central nervous system. The cell body of the sympathetic second neuron lies in ganglia close to the vertebral column, while the cell body of the parasympathetic second neuron lies close to or within the target organ. The myelinated axons of the first neurons are part of the peripheral nervous system and synapse with the second neurons. The non-myelinated post ganglionic axons from the second neurons end among or on cardiac muscle, smooth muscle, glandular cells or the cells of organs e.g., liver.

The ganglia associated with the autonomic nervous system are named after the specific location in which they are found. Many smaller, unnamed ganglia are found along the pathways of the autonomic nerves. Autonomic ganglia are classified as vertebral, collateral and terminal, depending on their topographical position. Vertebral ganglia are more or less segmentally distributed along the paired sympathetic chains which in turn lie along the ventral sides of the heads of the ribs. Collateral ganglia are found more peripherally and, in the abdominal cavity, are related to some of the larger arteries such as the celiac. Terminal ganglia are usually small and located in various organs.

The preganglionic neurons of the parasympathetic division are located in specific areas or nuclei of the brain stem or the lateral grey column in the sacral division of the spinal cord. It is therefore also referred to as the craniosacral division of the autonomic nervous system. The preganglionic neurons of the sympathetic division are located in the lateral grey columns of the thoracolumbar segment of the spinal cord. It is referred to as the thoracolumbar division of the autonomic nervous system.

Parasympathetic Division. Parasympathetic preganglionic axons leave the brain stem as part of cranial nerves III, VII, IX, and X. The axons in nerves III, VII, and IX are distributed to the head region, whereas those of cranial nerve $X$ are distributed to the cervical, thoracic and abdominal viscera. Preganglionic fibers from the sacral division leave the spinal cord with the ventral roots of the sacral spinal nerves as the pelvic nerve. Its fibres become part of the pelvic plexus.
For the detail of the distribution of the parasympathetic fibers, see cranial nerves III, VII, IX and $\mathrm{X}$, as well as the pelvic nerves of the lumbosacral plexus under the peripheral nervous system.

Sympathetic Division. The preganglionic fibers leave the thoracolumbar division of the spinal cord via the ventral roots of the spinal nerves and for a short distance are part of the corresponding spinal nerves. These fibers then leave the spinal nerves as one or more communicating rami that join the sympathetic trunk (Figs. 9-1 and 9-2). The trunk is a paired strand of pre- and postganglionic sympathetic and visceral afferent nerve fibers located ventrolateral to the bodies of the vertebrae in the thoracic and lumbar regions. Continuations of the trunks into the sacral region lie ventral to the sacral vertebral bodies. The trunks also continue cranially into the cervical region. They accompany the vagus nerves in the carotid sheaths. Each trunk ends in a cranial cervical ganglion. With the exception of certain ganglia, sympathetic chain ganglia are located at segmental intervals along the sympathetic trunk (Figs. 9-1 and 9-2). Preganglionic fibers may proceed cranially or caudally in the trunk before synapsing in a ganglion. Thus, preganglionic fibers from a given segmental level of the spinal cord are distributed to vertebral ganglia at many different levels. Postganglionic fibers may also pass cranially or caudally in the trunk before leaving it. The part of the sympathetic trunk that joins adjacent ganglia is the interganglionic segment. Furthermore, adjacent ganglia may fuse eg. the celiac and cranial mesenteric ganglia fuse to form a single celiacomesenteric ganglion.

Preganglionic fibers may also leave the sympathetic trunk (as for example the splanchnic nerves) and synapse in collateral ganglia such as the celiac and mesenteric ganglia.

Postganglionic fibers go to the smooth muscle of blood vessels, erector pili muscles, sweat glands and many other organ tissues e.g., liver. The fibers may be distributed in a number of different ways:

They may return via communicating rami to the spinal nerve at the same level. These are distributed to smooth muscle (vasomotor and pilomotor fibers) and sweat glands by way of the spinal nerves.

They may pass either cranially or caudally in the sympathetic trunk and join adjacent spinal nerves via their respective communicating rami.

They may leave the vertebral ganglia, such as the cervicothoracic ganglion, and go directly to an organ such as the heart (cardiac nerves), bronchi and lungs.

They may leave vertebral or collateral ganglia for distribution as plexuses along blood vessels of the head and abdominal viscera. 
Sympathetic Distribution to the Head and Neck. In general, sympathetic fibers to the head supply nasal and salivary glands and smooth muscle such as the vascular walls, dilator of the pupil and erector pili muscles.

Preganglionic fibers from the cranial thoracic spinal segments pass to the head as the cervical sympathetic trunk. Just caudal to the larynx the cervical sympathetic trunk separates from the vagus nerve. The fibers synapse in the cranial cervical ganglion, ganglion cervicale craniale. The latter is about $8 \mathrm{~mm}$ long and lies dorsal to the thyroid gland and larynx, between the common carotid artery and vagus nerve. Postganglionic fibers continue as plexuses along the arteries to the head. The internal carotid nerve, $n$. caroticus internus, leaves the cranial pole of the ganglion as a distinct bundle of postganglionic fibers. It accompanies the internal carotid artery to the cranial cavity where it forms plexuses around the arteries and venous sinuses. The deep petrosal nerve, $n$. petrosus profundus, leaves the cavernous plexus and passes through the petrous temporal bone. The latter nerve joins the major petrosal nerve to form the nerve of the pterygoid canal.

The vertebral nerve, $n$. vertebralis, originates from the cervicothoracic ganglion (see below). Postganglionic fibers leave the ganglion and accompanies the vertebral artery through the transverse foramina of the cervical vertebrae At each intervertebral foramen it gives off communicating rami to the cervical spinal nerves and fibers which form plexuses around the blood vessels. The fibers are distributed to the smooth muscle of the blood vessels and erector pili muscles.

\section{Sympathetic Distribution to the Thorax.} Postganglionic fibers from the thoracic vertebral ganglia are distributed via the spinal nerves and intercostal blood vessels to the thoracic wall and surrounding structures.

Fusion of vertebral ganglia in the cranial thoracic and caudal cervical regions has resulted in the formation of the middle cervical and cervicothoracic ganglia. The cervicothoracic ganglion, ganglion cervicothoracicum, is the largest autonomic ganglion, measuring $10 \mathrm{~mm}$ in length and $2 \mathrm{~mm}$ in width. It lies in the first intercostal space on the lateral surface of the longus colli muscle. Postganglionic fibers from the cervicothoracic ganglion go to the caudal cervical and cranial thoracic spinal nerves (brachial plexus), to the subclavian artery and its branches, to the thoracic viscera and they give rise to the vertebral nerves to the neck.

The sympathetic trunk divides cranioventrally to the cervicothoracic ganglion, forming a subclavian loop, ansa subclavia, around the subclavian artery.
The small middle cervical ganglion, ganglion cervicale medium, lies medial to the subclavian artery at the junction of the vagosympathetic trunk and the ansa subclavia. In some animals it is incorporated in, or attached to, the cervicothoracic ganglion. Postganglionic fibers from the ansa subclavia and middle cervical ganglion go to the viscera of the thorax.

Since sympathetic and parasympathetic fibers appear to intermix and form plexuses in the region of the heart and lungs, it is difficult to designate any given grossly dissectable nerve as being one or the other.

Sympathetic Distribution to the Abdomen and Pelvis. Branches from the thoracic and abdominal sympathetic trunk supply fibers to the abdominal and pelvic regions. Postganglionic fibers from the lumbar vertebral ganglia are distributed to the abdominal wall via lumbar spinal nerves or lumbar spinal arteries. In addition to these, preganglionic fibers leave the ventral aspect of the seventh, eight and ninth thoracic vertebral ganglia. They join to form the major splanchnic nerve, $n$. splanchnicus major. The latter is larger than the caudal continuation of the sympathetic trunk and passes caudoventrally, crossing the lateral surface of the psoas muscle. Both left and right major splanchnic nerves pass through the lumbocostal arch to enter the abdomen. Other branches designated as lesser splanchnic nerves, nn. splanchnicus minor, may leave the sympathetic trunk just caudal to the greater splanchnic nerve. The major splanchnic nerves supply preganglionic fibers to the celiacomesenteric ganglion. The latter is situated around the roots of the celiac and cranial mesenteric arteries. Postganglionic fibers from the ganglion form a dense plexus around the vessels and are distributed with the arteries to the abdominal viscera. The minor splanchnic nerves mostly supply postganglionic fibres to the adrenal glands.

Immediately caudal to the origin of the splanchnic nerves the lumbar sympathetic trunk is small, but as it proceeds caudally it becomes larger.

Lumbar splanchnic nerves, nn. splanchnici lumbales, arise from the lumbar sympathetic trunk and go to ganglia associated with aortic, adrenal, gonadal and renal plexuses. One of the lumbar splanchnic nerves passes caudoventrally to end in the caudal mesenteric ganglion. The latter lies at the root of the caudal mesenteric artery. Postganglionic fibers from the ganglion form a dense plexus around the artery and are distributed to the viscera via the blood vessels.

The right and left hypogastric nerves, nn. hypogastrici, represent largely postganglionic connections between the caudal mesenteric ganglion and 
the pelvic plexuses. They are usually grossly dissectable nerves. From the pelvic plexuses the fibers are distributed to the pelvic viscera and genital organs.
In the sacral region the two sympathetic trunks lie close to each other. Postganglionic fibers are distributed to the pelvic wall and tail via the sacral spinal nerves or blood vessels. 


\section{Chapter 11. Sensory Organs}

Eye. The eye, oculus, develops as a neuroectodermal outgrowth of the embryonic prosencephalon. The definitive eye and adnexa are contained within the orbit. The eye bulb is associated with the extraocular muscles that move it, eyelids and conjunctiva that protect it, and a lacrimal gland that moistens it. It is surrounded by periorbital fascia and fat that cushions it.

At birth the eye of the woodchuck appears merely as a darkened area on the face. When the animals are two weeks old the eye slits are visible, but closed. The eyes open when the pups are about four weeks old (Hamilton 1934; Baldwin 1985).

The orbit, orbita, is the conical cavity that contains the eyeball and its adnexa. It is bounded by bone along its dorsal, medial and ventral boundaries, while the lateral side is open. The orbital ligament and the temporal muscle close the opening laterally. The orbital margin outlines the base of the cone, the optic canal the apex of the cone. The axis (a line from the center of the base to the apex) of the orbit is directed rostrolaterally and dorsally. The circumference of the orbital margin is approximately $55 \mathrm{~mm}$. It is bony for $45 \mathrm{~mm}$ of its circumference and is laterally completed by the orbital ligament. The orbital ligament, ligamentum orbitale, is a thick fibrous band that extends between the zygomatic process of the frontal bone and the frontal process of the zygomatic bone.

The eyeball occupies the base of the orbit and projects some distance rostral to the orbital margin. Apart from the eye and its adnexa the orbit also contains the zygomatic salivary gland g. zygomaticus.

The periorbita, periorbita, is a conical fascial sheath that extends from the optic canal to the orbital margin. It surrounds the eyeball and associated structures. The periorbita is attached to the bony orbit at the optic canal, the lacrimal fossa and at the orbital margin. The orbital fat body lies intraand extra-orbitally. It cushions the contents of the orbit and permits the movement of the eyeball.

The upper eyelids, palpebra superior, and lower eyelid, palpebra inferior, are mobile folds of skin that can be drawn over the anterior part of the eyeball. The lids join at their medial and lateral aspects to form the lateral and medial canthus of the eye, commissura palpebrarum lateralis et medialis. The opening between the lids is the rima palpebra- rum. The upper and lower lacrimal ducts open onto the bulbar surfaces of the lid margins.

The skin of the face continues onto the outer surface of the eyelids. At the palpebral margin the epidermis of the skin changes abruptly to the epithelium of the palpebral conjunctiva. Long hairs (eyelashes, cilia) project from the upper and lower lid margins. Dorsal to the medial canthus of the eye there is a tuft of long, tactile hairs.

The conjunctiva, tunica conjunctiva, of the eye extends from the palpebral margin to the limbus of the cornea. It consists of palpebral and bulbar parts. The palpebral conjunctiva, tunica conjunctiva palpebrarum, lines the bulbar (inner) aspect of the eyelid. At the level of the orbital rim the palpebral conjunctiva reflects onto the eyeball to become the bulbar conjunctiva, tunica conjunctiva bulbi. The point of reflection is the conjunctival fornix and the potential space between the palpebral and bulbar conjunctiva is the conjunctival sac, saccus conjunctivae.

The third eyelid, plica semilunaris conjunctivae, is a poorly developed semilunar fold of the conjunctiva. It arises as a fold from the conjunctiva at the medial canthus of the eye. Its free edge is slightly concave and in contrast to the rest of the conjunctiva it is usually pigmented and reinforced by a small cartilage. The third eyelid can only extend to cover the medial corner of the cornea.

The lacrimal apparatus, apparatus lacrimalis, consists of the lacrimal gland and the nasolacrimal duct system.

The lacrimal gland, glandula lacrimalis, is an oval, lobulated gland that lies deep to the periorbita on the dorsolateral aspect of the eyeball. It is flattened between the eyeball and the orbital ligament. The gland is approximately $7 \mathrm{~mm}$ long and $5 \mathrm{~mm}$ wide. Its secretion empties into the dorsolateral conjunctival fornix through small secretory ducts.

The lacrimal punctum, punctum lacrimale, is the spot where the lacrimal canaliculi open. They are located on the bulbar conjunctiva of the upper and lower eyelids, approximately $2 \mathrm{~mm}$ from the lid margins and $2 \mathrm{~mm}$ from the medial canthus of the eye.

The lacrimal canaliculus, canaliculus lacrimalis, conducts tears from the punctum to the lacrimal sac. The dorsal lacrimal canaliculus passes medially along the upper eyelid and turns ventrally at the 
medial canthus of the eye to open into the lacrimal sac. The ventral lacrimal canaliculus passes ventromedially to open into the lacrimal sac.

The lacrimal sac, saccus lacrimalis, is the somewhat dilated origin of the nasolacrimal duct. It lies medioventral to the medial canthus of the eye, in the opening of the lacrimal foramen of the skull.

The nasolacrimal duct, ductus nasolacrimalis, extends between the lacrimal sac and the vestibule of the nasal cavity. It lies in a bony canal for the greater part of its course. From the lacrimal foramen it arches ventromedially, between the infraorbital canal and the root of the upper incisor tooth and then turns rostrally. It passes rostromedially along the ventral border of the upper incisor and leaves the bony canal at the rostral border of the ventral turbinate bone. It passes for a short distance in the mucosa of the nasal cavity before it opens at the nasolacrimal opening in the vestibule of the nose. The nasolacrimal opening lies on the medial surface of the alar cartilage, approximately $3 \mathrm{~mm}$ from the nares.

The eyeball, bulbus oculi, consists of three concentric layers: an outer fibrous tunic, a middle vascular tunic and an inner nervous tunic. The eyeball of the woodchuck is almost spherical. It measures approximately $15 \mathrm{~mm}$ along its vertical diameter and $12 \mathrm{~mm}$ along its sagittal diameter. The transparent cornea forms the anterior one-fifth of the eye ball and is approximately $7 \mathrm{~mm}$ in diameter. The anterior pole of the eyeball, polus anterior, is the vertex of the cornea. The point directly opposite to it is the posterior pole deep in the orbit. The line connecting the two poles is the axis of the eyeball. The equator, equator, of the eyeball is its maximum circumference and is located approximately midway between the two poles.

The fibrous tunic, tunica fibrosa bulbi, of the eyeball is divided into the sclera and the cornea. The junction of the cornea and sclera is the limbus of the cornea. The sclera, sclera, is the fibrous coat of the eyeball which gives the eye its shape when it is tensed by intraocular pressure. The extra-ocular muscles of the eye are attached to its outer surface and the ciliary muscle is attached to a small circular ridge on its inner surface. The optic nerve leaves the eyeball through a sievelike area in the sclera, the area cribrosa, which is approximately $2 \mathrm{~mm}$ wide and $7 \mathrm{~mm}$ long. It lies horizontally, dorsal to the caudal pole of the eyeball. The dura mater that surrounds the optic nerve is not continuous with the outer layer of the sclera, but ends approximately $1 \mathrm{~mm}$ from the area cribrosa.

The cornea, cornea, forms the transparent, avascular, anterior part of the fibrous tunic. It is slightly oval and measures $7 \mathrm{~mm}$ along its dorsoventral axis and $9 \mathrm{~mm}$ along its mediolateral axis. Since its ra- dius of curvature is smaller than the rest of the eye, it bulges forward.

The vascular tunic, tunica vasculosa bulbi, is the middle coat of the eyeball and consists of the choroid, the ciliary body and the iris.

The choroid, choroidea, is a pigmented, vascular layer that envelops the posterior part of the eyeball, except over the area cribrosa. It is anteriorly continuous with the ciliary body. The choroid of the woodchuck does not have a macroscopically discernible tapetum lucidum.

The ciliary body, corpus ciliare, is the thickened middle part of the vascular tunic, between the choroid and the iris. It consists of many small, parallel folds that are attached to a base plate, lamina $b a-$ salis. The ciliary muscle is attached to a thickened scleral ring. The lens is fixed in position by a delicate suspensory apparatus, the zonula ciliaris.

The iris, iris, is the most anterior segment of the vascular tunic. It is a thin, circular diaphragm that rests against the anterior surface of the lens. The central opening of the iris, the pupil, is circular. Sphincter and dilator muscles control the diameter of the pupil.

The retina, tunica interna bulbi, is the innermost tunic of the eyeball. It is divided into optic, ciliary and iridal parts. The optic part, pars optica retinae, is the visual segment of the retina and the ciliary and iridal parts the non-visual segment of the retina.

The iridal part of the retina lines the posterior surface of the iris. At the ciliary margin of the iris it reflects onto the ciliary body to become the ciliary part of the retina.

The demarcation between the visual and nonvisual retina is the ora serrata. The optic part of the retina lies posteriorly to the ora serrata and is clearly demarcated from the ciliary part. The axons of the ganglion layer of the retina arc toward the optic line. Here they become myelinated, turn and pass through the area cribrosa of the sclera to form the optic nerve. The intraocular myelinated portion of the nerve forms the optic line. The latter is slightly elevated and represents the optic disc of other animals. It is approximately $0.5 \mathrm{~mm}$ wide and 5 to 7 mm long.

The lens, lens, is a soft, transparent and nearly spherical structure suspended by the zonule, and is in contact with the posterior surface of the iris. It is circular in transverse section and slightly ellipsoidal in sagittal section. The posterior surface is more convex than the anterior surface. The dorsoventral diameter of the lens is approximately 5 to $6 \mathrm{~mm}$ and it measures 4 to $5 \mathrm{~mm}$ along its anteroposterior axis.

Since the lens increases in weight linearly after the first year by cellular addition, it can be used to 
determine the age of the animal (Davis 1964; Nuckle and Bergeron 1983).

The three chambers of the eye are the anterior chamber, the posterior chamber and the vitreous chamber.

The anterior chamber, camera anterior bulbi, is the space bounded anteriorly by the cornea and posteriorly by the iris. It is filled with the aqueous humor. The anterior chamber is in direct communication with the posterior chamber through the pupil of the iris.

The posterior chamber, camera posterior bulbi, is posterior to the iris and anterior to the lens, zonule and anterior face of the vitreous body. It is also filled with aqueous humor.

The vitreous chamber, camera vitrea bulbi, is the largest of the three chambers. It is bounded anteriorly by the lens and zonule. The vitreous chamber contains the vitreous body, corpus vitreum, a soft, clear gel that conforms to the shape of the cavity it occupies.

Extraocular muscles of the eye: See under the muscles of the head and neck.

Blood supply: See under the arteries and veins of the head and neck.

Innervation: See the optic, oculomotor, trochlear and abducens nerves, as well as the branches of the trigeminal, facial and glossopharyngeal nerves under the nerves of the head and neck.

Ear. The ear is divided into external, middle and inner ear.

The external ear, auris externa, consists of the pinna and the external acoustic meatus. At birth the ears are barely discernable and the external acoustic meatus is closed (Hamilton 1934). The ear canal opens when the pups are about three weeks old (Baldwin 1985).

The pinna or external ear, auricula, is a funnelshaped cartilage that is covered on both surfaces by skin. It is approximately $40 \mathrm{~mm}$ long and is attaced to the opening of the external acoustic meatus of the temporal bone by means of an annular cartilage. The opening of the ear canal faces dorsolaterally. The outer surface faces medially and the concave inner surface faces laterally.

The auricular cartilage, cartilago auriculae, is triangular in shape. Its distal part is flattened and its dorsal border is rounded. The proximal $7 \mathrm{~mm}$ of the rostral border of the cartilage is elongated to form the tragus. The latter is rolled to form an incomplete cartilaginous tube. The tube or canal is completed by skin to form the opening into the ear canal.

The distal rostral border of the auricular cartilage is flattened and almost straight. The caudal border is folded or rolled into a narrow tube. The tube is incomplete along its rostral border.

The annular cartilage, cartilago anularis, is interposed between the auricular cartilage and the external acoustic meatus. It is a C-shaped cartilage and is incomplete medially. The annular cartilage is approximately $5 \mathrm{~mm}$ wide and completes the cartilaginous part of the ear canal. It is attached to the external acoustic meatus by connective tissue.

The opening of the ear canal, meatus acusticus externus, extends from the external pore to the tympanic membrane. It is approximately $5 \mathrm{~mm}$ long and $4 \mathrm{~mm}$ in diameter and forms the bony part of the external ear canal. A thin membrane resembling skin lines the canal.

The middle ear, auris media, consists of the tympanic cavity, the auditory ossicles, and the tympanic membrane. The tympanic cavity contains various nerves and blood vessels that pass through it and the auditory tube from the pharynx that opens into it.

The tympanic membrane, membrana tympani, separates the external ear canal from the middle ear cavity. The malleus, first in the chain of ossicles, is attached to the inner surface of the tympanic membrane.

The tympanic cavity, cavum tympani, is divided into tegmental, labyrinthal and mastoid parts. The tegmental part lies dorsal to a frontal plane through the osseus external acoustic meatus. It contains the epitympanic recess. The latter houses the head of the malleus and the body of the incus.

The labyrinthal part bears cochlear and vestibular windows. The promontory is a rounded elevation on the medial wall of the tympanic cavity. It contains the cochlea and has a cochlear window with a secondary tympanic membrane.

The mastoid part contains the tympanic bulla. The cavity of the bulla is incompletely divided into cells by bony trabeculae. The auditory tube opens into the dorsal aspect of the tympanic bulla, rostral to the promontorium. The opening lies dorsal to a shelf of bone, separating the opening from the rest of the bulla. The opening is therefore not visible when the bulla is opened from the ventral aspect.

There are three auditory ossicles in the middle ear, the malleus, incus and stapes. They extend as a connected chain between the tympanic membrane and the vestibular window of the petrous temporal bone. Two muscles are associated with the ossicles: the tensor tympany with the malleus and the stapedius with the stapes. The malleus has a long manubrium, which is tightly attached to the tympanum, and a round head that articulates with the body of the incus. The incus has two projecting crura. The short crus lies in the epitympanic recess, the long crus articulates with the stapes. The stapes is a stirrup-shaped bone with a head, two crura and 
a base. The base of the stapes fits into and is attached within the vestibular window of the petrous temporal bone.

The inner ear, auris interna, lies within the petrous temporal bone. It consists of a membranous labyrinth, labyrinthus membranaceus, contained within a bony labyrinth labyrinthus osseus.

The membranous labyrinth consists of an en- dolymphatic duct, a sacculus, three semicircular ducts and a cochlear duct, all of which are filled with endolymph.

The bony labyrinth consists of a vestibule, three semicircular canals and a cochlea, all of which contain perilymph. These fluids, perilymph and endolymph are different chemically and are kept separated from each other. 
Ahl, A. S. 1987. Relationship of vibrissal length and habits in the Sciuridae. Journal of Mammalogy 68:848-853

Armitage, K. B. 1975. Social behaviour and population dynamics of marmots. Oikos 26:341-354

Armitage, K.B. 1991. Social and population dynamics of yellowbellied marmots: results from long-term research. Annual Review of Ecology and Systematics 22:379-407.

Armitage, K. B. 1999. Evolution of sociality in marmots. Journal of Mammalogy 80:1-10.

Baldwin, B. H. 1985. Studies on neonatal woodchuck development. Progress Report, Breeding and Experimental Facility for Woodchucks (Marmota monax), United States Government Contract \# AI 02651 (principal investigator: B.C. Tennant, Department of Clinical Sciences, New York State College of Veterinary Medicine, Cornell University, Ithaca, New York).

Baldwin, B. H., B. C. Tennant, T. J. Reimers, R. G. Cowan, and P. W. Concannon. 1985. Circannual changes in serum testosterone concentrations of adult and yearling woodchucks (Marmota monax). Biology of Reproduction 32:804-812.

Barash, D. P. 1989. Marmots: social behaviour and ecology. Stanford University Press, Palo Alto, California.

Black, C. C. 1963. A review of North American tertiary Sciuridae. Bulletin of the Museum of Comparative Zoology 130:111248.

Blumstein, D. T., and K. B. Armitage. 1998. Life history consequences of social complexity: a comparative study of ground dwelling sciurids. Behavioral Ecology 9:8-19.

Bryant, M. D. 1945. Phylogeny of Nearctic Sciuridae. American Midland Naturalist 33:257-390.

Bugge, J. 1971. The cephalic arterial pattern in sciuromorphs with special reference to the systematic classification of rodents. Acta Anatomica 80:336-361.

Christian, J. J. 1962. Seasonal changes in the adrenal glands of woodchucks (Marmota monax). Endocrinology 71:431-447

Christian, J. J., E. Steinberger, and T. D. McKinney. 1972. Annual cycle of spermatogenesis and testis morphology in woodchucks. Journal of Mammalogy 53:708-716.

Concannon, P., B. Baldwin, J. Lawless, W. Hornbuckle, and B. Tennant. 1983. Corpora lutea of pregnancy and elevated serum progesterone during pregnancy and postpartum anestrus in woodchucks (Marmota monax). Biology of Reproduction 29:1128-1134.

Concannon, P., B. Baldwin, and B. Tennant. 1984. Serum progesterone profiles and corpora lutea of pregnant, postpartum, barren and isolated females in a laboratory colony of woodchucks (Marmota monax). Biology of Reproduction 30: 945-951.

Courser, W., P. Sargent, L. E. Brownhill, and K. Benirschke. 1963. The somatic chromosomes of the north eastern American woodchuck (Marmota monax). Cytologia 28:108-111.

Davis, D. E. 1964. Evaluation of characters for determining age of woodchucks. Journal of Wildlife Management 28:9-15.

Davis, D. E. 1967. The annual rhythm of fat deposition on woodchucks (Marmota monax). Physiological Zoology 40:391-402.

Doetsch, G. S., and A. L. Towe. 1981. The pyramidal system of the woodchuck. Brain, Behavior and Evolution 19:37-55.

Frink, R., P. P. Krupp, and R. A. Young. 1977. Seasonal variation in the morphology of thyroid follicular (C) cells in the wood- chuck, Marmota monax: a light and electron microscopic study. Anatomical Record 189:397-412.

Garon, O., and J. Pierard. 1972. Etude morphologique comparee de l'estomac de deux rongeurs Ondatra zibethicus et Marmota monax (Mammalia:Rodentia). Canadian Journal of Zoology 50:239-245

Gilbert, P. W. 1940. Studies on the morphology of burrowing sciurids with special reference to the forelimb of Marmota and Cynomys. Ph.D. dissertation, Cornell University, Ithaca, New York.

Godman, J. D. 1842. Marmot; Arctomys; Gmel. Species I. The Maryland Marmot, Arctomys Monax Gmel. American Natural History 1:98-121.

Guthrie, D. A. 1963. The carotid circulation in the Rodentia. Bulletin of the Museum for Comparative Zoology, Harvard University 128:455-481

Hamilton, W. J., Jr. 1934. The life history of the rufescent woodchuck (Marmota monax rufescens, Howell). Annals of the Carnegie Museum 23:85-119.

Hill, J. E. 1937. Morphology of the pocket gopher, mammalian genus Thymomys. University of California Publications in Zoology 42:81-172.

Hoffman, R. S., J. W. Koeppl, and C. F. Nadler. 1979. The relationship of the Amphiberigian marmots (Mammalia: Sciuridae). Occasional Papers of the Museum of Natural History, University of Kansas 831-:56.

Howell, A. H. 1915. Revision of the American marmots. North American Fauna 37:14-79.

Jones, C. W. 1937. The ear muscles of the woodchuck. Journal of Mammalogy 18:517.

Larsell, O. 1953. The cerebellum of the cat and monkey. Journal of Comparative Neurology 99:135-199.

Meier, P. T. 1983. Relative brain size within the North American Sciuridae. Journal of Mammalogy 64:642-647.

Meinertz, T. 1943. Das superfizielle Fascialisgebiet der Nager. 1V. Die Sciuriden. 2. Marmota marmota (L). Zeitschrift fur Anatomie und Entwichlungsgeschichte 112:350-381.

Miller, G. S., Jr., and R. Kellogg. 1955. List of North American Recent mammals. United States National Museum Bulletin 205.

Mossman, H. W., J. W. Lawlah, and J. A. Bradley. 1932. The male reproductive tract of the Sciuridae. American Journal of Anatomy 51:89-155.

Nomina Anatomica Veterinaria. 1994. $4^{\text {th }}$ edition. World Association of Veterinary Anatomists, Vienna, Austria.

Nuckle, J., and J. M. Bergeron. 1983. Eye lens weight as a criterion for age classification of wild woodchucks. Journal of Wildlife Management 47:846-850.

Panic, R., D. W. Scott, W. A. Anderson, and B. C. Tennant. 1992. Microscopic anatomy of the skin of the woodchuck (Marmota monax): comparison of woodchuck hepatitis virus-infected and noninfected animals. Cornell Veterinarian 82:387-404.

Pocock, R. I. 1923. The classification of Sciuridae. Proceedings of the Zoological Society of London 1:209-246.

Ramousse, R. 1997. Bibliographia marmotarum. International Marmot Network, Université Claude Bernard, Lyon, France.

Rasmussen, A. T. 1918. Cyclic changes in the interstitial cells of the ovary and testis in the woodchuck (Marmota monax). Endocrinology 2:353-403. 
Rausch, R. L., and J. G. Bridgens. 1989. Structure and function of sudoriferous facial glands in Nearctic marmots, Marmota spp. (Rodentia: Sciuridae). Zoologischer Anzeiger 223:265282.

Ryan, J. M. 1989. Comparative myology and phylogenetic systematics of the Heteromyidae (Mammalia, Rodentia). Miscellaneous Publications of the Museum of Zoology, University of Michigan 176:1-103.

Seton, E. T. 1929. Lives of game animals. Doubleday, Doran and Company, Inc., New York.

Smith, J. D., and G. W. Hearn. 1979. Ultrastructure of the apocrine-sebaceous anal scent gland of the woodchuck, Marmota monax: evidence for apocrine and merocrine secretiomn by a single cell type. Anatomical Record 193:269-292.

Snyder, R. L., D. E. Davis, and J. J. Christian. 1961. Seasonal changes in the weights of woodchucks. Journal of Mammalogy 42:297-312.

Stark, D., and H. Wehrli. 1935. Die Kaumuskulatur von Marmota marmota L. Zeitschrift für Saugertierkunde 10:33-38

Steppan, S. J., M. R. Akhverdyan, E. A. Lyapunova, D. G. Fraser, N. N. Vorontsov, R. S. Hoffmann, and M. J. Braun. 1999. Molecular phylogeny of the marmots (Rodentia: Sciuridae): tests of evolutionary and biogeographic hypotheses. Systematic Biology 48:715-734.

Summers, J., J. M. Smolec, and R. Snyder. 1978. A virus similar to human hepatitis B virus associated with hepatitis and hep- atomas in woodchucks. Proceedings of the National Academy of Science 75:4533-4537.

Tandler, J. 1899. Zur vergleichenden Anatomie der Kopfarterien bei den Mammalia. Denkschriften der Kaiserlichen Akaemie der Wissenschaften, Mathematisch-Naturwissenschaftliche Classe 67:667-784.

Thorington, R. W., Jr., and K. Darrow. 1996. Jaw muscles of old world squirrels. Journal of Morphology 230:145-165.

Tuckerman, F. 1889. On the gustatory organs of Arctomys monax. Anatomischer Anzeiger 4:334-335.

Wahlert, J. H. 1974. The cranial foramina of protrogomorphous rodents: an anatomical and phylogenetic study. Bulletin of the Museum of Comparative Zoology, Harvard University 146:363-410.

Walro, J. M., P. T. Meier, and G. E. Svendson. 1983. Anatomy and histology of the scent glands associated with the oral angle in woodchucks. Journal of Mammalogy 64:701-703.

Wehrli, H. 1935. Zur Osteologie der Gattung Marmota Blumenb.(Arctomys Schreb.). Zeitschrift für Saugetierekunde 10: $1-32$.

Whittaker, J. O., Jr., and W. J. Hamilton, Jr. 1998. Mammals of eastern United States. 3rd edition. Comstock Publishing Associates, Cornell University, Ithaca, New York.

Wood, A. E. 1969. The third upper premolar of the woodchuck (Marmota). Journal of Mammalogy 50:608-609.

Young, R., and E. Sims. 1979. The woodchuck, Marmota monax, as a laboratory animal. Laboratory Animal Science 29:770780. 


\section{Index}

Abdomen, 71, 86

Abdomen, figure of, 92, 93, 94

Abdomen, nerves, 168

Abducent nerve, 157

Accessory nerve, 161

Adrenal glands, 118

Alarm calls, 4

Alimentary canal, 87

Alveolar process, 20, 21

Anatomical terms, 7

Anatomy, heart, 119

Ankle, 77

Antebrachium muscles, 61

Aorta, 123

Appendicular skeleton, 27

Arterial system, 123

Arteries, 124, 125

Atlas, 23

Atria, 119

Autonomic nervous system, 167

Axial skeleton, 11

Axis, 24

Basal nuclei, 150

Basisphenoid bone, 18

Bifula, 36

Bile ducts, 97

Bladder, 109

Bladder, figure of, 111

Blood vessels, figure of, 133, 134, 135

Bone, cranium, 11

Brachial plexus, 162

Brachial plexus, figure of, 159

Brain, 145

Brain, arteries, 127

Brain, figure of, 134, 146, 147, 148, 149, 153

Brain, subdivision, 149

Breeding characteristics, 3

Brown fat, ix

Cancer, liver, ix

Cardiac veins, 132

Cardiovascular system, 119

Carpal joint, 41

Carpus, 30

Carpus extensors, 61

Carpus flexors, 61

Caudal constrictors, 54

Caudal vertebrae, 26

Central nervous system, 145

Cerebellum, 152
Cerebral cortex, 149

Cerebrospinal fluid, 153

Cerebrum, 149

Cervical plexus, 162

Cervical vertebrae, 24

Cheek, 45, 81

Chest, 104

Chest, figure of, 105

Classification of, 1

Clavicle, figure of, 30

Clavicula, 28

Clavicular joints, 40

Clavicular ligaments, 40

Claws, 9

Coat, 8

Cochlear nerve, 160

Colon, 91, 92

Condyle, 29

Cornell University, ix

Costovertebral ligaments, 40

Cranial ligaments, 43

Cranial nerve, 155

Cranial veins, 132

Cranium, 11, 43

Crus, 77

Deep veins, 138

Den, 4

Description, 3

Diaphragm, 71, 72

Diencephalons, 151

Digestive system, 81

Digit extensors, 62, 78

Digit flexors, 62

Digits, 32

Dilator, pharynx, 55

Directional terms, 7

Directional terms, figure, 8

Distribution, 3, 4

Distribution, figure of, 3

Drainage, nervous system, 137

Duodenum, 90

Ear, 48, 49, 172

Elbow extensors, 61

Elbow flexors, 60

Elbow joint, 40

Elbow muscles, 60

Encephalon, 145

Endocrine organs, 118

Enemies, 5 
Epaxial muscles, 66

Epithelium, 81

Esophagus, 104

Ethmoid bone, 19

Extensors, ankle, 77

Extensors, carpus, 61

Extensors, digit, 62, 78

Extensors, elbow, 61

Eye, 49,170

Eyelids, 48

Facial bones, 20

Facial nerve, 157

Farm pest, 5

Feet, 9, 78

Female genital organs, 115

Femur, figure of, 35

Fiber tracts, 150

Fibula, figure of, 37

Flexors, ankle, 77

Flexors, carpus, 61

Flexors, digit, 62, 78

Flexors, elbow, 60

Food, 5

Foot pads, 9

Forepaw, figure of, 33, 34

Fourth ventricle, 152

Frontal bone, 18

Fur, 8

Gall bladder, 97

Garden pest, 5

Genital glands, 115

Genital organs, 111, 115

Genitals, figure of, 112, 113, 114

Glands, 9

Glands, adrenal, 118

Glands, genital, 115

Glands, mammary, 10

Glands, parathyroid, 118

Glands, pineal, 118

Glands, salivary, 85

Glands, thyroid, 118

Glosspharyngeal nerve, 160

Habitat, 4

Habits, 4

Hair, 8

HBV, ix, $\mathrm{x}$

Head muscles, 45

Head, 45

Head, arteries, 125

Head, figure of, 48, 54, 82, 83, 85, 101, 133, 157

Head, nerves, 168

Head, veins, 132

Hearing, 172

Heart, 119

Heart, figure of, 106, 121, 122

Hepatitis $\mathrm{B}$ virus, $\mathrm{ix}, \mathrm{x}$

Hibernation, ix, 3, 7
Hind paw, figure of, 37, 38

Hip, 74

History of, 1

Human relations, 5

Humerus, 28

Humerus, figure of, 31

Hyoid apparatus, 23, 39, 52

Hyoid apparatus, figure of, 23

Hypaxial trunk muscles, 69

Hypoglossal nerve, 161

Hypophysis, 118

Ileum, 91

Incisive bone, 21

Integument, 8

Intestines, 90

Intestines, figure of, 90, 92, 93, 94

Ischium, 34

Jaw, figure of, $6,20,21,22$

Jaws, 50, 51

Jejunum, 91

Joints, 39

Kidney, 108

Kidney, figure of, 109

Knee joint, 42

Lacrimal bone, 20

Large intestines, 91

Larynx, 55, 102, 103

Larynx, figure of, 102, 103

Lateral ventricles, 151

Latissimus dorsi, 56

Leg joints, 43

Leg, figure of, $35,36,37,57,59$

Ligaments, 39

Ligaments, liver, 96

Ligaments, metacarpal, 41

Ligaments, phalangeal joints, 42

Ligaments, radioulnar joints, 41

Limb of pelvis, figure of, 68

Lips, 45, 81

Litters, 7

Liver cancer, ix

Liver, 93-95

Liver, figure of, 96, 97, 99

Liver, ligaments, 96

Lobes, liver, 95

Lubosacral plexus, 159

Lumbar vertebrae, 25

Lumbosacral plexus, 164

Lungs, 105

Lungs, figure of, 106

Lymph nodes, figure of, 136

Lymphatic system, 141

Male genital organs, 111

Mammary glands, 10

Mandible, 21

Mandible, figure of, 21, 22

Mandibular muscle, 56 
Manus arteries, 129

Manus, figure of, 63, 64

Manus muscles, 63

Manus rotator, 63

Marmota, x

Mastication, 50

Mating, 4

Maxilla, 20

Maxillary bone, 20

Measurements, 5

Medulla spinalis, 153

Mesencephalon, 151

Metacarpal joints, 41

Metacarpus, 31

Midbrain, 151

Middle constrictor, 54

Mouth, 81

Mouth, figure of, 82,83

Muscles, abdomen, 71

Muscles, ankle, 77

Muscles, antebrachium, 61

Muscles, ear, 48, 49

Muscles, elbow, 60

Muscles, epaxial, 66

Muscles, eye, 49

Muscles, eyelids, 48

Muscles, head, figure of, 48

Muscles, hip, 74

Muscles, hyoid apparatus, 52

Muscles, jaws, 50, 51

Muscles, larynx, 55

Muscles, manus, 63

Muscles, neck, 69

Muscles, neck, figure of, 51, 53

Muscles, pes, 79

Muscles, shoulder, 60

Muscles, skin, 10

Muscles, soft palate, 54

Muscles, sublumbar, 73

Muscles, tail, 70

Muscles, thigh, 74, 75

Muscles, thoracic limb, 56

Muscles, thoracic wall, 70

Muscles, tongue, 52

Muscles, trunk, 65, 69

Muscles, trunk, figure of, 46, 47

Muscles, vertebra, 65

Muscular system, 45

Myelencephalon, 152

Nasal bone, 20

Nasal cavity, 100

National Cancer Institute, ix

National Institute of Allergy and Infectious Diseases, ix

Neck muscles, 45, 69

Neck muscles, figure of, 48, 51, 53

Neck, arteries, 125
Neck, figure of, 56, 51, 53, 133, 158

Neck, nerves, 168

Neck, veins, 132

Neocortex, 149

Nerve, abdomen, 168

Nerve, abducent, 157

Nerve, accessory, 161

Nerve, cochlear, 160

Nerve, cranial, 155

Nerve, facial, 157

Nerve, glosspharyngeal, 160

Nerve, head, 168

Nerve, hypoglossal, 161

Nerve, neck, 168

Nerve, oculomotor, 155

Nerve, olfactory, 155

Nerve, optic, 155

Nerve, parasympathetic, 167

Nerve, pelvic limb, 165

Nerve, pelvis, 164, 168

Nerve, spinal, 161

Nerve, sympathetic, 167

Nerve, thorax, 168

Nerve, trigeminal, 155

Nerve, trochlear, 155

Nerve, vagus, 160

Nerve, vestibulo-cochlear, 160

Nervous system, autonomic, 167

Nervous system, central, 145

Nervous system, peripheral, 154

Nose, 45,100

Number of species, 1

Occipital bone, 14

Oculomotor nerves, 155

Olfactory nerves, 155

Omotransversarius muscle, 58

Optic nerves, 155

Organs, endocrine, 118

Organs, sensory, 170

Palate, 81

Palatine bone, 21

Palatine process, 21

Paranasal sinuses, 100

Parasympathetic nerves, 167

Parathyroid glands, 118

Parietal bone, 15

Patella, 36

Paw, figure of, 9, 32, 33, 34, 37, 38, 63, 64

Paws, joints, 41

Pectoral muscle, 58

Pelvic arteries, 131

Pelvic canal, 34

Pelvic diaphragm, 72

Pelvic girdle, 32

Pelvic limb, 35, 42, 73

Pelvic limb, arteries, 131

Pelvic limb, figure of, 68, 73 
Pelvic limb, nerves, 165

Pelvic limb, veins, 140

Pelvic nerves, 164

Pelvis, 42

Pelvis, arteries, 130

Pelvis, figure of, 34,73

Pelvis, nerves, 168

Pericardium, 119

Perimeum, 72

Peripheral nervous system, 154

Peritoneum, 86

Pes, 79

Pes, arteries, 132

Phalangeal joints, 42

Phalanges, 32

Pharynx, 55, 101

Phylogeny, 1

Pineal glands, 118

Platysma, 45

Pleurae, 104

Plexus, brachial, 162

Plexus, cervical, 162

Plexus, lumbosacral, 164

Pons, 152

Population, 5

Presphenoid bone, 18

Pterygoid bone, 19

Pubis, 33

Pulmonary veins, 132

Radioulnar joints, 41

Radius, 29

Radius, figure of, 32

Relationship to humans, 5

Respiratory system, 100

Rhomboid muscle, 56

Rib, figure of, 28

Ribs, 27, 40

Rostral constrictors, 54

Rotator, manus, 63

Sacral vertebrae, 25

Sacrum, figure of, 26

Sagittal crest, 15

Salivary glands, 85

Scapula, 27

Scapula, figure of, 29

Sciuridae, $x$

Sensory organs, 170

Sesamoid bones, 32, 38

Shoulder, 27, 40, 60

Sight, 170

Sinuses, 100, 137

Size, 3

Skeleton, 11

Skeleton, figure of, 12

Skin, 8, 10

Skull, 11, 39

Skull, figure of, 12, 13. 14, 16, 17, 19, 20
Small intestines, 90

Soft palate, 54

Spermophilus, $\mathrm{x}$

Spinal column, 23, 39, 65

Spinal cord, 153

Spinal cord, arteries, 127

Spinal cord, figure of, 153, 154

Spinal nerves, 161

Spine, 145

Spleen, 143

Spleen, figure of, 89

Squamous, 16

Sternum, 26

Sternum, figure of, 27

Stomach, 87,88

Stomach, figure of, $87,88,89,99$

Subclavian muscle, 58

Sublumbar muscles, 73

Superficial veins, 138

Sympathetic nerves, 167

Tail, 70, 130

Talus, 36

Tarsal joints, 43

Teeth, 5, 6, 82, 83, 84

Teeth, figure of, 6

Temporal bone, 16

Territory, 5

Thigh, 74

Third ventracle, 151

Thoracic cavity, 104

Thoracic cavity, figure of, 120

Thoracic limb, 28, 40, 56, 138

Thoracic limb, figure of, 57, 59

Thoracic vertebrae, 24

Thoracic wall muscles, 70

Thorax nerves, 168

Thorax, figure of, 56, 66, 105, 106

Thymus, 143

Thyroid glands, 118

Thyroid, 103

Tibia, 36

Tibia, figure of, 36

Toe extensors, 62

Toe flexors, 62

Toes, 78

Tongue, 52, 81

Tongue, figure of, 84

Trachea, 103

Trachea, figure of, 102, 103

Trapezius muscle, 56

Trigeminal nerve, 155

Trochlear nerve, 155

Trunk, 65

Trunk, arteries, 127

Trunk muscles, 65, 69

Trunk muscles, figure of, 46, 47

Tympanic bone, 16, 17 
Ulna, 29

Ulna, figure of, 33

Ureter, 108

Urethra, 110

Urinary bladder, 109

Urinary bladder, figure of, 111

Urogenital apparatus, 108

Urogenital tract, 110

Vagus nerve, 160

Vascular system, 122

Veins, cardiac, 132

Veins, deep, 138

Veins, figure of, 136

Veins, nervous system, 137

Veins, pelvic limb, 140

Veins, pulmonary, 132

Veins, sinuses, 137
Veins, superficial, 138

Veins, thoracic limb, 138

Vena cava, 132

Venous system, 132

Ventral concha, 21

Ventricles, 121

Ventricles, lateral, 151

Ventriculus quartus, 152

Ventriculus tertius, 151

Vertebra, 23, 39

Vertebra, figures of, 24, 25, 26, 27

Vertebra, muscles, 65

Vestibulo-cochlear nerve, 160

Whiskers, 9

Woodchucks, figure of, 2

Zygomatic bone, 21

Zygomatic muscle, 45

Zygomatic process, 20 




\section{Special Publications of the American Society of Mammalogists}

If available, Nos. 1-11 Specially Priced

No. 1. The Natural History and Behavior of the California Sea Lion Richard S. Peterson and George A. Bartholomew. 1967. (out of print)

No. 2. Biology of Peromyscus (Rodentia)

John A. King, Editor. 1968. (hardback)

No. 3. The Life History and Ecology of the Gray Whale (Eschrichtius robustus) Dale W. Rice and Allen A. Wolman. 1971. (out of print)

No. 4. Population Ecology of the Little Brown Bat, Myotis lucifugus, in Indiana and North-central Kentucky

Dale W. Rice and Allen A. Wolman. 1971. (softcover)

No. 5. Ecology and Behavior of the Manatee (Trichechus manatus) in Florida Daniel S. Hartman. 1979. (out of print)

No. 6. Locomotor Morphology of the Vampire Bat, Desmodus rotundus J. Scott Altenbach. 1979. (hardback)

No. 7. Advances in the Study of Mammalian Behavior John F. Eisenberg and Devra G. Kleiman, Editors. 1983. (hardback)

No. 8. Biology of the New World Microtus Robert H. Tamarin, Editor. 1985. (hardback)

No. 9. Dispersal in Rodents: A Resident Fitness Hypothesis Paul K. Anderson. 1989. (hardback)

No. 10. Biology of the Heteromyidae Hugh H. Genoways and James H. Brown, Editors. 1993. (hardback)

No. 11. Seventy-five years of Mammalogy (1919-1994) Elmer C. Birney and Jerry R. Choate, Editors. 1994. (hardback)

No. 12. The Wild Mammals of Montana Kerry R. Foresman. 2001. \$27.95 (softcover)

No. 13. Anatomy of the Woodchuck (Marmota monax) A.J. Bezuidenhout and H.E. Evans. 2005. \$45.00 (softcover)

To purchase available Special Publications, please visit the American Society of Mammalogists' website at www.mammalsociety.org. 


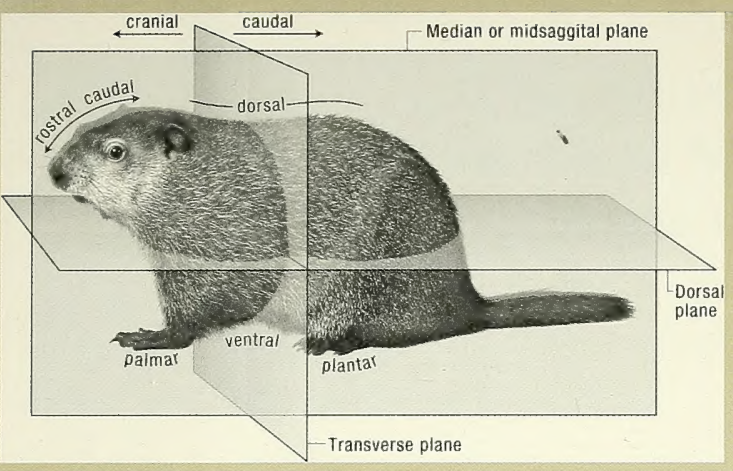

ANATomy of the Woodchu

100125828

the first such account on a species of increasing interest to mammalogists and scientists concerned with animal and human health.

The book is divided into 11 chapters and richly illustrated with numerous figures of remarkable detail and clarity. Dr. Bud C. Tennant, the James Law Professor of Comparative Medicine at Cornell University notes that "this volume represents a quantum advance in the anatomical information currently available on the woodchuck" and will be "a bridge between the biomedical research community and those studying ecology and evolution" of this interesting genus.

Abraham J. Bezuidenhout (left) is a graduate (BVSc) and a Doctor of Veterinary Science (DVSc) of the University of Pretoria, South Africa. He was in private veterinary practice for 5 years and on the Faculty of Veterinary Science in Anatomy at Onderstepoort Veterinary Institute, South Africa, for 19 years. In 1989, he was appointed Visiting Professor in the Department of Anatomy at Cornell University to study the anatomy of the woodchuck. Dr. Bezuidenhout is now a Senior Lecturer in the Department of Biomedical Sciences in the College of Veterinary Medicine at Cornell University. His publications include research on the anatomy of the ostrich and elephant and a book on the anatomy of the dromedary.

Howard E. Evans is an Emeritus Professor of Veterinary and Comparative Anatomy in the College of Veterinary Medicine at Cornell University where he has taught since 1950 . He continues to teach the "Literature of Natural History" and lead naturalhistory tours for Cornell University. His research has focused on the anatomy of fishes, reptiles, birds, and mammals. Professor Evans is the coauthor of "Anatomy of the Dog" and "Guide to the Dissection of the Dog," both of which are widely used in the veterinary profession.

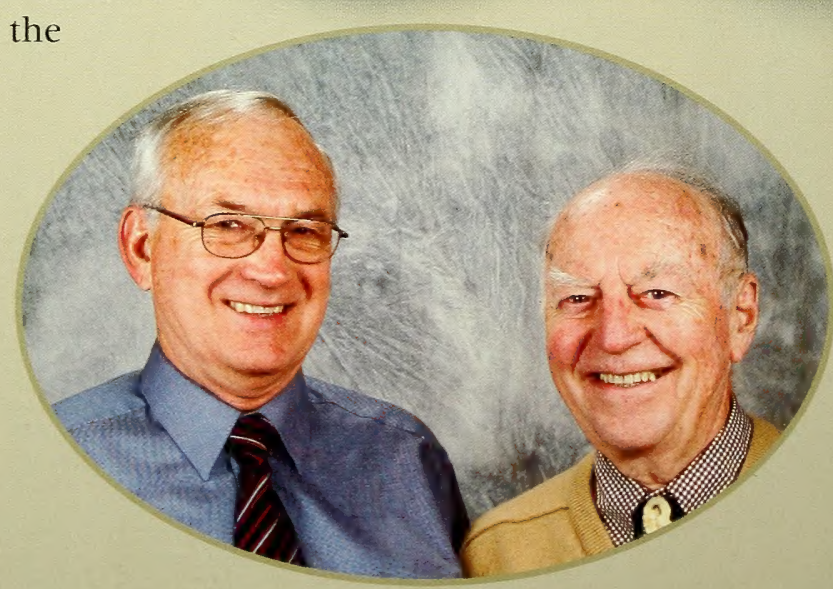

